U.S. GEOLOGICAL SURVEY URBAN-STORMWATER DATA BASE OF

CONSTITUENT STORM LOADS; CHARACTERISTICS OF RAINFALL, RUNOFF, AND ANTECEDENT CONDITIONS; AND BASIN CHARACTERISTICS

By Martha H. Mustard, Nancy E. Driver, John Chyr, and Brian G. Hansen

U.S. GEOLOGICAL SURVEY

Water Resources Investigations Report 87-4036

Denver, Colorado

1987 
DEPARTMENT OF THE INTERIOR

DONALD PAUL HODEL, Secretary

U.S. GEOLOGICAL SURVEY

Dallas L. Peck, Director

For additional information write to:

District Chief

U.S. Geological Survey

Water Resources Division

Box 25046, Mail Stop 415

Federal Center

Denver, CO 80225
Copies of this report can be purchased from:

U.S. Geological Survey

Books and Open-File Reports Section Federal Center

Box 25425

Denver, CO 80225 
ihstract-1

lntroduction-

Study areas-.....

Description of data base-1.

Constituent storm loads-_. 9

Characteristics of rainfall, runoff, and antecedent conditions-.... 10

Basin characteristics-_.

Quality assurance-1... 12

Constituent storm loads-1.

Characteristics of rainfall, runoff, and antecedent conditions-.... 13

Basin characteristics-1. 13

Magnetic-tape data storage and format_... 13

Constituent storm-loads files-1. 14

Files of characteristics of rainfall, runoff, and antecedent

conditions-...

Basin-characteristics files-1.

References cited-1. 20

Hydrologic data-..... 22

\section{FIGURES}

Figure 1. Page

Figure 1. Map showing locations of the urban-stormwater study areas----- 3

2. Example of a storm-loads file-_........ 16

3. Example of a file of characteristics of rainfall, runoff, and antecedent conditions-1... 18

4. Example of a basin-characteristics file-_... 19

\section{TABLES}

Table 1. Station numbers, station names, labels, and dataset names

by metropolitan area-1... 4

2-26. Constituent loads during storm-runoff periods at station:

2. 01589460 Hampden storm sewer at Baltimore, Md.-....- 23

3. 01589470 Reservoir Hill storm sewer at Baltimore, Md.--- 24

4. 01589475 Bolton Hill storm sewer at Baltimore, Md.-.-.- 25

5. 02306021 St. Louis Street drainage ditch at Tampa, Fla.- 26

6. 02307731 Allen Creek near Largo, Fla._-_.......... 26

7. 03226900 Fishinger Road Creek at Upper Arlington, Ohio-- 27

8. 03227050 Norman ditch at Chambers Circle

at Columbus, Ohio-..... 29

9. 04086941 Lincoln Creek at 54 th Street at Milwaukee, Wis. 30

10. 04086943 Lincoln Creek tributary at Milwaukee, Wis.---- 31

11. 04086945 Lincoln Creek tributary at Milwaukee, Wis.---- 32

12. 04087056 Tributary to Little Menomonee River tributary

at Milwaukee, Wis.... 33

13. 04087057 Little Menomonee River tributary

at Milwaukee, Wis.

14. 04087115 Honey Creek tributary at West Allis, Wis.-.-- 35 
Tables 2-26. Constituent loads during storm-runoff periods at station: --Continued

Table 15. 04087133 Menomonee River tributary at West Allis, Wis.-- 36

16. 04232040 Irondequoit Creek near Pittsford, N.Y.-...-.- 37

17. 04232046 Thomas Creek at Fairport, N.Y._-_... 38

18. 06412500 Rapid Creek above Canyon Lake near Rapid City, S. Dak.-... 39

19. 06413700 Rapid Creek above water-treatment plant at Rapid City, S. Dak.-...- 40

20. 06414000 Rapid Creek at Rapid City, S. Dak.-.....- 41

21. 06414700 Rapid Creek at East Main Street at Rapid City, S. Dak.-... 42

22. 06416000 Rapid Creek below Hawthorn ditch at Rapid City, S. Dak.- 43

23. 06710200 Big Dry Creek tributary at Littleton, Colo.---- 44

24. 06710225 Big Dry Creek tributary at Easter Street near Littleton, Colo.... 45

25. 06710610 Rooney Gulch at Rooney Ranch near Morrison, Colo.-...- 46

26. 06711586 Asbury Park storm drain at Asbury Avenue at Denver, Colo..-.... 47

27. Constituent loads during storm-runoff periods during 1976-77 at station 06711635 North Avenue storm drain at Denver Federal Center at Lakewood, Colo..-

28. Constituent loads during storm-runoff periods during 1980-81 at station 06711635 North Avenue storm drain at Denver Federal Center at Lakewood, Colo....

29-99. Constituent loads during storm-runoff periods at station:

29. 06711637 North Avenue storm drain at Denver

Federal Center North Avenue at Lakewood, Colo......- 50

30. 06713010 Cherry Knolls storm drain at Denver, Colo.-.-- 51

31. 06714100 36th Street storm drain at Denver, Colo.-.-.- 52

32. 06720330 Grange Hall Creek at Grant Park at Northglenn, Colo.... 52

33. 06720415 Grange Hall Creek at Northglenn, Colo.-.....- 53

34. 06720420 Storm drain at 116 th Avenue and Claude Court at Northglenn, Colo........ 54

35. 06893520 Blue River near Gregory Boulevard at Kansas City, Mo.- 55

36. 06893566 Blue River at Coal Mine Road at Kansas City, Mo.-... 56

37. 06893575 Blue Ridge Mall storm sewer at Kansas City, Mo. 56

38. 08074400 Lazybrook Street storm sewer at Houston, Tex.-- 57

39. 08155300 Barton Creek at Loop 360 at Austin, Tex.-...- 57

40. 08156800 Shoal Creek at West 12 th Street at Austin, Tex. 58

41. 08329900 North floodway channel near Alameda, N. Mex.--- 59

42. 10167220 Bells Canyon conduit at 1000 East and 11000 South at Sandy, Utah- 60

43. 10168000 Little Cottonwood Creek at Jordan River near Salt Lake City, Utah- 
-499 . Cinstituent loals during storm-runoff periods at station:

- - Cont inued

lible 44. 10168840 Holladay drain at 4800 South

Page at Big Cottonwood Creek near Murray, Utah--.-.-.-.-- 63

45. 10169500 Big Cottonwood Creek at Jordan River near Salt Lake City, Utah----

46. $1017090021 \mathrm{st}$ South conduit at Jordan River

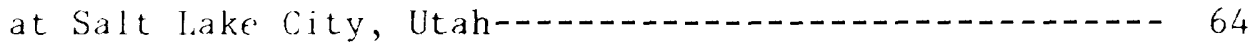

47. 10172372 8th South middle conduit at Jordan River at Salt Lake City, Utah- 65

48. 10172373 8th South north conduit at Jordan River at Salt Lake City, Utah-- 66

49. 10172520 North Temple conduit at Jordan River at Salt Lake City, Utah-..... 67

50. 12119725 Lake Hills storm-sewer outfall at Bellevue, Wash.-. 68

51. 12119730148 th Avenue Southeast storm sewer below Lake $\mathrm{Hill}$ ls Boulevard at Bellevue, Wash........ 70

52. 12120005 Surrey Downs storm-sewer outfall at Bellevue, Wash............ 72

53. 14206330 Beaverton Creek tributary at Southwest Murray Boulevard in Beaverton, Oreg.---- 74

54. 14206900 Fanno Creek at Southwest 56th Avenue in Portland, Oreg. 75

55. 14211120 Willamette River tributary on Southeast River Road in Oak Grove, Oreg.-....... 76

56. 14211301 Tryon Creek tributary at Dolph Court at Portland, Oreg.

57. 15274820 South Branch South Fork Chester Creek tributary near Baxter Road at Anchorage, Alaska------- 78

58. 15275035 North Fork Chester Creek tributary near 20th Avenue at Anchorage, Alaska-_........ 80

59. 15275055 Chester Creek tributary near 36th Avenue at Anchorage, Alaska-- 81

60. 212553158011000 Drain B in Mililani, Hawaii-_-_-_---- 82

61. 212604158012700 Drain A in Mililani, Hawaii-_........ 82

62. 254031080191100 Apartment site at South Miami, Fla.---- 83

63. 261002080070100 Commercial basin at Fort Lauderdale, Fla. 84

64. 261615080055900 Residential area at Pompano Beach, F1a.- 86

65. 261629080072400 Highway basin near Pompano Beach, Fla.-- 88

66. 364155119445000 Industrial urban-runoff site at Fresno, Calif.... 90

67. 364746119445400 Single-dwelling residential urbanrunoff site at Fresno, Calif.-_..- 91

68. 364818119443800 Multi-dwelling residential urbanrunoff site at Fresno, Calif._. 92

69. 364818119464700 Commercial urban-runoff site at Fresno, Calif.

70. 394236105042400 Villa Italia storm drain at Lakewood, Colo... 96 
Tables 29-99. Constituent loads during storm-runoff periods at station:

--Continued

Table

71. 394322105073601 Denver Federal Center rainfa11-

Page runoff simulation plot 1 at Lakewood, Colo........ 98

72. 394322105073602 Denver Federal Center rainfal1runoff simulation plot 2 at Lakewood, Colo......... 98

73. 394322105073603 Denver Federal Center rainfa11runoff simulation plot 3 at Lakewood, Colo.........- 99

74. 394322105073604 Denver Federal Center rainfal1runoff simulation plot 4 at Lakewood, Colo.-.....-- 99

75. 394322105073605 Denver Federal Center rainfa11runoff simulation plot 5 at Lakewood, Colo.-...-.- 100

76. 394322105073606 Denver Federal Center rainfa11runoff simulation plot 6 at Lakewood, Colo........- 100

77. 394322105073607 Denver Federal Center rainfallrunoff simulation plot 7 at Lakewood, Colo._-..-.-- 101

78. 394322105073608 Denver Federal Center rainfallrunoff simulation plot 8 at Lakewood, Colo.-...-.--- 101

79. 394322105073609 Denver Federal Center rainfallrunoff simulation plot 9 at Lakewood, Colo.-.-.-.- 102

80. 4046531115458019 th West conduit at 536 North at Salt Lake City, Utah--103

81. 404713073273001 Recharge basin at Plainview, N.Y.------ 104

82. 404815073294601 Recharge basin at Syosset, N.Y.-.-.-- 105

83. 405124073292601 Recharge basin at Laurel Hollow, N.Y.--- 106

84. 405135073055101 Recharge basin at Centereach, N.Y.----- 107

85. 415302088033804 Main inlet of Lake Ellyn

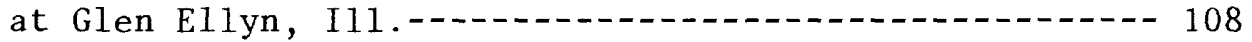

86. 415311088032906 Submerged outlet of Lake Ellyn at Glen Ellyn, Il1.-... 109

87. 415311088032907 Surface outlet of Lake Ellyn at Glen Ellyn, Ill.-...- 110

88. 430403077311500 Tributary to Barge Canal tributary near Pittsford, N.Y.

89. 430428077261100 White Brook tributary near Fairport, N.Y.-- 112

90. 430649077285500 Irondequoit Creek tributary at East Rochester, N.Y.-.-113

91. 445032092552801 Iverson Avenue storm sewer in Cottage Grove, Minn.-... 114

92. 445210093271701 Valley View Road storm sewer in Eden Prairie, Minn.--16

93. 445937093230701 Wesley Park storm sewer in Golden Valley, Minn.-... 118

94. 450011093221901 Sandberg Avenue storm sewer in Golden Valley, Minn.-... 121

95. 450100093205501 State Highway 100 at Scott Avenue

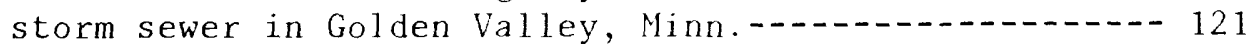

96. 450541093201201 Estates Drive storm sewer in Brooklyn Park, Minn...... 123

97. 450545093211501 Yates Avenue storm sewer in Brooklyn Park, Minn 
Tables 29-99. Constituent loads during storm-runoff periods at station: --Continued

Table 98. 473542122083001 148th Avenue Southeast detention-

basin number 3 outlet at Bellevue, Wash........... 126

99. 473544122083001 148th Avenue Southeast detention-

basin number 5 outlet at Bellevue, Wash.-.- 127

100-123. Characteristics of rainfall, runoff, and antecendent

conditions at station:

100. 01589460 Hampden storm sewer at Baltimore, Md.---1---- 128

101. 01589470 Reservoir Hill storm sewer at Baltimore, Md.--- 129

102. 01589475 Bolton Hill storm sewer at Baltimore, Md.----- 130

103. 02306021 St. Louis Street drainage ditch at Tampa, Fla.- 131

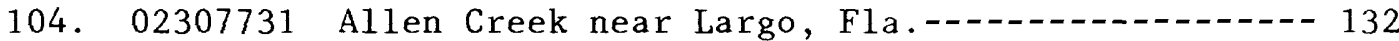

105. 03226900 Fishinger Road Creek at Upper Arlington, Ohio-- 133

106. 03227050 Norman ditch at Chambers Circle

at Columbus, Ohio--n 135

107. 04086941 Lincoln Creek at 54th Street at Milwaukee, Wis. 136

108. 04086943 Lincoln Creek tributary at Milwaukee, Wis.----- 137

109. 04086945 Lincoln Creek tributary at Milwaukee, Wis.---- 138

110. 04087056 Tributary to Little Menomonee River tributary

at Milwaukee, Wis.-- 139

111. 04087057 Little Menomonee River tributary

at Milwaukee, Wis.-n 140

112. 04087115 Honey Creek tributary at West Allis, Wis.----- 141

113. 04087133 Menomonee River tributary at West Allis, Wis.-- 142

114. 04232040 Irondequoit Creek near Pittsford, N.Y.------- 143

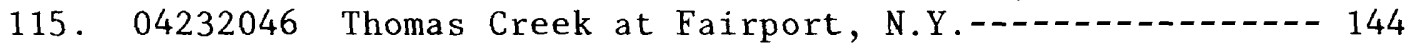

116. 06413700 Rapid Creek above water-treatment plant

at Rapid City, S. Dak.-. 145

117. 06414000 Rapid Creek at Rapid City, S. Dak.--14--146

118. 06414700 Rapid Creek at East Main Street

at Rapid City, S. Dak.-147

119. 06416000 Rapid Creek below Hawthorn ditch at Rapid City, S. Dak.-148

120. 06710200 Big Dry Creek tributary at Littleton, Colo.--- 149

121. 06710225 Big Dry Creek tributary at Easter Street near Littleton, Colo.-... 150

122. 06710610 Rooney Gulch at Rooney Ranch near Morrison, Colo.--..- 151

123. 06711586 Asbury Park storm drain at Asbury Avenue at Denver, Colo.--1.-- 152

124. Characteristics of rainfall, runoff, and antecendent conditions during 1976-77 at station 06711635 North Avenue storm drain at Denver Federal Center at Lakewood, Colo.----- 152

125. Characteristics of rainfal1, runoff, and antecendent conditions during 1980-81 at station 06711635 North Avenue storm drain at Denver Federal Center at Lakewood, Colo.----- 153

126-196. Characteristics of rainfall, runoff, and antecendent conditions at station:

126. 06711637 North Avenue storm drain at Denver Federal

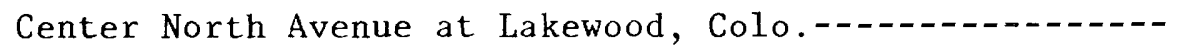


Tables 126-196. Characteristics of rainfall, runoff, and antecendent conditions at station:--Continued

Table

127. 06713010

Cherry Knolls storm drain at Denver, Colo

128. 06714100 36th Street storm drain at Denver, Colo.--.--- 157

129. 06720330 Grange Hall Creek at Grant Park

at Northglenn, Colo.-. 157

130. 06720415 Grange Hall Creek at Northglenn, Colo........- 158

131. 06720420 Storm drain at 116th Avenue and Claude Court at Northglenn, Colo..- 159

132. 06893520 Blue River near Gregory Boulevard at Kansas City, Mo.-. 160

133. 06893566 Blue River at Coal Mine Road at Kansas City, Mo.... 161

134. 06893575 Blue Ridge Mall storm sewer at Kansas City, Mo.-... 162

135. 08074400 Lazybrook Street storm sewer at Houston, Tex.-- 162

136. 08155300 Barton Creek at Loop 360 at Austin, Tex.--.--- 163

137. 08156800 Shoal Creek at West 12th Street at Austin, Tex. 163

138. 08329900 North floodway channel near Alameda, N. Mex.--- 164

139. 10167220 Bells Canyon conduit at 1000 East and 11000 South at Sandy, Utah-165

140. 10168000 Little Cottonwood Creek at Jordan River near Salt Lake City, Utah-166

141. 10168840 Holladay drain at 4800 South at Big Cottonwood Creek near Murray, Utah-_-_-_- 167

142. 10169500 Big Cottonwood Creek at Jordan River near Salt Lake City, Utah-168

143. 1017090021 st South conduit at Jordan River at Salt Lake City, Utah-168

144. 10172372 8th South middle conduit at Jordan River at Salt Lake City, Utah-_.-169

145. 101723738 th South north conduit at Jordan River at Salt Lake City, Utah-10

146. 10172520 North Temple conduit at Jordan River at Salt Lake City, Utah-_...- 171

147. 12119725 Lake Hills storm-sewer outfall at Bellevue, Wash.-. 172

148. 12119730 148th Avenue Southeast storm sewer below Lake Hills Boulevard at Bellevue, Wash.-...-.- 174

149. 12120005 Surrey Downs storm-sewer outfall at Bellevue, Wash. 176

150. 14206330 Beaverton Creek tributary at Southwest Murray Boulevard in Beaverton, Oreg.---- 178

151. 14206900 Fanno Creek at Southwest 56th Avenue in Portland, Oreg. 179

152. 14211120 Willamette River tributary on Southeast River Road in Oak Grove, Oreg.-...... 180

153. 14211301 Tryon Creek tributary at Dolph Court at Portland, Oreg. 181

154. 15274820 South Branch South Fork Chester Creek tributary near Baxter Road at Anchorage, Alaska---.-- 182 
Tables 126-196. Characteristics of rainfall, runoff, and antecendent conditions at station:--Continued

Table 155. 15275035 North Fork Chester Creek tributary

near 20th Avenue at Anchorage, Alaska-_...- 184

156. 15275055 Chester Creek tributary near 36th Avenue

at Anchorage, Alaska-- 185

157. 212553158011000 Drain B in Mililani, Hawaii-_...... 186

158. 212604158012700 Drain A in Mililani, Hawaii-_._. 186

159. 254031080191100 Apartment site at South Miami, Fla.-.-- 187

160. 261002080070100 Commercial basin

at Fort Lauderdale, Fla.... 188

161. 261615080055900 Residential area at Pompano Beach, Fla.- 190

162. 261629080072400 Highway basin near Pompano Beach, Fla.-- 192

163. 364155119445000 Industrial urban-runoff site

at Fresno, Calif.... 194

164. 364746119445400 Single-dwelling residential urban-

runoff site at Fresno, Calif._._._.-. 195

165. 364818119443800 Multi-dwelling residential urban-

runoff site at Fresno, Calif._._._. 196

166. 364818119464700 Commercial urban-runoff site at Fresno, Calif.

167. 394236105042400 Villa Italia storm drain

at Lakewood, Colo.-... 200

168. 394322105073601 Denver Federal Center rainfal1-

runoff simulation plot 1 at Lakewood, Colo.---.----- 202

169. 394322105073602 Denver Federal Center rainfal1-

runoff simulation plot 2 at Lakewood, Colo.-.-.-.-- 202

170. 394322105073603 Denver Federal Center rainfal1-

runoff simulation plot 3 at Lakewood, Colo.-...... 203

171. 394322105073604 Denver Federal Center rainfal1-

runoff simulation plot 4 at Lakewood, Colo.--.-.-.-- 203

172. 394322105073605 Denver Federal Center rainfall-

runoff simulation plot 5 at Lakewood, Colo.-.-.-.-- 204

173. 394322105073606 Denver Federal Center rainfal1-

runoff simulation plot 6 at Lakewood, Colo.-.-.-.-- 204

174. 394322105073607 Denver Federal Center rainfal1-

runoff simulation plot 7 at Lakewood, Colo.---.-.--- 205

175. 394322105073608 Denver Federal Center rainfal1-

runoff simulation plot 8 at Lakewood, Colo.-...-.-. 205

176. 394322105073609 Denver Federal Center rainfallrunoff simulation plot 9 at Lakewood, Colo.-.-.-- 206

177. 4046531115458019 th West conduit at 536 North at Salt Lake City, Utah- 207

178. 404713073273001 Recharge basin at Plainview, N.Y.-.... 208

179. 404815073294601 Recharge basin at Syosset, N.Y.--..- 209

180. 405124073292601 Recharge basin at Laurel Hollow, N.Y.--- 210

181. 405135073055101 Recharge basin at Centereach, N.Y.----- 211

182. 415302088033804 Main inlet of Lake Ellyn at Glen Ellyn, Ill. 212

183. 415311088032906 Submerged outlet of Lake Ellyn at Glen Ellyn, Ill..... 213

184. 415311088032907 Surface outlet of Lake Ellyn at Glen Ellyn, Ill. 214 
Tables 126-196. Characteristics of rainfall, runoff, and antecendent conditions at station:--Continued

Table 185. 430403077311500 Tributary to Barge Canal tributary

near Pittsford, N.Y.--15

186. 430428077261100 White Brook tributary

near Fairport, N.Y.--16

187. 430649077285500 Irondequoit Creek tributary

at East Rochester, N.Y.-...- 217

188. 445032092552801 Iverson Avenue storm sewer

in Cottage Grove, Minn.--18

189. 445210093271701 Valley View Road storm sewer

in Eden Prairie, Minn.-- 220

190. 445937093230701 Wesley Park storm sewer

in Golden Valley, Minn.-- 222

191. 450011093221901 Sandberg Avenue storm sewer

in Golden Valley, Minn.--.- 223

192. 450100093205501 State Highway 100 at Scott Avenue storm sewer in Golden Valley, Minn.---.--- 225

193. 450541093201201 Estates Drive storm sewer

in Brooklyn Park, Minn.-- 227

194. 450545093211501 Yates Avenue storm sewer

in Brooklyn Park, Minn.--1 229

195. 473542122083001 148th Avenue Southeast detention-

basin number 3 outlet at Bellevue, Wash.-.-.-.-230

196. 473544122083001 148th Avenue Southeast detention-

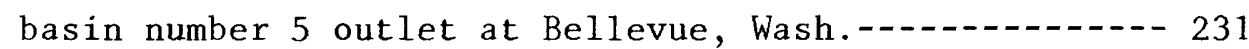

197-220. Basin characteristics of station:

197. 01589460 Hampden storm sewer at Baltimore, Md.-------- 232

198. 01589470 Reservoir Hill storm sewer at Baltimore, Md.--- 233

199. 01589475 Bolton Hill storm sewer at Baltimore, Md.----- 234

200. 02306021 St. Louis Street drainage ditch at Tampa, Fla.- 235

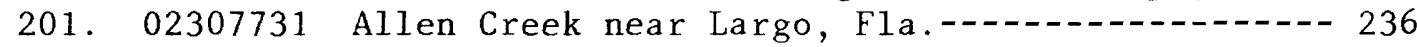

202. 03226900 Fishinger Road Creek at Upper Arlington, Ohio-- 237

203. 03227050 Norman ditch at Chambers Circle

at Columbus, Ohio-.... 238

204. 04086941 Lincoln Creek at 54 th Street

at Milwaukee, Wis.-- 239

205. 04086943 Lincoln Creek tributary at Milwaukee, Wis.---- 240

206. 04086945 Lincoln Creek tributary at Milwaukee, Wis.----- 241

207. 04087056 Tributary to Little Menomonee River tributary

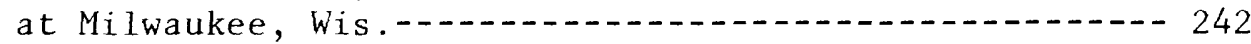

208. 04087057 Little Menomonee River tributary

at Milwaukee, Wis.

209. 04087115 Honey Creek tributary at West Allis, Wis.----- 244

210. 04087133 Menomonee River tributary at West Allis, Wis.-- 245

211. 04232040 Irondequoit Creek near Pittsford, N.Y.-....-. 246

212. 04232046 Thomas Creek at Fairport, N.Y._...... 247

213. 06413700 Rapid Creek above water-treatment plant

at Rapid City, S. Dak. 248

214. 06414000 Rapid Creek at Rapid City, S. Dak.-............. 249

215. 06414700 Rapid Creek at East Main Street

at Rapid City, S. Dak. 250 
Tables 197-220.--Basin characteristics of station:--Continued

Table 216. 06416000 Rapid Creek below Hawthorn ditch

at Rapid City, S. Dak._. 251

217. 06710200 Big Dry Creek tributary at Littleton, Colo.---- 252

218. 06710225 Big Dry Creek tributary at Easter Street

near Littleton, Colo._. 253

219. 06710610 Rooney Gulch at Rooney Ranch

near Morrison, Colo.-_- 254

220. 06711586 Asbury Park storm drain

at Asbury Avenue at Denver, Colo... 255

221. Basin characteristics during 1976-77 of station 06711635

North Avenue storm drain at Denver Federal Center at

Lakewood, Colo.-. 256

222. Basin characteristics during 1980-81 of station 06711635

North Avenue storm drain at Denver Federal Center at

Lakewood, Colo.-. 257

223-293. Basin characteristics of station:

223. 06711637 North Avenue storm drain at Denver

Federal Center North Avenue at Lakewood, Colo-_-.-.-- 258

224. 06713010 Cherry Knolls storm drain at Denver, Colo.--.- 259

225. 06714100 36th Street storm drain at Denver, Colo.---.-- 260

226. 06720330 Grange Hall Creek at Grant Park

at Northglenn, Colo._. 261

227. 06720415 Grange Hall Creek at Northglenn, Colo.-.-.-.- 262

228. 06720420 Storm drain at 116th Avenue and Claude Court at Northglenn, Colo.

229. 06893520 Blue River near Gregory Boulevard

at Kansas City, Mo..- 264

230. 06893566 Blue River at Coal Mine Road

at Kansas City, Mo..-. 265

231. 06893575 Blue Ridge Mall storm sewer

at Kansas City, Mo. 266

232. 08074400 Lazybrook Street storm sewer at Houston, Tex.-- 267

233. 08155300 Barton Creek at Loop 360 at Austin, Tex.--.-. 268

234. 08156800 Shoal Creek at West 12 th Street

at Austin, Tex. 269

235. 08329900 North floodway channel near Alameda, N. Mex.--- 270

236. 10167220 Bells Canyon conduit at 1000 East and 11000 South at Sandy, Utah-1 271

237. 10168000 Little Cottonwood Creek at Jordan River near Salt Lake City, Utah- 272

238. 10168840 Holladay drain at 4800 South
at Big Cottonwood Creek near Murray, Utah- 273

239. 10169500 Big Cottonwood Creek at Jordan River near Salt Lake City, Utah-_ 274

240. 10170900 21st South conduit at Jordan River
at Salt Lake City, Utah- 275

241. 10172372 8th South middle conduit at Jordan River
at Salt Lake City, Utah- 276

242. 10172373 8th South north conduit at Jordan River
at Salt Lake City, Utah- 277

243. 10172520 North Temple conduit at Jordan River
at Salt Lake City, Utaho 278 
Tables 223-293. Basin characteristics of station:--Continued

Table 244. 12119725 Lake Hills storm-sewer outfall at Bellevue, Wash.-1 279

245. 12119730148 th Avenue Southeast storm sewer below Lake Hills Boulevard at Bellevue, Wash.-.-.- 280

246. 12120005 Surrey Downs storm-sewer outfall at Bellevue, Wash....... 281

247. 14206330 Beaverton Creek tributary at Southwest Murray Boulevard in Beaverton, Oreg.-..- 282

248. 14206900 Fanno Creek at Southwest 56th Avenue in Portland, Oreg. 283

249. 14211120 Willamette River tributary on Southeast River Road in Oak Grove, Oreg.-.. 284

250. 14211301 Tryon Creek tributary at Dolph Court at Portland, Oreg. 285

251. 15274820 South Branch South Fork Chester Creek tributary near Baxter Road at Anchorage, Alaska--.-.- 286

252. 15275035 North Fork Chester Creek tributary near 20th Avenue at Anchorage, Alaska-- 287

253. 15275055 Chester Creek tributary near 36th Avenue at Anchorage, Alaska-..... 288

254. 212553158011000 Drain B in Mililani, Hawaii-........ 289

255. 212604158012700 Drain A in Mililani, Hawaii-............. 290

256. 254031080191100 Apartment site at South Miami, Fla.---- 291

257. 261002080070100 Commercial basin at Fort Lauderdale, Fla..... 292

258. 261615080055900 Residential area at Pompano Beach, Fla.- 293

259. 261629080072400 Highway basin near Pompano Beach, Fla.-- 294

260. 364155119445000 Industrial urban-runoff site

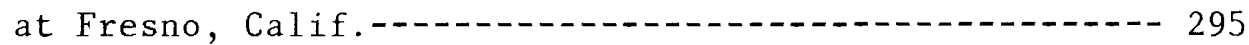

261. 364746119445400 Single-dwelling residential urban-

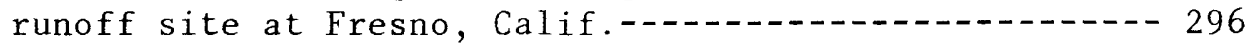

262. 364818119443800 Multi-dwelling residential urban-

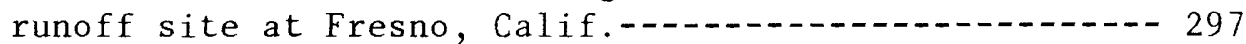

263. 364818119464700 Commercial urban-runoff site

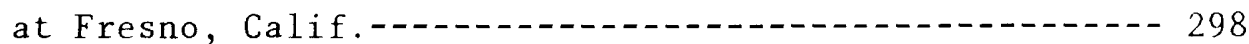

264. 394236105042400 Villa Italia storm drain

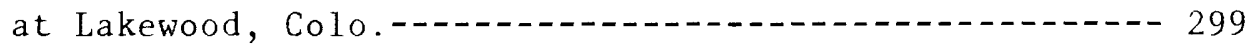

265. 394322105073601 Denver Federal Center rainfallrunoff simulation plot 1 at Lakewood, Colo.-.-.-.-.- 300

266. 394322105073602 Denver Federal Center rainfallrunoff simulation plot 2 at Lakewood, Colo.---.----- 301

267. 394322105073603 Denver Federal Center rainfallrunoff simulation plot 3 at Lakewood, Colo.--.-.-.-- 302

268. 394322105073604 Denver Federal Center rainfallrunoff simulation plot 4 at Lakewood, Colo.--1.-.-.- 303

269. 394322105073605 Denver Federal Center rainfallrunoff simulation plot 5 at Lakewool, Colo.-...... 304

270. 394322105073606 Denver Federal Center rainfallrunoff simulation plot 6 at Lakesood, Colo........ 305

271. 394322105073607 Denver Federal Center rainfallrunoff simulation plot 7 at lakewood, Colo..........306 
Tables 223-293. Basin characteristics of station:--Continued

Table 272. 394322105073608 Denver Federal Center rainfallrunoff simulation plot 8 at Lakewood, Colo.-.....-- 307

273. 394322105073609 Denver Federal Center rainfallrunoff simulation plot 9 at Lakewood, Colo.-......- 308

274. 4046531115458019th West conduit at 536 North at Salt Lake City, Utah-... 309

275. 404713073273001 Recharge basin at Plainview, N.Y.-.-.- 310

276. 404815073294601 Recharge basin at Syosset, N.Y.-....- 311

277. 405124073292601 Recharge basin at Laurel Hollow, N.Y.-.- 312

278. 405135073055101 Recharge basin at Centereach, N.Y.---.- 313

279. 415302088033804 Main inlet of Lake Ellyn at Glen E1lyn, I11... 314

280. 415311088032906 Submerged outlet of Lake Ellyn at Glen Ellyn, I11.... 315

281. 415311088032907 Surface outlet of Lake Ellyn at Glen Ellyn, Ill. 316

282. 430403077311500 Tributary to Barge Canal tributary near Pittsford, N.Y.

283. 430428077261100 White Brook tributary near Fairport, N.Y. 318

284. 430649077285500 Irondequoit Creek tributary at East Rochester, N.Y. 319

285. 445032092552801 Iverson Avenue storm sewer in Cottage Grove, Minn. 320

286. 445210093271701 Valley View Road storm sewer in Eden Prairie, Minn.... 321

287. 445937093230701 Wesley Park storm sewer in Golden Valley, Minn. 322

288. 450011093221901 Sandberg Avenue storm sewer in Golden Valley, Minn.-... 323

289. 450100093205501 State Highway 100 at Scott Avenue storm sewer in Golden Valley, Minn.-... 324

290. 450541093201201 Estates Drive storm sewer in Brooklyn Park, Minn. 325

291. 450545093211501 Yates Avenue storm sewer in Brooklyn Park, Minn. 326

292. 473542122083001 148th Avenue Southeast detentionbasin number 3 outlet at Bellevue, Wash..... 327

293. 473544122083001 148th Avenue Southeast detentionbasin number 5 outlet at Bellevue, Wash... 328 
For the convenience of readers who prefer metric (International System) units rather than the inch-pound units used in this report, the following factors may be used:

To convert inch-pound unit

$\begin{array}{cc}\text { Multiply by } & \text { To obtain metric unit } \\ 0.4047 & \text { hectare } \\ 1,233 & \text { cubic meter } \\ 0.02832 & \text { cubic meter per } \\ & \text { second } \\ 25.40 & \text { millimeter } \\ 25.40 & \text { millimeter per hour } \\ 0.1894 & \text { meter per kilometer } \\ 0.4536 & \text { kilogram } \\ 2.590 & \text { square kilometer }\end{array}$

acre

acre-foot

cubic foot per second

inch

inch per hour

foot per mile

pound

square mile
2.590
25.40

25.40

0.1894 hectare

cubic meter

cubic meter per

$$
\text { second }
$$

millimeter

millimeter per hour meter per kilometer square kilometer

To convert degree Fahrenheit to degree Celsius, use the following equation:

$$
{ }^{\circ} \mathrm{C}=5 / 9\left({ }^{\circ} \mathrm{F}-32\right) \text {. }
$$




\title{
U.S. GEOLOGICAL SURVEY URBAN-STORMWATER DATA BASE OF CONSTITUENT \\ STORM LOADS; CHARACTERISTICS OF RAINFALL, RUNOFF, AND \\ ANTECEDENT CONDITIONS; AND BASIN CHARACTERISTICS
}

By Martha H. Mustard, Nancy E. Driver, John Chyr, and Brian G. Hansen

\begin{abstract}
A data base which consists of constituent storm loads, characteristics of rainfall, runoff, and antecedent conditions, and basin characteristics measured at most of the stations in the U.S. Geological Survey urbanstormwater data collection network in metropolitan areas throughout the United States has been compiled. Tables of these constituent storm loads and characteristics are presented in this report. The data base also is available on magnetic tape.

The data represent 1,144 storms at 97 stations in 21 metropolitan areas . Storm loads of 18 constituents and 15 characteristics of rainfall, runoff, and antecedent conditions are reported. Twenty-eight selected basin characteristics also are reported, including 11 categories of land use.
\end{abstract}

\section{INTRODUCTION}

The U.S. Geological Survey has been collecting urban rainfall, runoff, and water-quality data for several decades. Recently, data from the Survey's urban-stormwater data collection networks in 22 metropolitan areas throughout the United States were compiled. The data base includes concurrent time-series values of rainfall and discharge, water-quality analyses, and basin characteristics. The data base, which is described by Driver and others (1985), is available on magnetic tape.

The purpose of this report is to present storm-loads and characteristics data for most of the stations included in the Survey's urban-stormwater data base. These data can be used to perform statistical analyses of urbanstormwater loadings according to regional area, size of drainage area, land use of the drainage basin, and other categories. 
The constituents for which storm loads are given in this report are: chemical oxygen demand; solids, suspended; solids, settleable; nitrogen, total; ammonia, dissolved, as nitrogen; ammonia plus organic nitrogen, total, as nitrogen; nitrite plus nitrate, total, as nitrogen; phosphorus, total; phosphorus, dissolved; orthophosphate, dissolved, as phosphorus; chloride, dissolved; cadmium, total recoverable; copper, total recoverable; lead, total recoverable; zinc, total recoverable; fecal coliform; solids, dissolved; and sediment, suspended. All constituent storm loads are reported in pounds, except the loads of fecal coliform, which are reported in millions of colonies.

The characteristics of rainfall, runoff, and antecedent conditions included in this report are: total rainfa11, average for the drainage basin, in inches; maximum 5-minute rainfall rate, in inches per hour; maximum 15-minute rainfall rate, in inches per hour; maximum 60-minute rainfall rate, in inches per hour; number of preceding dry days; rainfall during the preceding day, in inches; rainfall during the preceding 3 days, in inches; rainfall during the preceding 7 days, in inches; runoff, in inches over the drainage basin; peak discharge, in cubic feet per second; base flow prior to the storm, in cubic feet per second; duration of runoff, in minutes; duration of rainfall, in minutes; time from beginning of rainfall to peak discharge, in minutes; and time since last street cleaning, in days.

The basin characteristics included in this report are: contributing drainage area, in acres; impervious area, in percentage of contributing drainage area; effective impervious area, in percentage of contributing drainage area; average basin slope, in feet per mile; main conveyance slope, in feet per mile; population density, in number of people per square mile; street density, in miles of lanes per square mile; 11 categories of land use in the contributing drainage basin, in percentage of the contributing drainage basin; detention storage, in acre-feet; percentage of contributing drainage area upstream from detention storage; percentage of contributing drainage area drained by a storm-sewer system; percentage of streets that have curb and gutter drainage; percentage of streets that have ditch and swale drainage; mean annual rainfall, in inches; 10-year 1-hour rainfall intensity, in inches per hour; mean minimum January temperature, in degrees Fahrenheit; 2-year 24-hour rainfall, in inches; and mean annual nitrogen precipitation load, in pounds of nitrogen per acre.

\section{STUDY AREAS}

Data in this report were collected at the areas shown in figure 1 . All stations included in the data base are listed alphabetically by State and metropolitan area in table 1 . Within a metropolitan area, the stations are listed numerically according to station number; all 8-digit station numbers precede all 15-digit station numbers. 


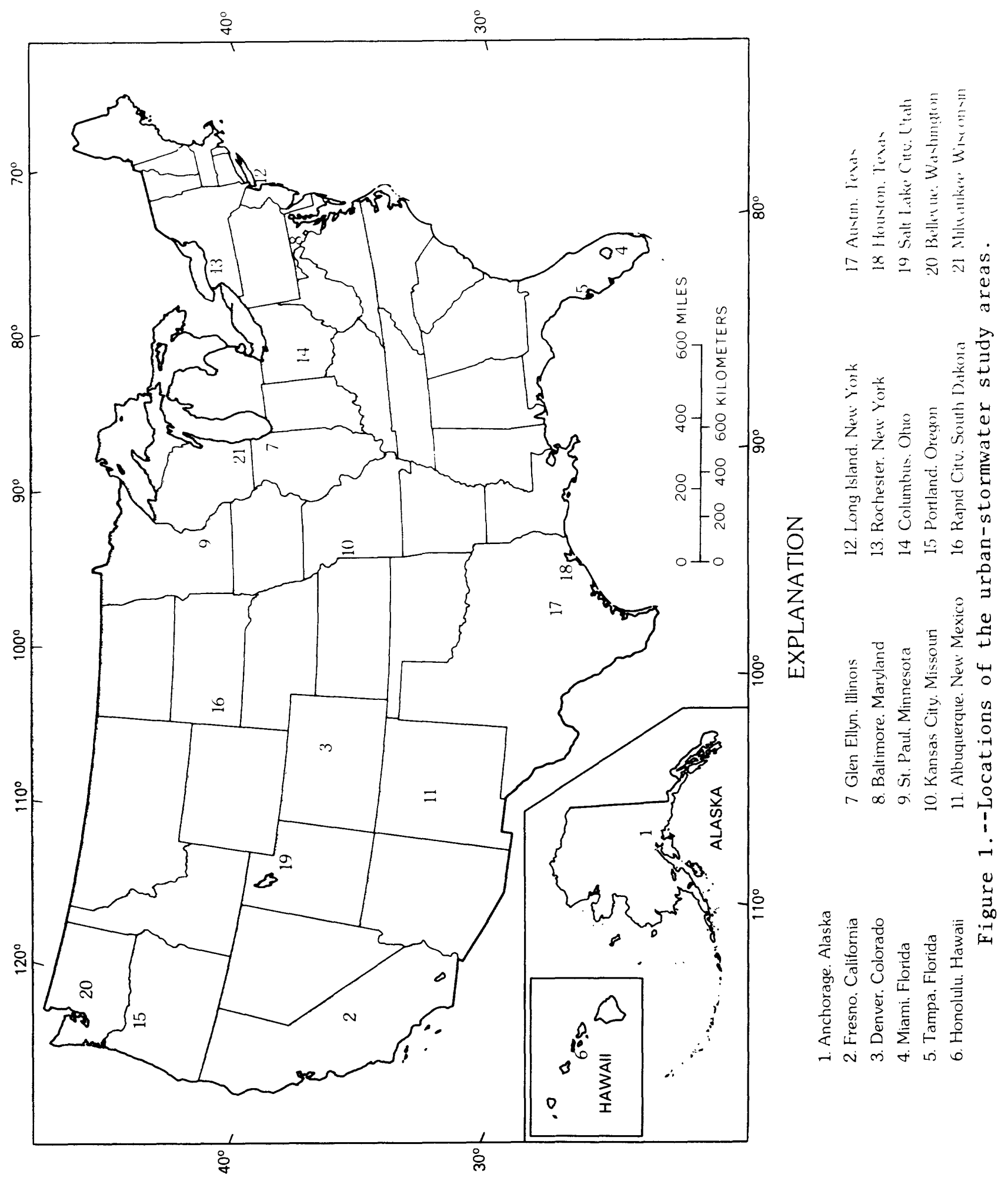




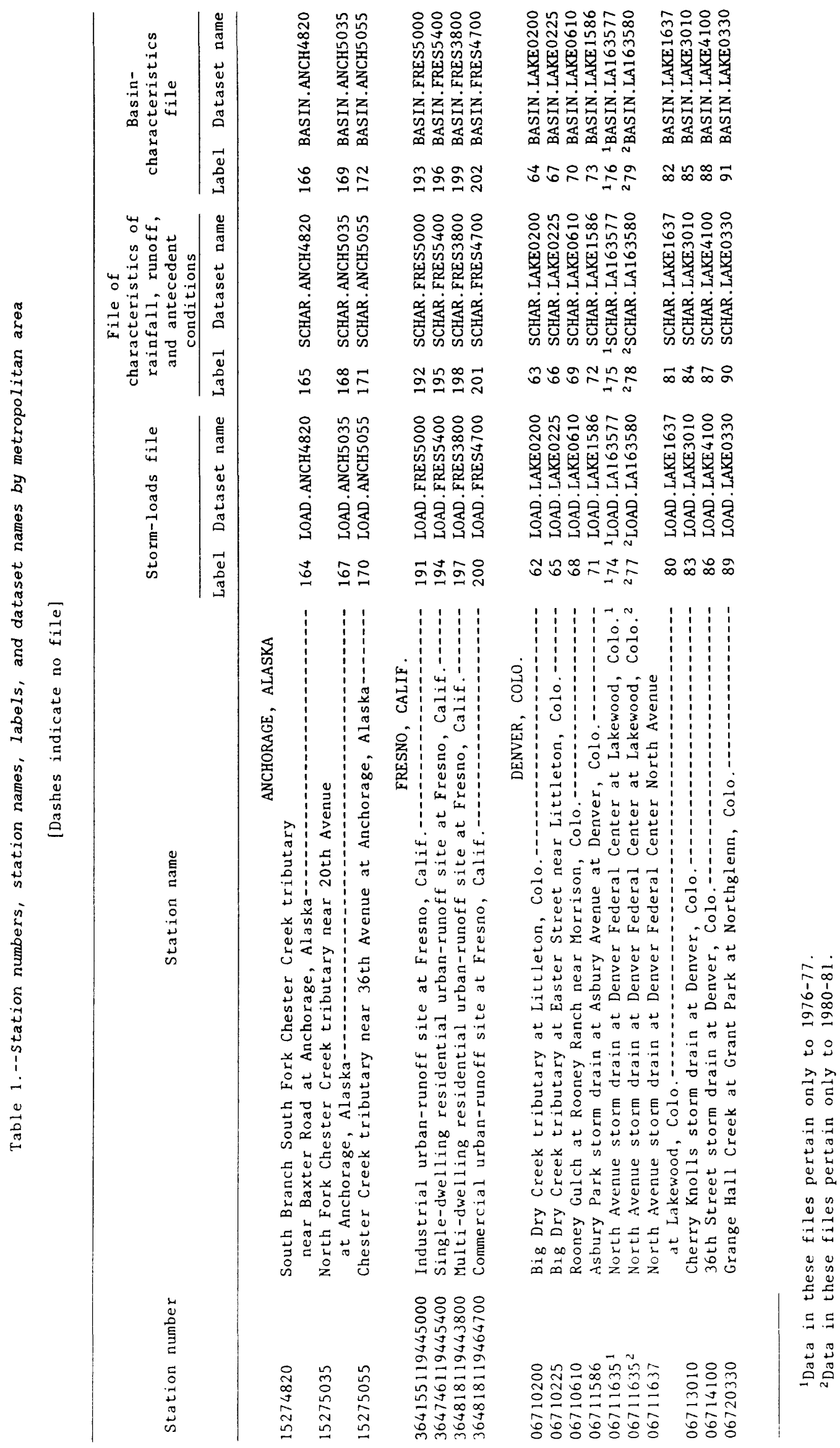




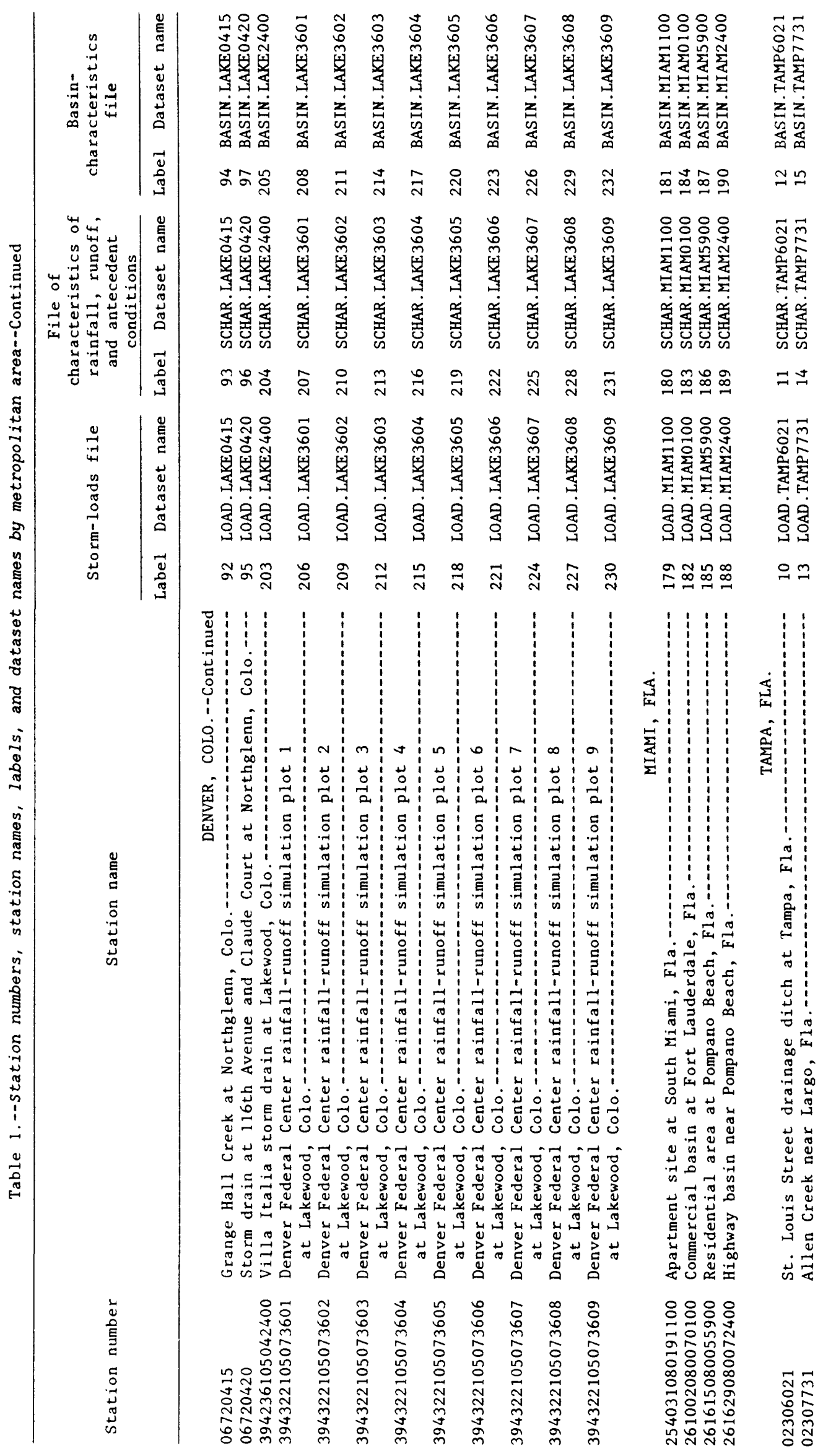




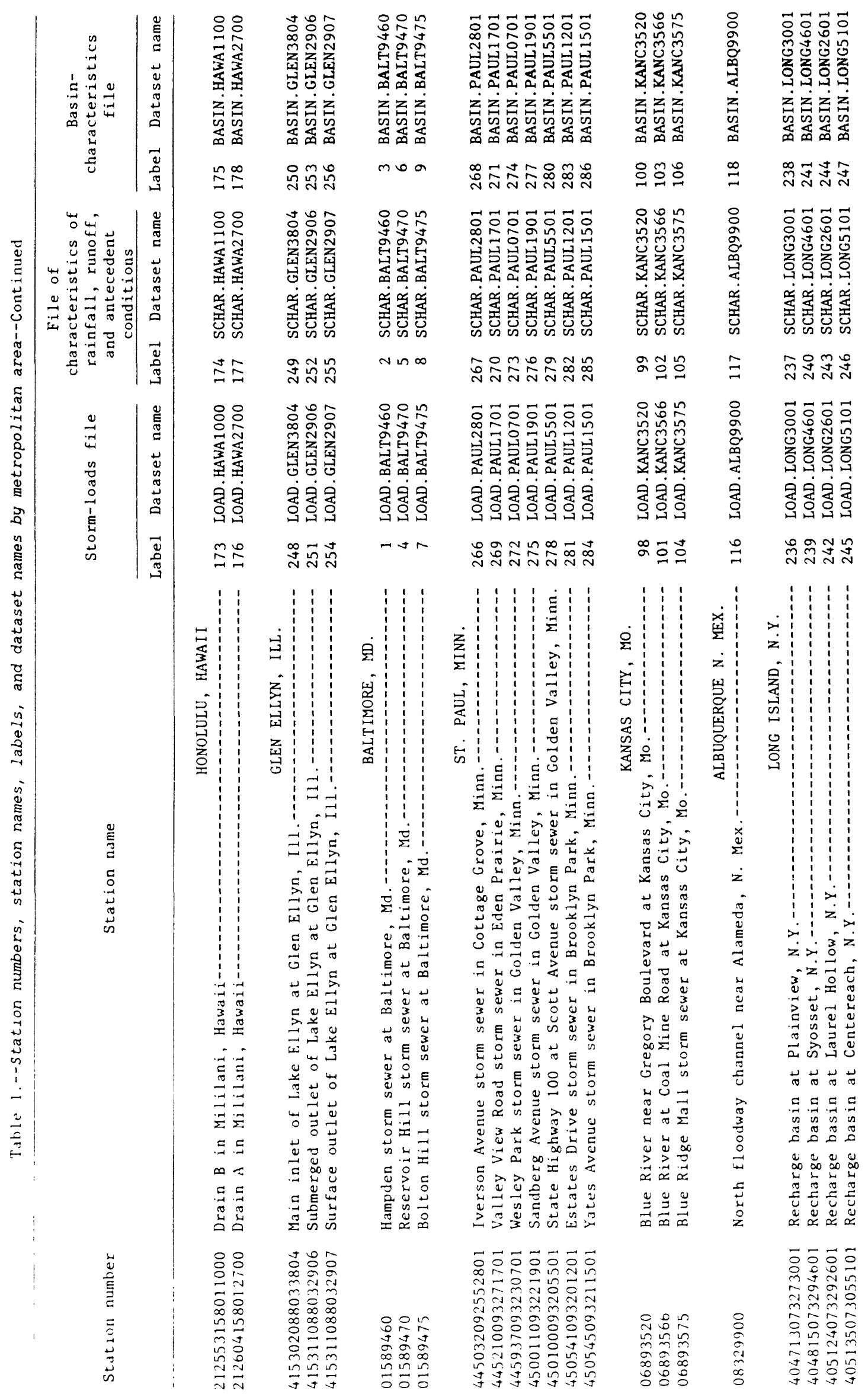




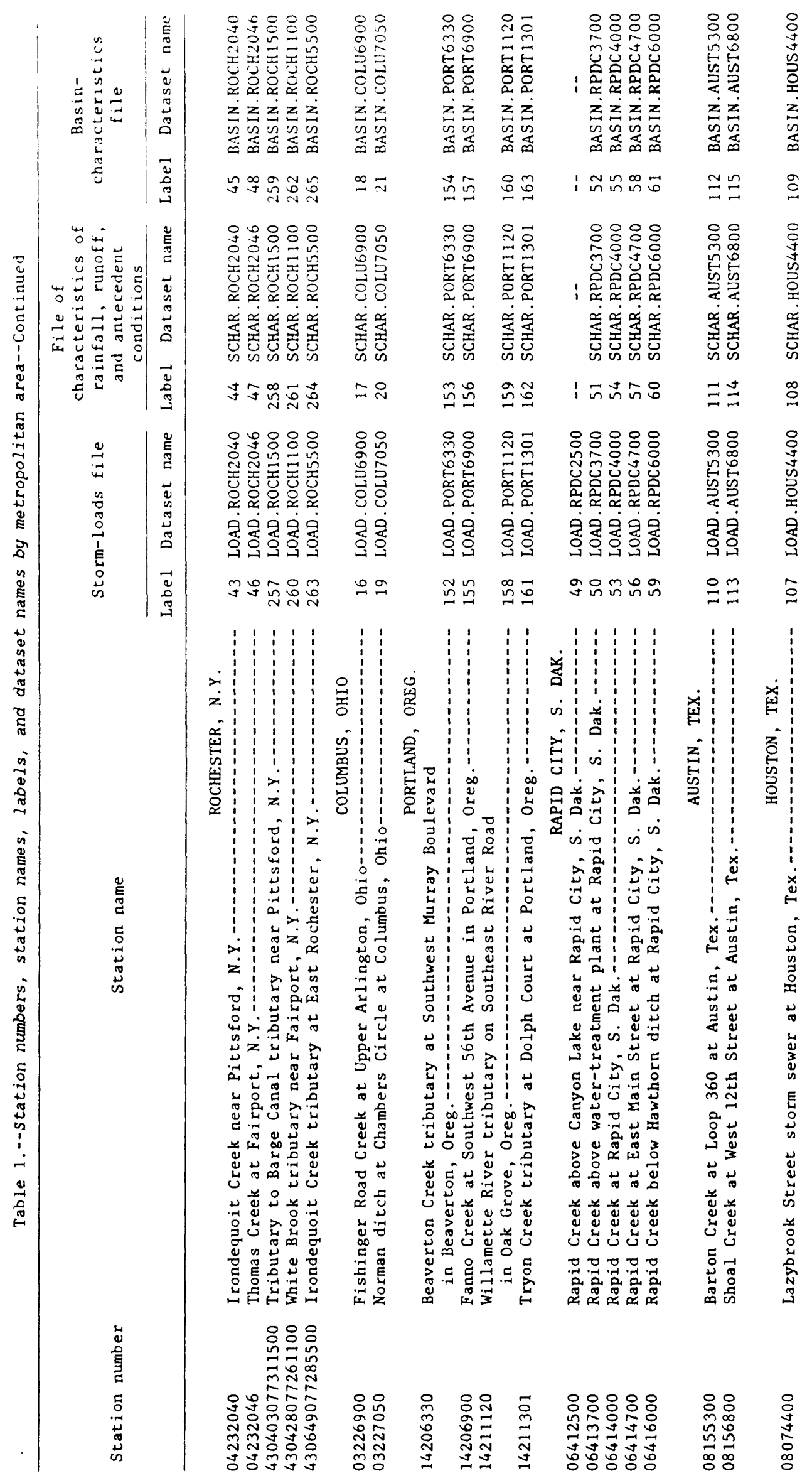




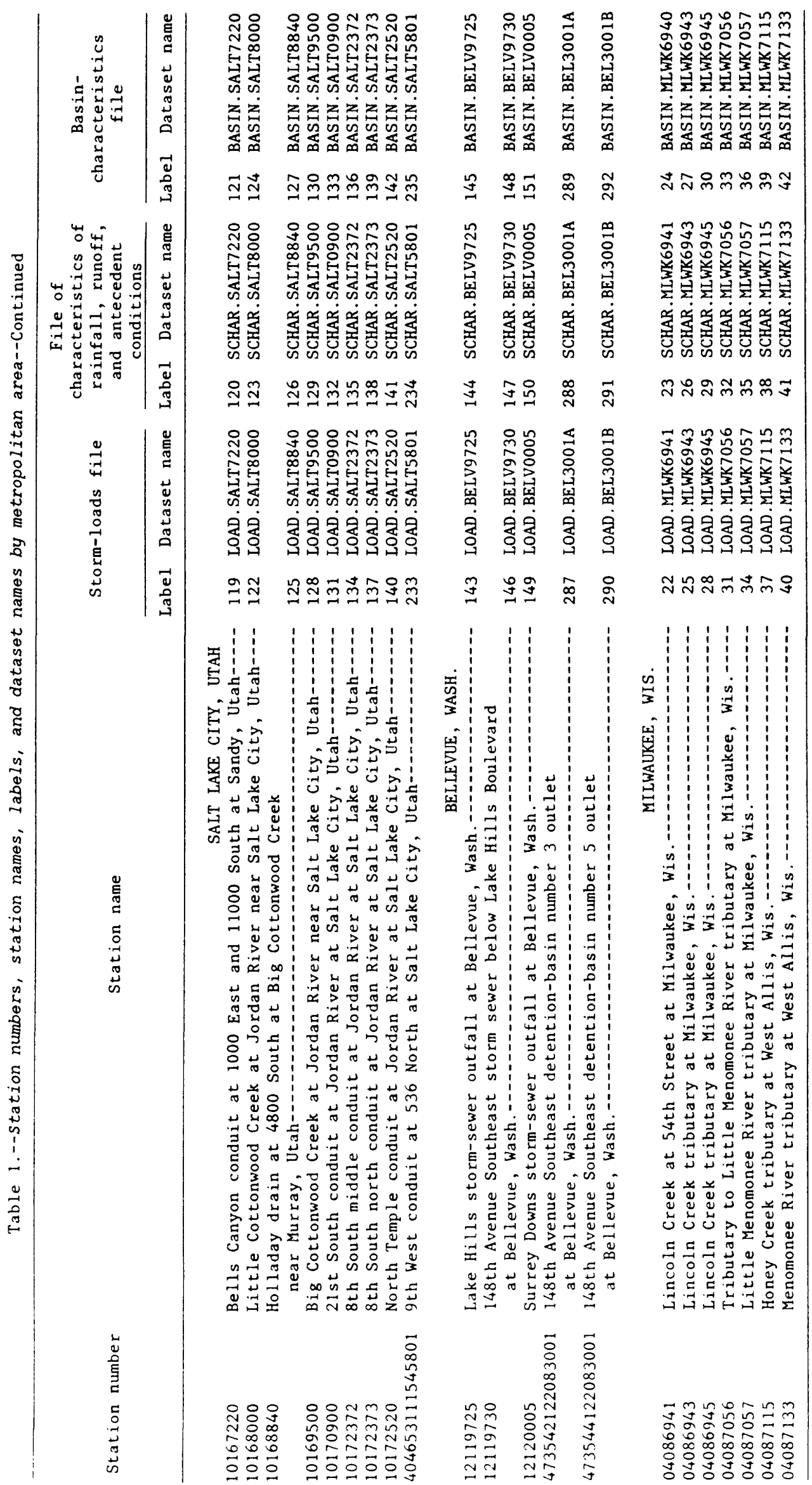


The constituent storm loads and the characteristics of rainfall, runoff, and antecedent conditions were compiled from two different sources. The first source vias U.S. Geological Survey project personnel involved in the data collection and interpretation for each study area. The second source was computer programs that calculated the values from the "U.S. Geological Survey urban-stormwater data base for 22 metropolitan areas throughout the United States" (Driver and others, 1985). Quality-assurance procedures then were performed on the data. All complete datasets that passed the qualityassurance procedures were retained; a complete dataset consists of corresponding quality-assured records of constituent storm loads and characteristics of rainfall, runoff, and antecedent conditions for a given storm from a common source. (See exception below in next paragraph.) Finally, duplicate datasets were eliminated; that is, if data from a given storm were reported from both sources, one of the pair was eliminated. Generally, preference was given to datasets reported from project personnel unless there was an overriding reason to retain the computed dataset. For example, if the computed dataset contained loads for significantly more constituents, it was retained and the reported dataset eliminated.

For every station included in this data base, there is a printed stormloads table, a corresponding storm-loads file on the data tape, a printed table of characteristics of rainfall, runoff, and antecedent conditions, a corresponding characteristics file on the data tape, and a printed table of basin characteristics, and a corresponding basin-characteristics file on the data tape. The exception is station 06412500 Rapid Creek above Canyon Lake near Rapid City, S. Dak. This station represents the upstream boundary of the urban area of Rapid City, S. Dak. Samples were collected and urban runoff was monitored at several stations downstream (06413700, 06414000, 06414700, and 06416000). The storm-load data for station 06412500 is included in this report to enable the reader to account for loads entering the urban area from upstream. There are no corresponding characteristics tables or files because the basin upstream of the station was not studied; the only purpose in including the station is to provide baseline data for the downstream stations.

\section{Constituent Storm Loads}

The constituent storm loads presented in this report were developed using two methods. The first method was to contact the U.S. Geological Survey project personnel directly involved in the data collection and interpretation for each study area (Driver and others, 1985, p. 216-219). In many cases, the project personnel already had calculated storm loads. This method provided storm-load data for some storms not included by Driver and others (1985).

The second method of determining storm loads was to use a computer program to calculate loads from the data contained in the Survey's urbanstormwater data base for 22 metropolitan areas throughout the United States (Driver and others, 1985). For each storm at each station, the program read all the appropriate water-quality sample analyses and all the appropriate unit values (time-series values) of discharge from the data base. The program 
then assigned a constituent concentration for every time for which there was a value of discharge. These assigned constituent concentrations were timedependent linear interpolations between analyzed samples. The concentrations assigned to times before the first sample were the concentrations of the first sample; similarly, the concentrations assigned to times after the last sample were the concentrations of the last sample. Next, the program developed timeseries values of instantaneous loading rates as the product of the time-series value of discharge and the assigned constituent concentrations and a conversion factor. These instantaneous loading rates then were multiplied by one-half the time period between the previous time and the following time in the time series; this produced a time series of loads. (For the first and last value, the time period was assumed to be one-half the time period between the current time-series value and the adjacent one plus one-half the regular interval of the time series.) Finally, the values in the time series of loads were summed to produce a total storm load for each constituent. The storm loads determined by this method were the total loads of the constituents carried by the total flow that occurred during the interval of the storm. There is no adjustment for baseload or for base flow.

The storm loads determined from the two methods were merged and qualityassurance procedures were performed. Only one dataset was retained for a given storm at a given station. The constituent loads during storm-runoff periods are shown in tables 2 through 99 in the "Hydrologic Data" section at the back of the report; they are presented in numerical order by station number; all 8-digit station numbers precede all 15-digit station numbers. A list of station numbers by State and metropolitan area is presented in table 1 .

There may be some inconsistency in the accounting of baseloads. The loads reported by the computed method included baseloads, if present. The loads reported by project personnel may or may not have included baseloads. The loads reported for stations 10168000 , 10168840, 10169500, 10170900, and 10172520 in the Salt Lake City metropolitan area included baseloads. (Christensen and others (1984) also report loads for these stations with baseloads excluded.) The loads reported for the Denver Federal Center rainfal1-runoff simulation plots 1-9 at Lakewood, Colo. (tables 71-79), excluded the loads applied in the simulated rainfall.

\section{Characteristics of Rainfall, Runoff, and Antecedent Conditions}

The characteristics of rainfall, runoff, and antecedent conditions presented in this report were developed using two methods. The first method was to contact the Survey project personnel directly involved in the data collection and interpretation for each study area (Driver and others, 1985, p. 216-219). In many cases, the project personnel already had calculated these characteristics. This method provided characteristics data for some storms not included in the report by Driver and others (1985).

The second method was to calculate the characteristics from data on the magnetic tape described by Driver and others (1985). A computer program was written using rainfall and discharge unit values and rainfall daily values. Eleven storm characteristics (total rainfall, in inches; preceding dry days; rain during preceding day, in inches; rain during preceding 3 days, in inches; 
rain during preceding 7 days, in inches; runoff, in inches; peak discharge, in cubic feet per second; base flow, in cubic feet per second; duration of runoff, in minutes; duration of rainfall, in minutes; and time from beginning of rainfall to peak discharge, in minutes) were calculated for each storm if the data were available.

Total rainfall was an accumulation of the rainfall data for each storm. If data from more than one rain gage were available for a station, then Thiessen coefficients (Linsley and Franzini, 1972) were multiplied by the total rainfall for each appropriate rain gage, and these products were summed. If data were missing from one of the rain gages for certain storms, then the average from the remaining rain gages was reported.

Preceding dry days were calculated from the rainfall daily-values file. Each day with less than 0.05 inch of rain was counted as a dry day. Many stations did not have these data available in the rainfall daily-values files in WATSTORE (U.S. Geological Survey, 1983).

Values of rain during the preceding day, preceding 3 days, and preceding 7 days also were calculated from the rainfall daily-values file. No weighting factors for more than one rain gage were used because there never was more than one rainfall daily-values file per runoff station.

Runoff, peak discharge, base flow, and duration of runoff were calculated from the discharge unit-values file. Runoff was an accumulation of the discharge values for each storm multipled by the appropriate time interval and conversion factor. Peak discharge was the largest discharge value for a storm. Base flow was the first discharge value for each storm in the data file. Duration of runoff was calculated from first to last nonzero discharge value for each storm in the unit-values file reported by Driver and others (1985).

Duration of rainfall was calculated from the rainfall unit-values file. The value was based on the duration of rainfall from the first to last nonzero rainfall value for a storm. If more than one rain gage was used at a station, the longest duration of rainfall was used.

The time from beginning of rainfall to peak discharge was calculated from the rainfall and discharge unit-values files. The value was based on the time of the first nonzero rainfall data to the time of the peak discharge value. If there was more than one rain gage, the gage with the longest duration of rainfall was used to compute the variable.

The characteristics determined from the two methods were merged and quality-assurance procedures were performed. Only one dataset was retained for a given storm at a given station. The characteristics of rainfall, runoff, and antecedent conditions are shown in tables 100 to 196 in the "Hydrologic Data" section at the back of the report; they are presented in numerical order by station number; all 8-digit station numbers precede all 15-digit station numbers. A list of station numbers by State and metropolitan area is presented in table 1 . There is no characteristics table for station 06412500 Rapid Creek above Canyon Lake near Rapid City, S. Dak. 


\section{Basin Characteristics}

The basin characteristics selected for publication in this report are a partial listing of the basin-characteristics files on the data tape described by Driver and others (1985) and four additional characteristics. Basincharacteristics data are shown in tables 197 to 293 in the "Hydrologic Data" section at the back of the report; they are presented in numerical order by station number; all 8-digit station numbers precede all 15-digit station numbers. A list of station numbers by State and metropolitan area is presented in table 1 . There is no basin-characteristics table for station 06412500 Rapid Creek above Canyon Lake near Rapid City, S. Dak.

\section{QUALITY ASSURANCE}

All data published in this report either have been verified by the U.S. Geological Survey project personnel reporting them or have been derived from published data that have undergone quality-assurance procedures (Driver and others, 1985, p. 9-12). In addition, the following quality-assurance procedures were performed.

\section{Constituent Storm Loads}

The computer program used to calculate constituent storm loads was tested by comparing results with hand-calculated values and with output from other programs written for different data bases. These tests included input data that could have presented particular problems. For example, the computer program was tested for storms that lasted several days and for storms that had missing water-quality sample analyses for some constituents.

In cases where storm loads were available from both sources (U.S. Geological Survey project personnel reports and computer programs), the pairs of values were compared. In most cases, there was a close but not exact relation. There are several reasons for the discrepancies. The first reason is the selection of exact beginning and ending times for a storm-runoff period. Although there are guidelines available to choose the ending time of a stormrunoff period, they are not always applicable to every situation, and the choice then becomes arbitrary. The second reason for the discrepancies is the method of calculating loads. There are several methods for calculating constituent storm loads; two methods are the method described in the "Description of Data Base" section of this report and a midpoint method that uses only discharge values corresponding to sampling times. A third reason for the discrepancies is the consideration of base flow and baseload. In data derived from the computer program, neither base flow nor baseload were considered. That is, the constituent storm loads reported included the baseloads. The loads reported by project personnel may or may not have considered base flow and baseloads. A fourth reason for the discrepancies is error, including data-entry error, conversion error, and inherent error in the data received.

To identify the errors, statistical summaries were compiled for each station for each constituent. The outliers then were double checked for dataentry errors, conversion errors, and plausibility. Storm datasets containing data that were unacceptable were deleted from the data base. 
The computer program used to calculate the characteristics was tested by comparing the output of selected datasets with hand-calculated values of the characteristics. In cases where the characteristics were available from both sources (U.S. Geological Survey project personnel and computer programs), the pairs of values were compared. In many cases, identical storm-runoff periods were not used by the two sources, and, therefore, comparisons were not valid. However, the comparisons did indicate some data-entry errors.

Other tests performed on the dataset included checks for logic: rainfall rate greatest for shortest period, rainfall during preceding days greatest for longest period, and peak discharge greater than base flow. Finally, the ratio of runoff to rainfall was checked for plausibility, allowing some leniency for base flow. If volume of base flow was included in the runoff calculations, the ratio may be greater than 1.00 . However, if the ratio was too large, the data were excluded because inclusion of the volume of base flow would significantly affect the results of any statistical analysis. Storm datasets containing data that were unacceptable were deleted from the data base.

\section{Basin Characteristics}

Most basin characteristics published in this report were obtained directly from the data tape described by Driver and others (1985). No additional quality-assurance procedures were performed on those characteristics. A description of procedures used for quality assurance of the data is given by Driver and others (1985, p. 9-12).

Four characteristics from other sources were added to the basincharacteristics tables and files. The source of these data are footnoted in the basin-characteristics tables.

\section{MAGNETIC-TAPE DATA STORAGE AND FORMAT}

All the constituent storm loads, characteristics of rainfall, runoff, and antecedent conditions, and basin characteristics shown in tables 2 through 293 also are available on magnetic tape. One storm-loads file and two characteristics files per station are available, with the exception of station 06412500 Rapid Creek above Canyon Lake near Rapid City, S. Dak. No characteristics files for this station are available because the drainage basin is not of interest (see "Description of Data Base" section). The files are arranged numerically by station number; all 8-digit station numbers precede all 15-digit station numbers. The storm-loads file for each station immediately precedes the characteristics files. The label numbers and dataset names for each file are shown in table 1 . All files are formatted into 80-character records. A value of negative nine $(-9)$ is a no-value indicator.

The magnetic tape is available on the U.S. Geological Survey's AMDAHL computer in Reston, Va. U.S. Geological Survey personnel may call Federal Telephone System (FTS) number 959-7154 or commercial number (703) 648-7154 for user-support information. The following program will provide a listing of 
data in a given file by substituting the label number for the question mark (?) after "//S" and after "F=" and substituting the dataset name for the question mark (?) after "DSN='AG40ZHA". More than one file can be listed by repeating this line for each file desired. The label numbers and dataset names are listed in table 1 . The job card must have a current account number in place of the asterisks; the logonid card must have a valid userid in place of the asterisks; and the password card must have a valid password in place of the asterisks.

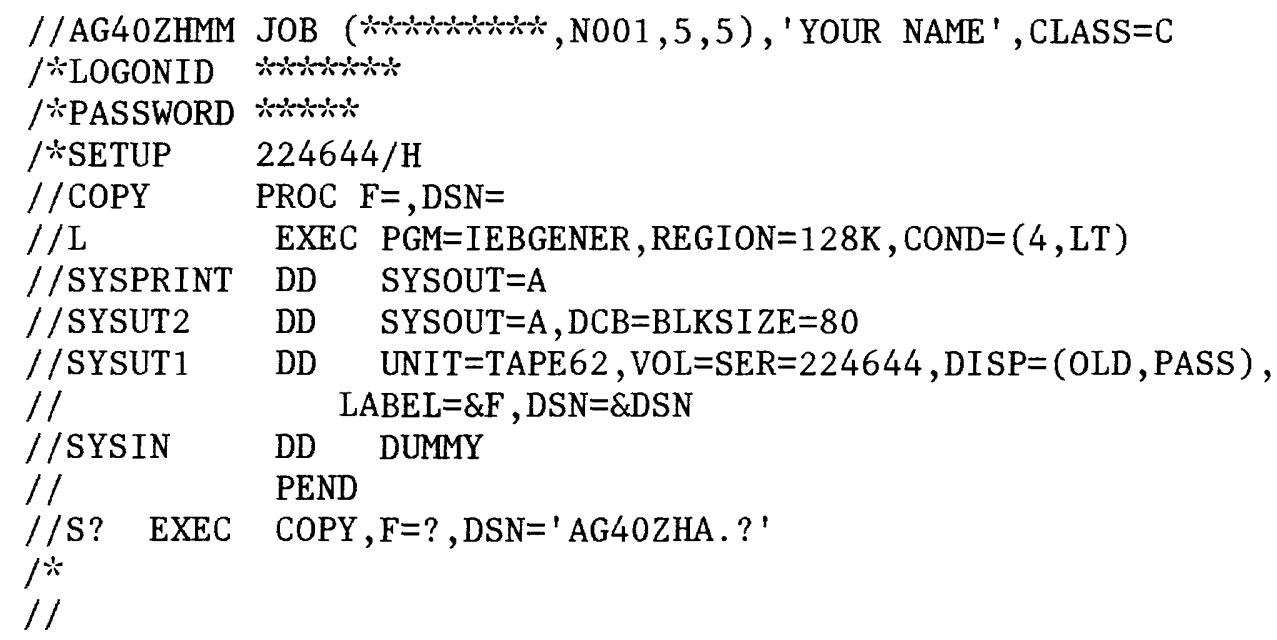

Persons not affiliated with the U.S. Geological Survey may obtain a copy of the tape through the National Water-Data Exchange (NAWDEX) by requesting a copy of tape 224644. Charges for NAWDEX services are assessed for those requests that result in charges for computer time, extensive personnel time, duplicating services, or other charges accrued by NAWDEX in the course of providing services. To request a copy of the data base, contact:

Program Office

National Water-Data Exchange (NAWDEX)

U.S. Geological Survey

421 National Center

12201 Sunrise Valley Drive

Reston, VA 22092

Telephone: FTS 959-5663

Commercial (703) 648-5663

(Hours: 7:45 a.m. to 4:15 p.m., Eastern time)

\section{Constituent Storm-Loads Files}

The constituent storm-loads files have three records (or lines) of values per storm. The first record contains: the station number; the beginning date; storm sequence number; and loads, in pounds, of chemical oxygen demand; solids, suspended; solids, settleable; nitrogen, total; and ammonia, dissolved as nitrogen. Columns 1 through 15 contain the station number (if there are only 8 digits in the station number, these digits appear in columns 2 through 9); columns 20 and 21 contain the year of the beginning date in integer format; columns 23 and 24 contain the month of the beginning date in integer format; columns 26 and 27 contain the day of the beginning date in integer 
format; and column 29 contains the sequence number of the storm. The sequence number always is zero unless there is more than one storm at the station with the identical beginning date; the sequence number is used to distinguish correspondence between storm-loads files and files of characteristics of rainfall, runoff, and antecedent conditions. The remainder of the record is five repetitions of a blank column followed by nine columns of storm-loads values in E9.2 format.

The second record of storm-loads values contains: the loads, in pounds, of ammonia plus organic nitrogen, total as nitrogen; nitrite plus nitrate, total as nitrogen; phosphorus, total; phosphorus, dissolved; orthophosphate, dissolved, as phosphorus; chloride, dissolved; cadmium, total recoverable; and copper, total recoverable. The second record contains eight repetitions of nine-column fields of E9.2 format separated by one blank column.

The third record of storm-loads values contains: the values for storm loads of lead, total recoverable, in pounds; zinc, total recoverable, in pounds; fecal coliform, in millions of colonies; solids, dissolved, in pounds; and sediment, suspended, in pounds; the source of the data; and a variable indicating whether there is data for the storm on the magnetic tape described by Driver and others (1985). The format is five repetitions of nine-column fields of E9.2 format separated by one blank column and two fields of A2. Column 51 contains either a "D", indicating that the source of the data was from U.S. Geological Survey project personnel, or a "C", indicating that the source of the data was from computer programs. Column 53 contains either a " $T$ ", indicating that the data for the storm is available on the magnetic tape described by Driver and others (1985) or " $\mathrm{N}$ ", indicating it is not available on the magnetic tape described by Driver and others (1985).

A printed example of a constituent storm-loads file is shown in figure 2 .

\section{Files of Characteristics of Rainfall, Runoff, and Antecedent Conditions}

The files of characteristics of rainfall, runoff, and antecedent conditions have two records (or lines) of values per storm. The first record contains: the station number; the beginning date of the storm; the storm sequence number; the total rainfall, in inches; the maximum 5-minute rainfall rate, in inches per hour; the maximum 15 -minute rainfall rate, in inches per hour; the maximum 60-minute rainfall rate, in inches per hour; the preceding number of dry days; the amount of rainfall during the preceding day, in inches; the amount of rainfall during the preceding 3 days, in inches; and the amount of rainfall during the preceding 7 days, in inches. Columns 1 through 15 contain the station number (if there are only 8 digits to the station number, they appear in columns 2 through 9); columns 17 and 18 contain the year of the beginning date in integer format; columns 20 and 21 contain the month of the beginning date in integer format; columns 23 and 24 contain the day of the beginning date in integer format; and column 26 contains the sequence number of the storm. The sequence number always is zero, unless there is more than one storm at the station with the identical beginning date; it is used to distinguish correspondence between storm-loads files and files of characteristics of rainfall, runoff, and antecedent conditions. The remainder of the record is $\mathrm{F} 7.3,3(\mathrm{~F} 6.2), \mathrm{I} 4$, and $3(\mathrm{~F} 6.2)$. 
Columns

12345678901234567890123456789012345678901234567890123456789012345678901234567890

1

2

01589460

$0.14 E+02$

$0.97 E+00$

01589460

$0.13 E+02$

$0.23 E+00$

01589460

$0.32 \mathrm{E}+01$

$0.46 \mathrm{E}-01$

01589460

$0.15 \mathrm{E}+01$

$0.29 \mathrm{E}-01$

01589460

$0.79 \mathrm{E}+00$

$0.46 \mathrm{E}-01$

01589460

$0.42 E+01$

$0.11 E+00$

01589460

$0.84 \mathrm{E}+01$

$0.13 E+00$

01589460

$0.41 E+01$

$0.16 \mathrm{E}+00$

01589460

$0.67 \mathrm{E}+01$

$0.24 \mathrm{E}+00$

01589460

$0.67 E+01$

$0.54 \mathrm{E}-01$
3

4

5

6

7

8
$8151500-0.90 \mathrm{E}+01$
$0.38 E+02 \quad 0.35 E+01-0.90 E+01-0.90 E+01-0.90 E+01$
$0.52 E+02-0.90 E+01$
$0.13 E+01-0.90 E+01 \quad 0.38 E+03-0.90 E+01 C T$
$8199800 \quad 0.77 E+02 \quad 0.97 E+02-0.90 E+01$
$0.22 E-01 \quad 0.33 E+00$
$0.13 E+01 \quad 0.56 E+00-0.90 E+01-0.90 E+01-0.90 E+01$
$0.31 E+00-0.90 E+01 \quad 0.36 E+02-0.90 E+01 \quad C \quad T$
$\begin{array}{lllllll}81 & 9 & 17 & 0 & 0.36 \mathrm{E}+02 & 0.14 \mathrm{E}+02 & -0.90 \mathrm{E}+01\end{array}$
$0.14 E+02-0.90 E+01$
$0.24 \mathrm{E}-02 \quad 0.41 \mathrm{E}-01$
$0.51 E+01 \quad 0.18 E+00-0.90 E+01-0.90 E+01-0.90 E+01$
$0.83 E+01-0.90 E+01$
$0.11 \mathrm{E}+00-0.90 \mathrm{E}+01 \quad 0.11 \mathrm{E}+02-0.90 \mathrm{E}+01 \mathrm{C} \mathrm{T}$
$819 \begin{array}{llllll}818 & 0 & -0.90 E+01 & 0.33 E+01 & -0.90 E+01\end{array}$
$0.15 \mathrm{E}-02 \quad 0.26 \mathrm{E}-01$
$0.54 \Sigma+01 \quad 0.53 E-01-0.90 E+01-0.90 E+01-0.90 E+01$
$0.69 \mathrm{E}+01-0.90 \mathrm{E}+01$
$0.40 \mathrm{E}-01-0.90 \mathrm{E}+01 \quad 0.41 \mathrm{E}+01-0.90 \mathrm{E}+01 \mathrm{C} \mathrm{T}$
$819 \begin{array}{llllll}9 & 27 & 0 & 0.16 \mathrm{E}+02 & 0.21 \mathrm{E}+02 & -0.90 \mathrm{E}+01\end{array}$
$0.16 \mathrm{E}-02 \quad 0.17 \mathrm{E}-01$
$0.13 E+01 \quad 0.14 E+00-0.90 E+01-0.90 E+01-0.90 E+01$
$0.21 E+01-0.90 E+01$
$\begin{array}{lllll}0.65 \mathrm{E}-01-0.90 \mathrm{E}+01 & 0.25 \mathrm{E}+02-0.90 \mathrm{E}+01 \mathrm{C} \mathrm{T}\end{array}$
$\begin{array}{lllllll}81 & 10 & 23 & 0 & 0.12 \mathrm{E}+03 & 0.39 \mathrm{E}+02 & -0.90 \mathrm{E}+01\end{array}$
$0.84 \mathrm{E}-03 \quad 0.16 \mathrm{E}-01$
$\begin{array}{llllll}0.33 E+01 & 0.30 E+00 & -0.90 E+01 & -0.90 E+01 & -0.90 E+01\end{array}$
$0.75 E+01-0.90 E+01$
$\begin{array}{lllll}0.15 \mathrm{E}+00-0.90 \mathrm{E}+01 & 0.84 \mathrm{E}+02-0.90 \mathrm{E}+01 \mathrm{C} \mathrm{T}\end{array}$
$811210-0.90 E+01 \quad 0.49 E+02-0.90 E+01$
$0.56 E-02 \quad 0.18 E-01$
$0.16 E+02 \quad 0.31 E+00-0.90 E+01-0.90 E+01-0.90 E+01$
$0.24 E+02-0.90 E+01$
$0.26 \mathrm{E}+00-0.90 \mathrm{E}+01 \quad 0.26 \mathrm{E}+02-0.90 \mathrm{E}+01 \mathrm{C} \mathrm{T}$
$8112140-0.90 \mathrm{E}+01 \quad 0.21 \mathrm{E}+02-0.90 \mathrm{E}+01$
$0.65 \mathrm{E}-02 \quad 0.39 \mathrm{E}-01$
$0.34 \mathrm{E}+02-0.90 \mathrm{E}+01$
$0.74 \mathrm{E}-02 \quad 0.44 \mathrm{E}-01$
$0.14 \mathrm{E}+01 \quad 0.40 \mathrm{E}+00-0.90 \mathrm{E}+01-0.90 \mathrm{E}+01-0.90 \mathrm{E}+01$
$0.81 \mathrm{E}+01-0.90 \mathrm{E}+01$
$0.31 E+00-0.90 E+01 \quad 0.49 E+02-0.90 E+01 C T$
$\begin{array}{lllllll}824 & 26 & 0 & -0.90 \mathrm{E}+01 & 0.41 \mathrm{E}+02 & -0.90 \mathrm{E}+01\end{array}$
$0.50 \mathrm{E}-02 \quad 0.40 \mathrm{E}-01$
$0.64 E+00 \quad 0.26 E+00-0.90 E+01-0.90 E+01-0.90 E+01$
$0.74 E+01-0.90 E+01$
$0.11 E+00-0.90 E+01 \quad 0.13 E+02-0.90 E+01 C T$
$0.54 \mathrm{E}-02 \quad 0.21 \mathrm{E}-01$

Figure 2.--Example of a storm-loads file. 
The second line of the characteristics file contains: the amount of runoff, in inches; the peak discharge, in cubic feet per second; the base flow, in cubic feet per second; the duration of runoff, in minutes; the duration of rainfall, in minutes; the time from beginning of rainfall to peak discharge, in minutes; the time since last street cleaning, in days; the source of the data; and a variable indicating whether there are data for the storm on the data tape described by Driver and others (1985). The format is F11.3, F8.2, F9.3, 3(I5), F7.1, and 2A2. Column 52 contains either a "D", indicating that the source of the data was from U.S. Geological Survey project personnel, or " $\mathrm{C}$ ", indicating that the source was from computer programs. Column 54 contains either a "T", indicating that the data for the storm are available on the magnetic tape described by Driver and others (1985), or " $N$ ", data are not available on the magnetic tape described by Driver and others (1985).

A printed example of a characteristics file is shown in figure 3 .

\section{Basin-Characteristics Files}

The basin characteristics files have eight records (or lines) of values per station. The first record contains the station number in columns 1 through 15; if there are only 8 digits to the station number, they appear in columns 2 through 9 .

The second record contains: the contributing drainage area, in acres; the impervious area, in percentage of contributing drainage area; the effective impervious area, in percentage of contributing drainage area; the average basin slope, in feet per mile; and the main conveyance slope, in feet per mile. The format of the record is F15.5, F12.2, F12.2, F12.4, F12.4.

The third record contains the population density, in number of people per square mile, and street density, in miles of lanes per square mile. The population density occurs in integer format in columns 47 through 57 and the street density occurs in integer format in columns 58 through 69 .

The fourth record contains the percentage of contributing drainage area with the following land uses: rural and pasture, agricultural, low-density residential, medium-density residential, and high-density residential. The format of the record is five repetitions of F12.2.

The fifth record contains the percentage of contributing drainage area with the following land uses: commercial, industrial, under construction, idle or vacant, and wetland. The format of the record is five repetitions of F12.2.

The sixth record contains: the percentage of contributing drainage area with parkland; detention storage, in acre-feet; the percentage of contributing drainage area upstream from detention storage; the percentage of contributing area drained by a storm-sewer system; and the percentage of streets that have curb and gutter drainage. The format of the record is five repetitions of F12.2.

The seventh record contains: the percentage of streets that have ditch and swale drainage; the mean annual rainfall, in inches; and the 10-year, 1-hour rainfall intensity, in inches per hour. The percentage of streets that 
Columns

12345678901234567890123456789012345678901234567890123456789012345678901234567890

1

2

3

4

5

6

7

8

Figure 3.--Example of a file of characteristics of rainfall, runoff, and antecedent conditions. 
have ditch and swale drainage occur in F12.2 format in columns 1 through 12; the mean annual rainfall occurs in F12.2 format in columns 25 through 36 ; and the 10-year, 1-hour rainfall intensity occurs in F12.2 format in columns 37 through 48 .

The eighth record contains: the mean minimum January temperature, in degrees Fahrenheit; the 2-year, 24-hour rainfall, in inches; and the mean annual nitrogen precipitation load, in pounds of nitrogen per acre. The format of the record is F5.1, F5.1, F6.2.

A printed example of a basin-characteristics file is shown in figure 4.

\section{Columns}

12345678901234567890123456789012345678901234567890123456789012345678901234567890

1

2

3

4

5

6

7

01589460

16.90000

72.00

72.00

206.0000

188.0000

0.00

0.00

0.00

0.00

0.00

0.00

0.00

35800

16.00

0.00

0.00

0.00

40.46

100.00

2.50

$24.9 \quad 3.3 \quad 7.00$

Figure 4.--Example of a basin-characteristics file. 


\section{REFERENCES CITED}

Blevins, D.W., 1984, Stormwater quality of the Blue River basin in Kansas City, Missouri: U.S. Geological Survey Water-Resources Investigations Report 84-4226, $131 \mathrm{p}$.

Christensen, R.C., Stephens, D.W., Pyper, G.E., McCormack, H.F., and Weig1, J.F., 1984, Quality and quantity of runoff and atmospheric deposition in urban areas of Salt Lake County, Utah, 1980-81: U.S. Geological Survey Water-Resources Investigations Report 84-4011, $223 \mathrm{p}$.

Driver, N.E., Mustard, M.H., Rhinesmith, R.B., and Middelburg, R.F., 1985, U.S. Geological Survey urban-stormwater data base for 22 metropolitan areas throughout the United States: U.S. Geological Survey Open-File Report 85-337, $219 \mathrm{p}$.

Ellis, S.R., 1978, Hydrologic data for urban storm runoff from three localities in the Denver metropolitan area, Colorado: U.S. Geological Survey Open-File Report 78-410, 135 p., 3 plates.

Ellis, S.R., Doerfer, J.T., Mustard, M.H., Blakely, S.R., and Gibbs, J.W., 1984, Analysis of urban storm-runoff data and the effects on the South Platte River, Denver metropolitan area, Colorado: U.S. Geological Survey Water-Resources Investigations Report 84-4159, $66 \mathrm{p}$.

Hershfield, D.M., 1961, Rainfall frequency atlas of the United States for durations from 30 minutes to 24 hours and return periods from 1 to 100 years: U.S. Weather Bureau Technical Paper No. 40, $71 \mathrm{p}$.

Linsley, R.K., and Franzini, J.B., 1972, Water-resources engineering (2d ed.): New York, McGraw-Hill Book Co., 690 p.

Lopez, M.A., and Giovannelli, R.F., 1984, Water-quality characteristics of urban runoff and estimates of annual loads in the Tampa Bay area, Florida, 1975-80: U.S. Geological Survey Water-Resources Investigations Report 83-4181, $76 \mathrm{p}$.

Mattraw, H.C., Jr., and Miller, R.A., 1981, Stormwater quality processes for three land-use areas in Broward County, Florida: U.S. Geological Survey Water-Resources Investigations 81-23, $56 \mathrm{p}$.

Miller, T.L., and McKenzie, S.W., 1978, Analysis of urban storm-water quality from seven basins near Portland, Oregon: U.S. Geological Survey OpenFile Report 78-662, $8 \mathrm{p}$.

Mustard, M.H., Ellis, S.R., and Gibbs, J.W., 1985, Runoff characteristics and washoff loads from rainfall-simulation experiments on a street surface and a native pasture in the Denver metropolitan area, Colorado: U.S. Geological Survey Open-File Report 84-820, 44 p.

National Atmospheric Deposition Program, NADP Subcommittee Number 3--Data Management and Analysis, 1985, NADP annual data summary - precipitation chemistry in the United States - 1982: Fort Collins, Colorado State University, Natural Resources Ecology Laboratory, $135 \mathrm{p}$.

National Oceanic and Atmospheric Administration, 1980, Climates of the states, 2d ed.: Detroit, Gale Research Company, 2 v.

Payne, G.A., Ayers, M.A., and Brown, R.G., 1982, Quality of runoff from small watersheds in the Twin Cities metropolitan area, Minnesota--Hydrologic data for 1980: U.S. Geological Survey Open-File Report 82-504, 289 p.

Striegl, R.G., and Cowan, E.A., 1987, Relations between quality of urban runoff and quality of Lake Ellyn at Glen Ellyn, Illinois: U.S. Geological Survey Water-Supply Paper 2301. 
U.S. Geological Survey, 1983, Daily values in WATSTORE (National Water Data Storage and Retrieval System): Reston, Va., available from WATSTORE. Program office, computer tape.

Zarriello, P.J., Harding, W.E., Yager, R.M., and Kappel, W.M., 1985, Quantity and quality of storm runoff in the Irondequoit Creek basin near Rochester, New York, Part 1--Data collection network and methods, quality-assurance program, and description of available data: U.S. Geological Survey Open-File Report 84-610, 44 p. 
HYDROLOGIC DATA 


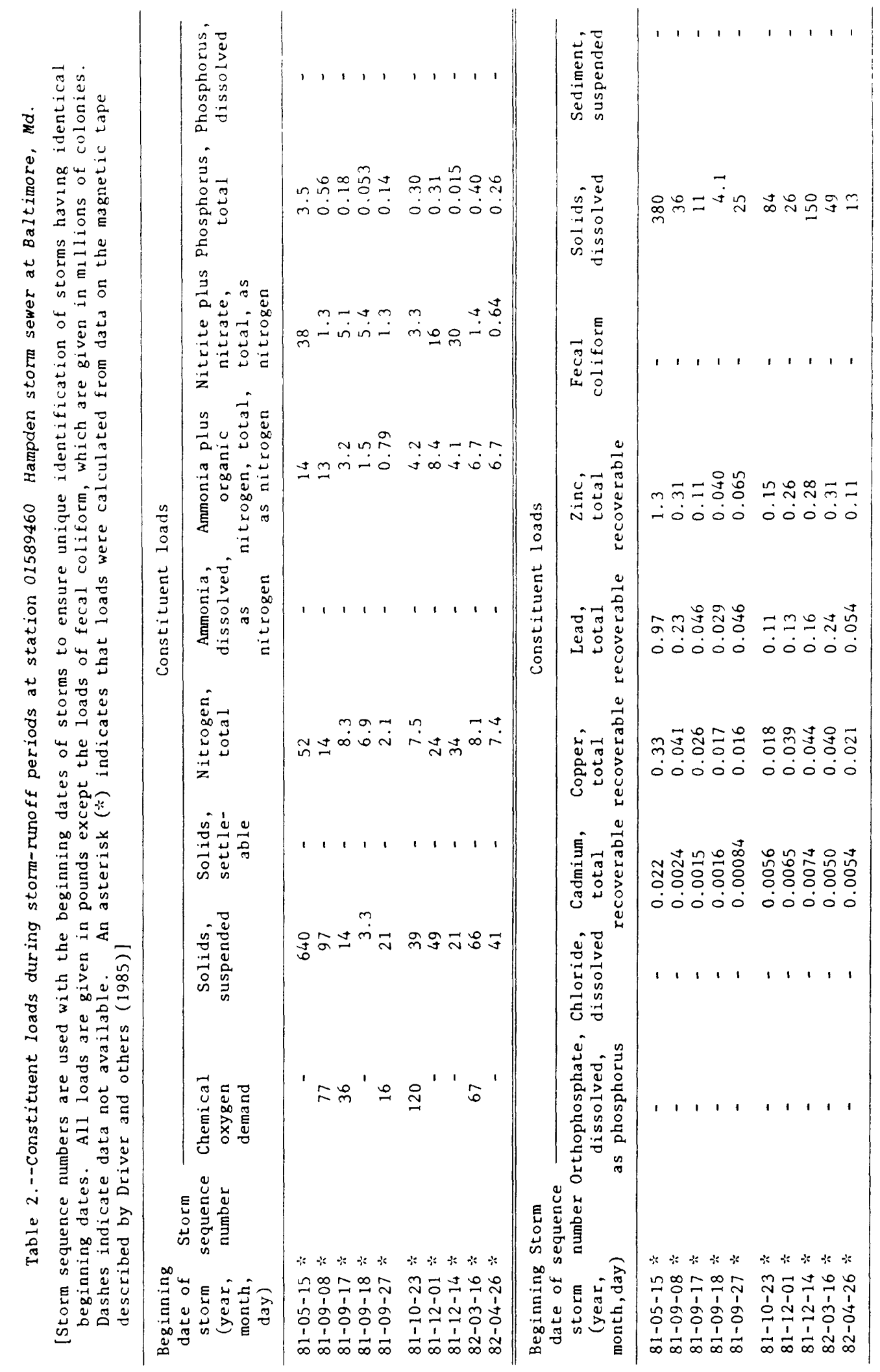




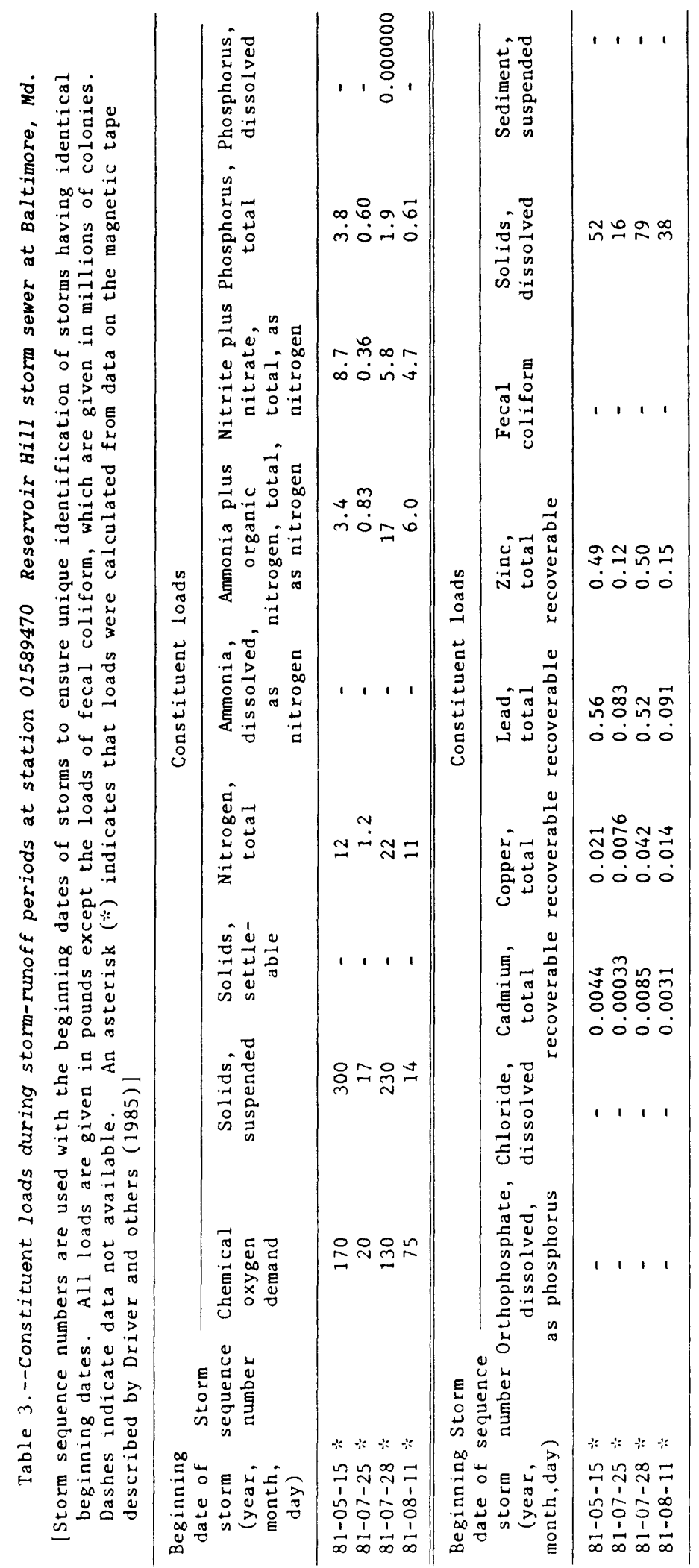




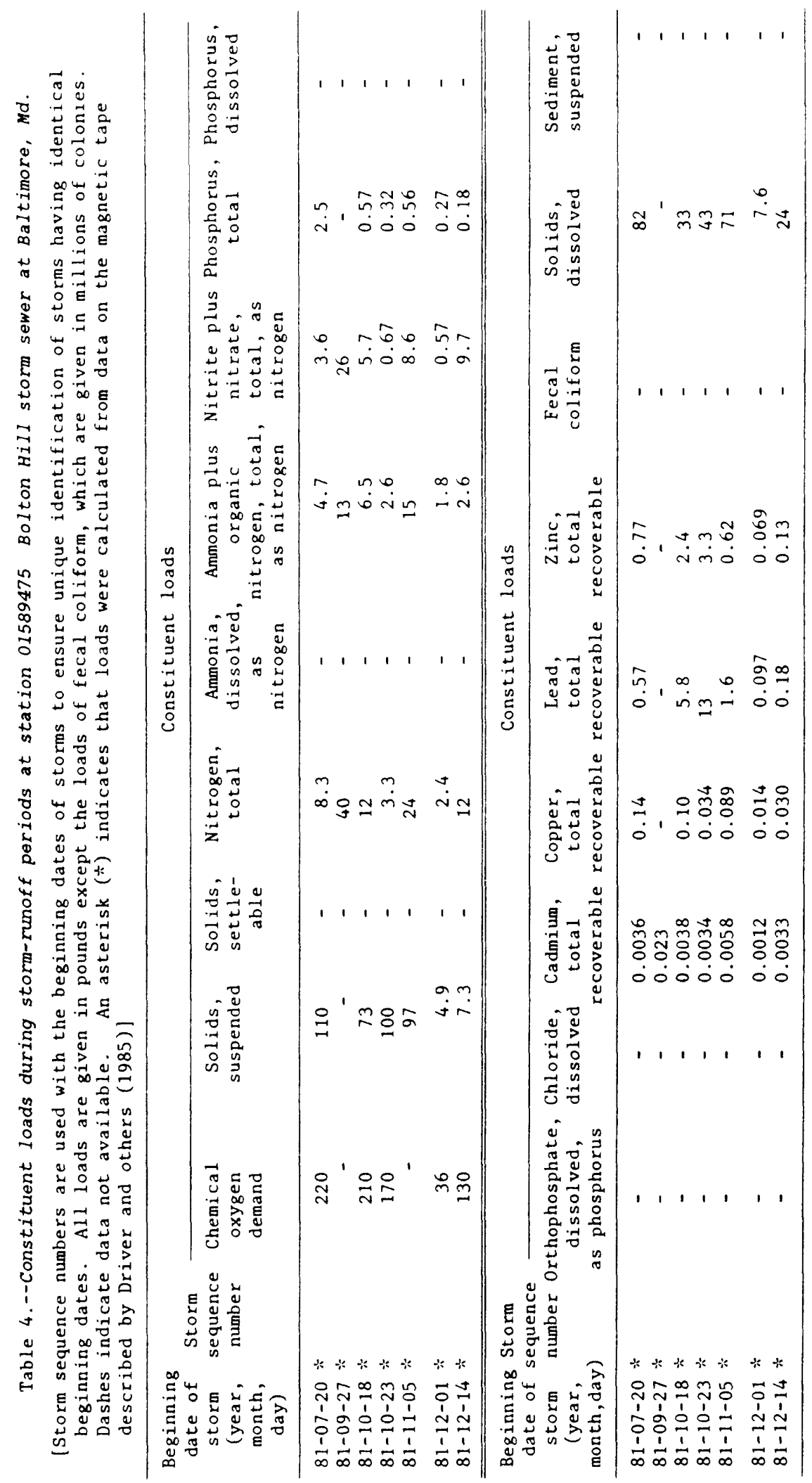




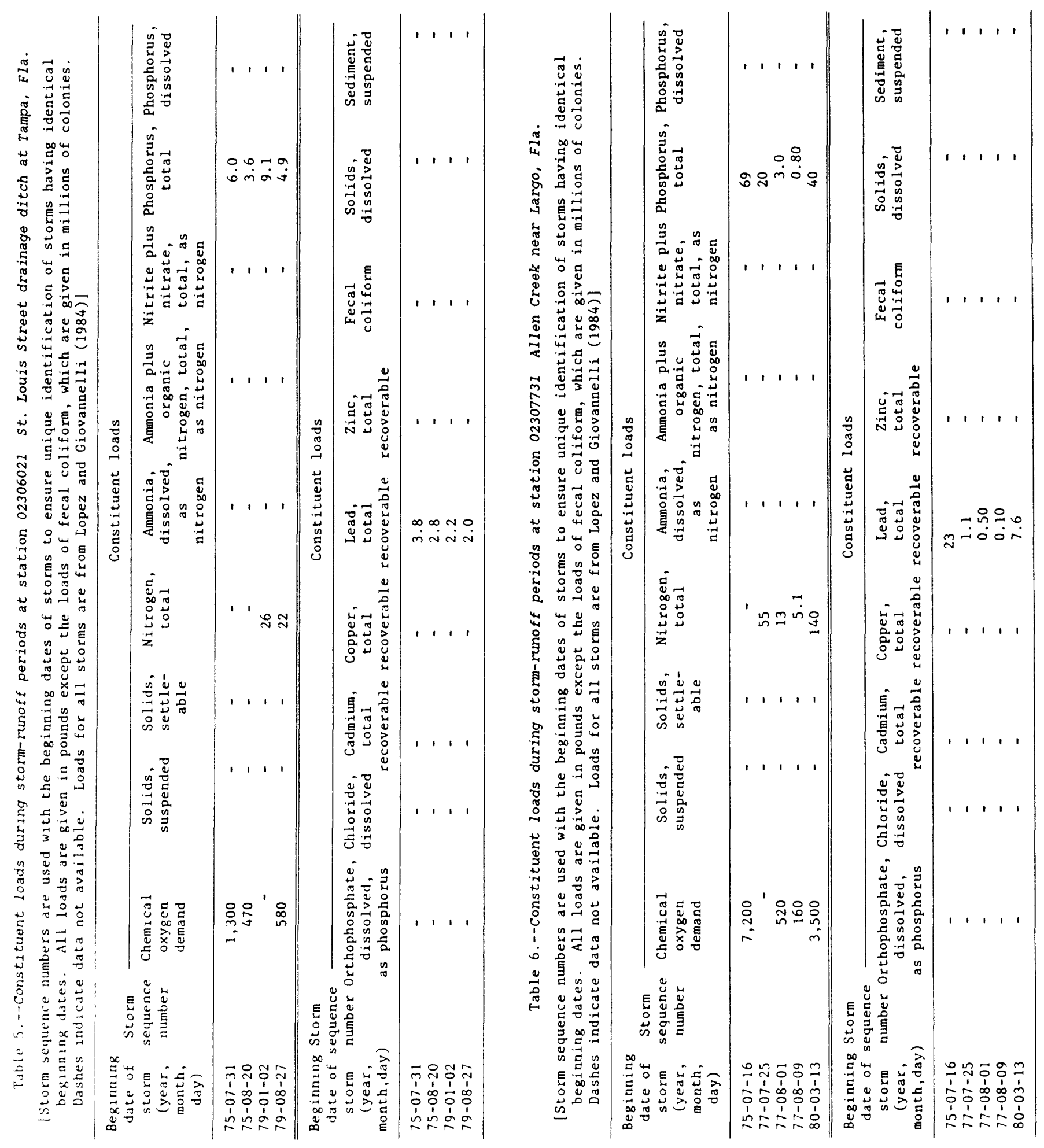




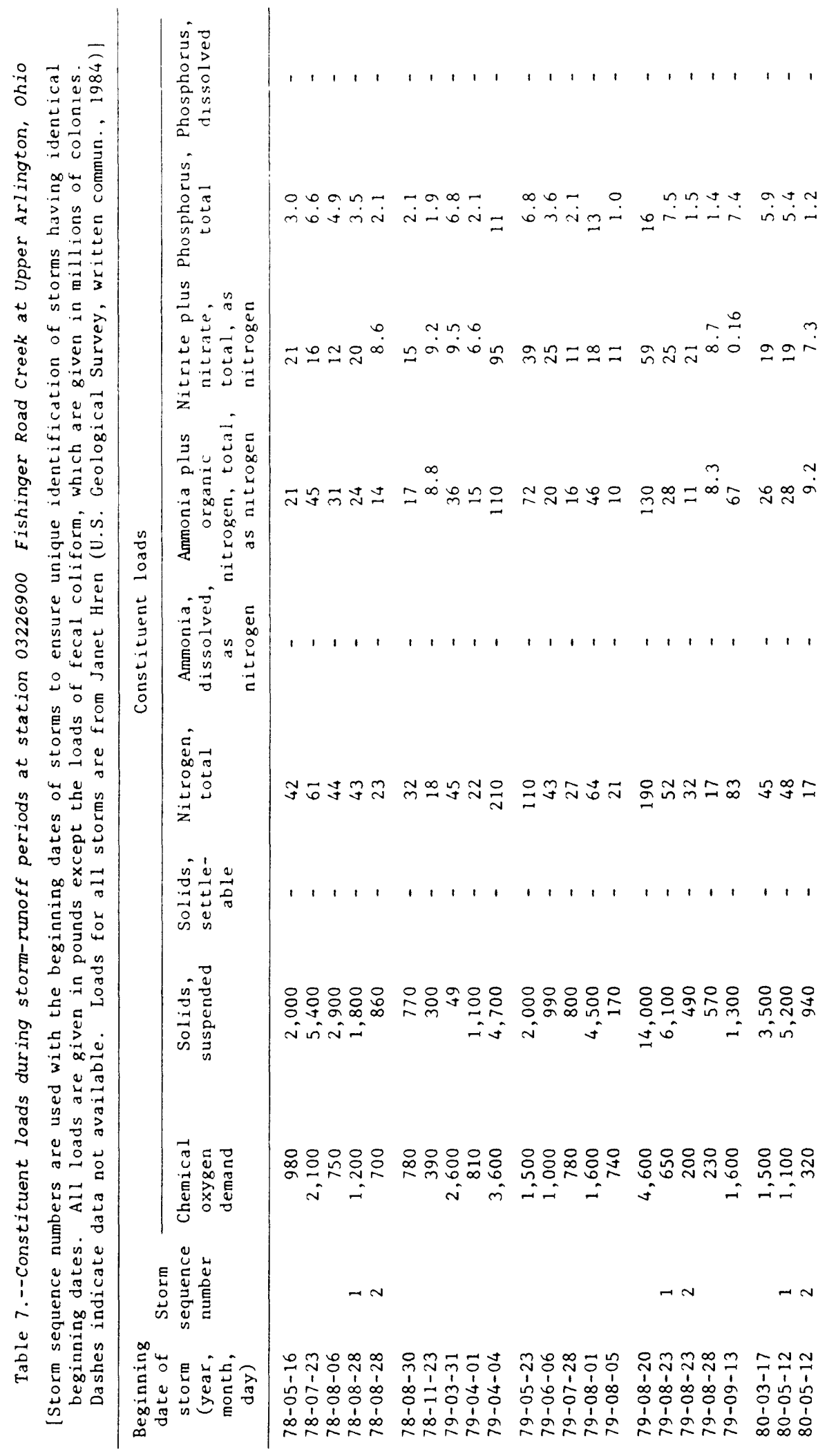




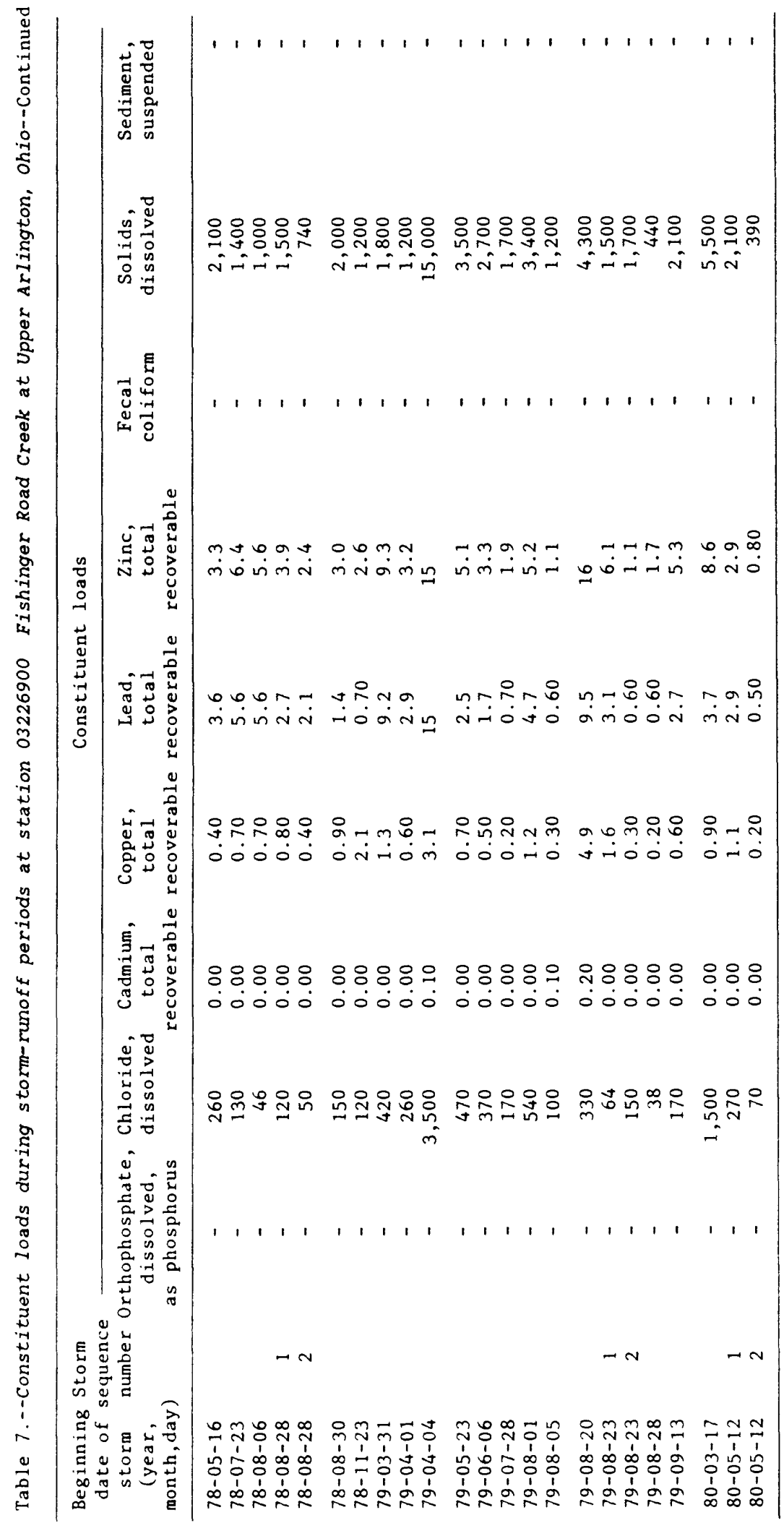




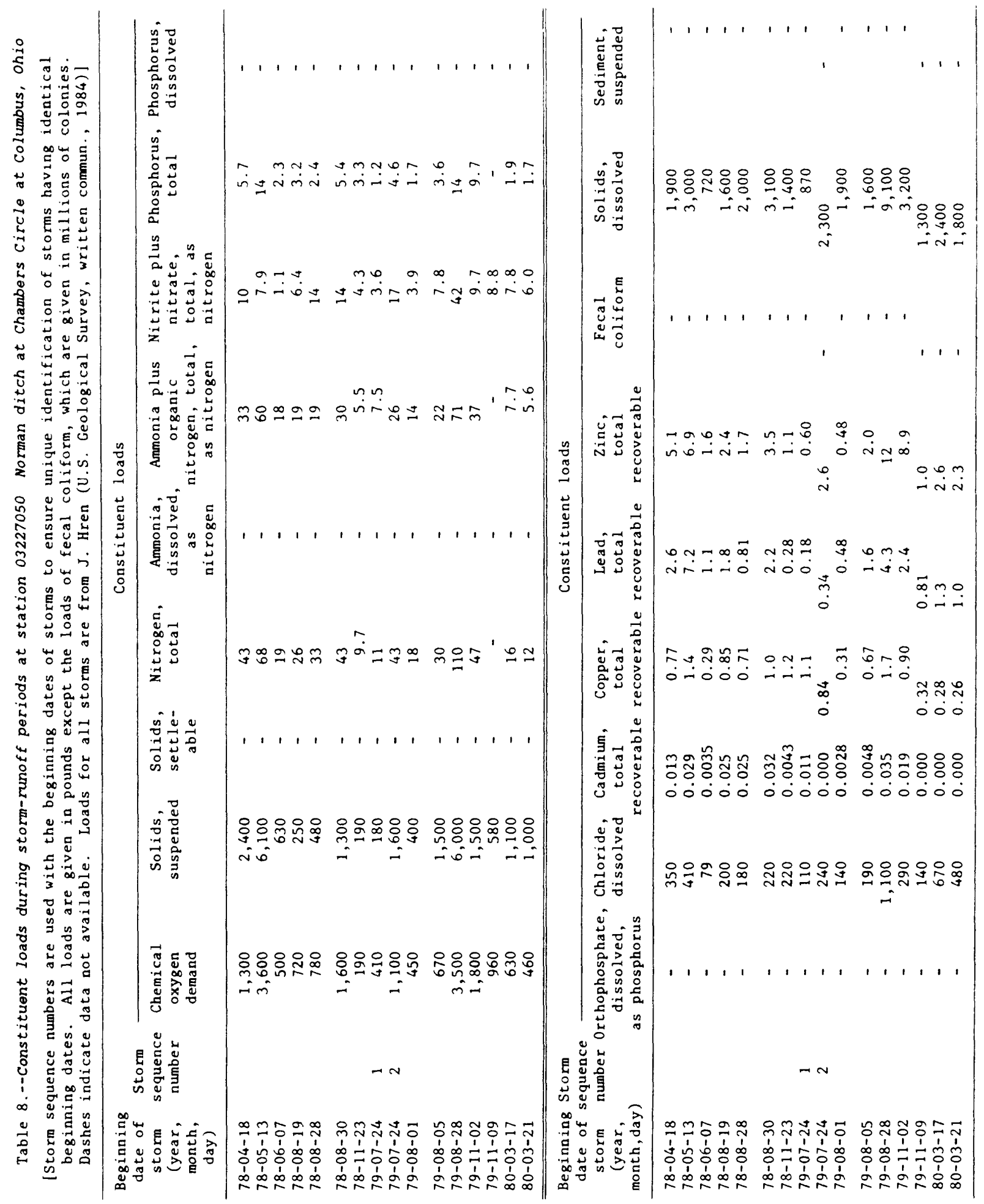




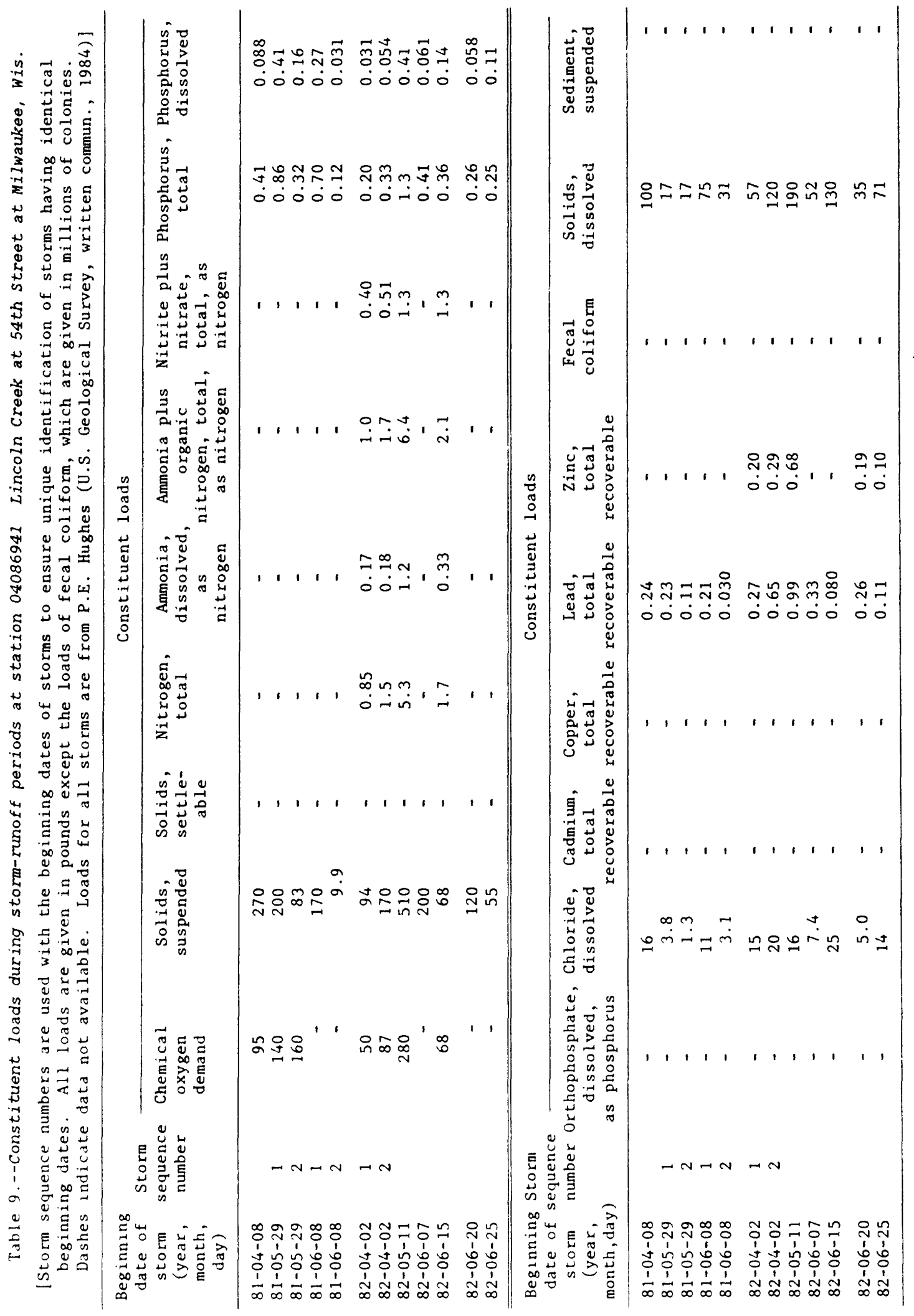




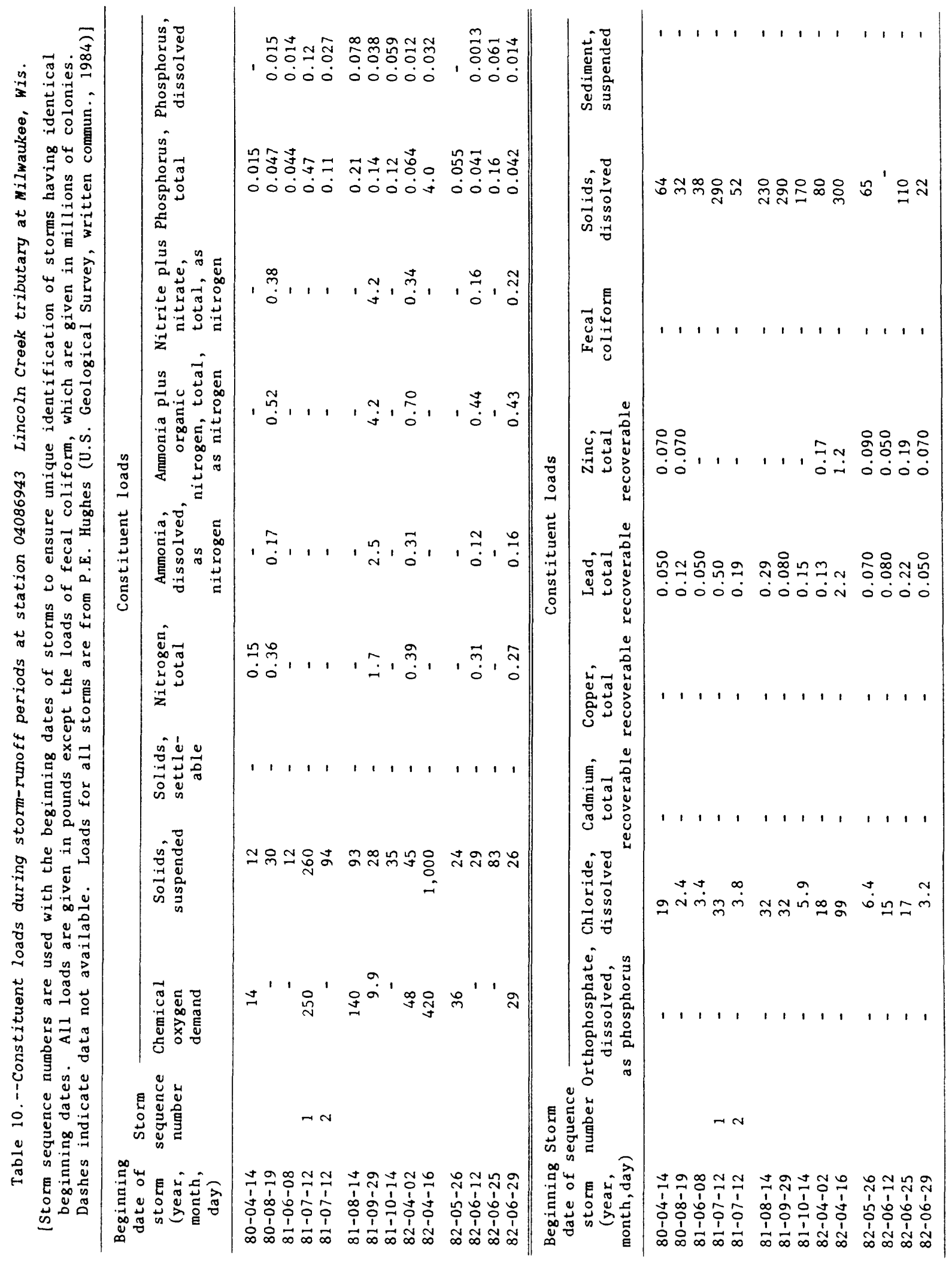




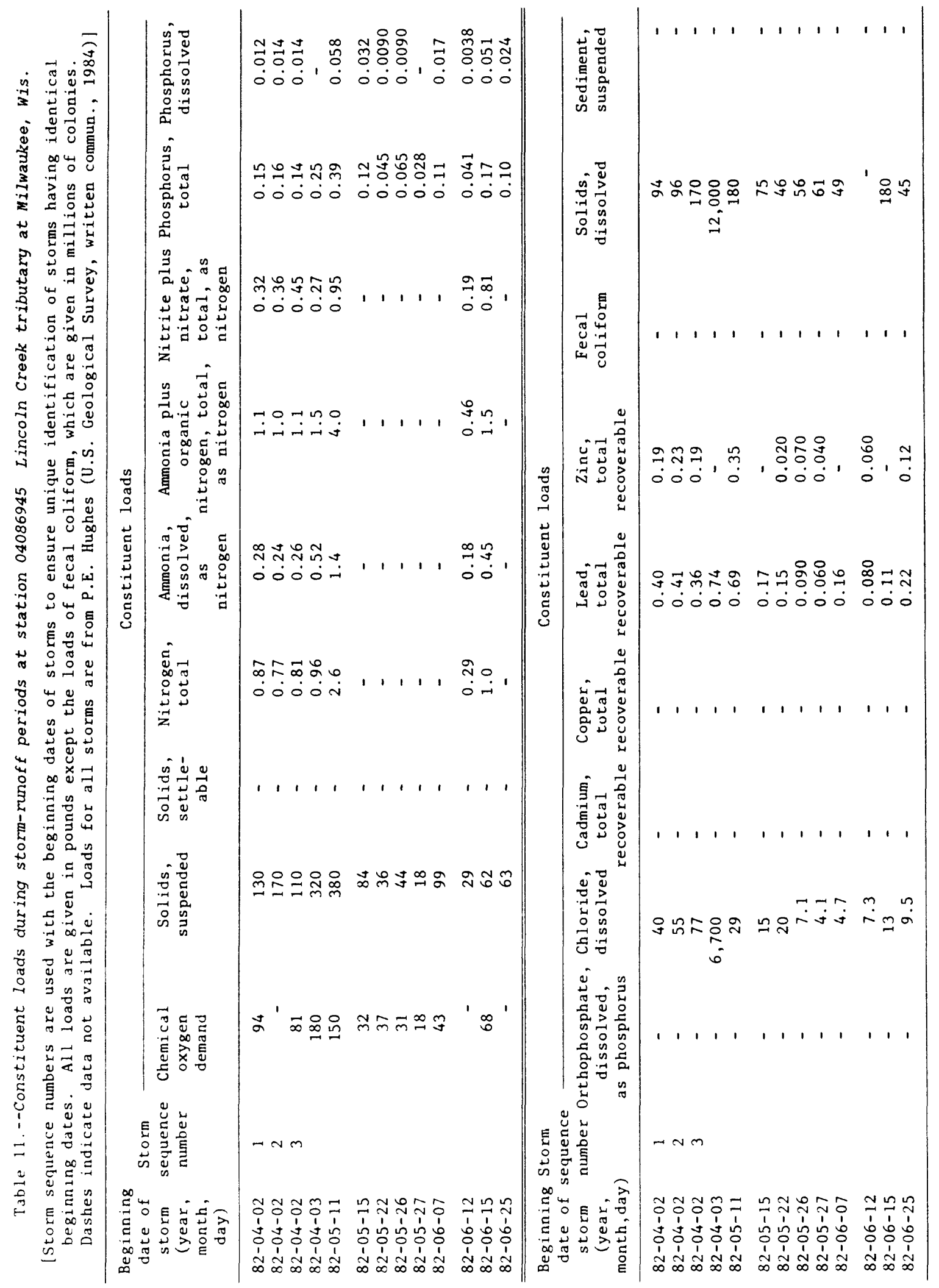




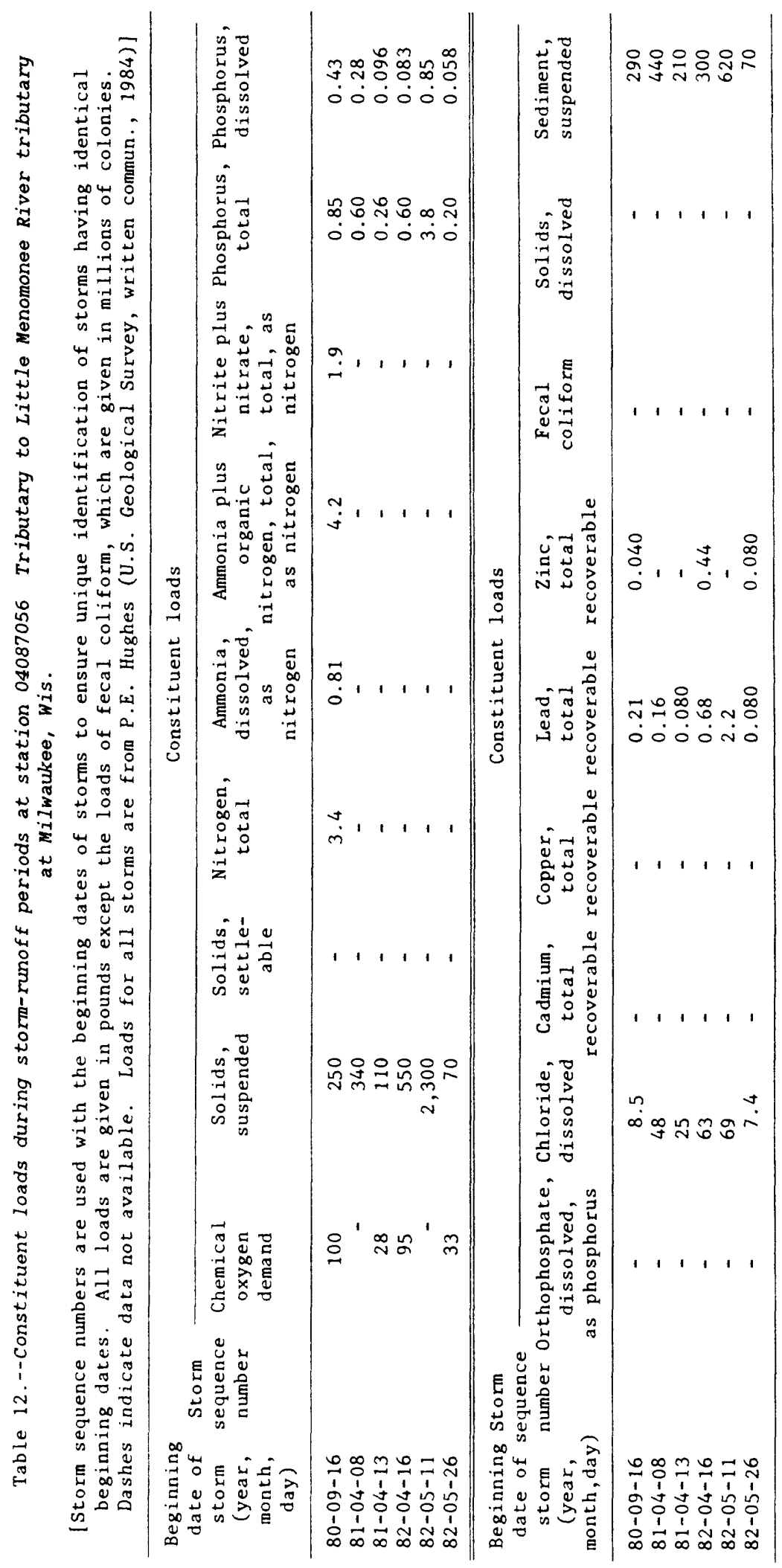




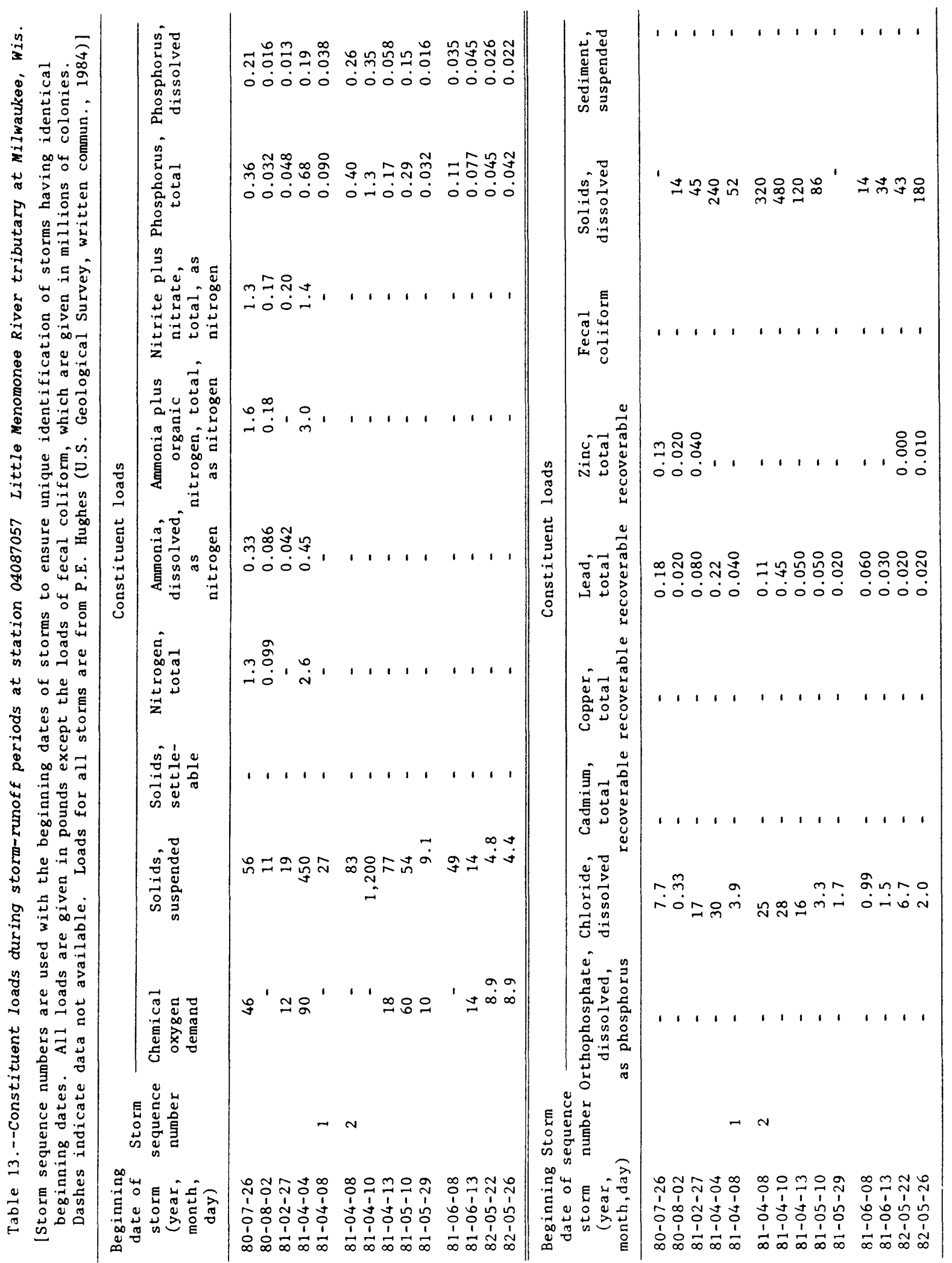




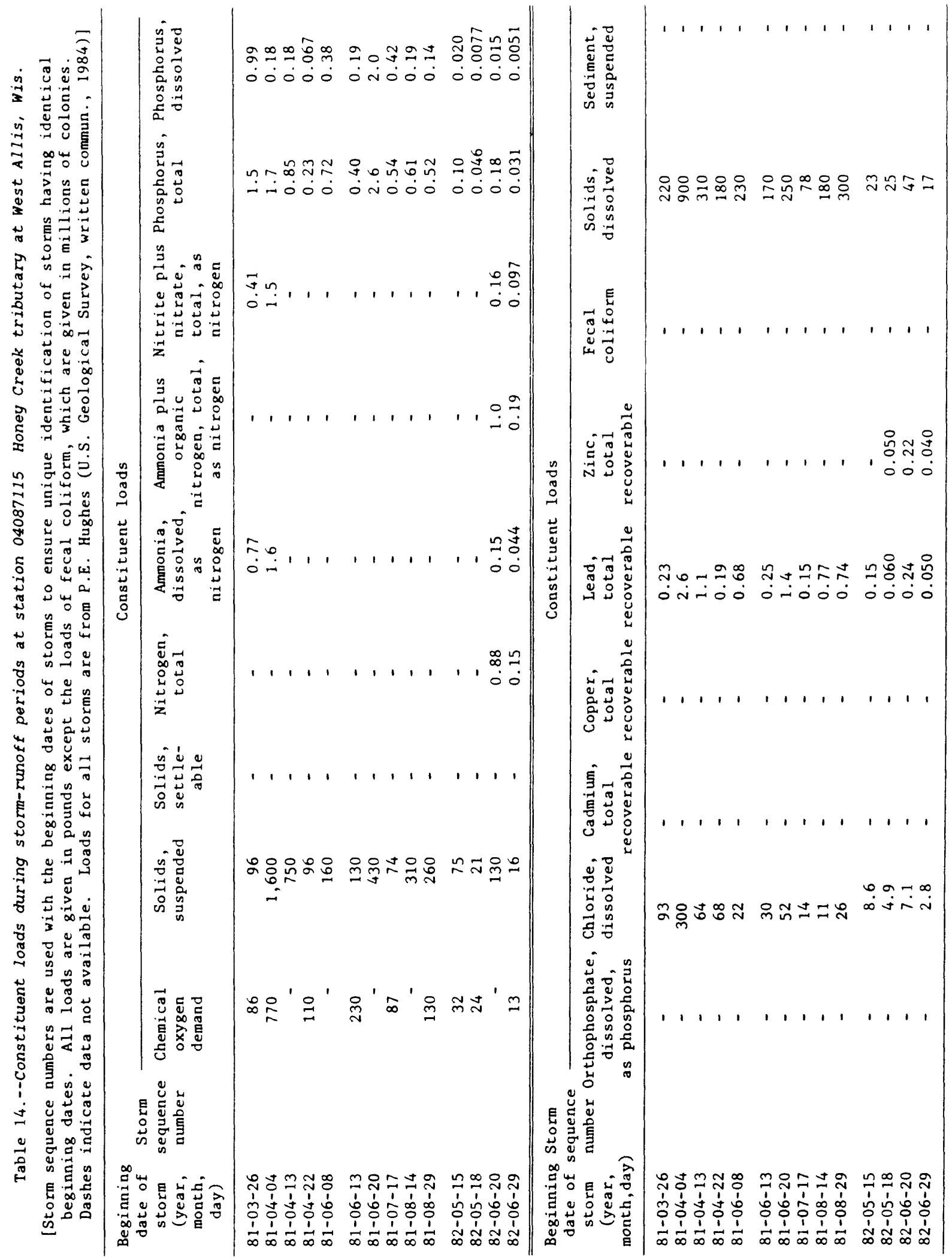




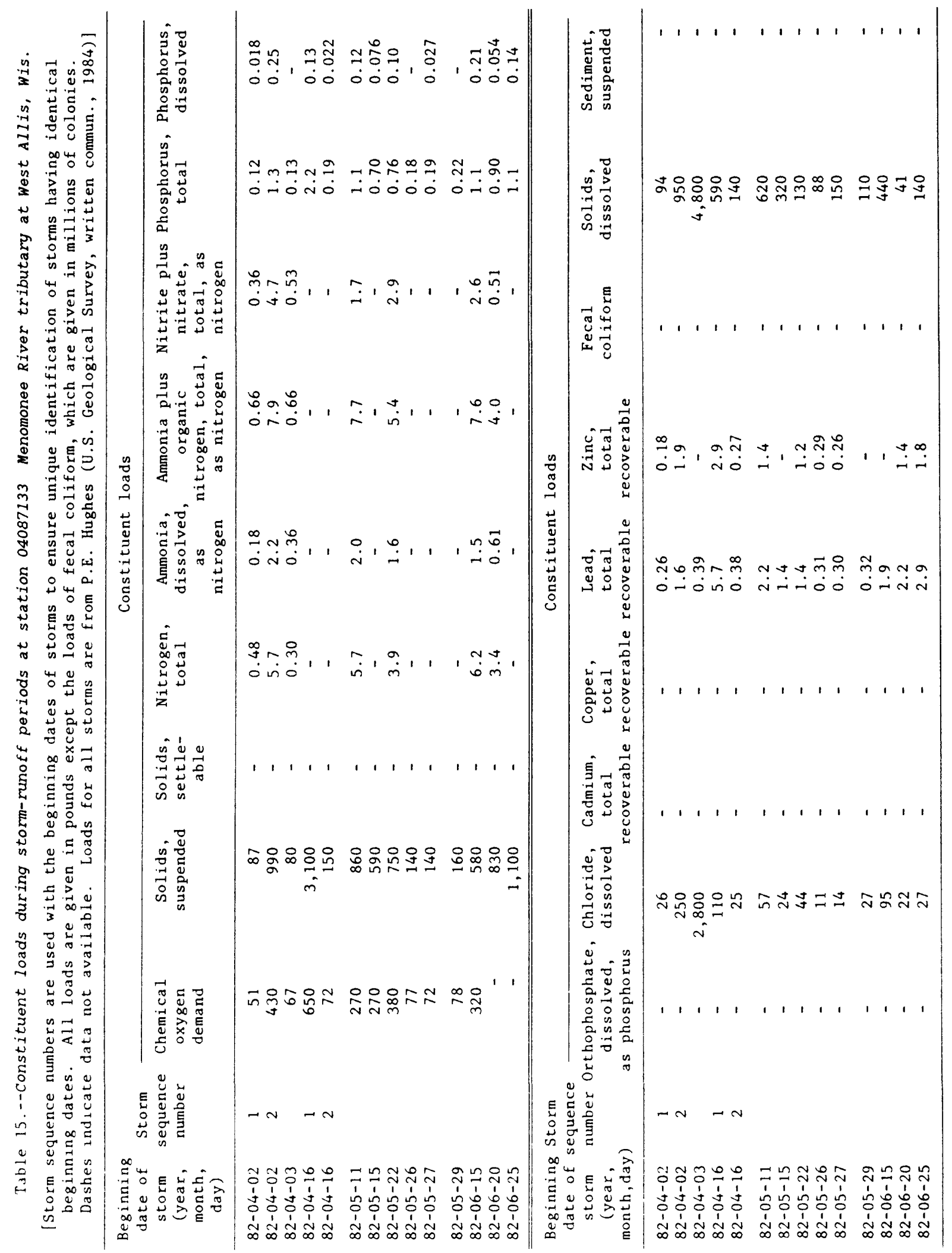




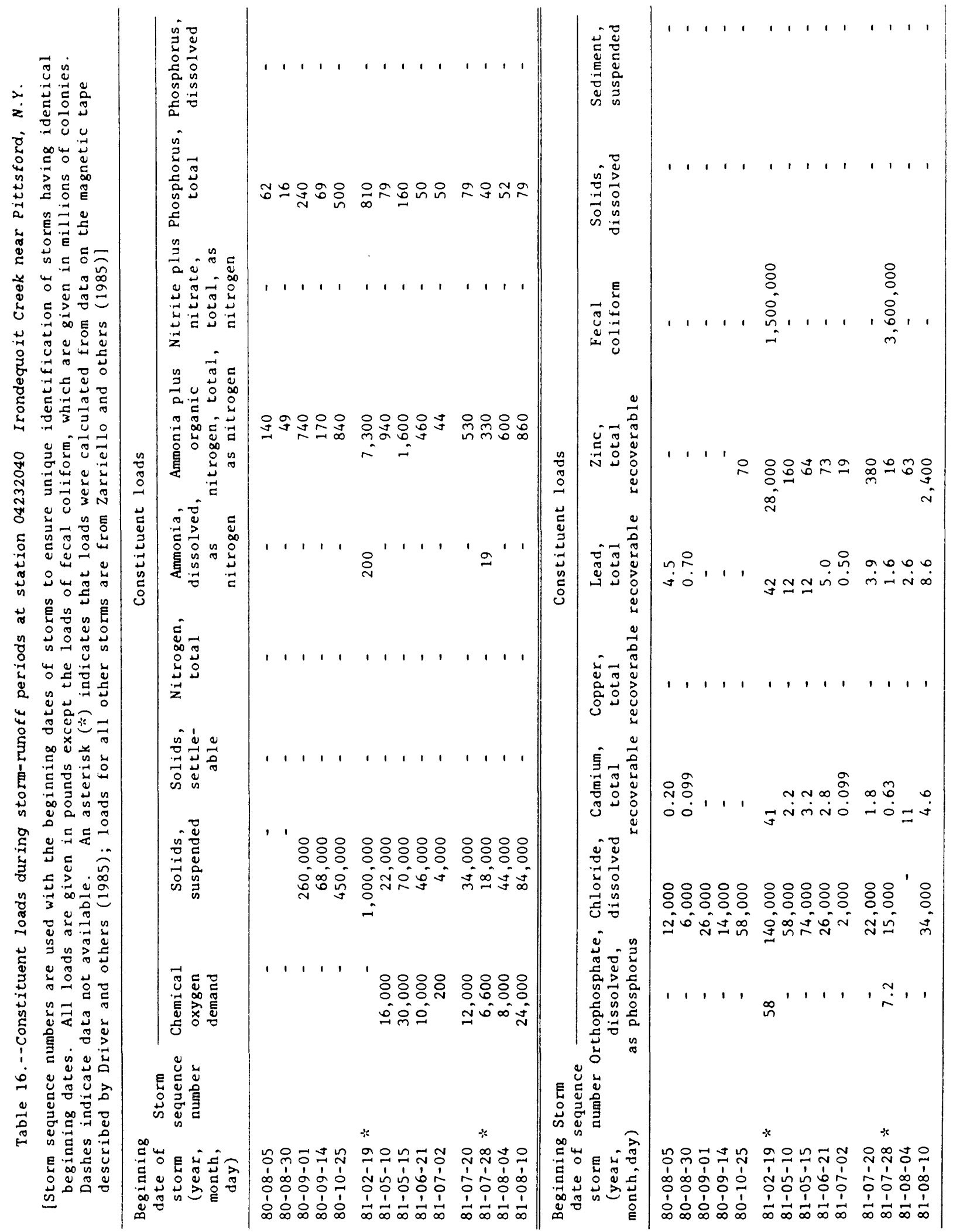




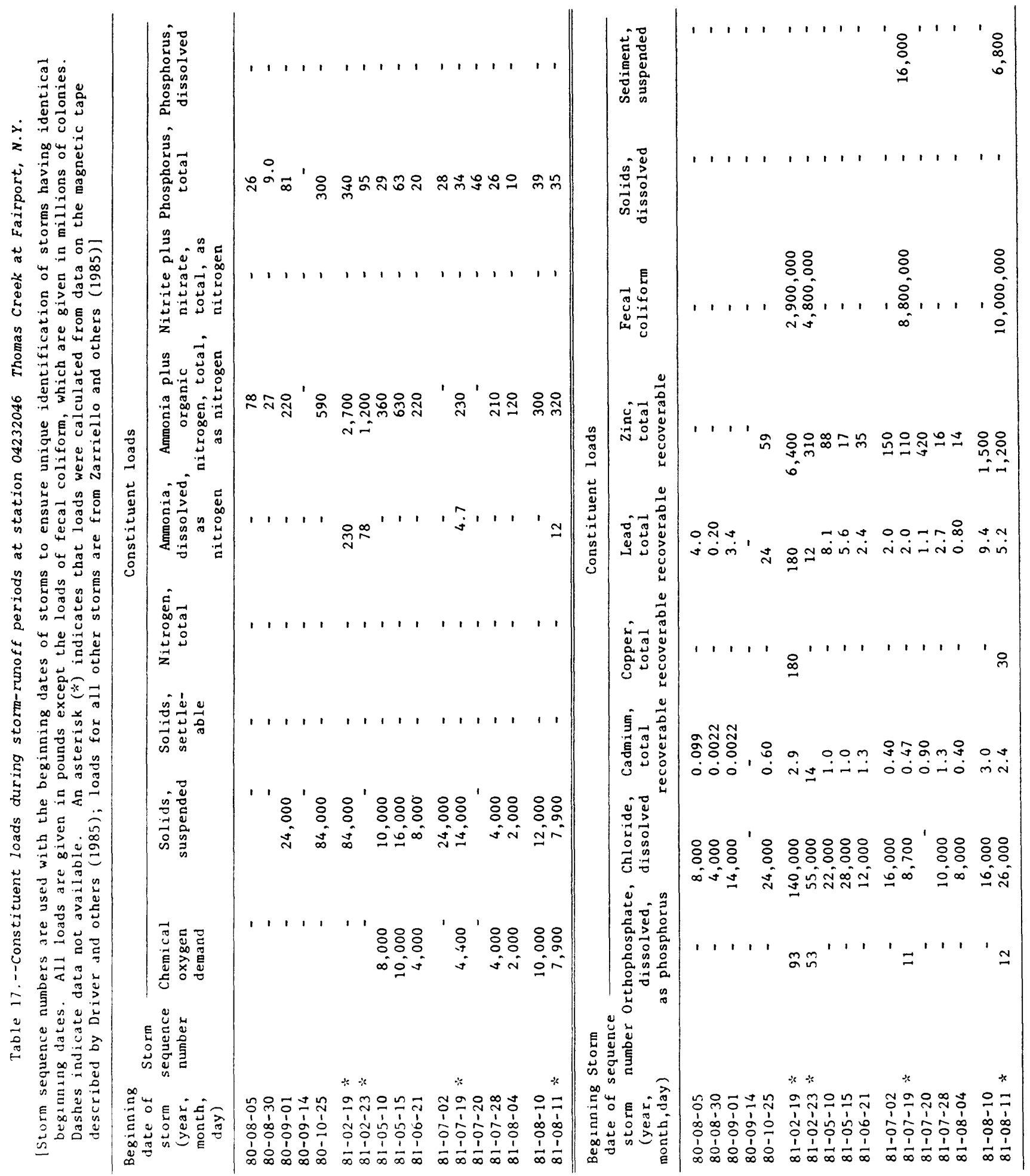




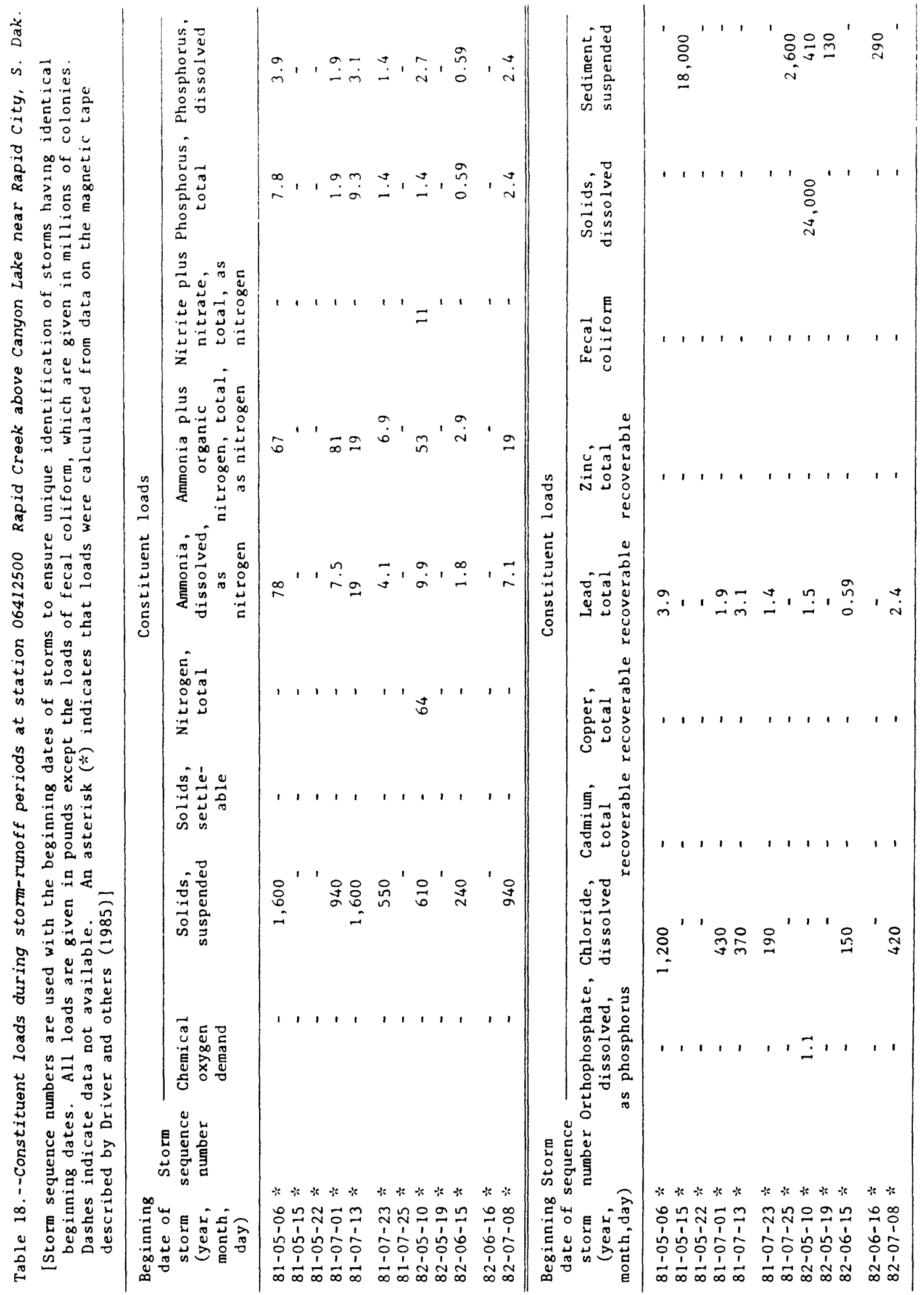




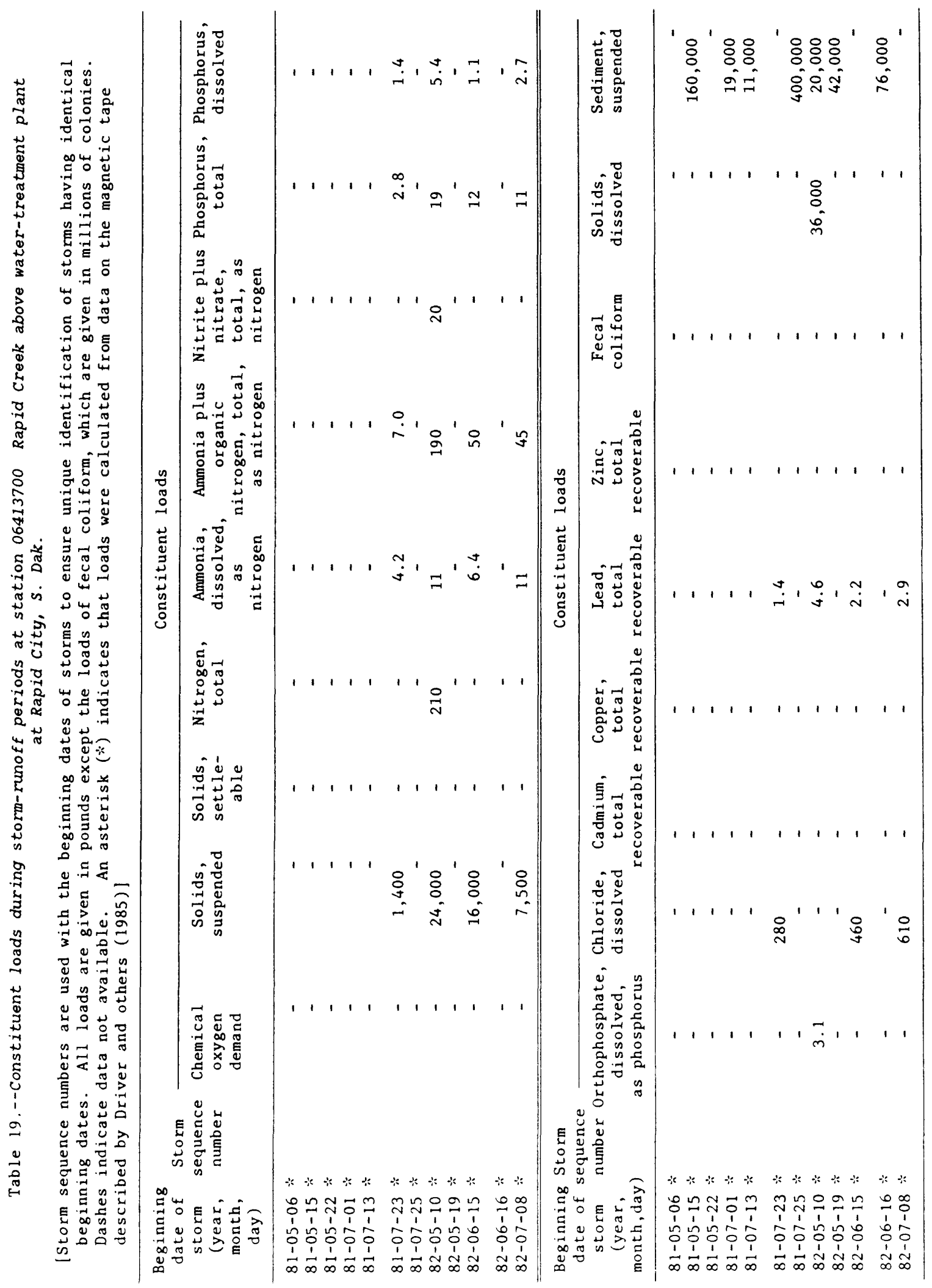




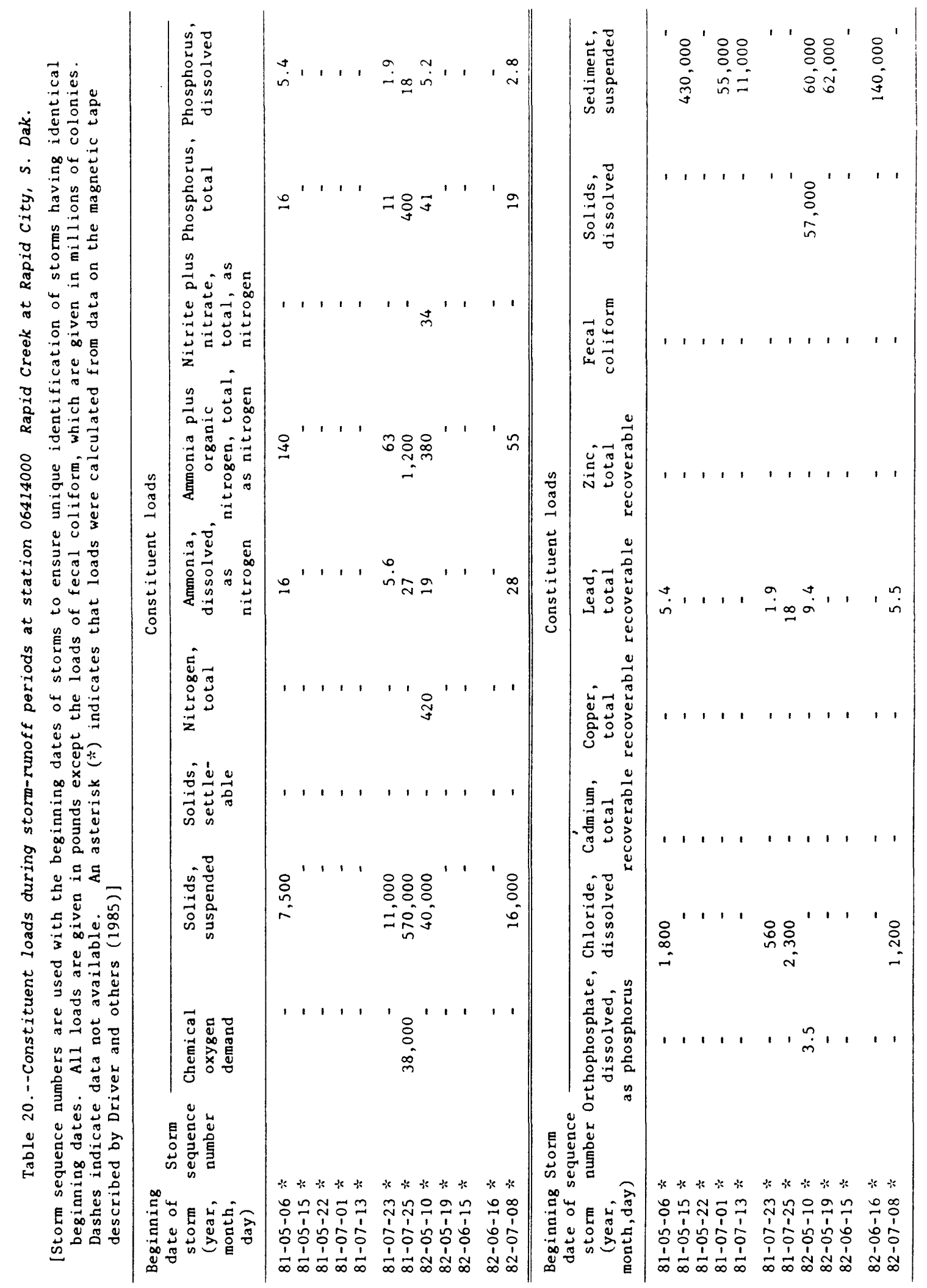




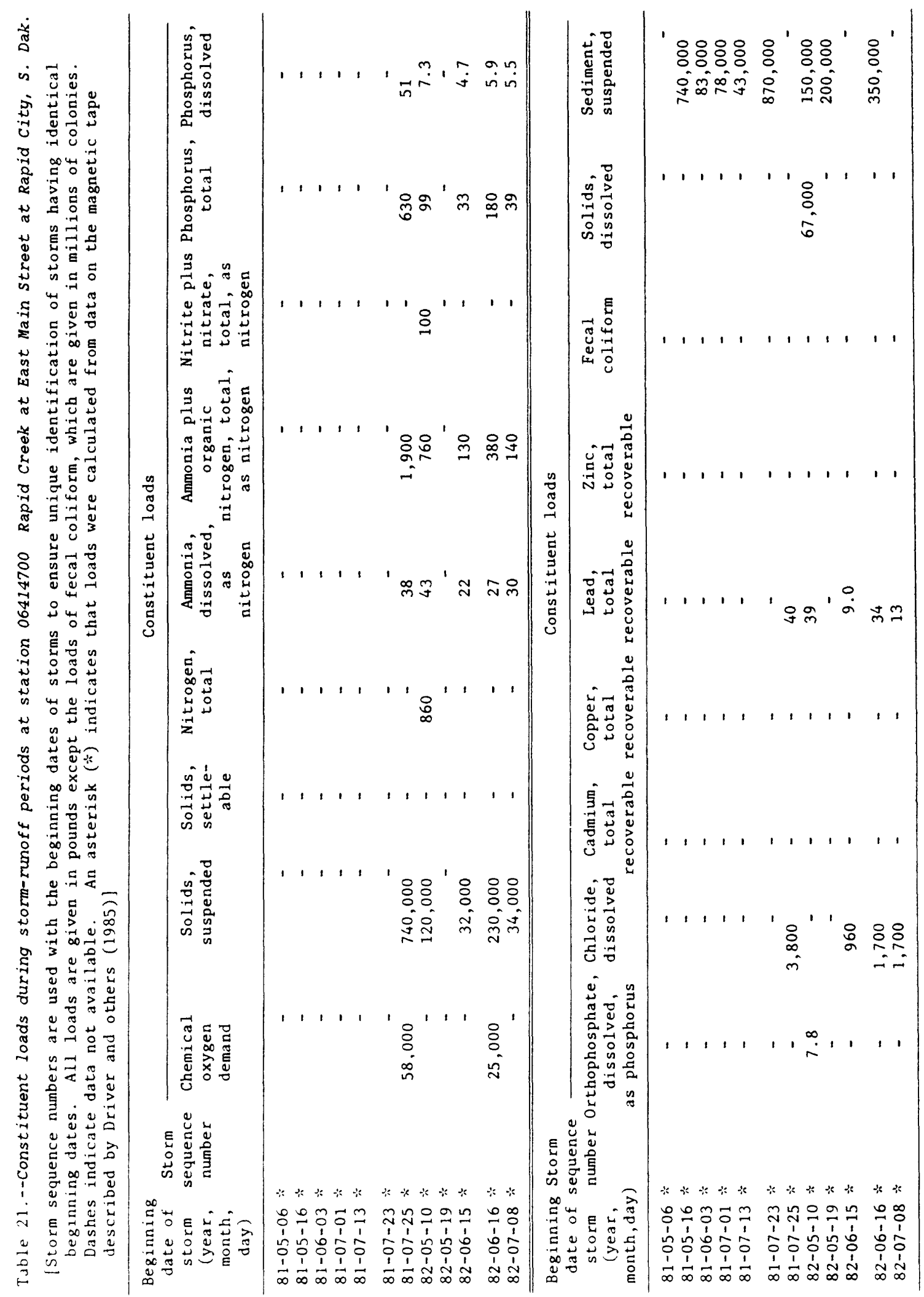




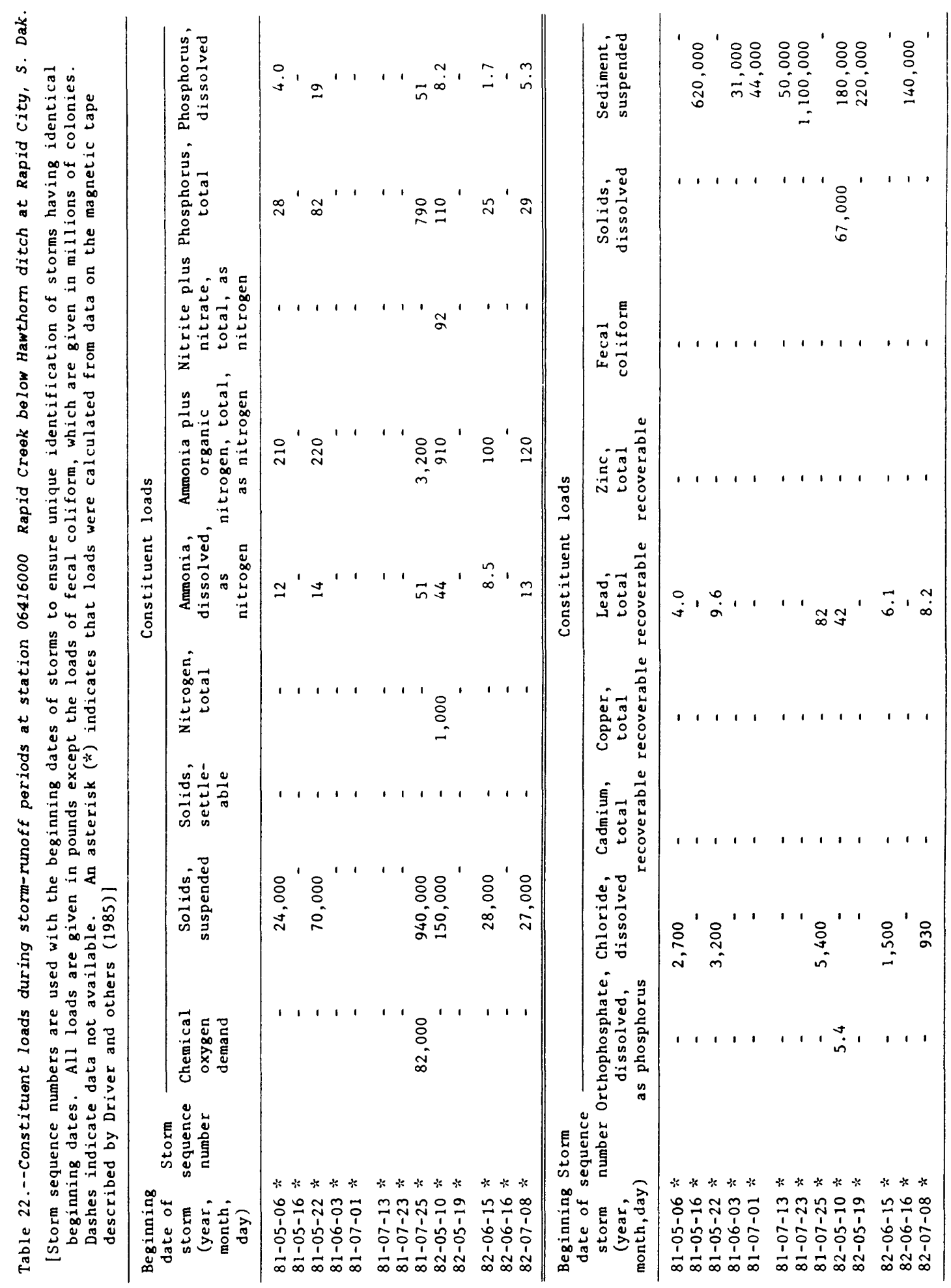




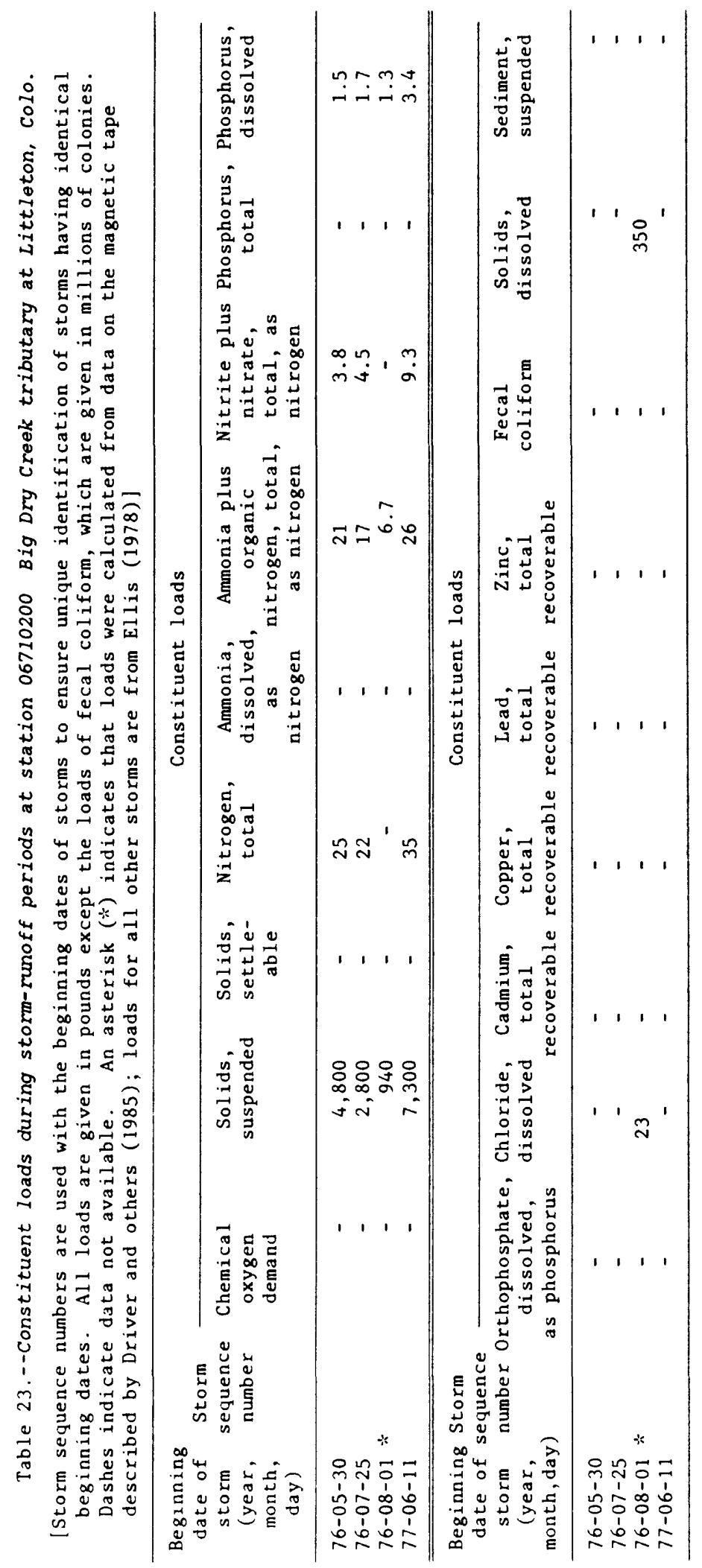




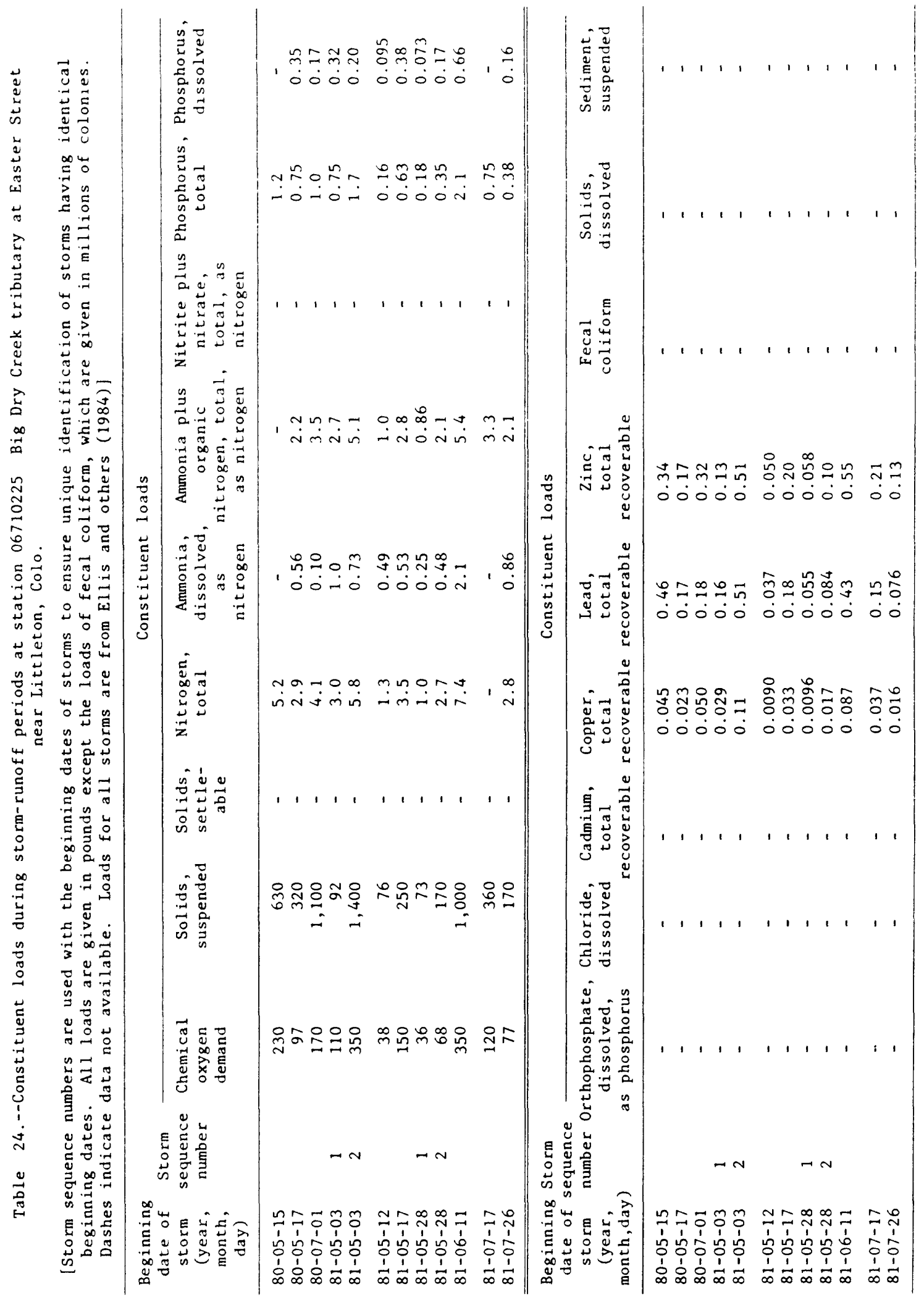




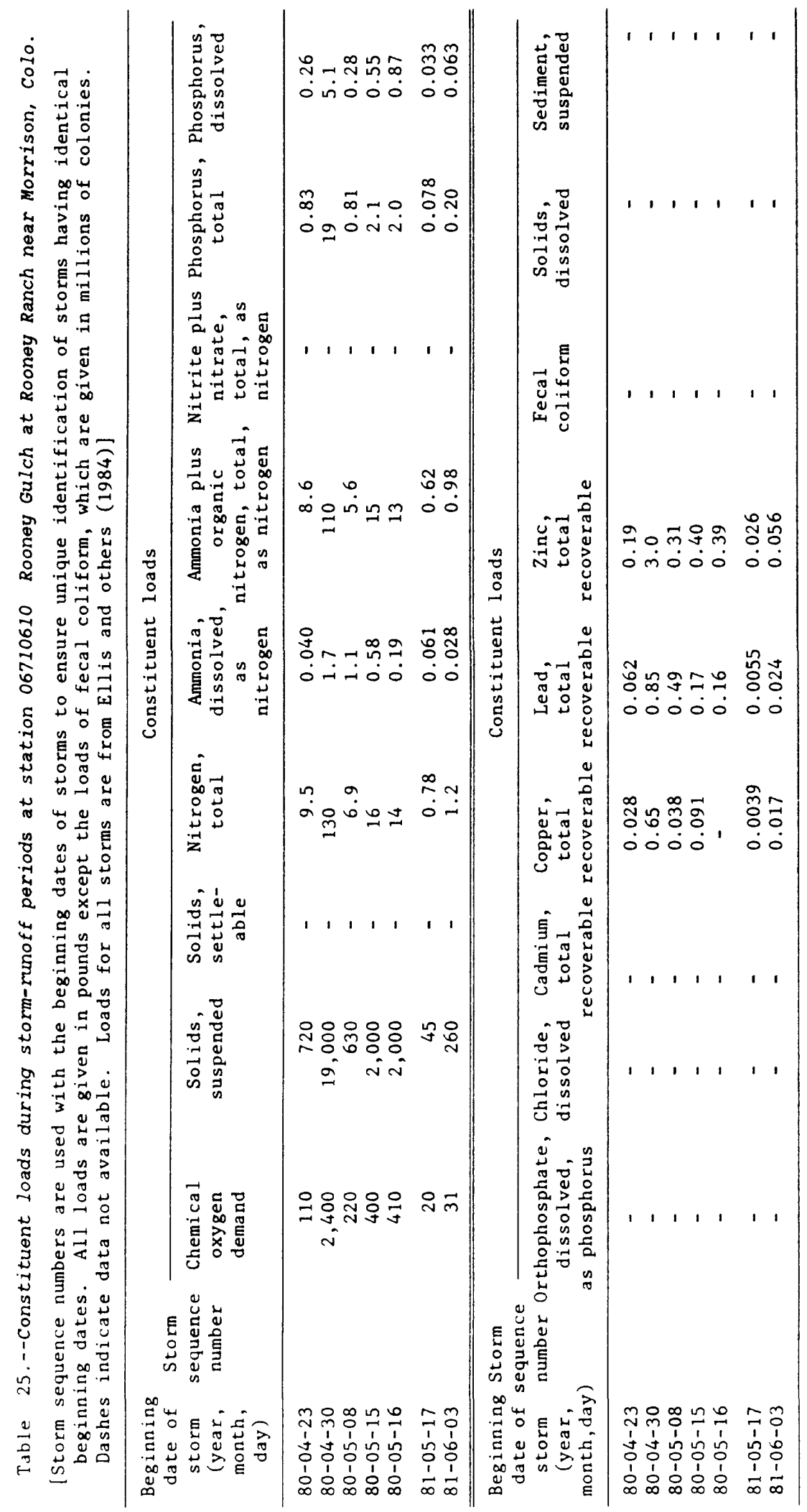



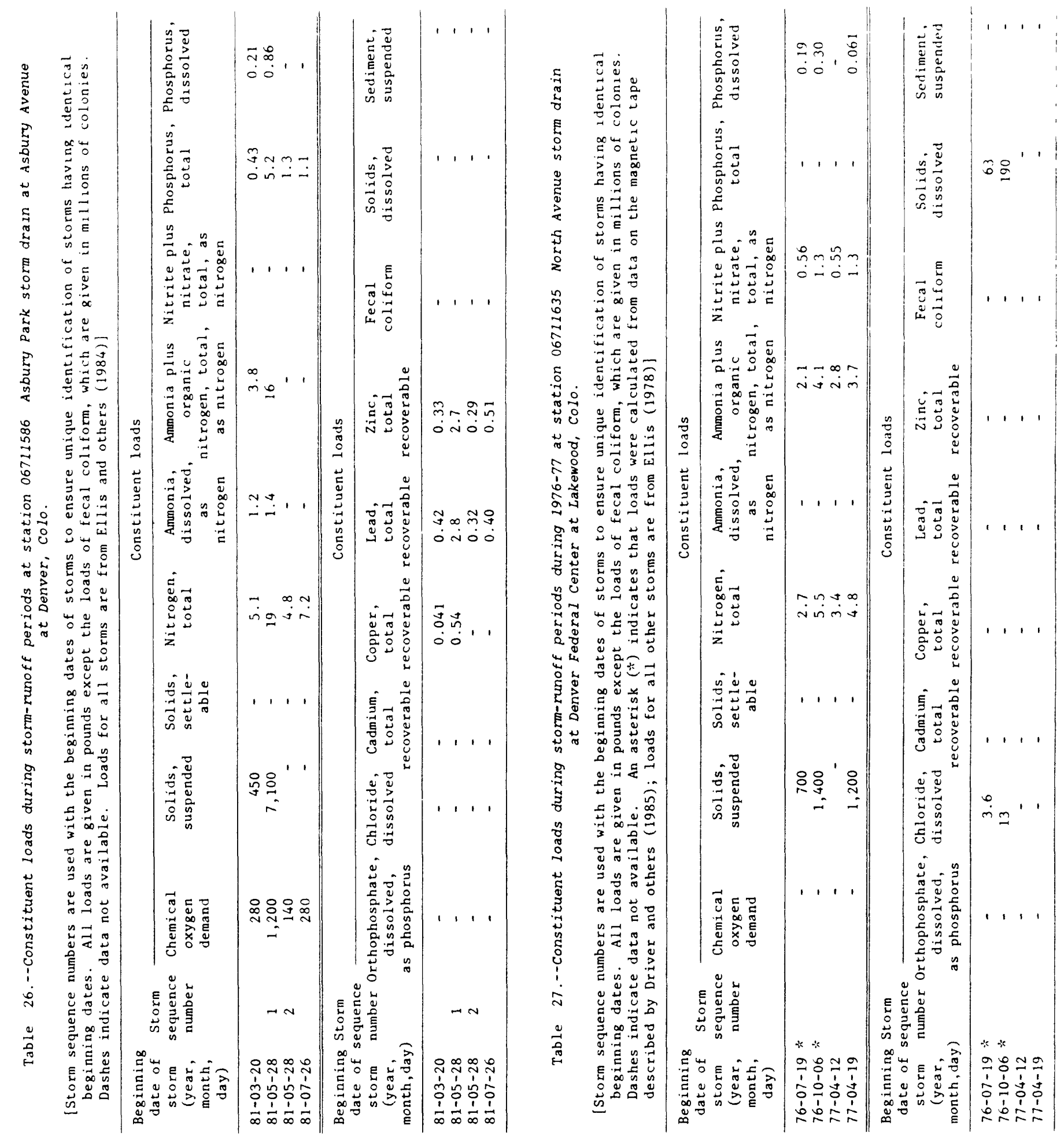


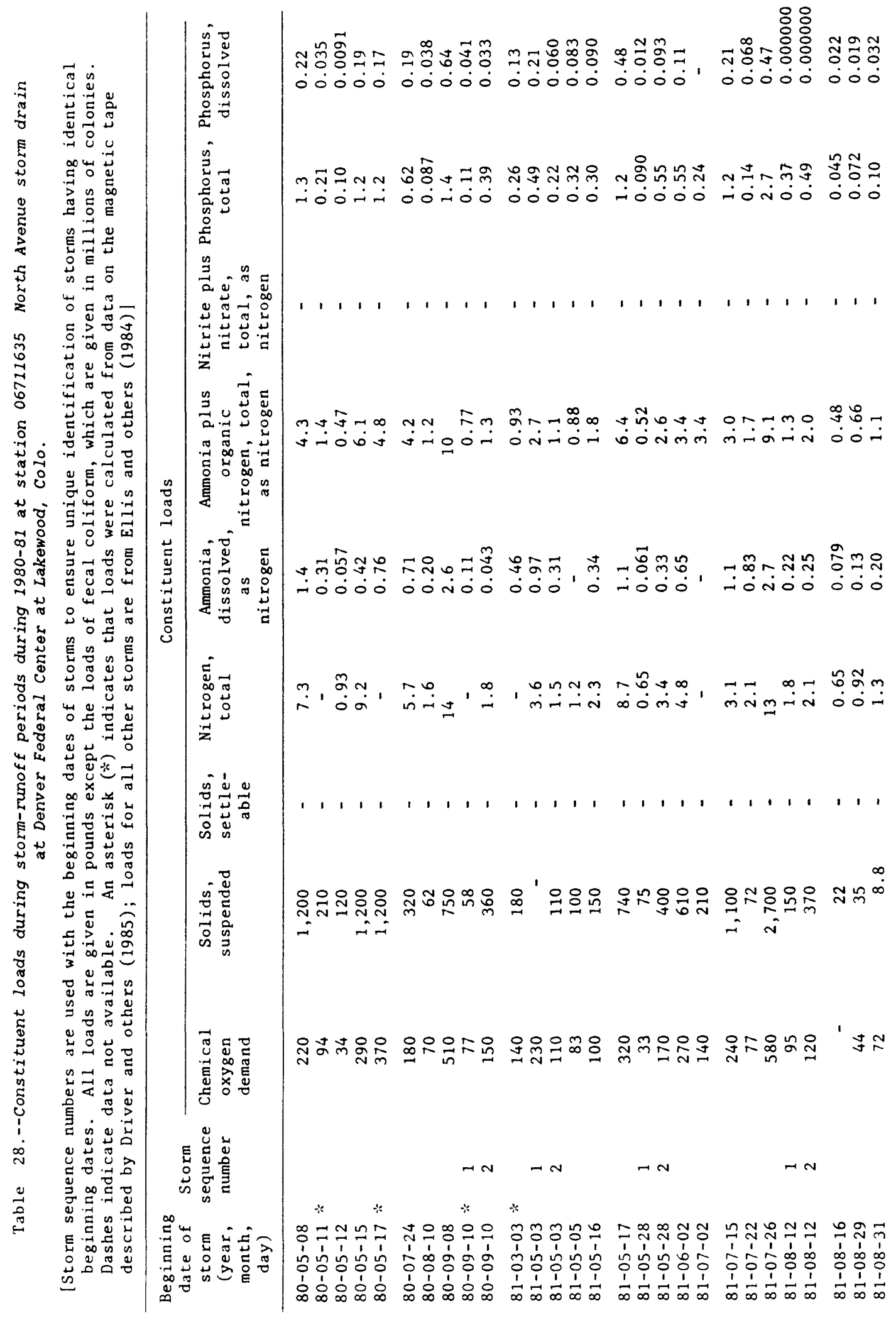




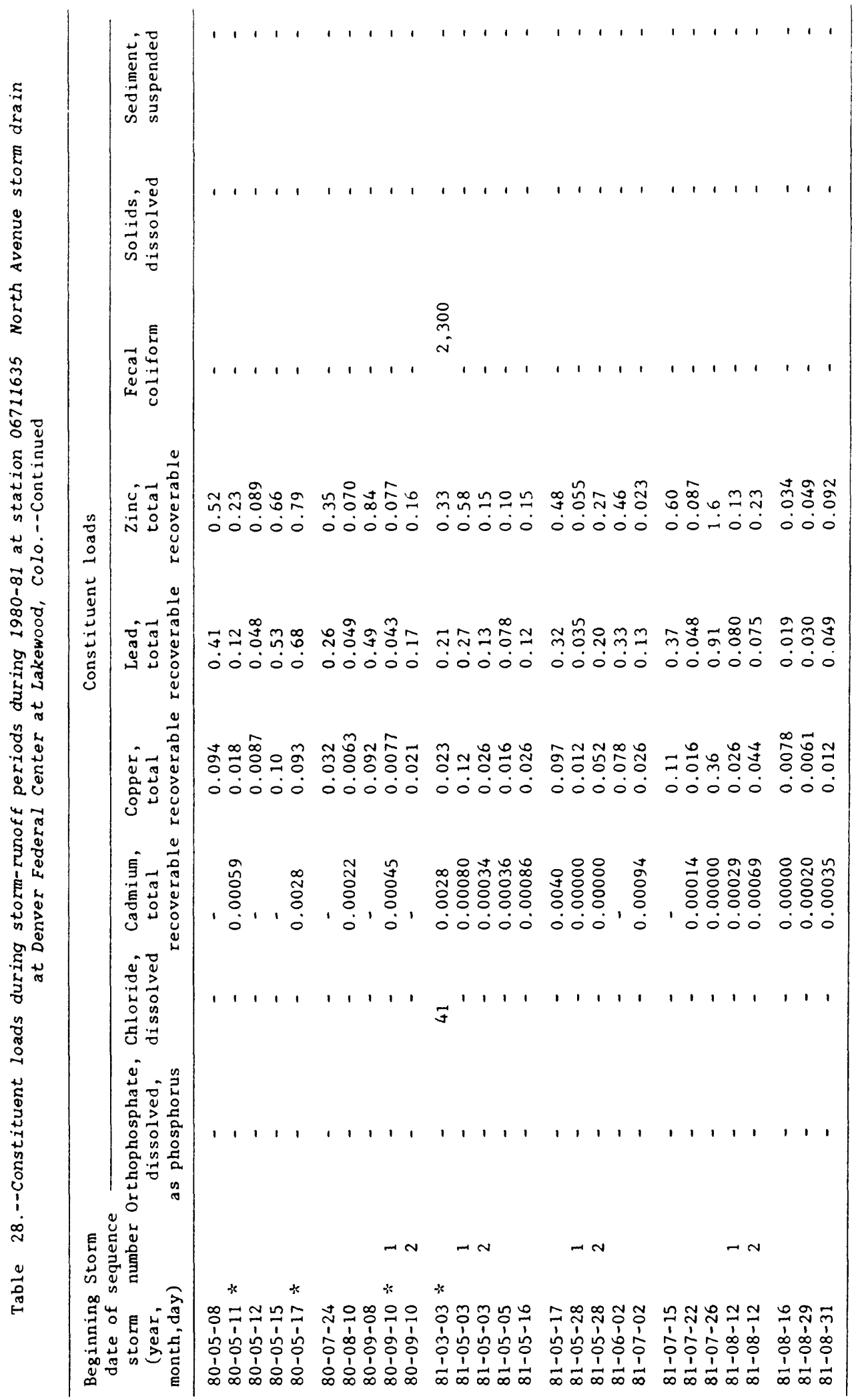




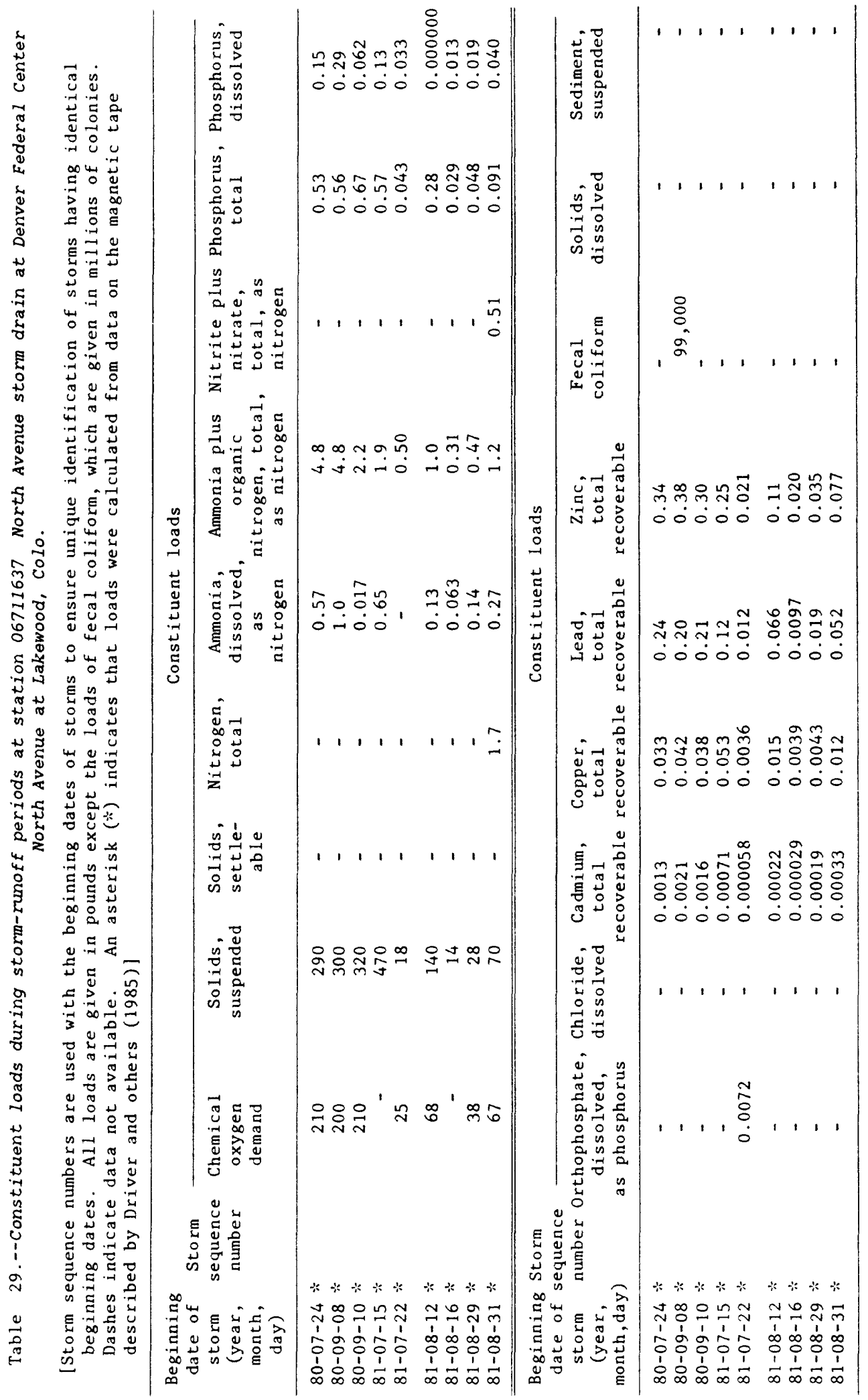




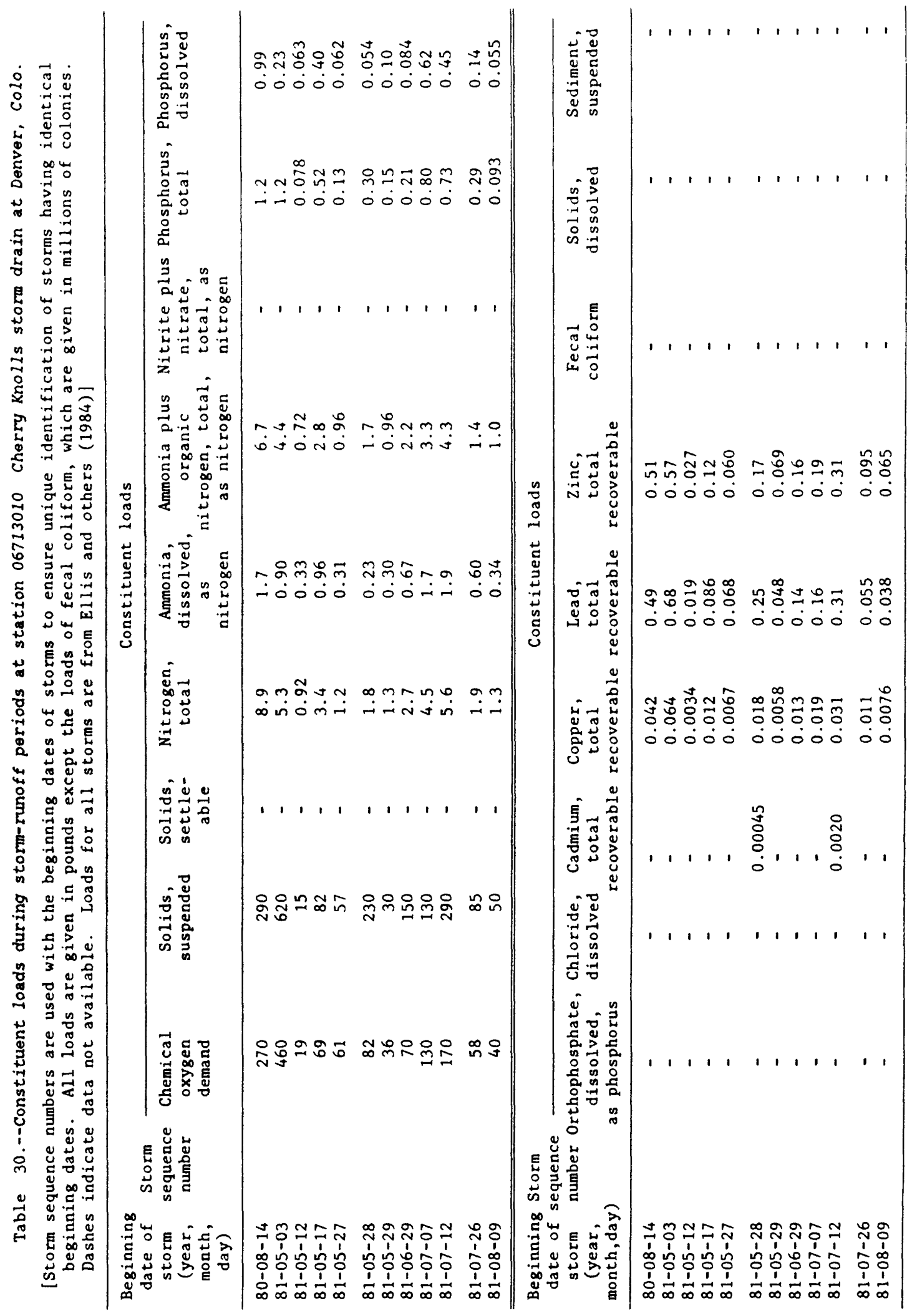




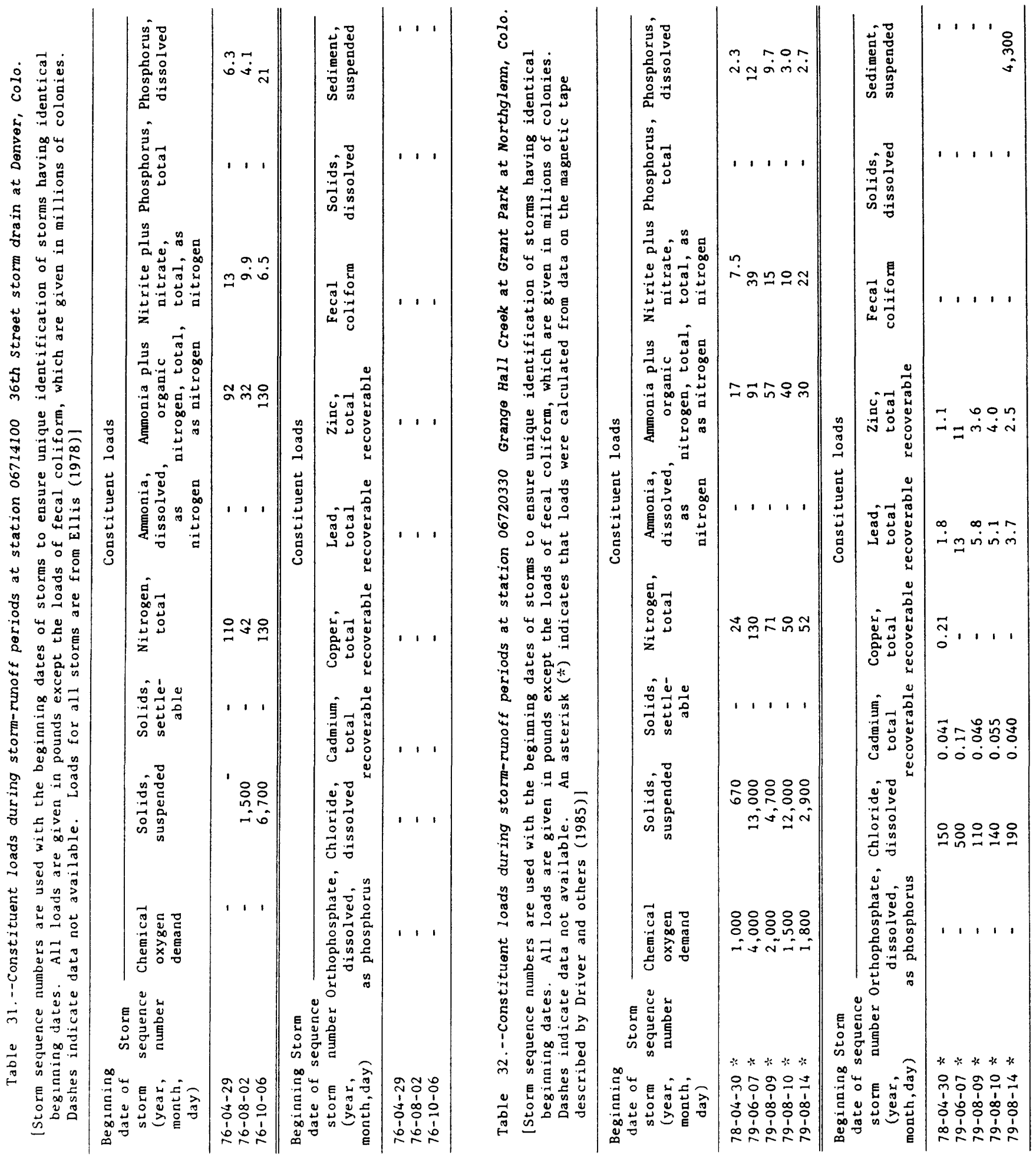




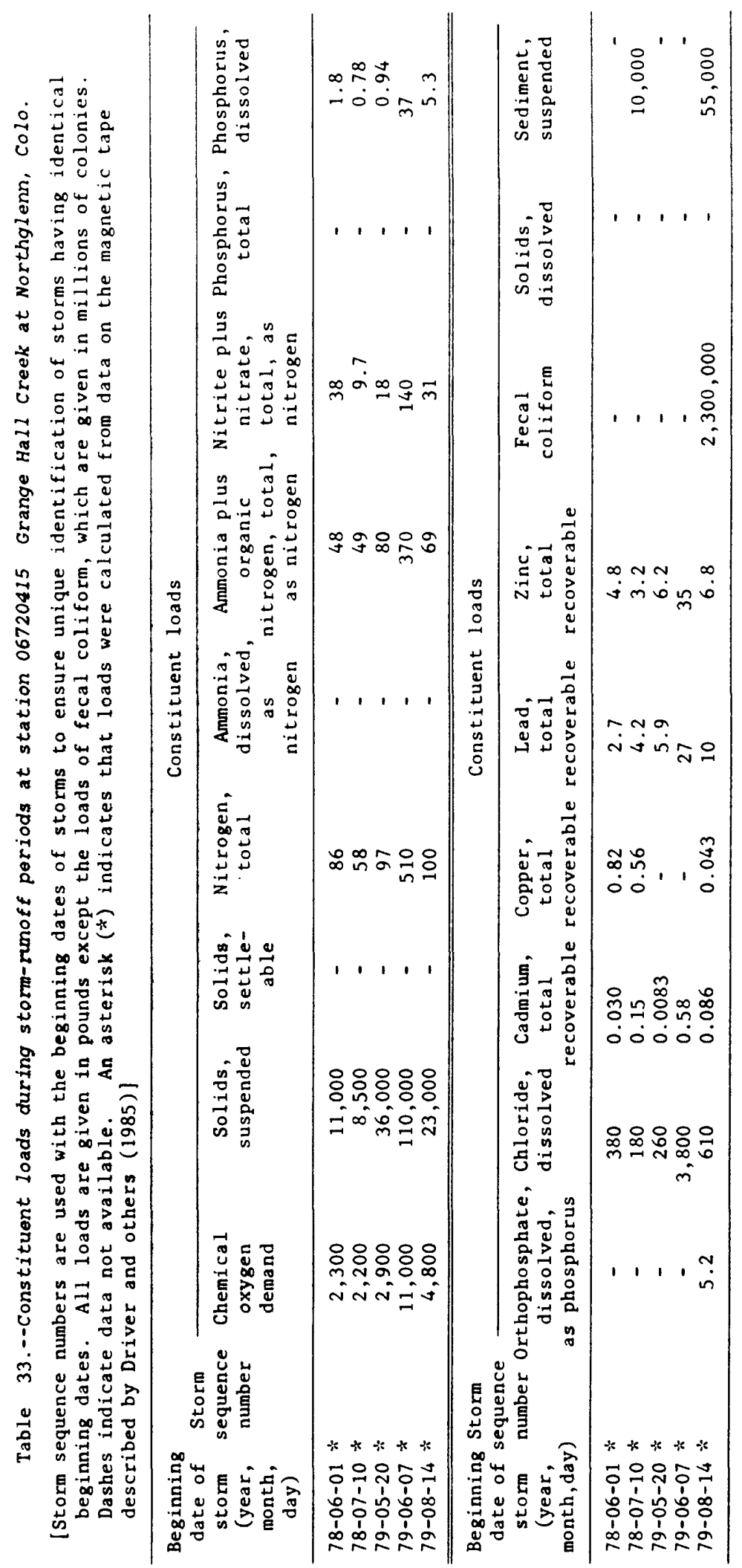




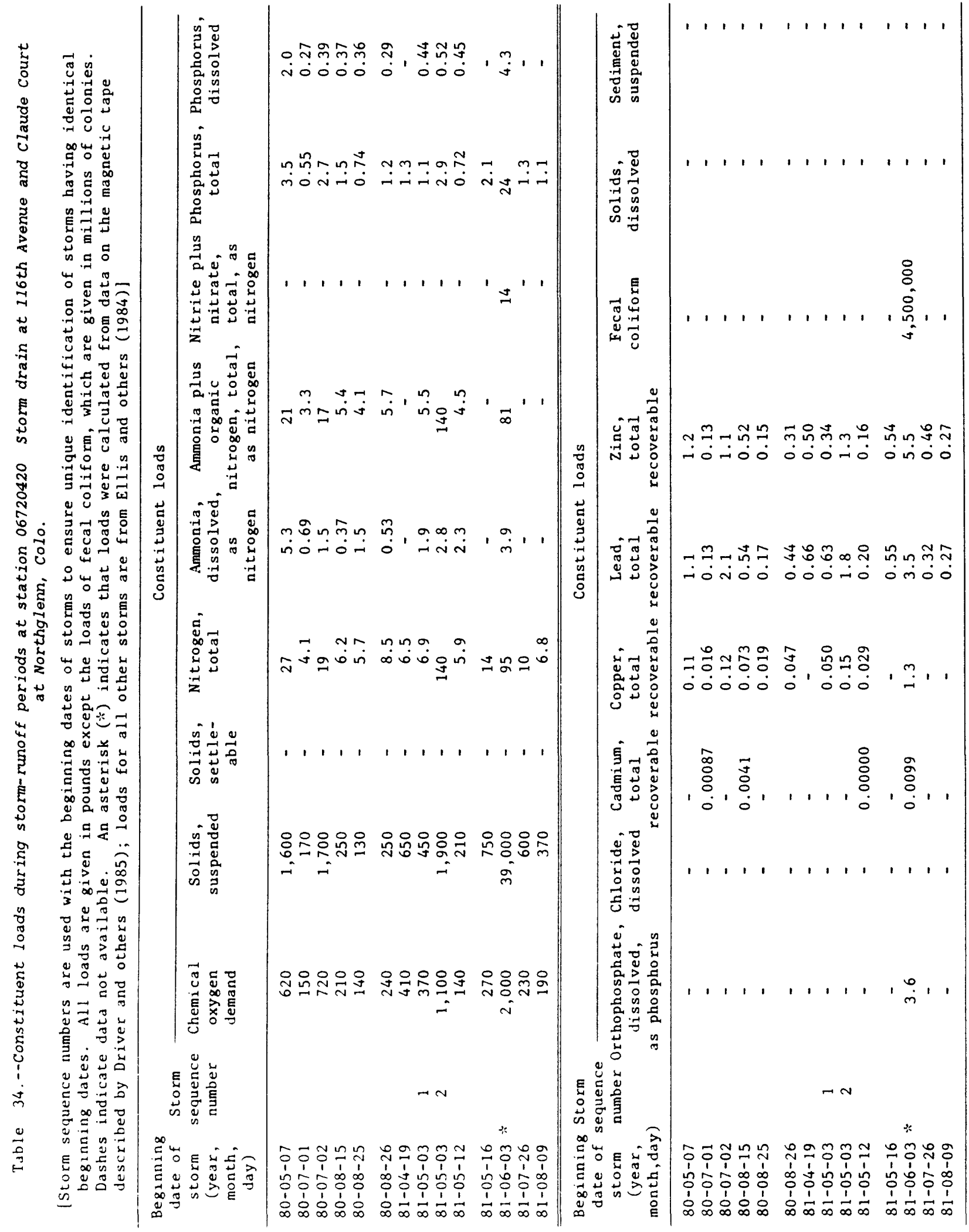




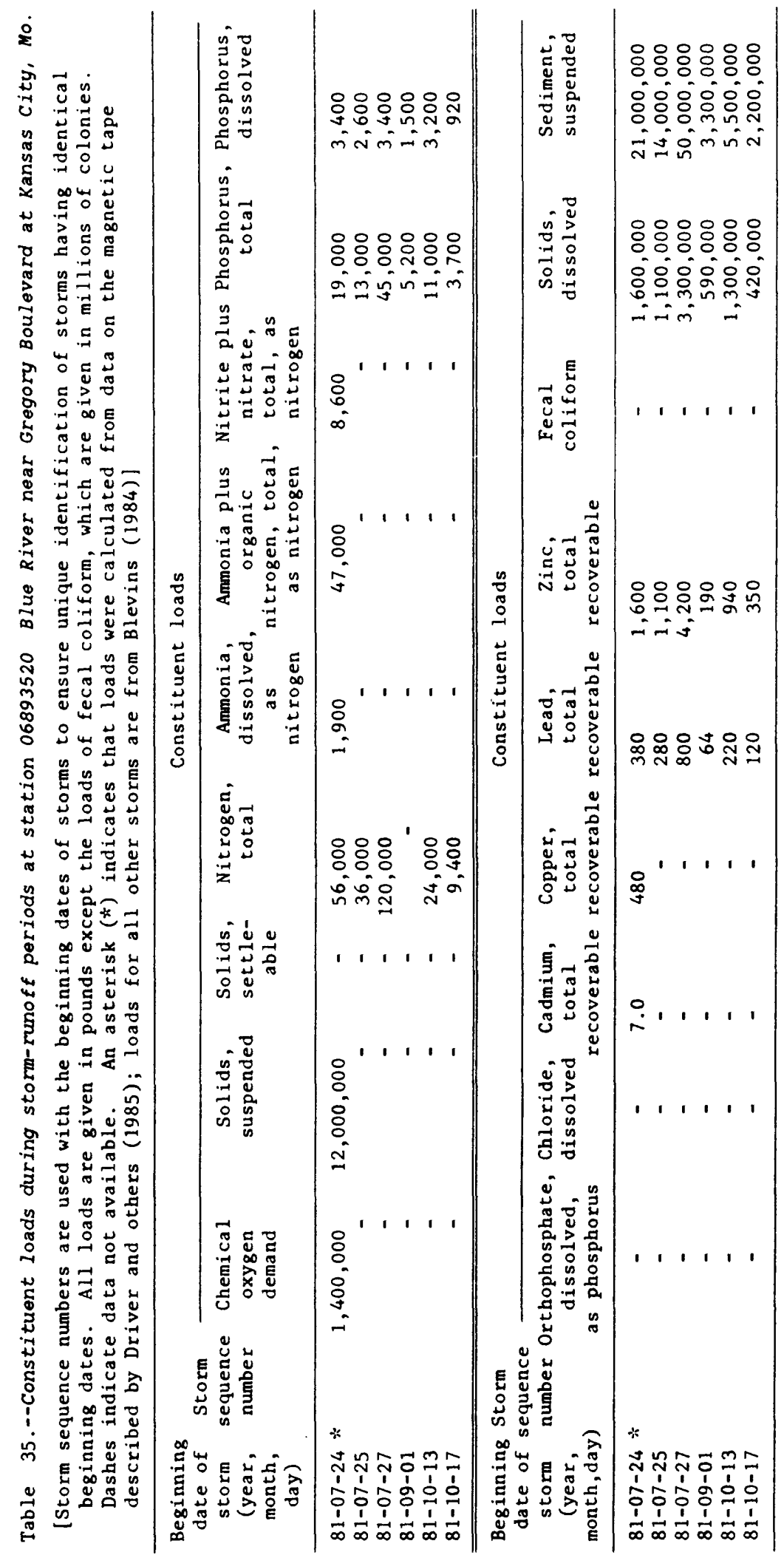




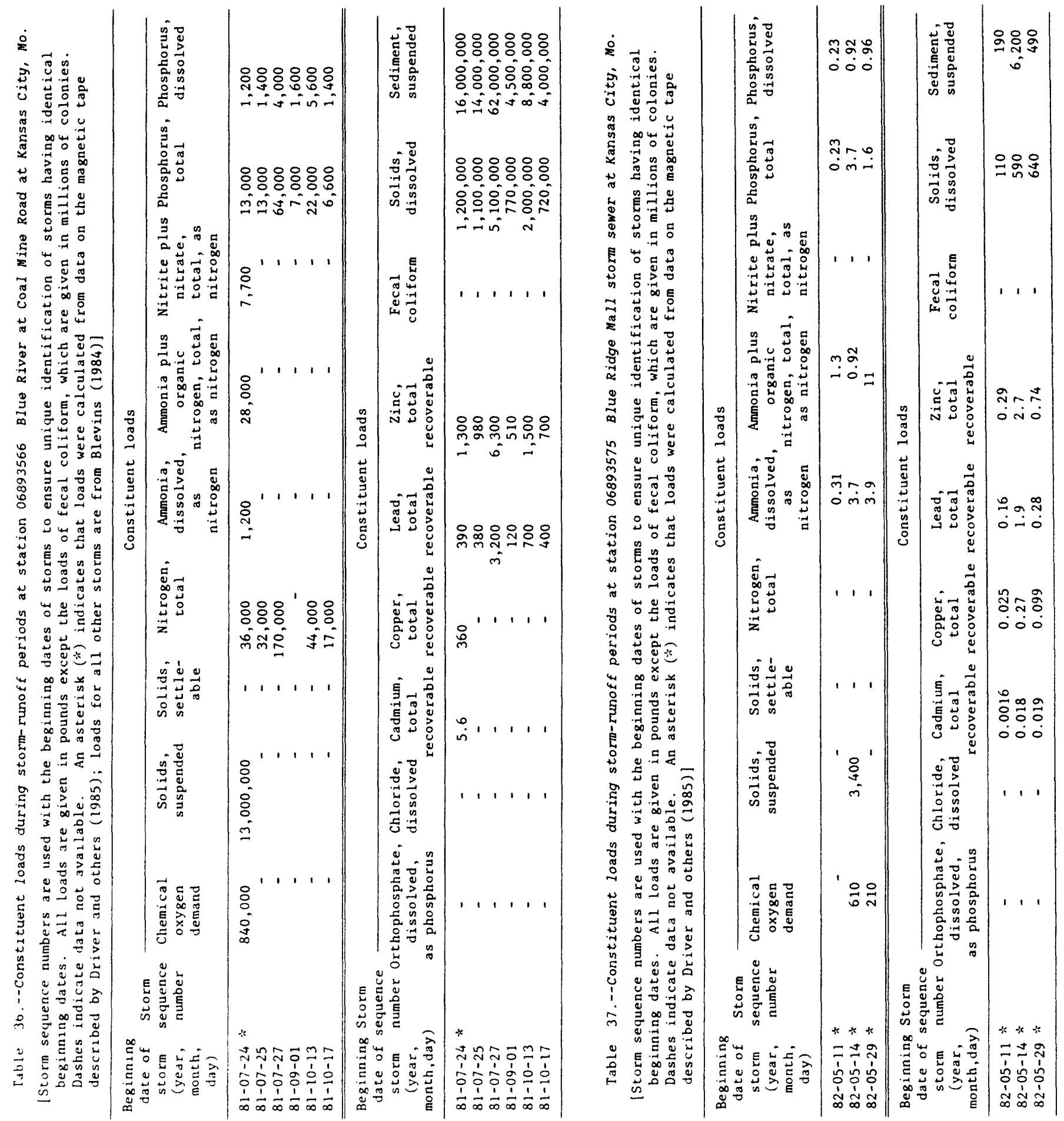




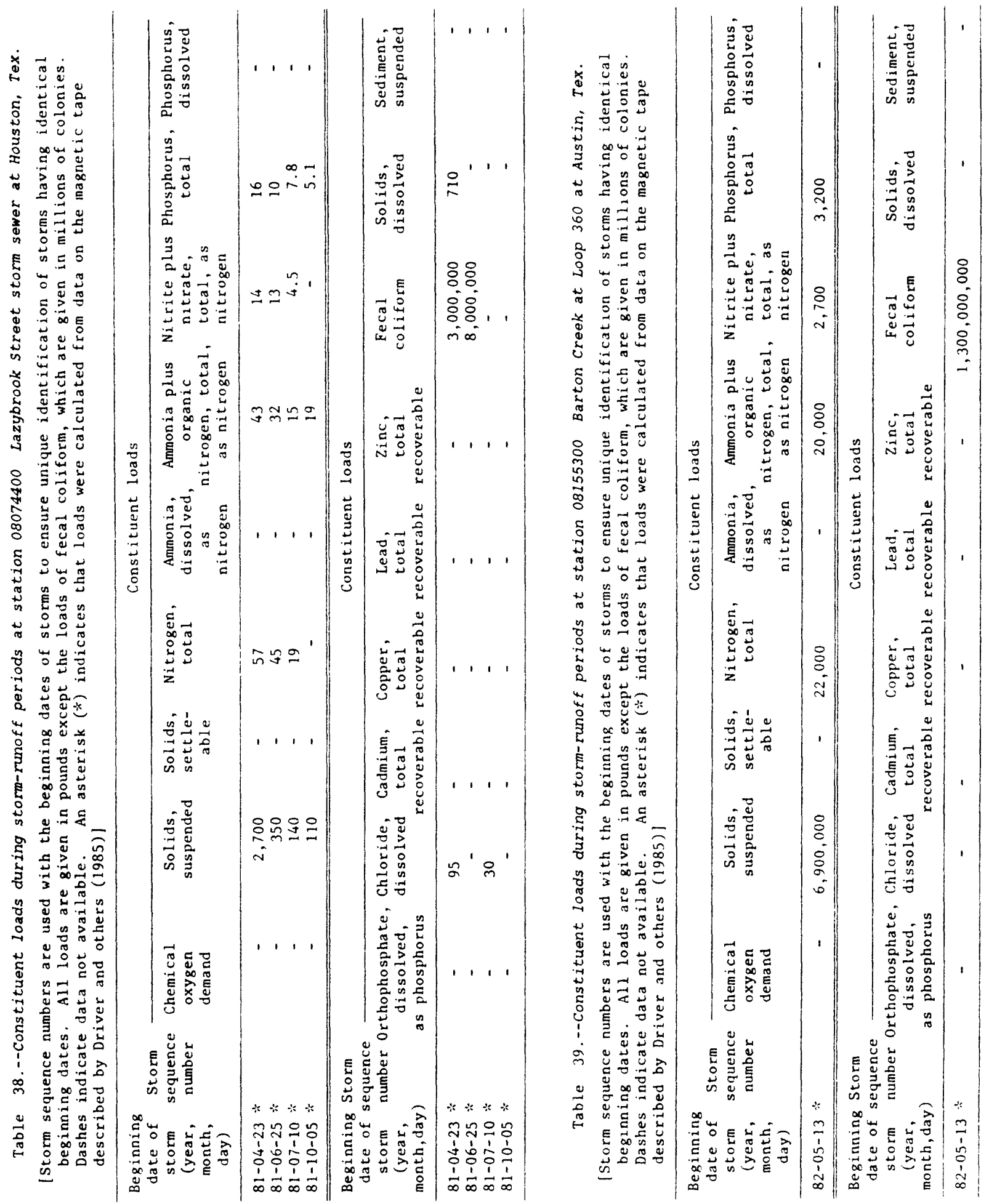




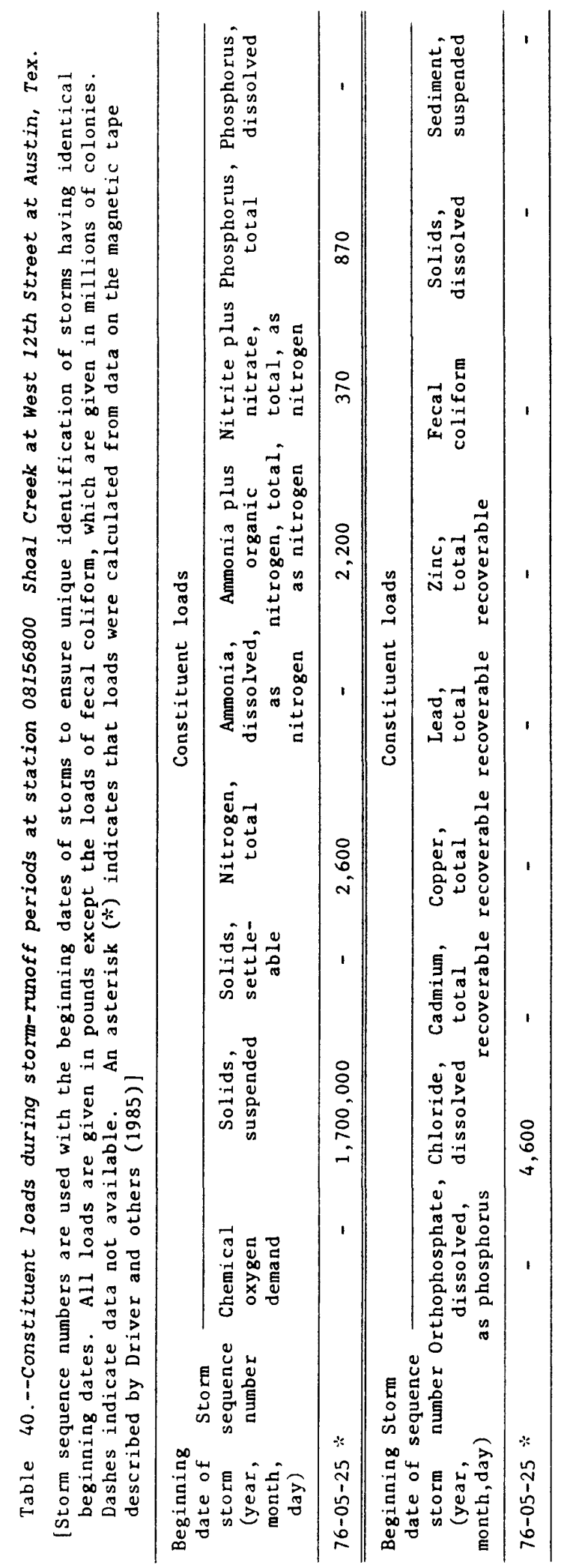




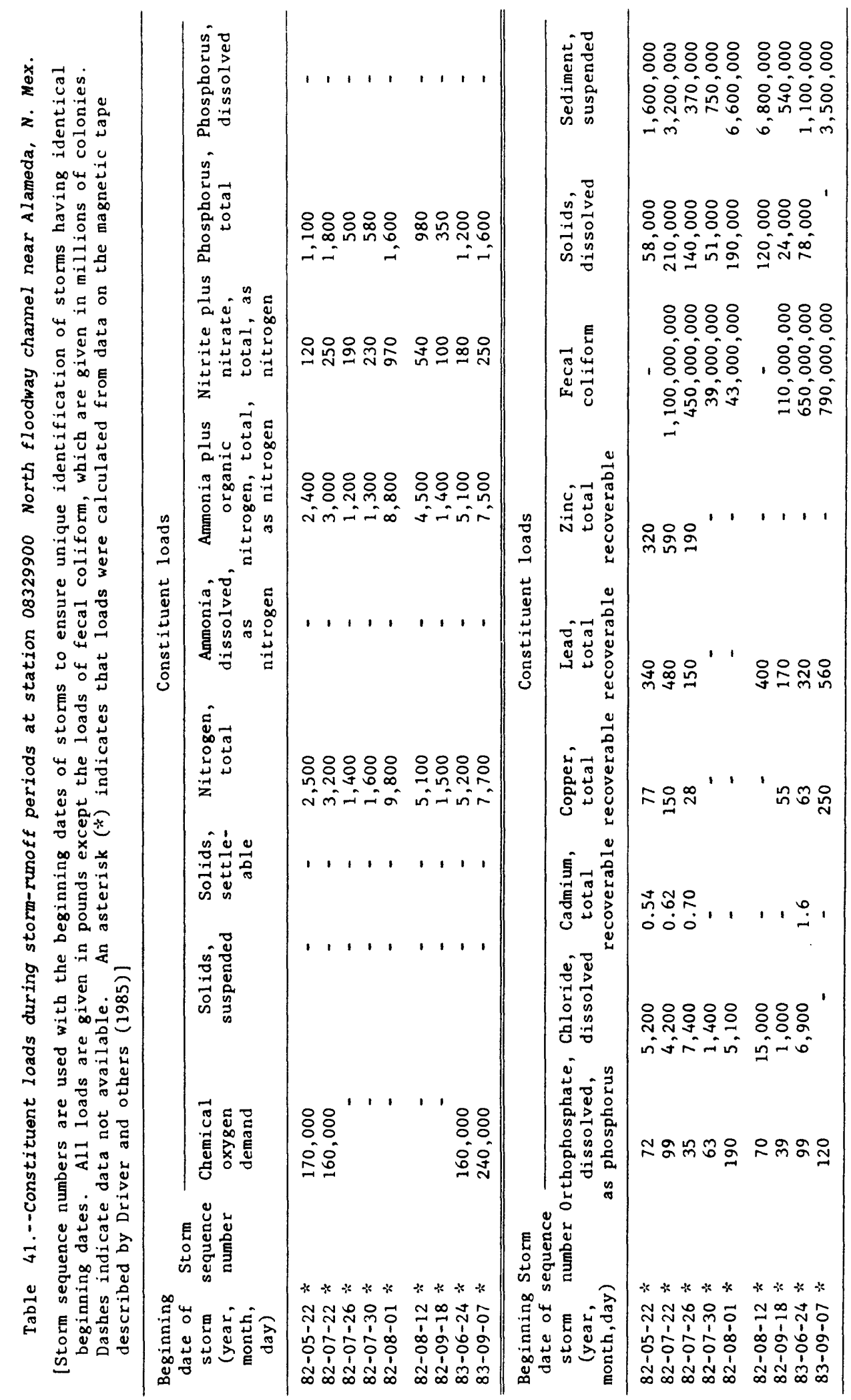




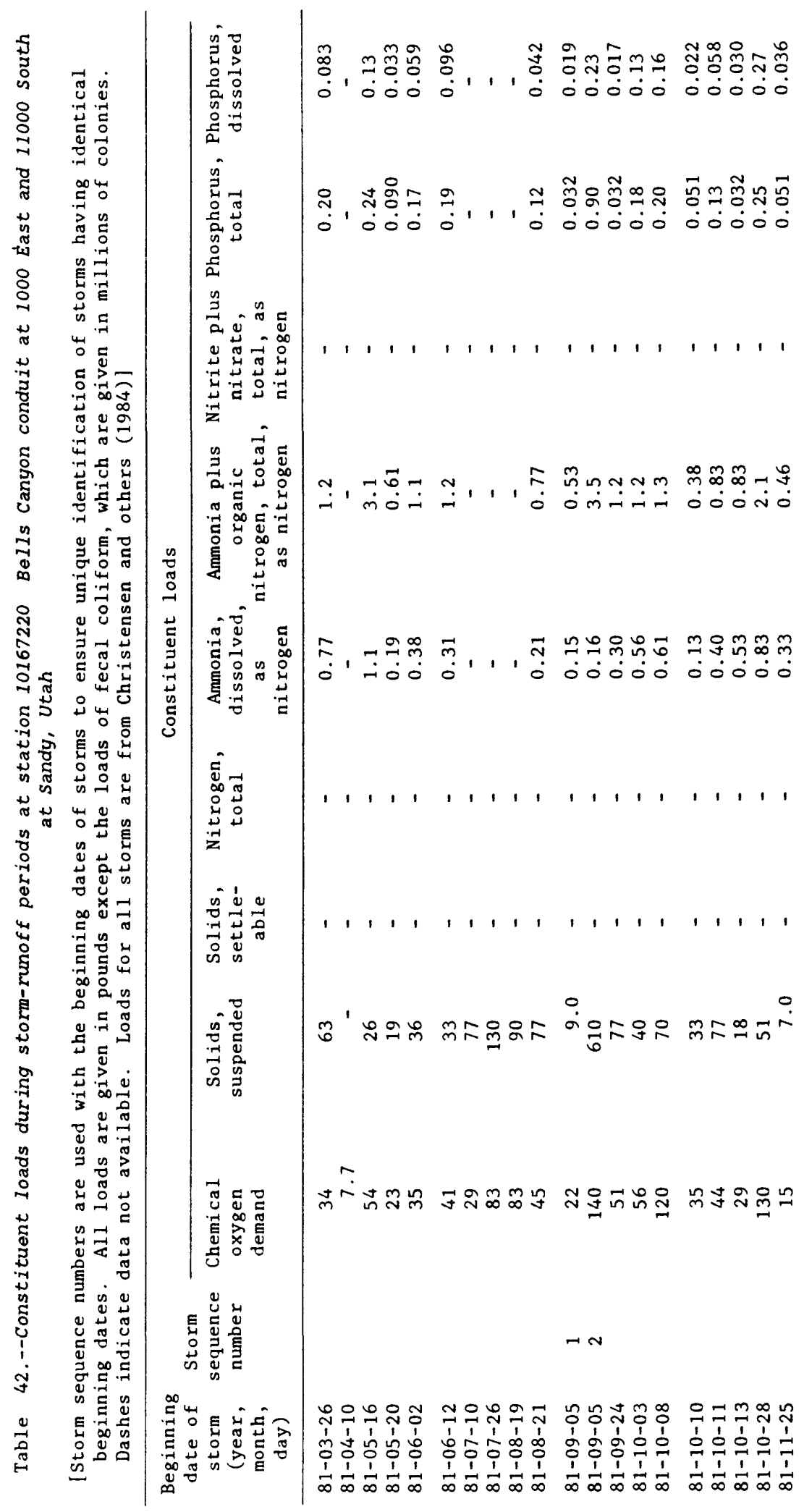




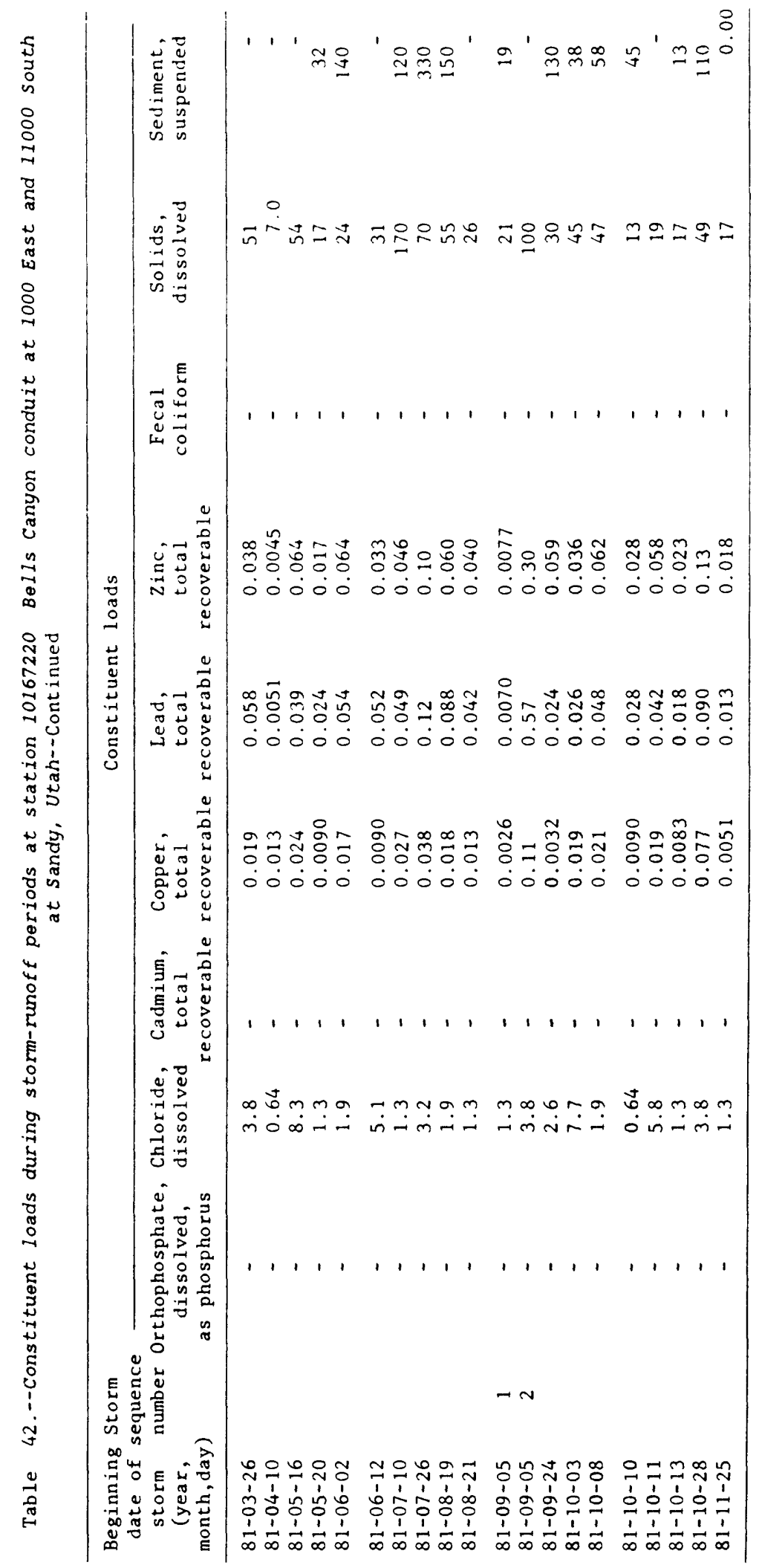




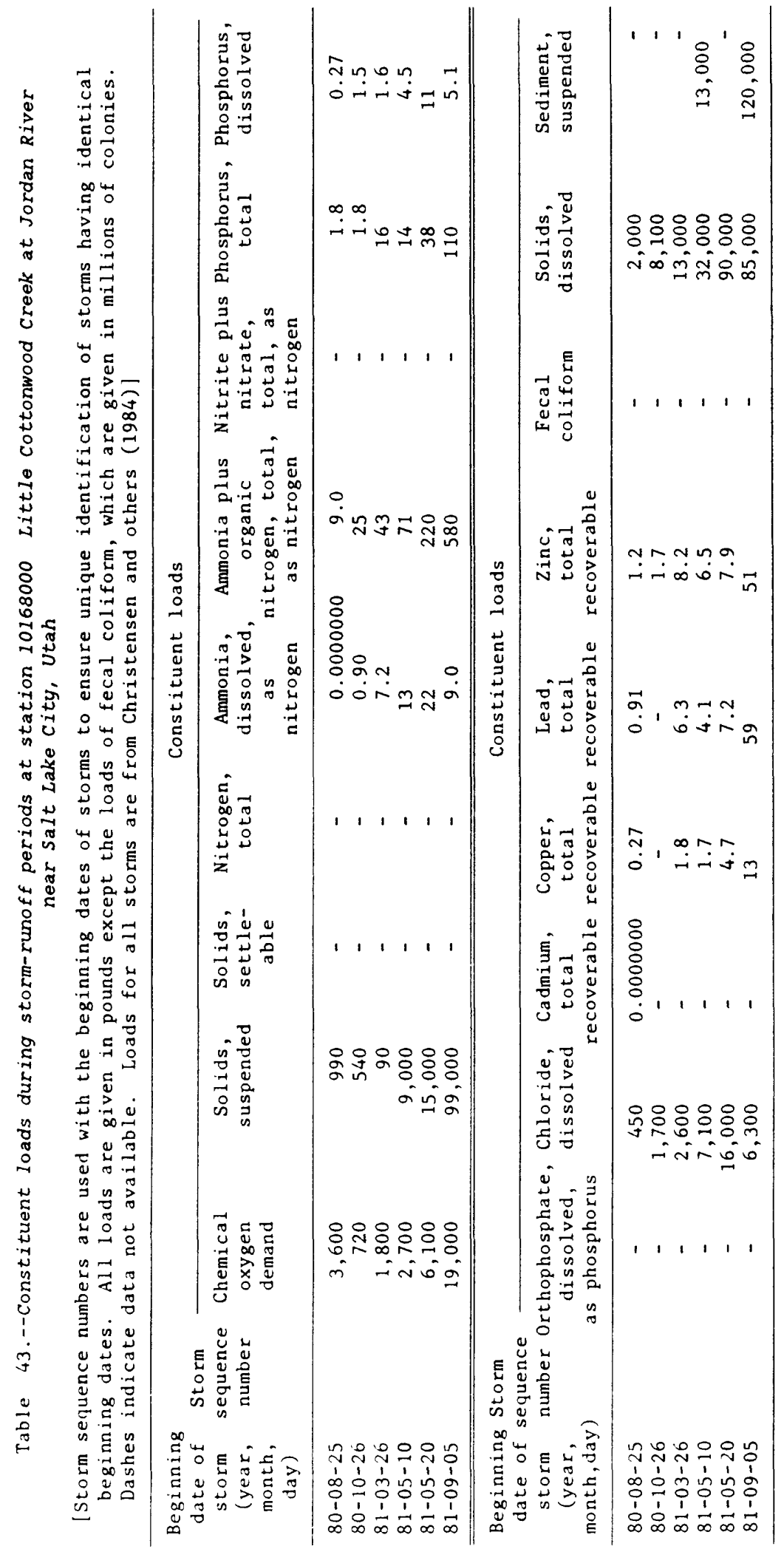




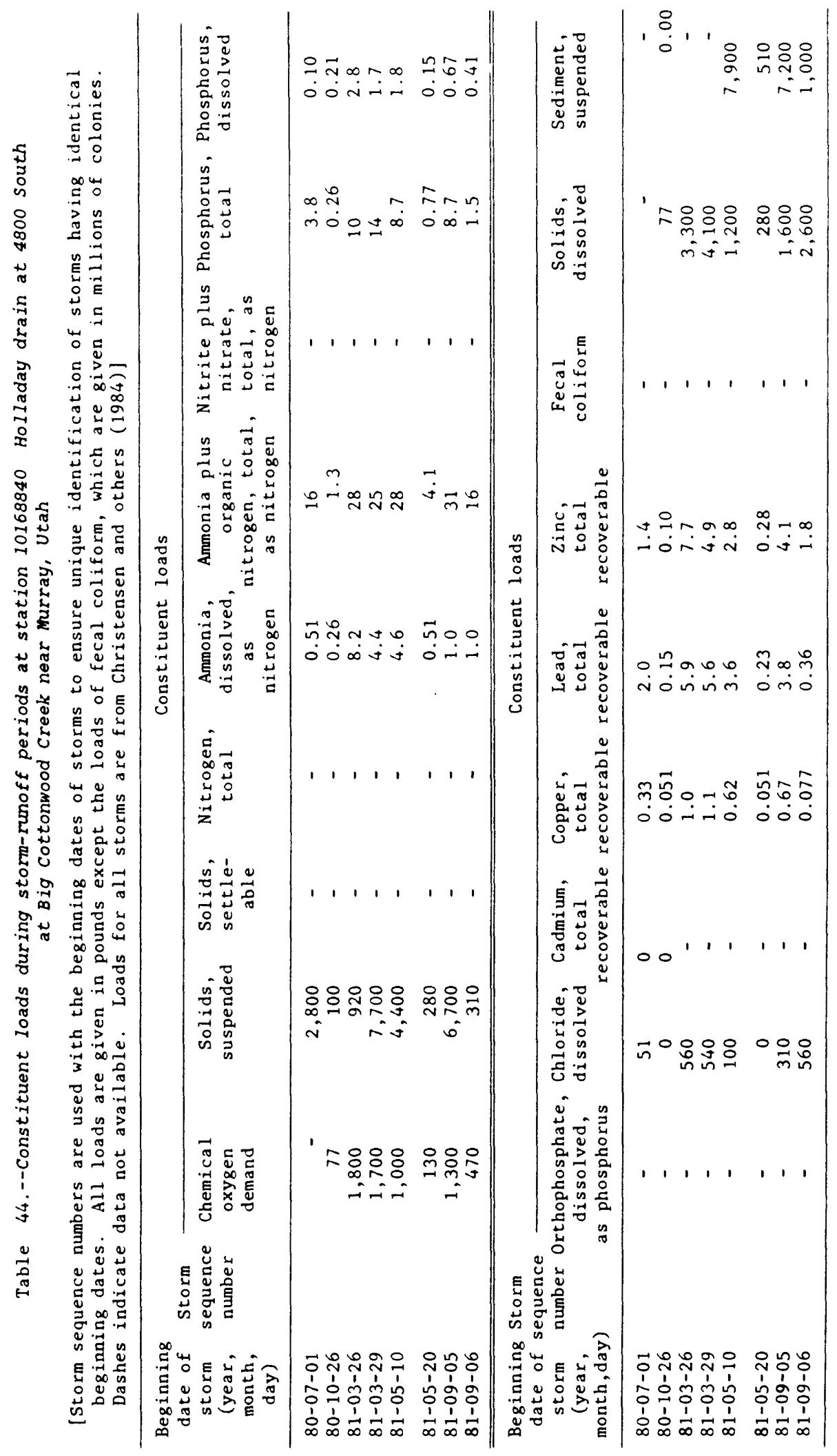




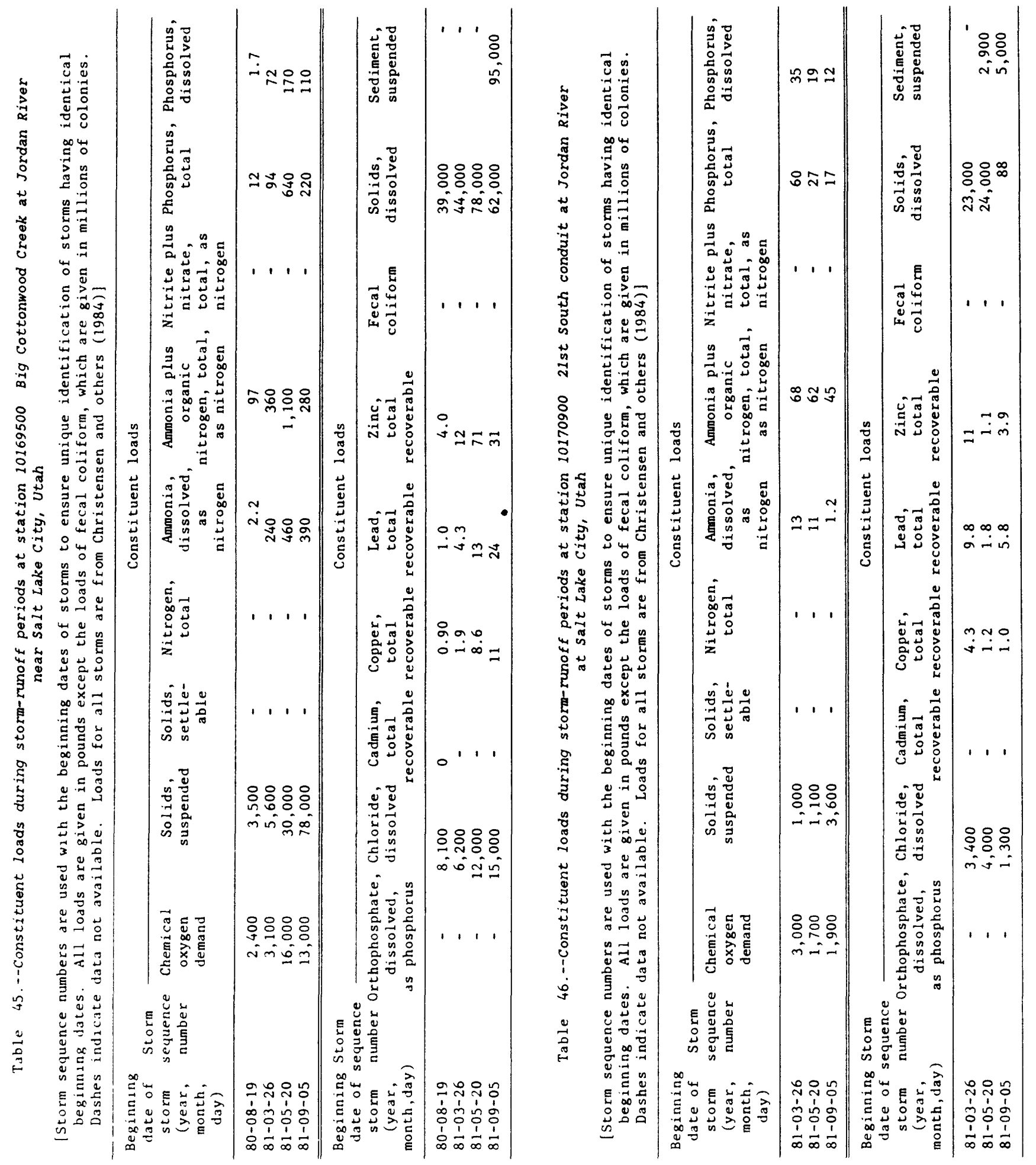




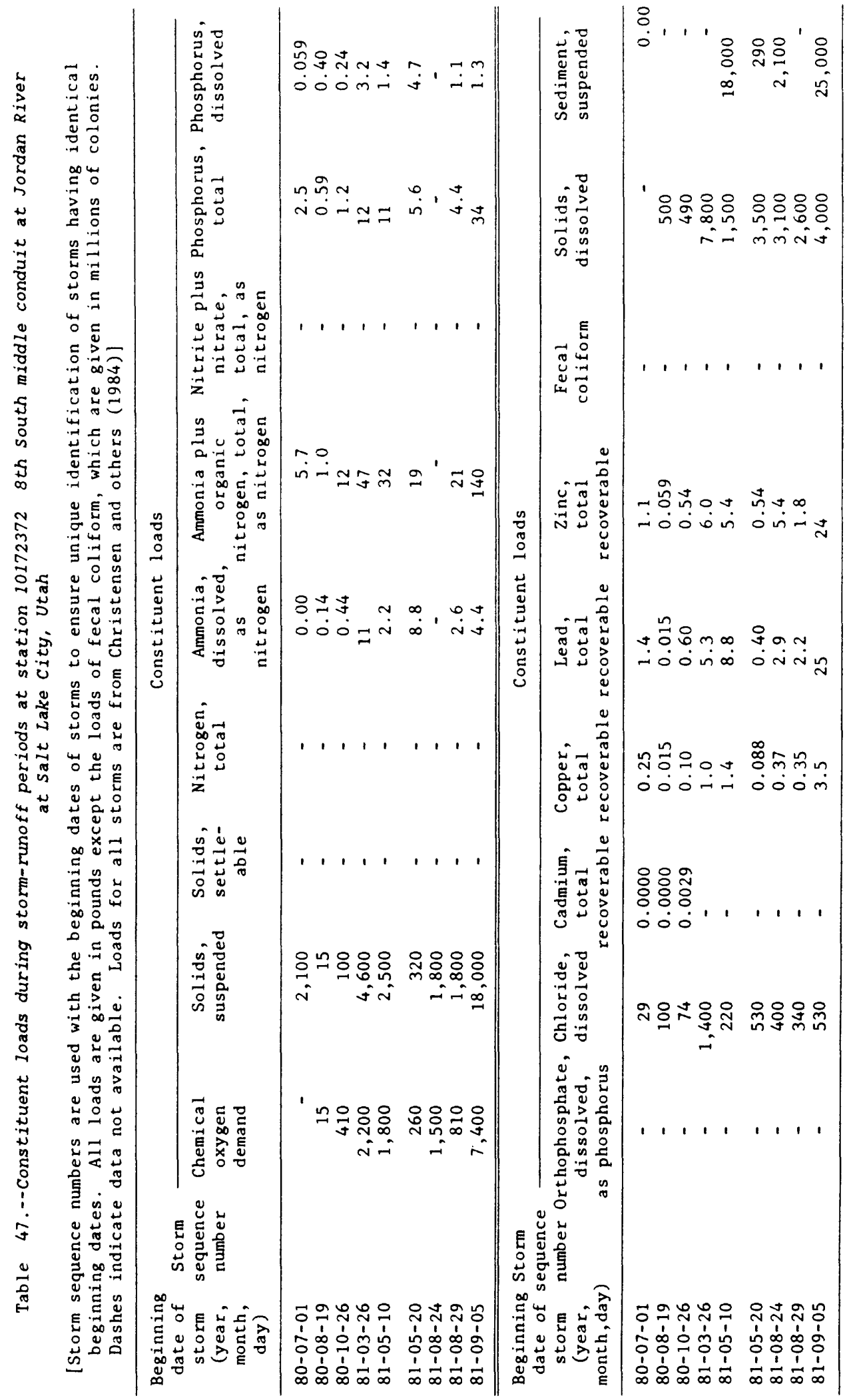




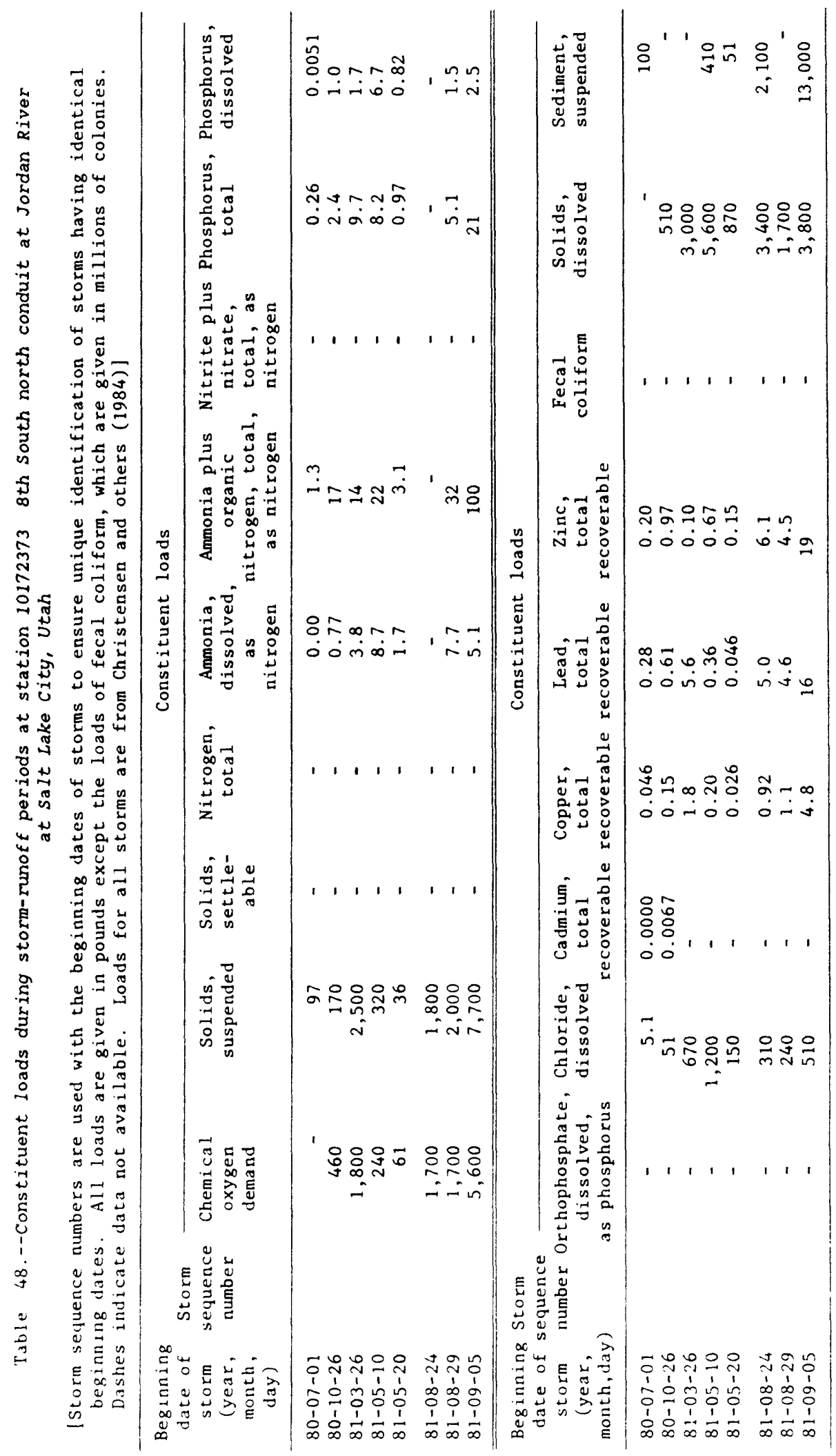




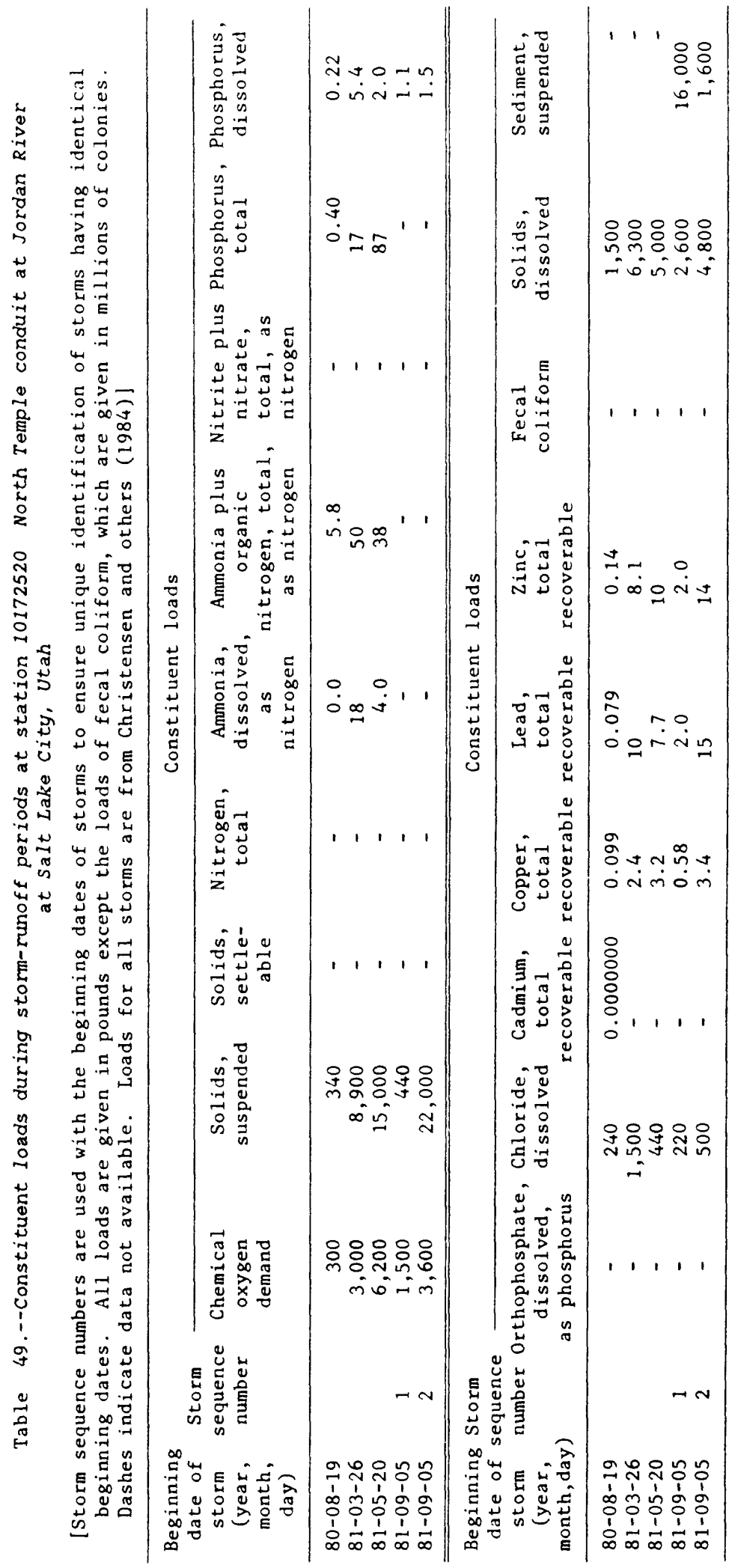




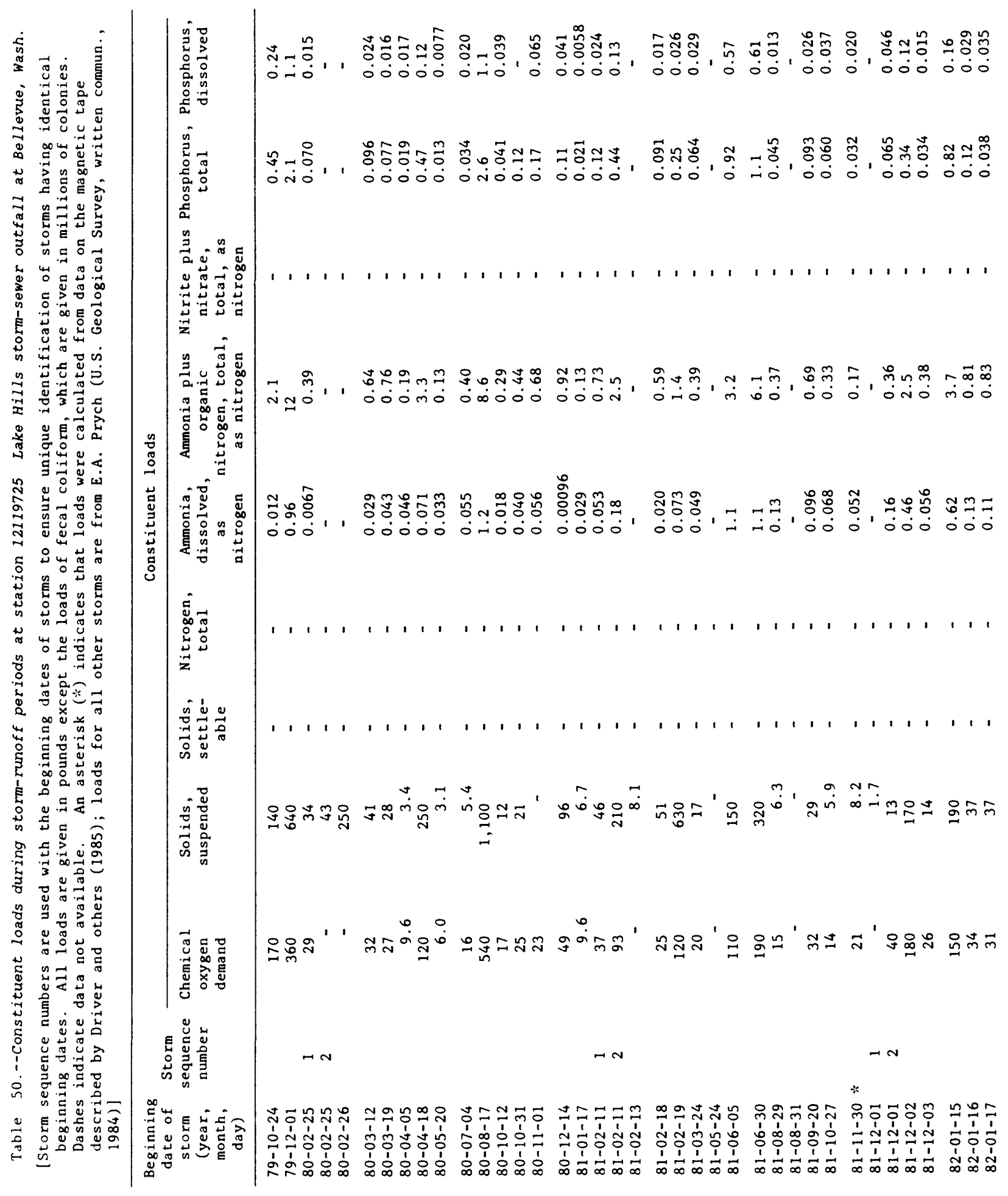




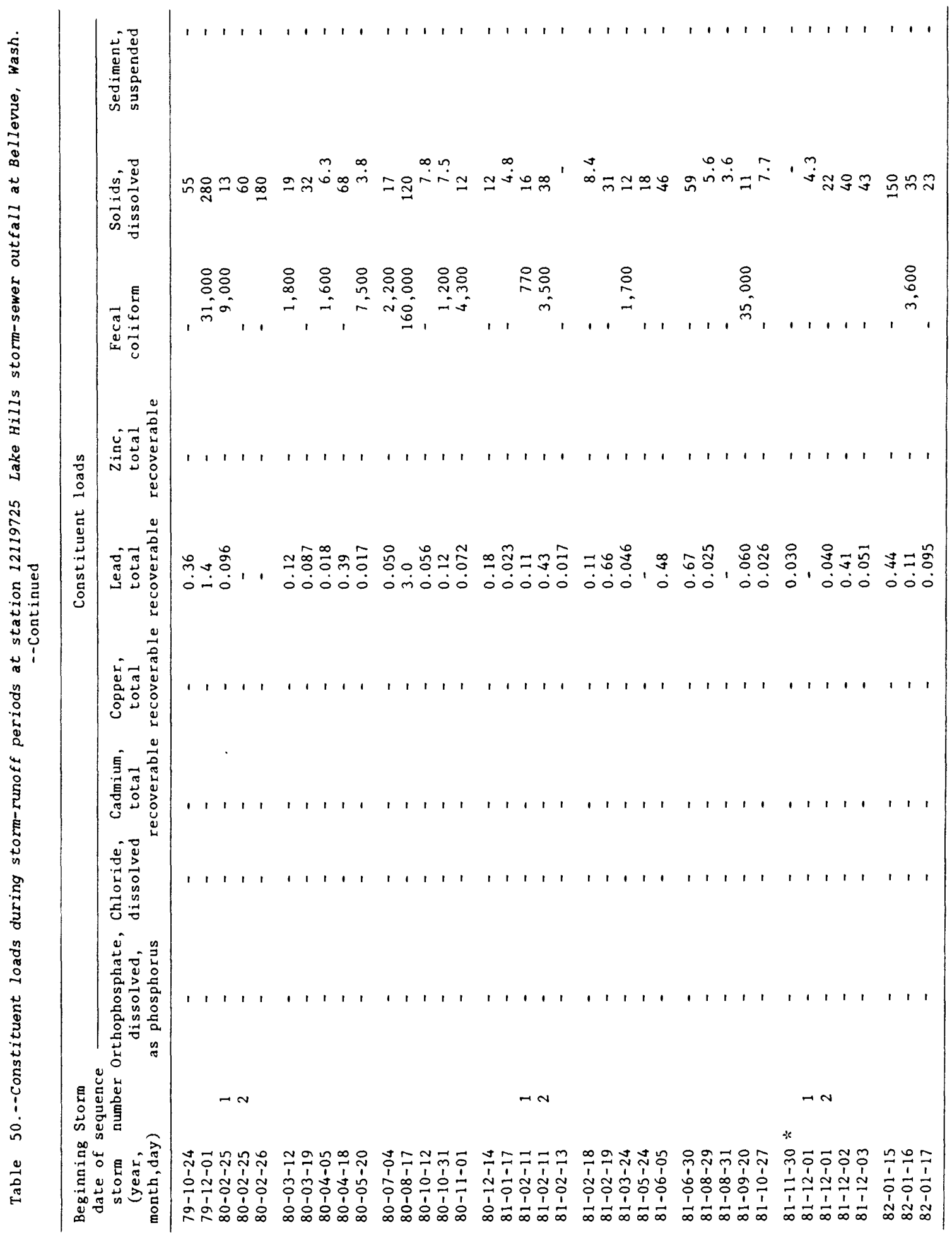




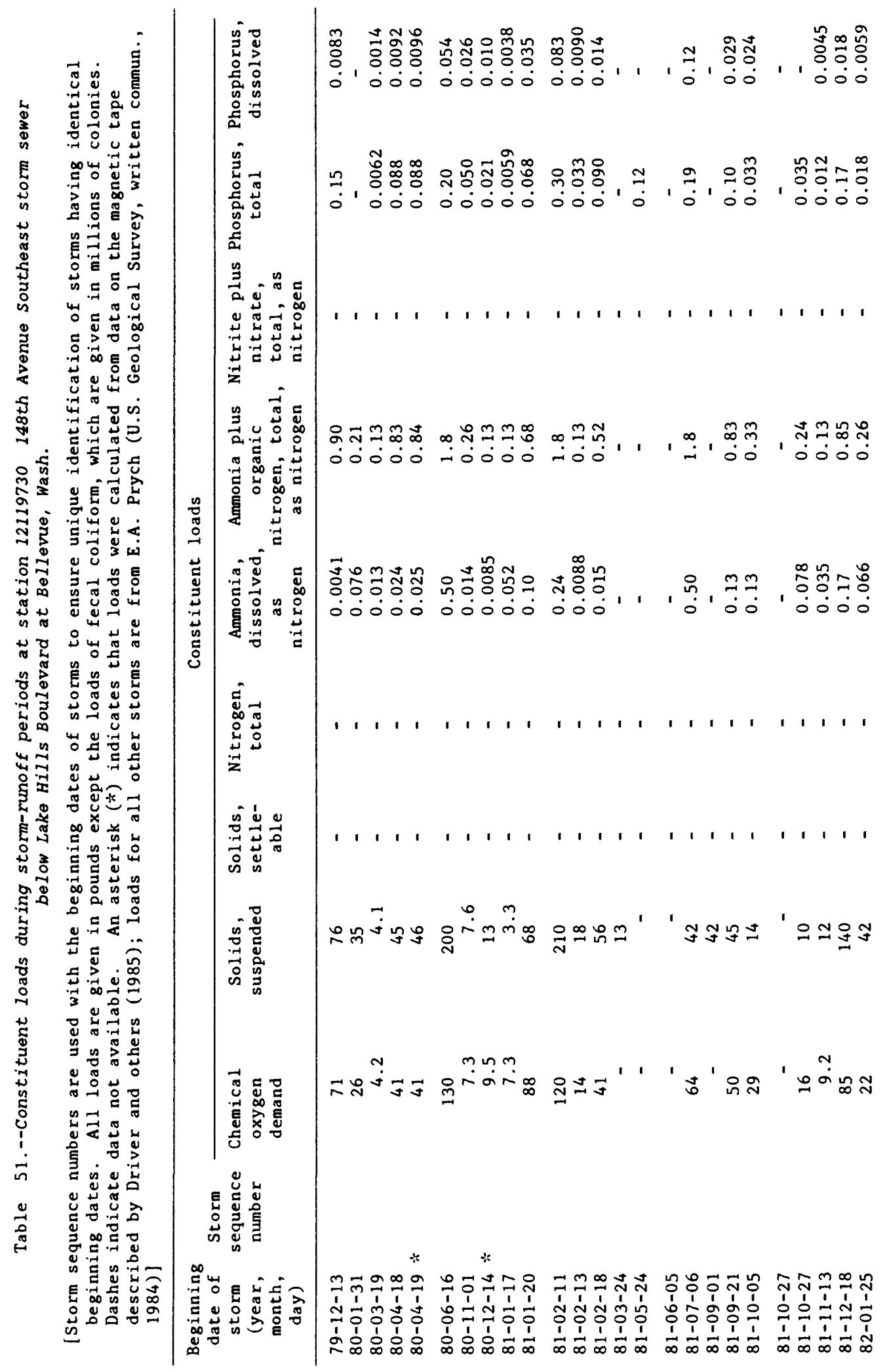




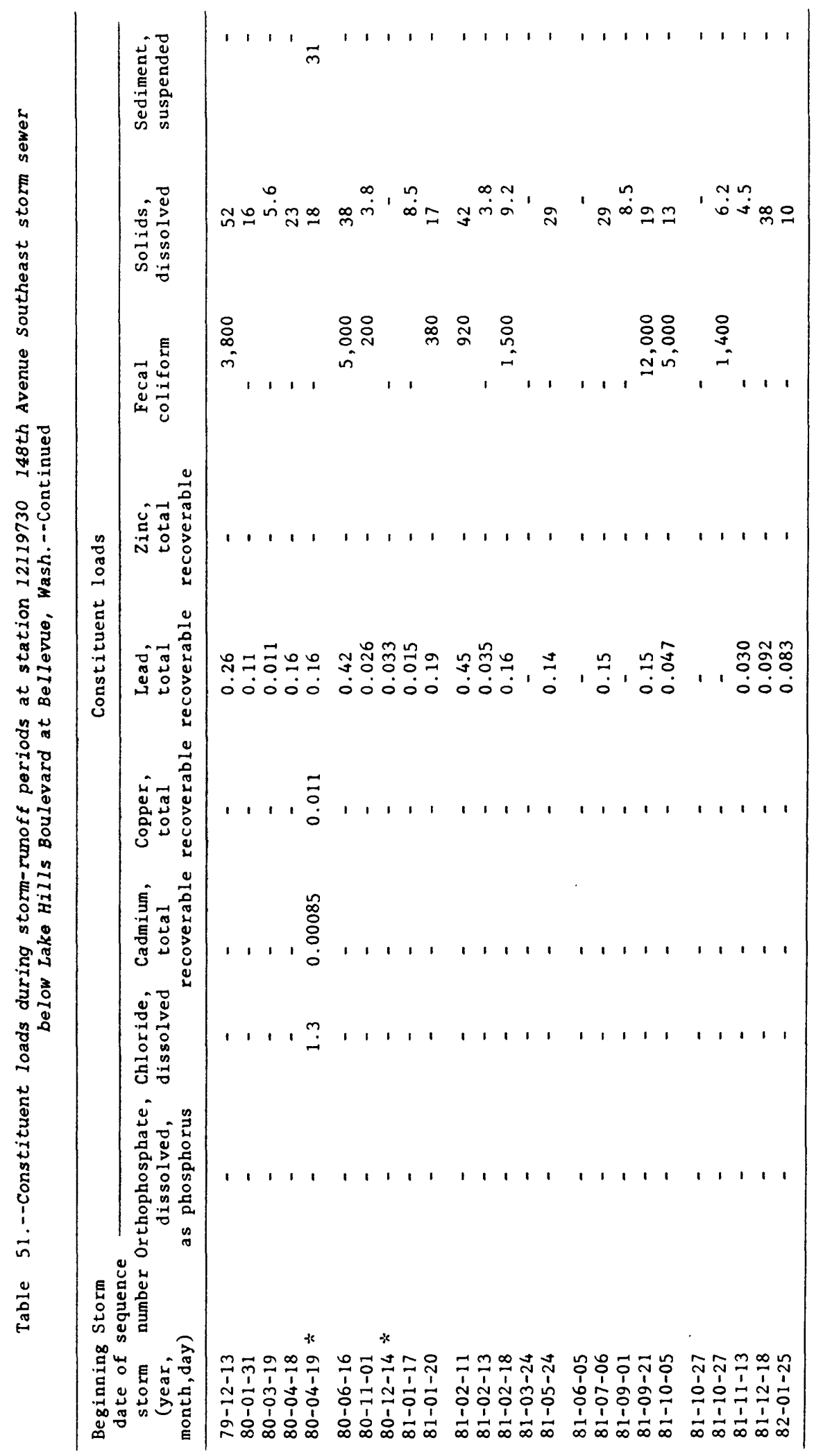




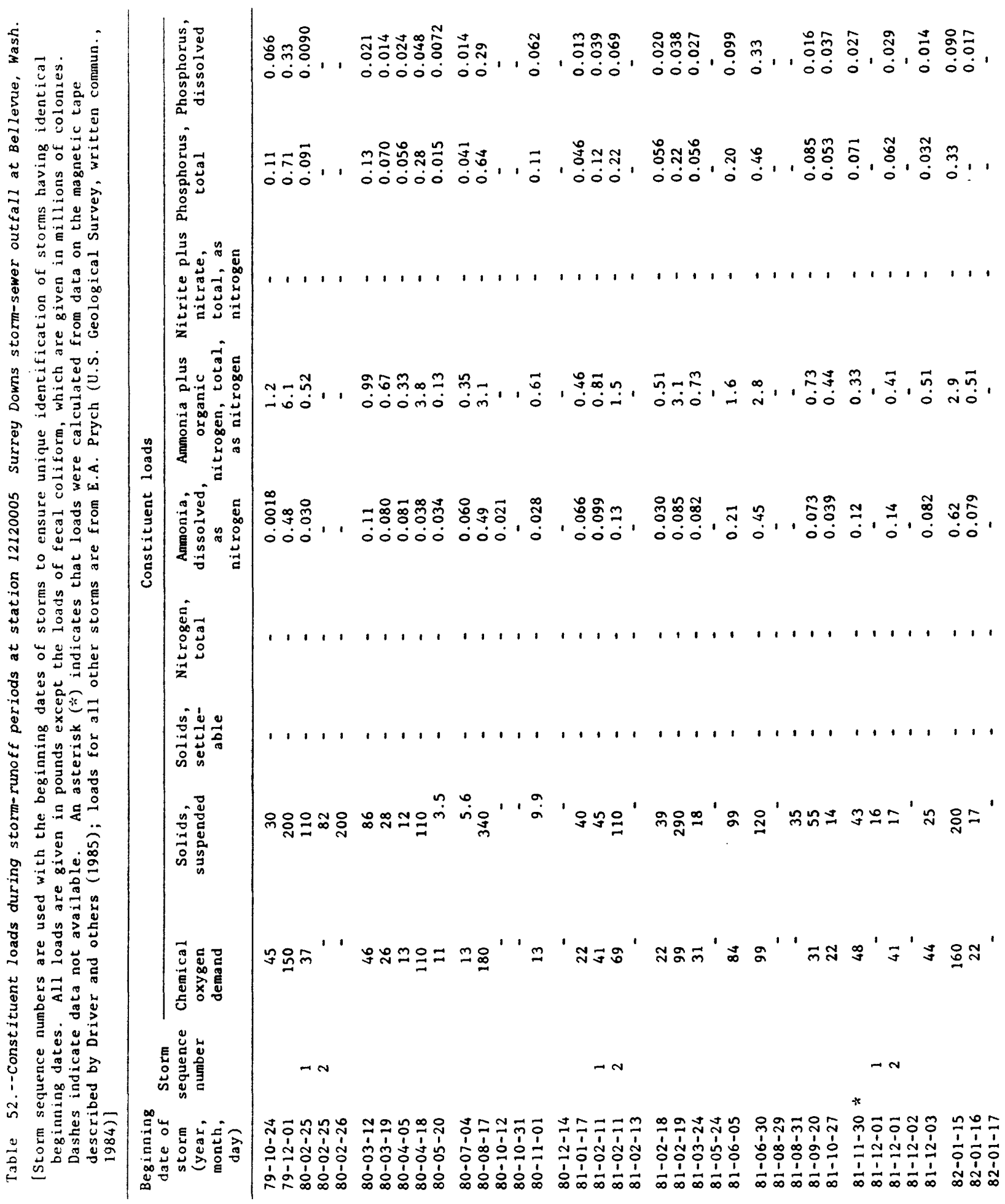




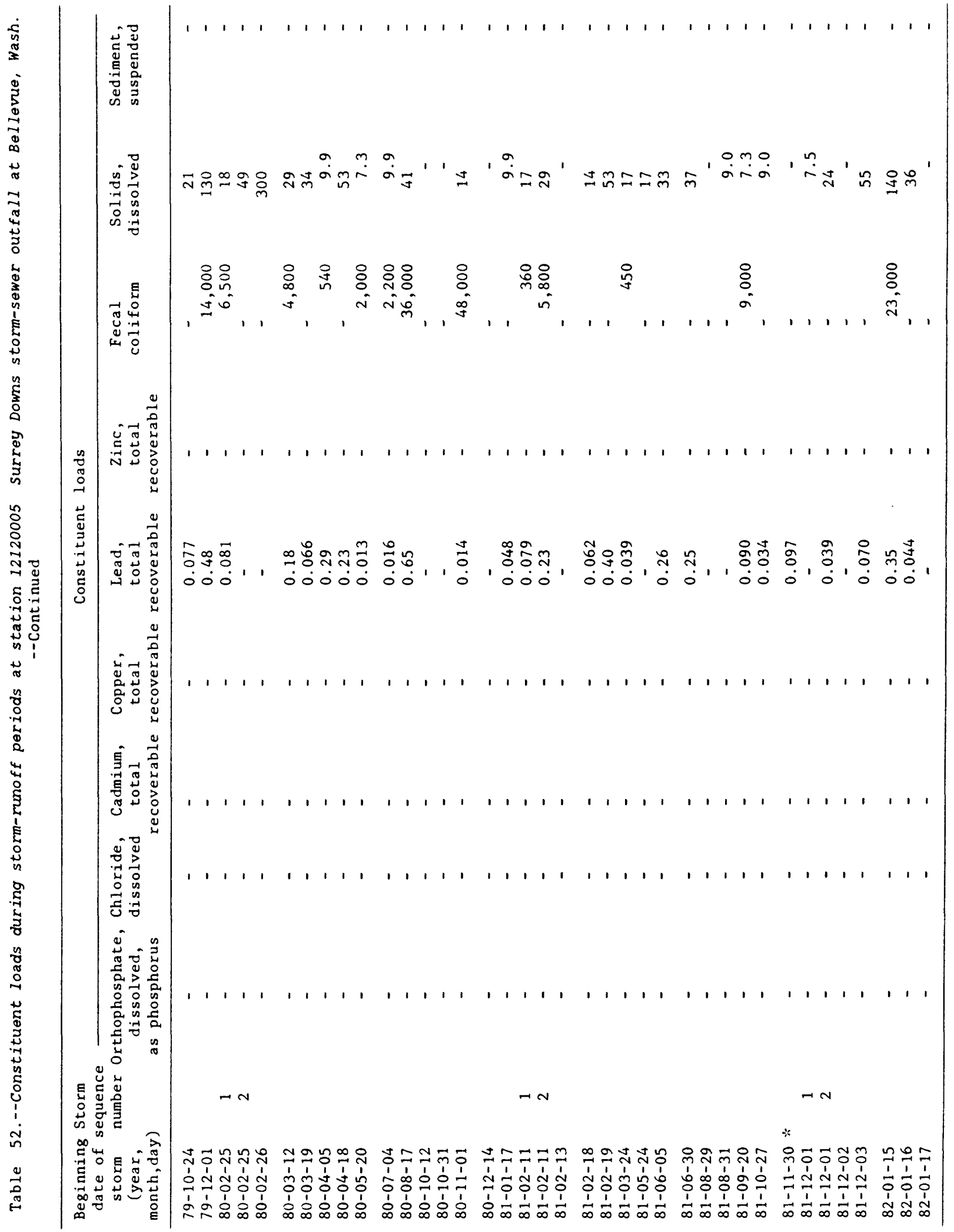




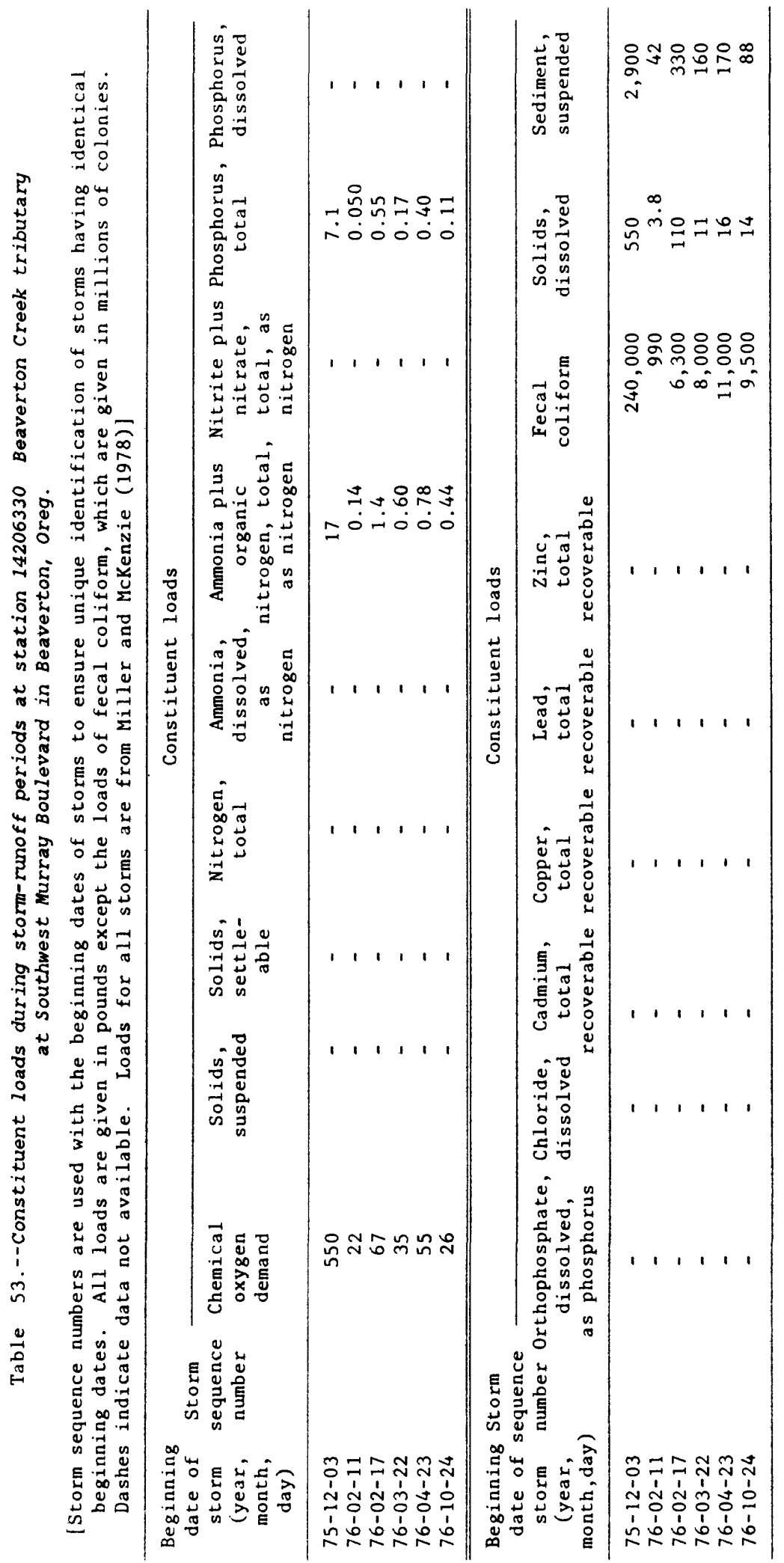




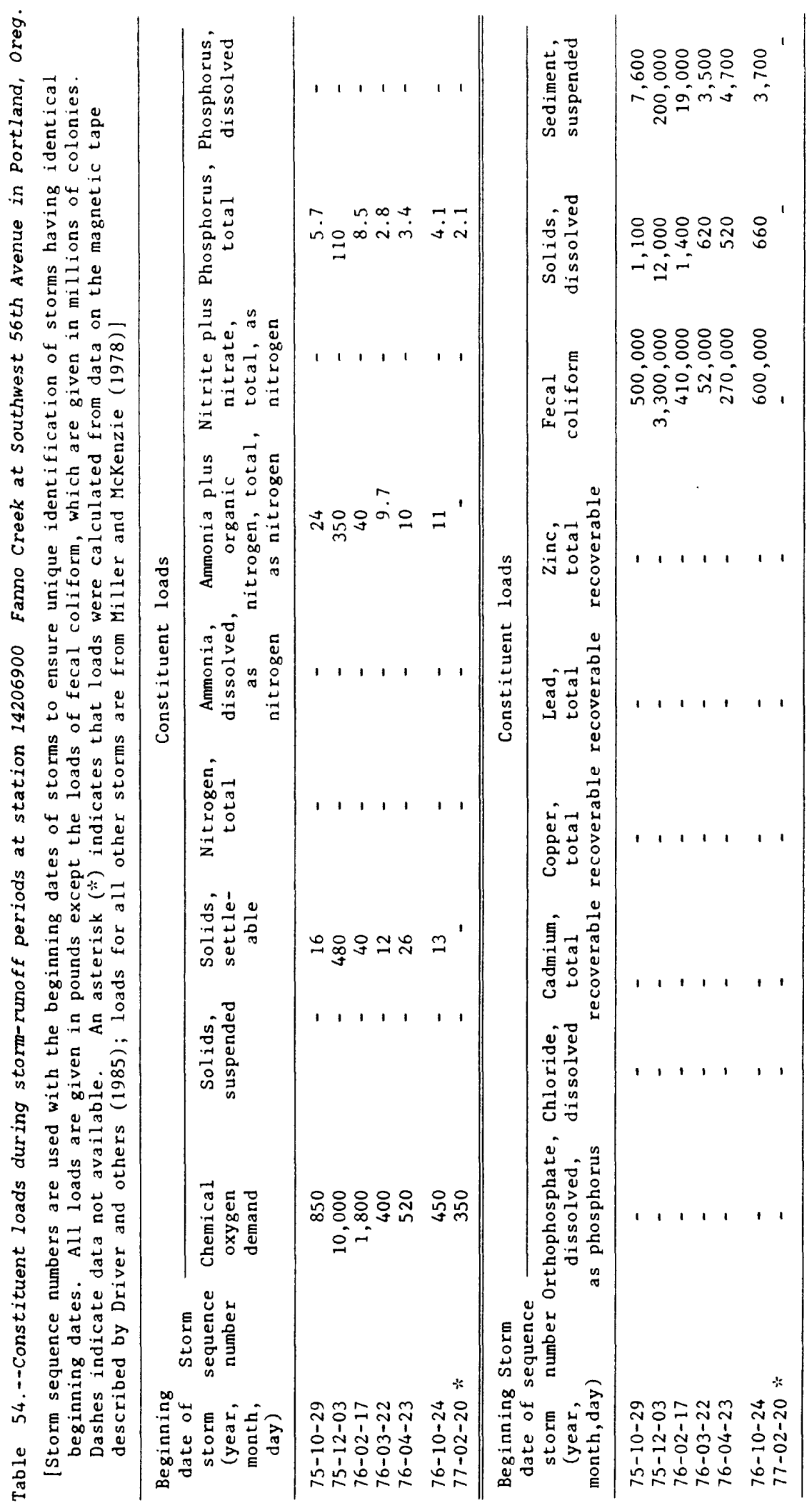




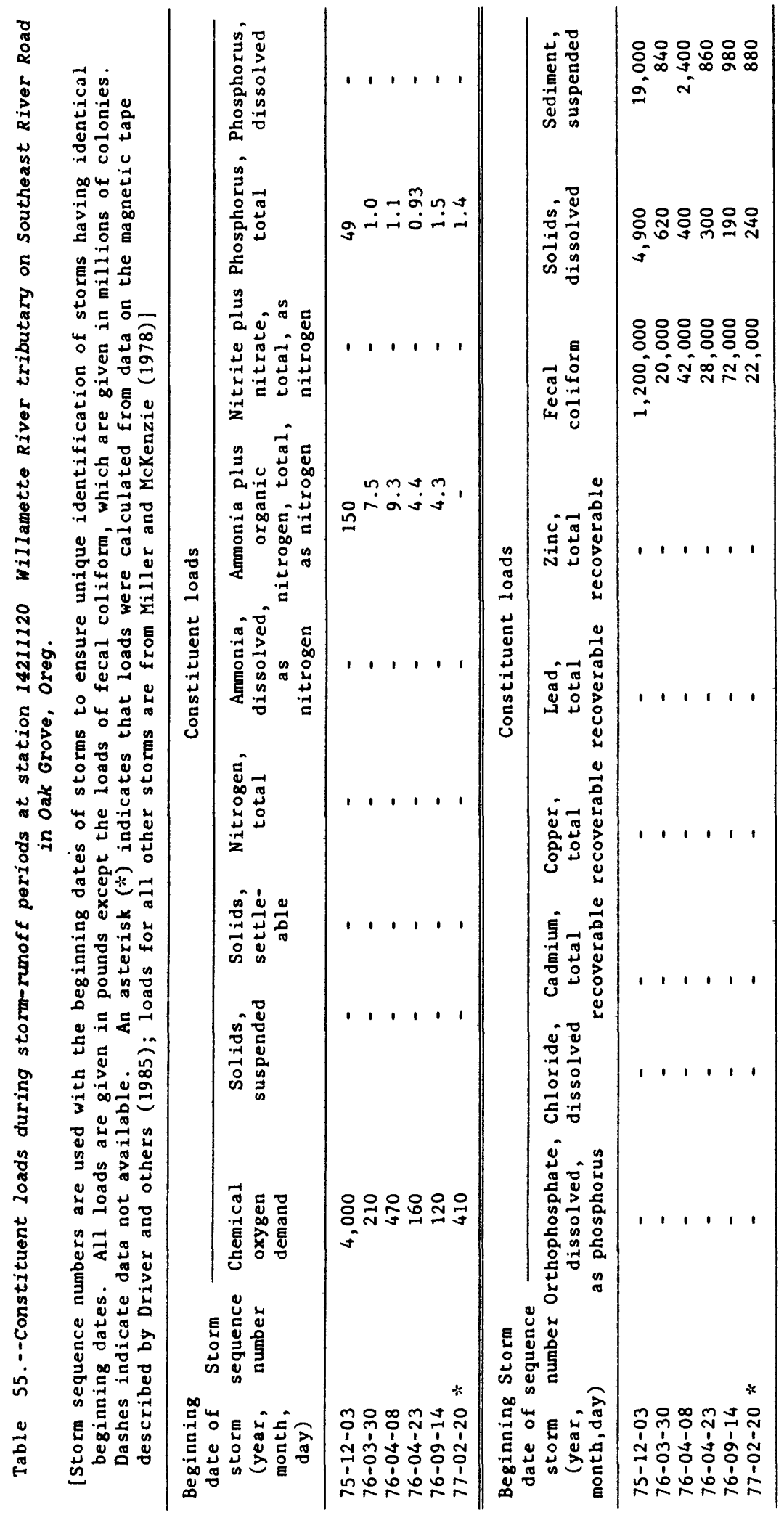




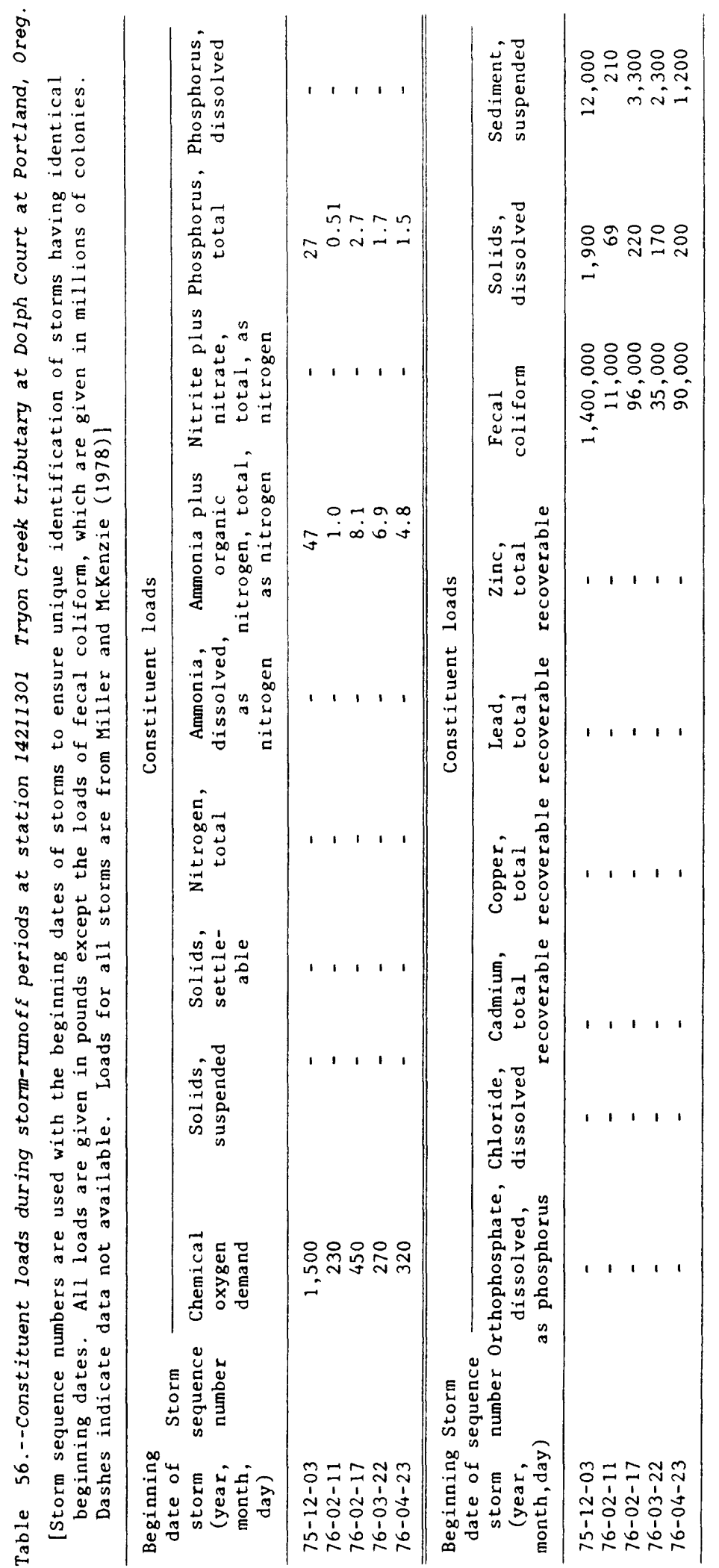




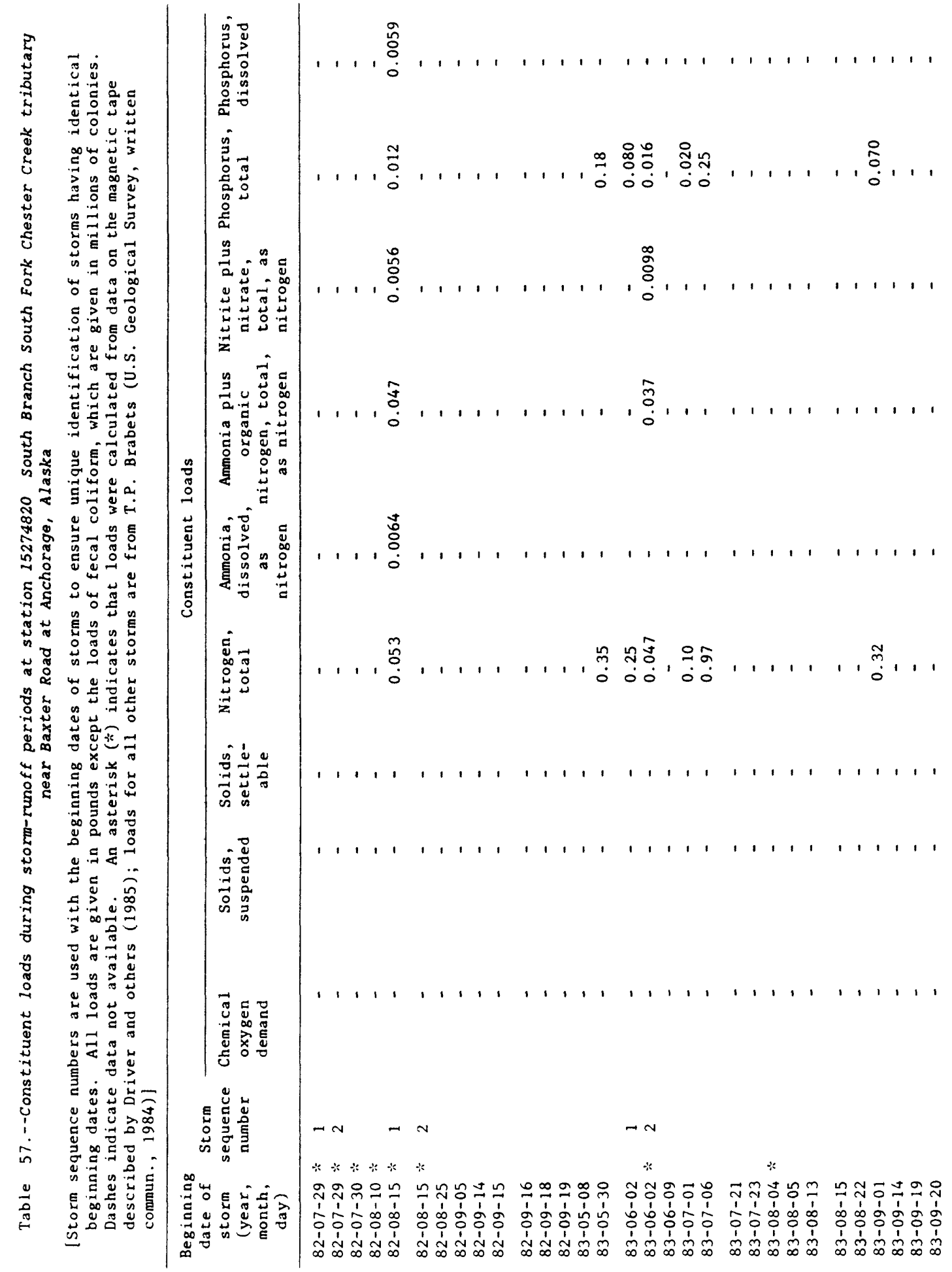




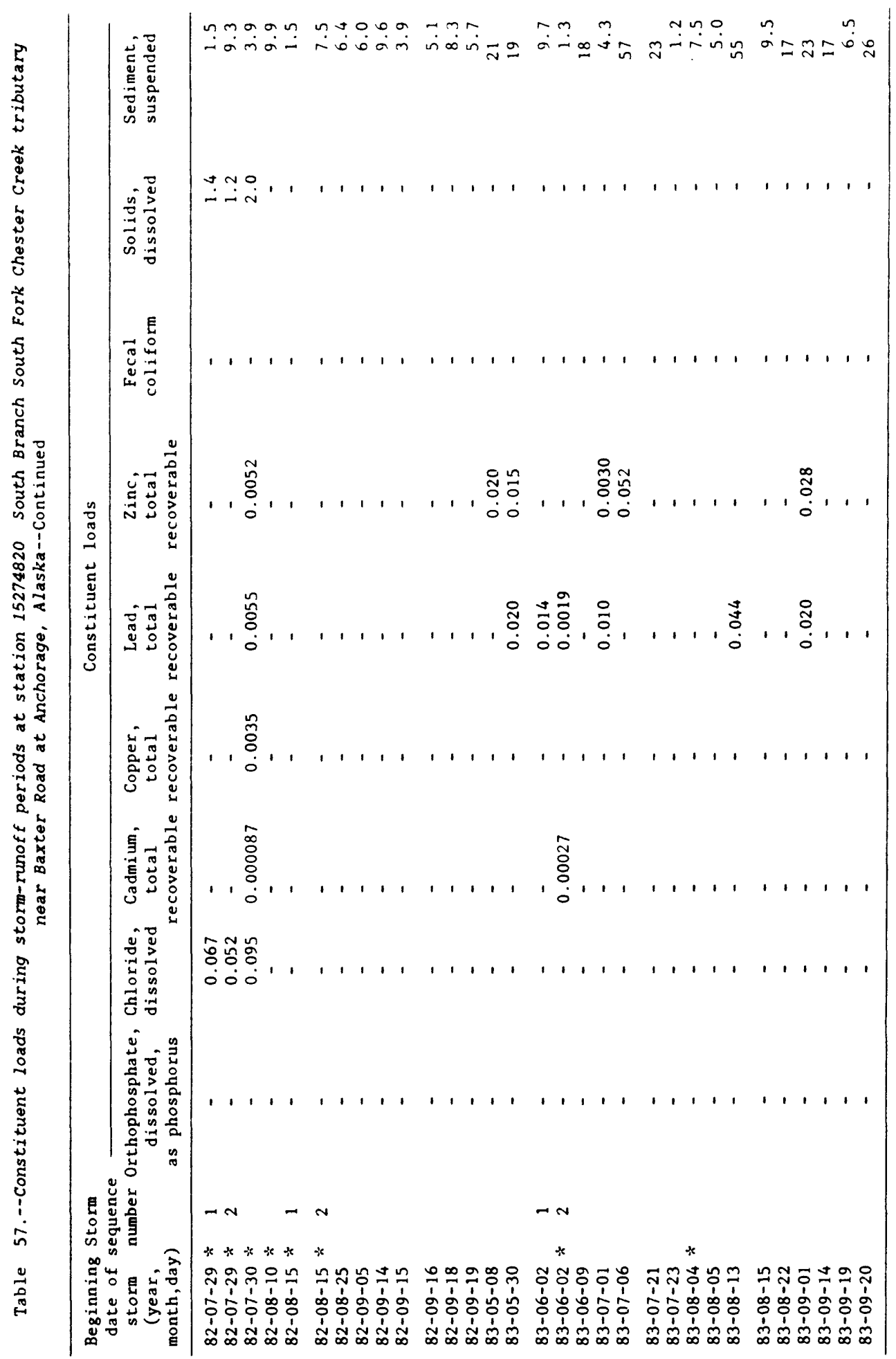




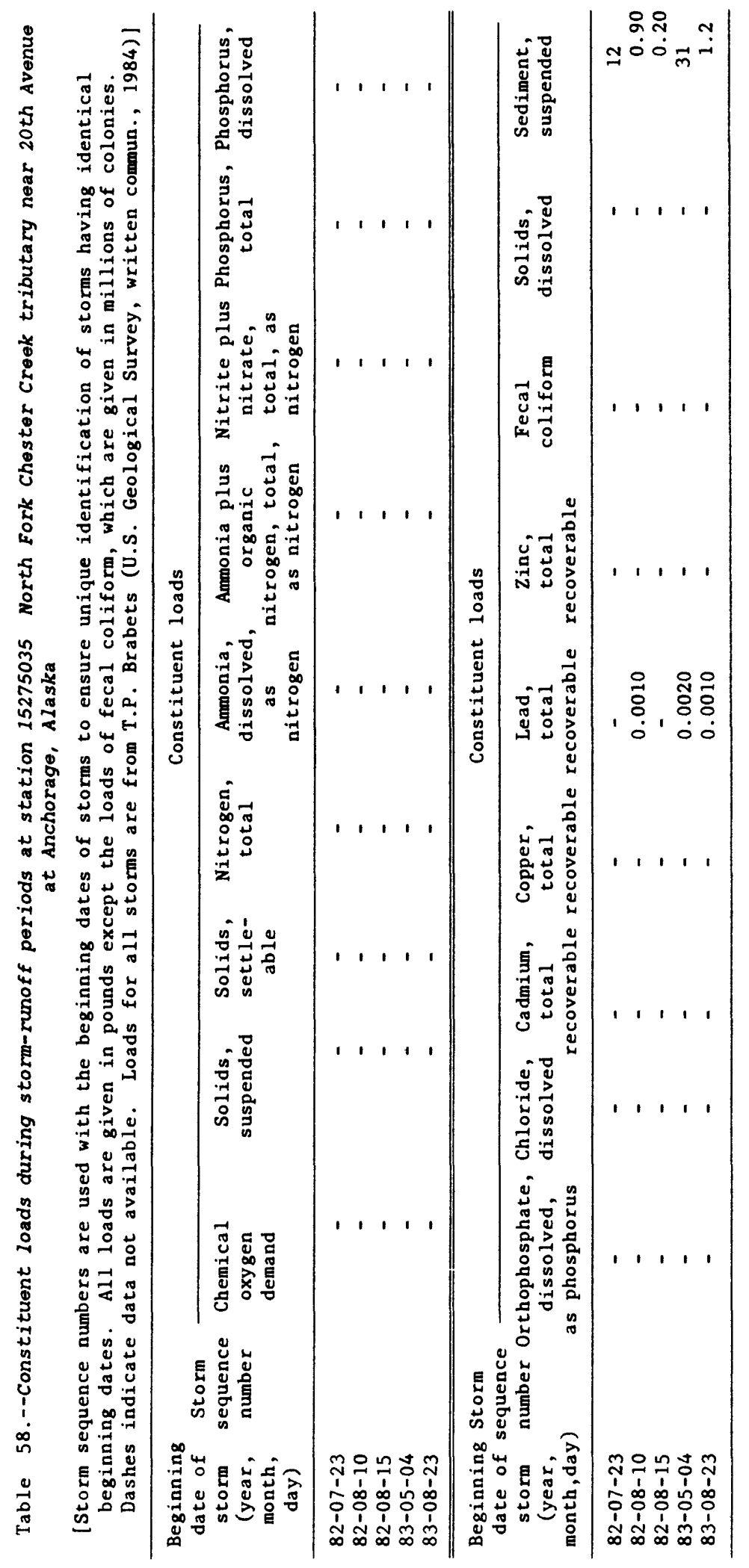




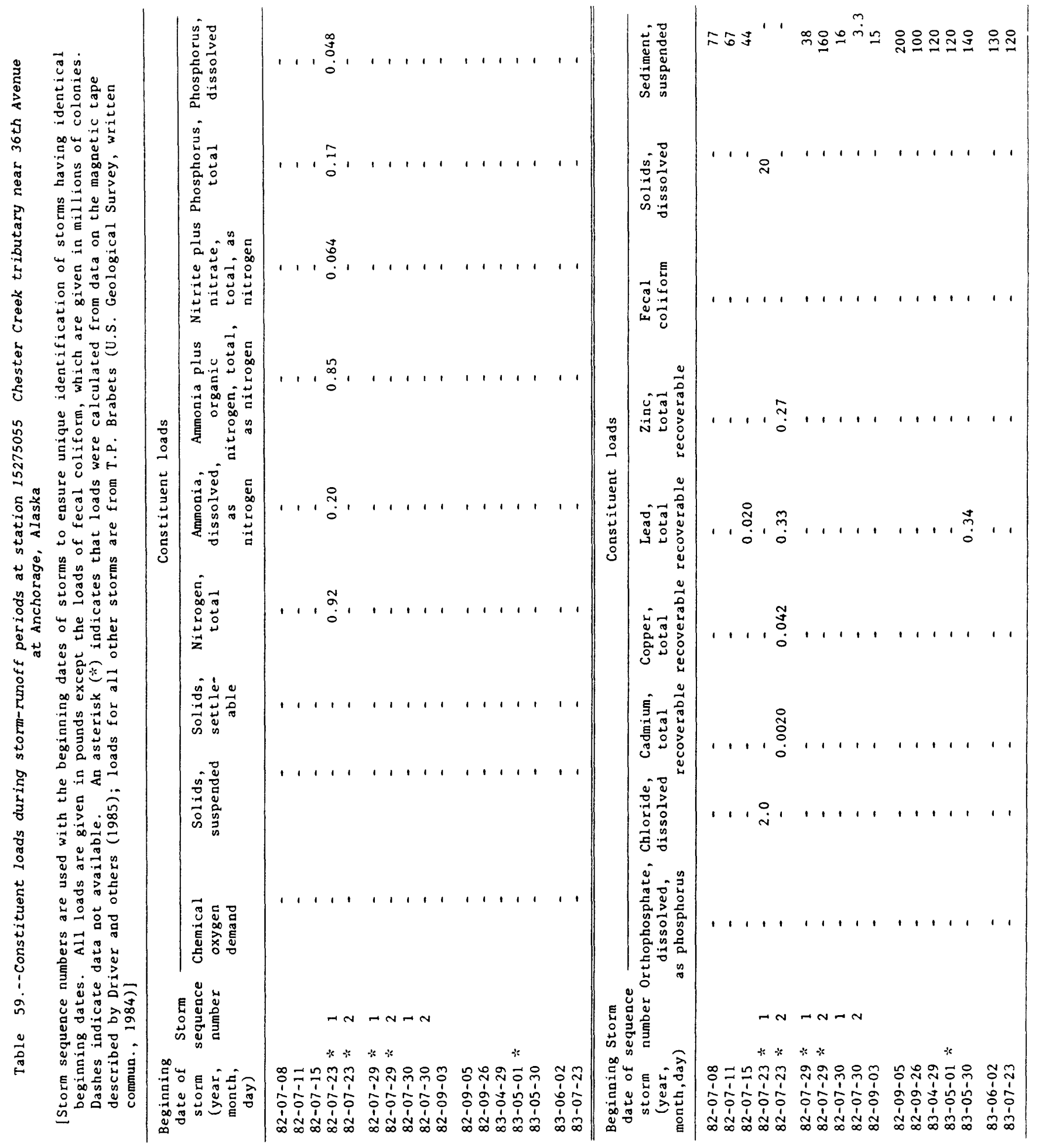



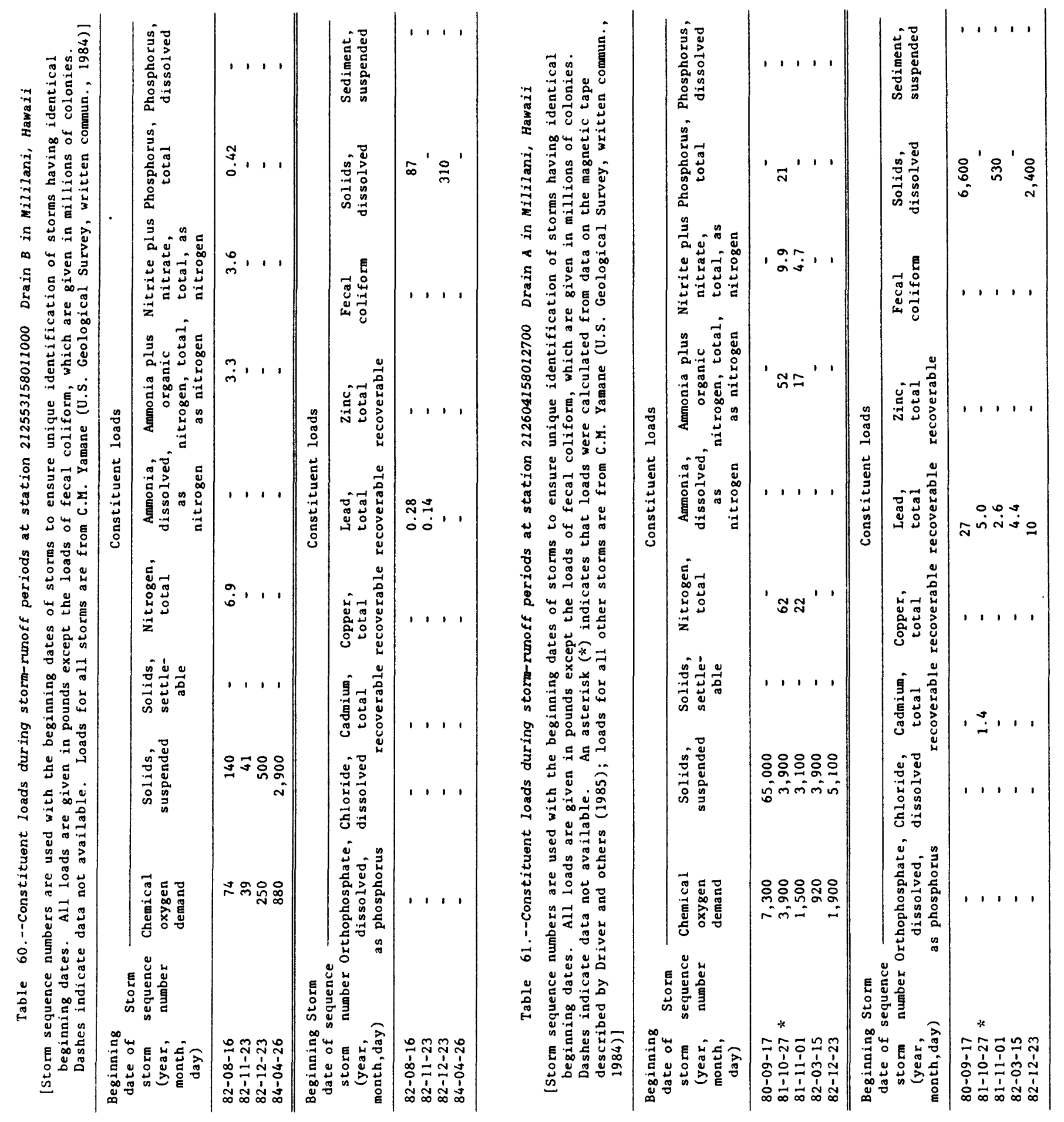


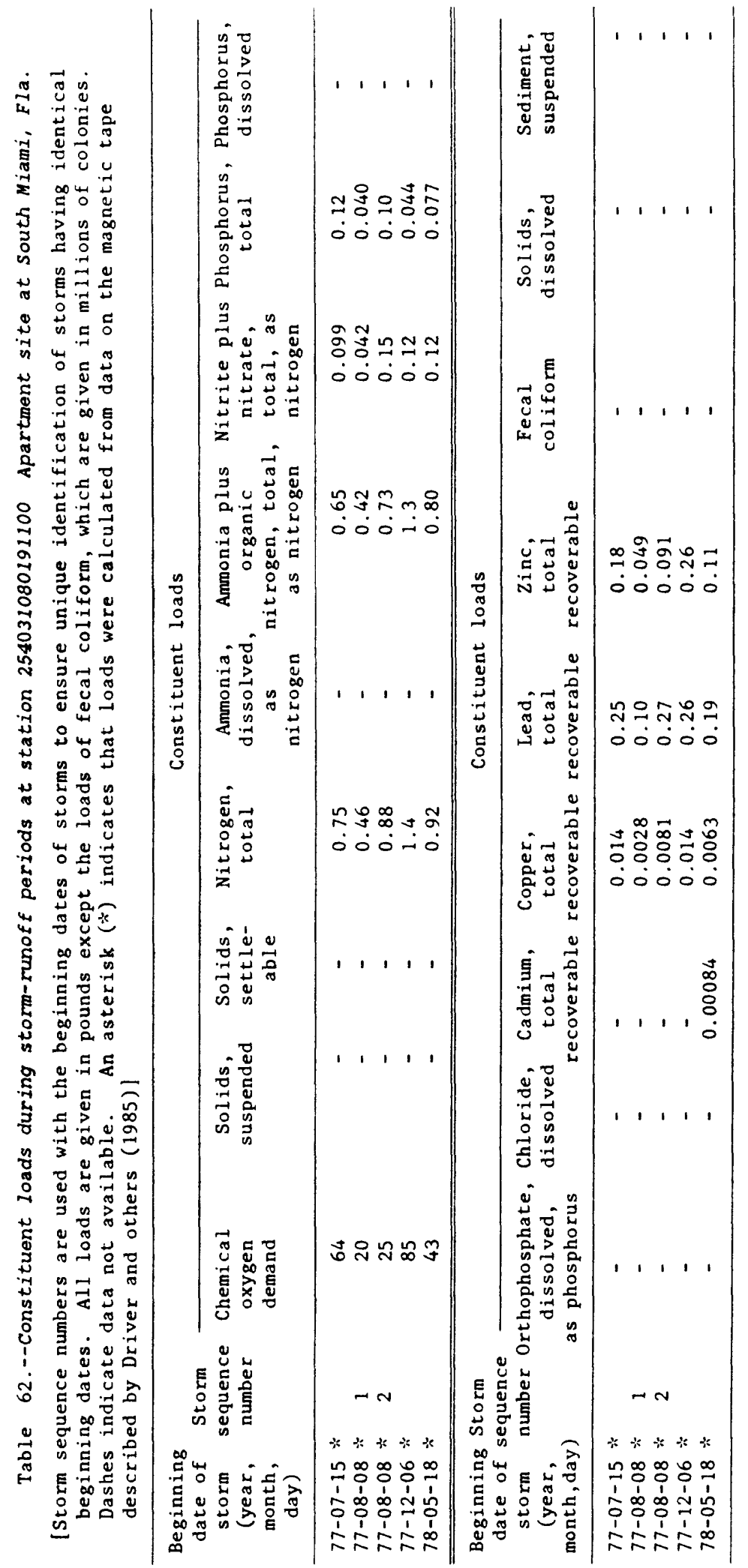




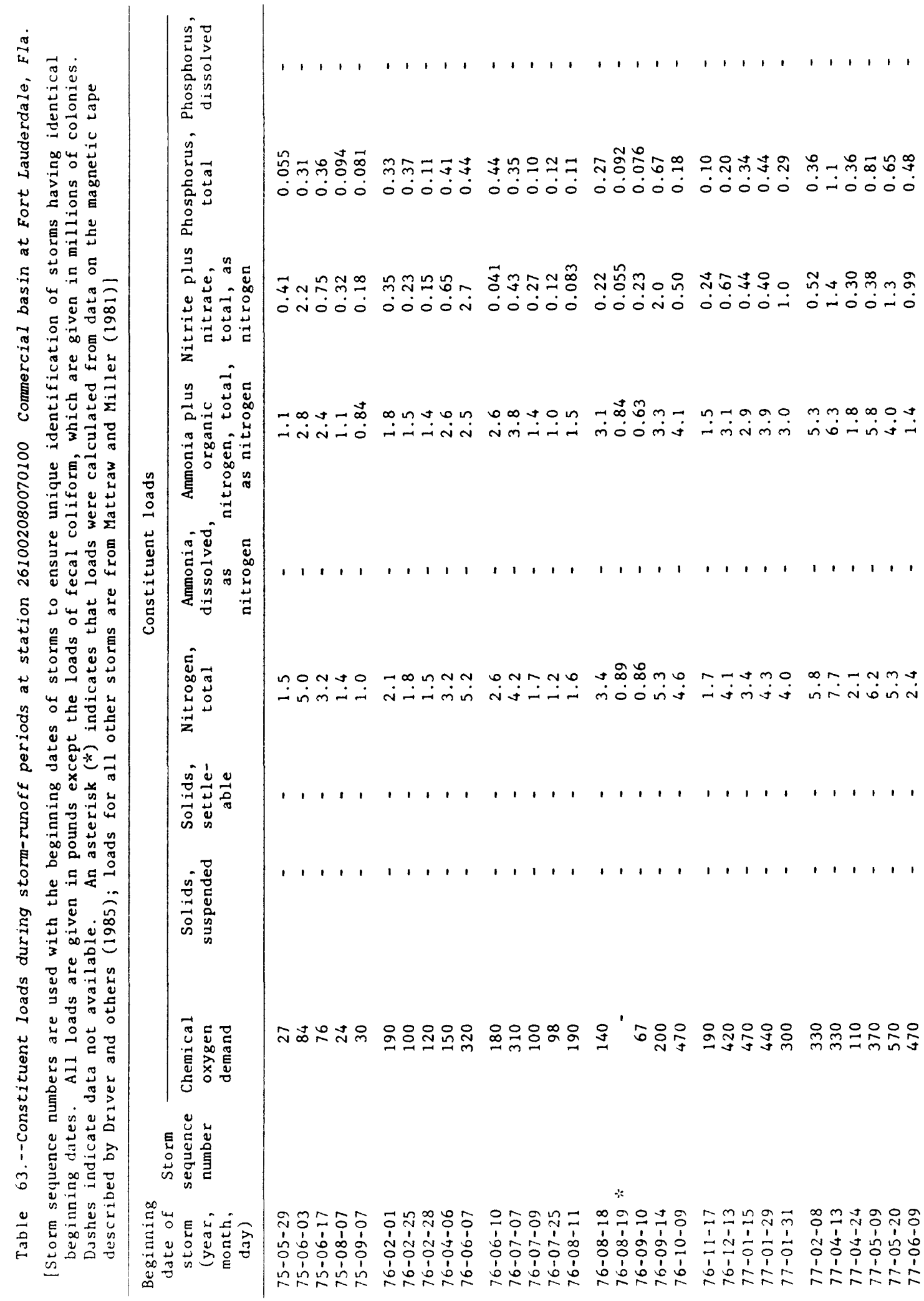




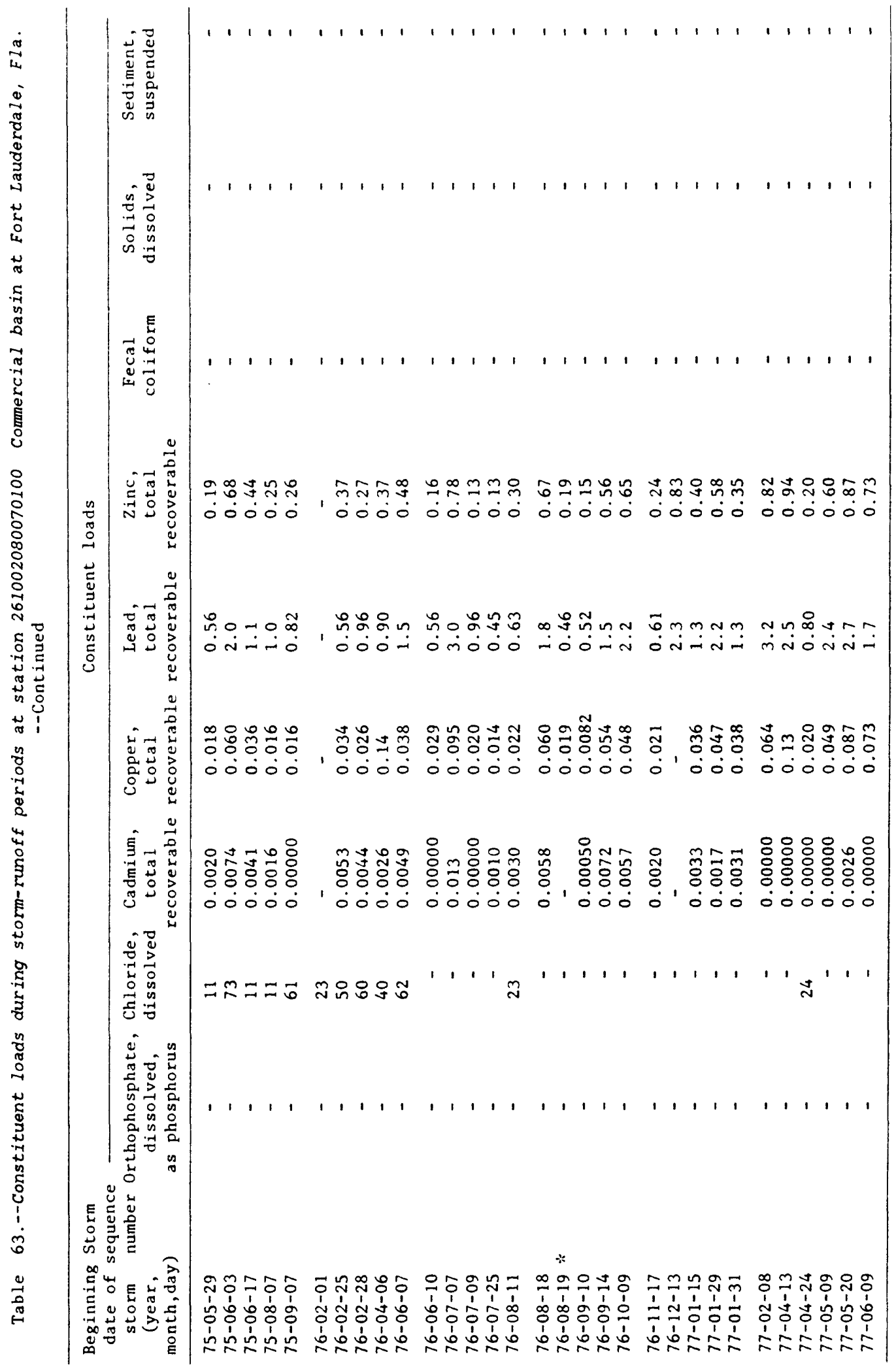




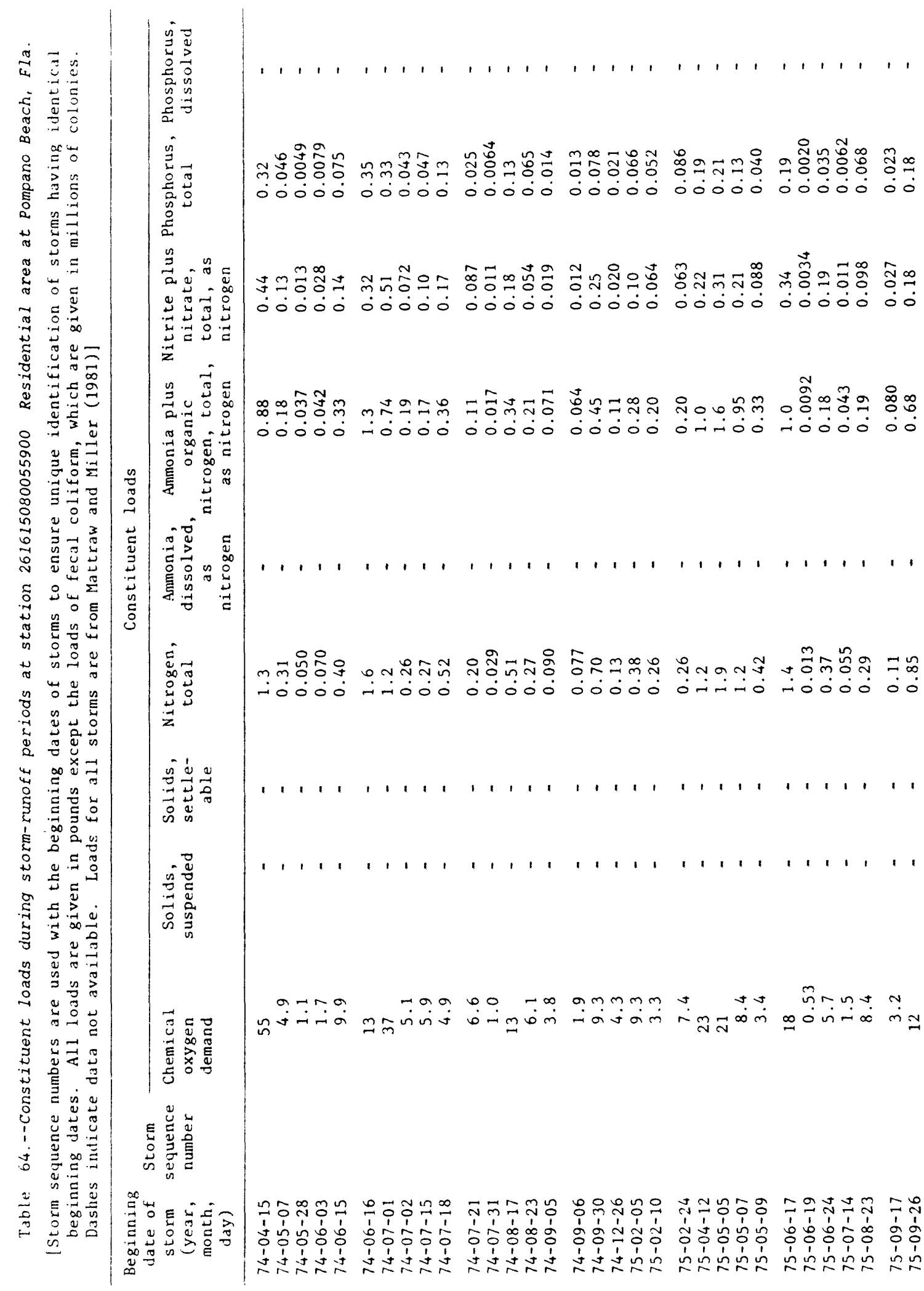




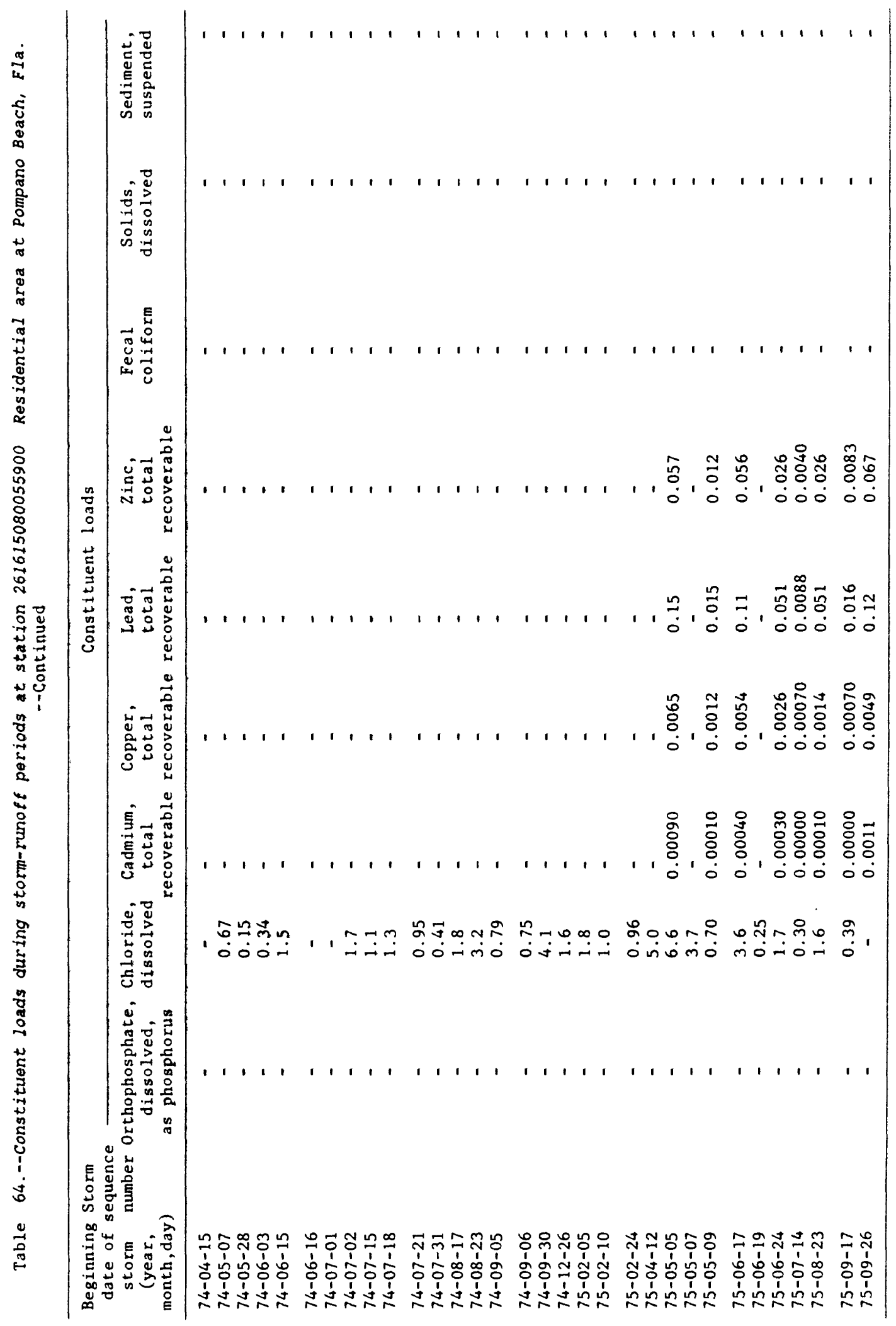




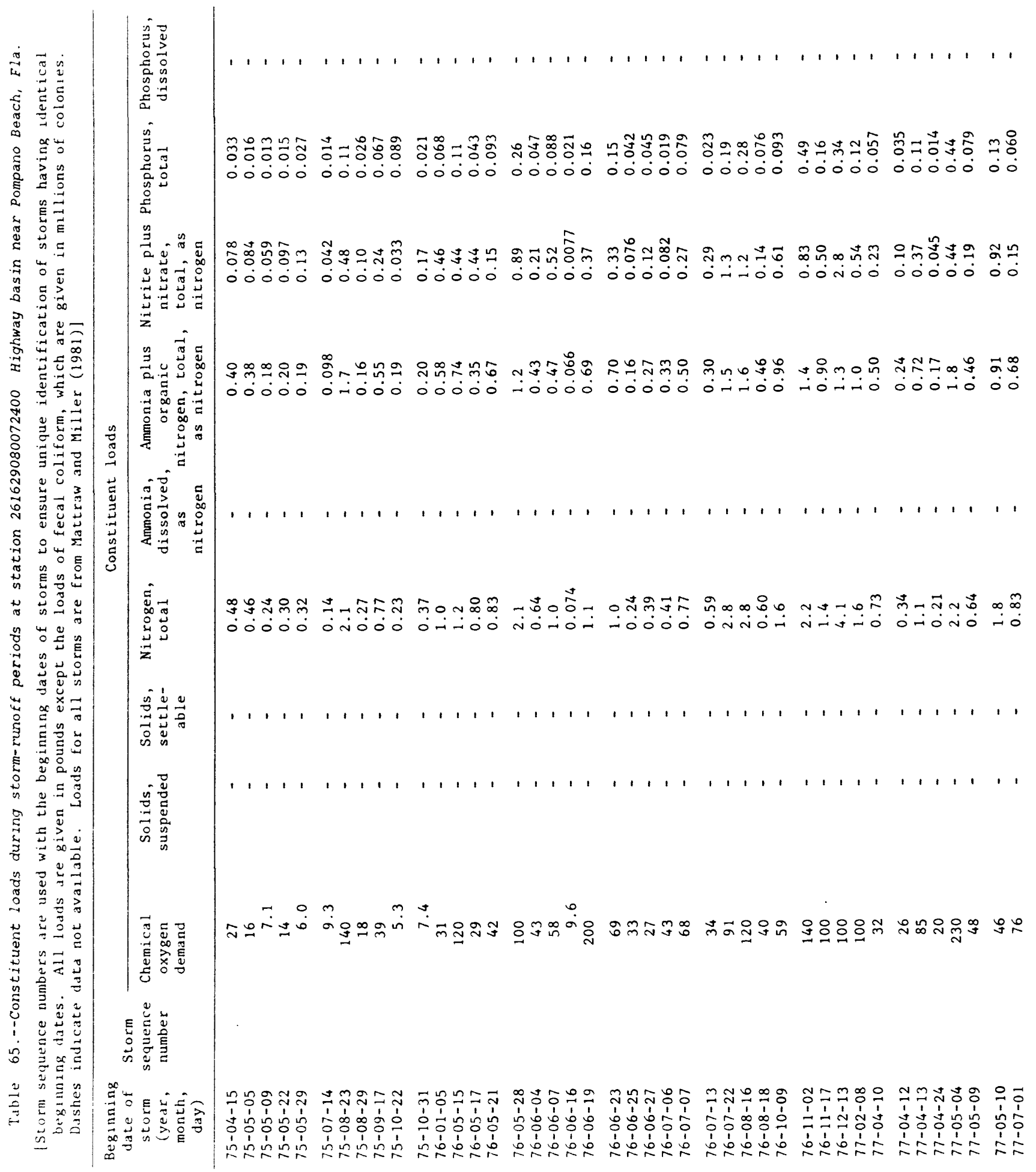




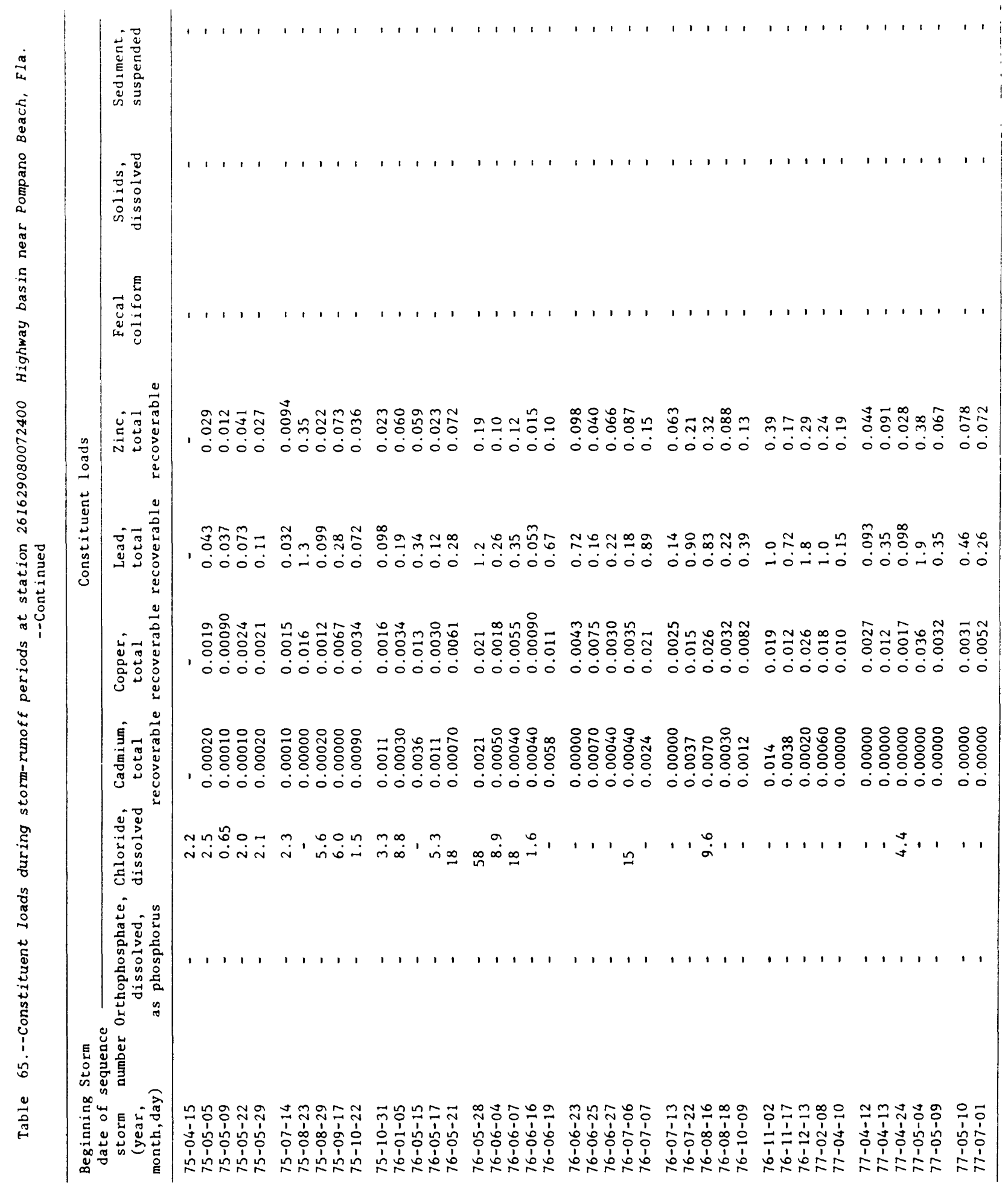




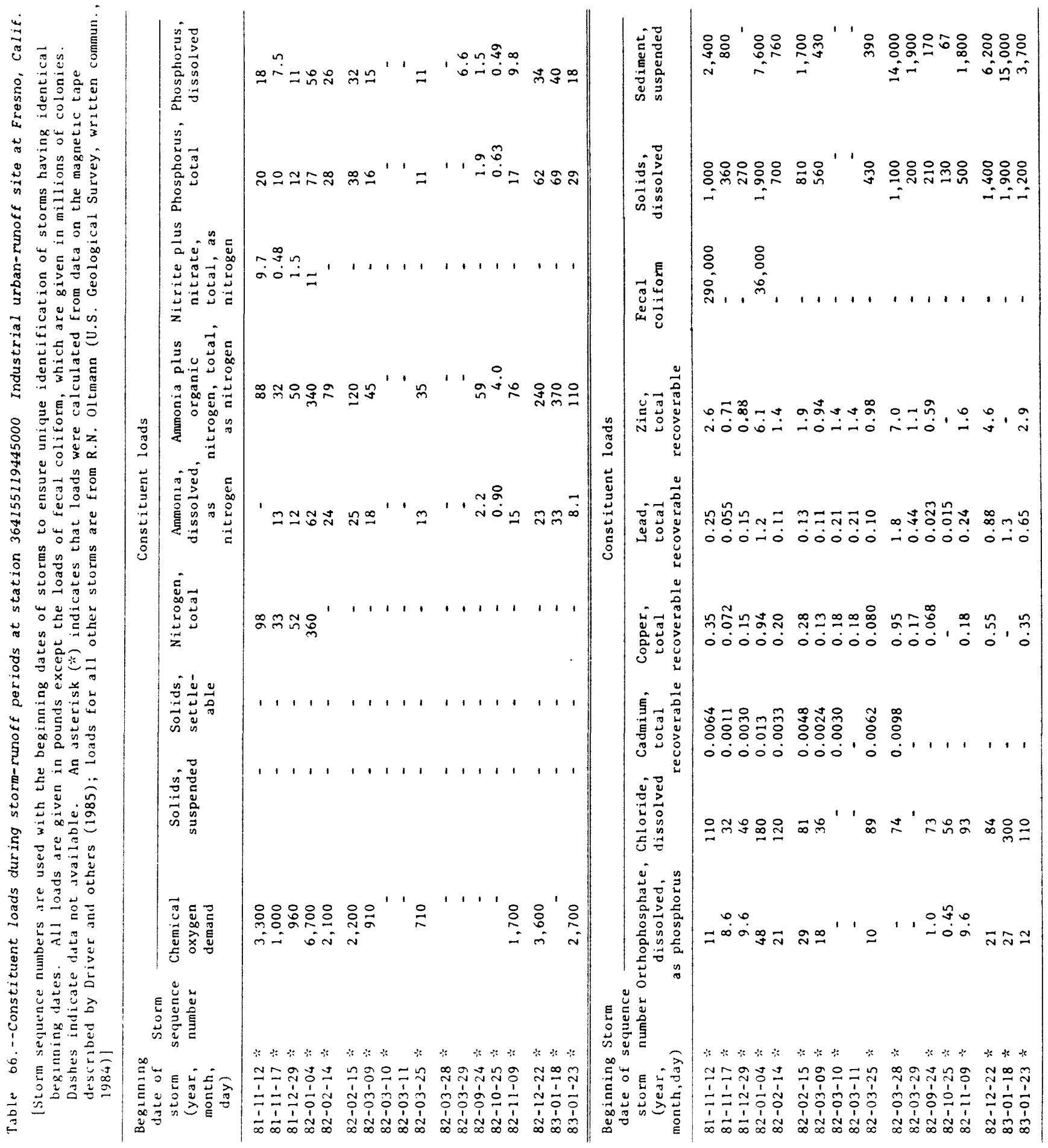



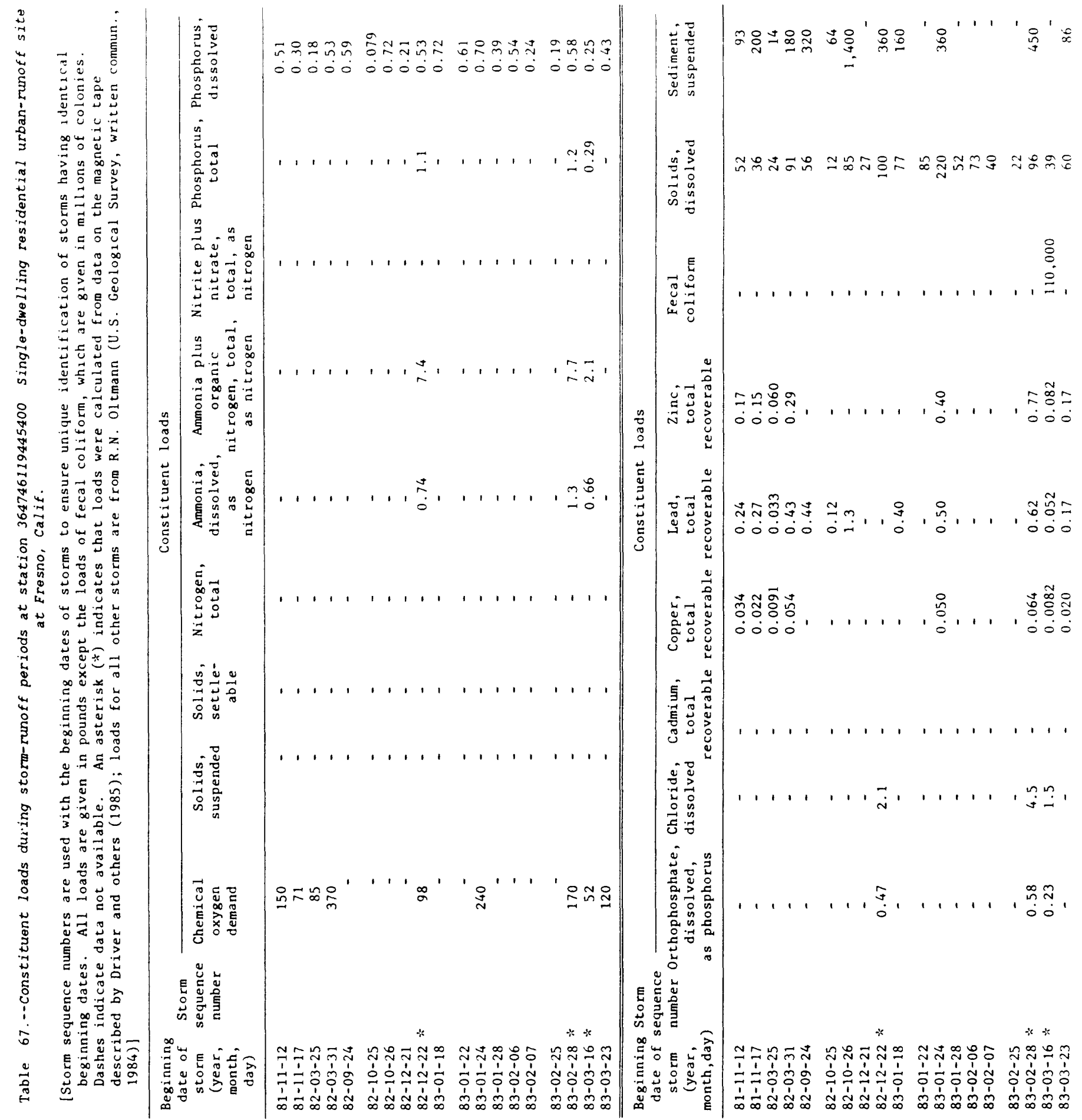


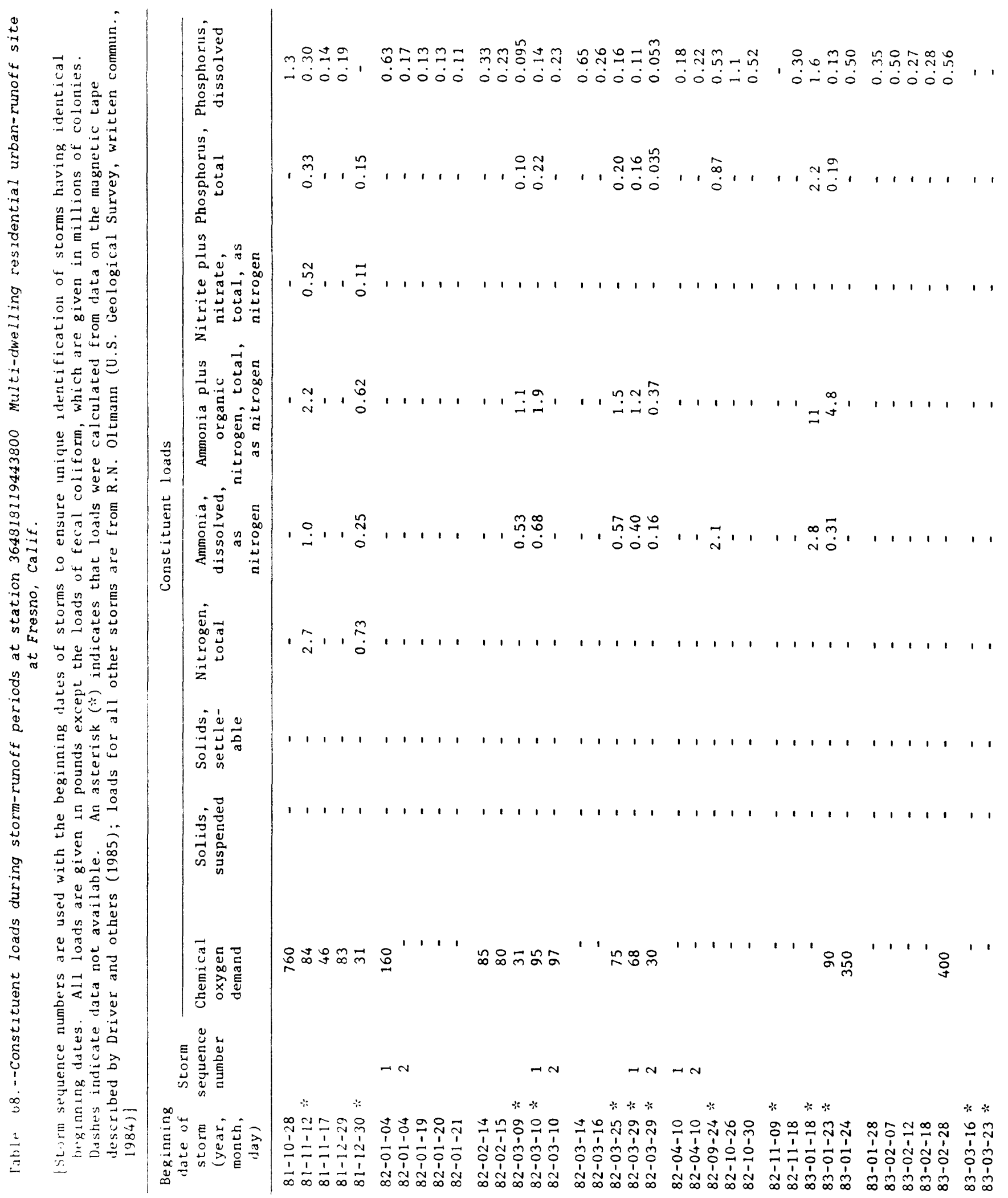




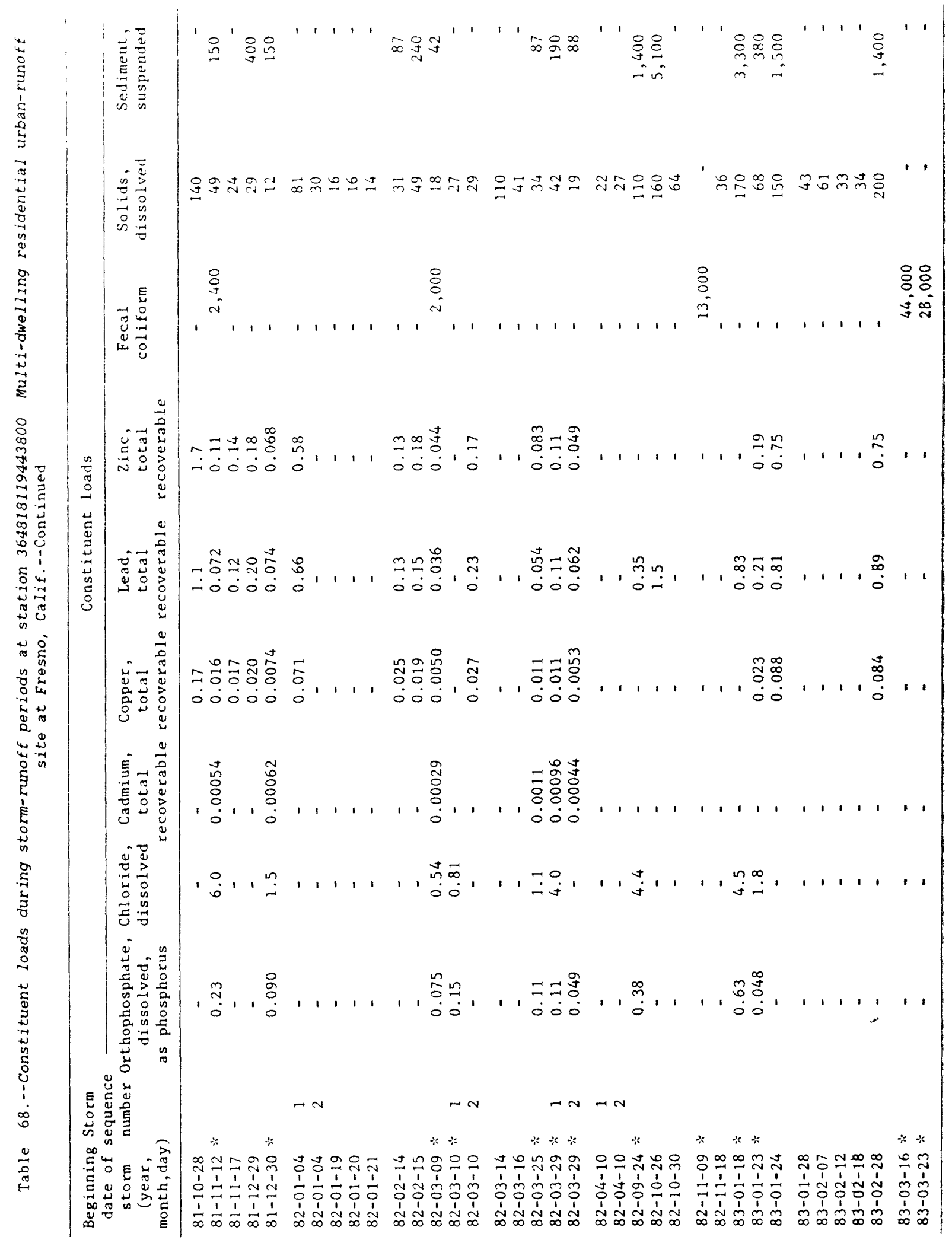




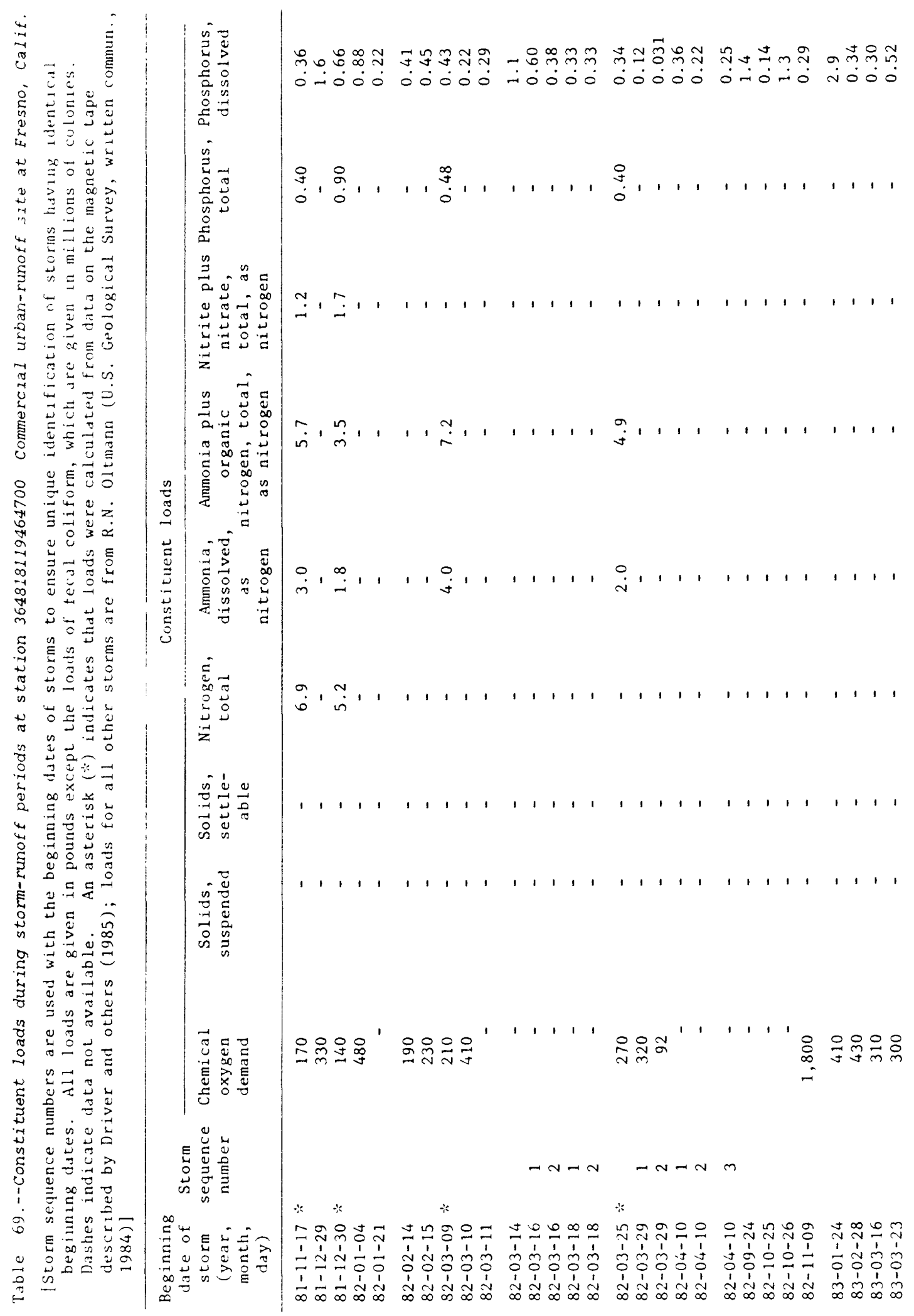




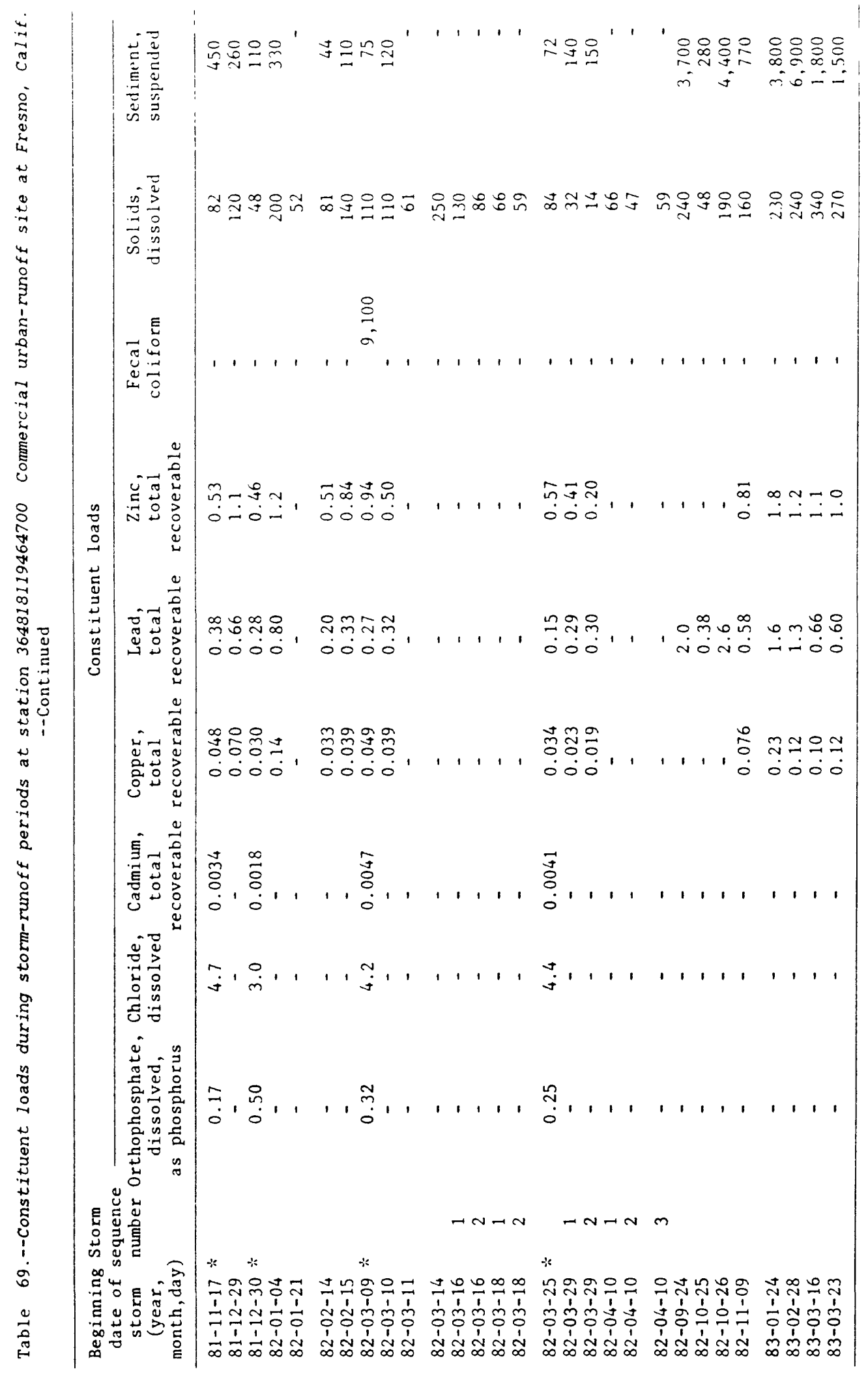




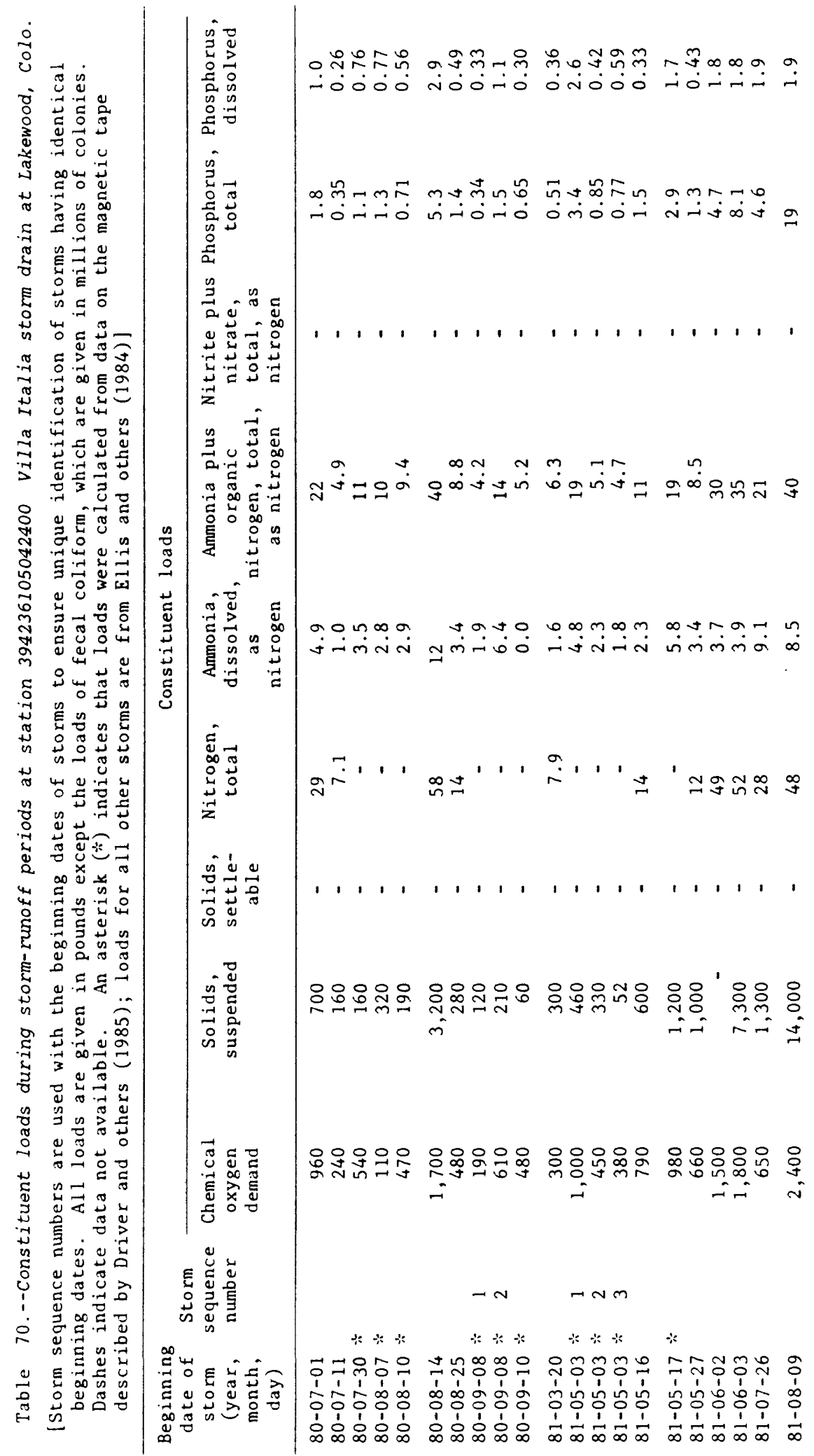




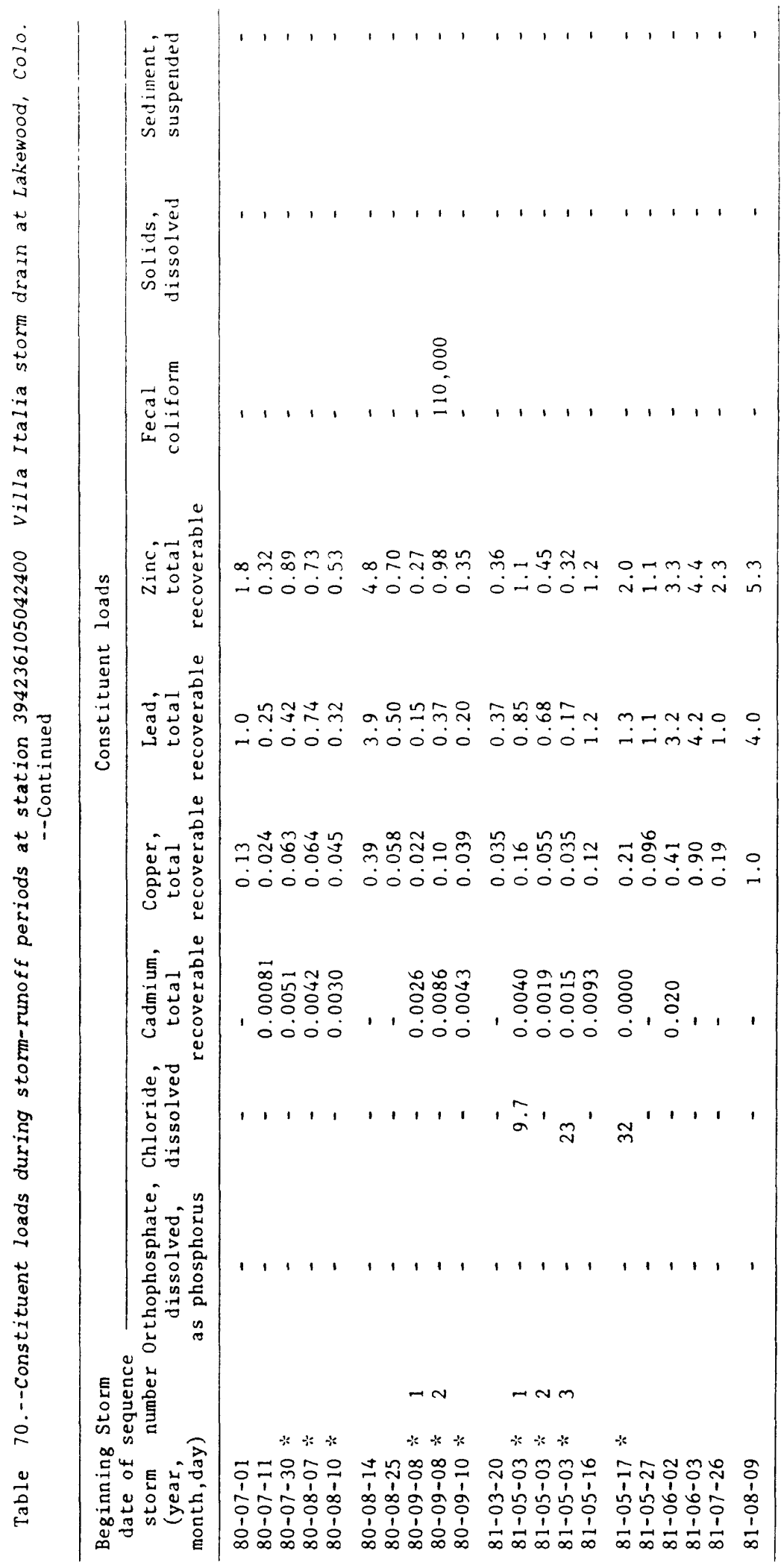




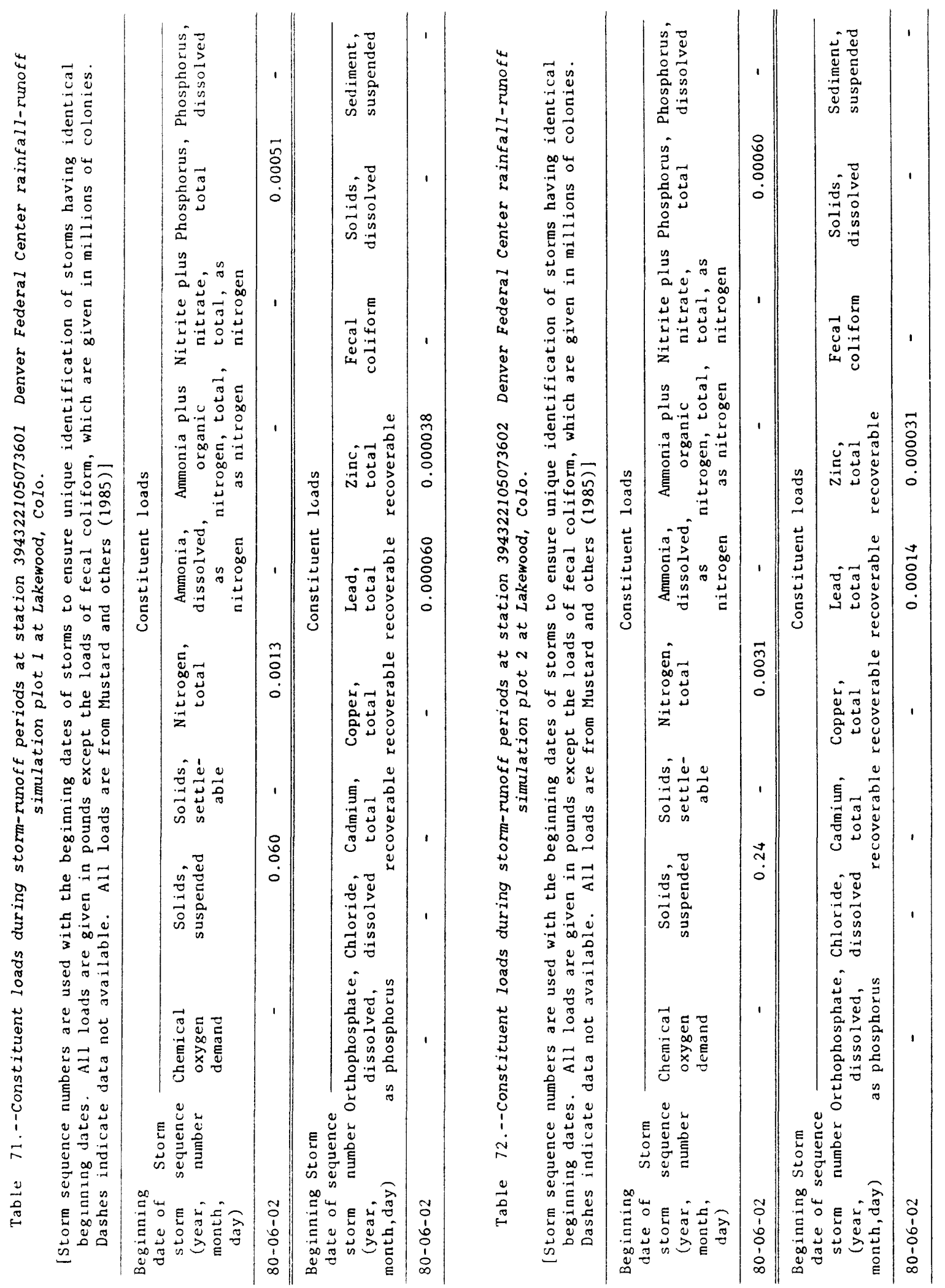



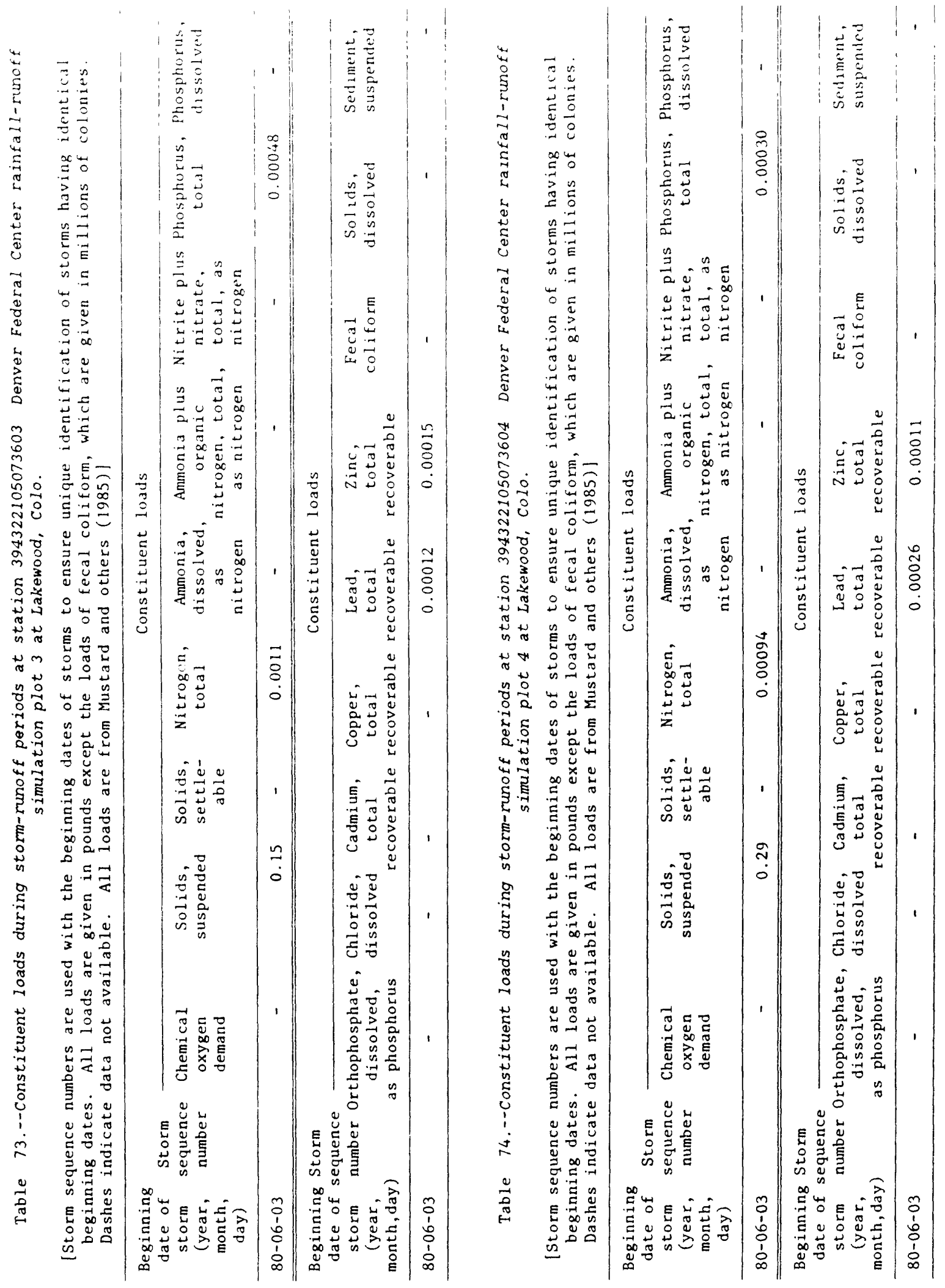


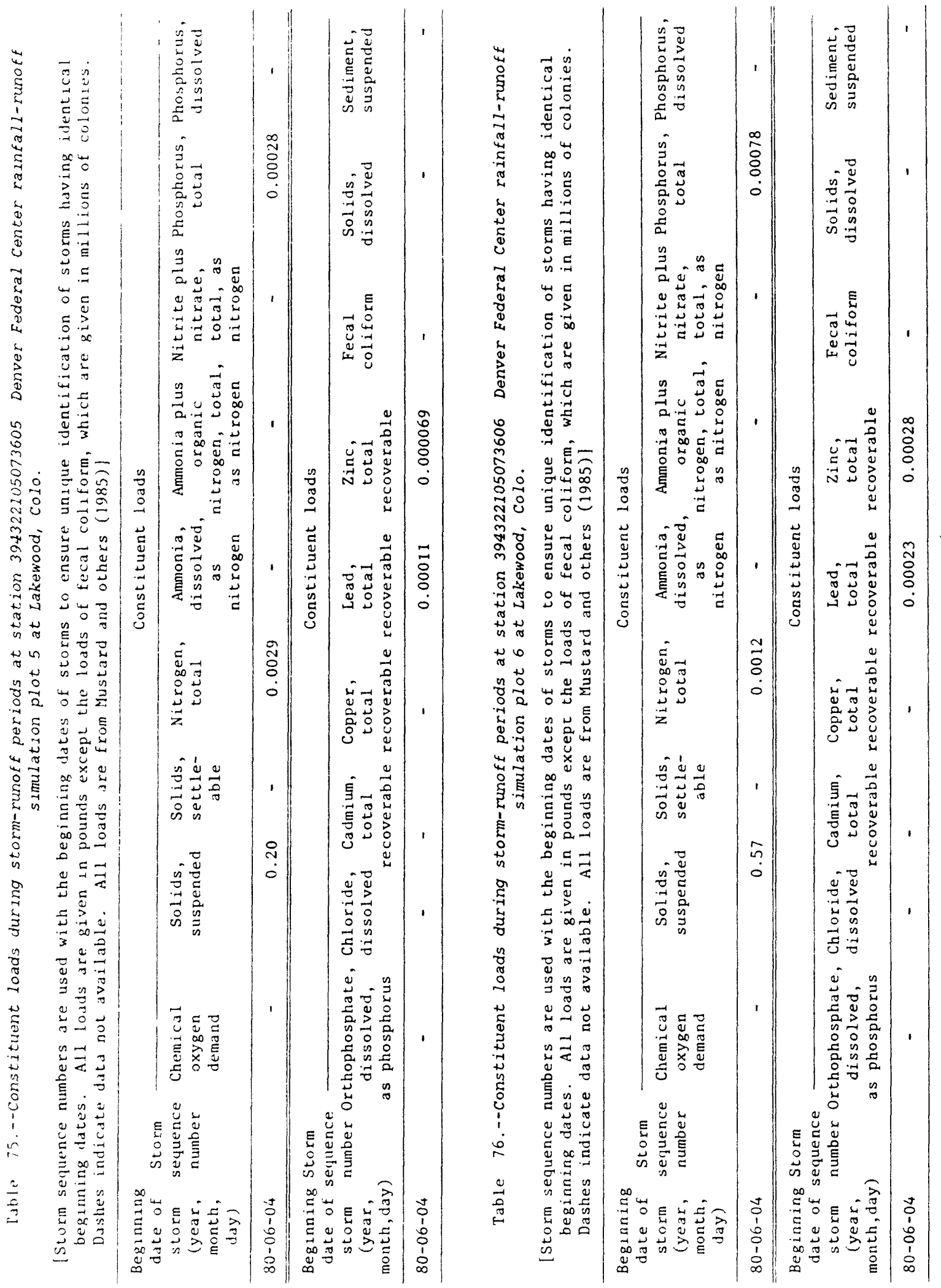




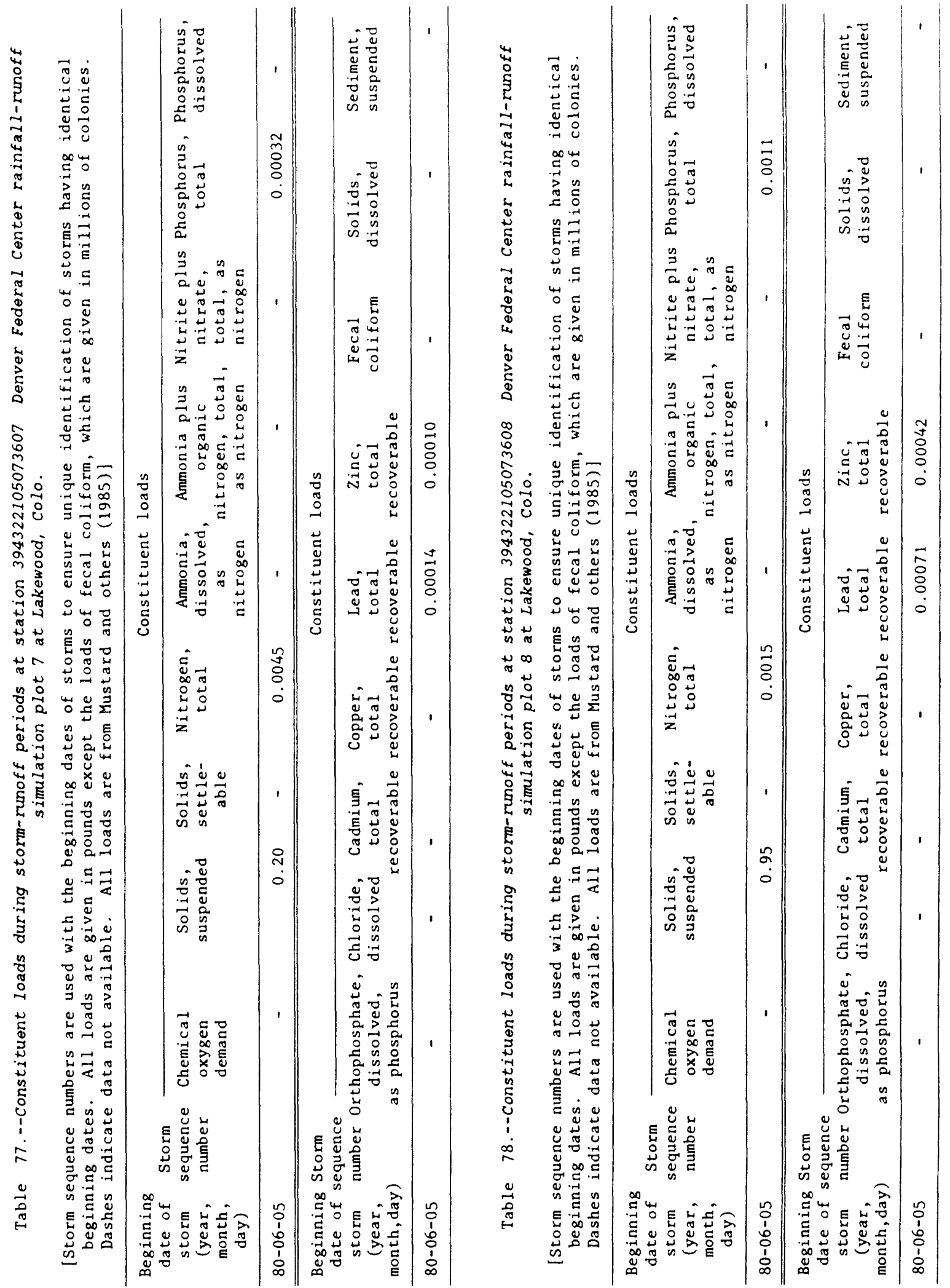




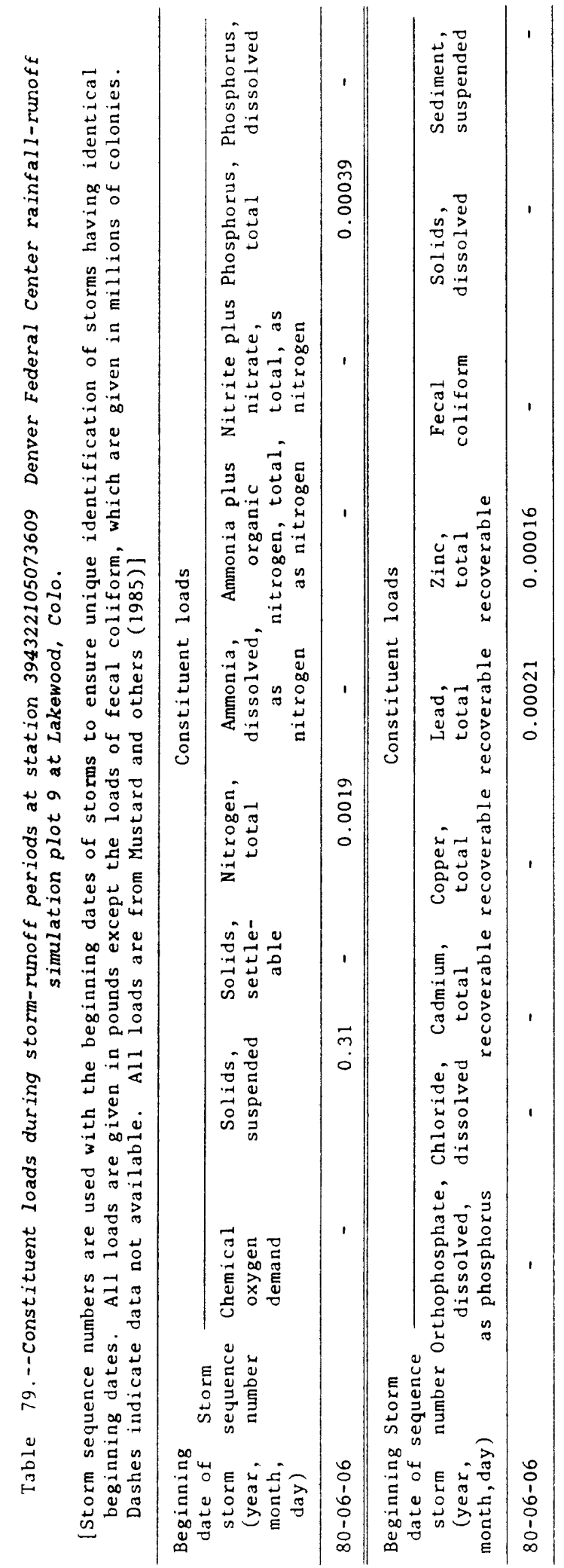




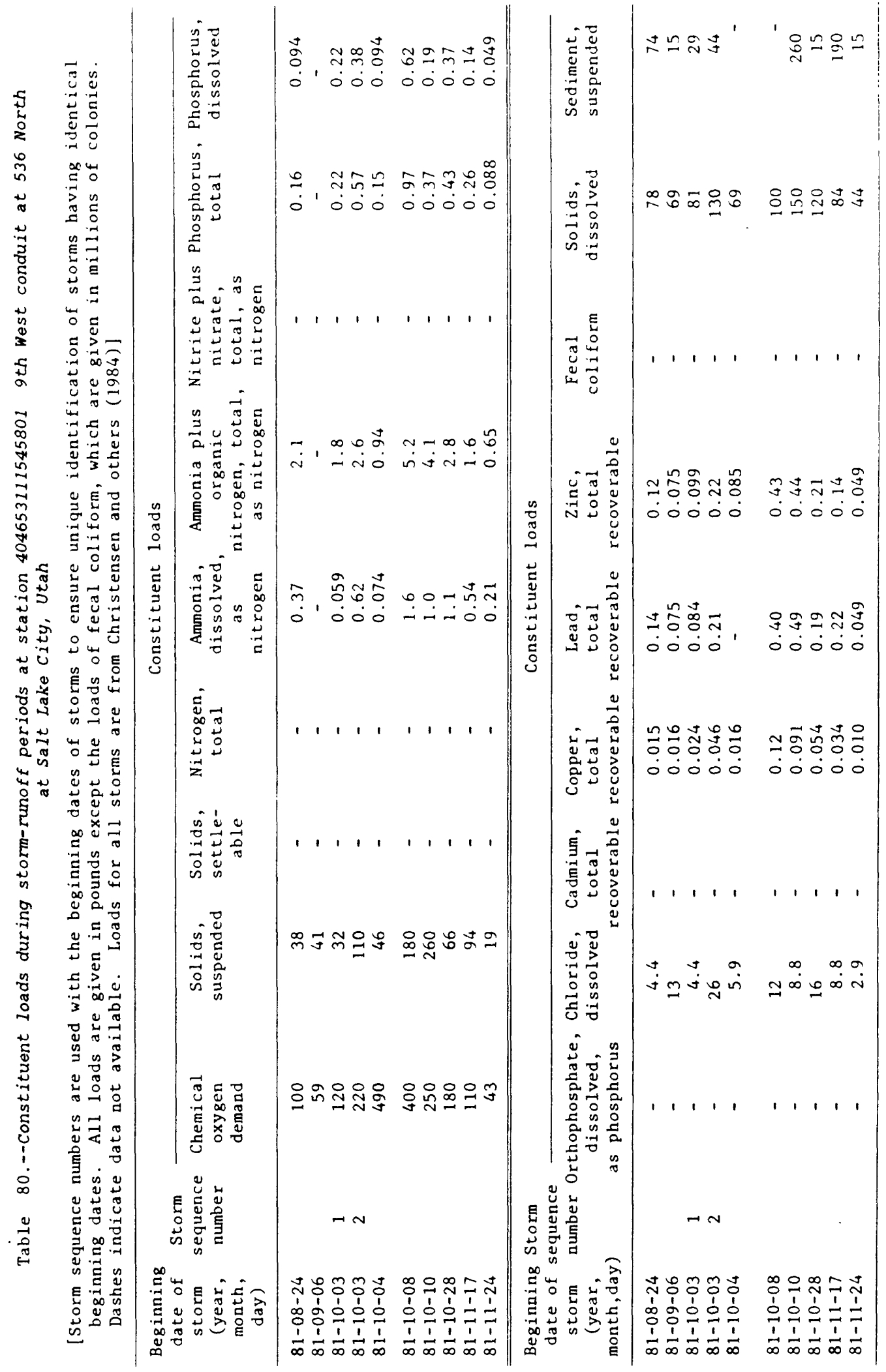




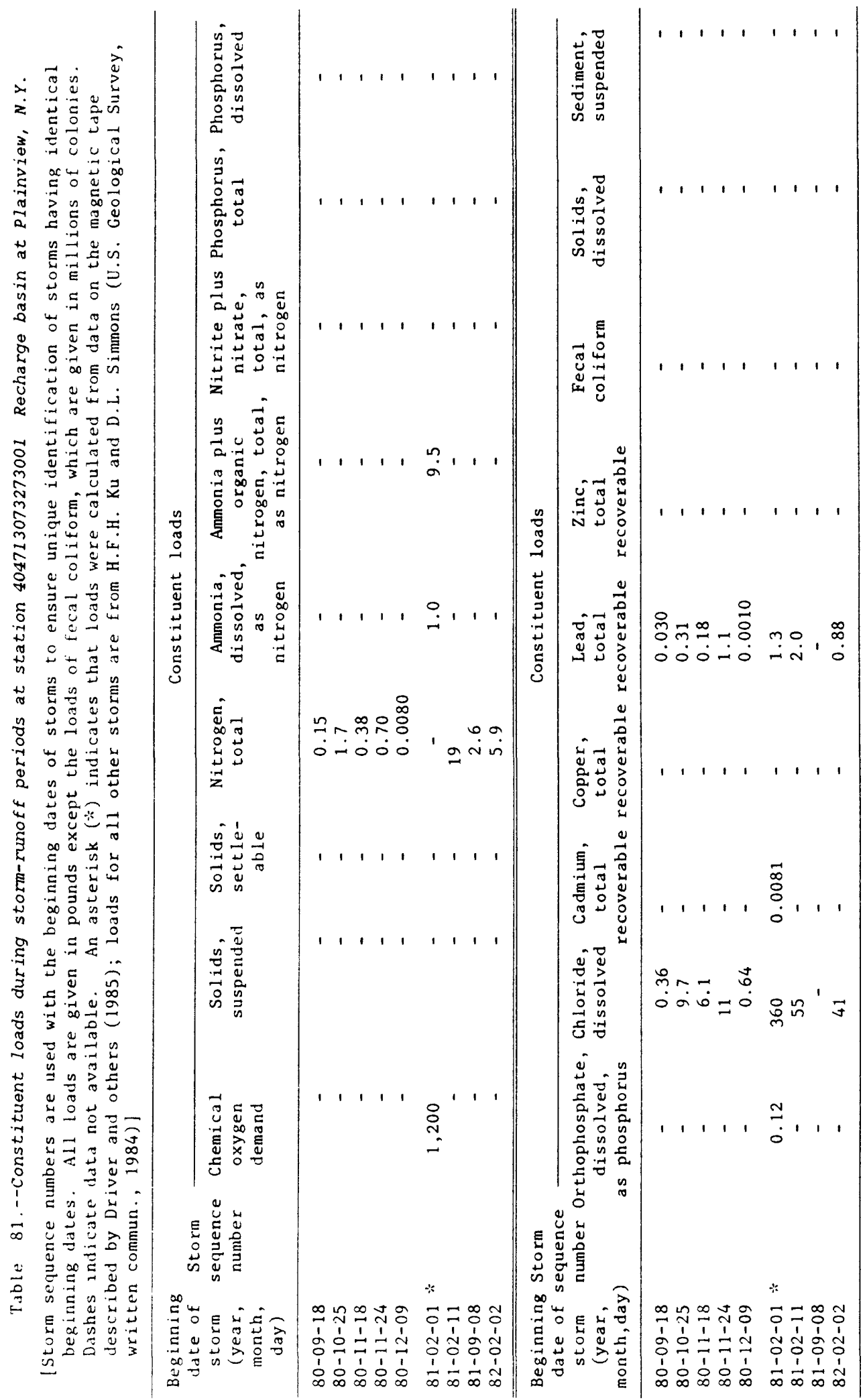




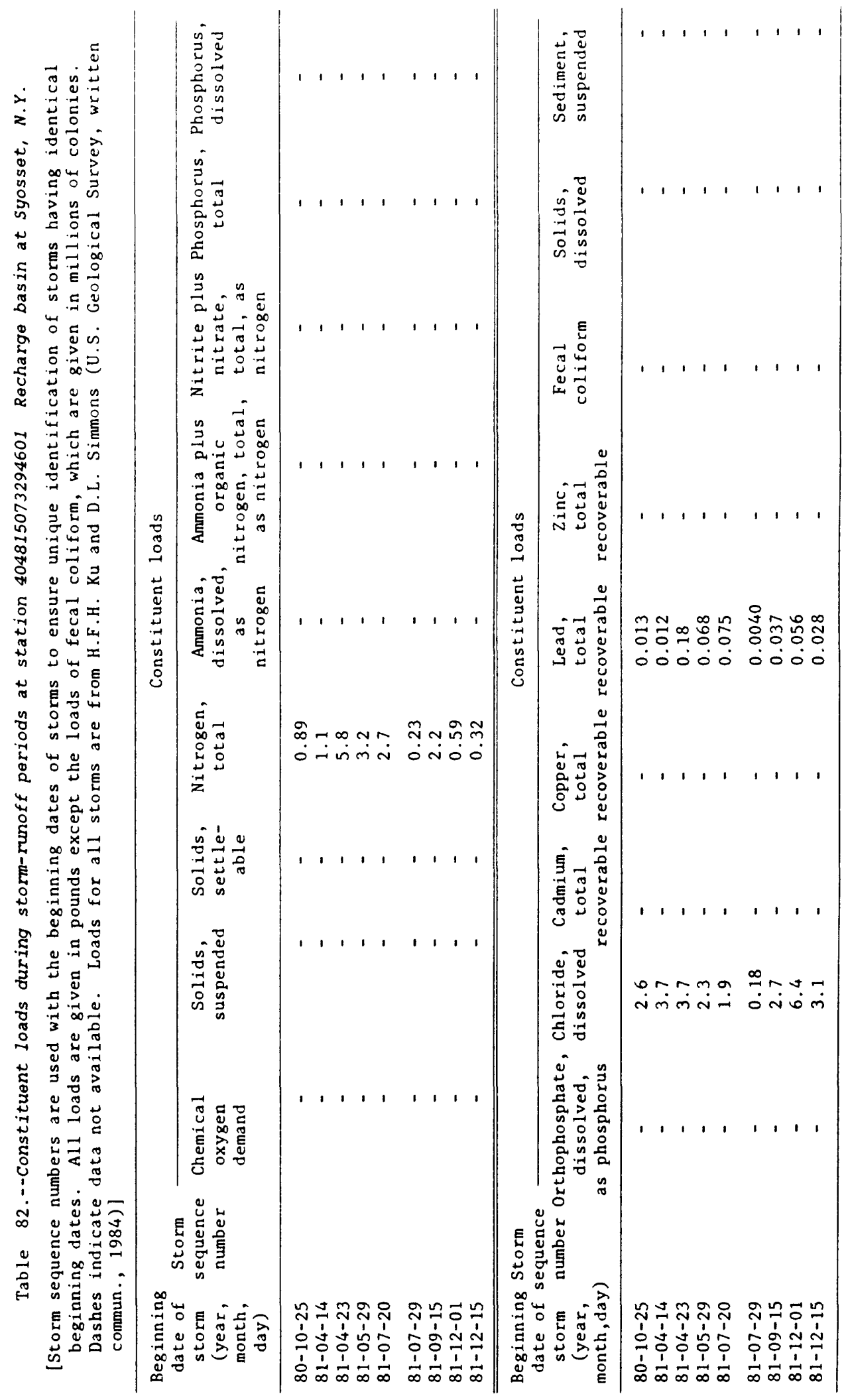




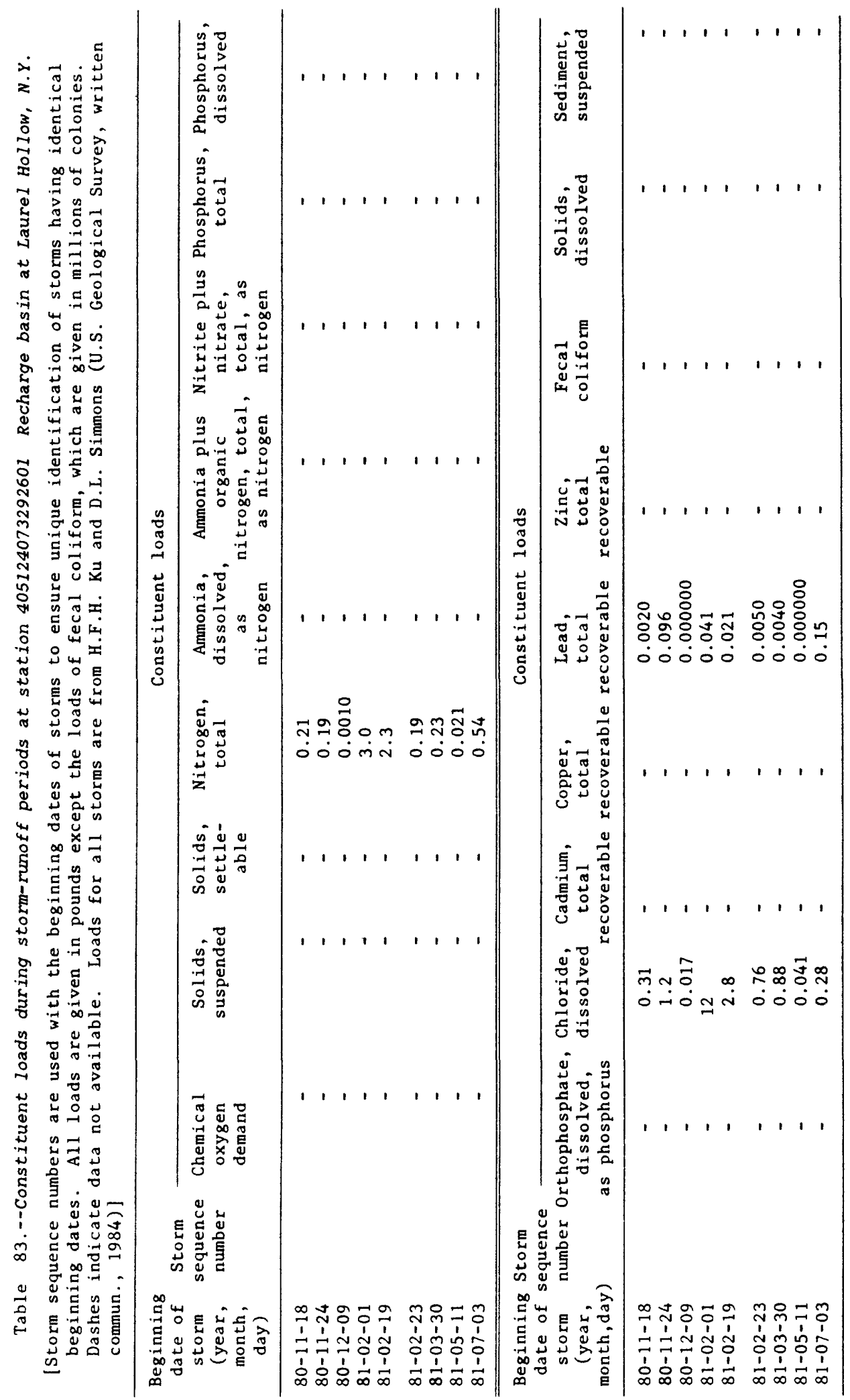




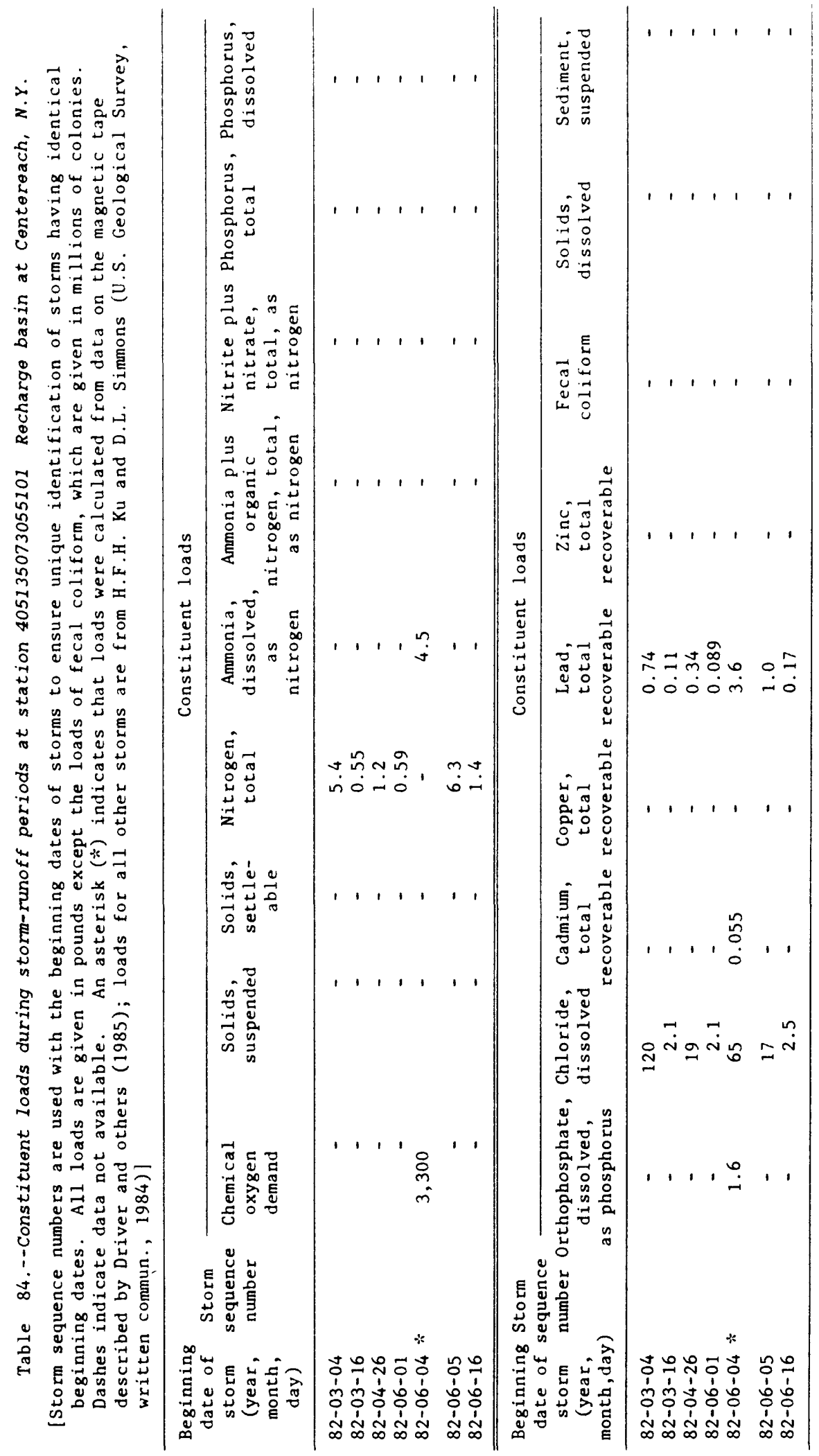




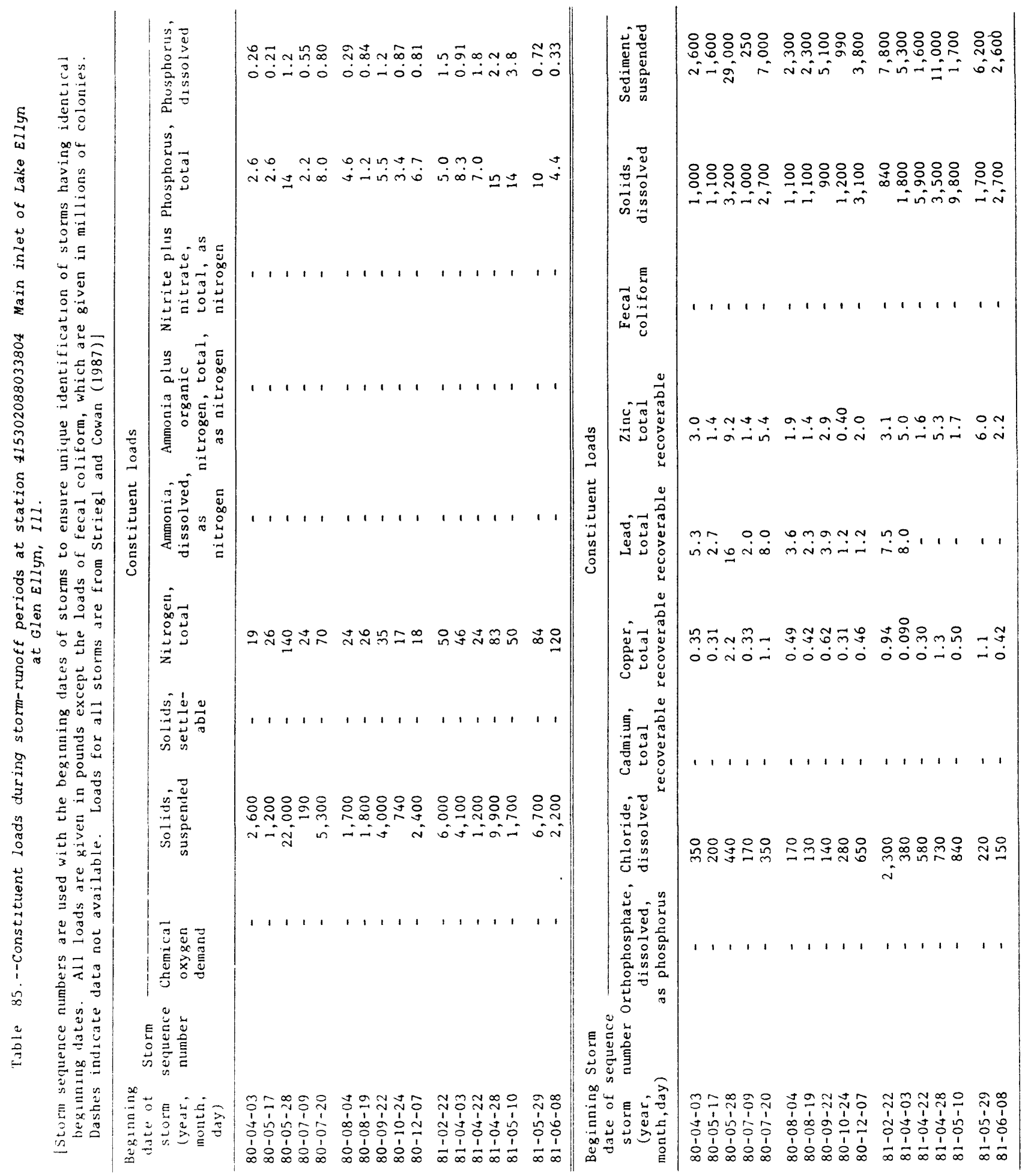




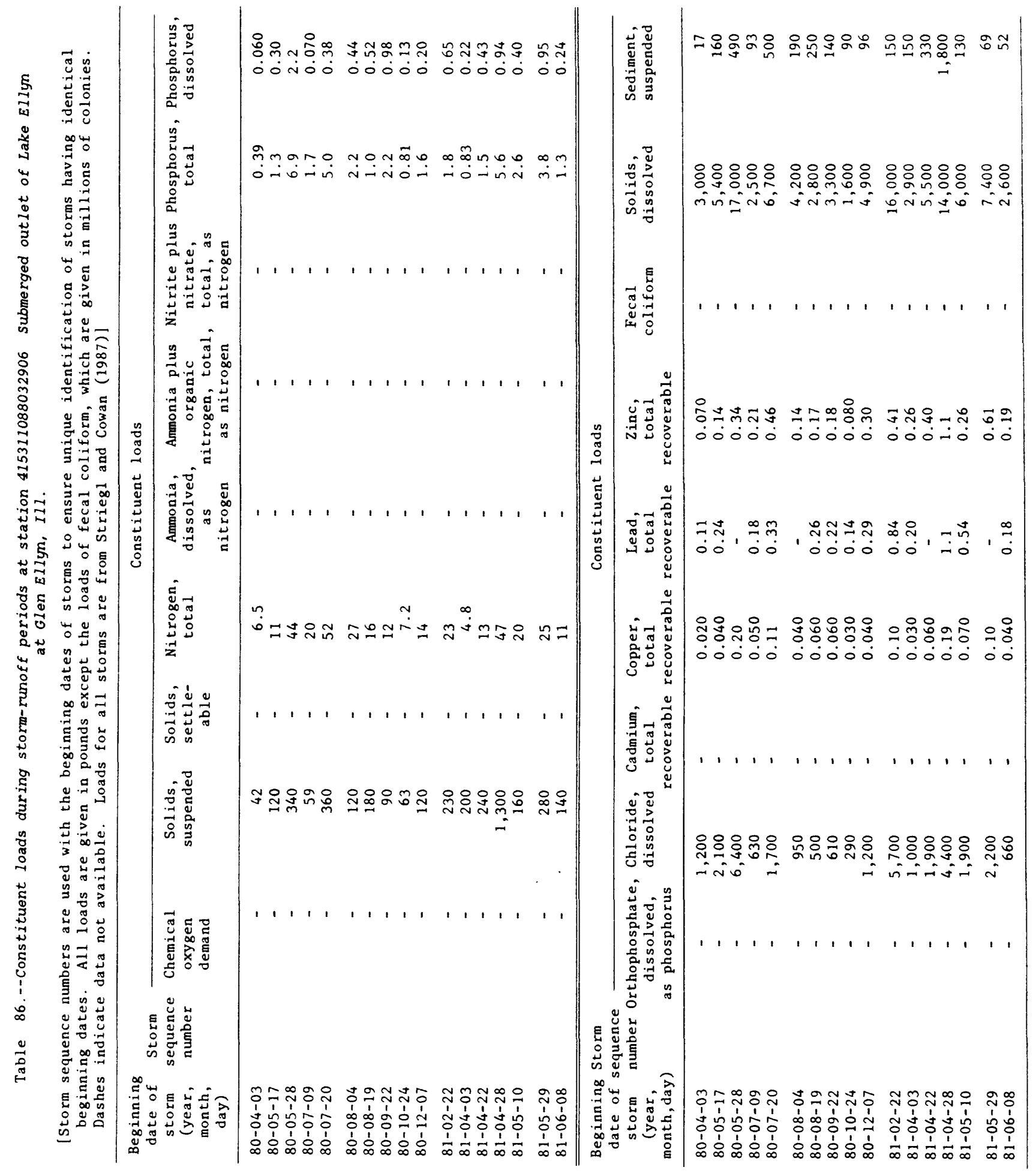




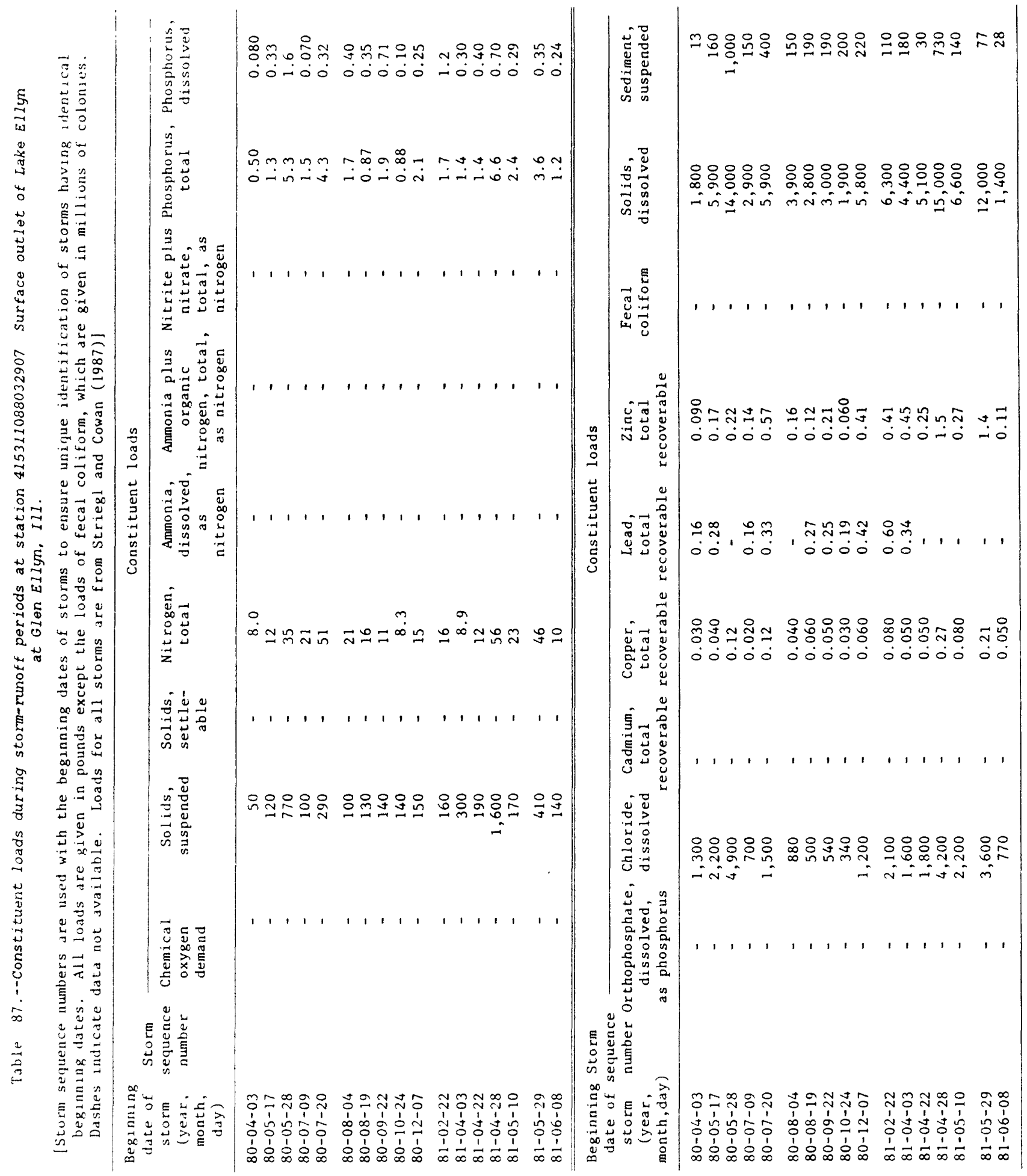




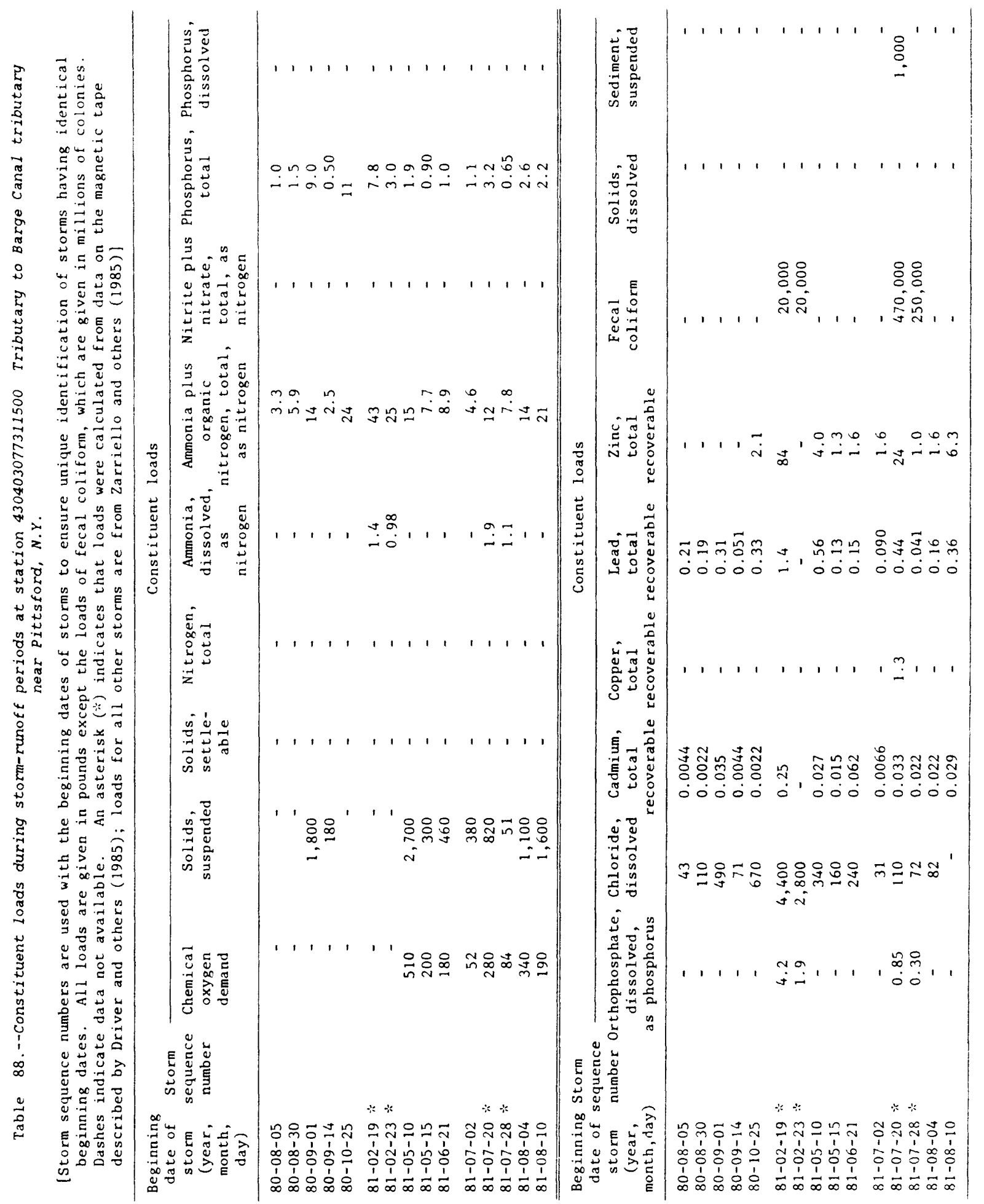




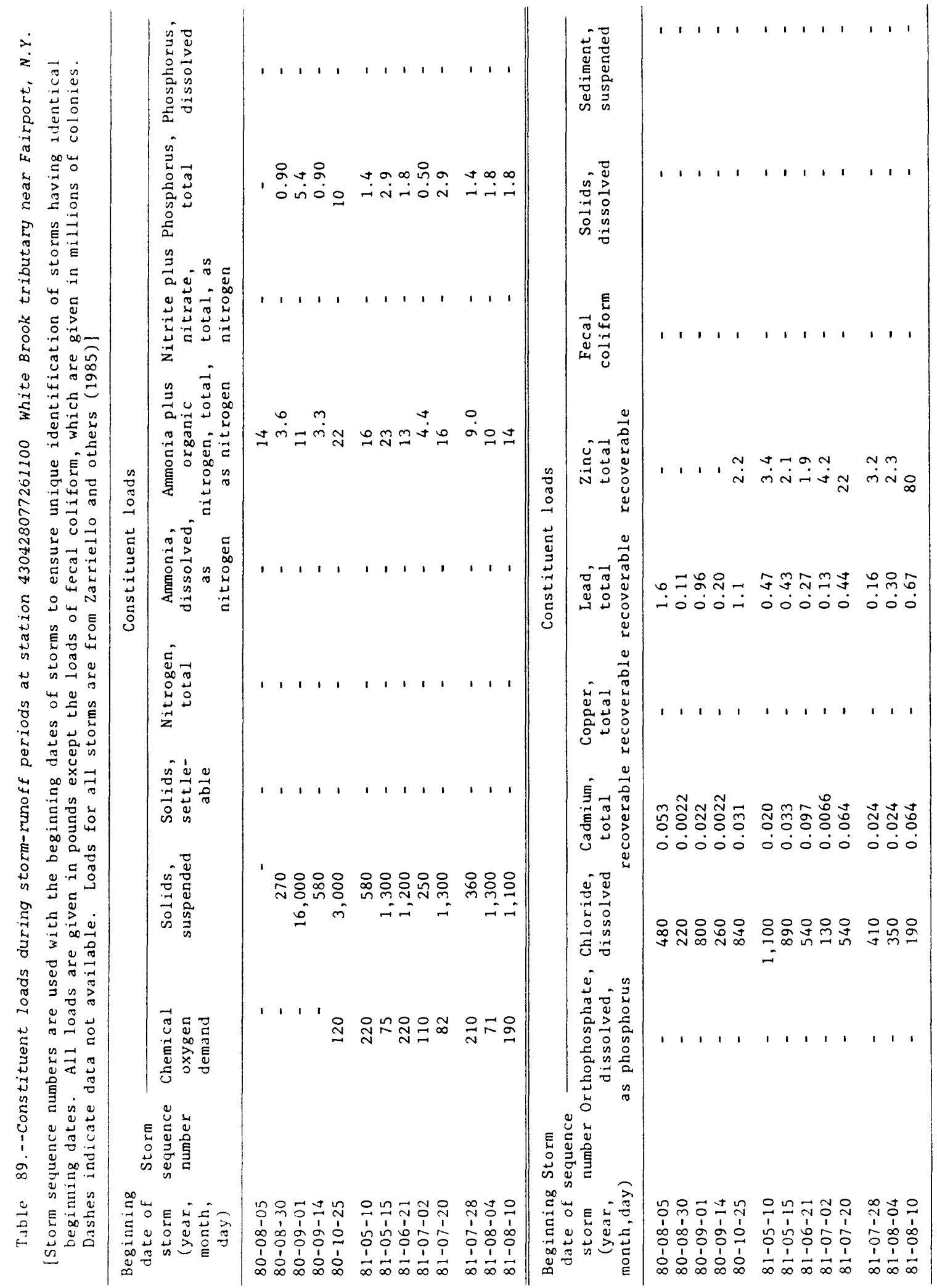




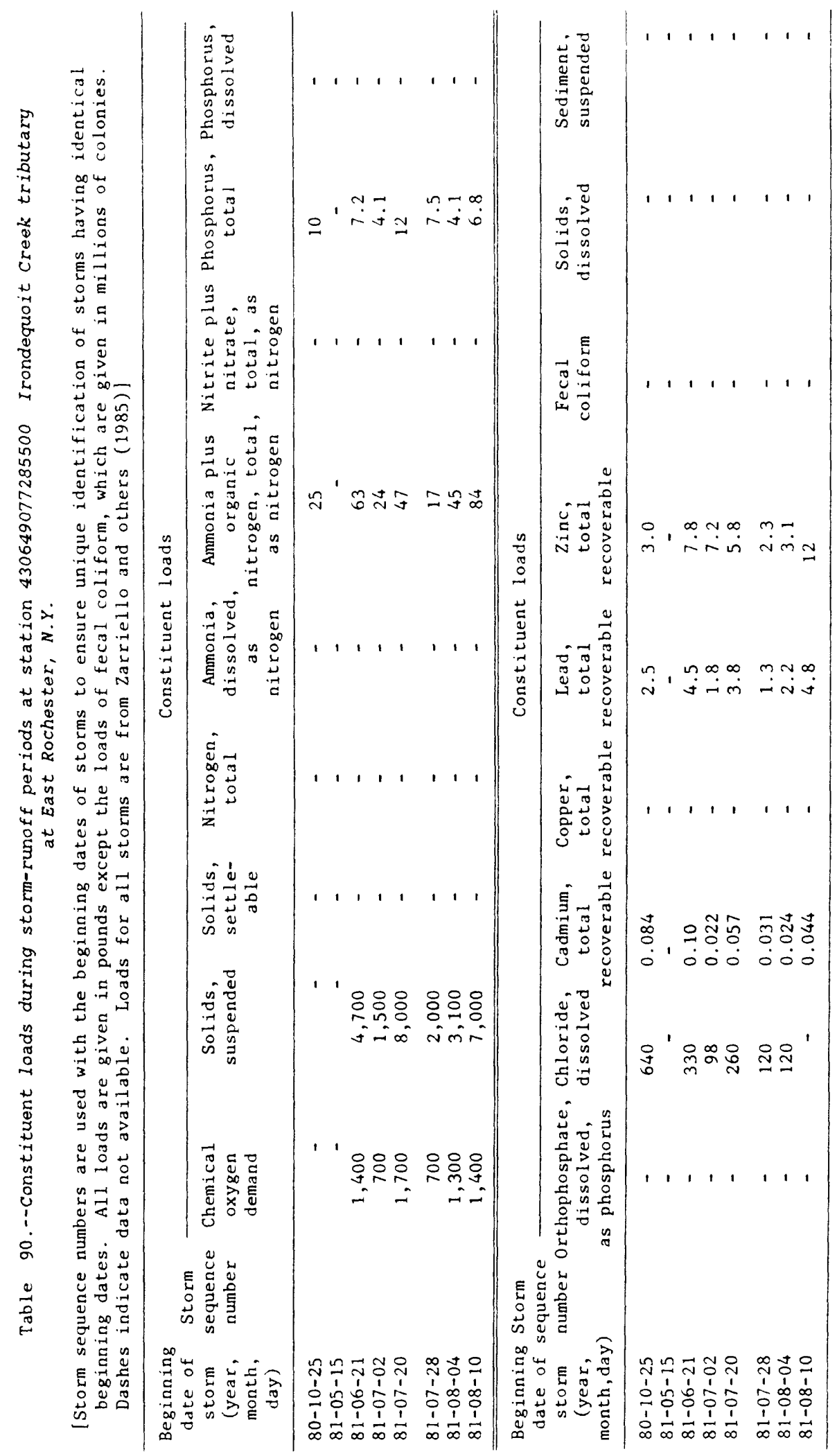




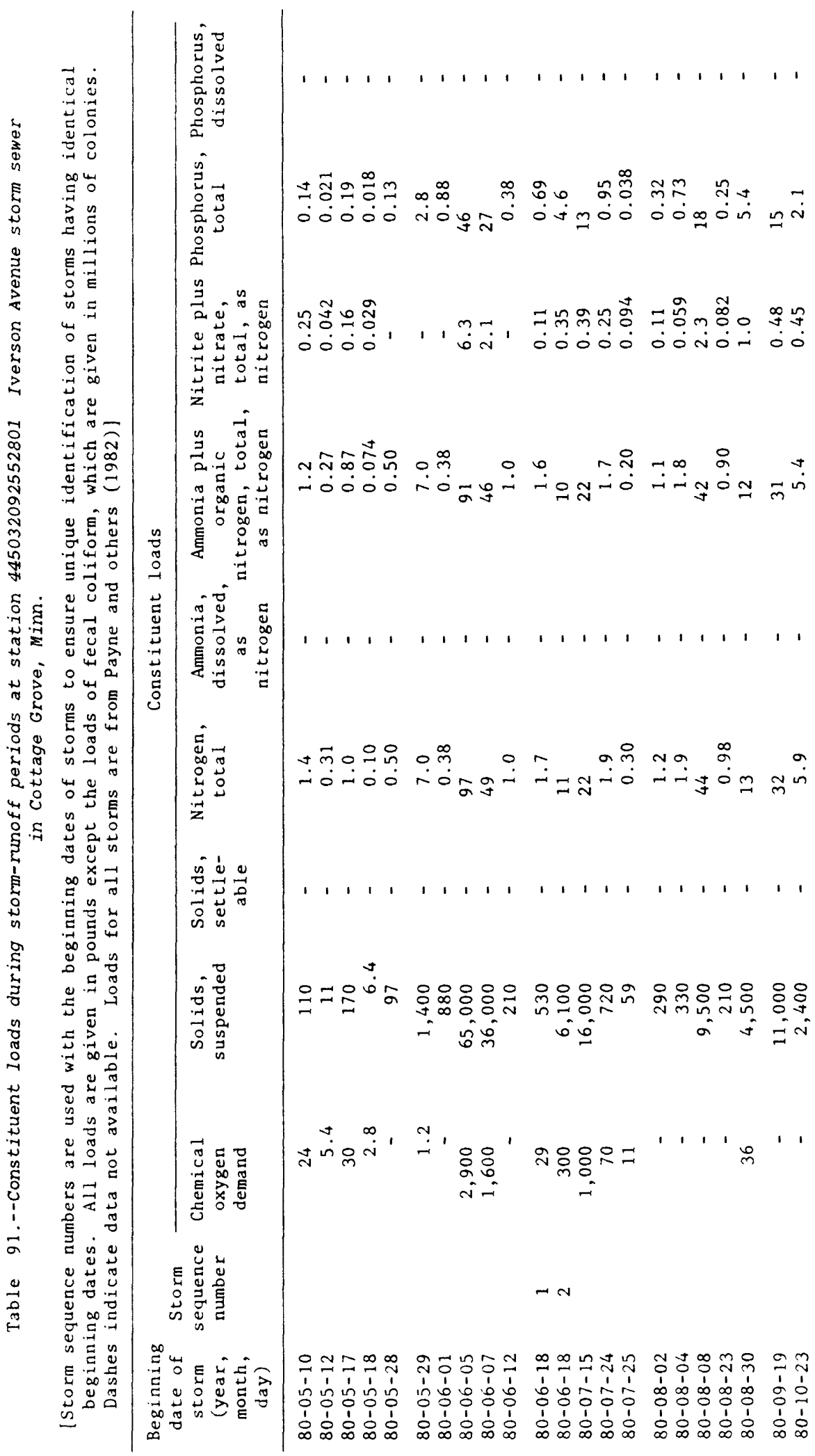




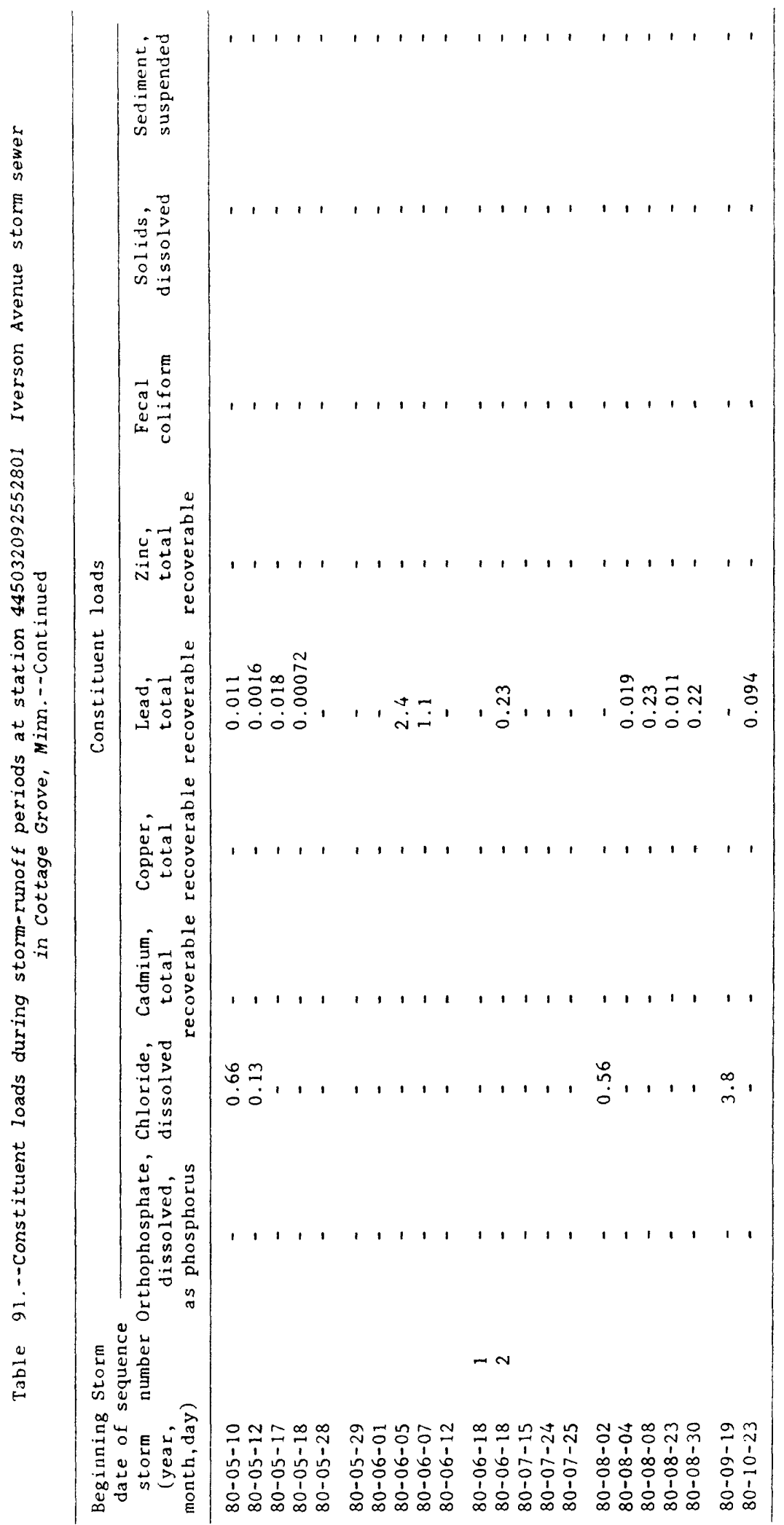




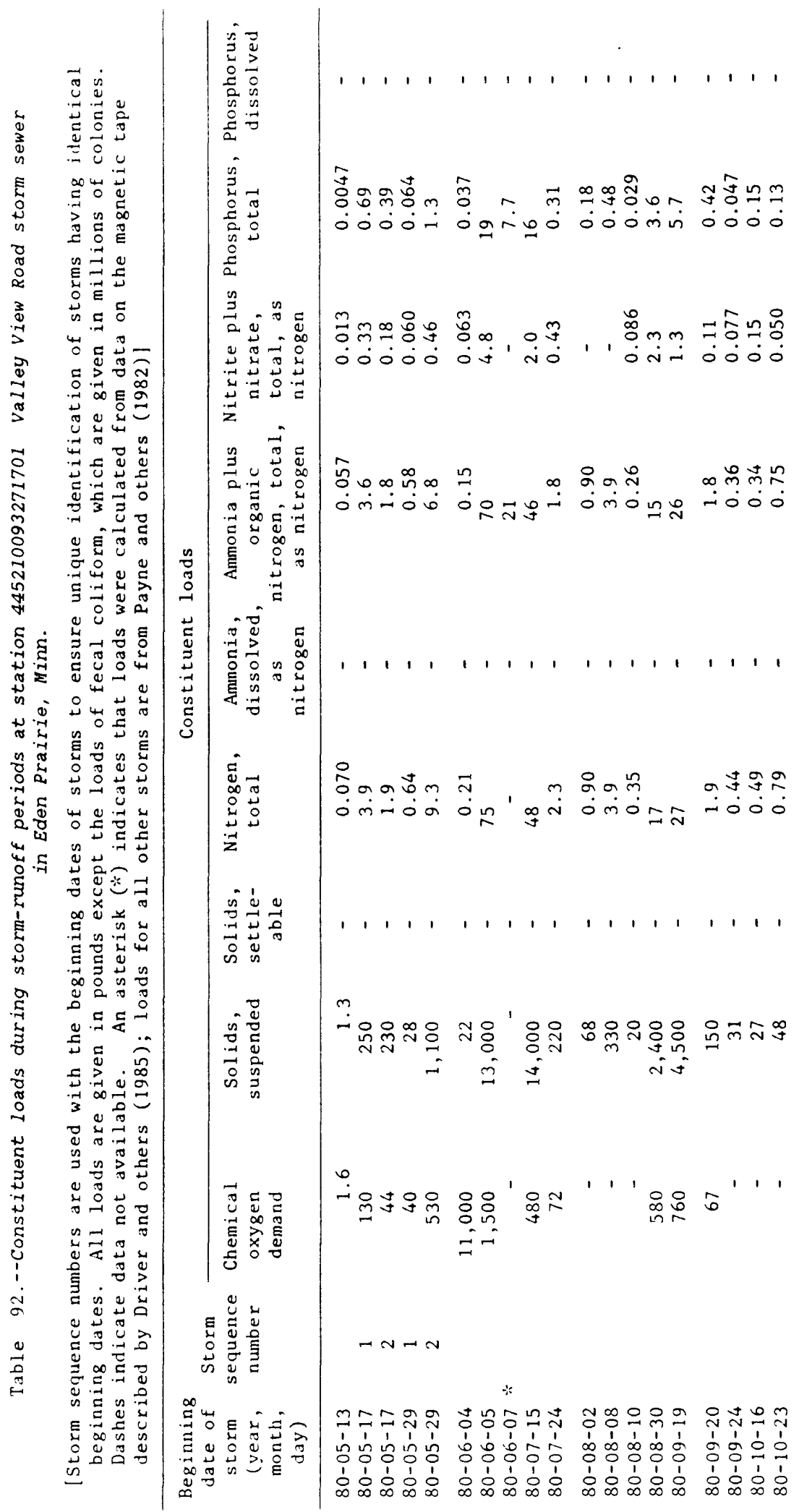




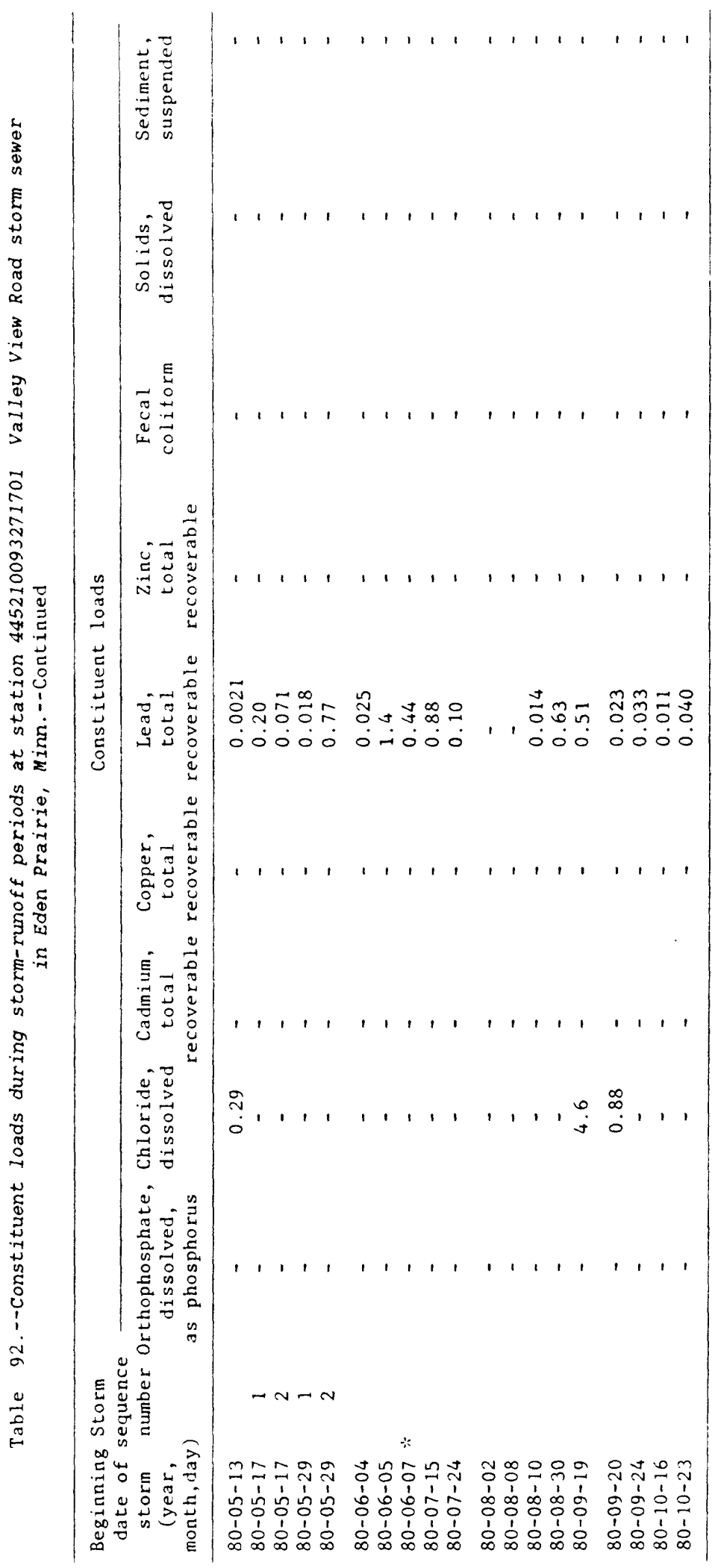




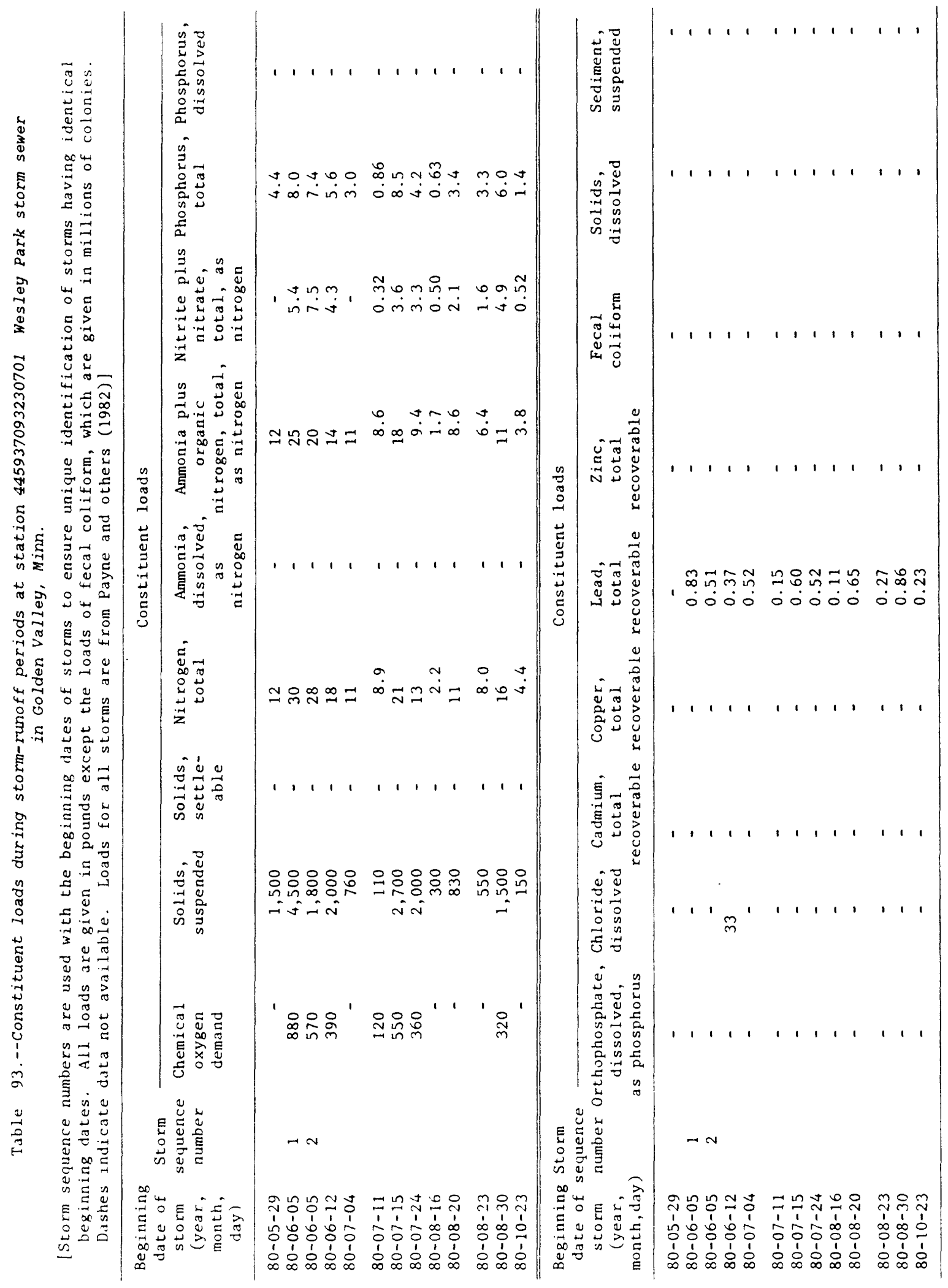




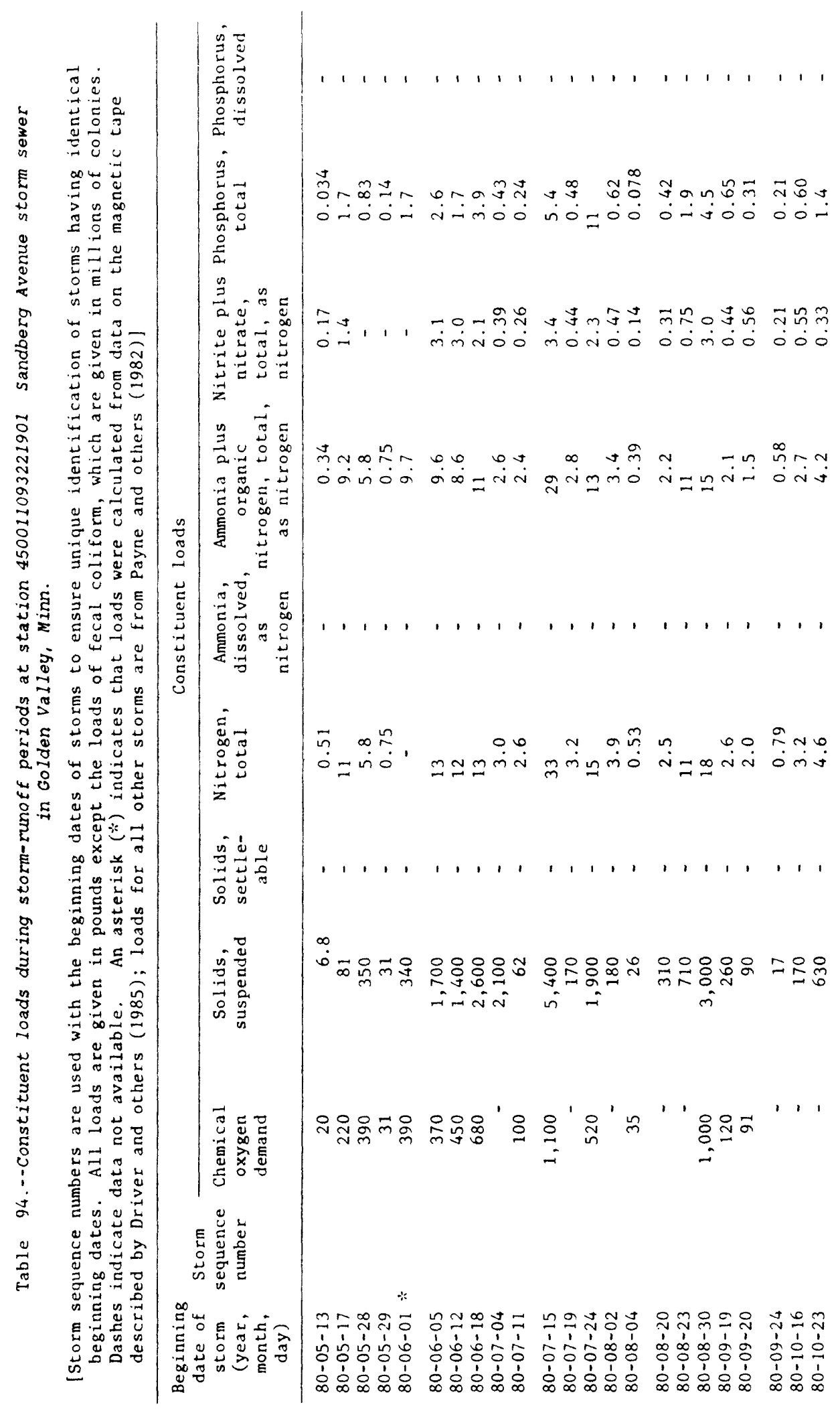




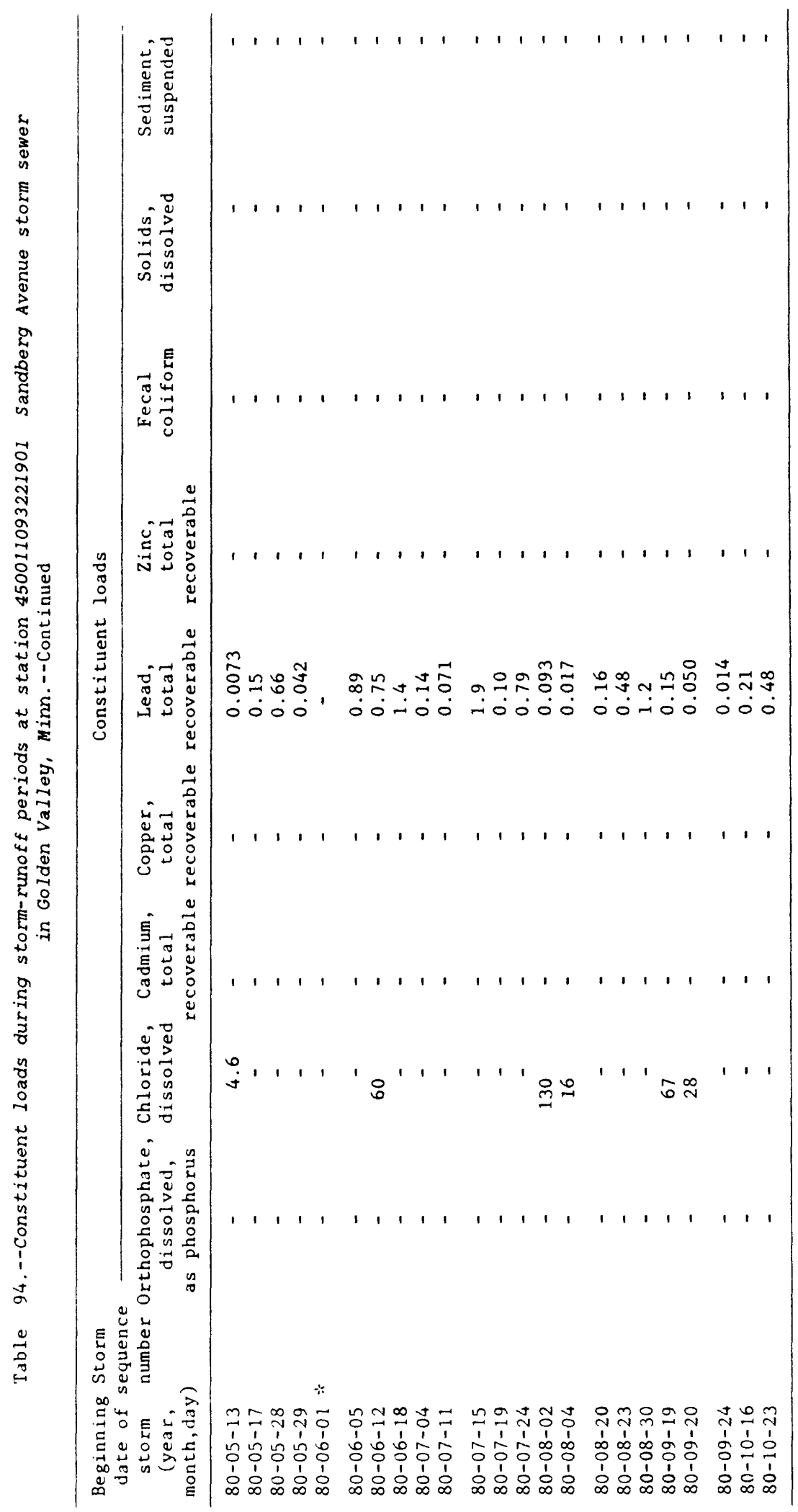




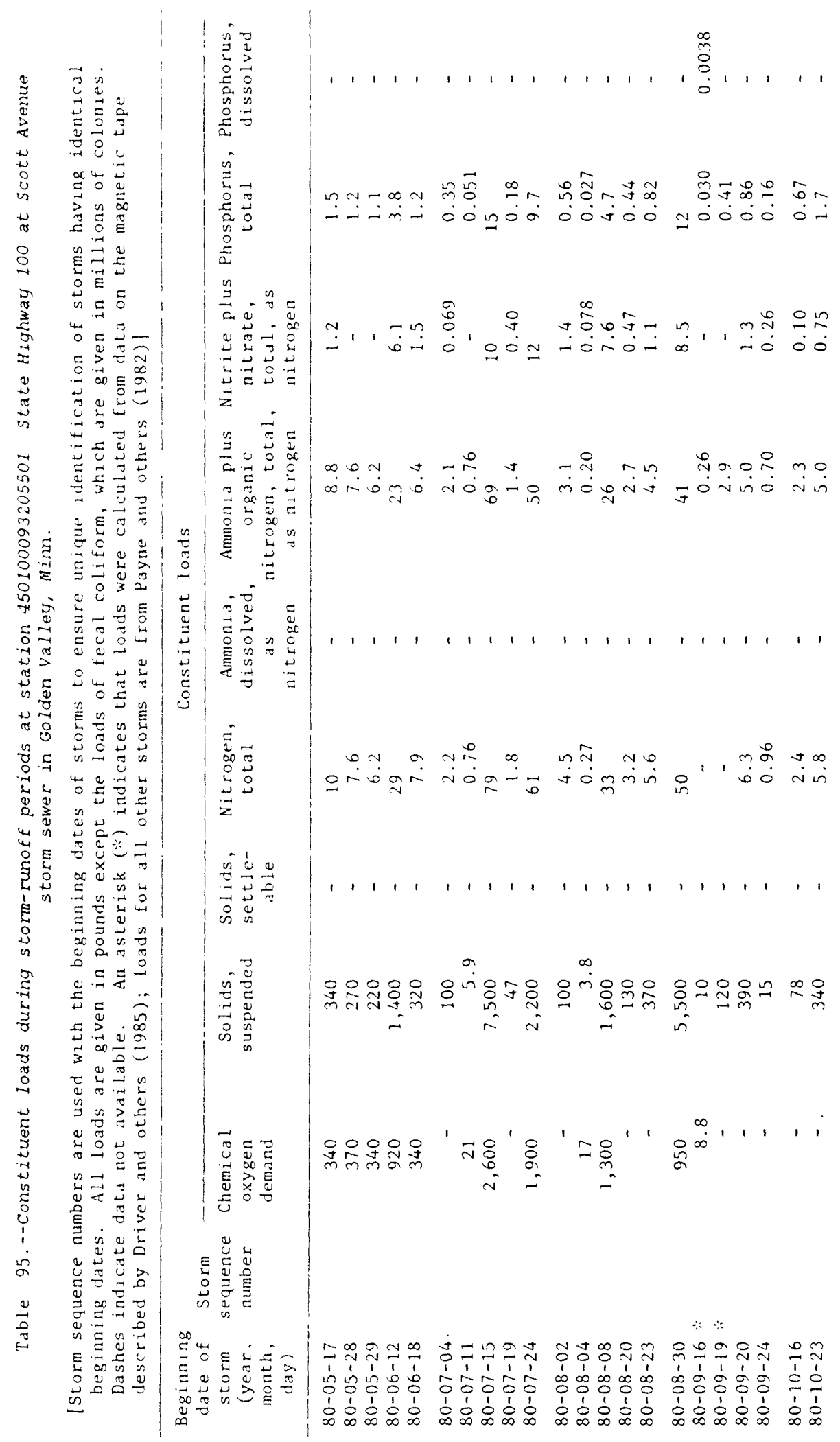




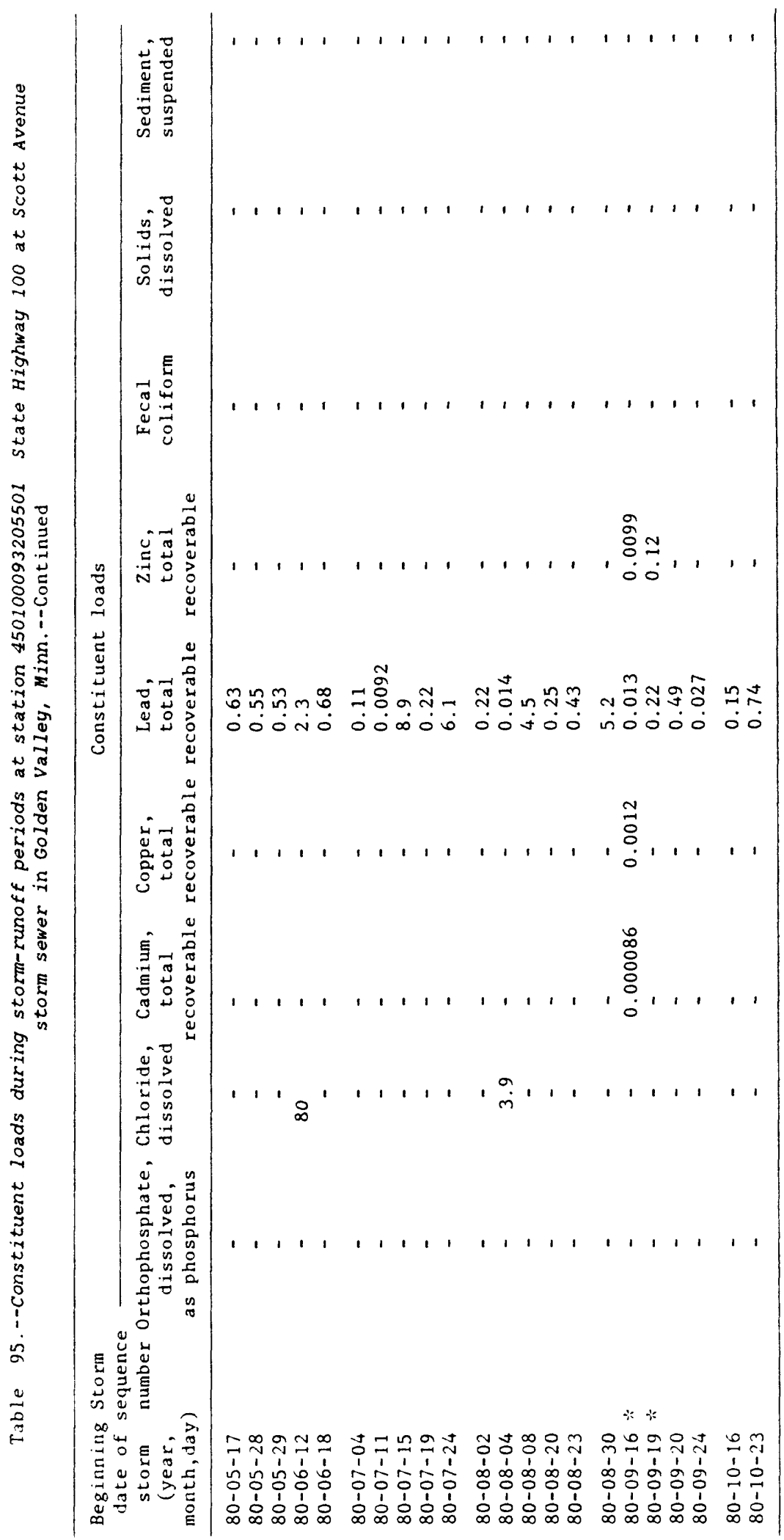




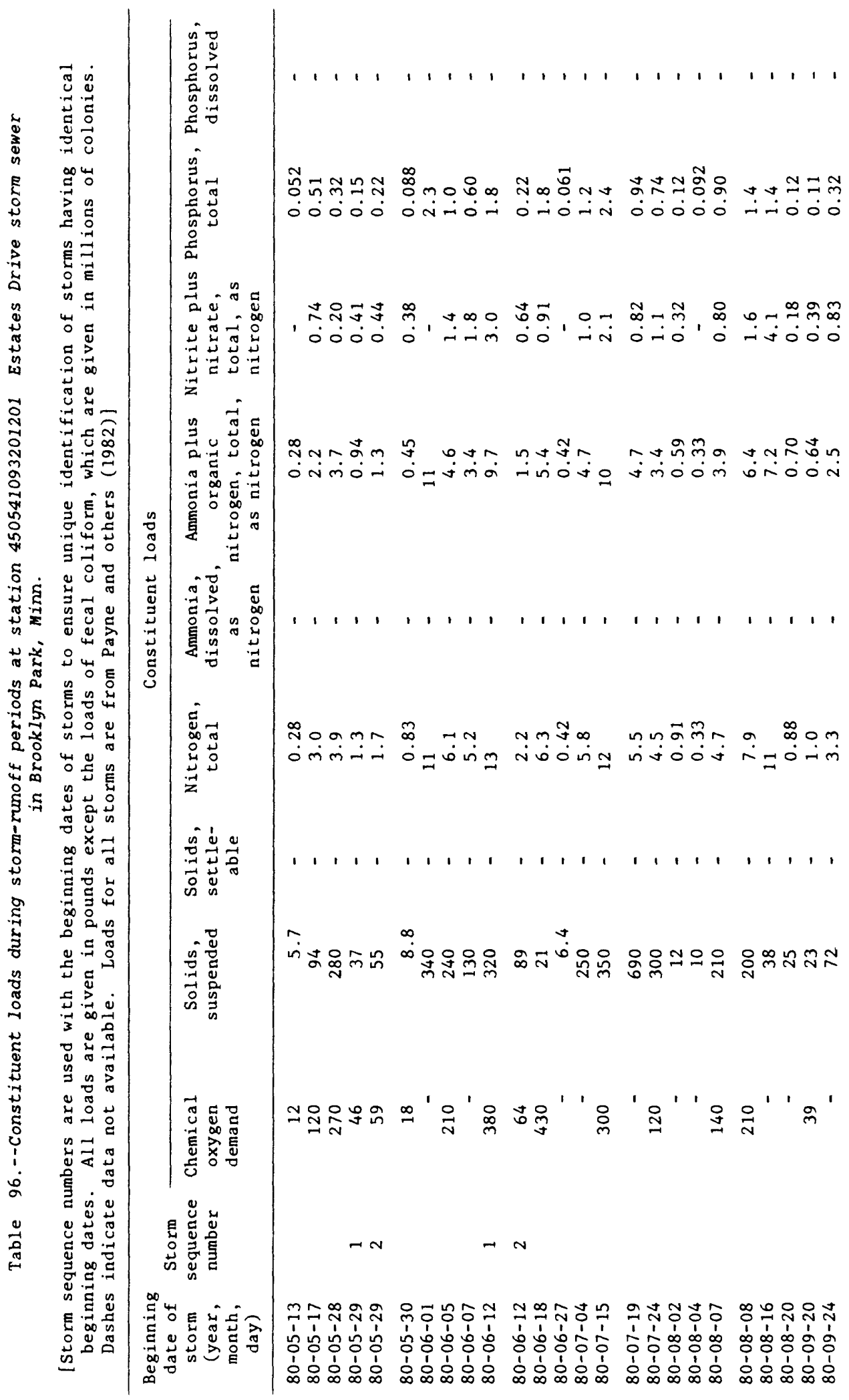




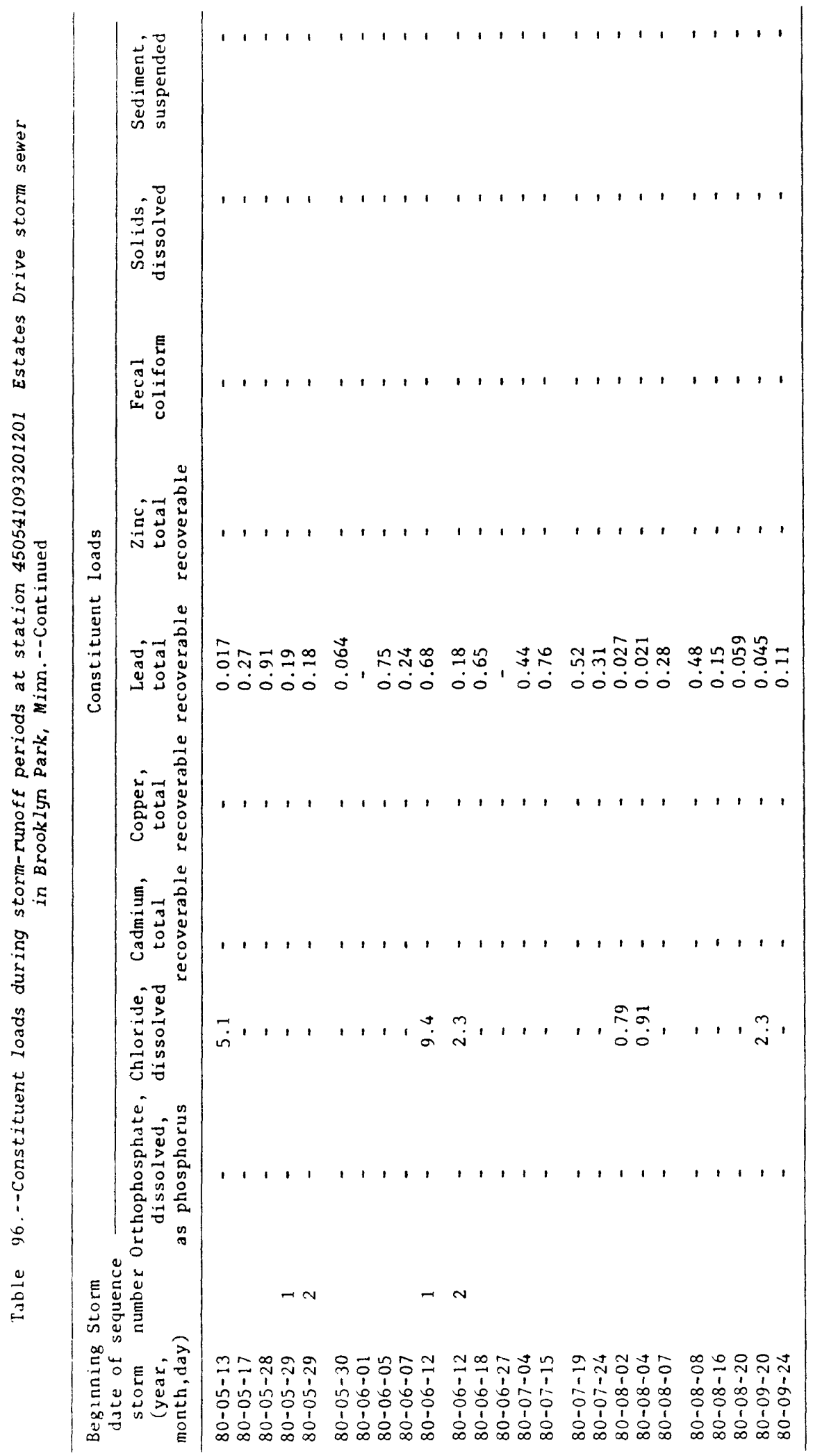




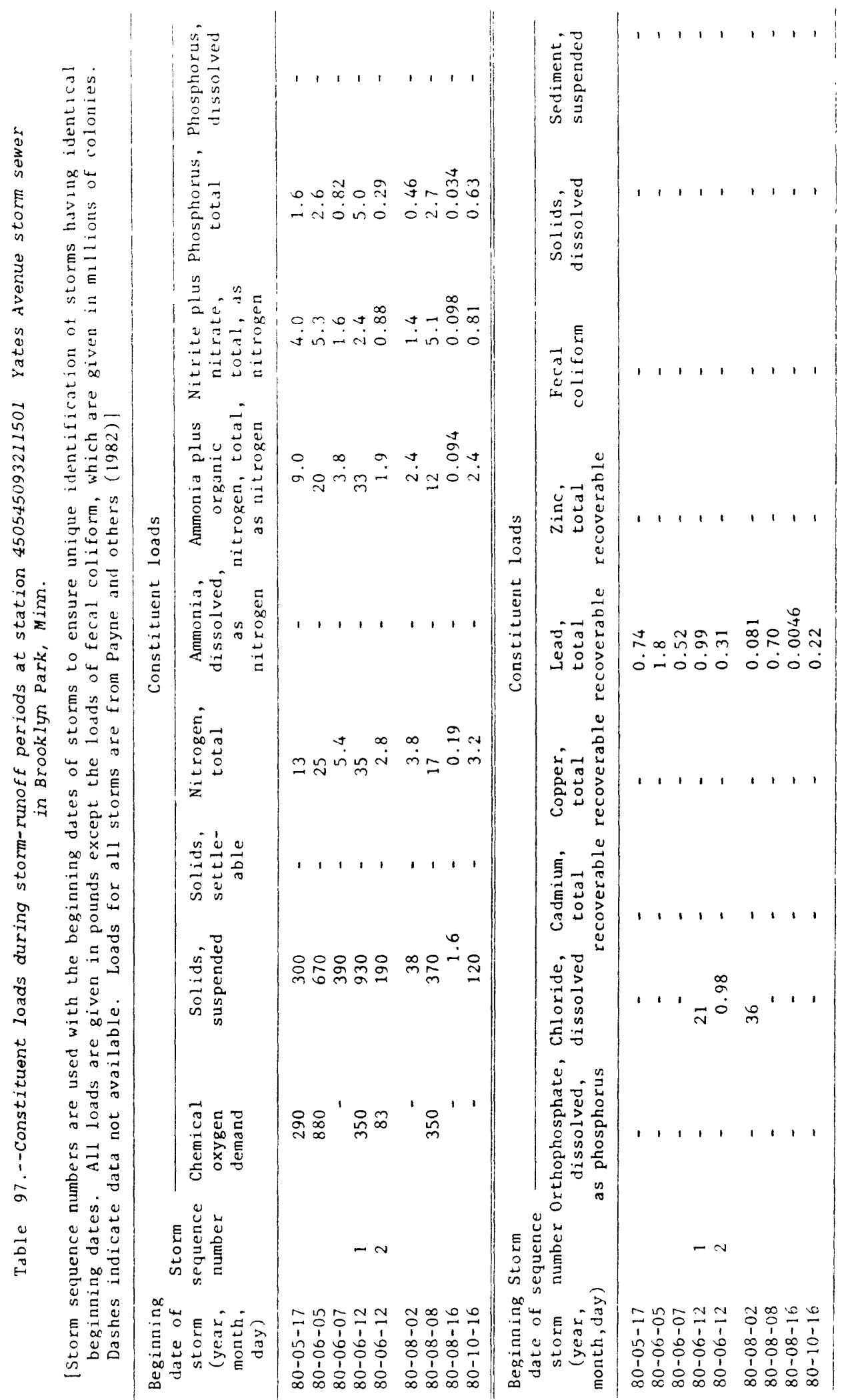




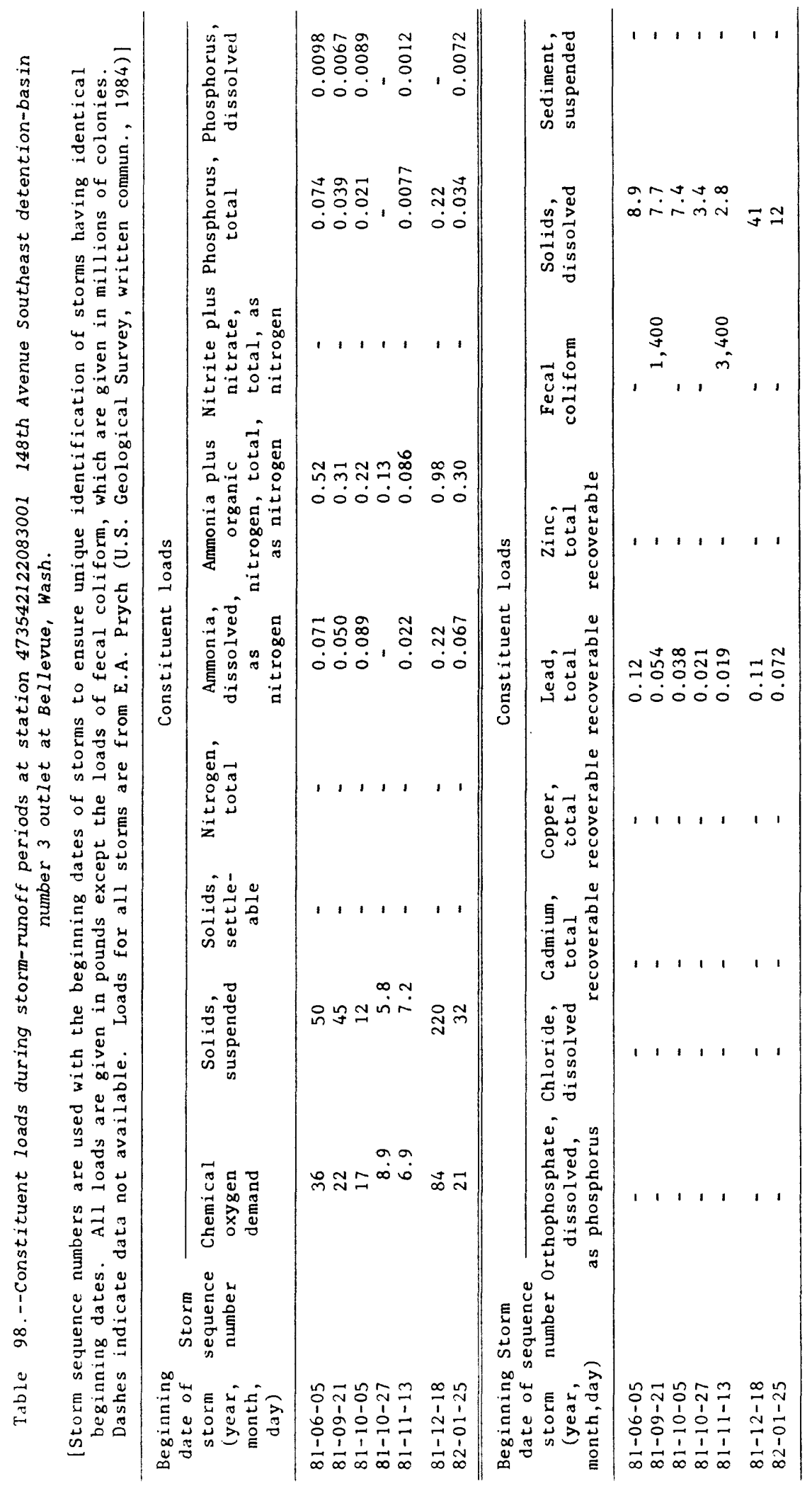




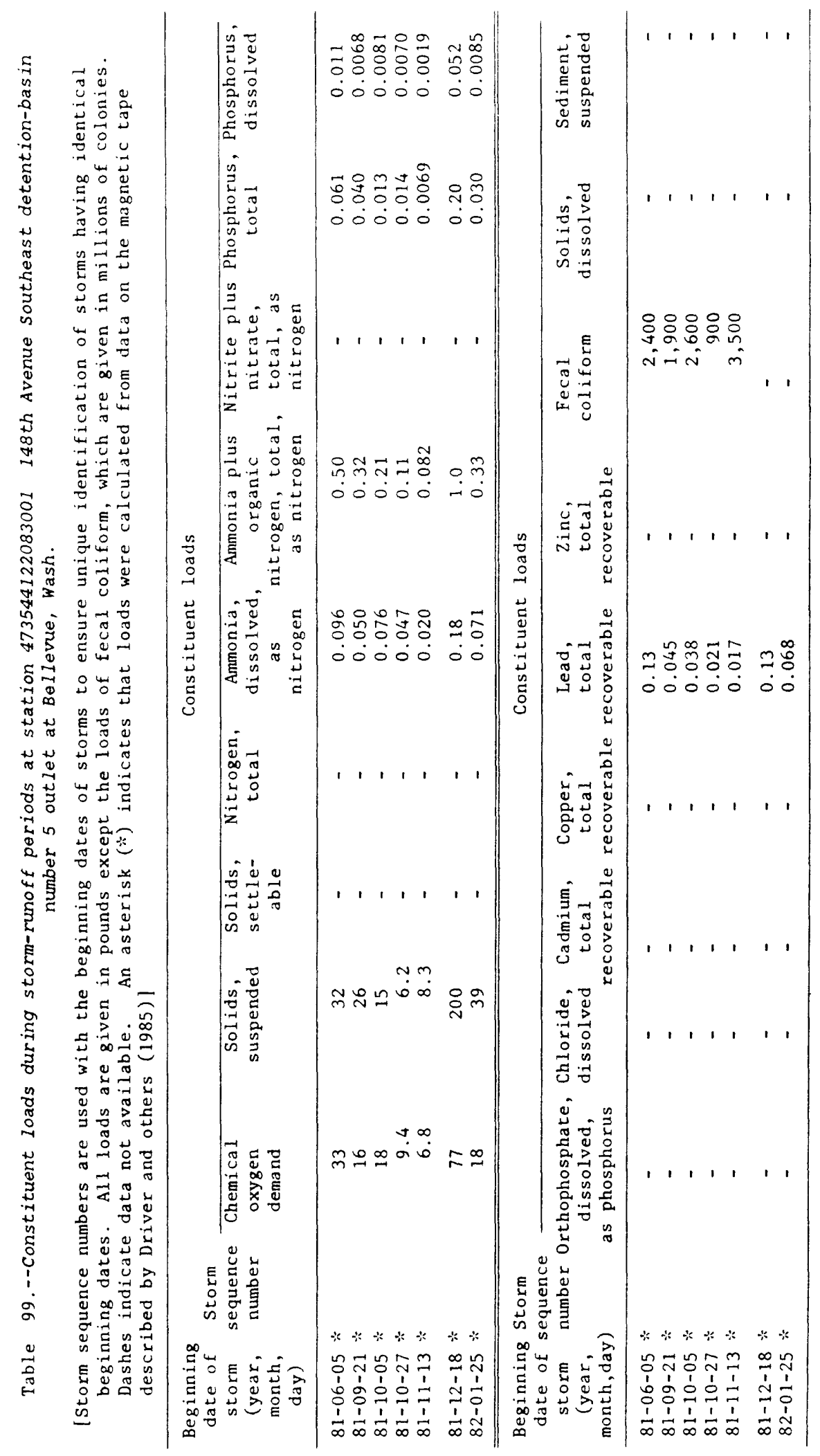




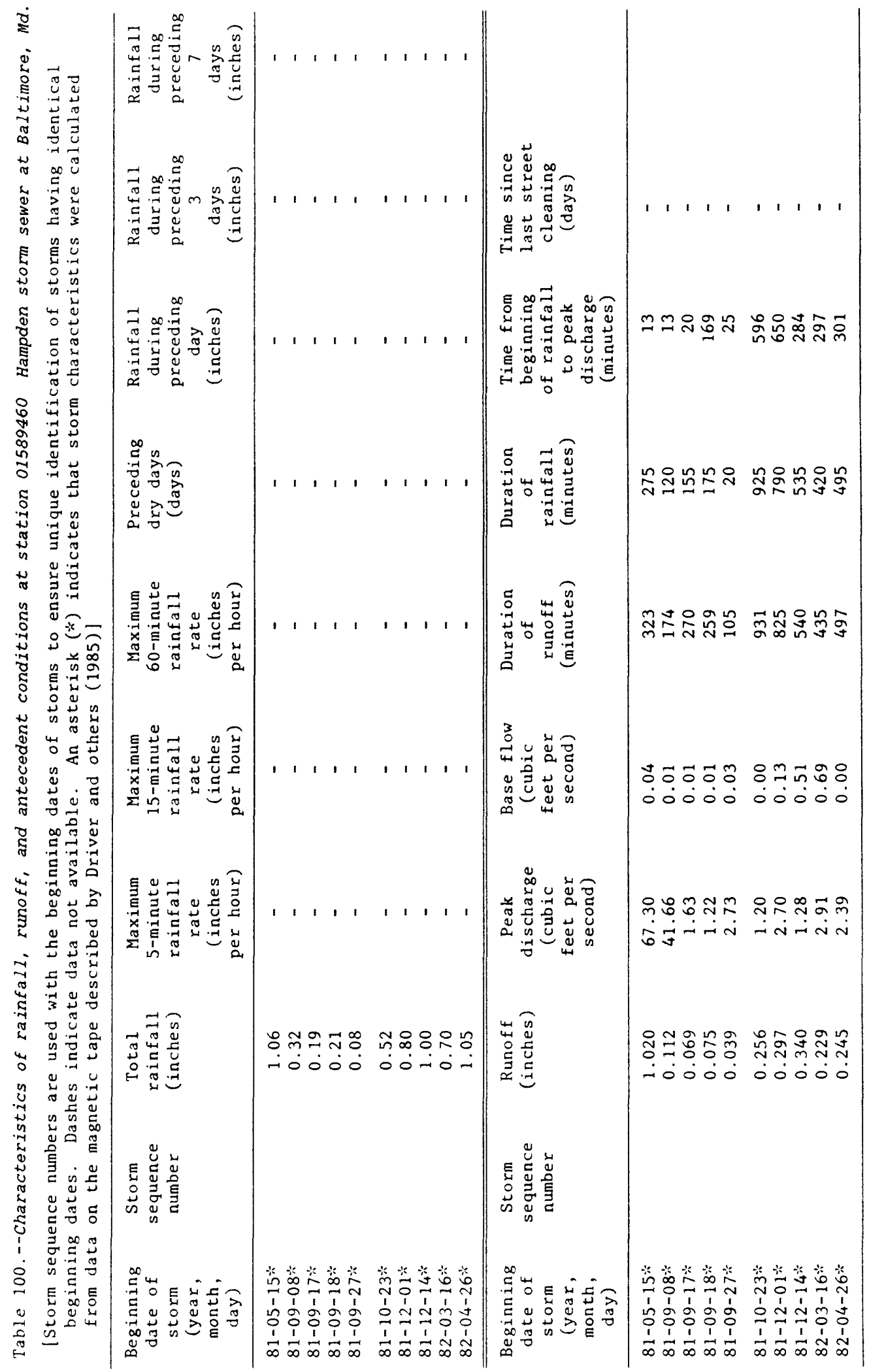




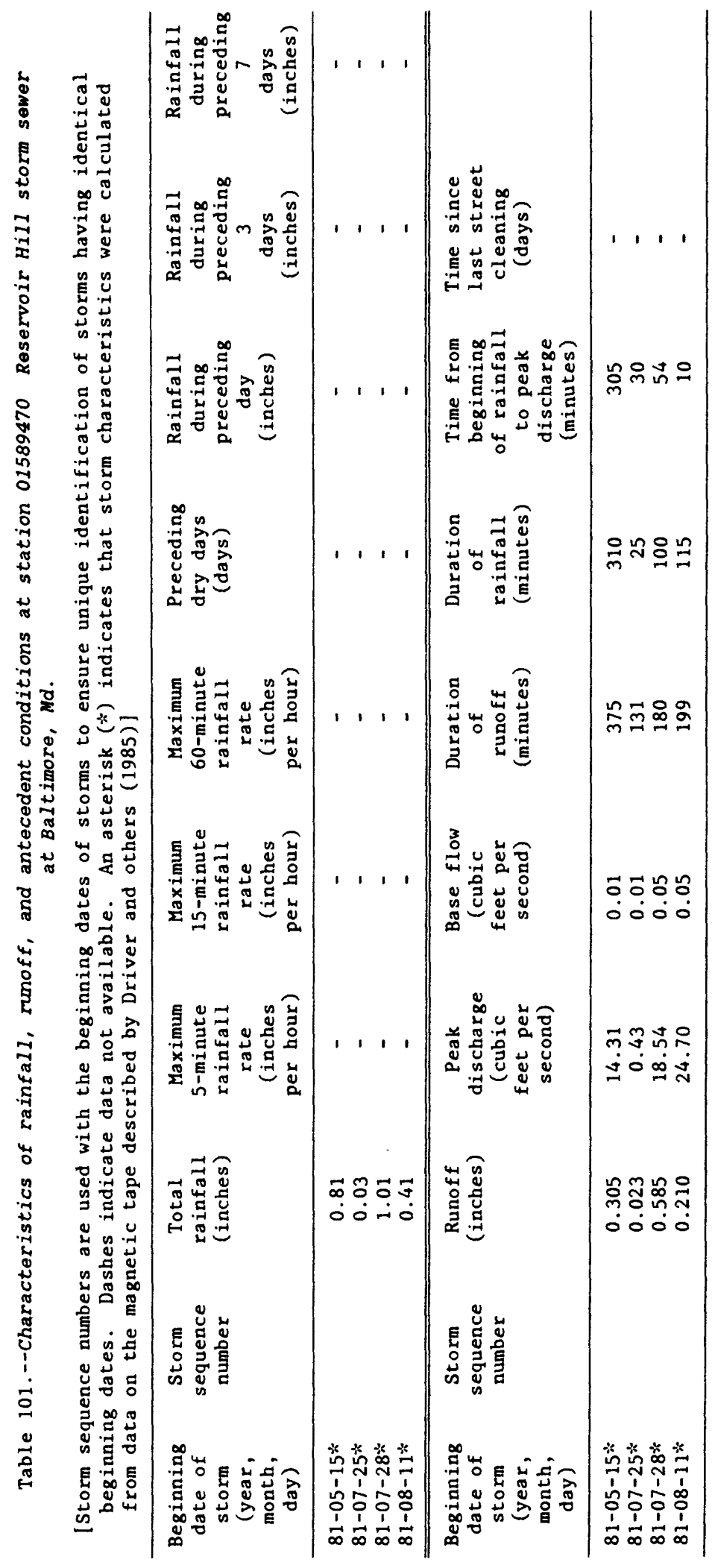




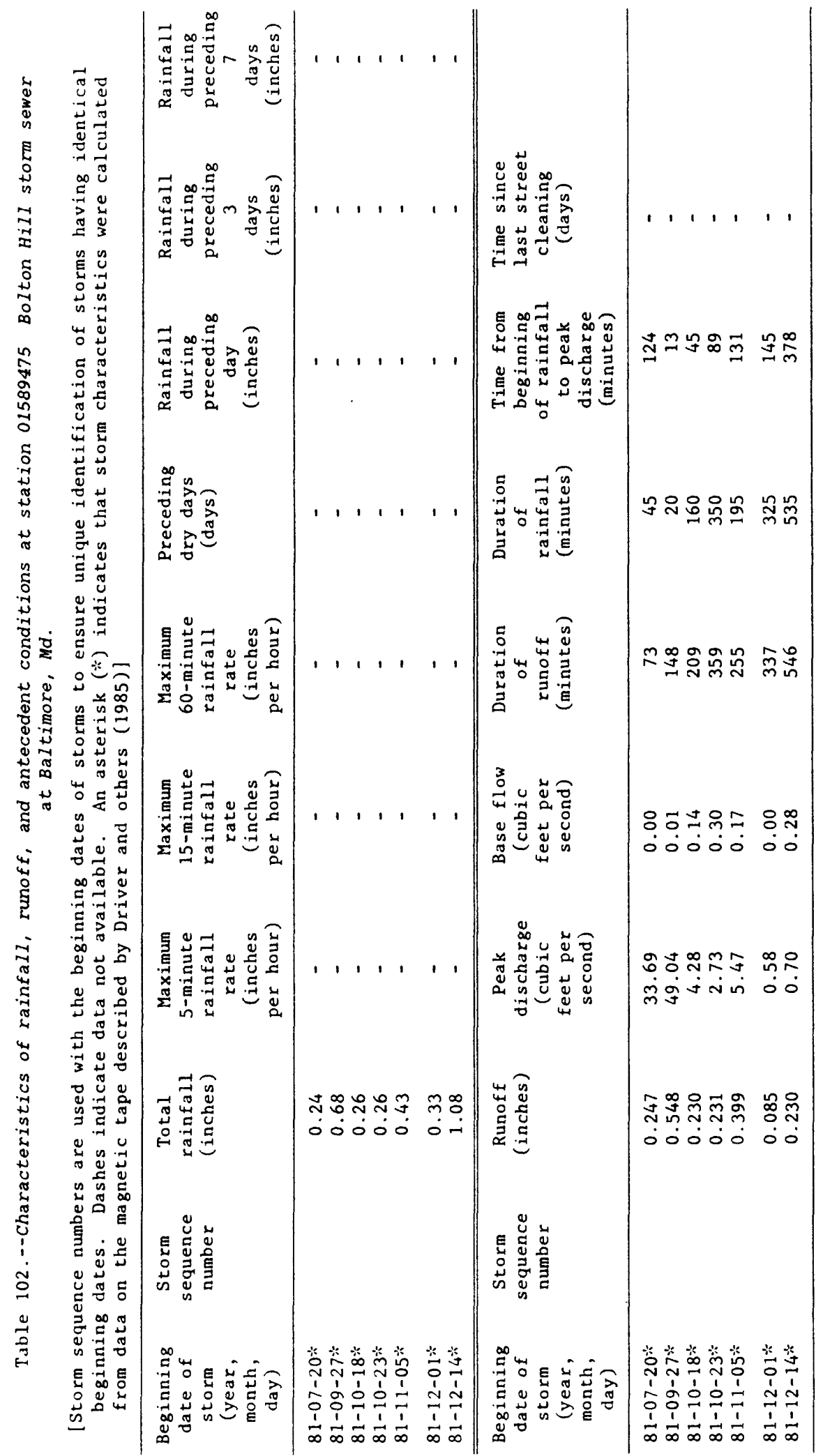




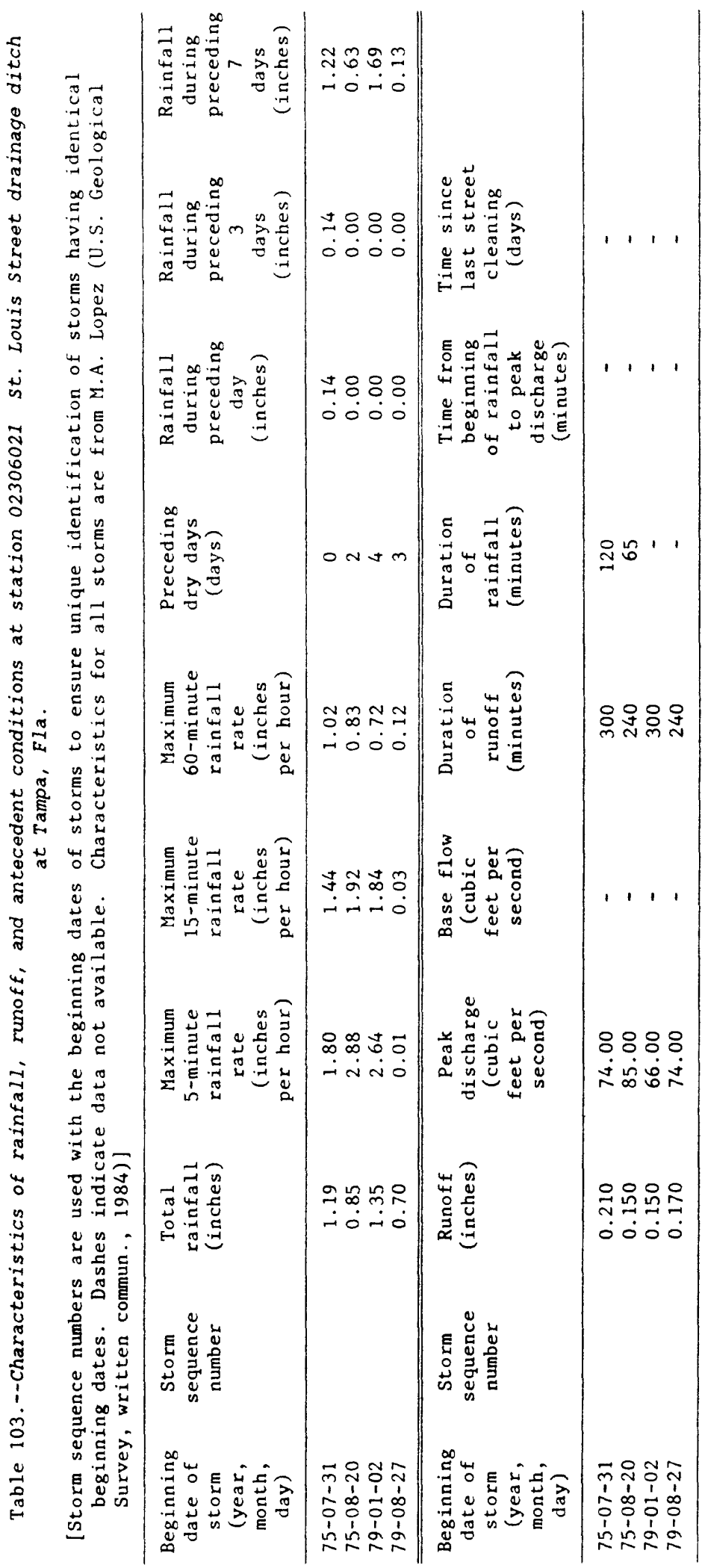




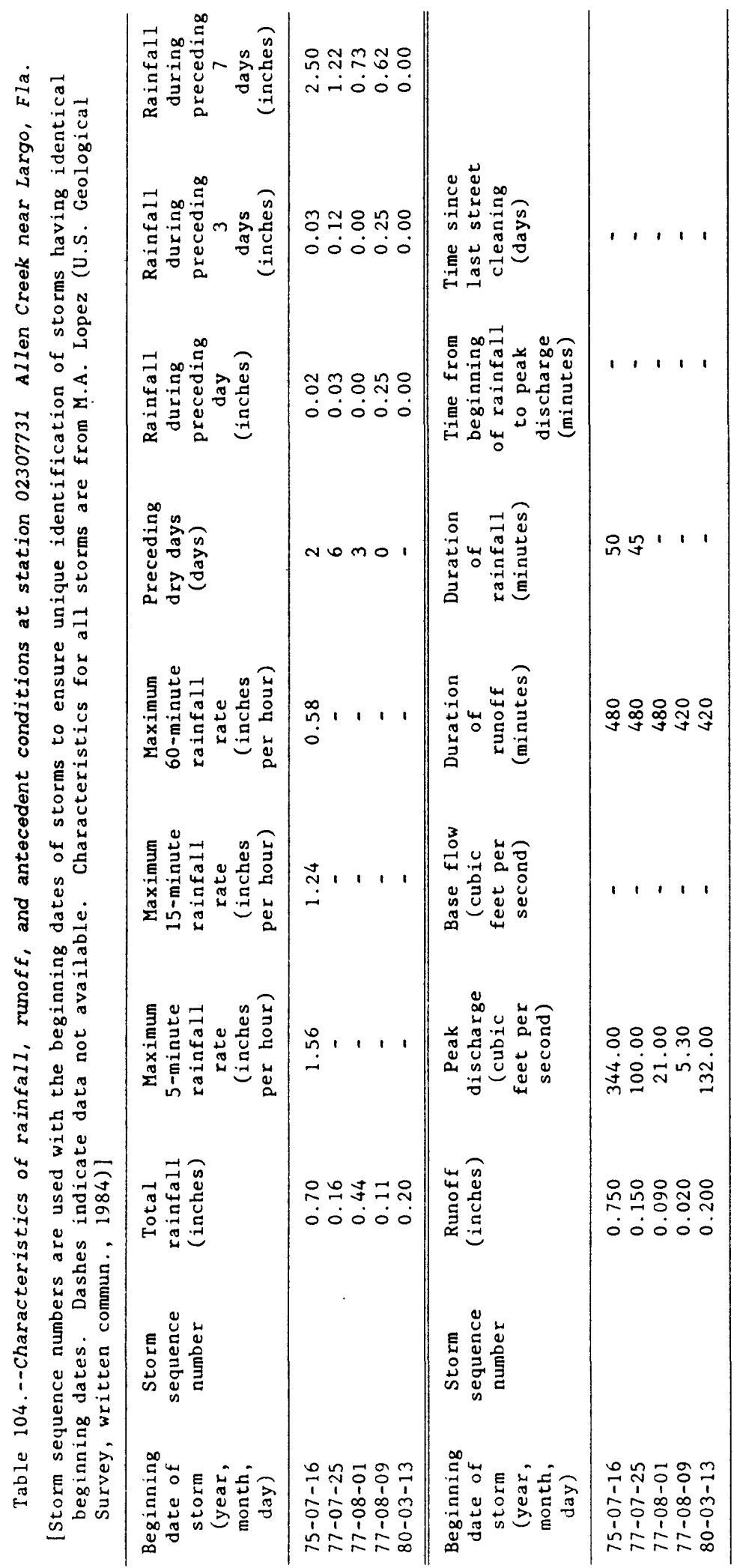




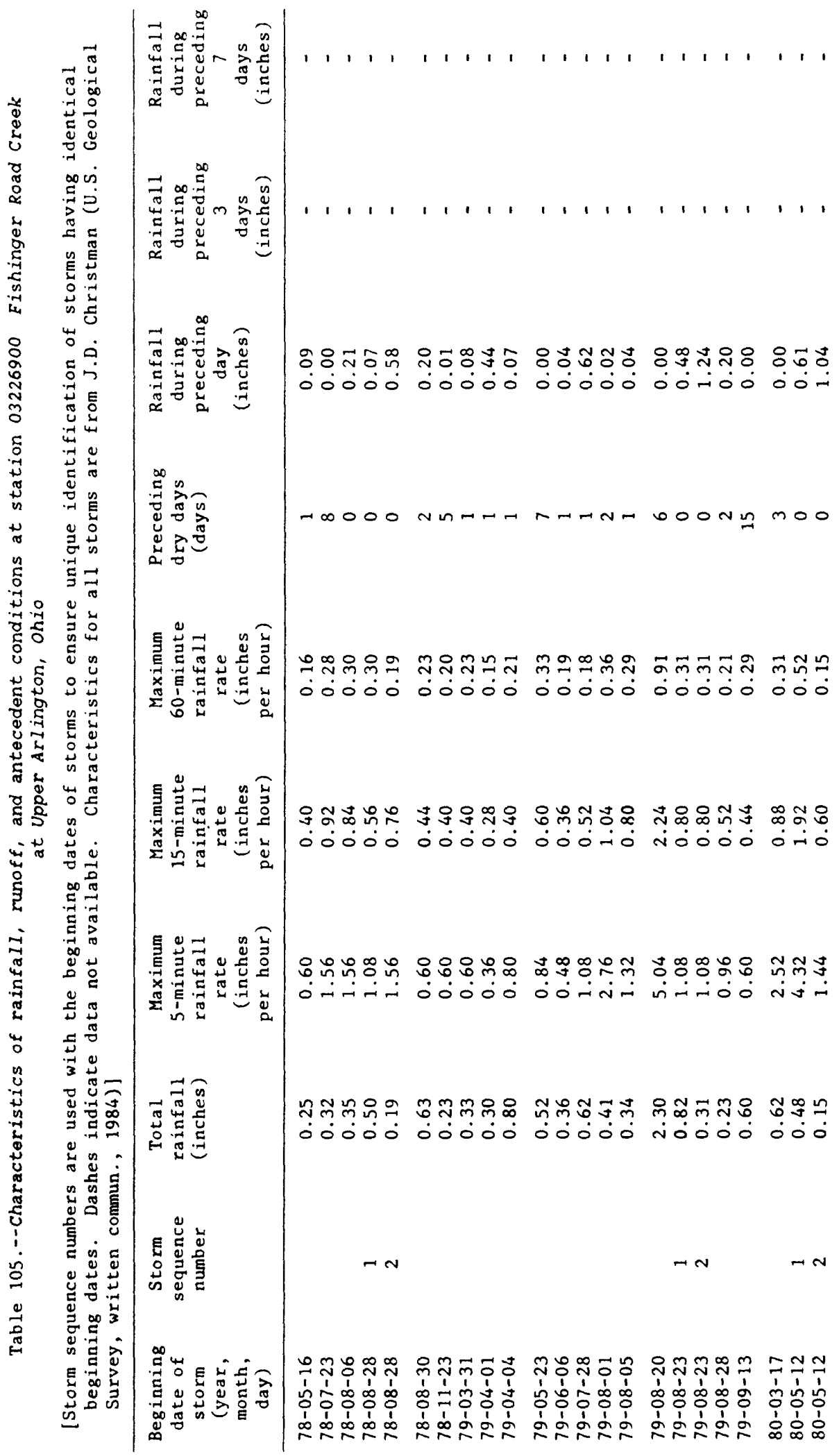




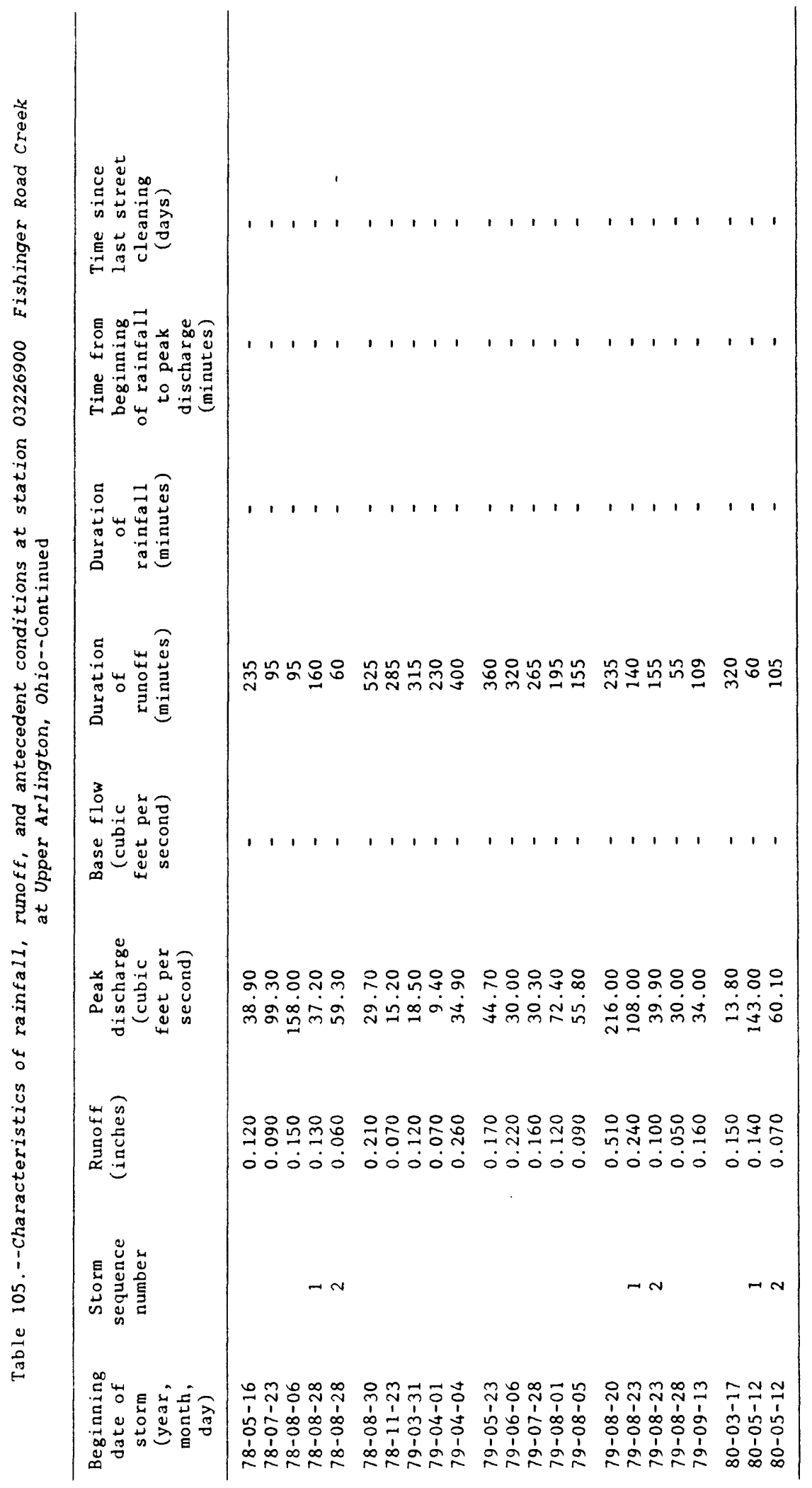




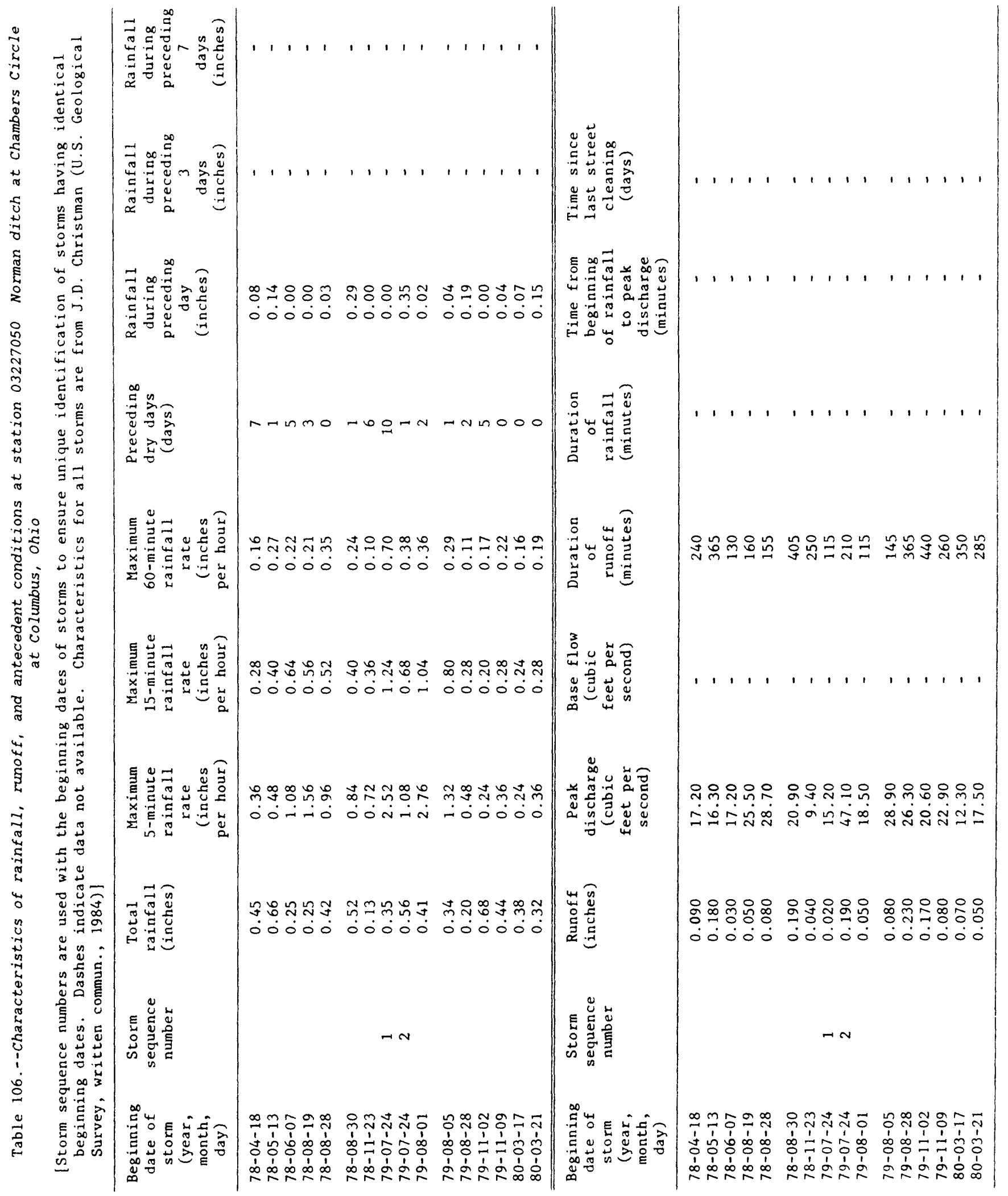




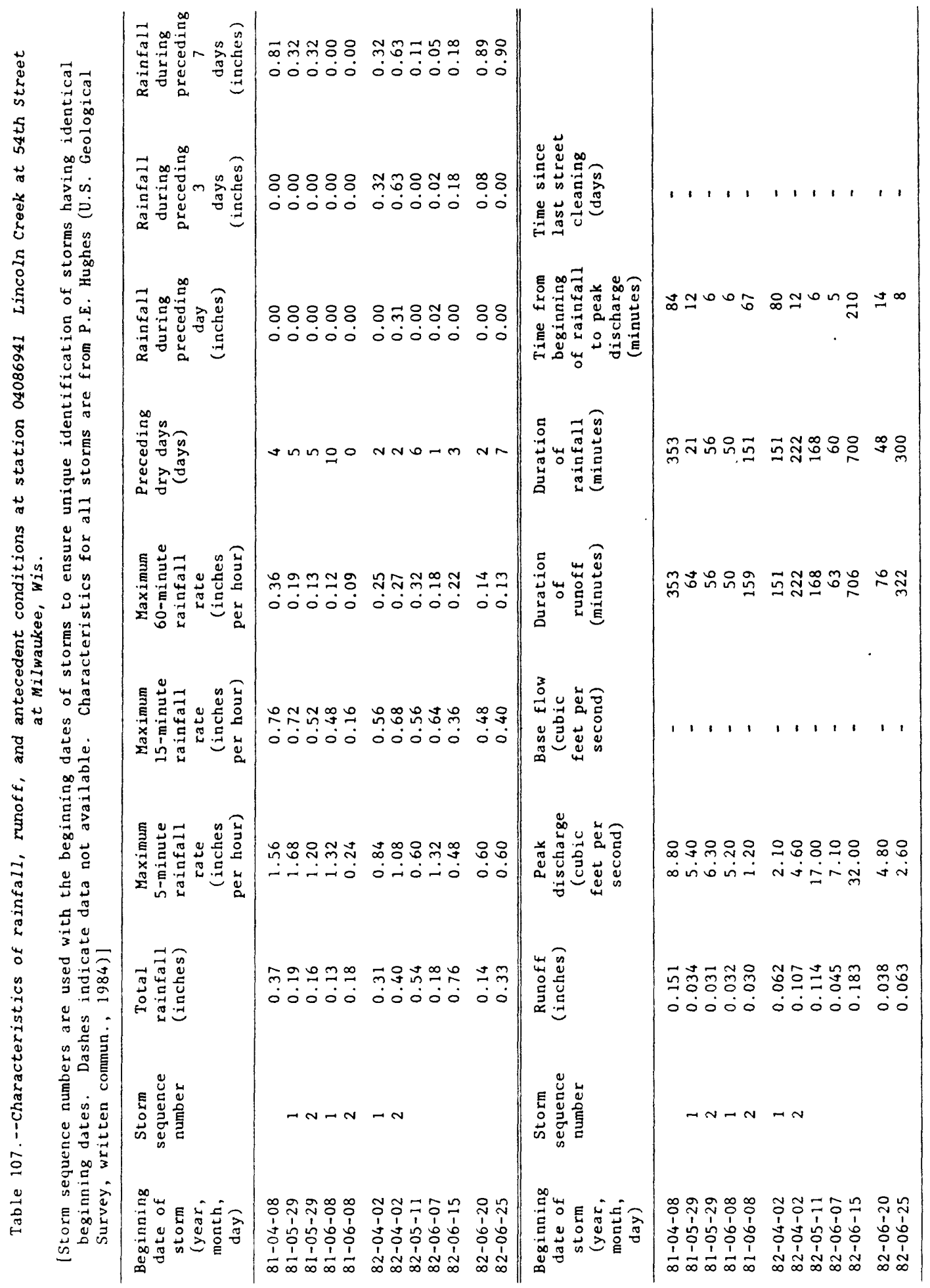




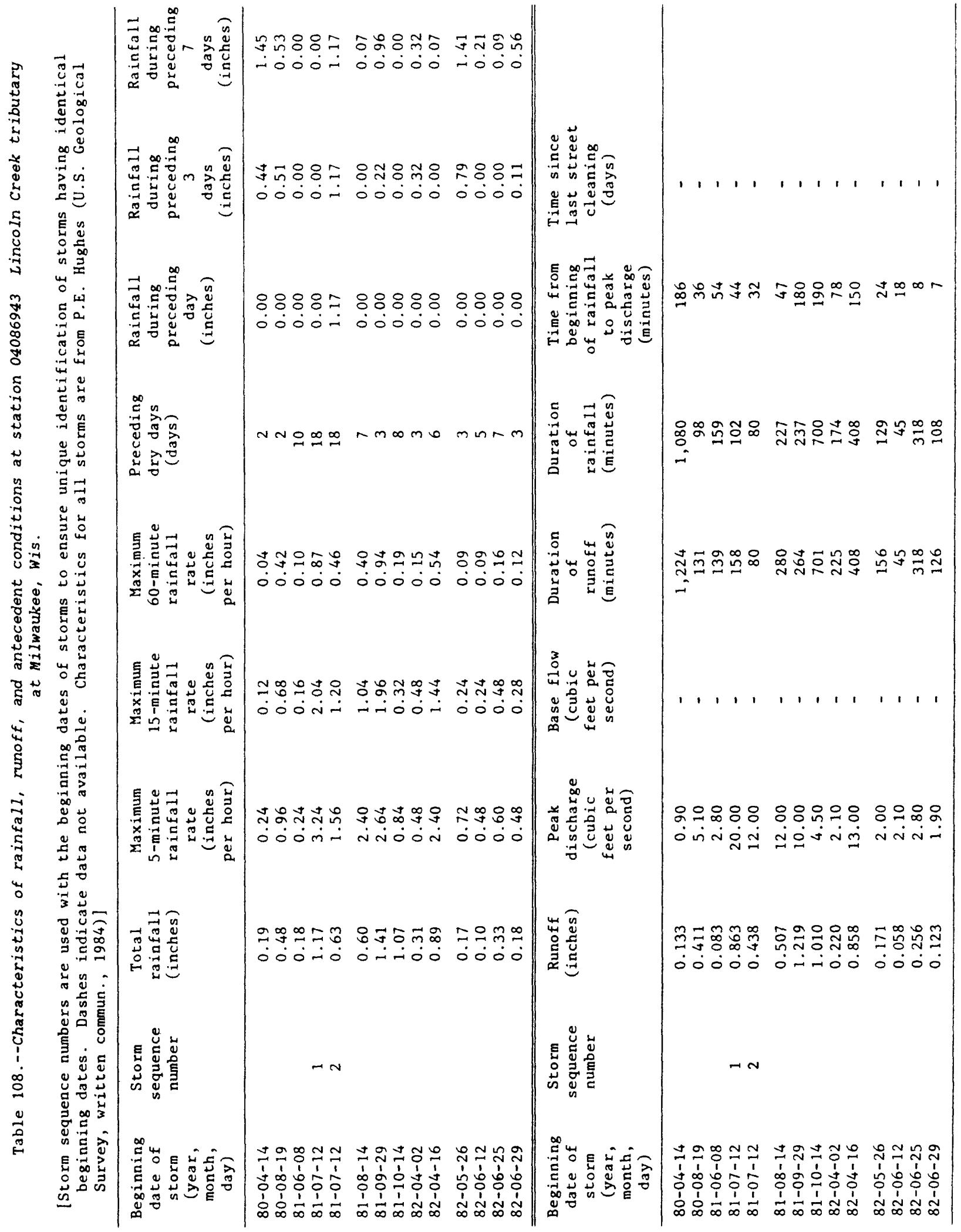




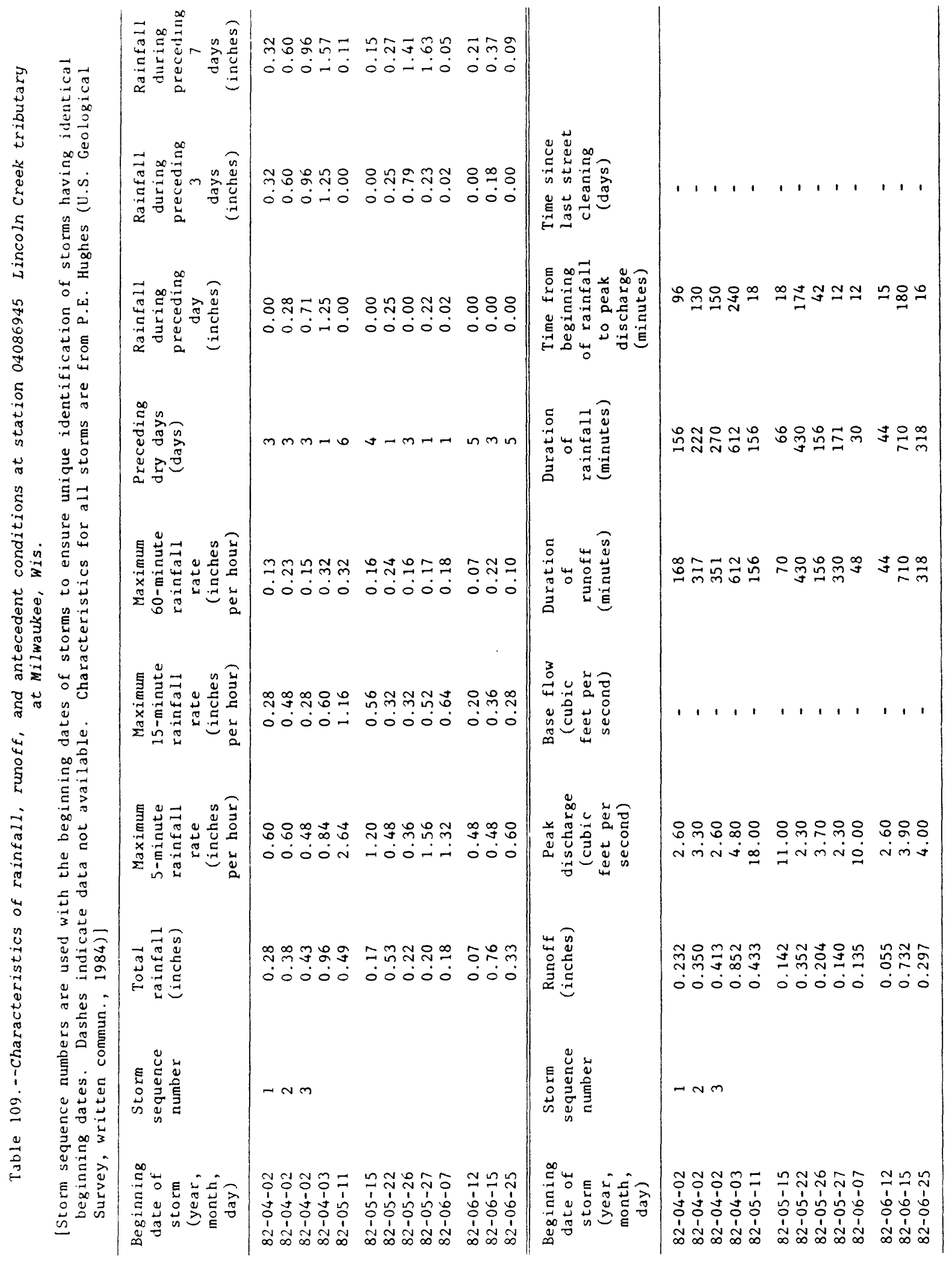




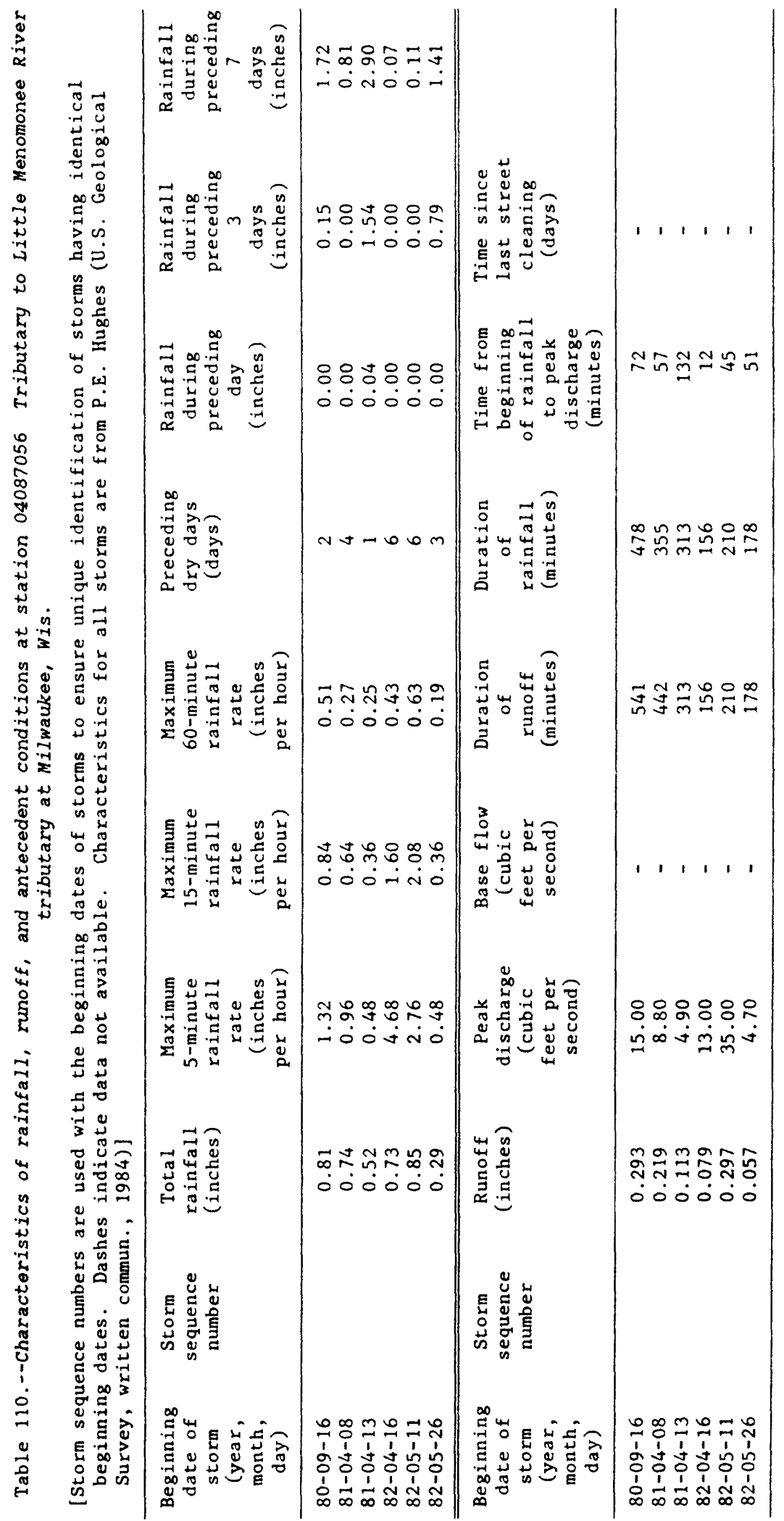




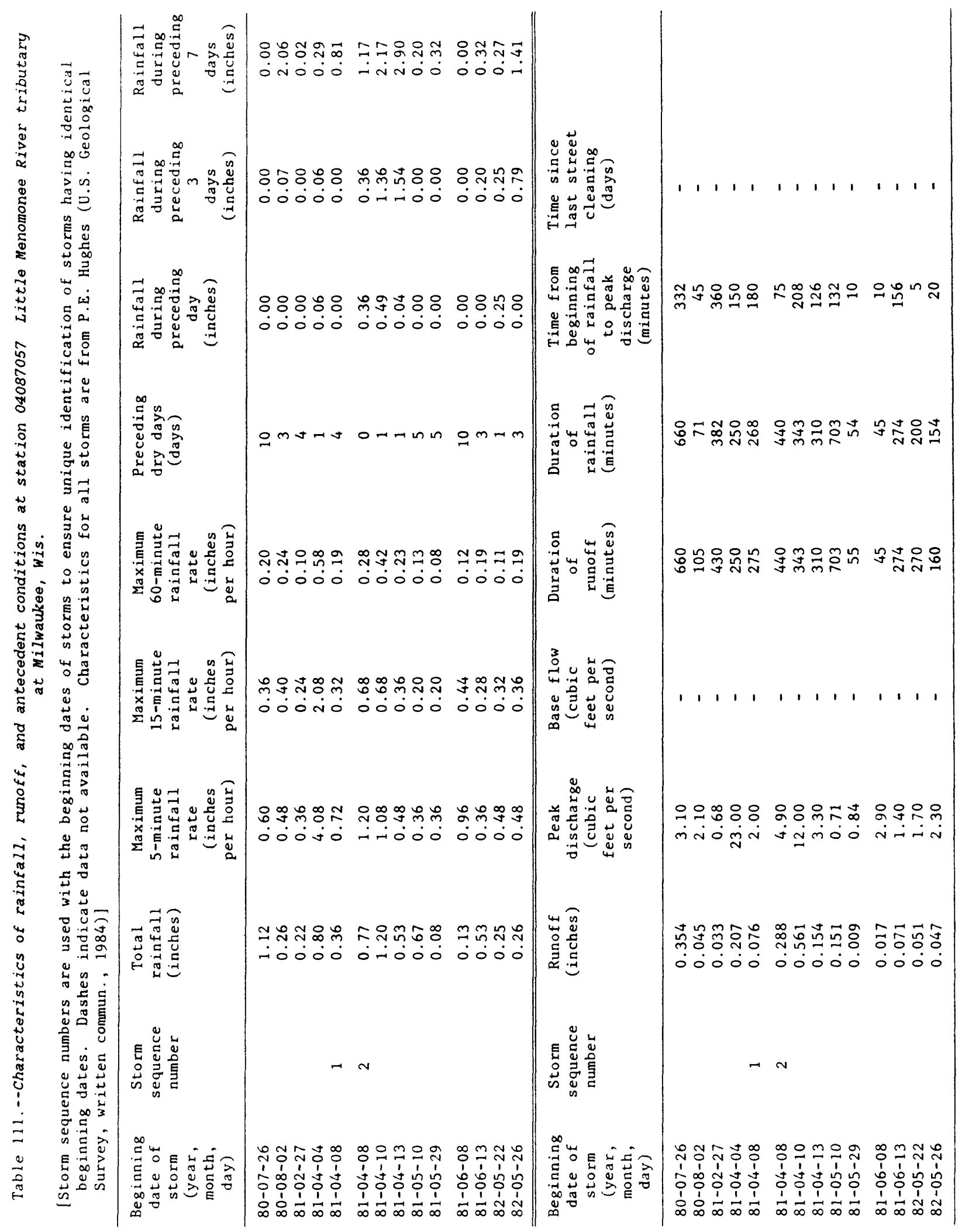




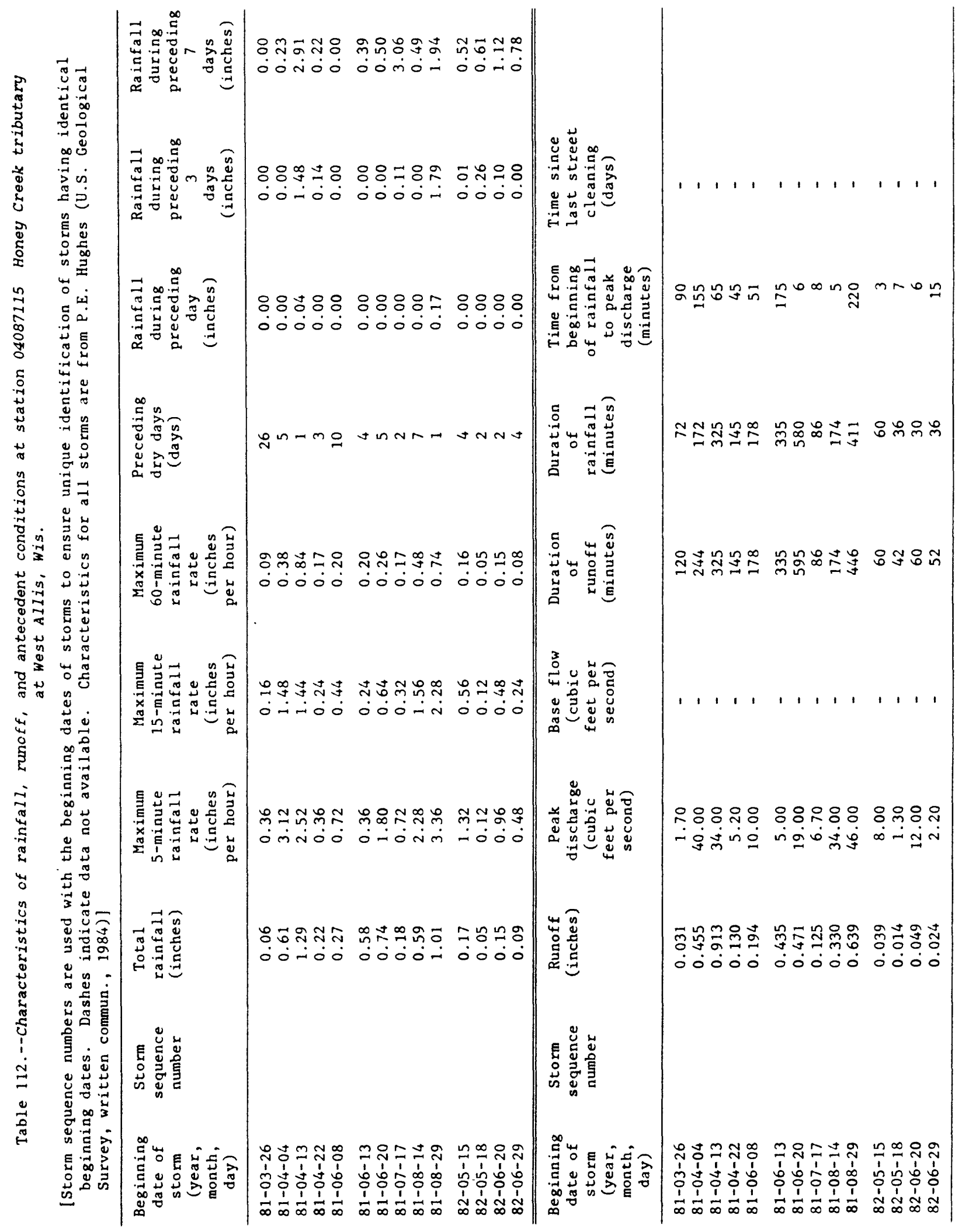




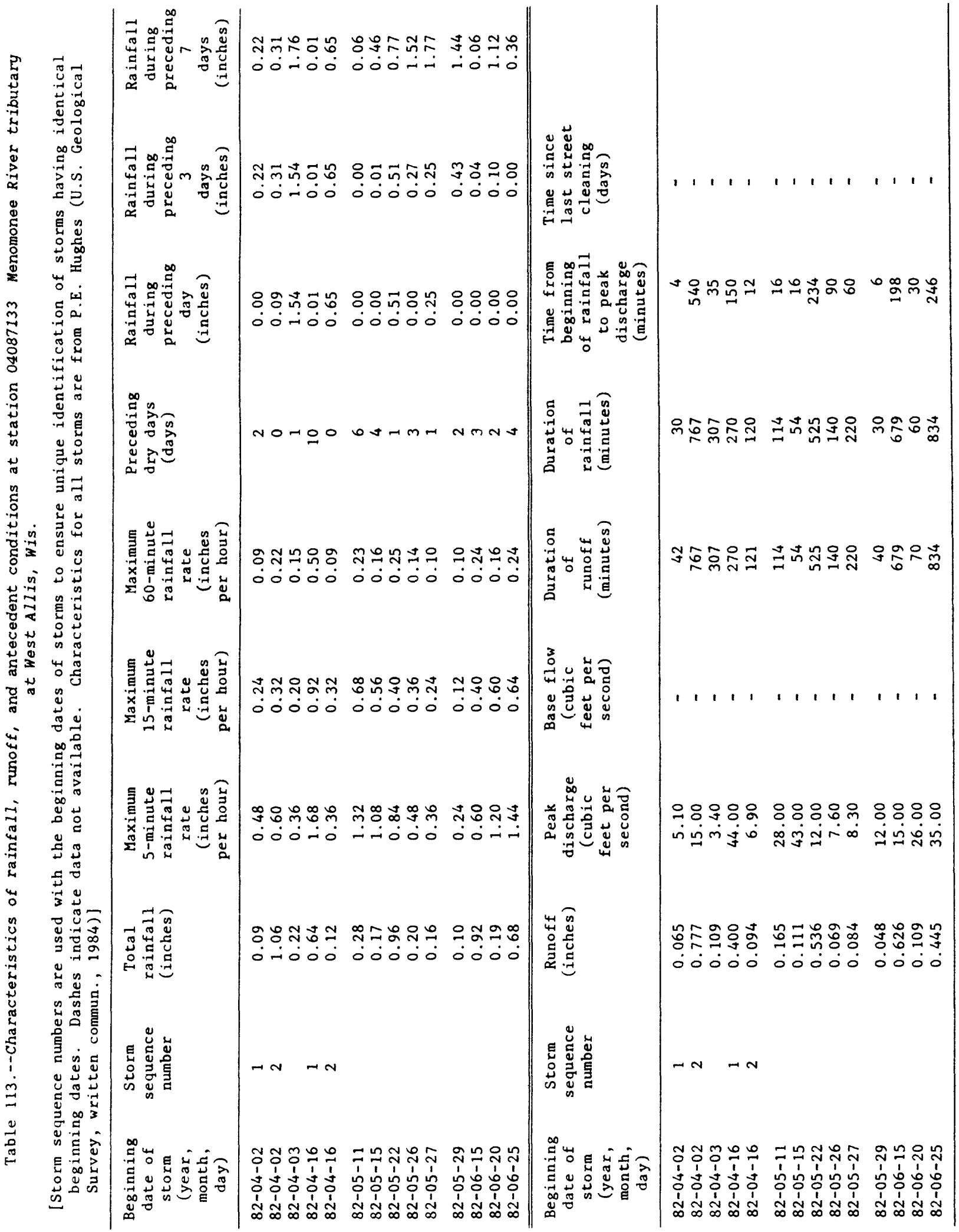




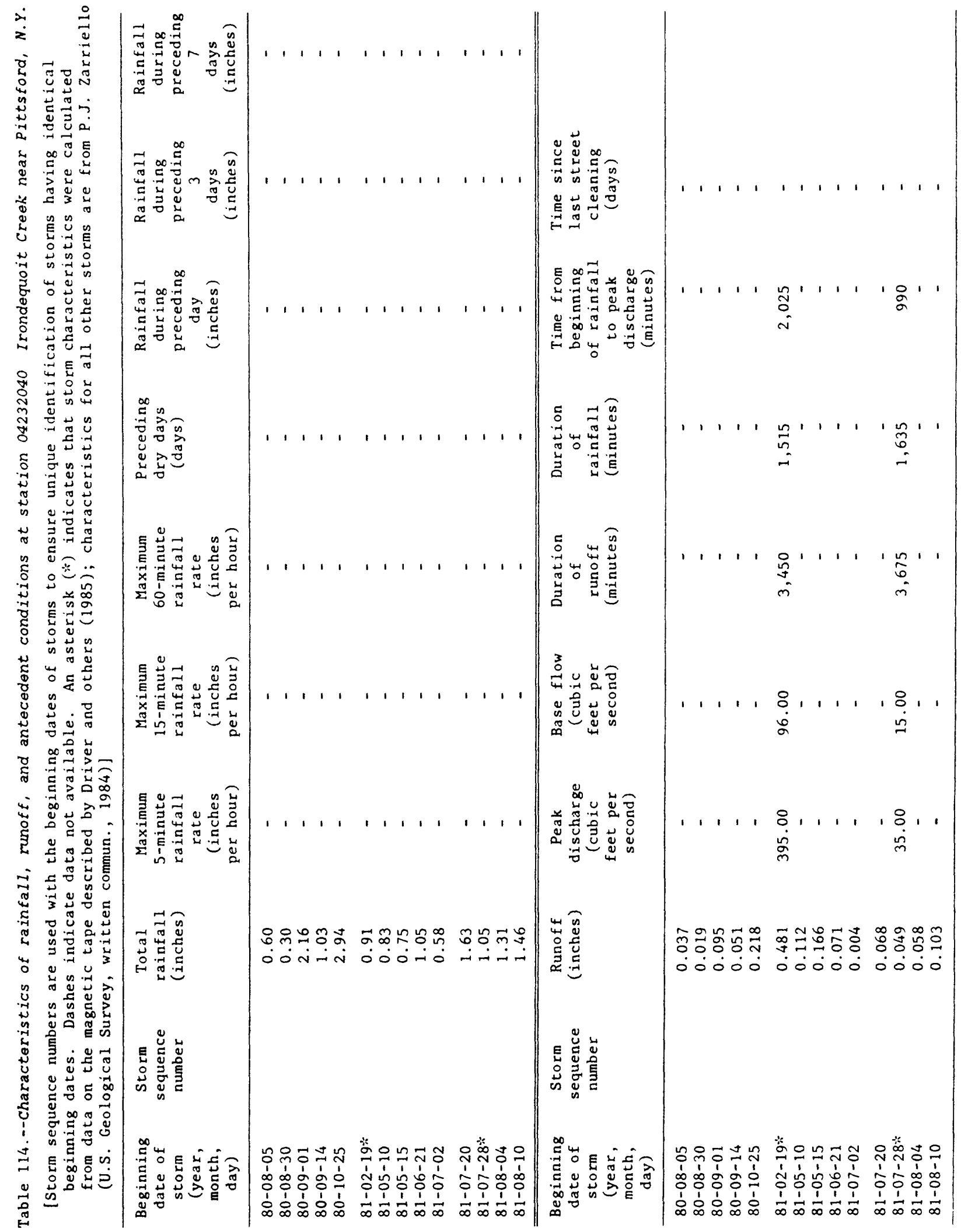




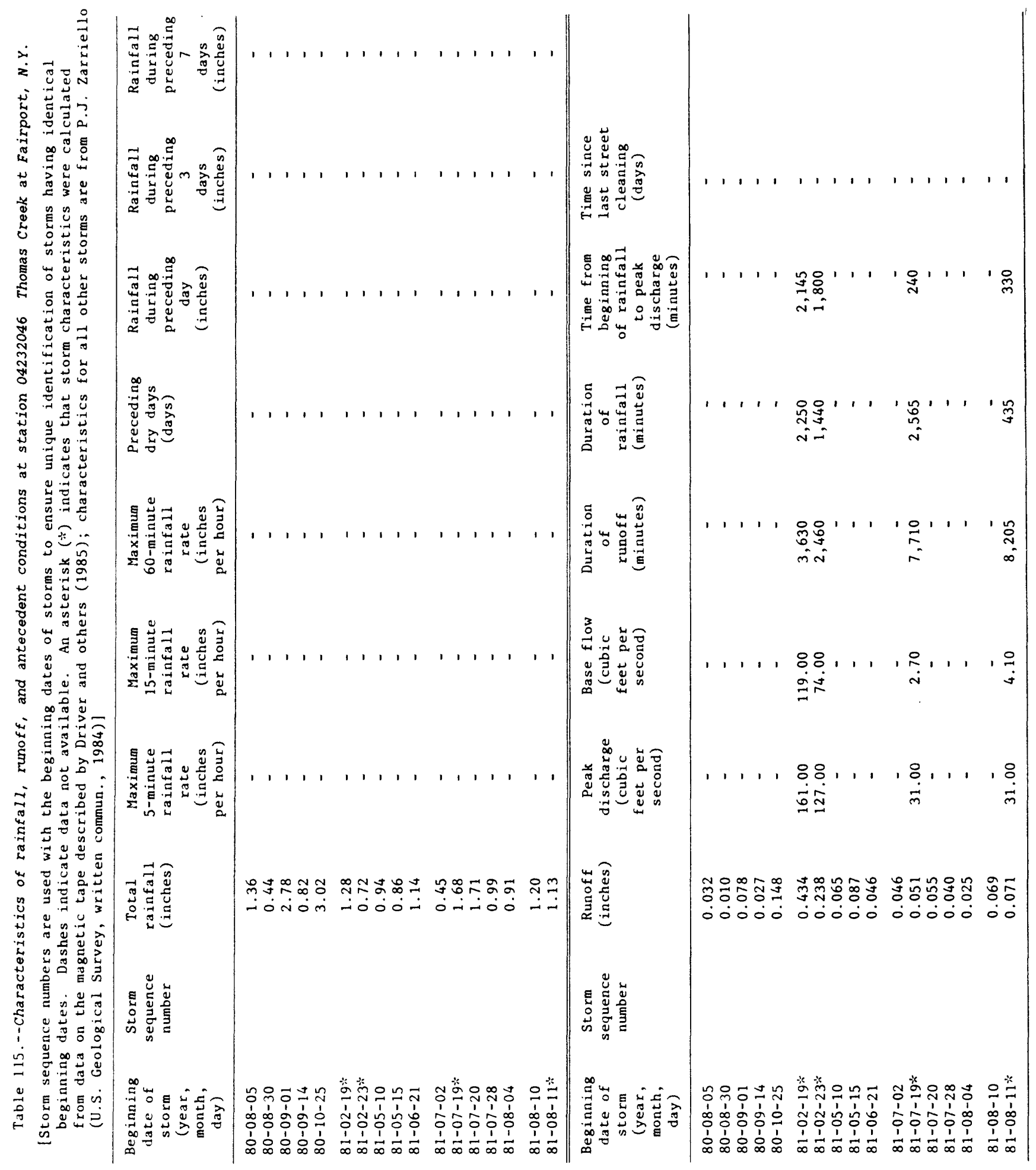




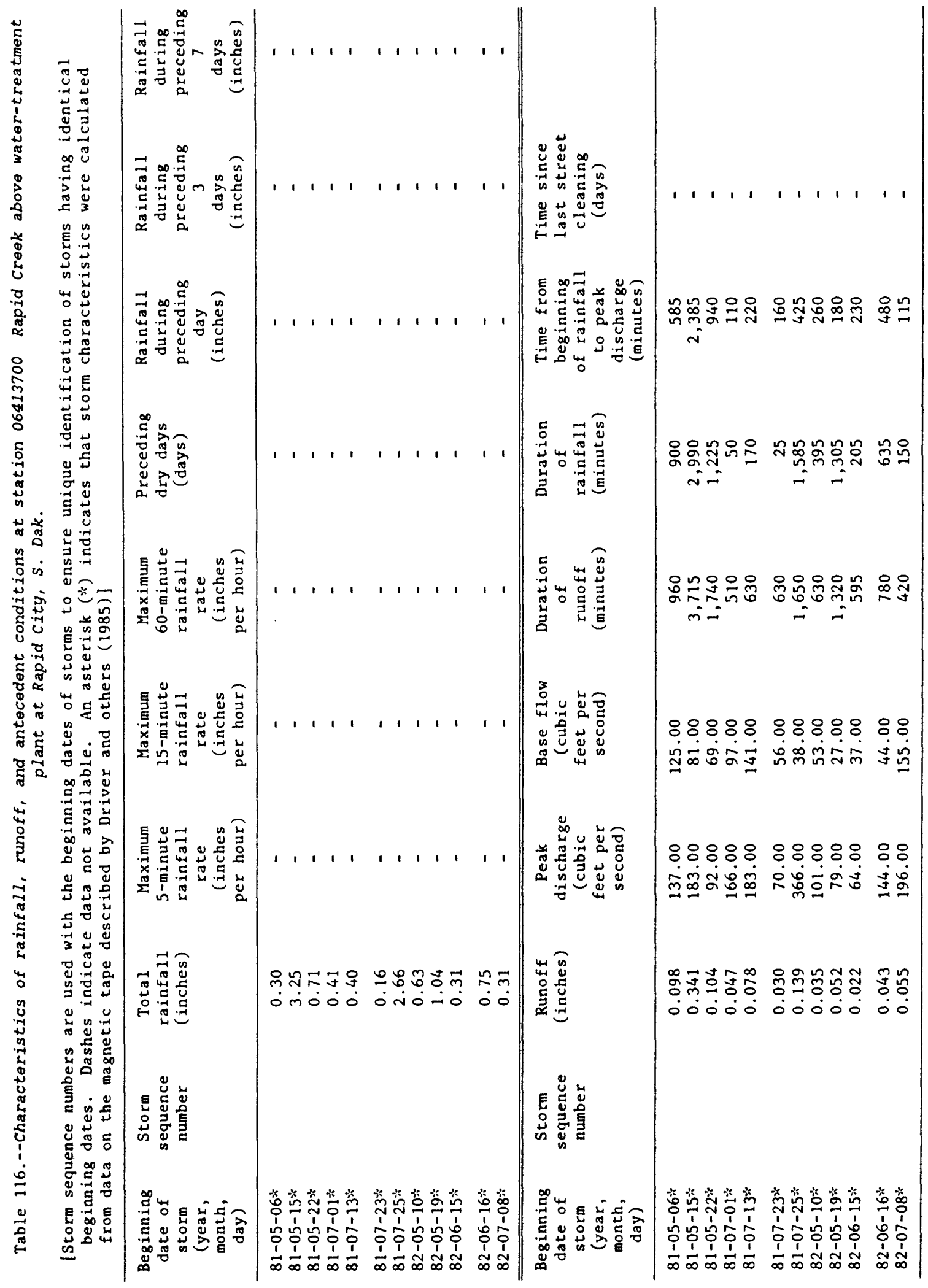




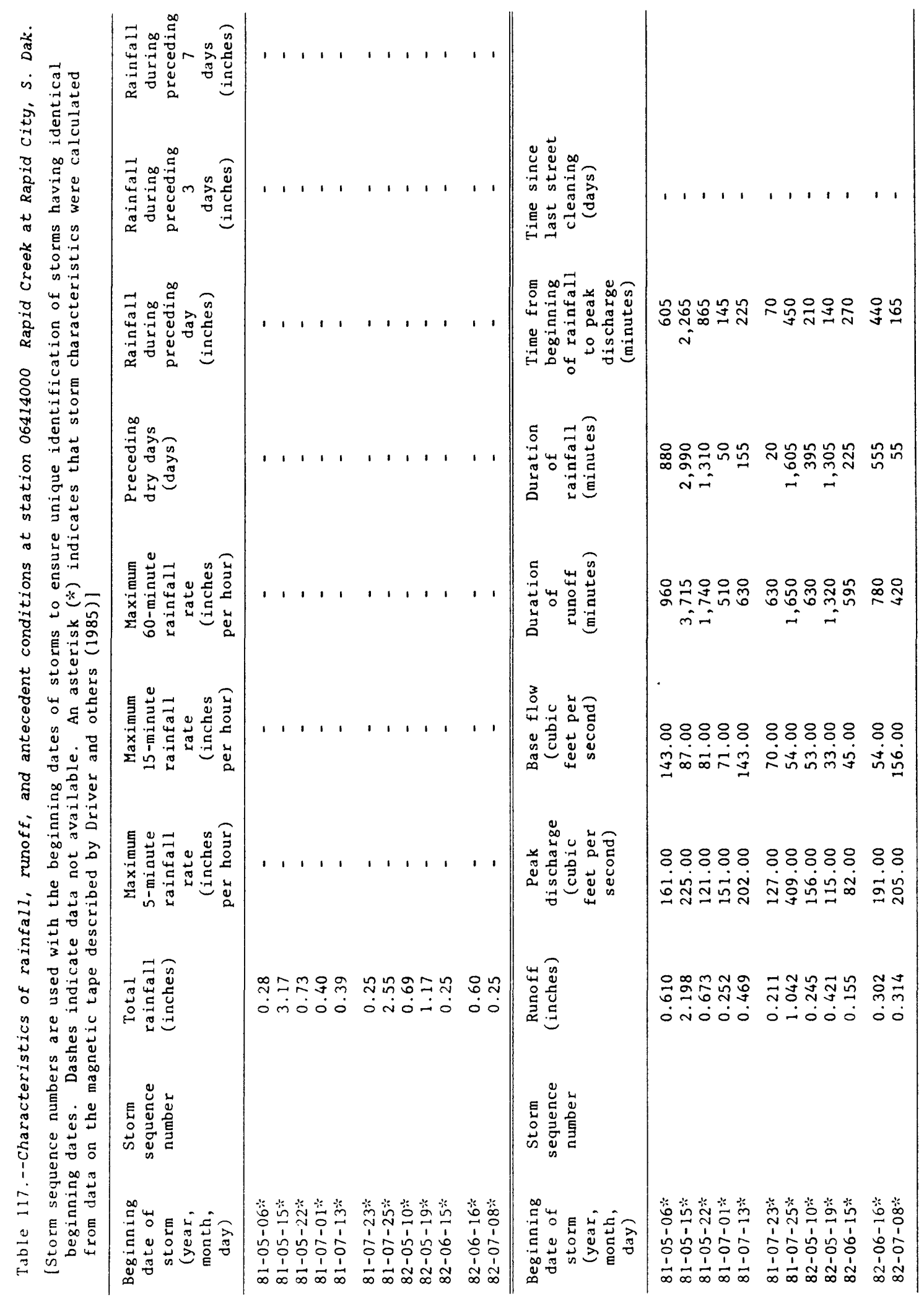




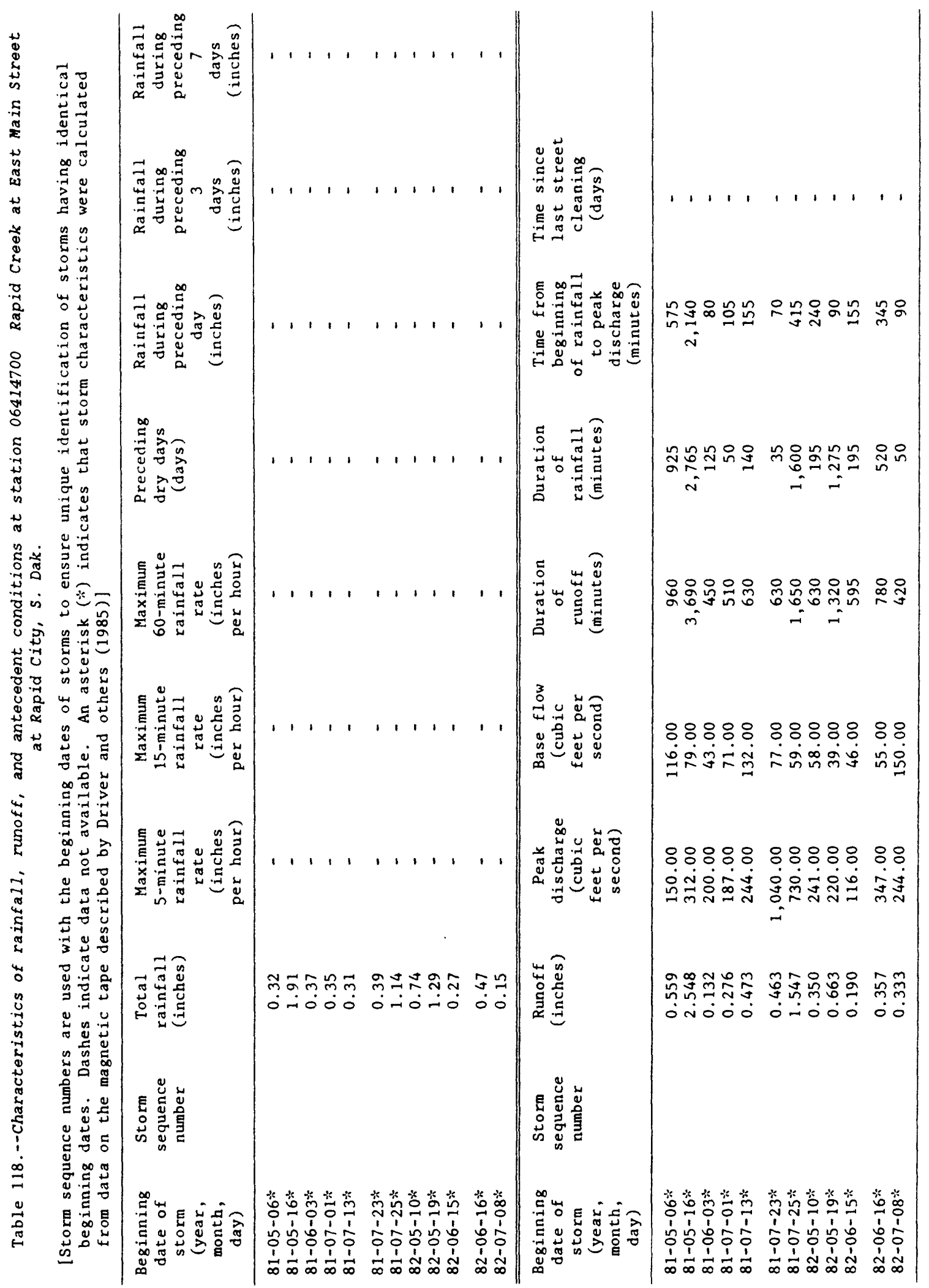




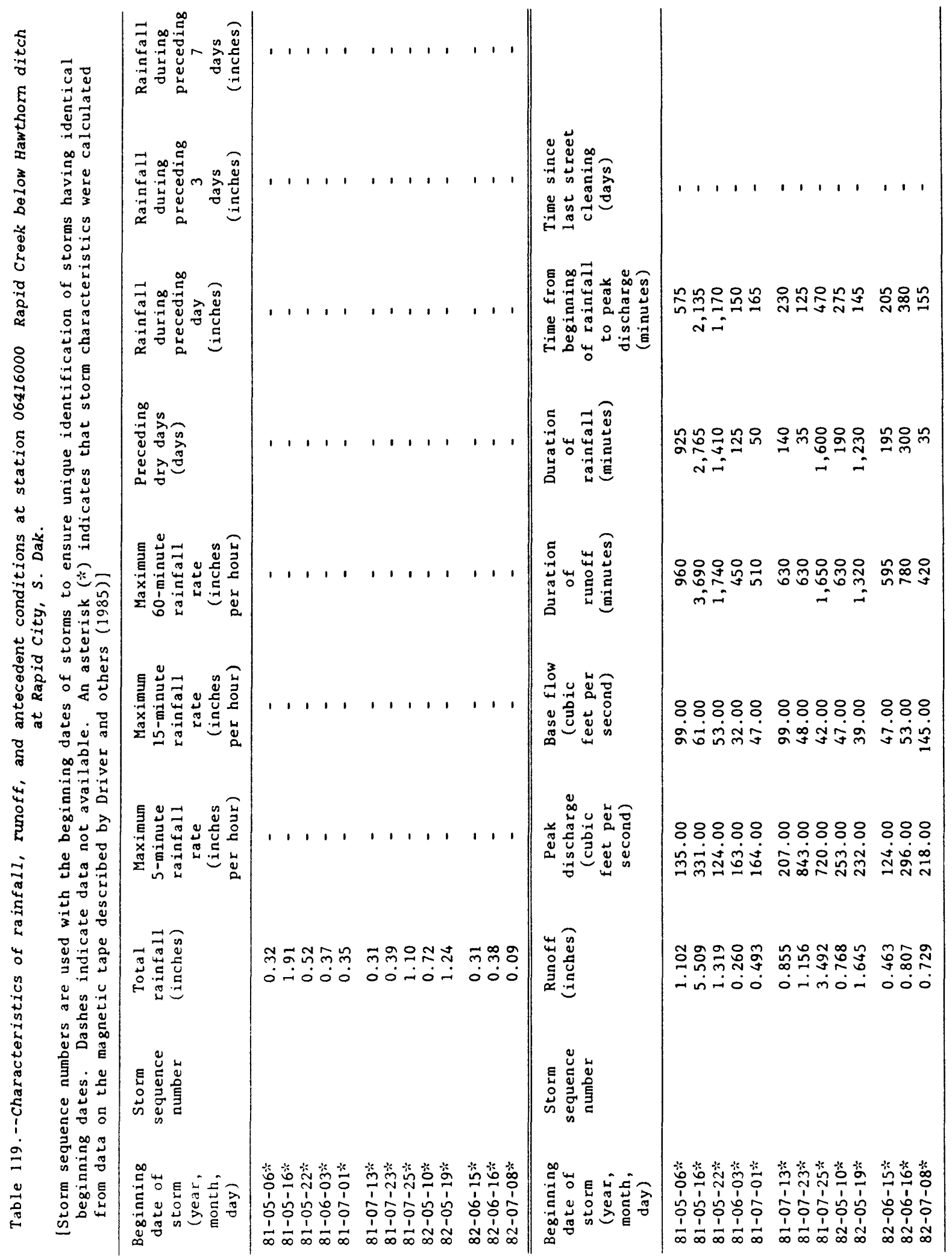




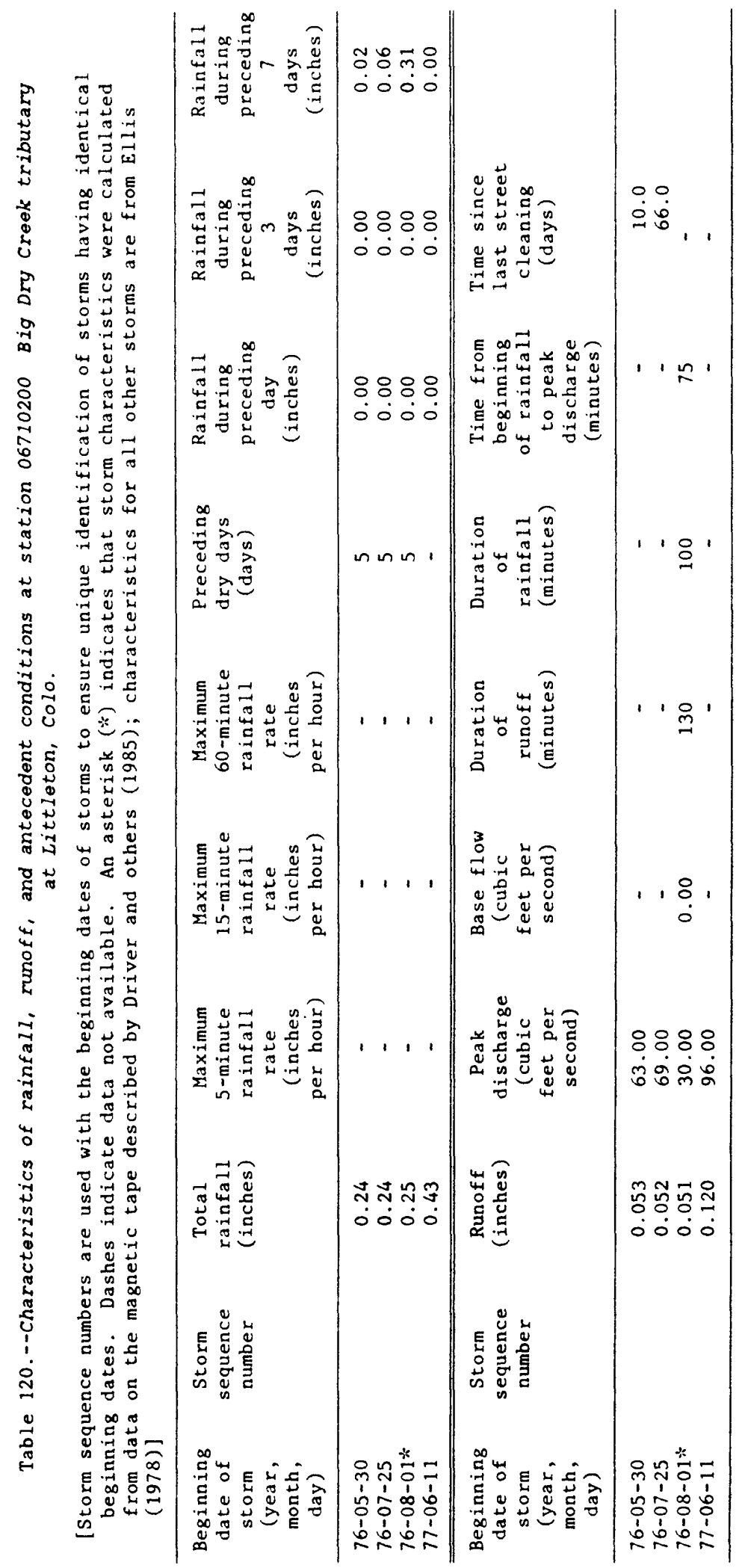




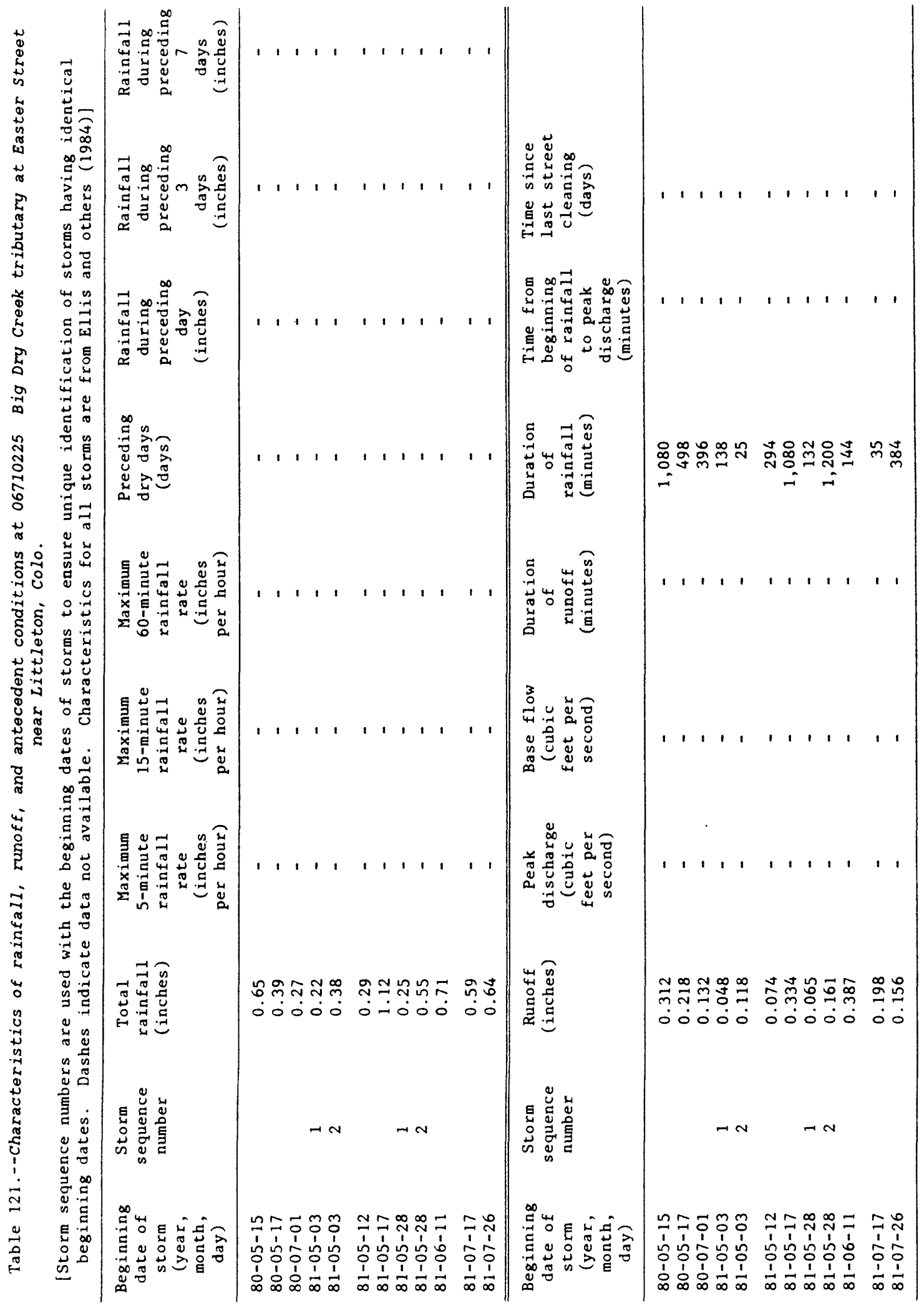




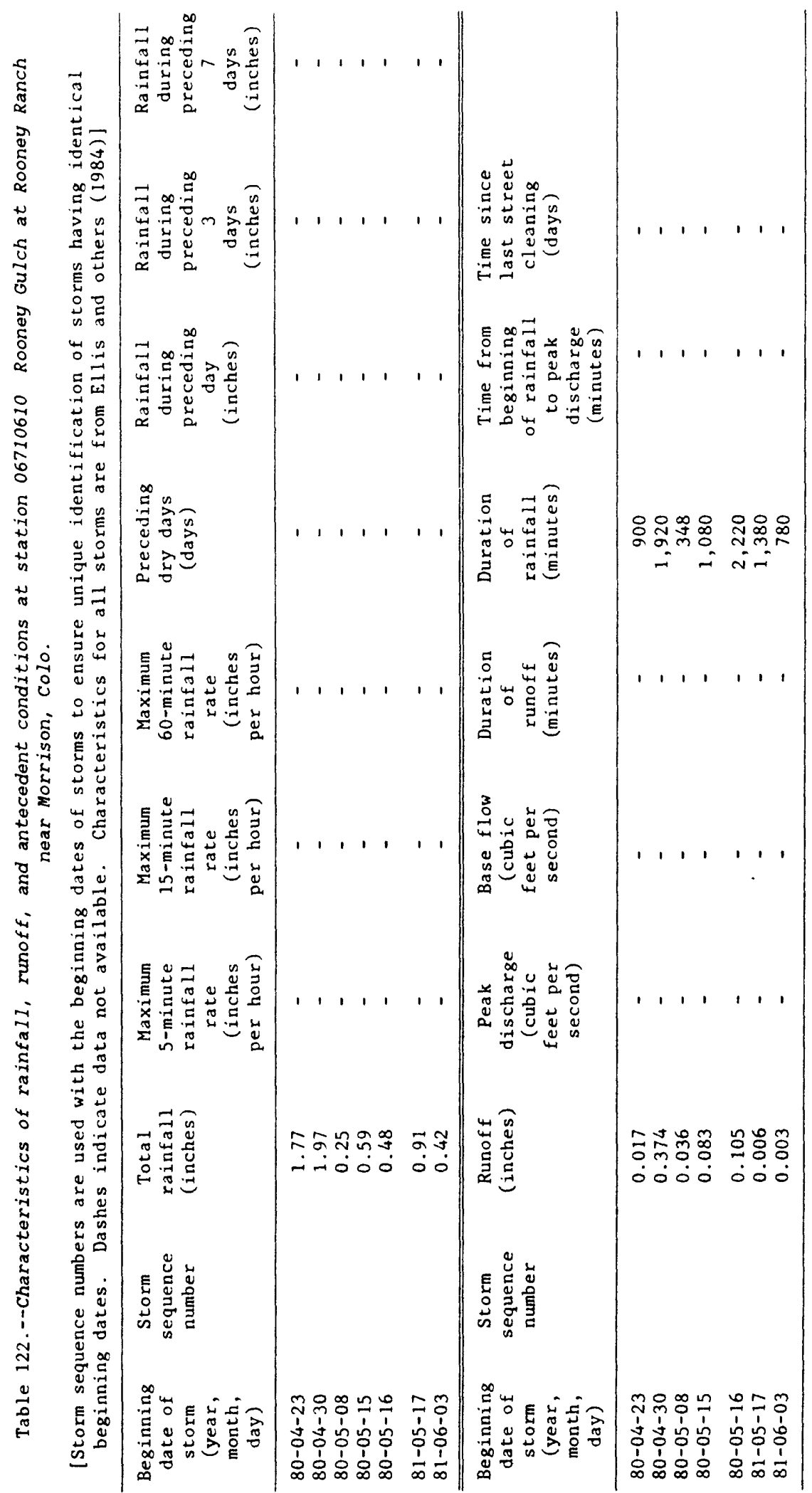



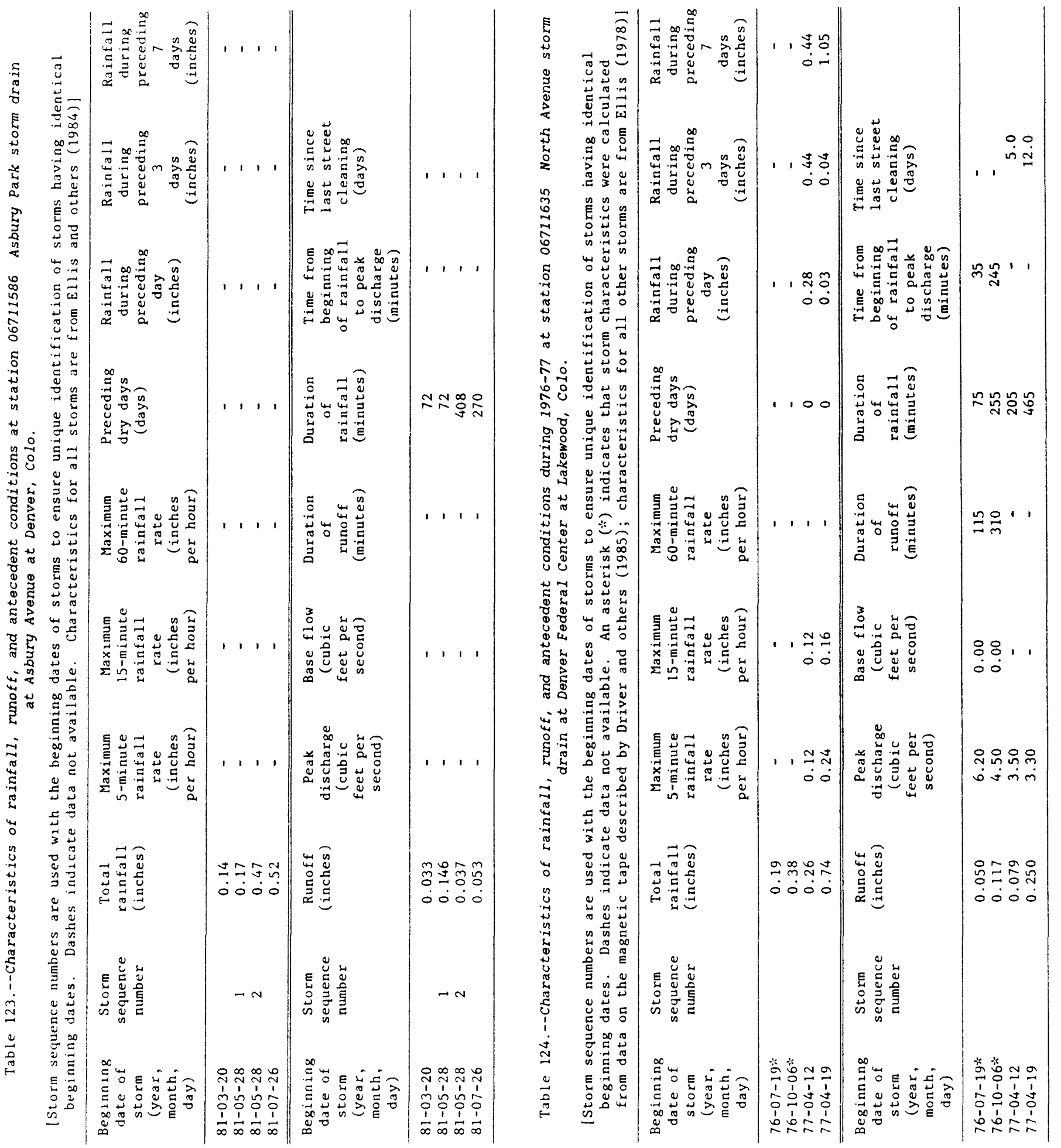


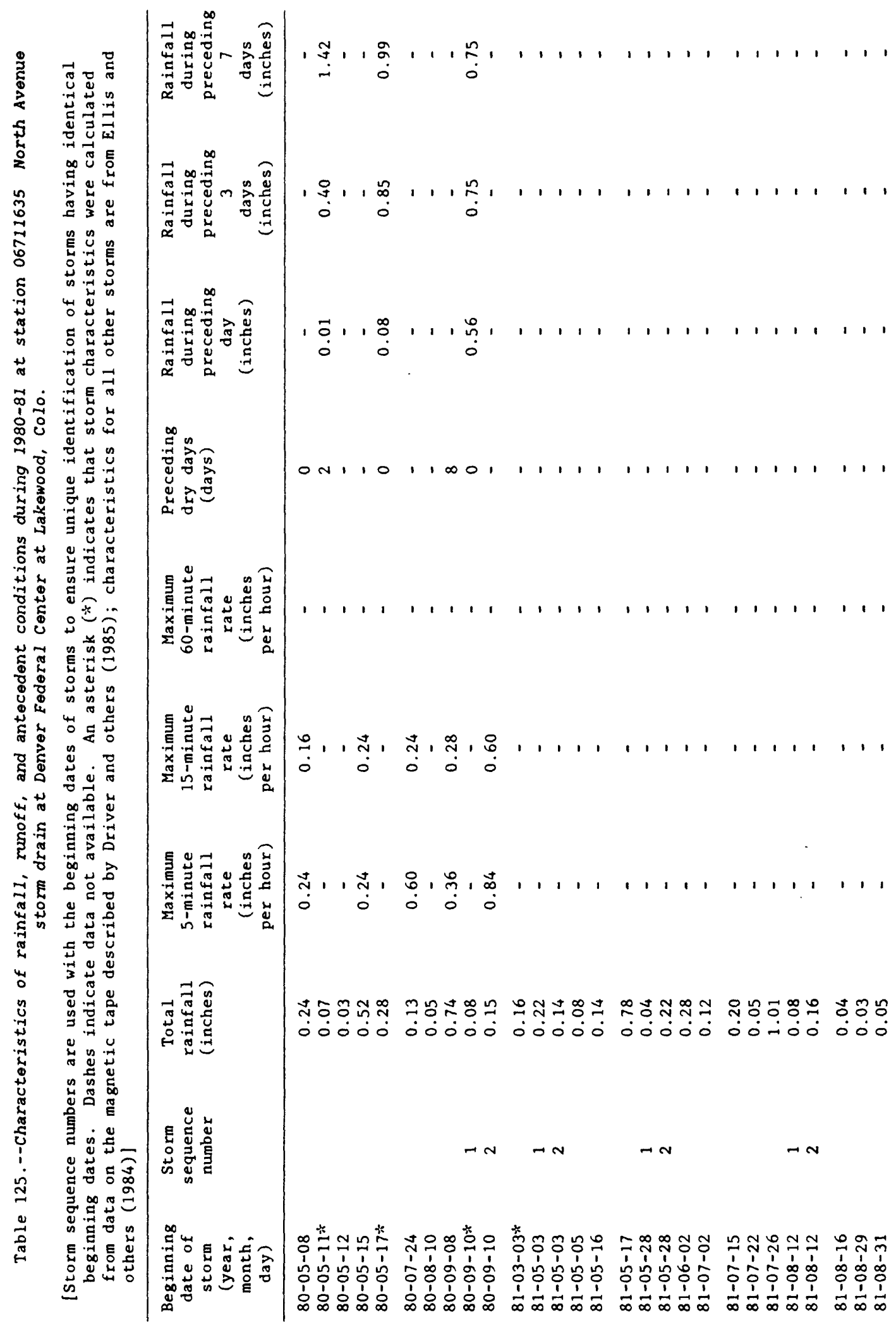




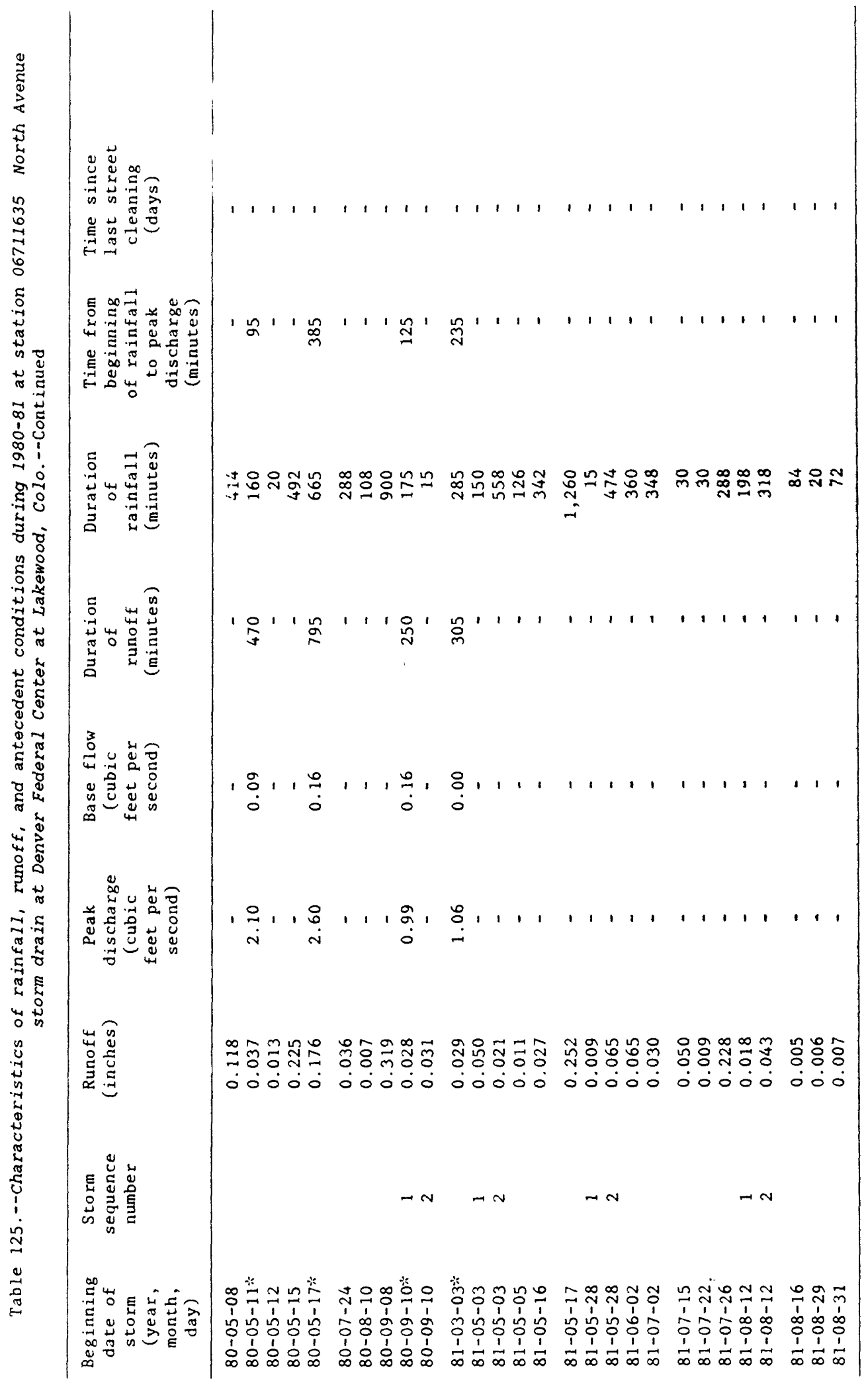




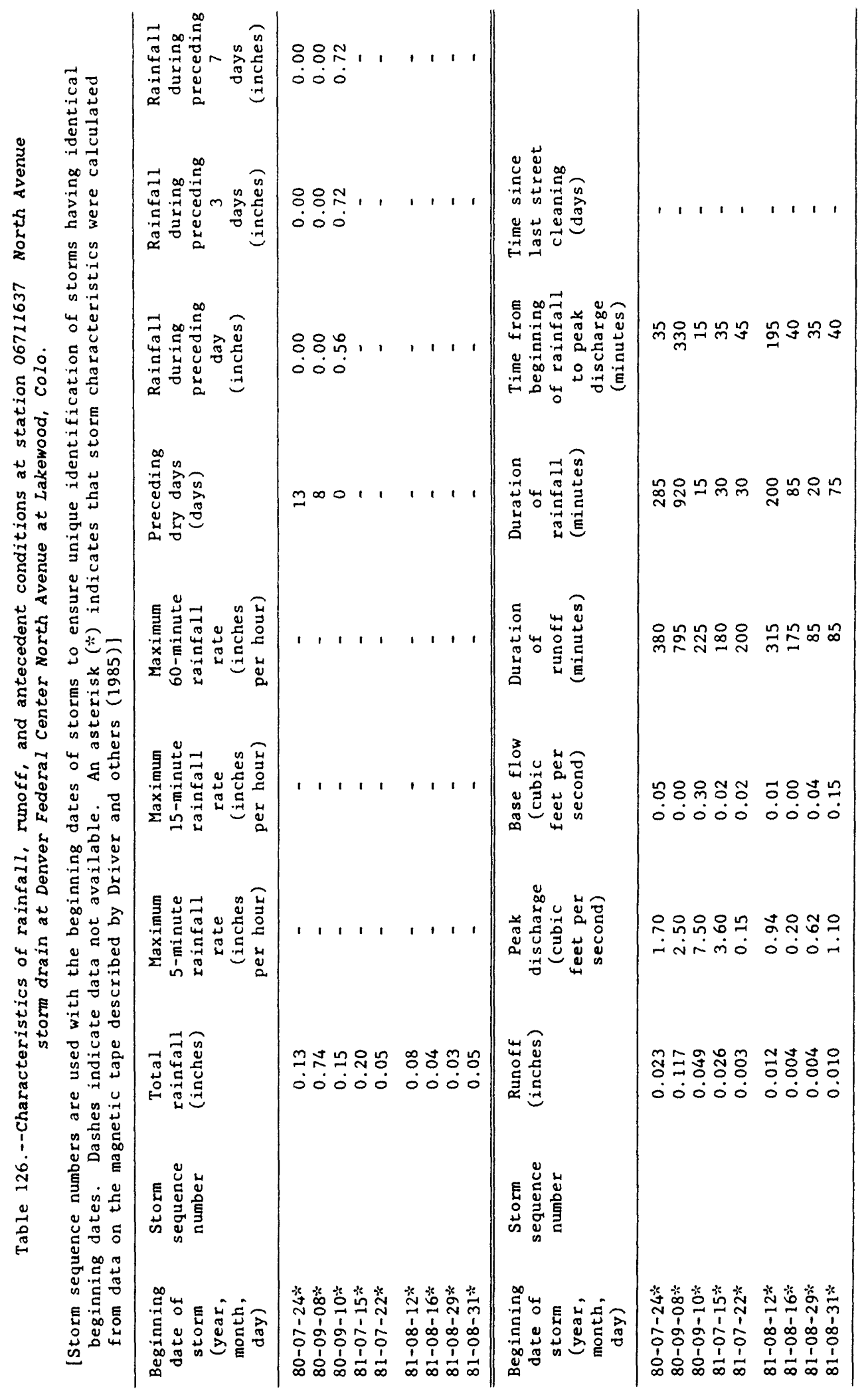




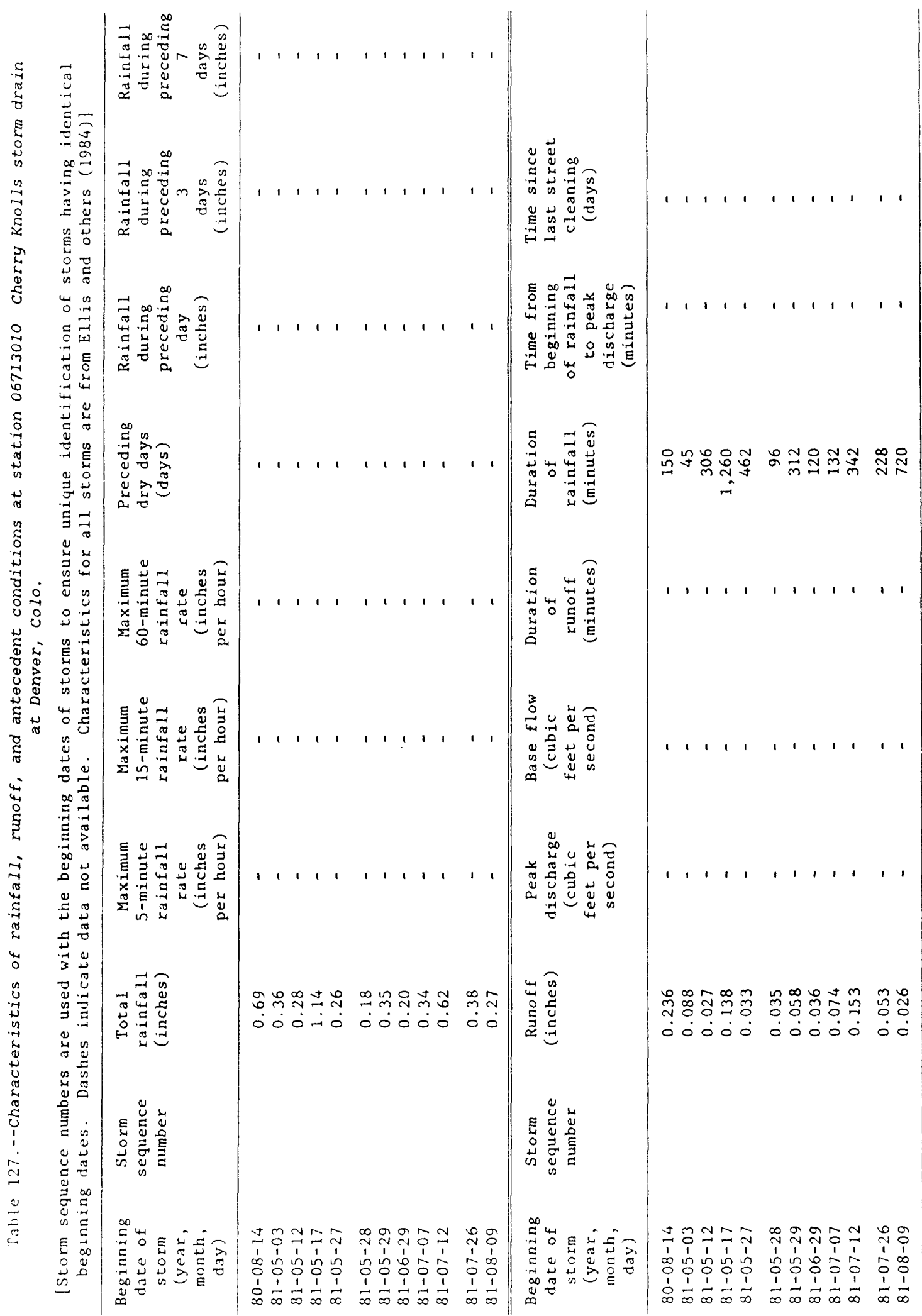



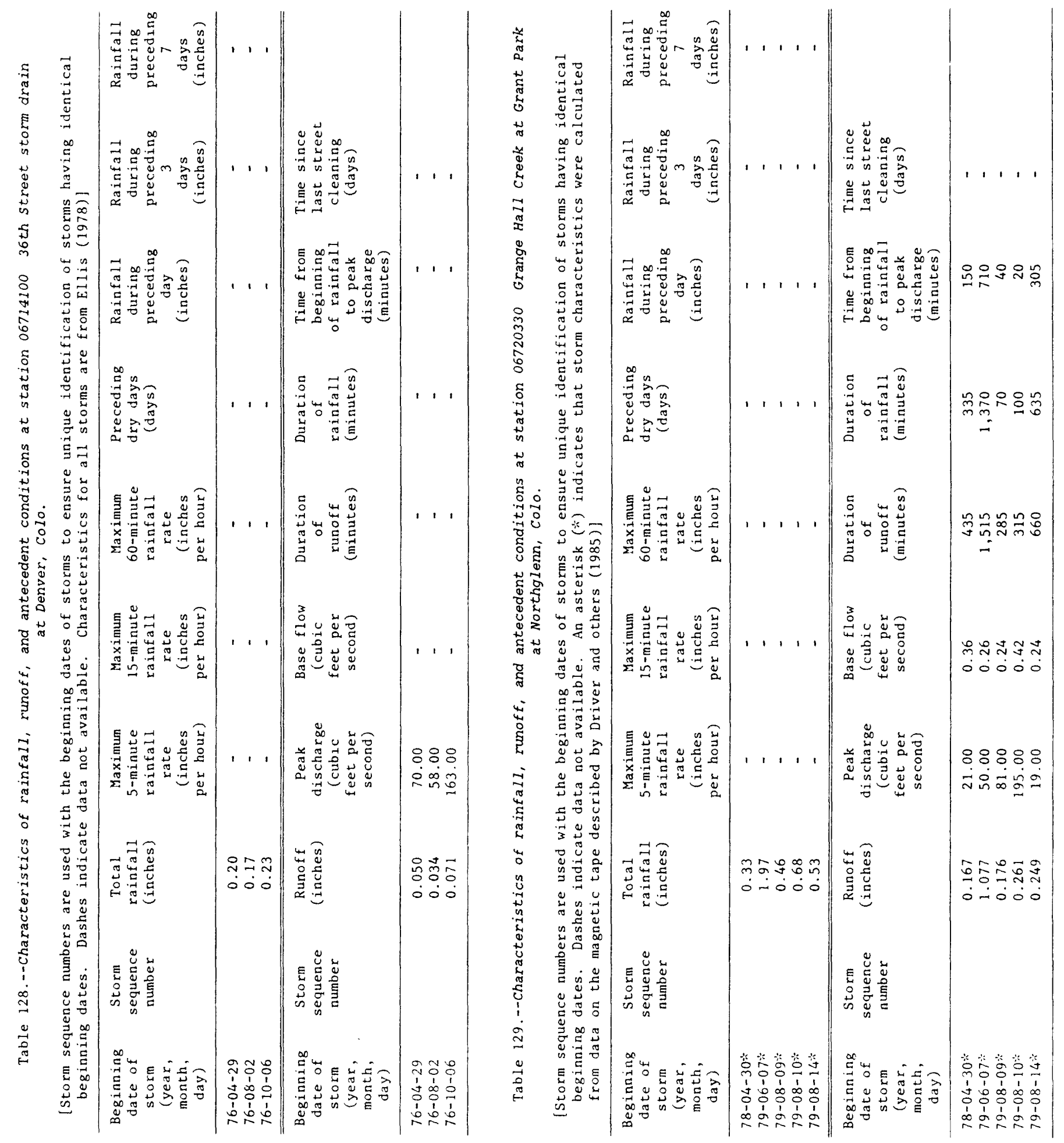


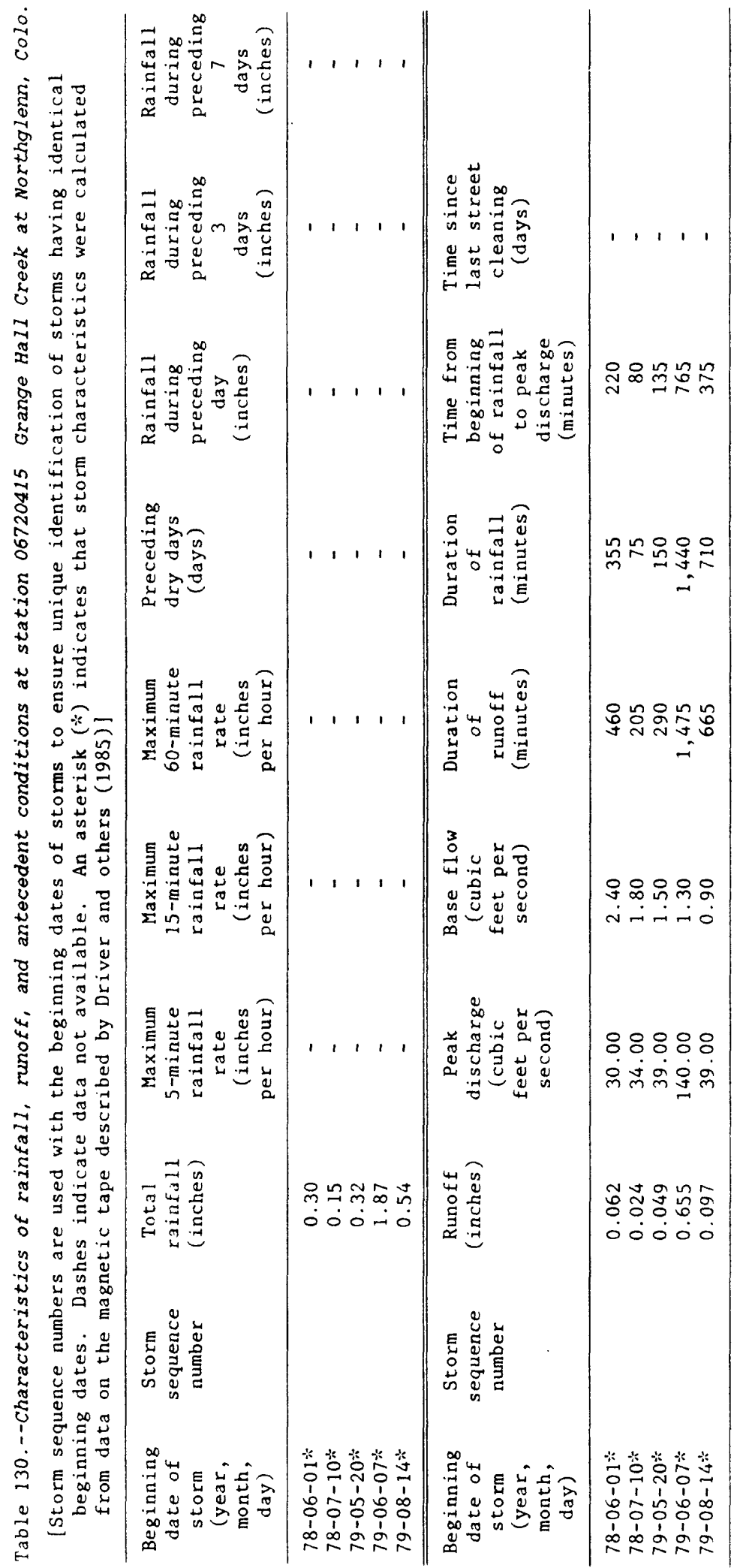




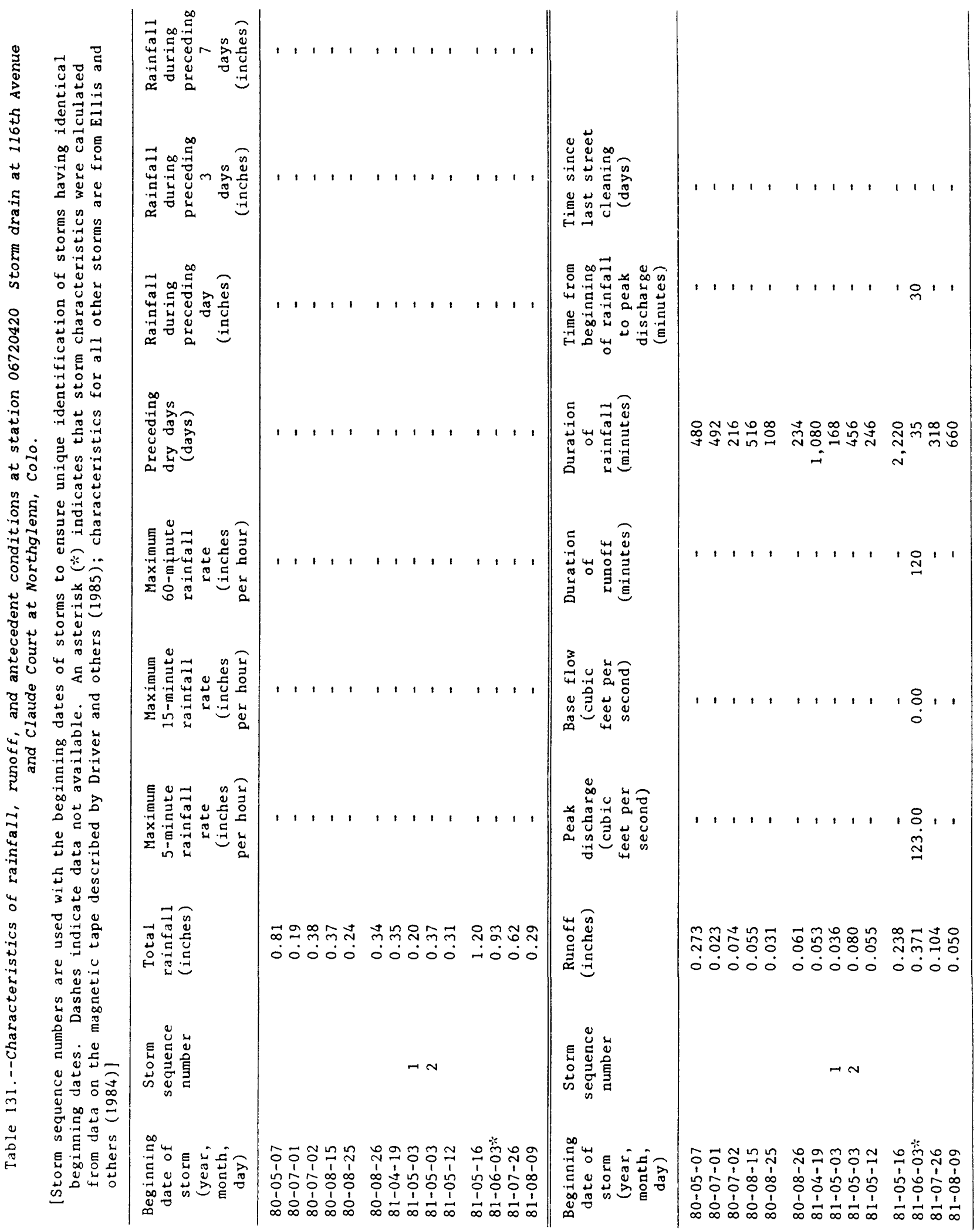




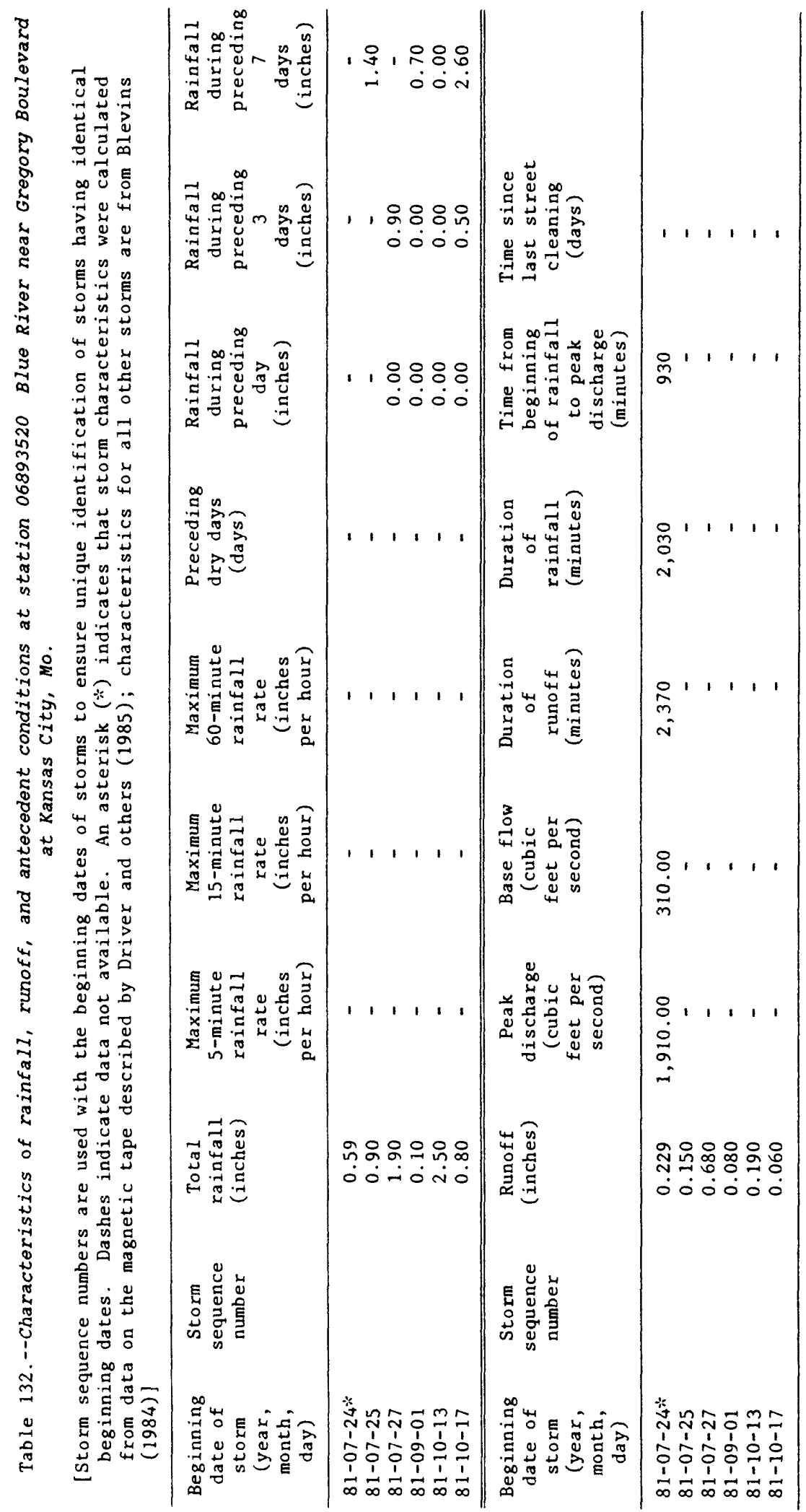




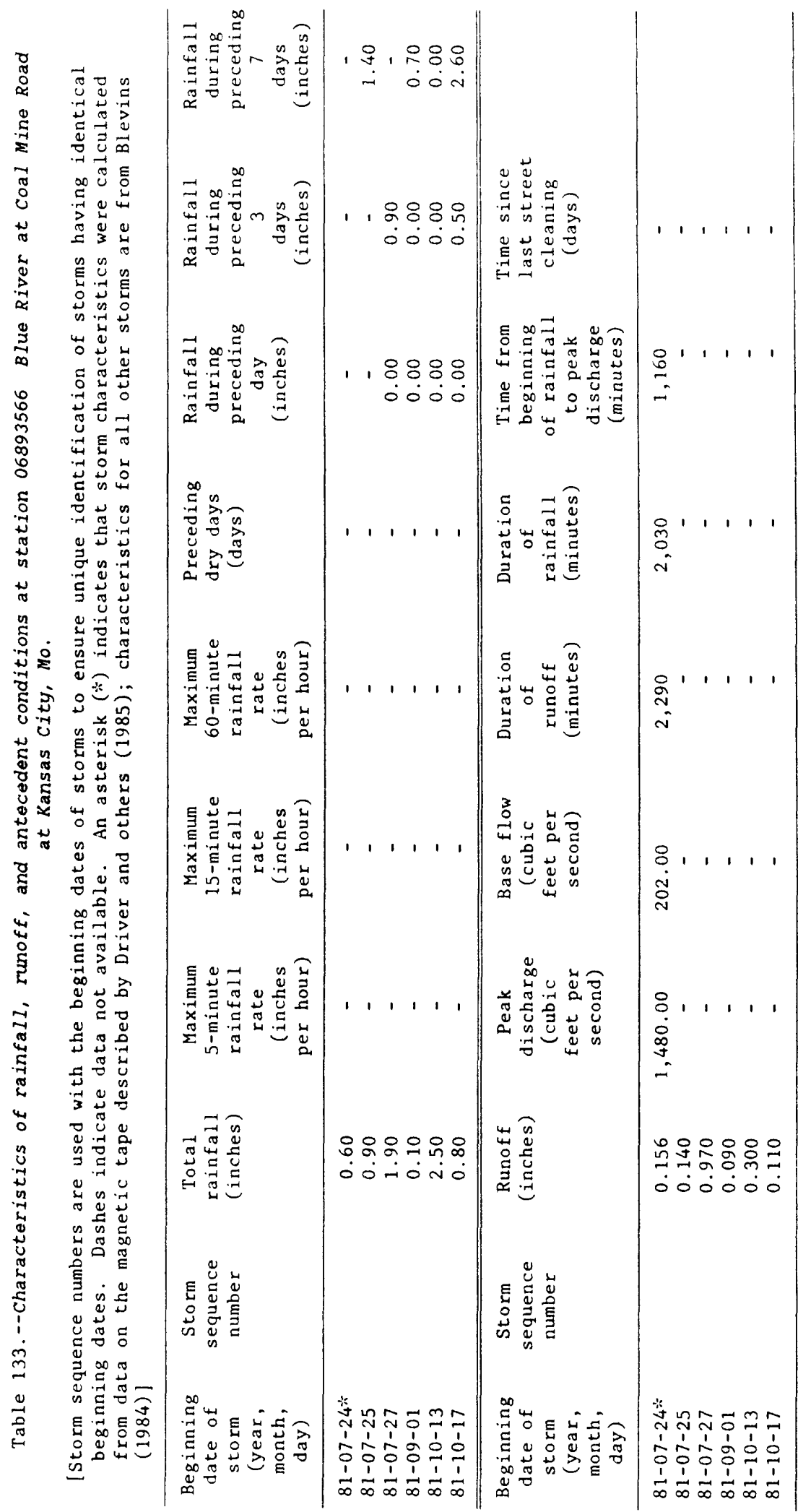



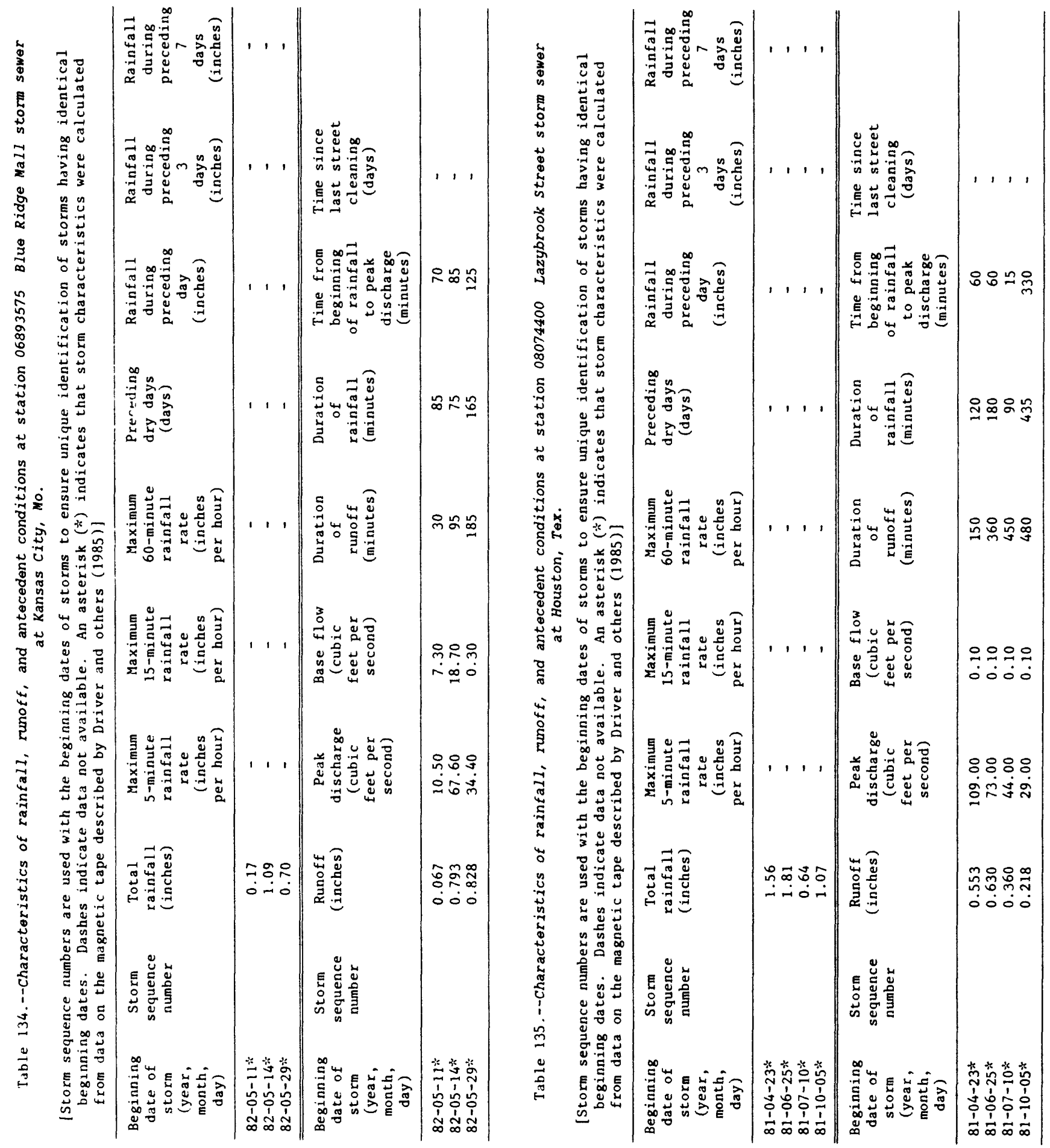


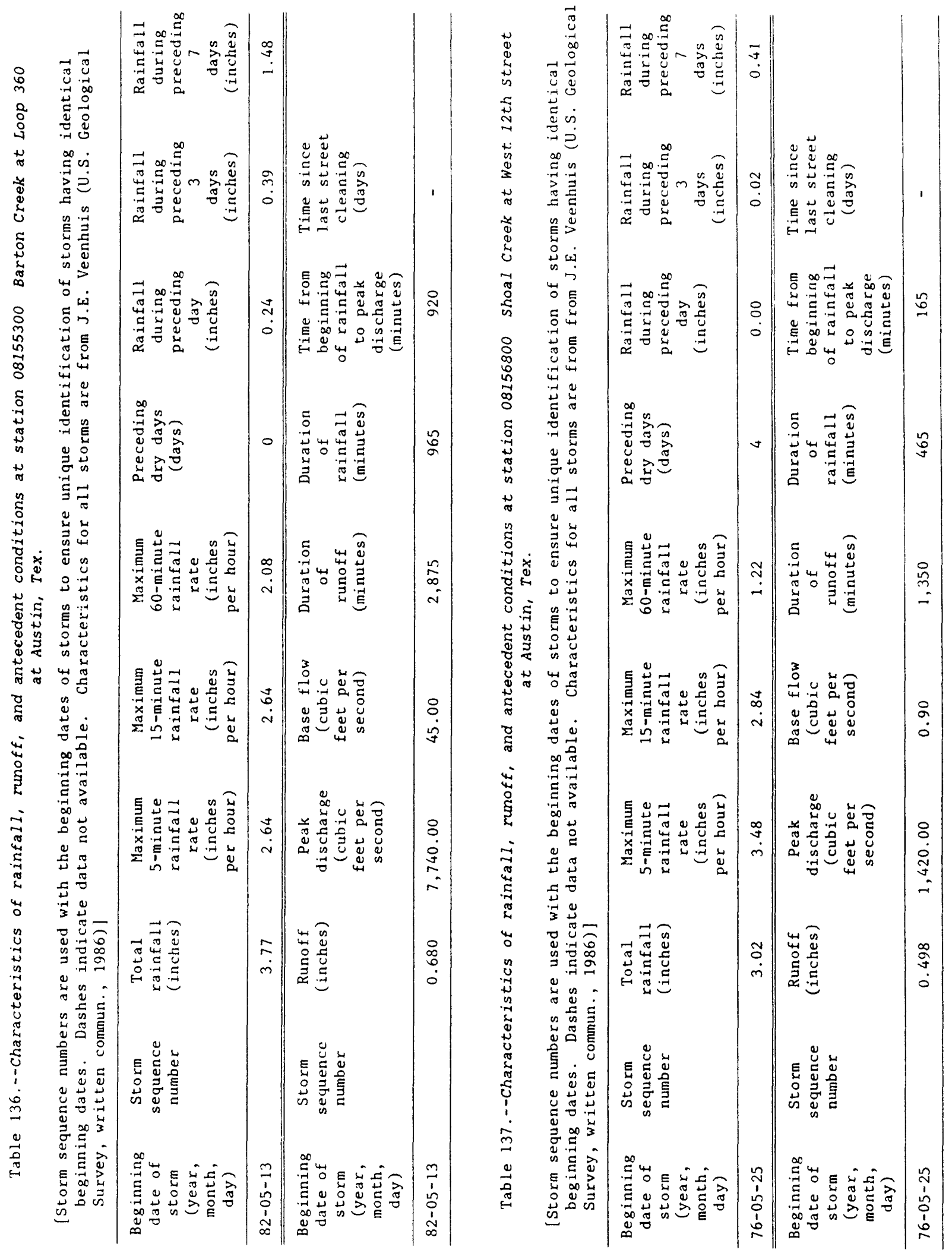




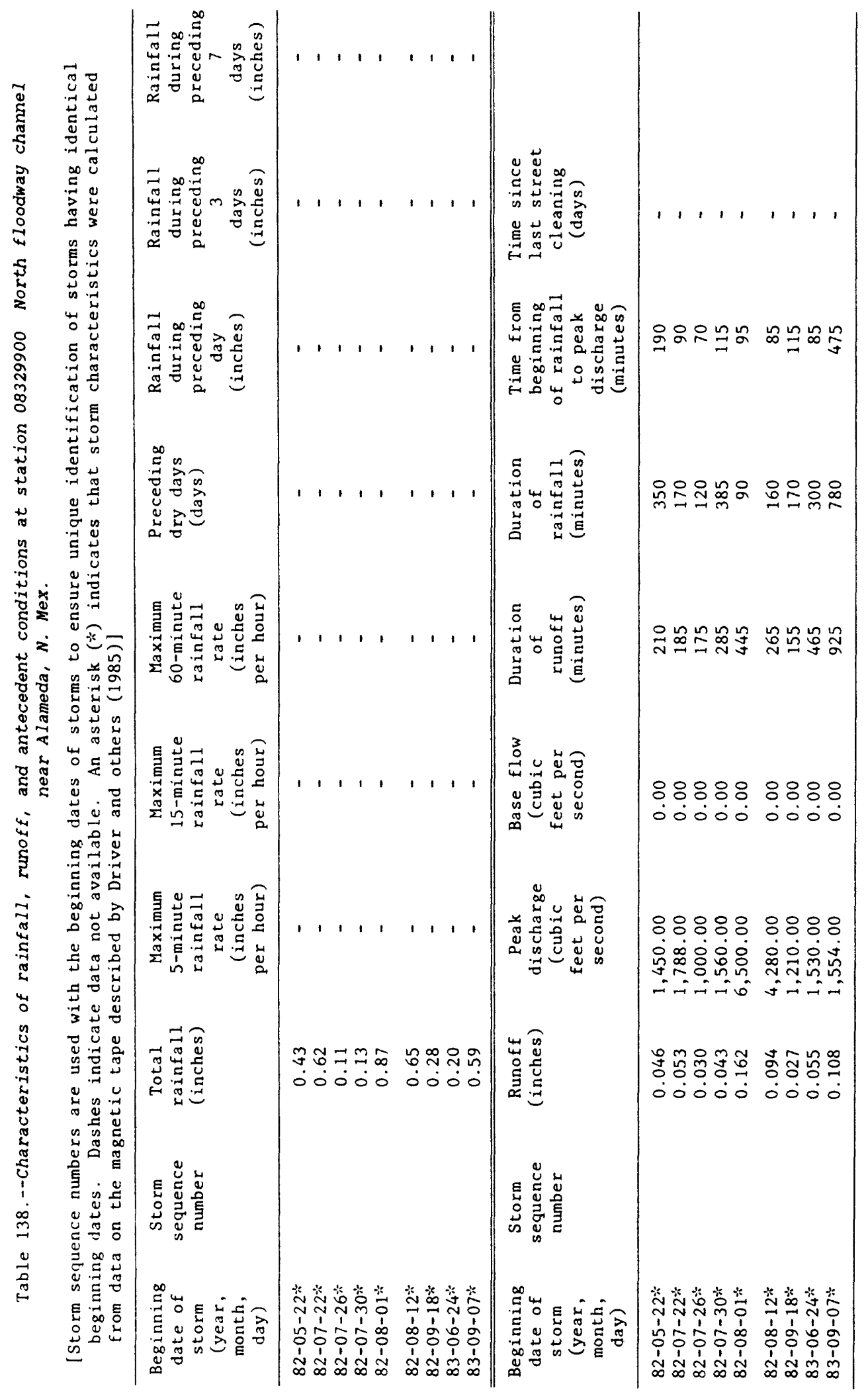




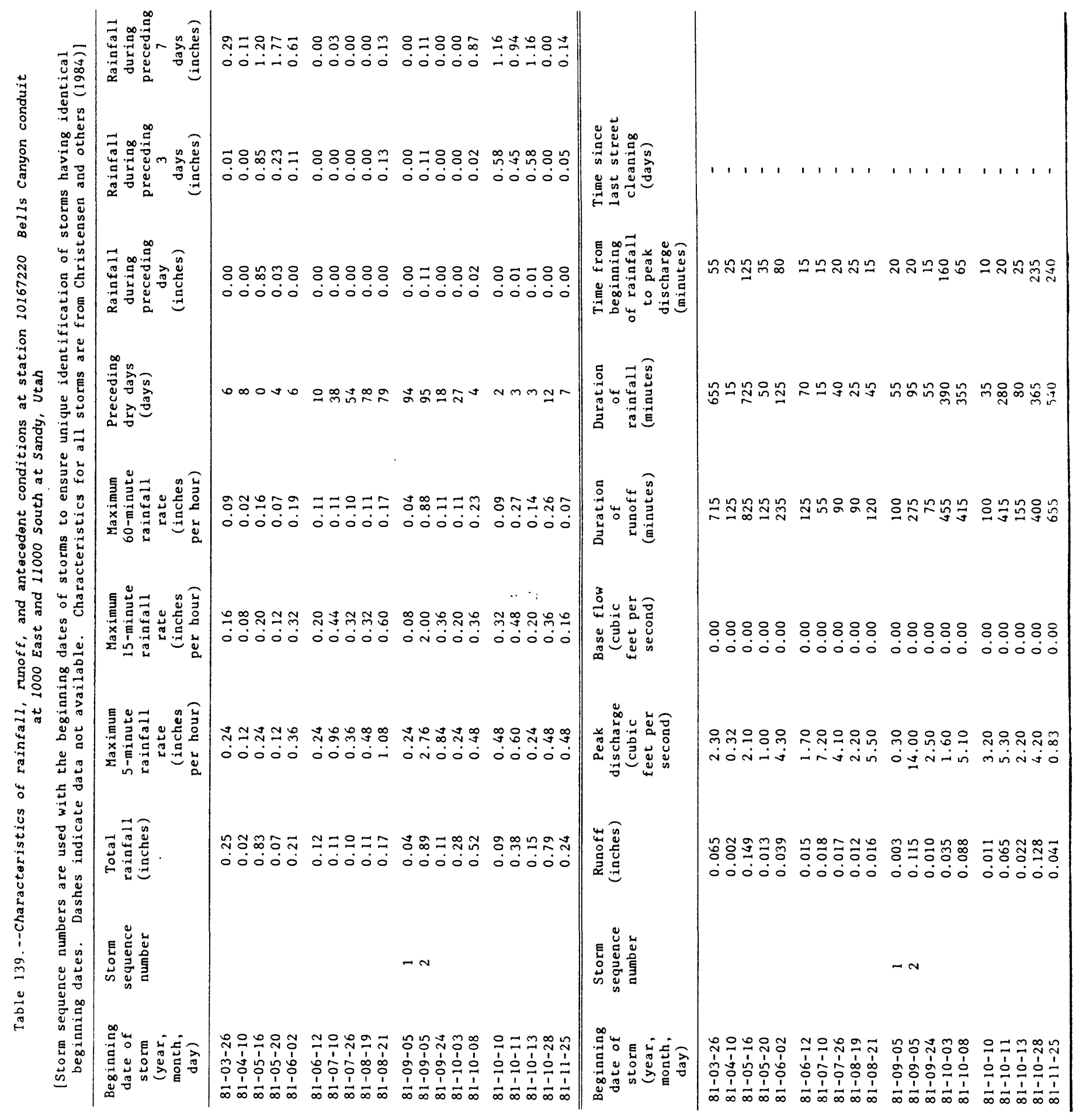




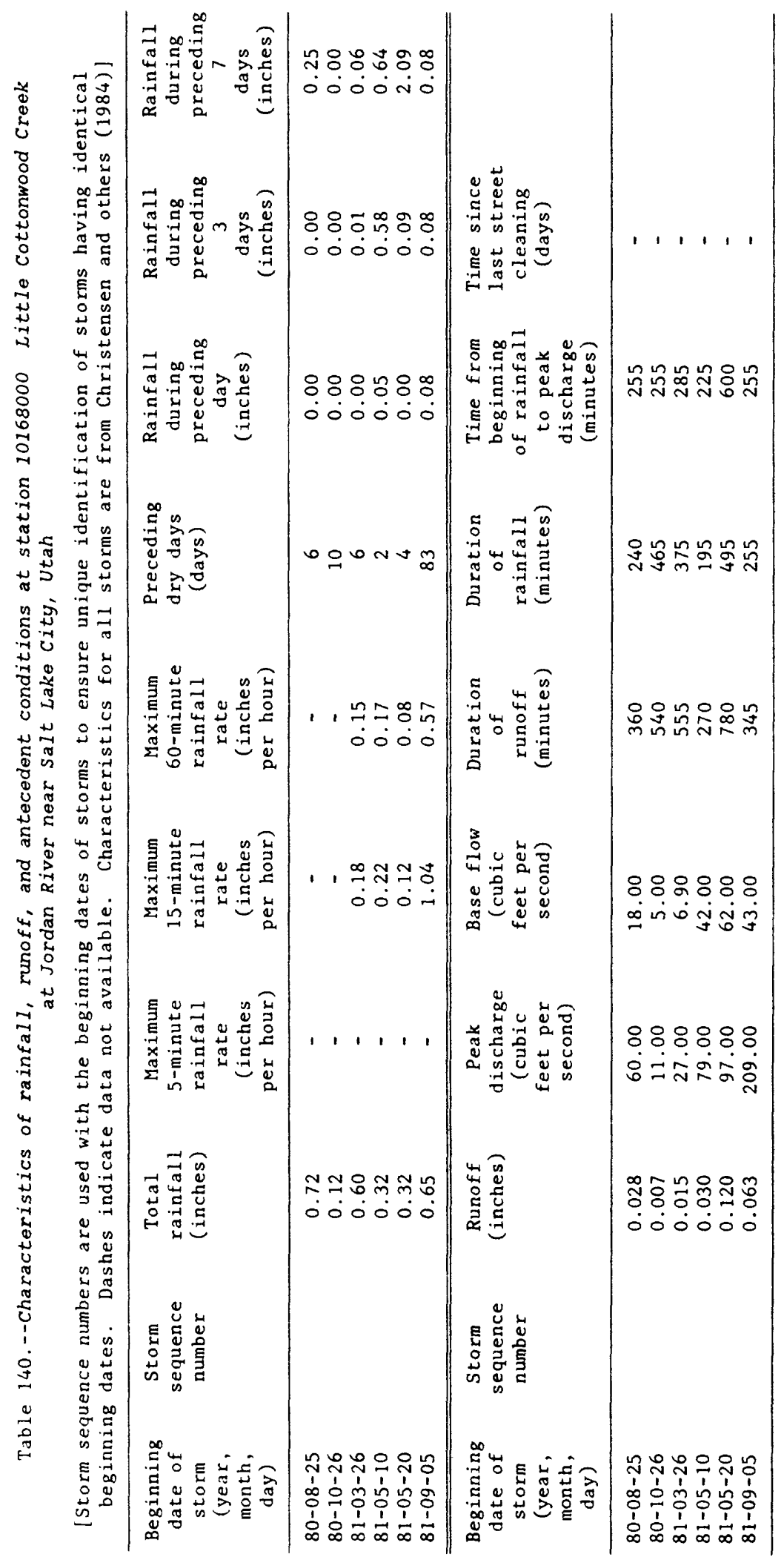




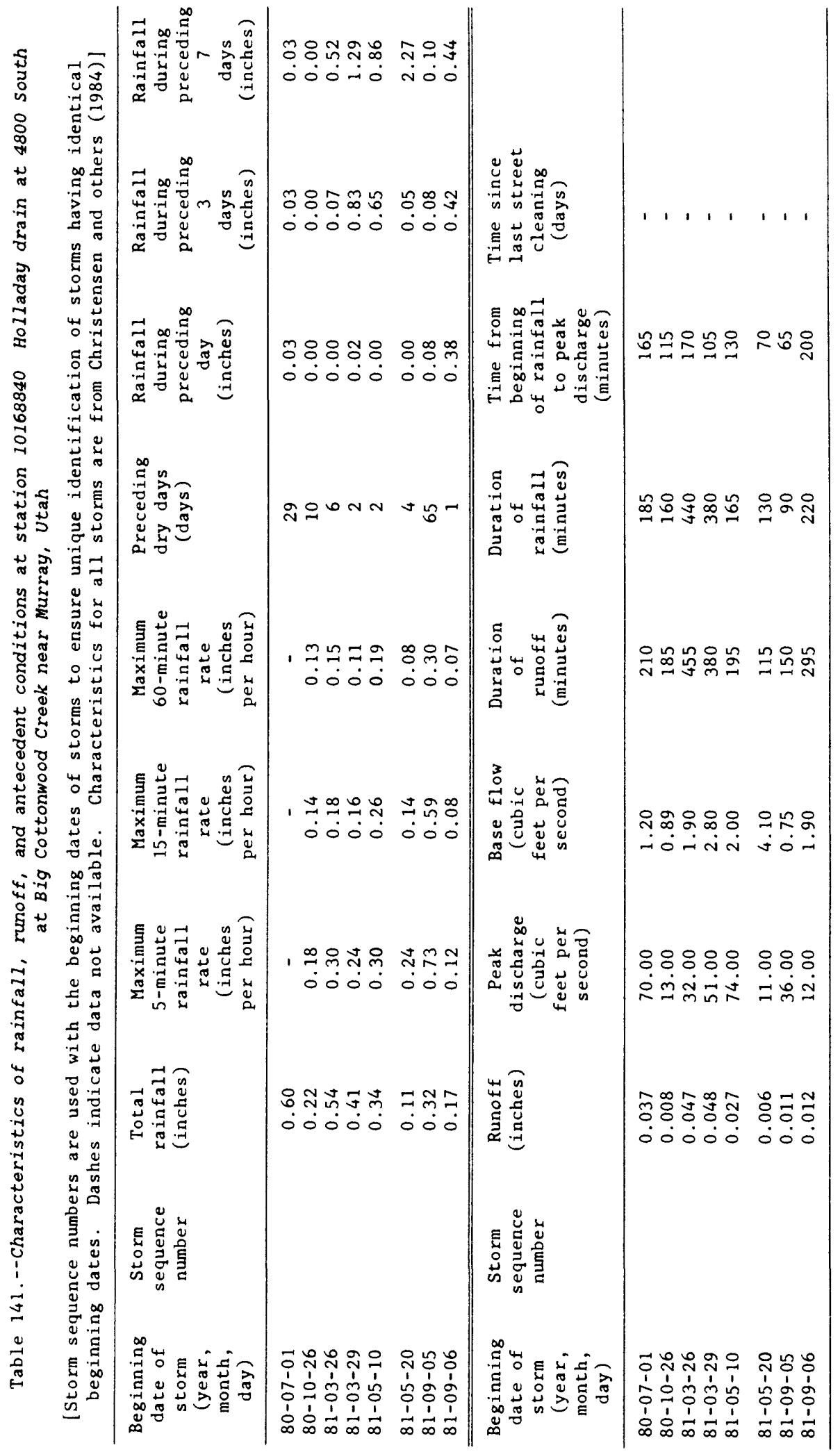



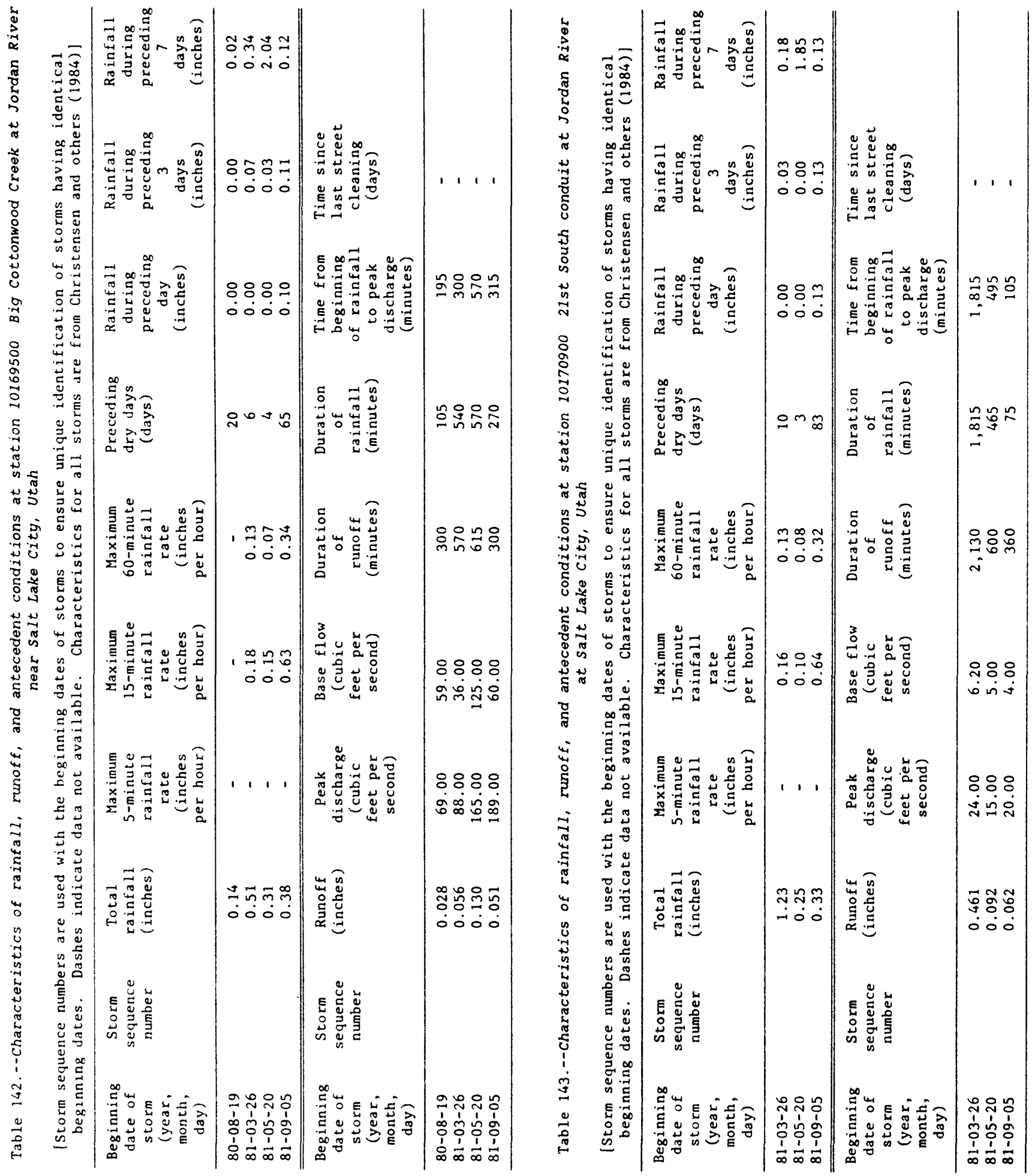


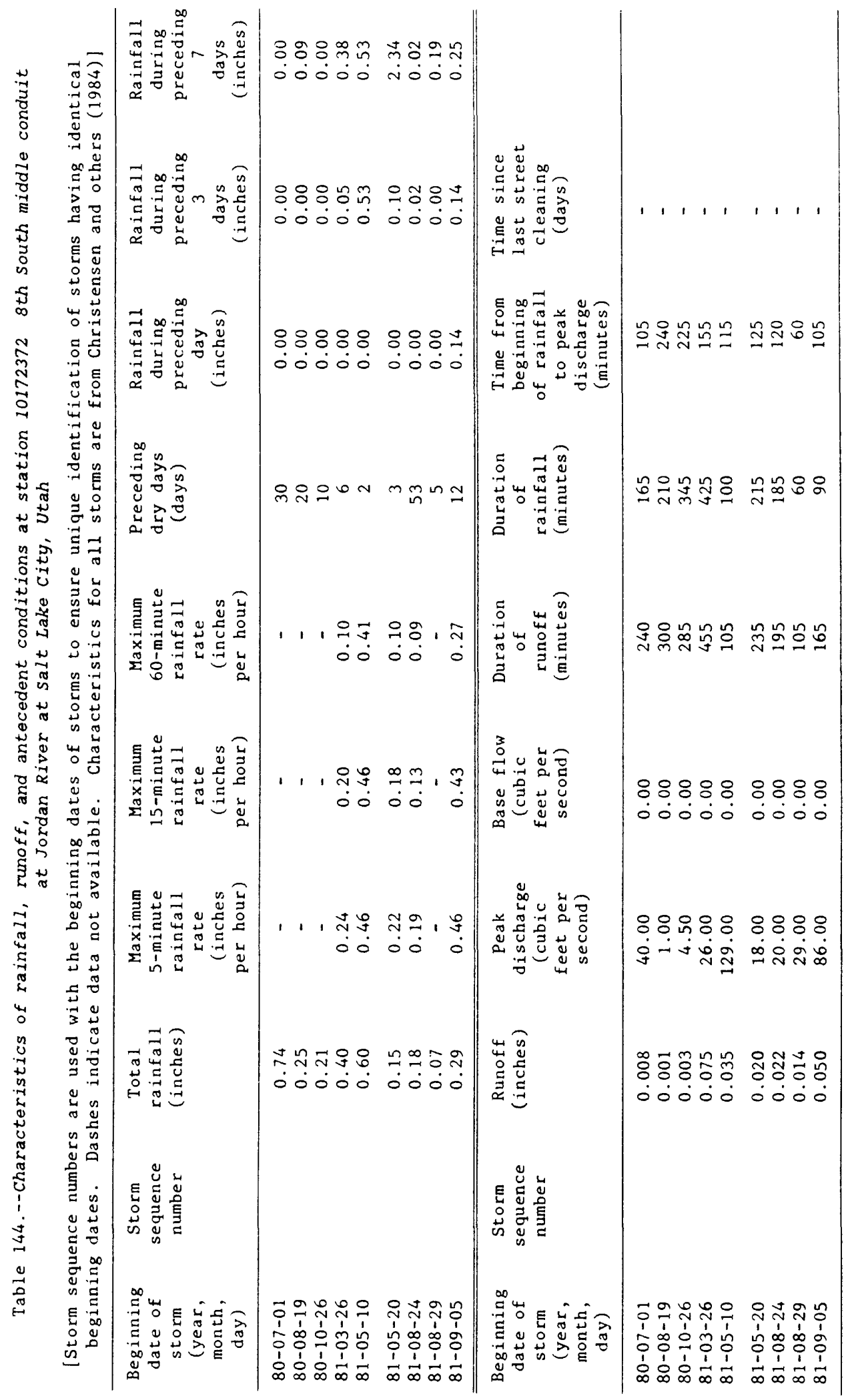




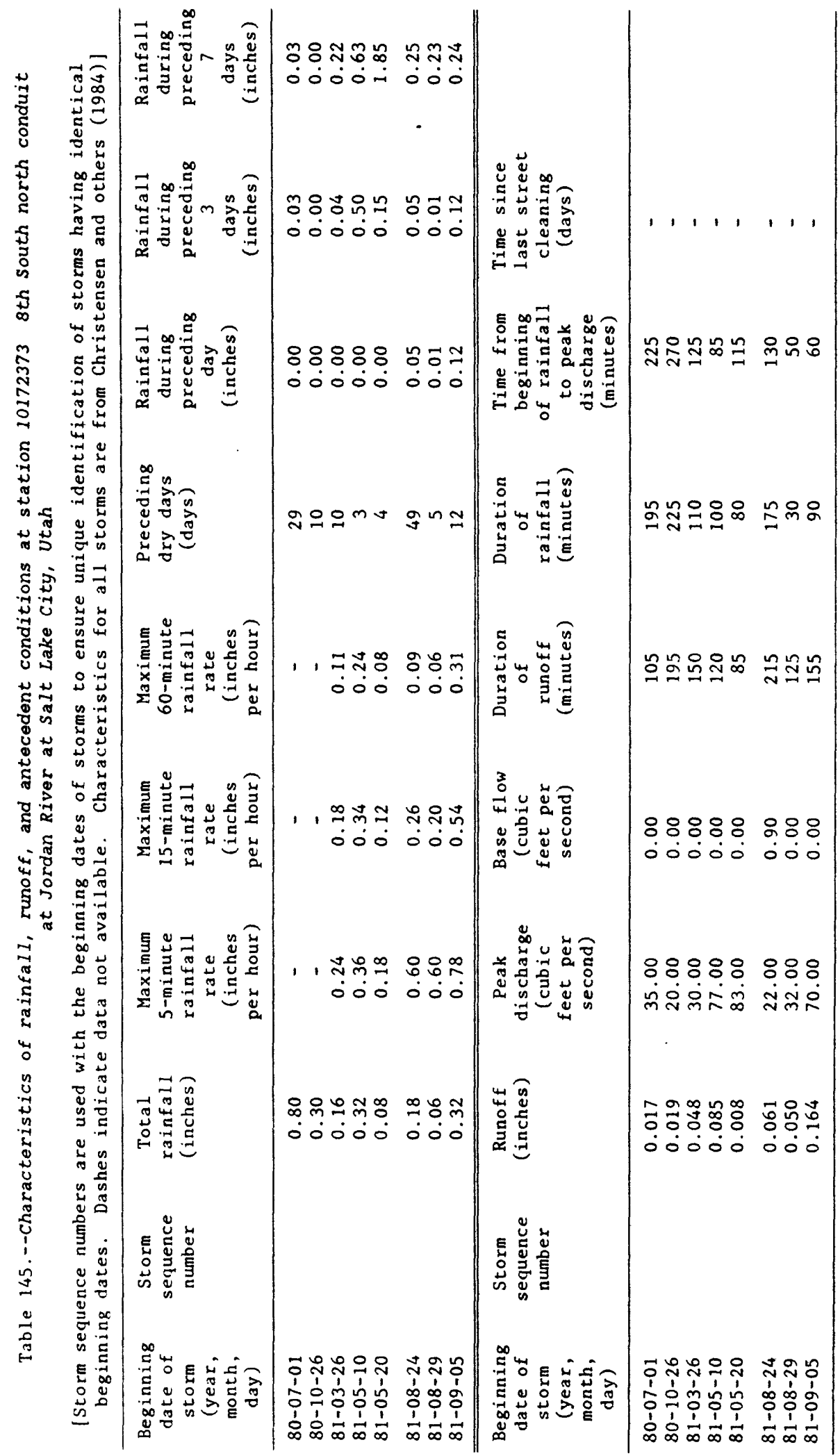




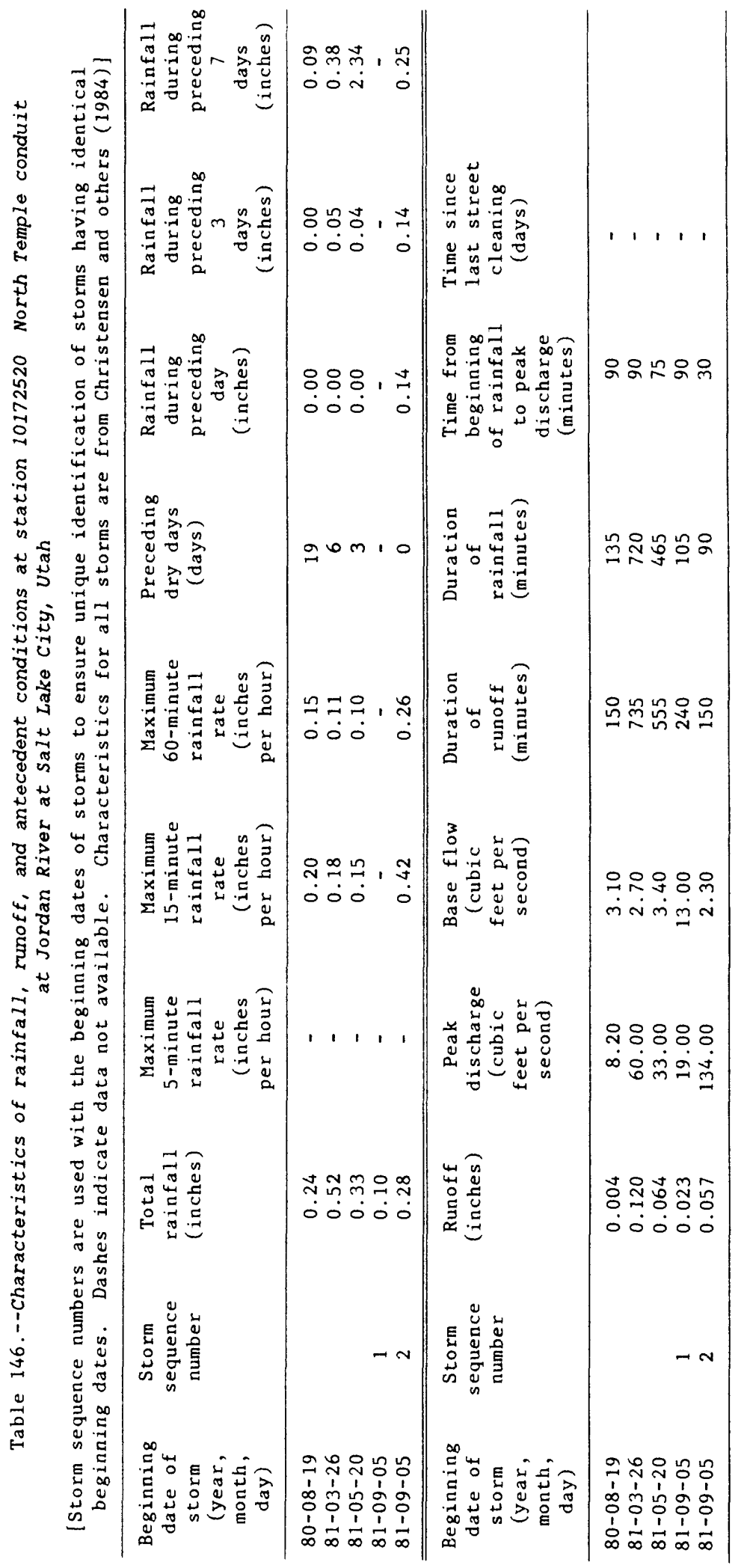




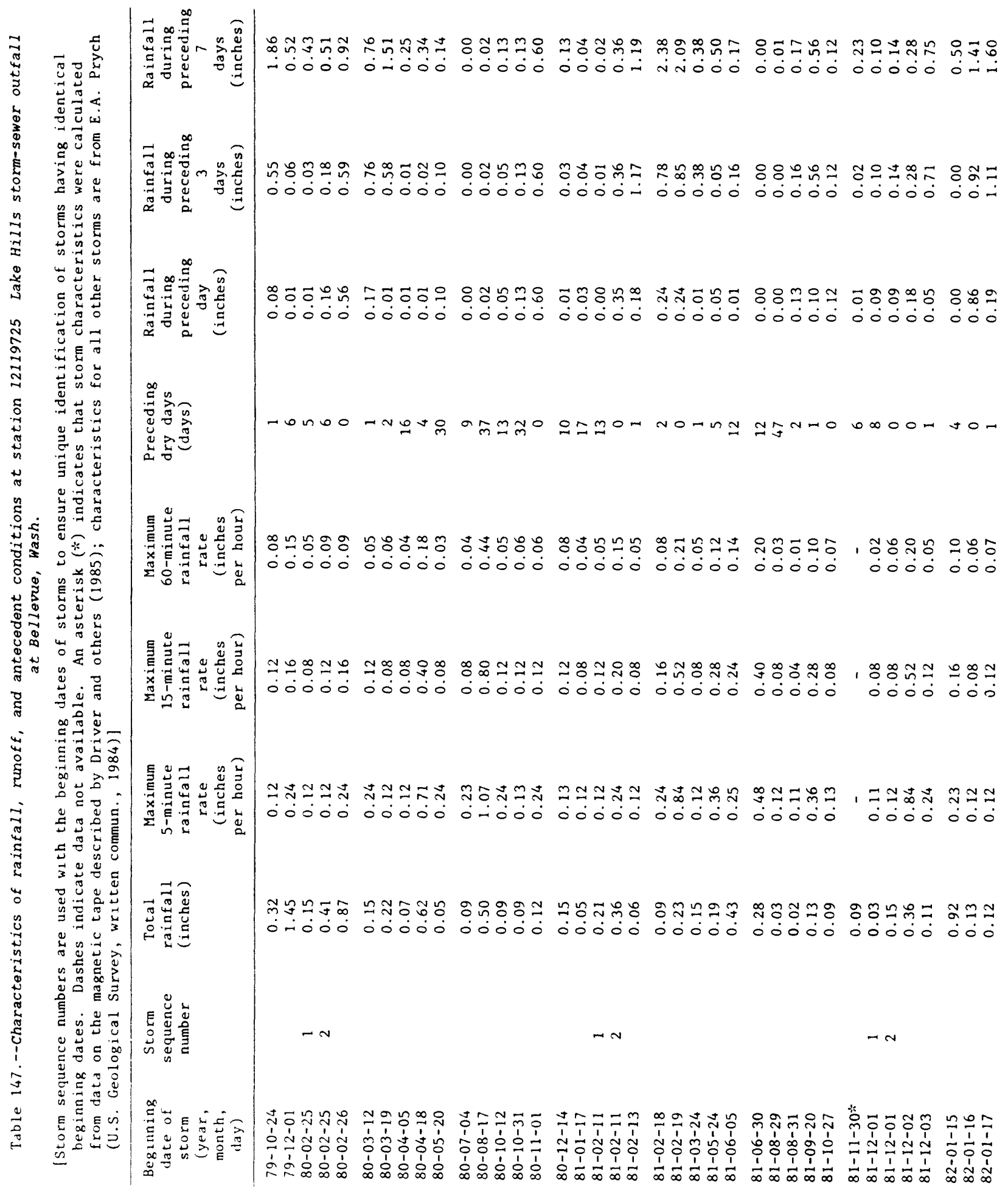




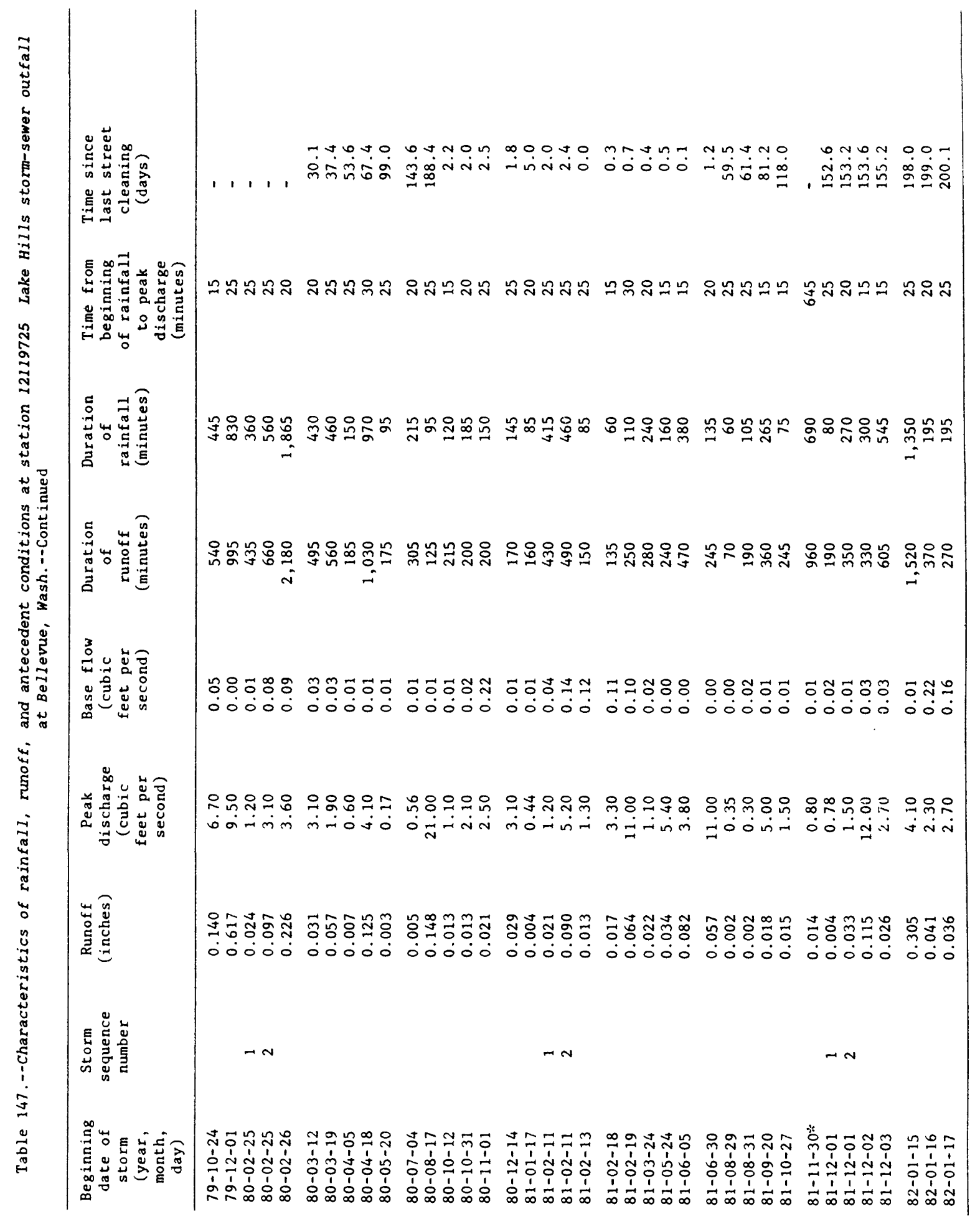




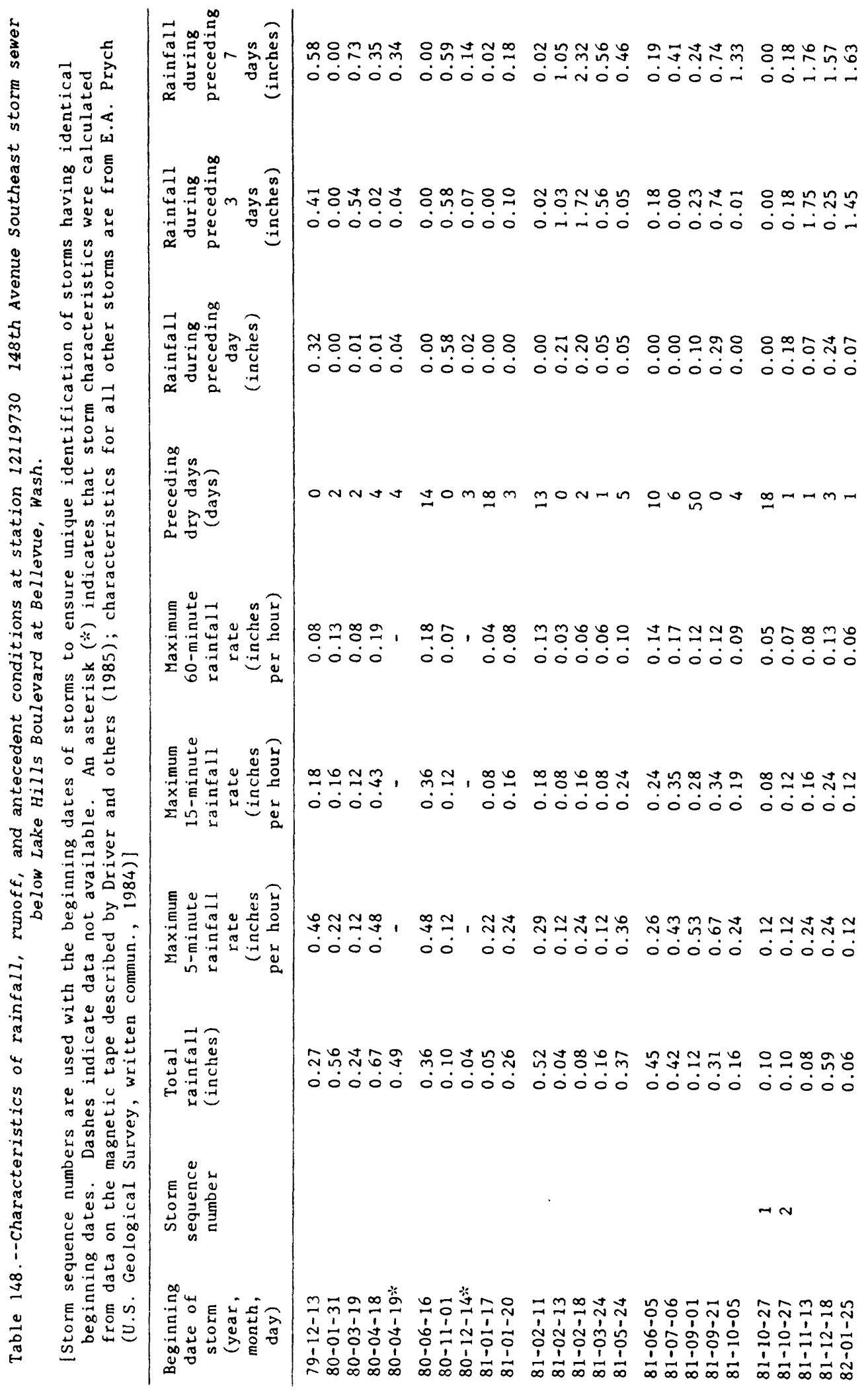




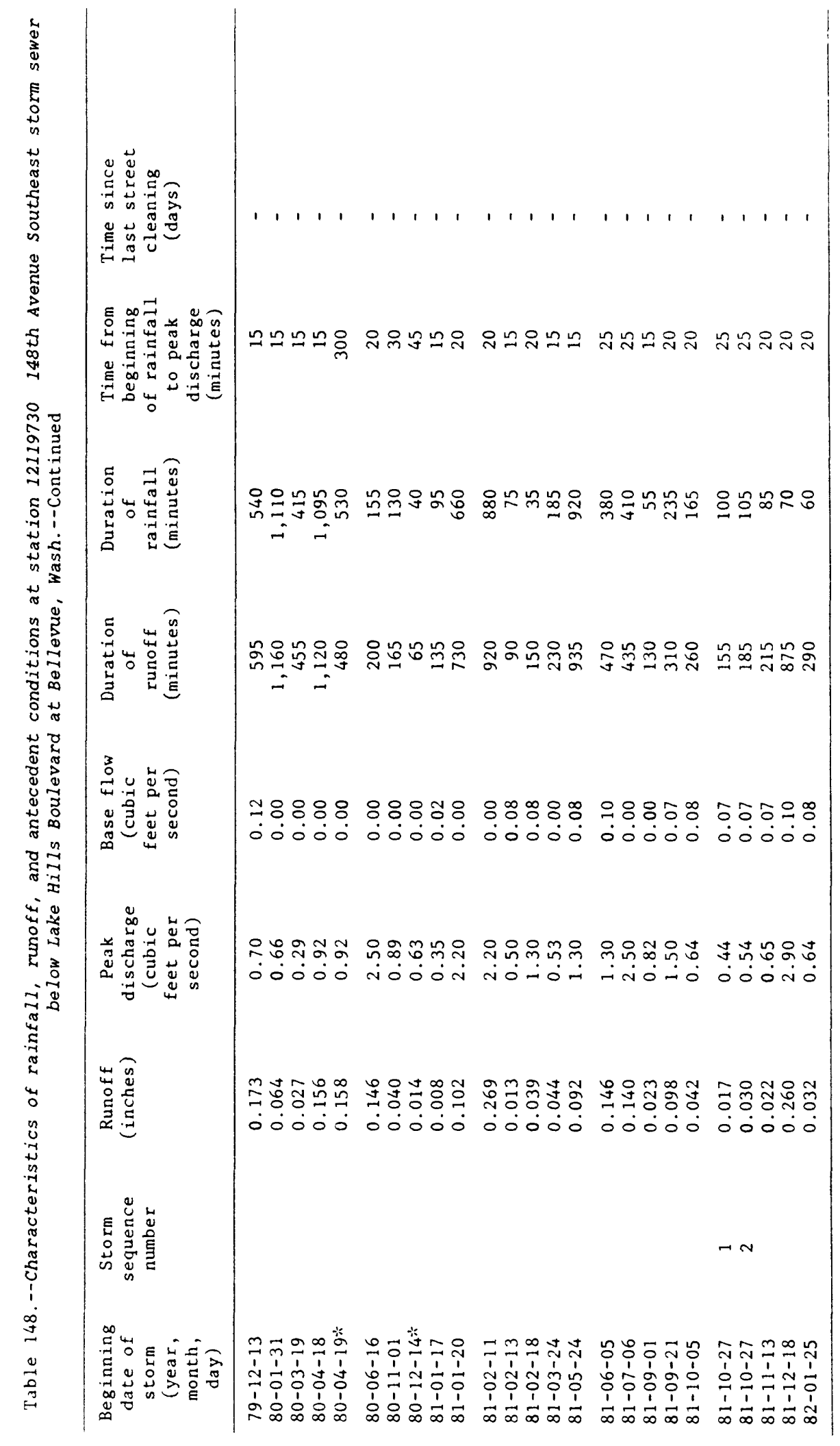




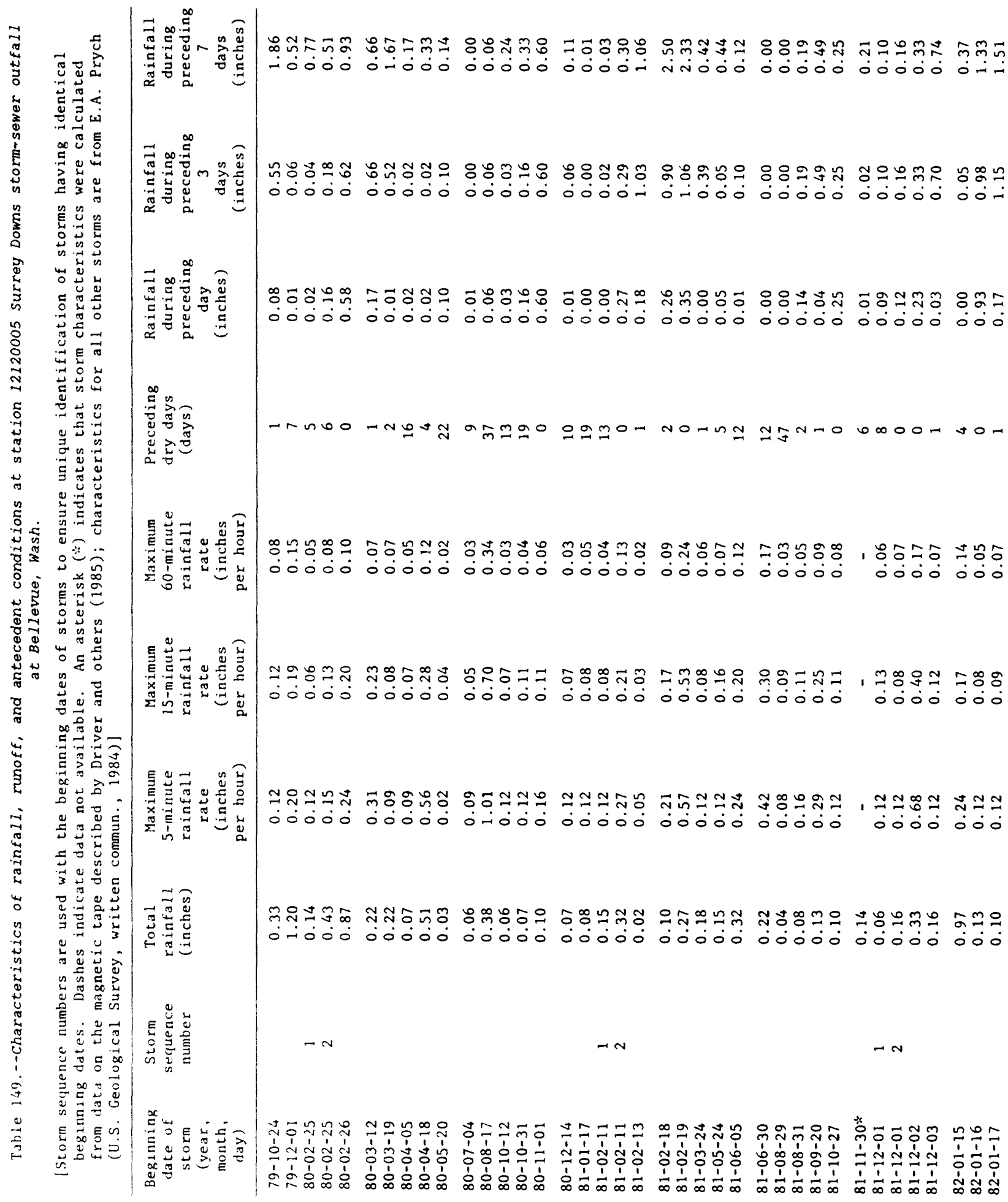




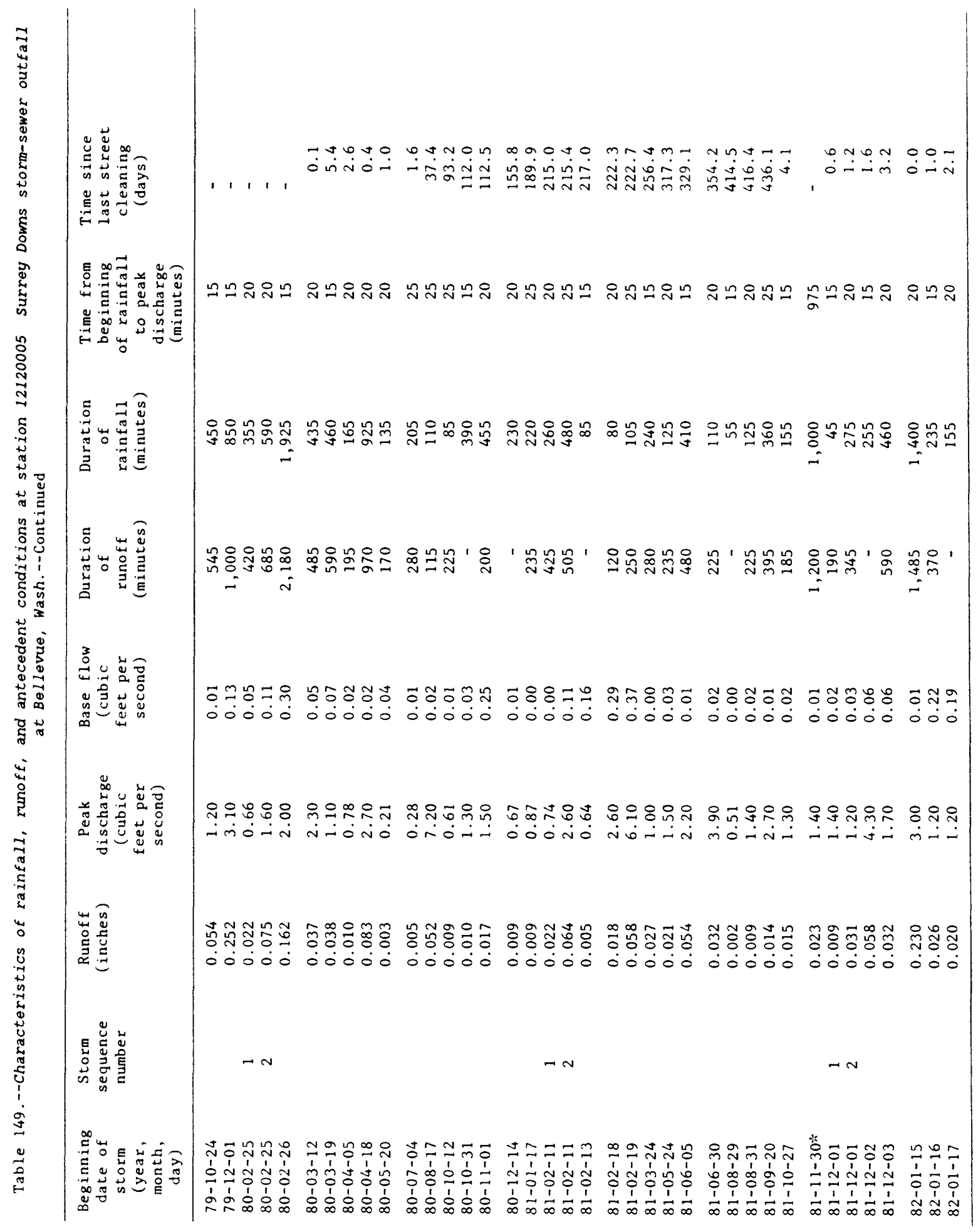




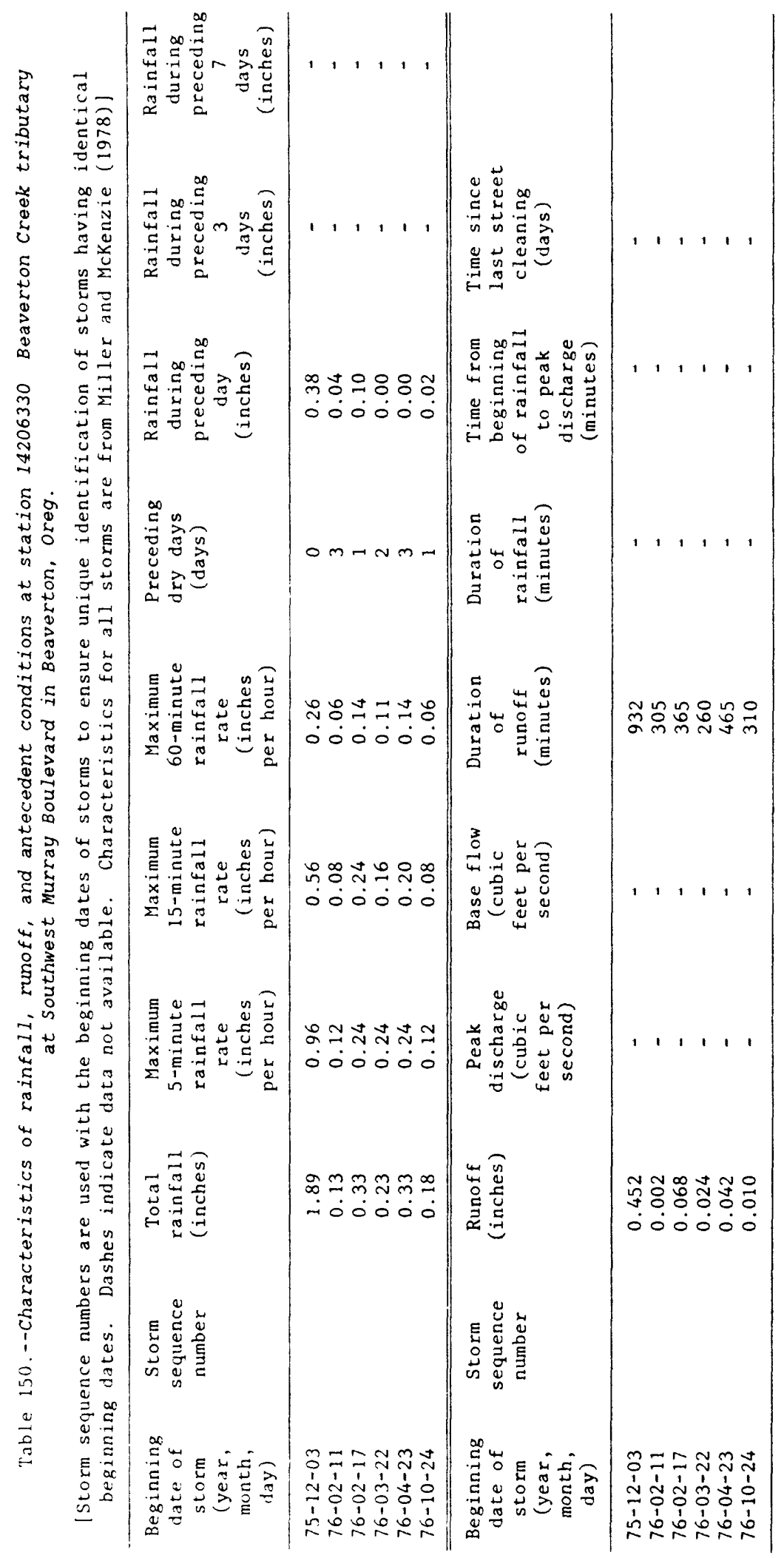




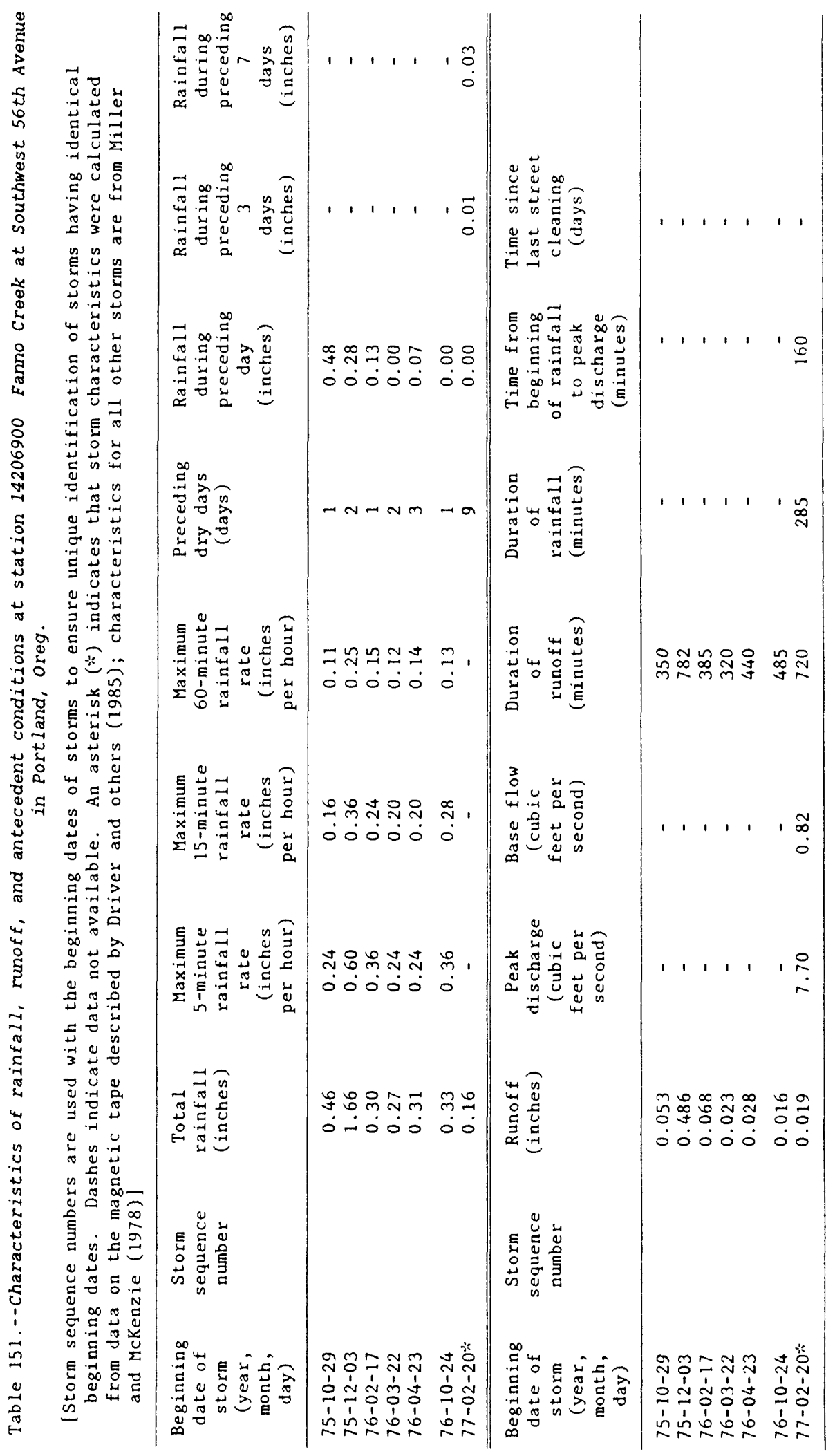




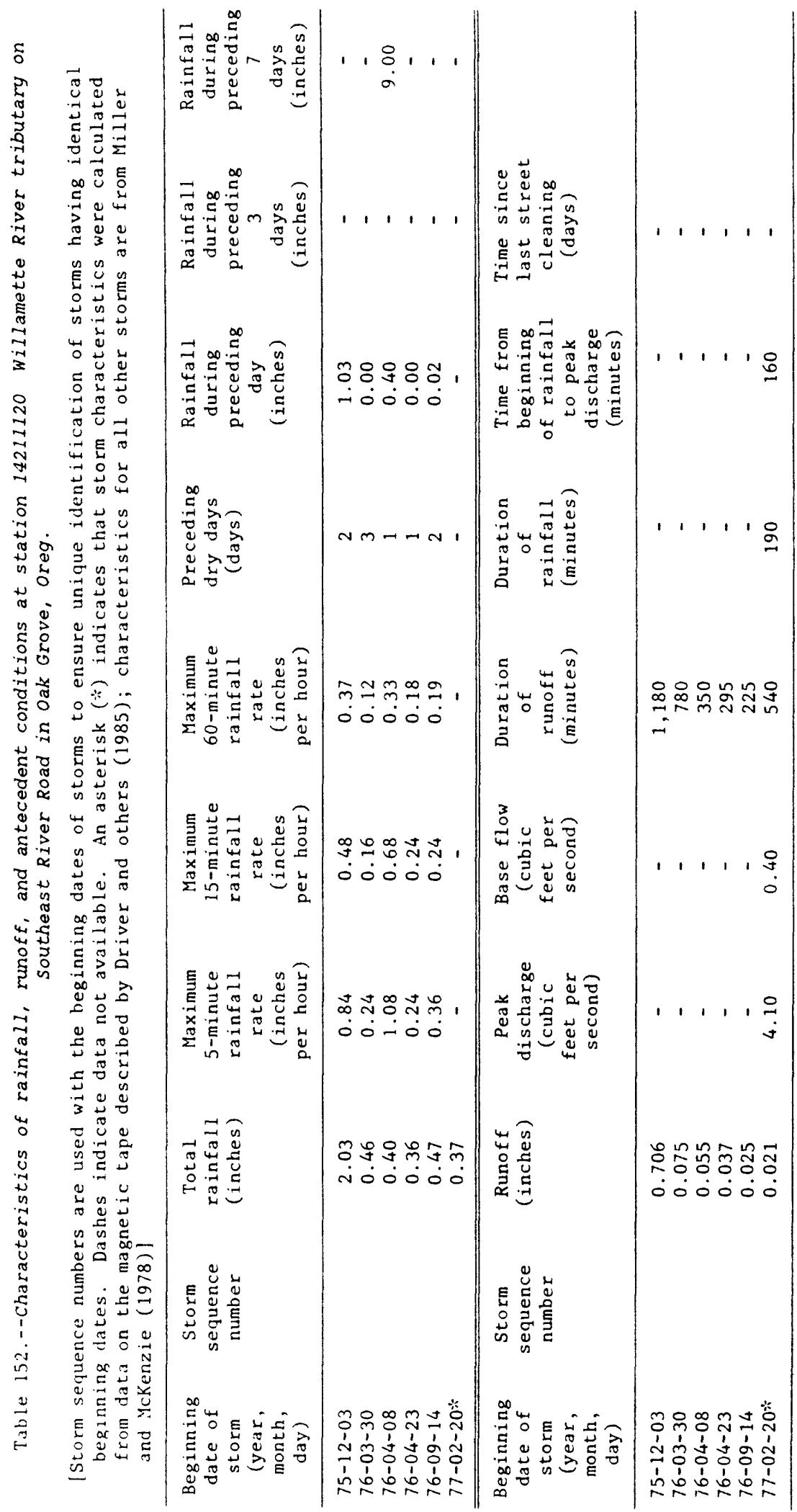




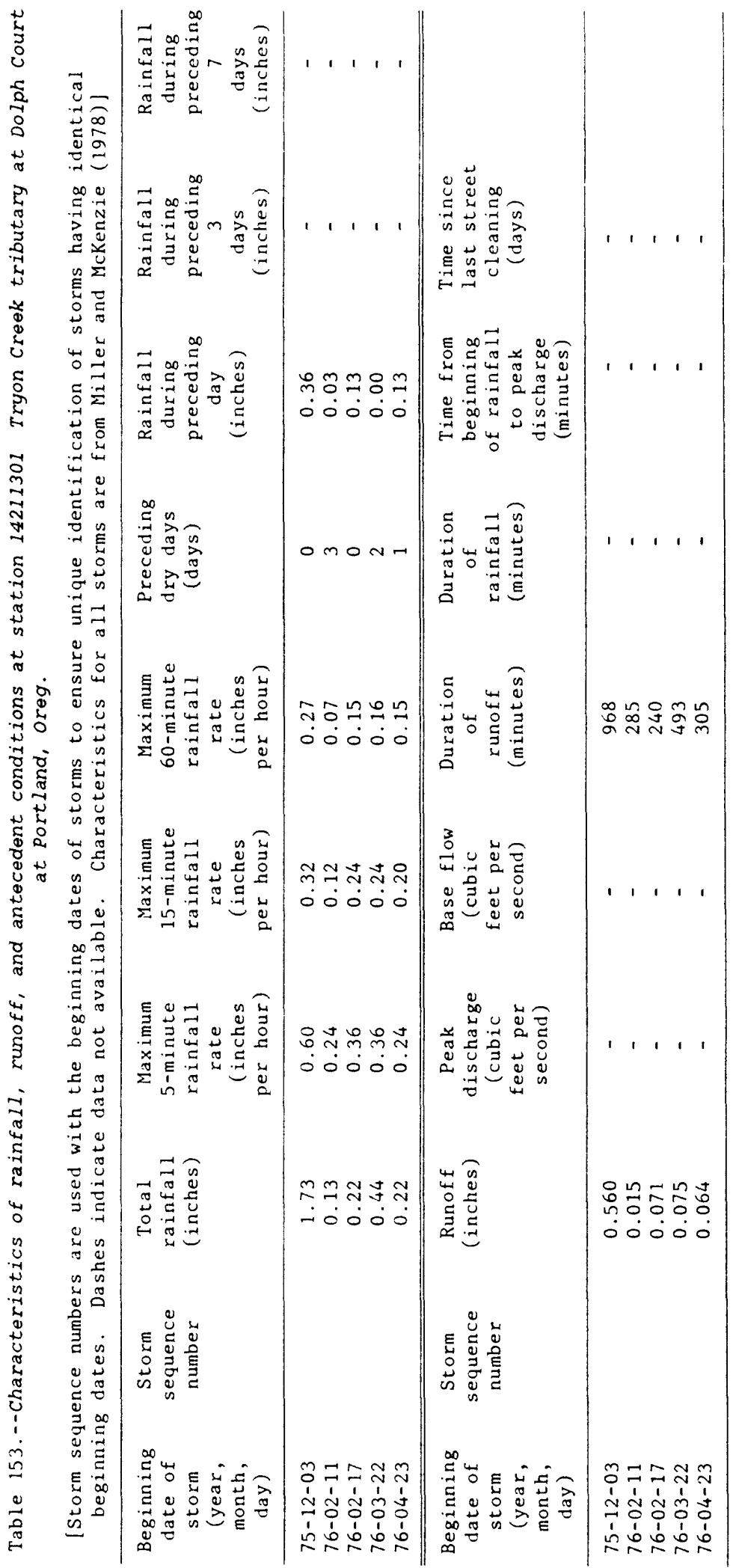




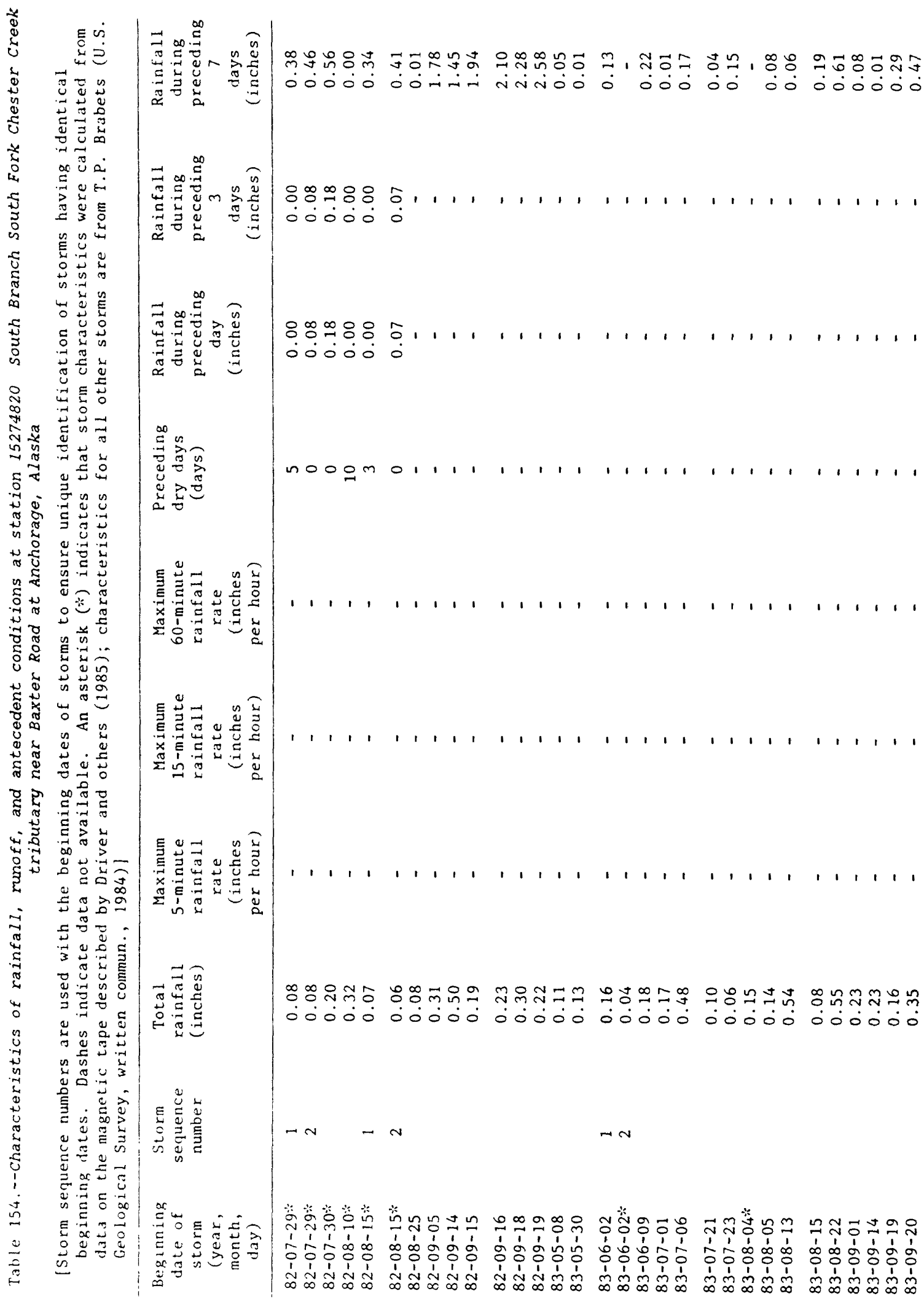




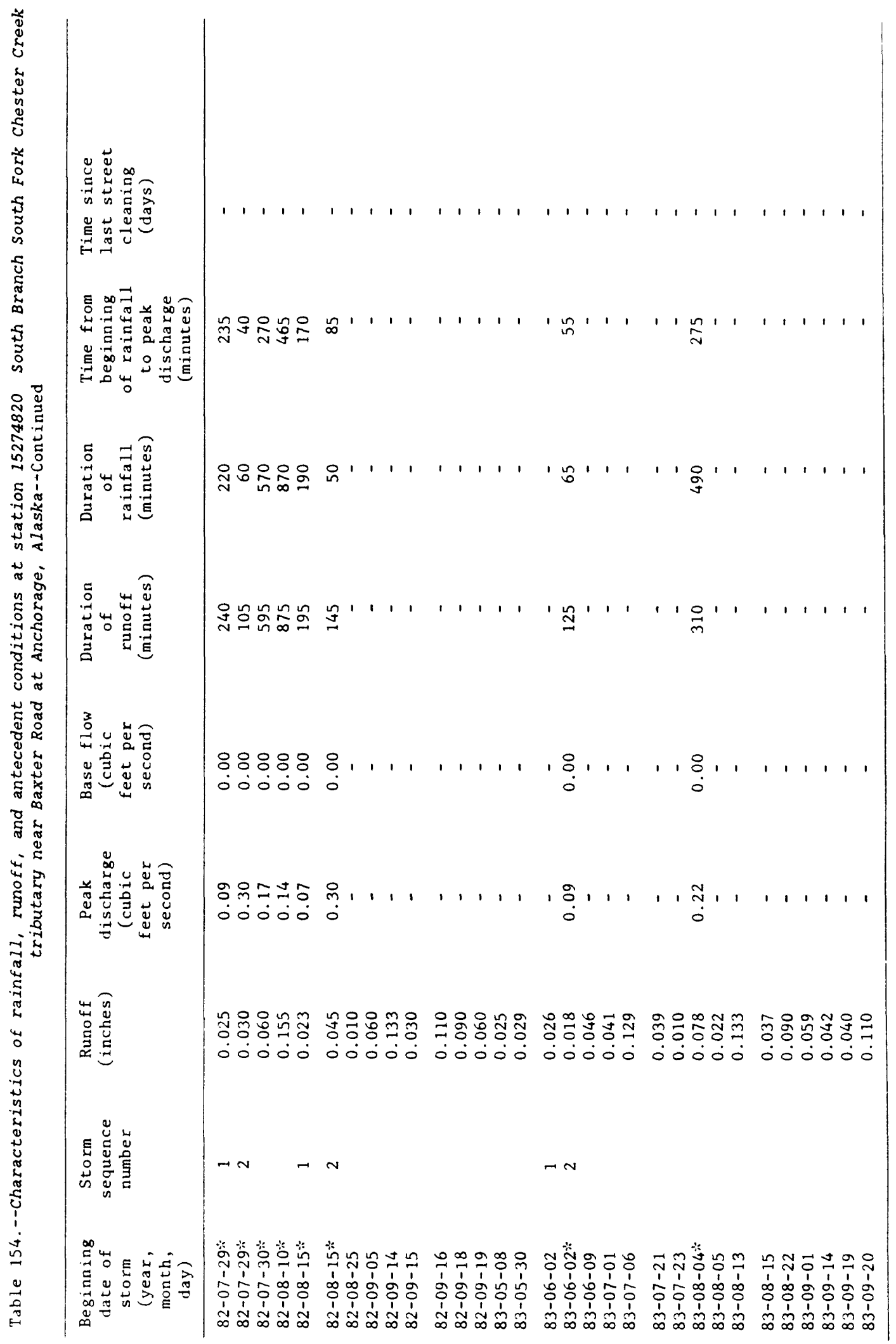




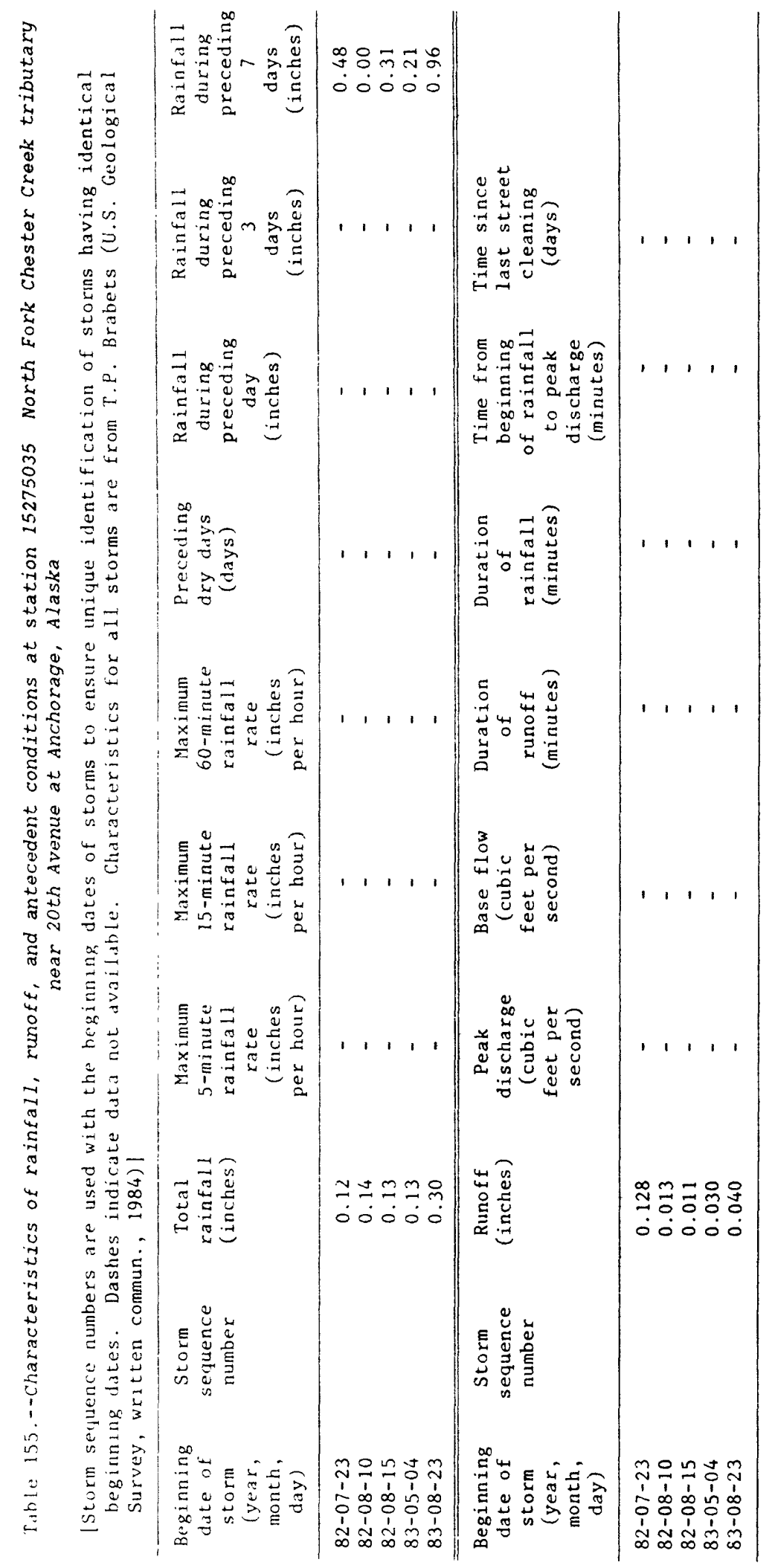




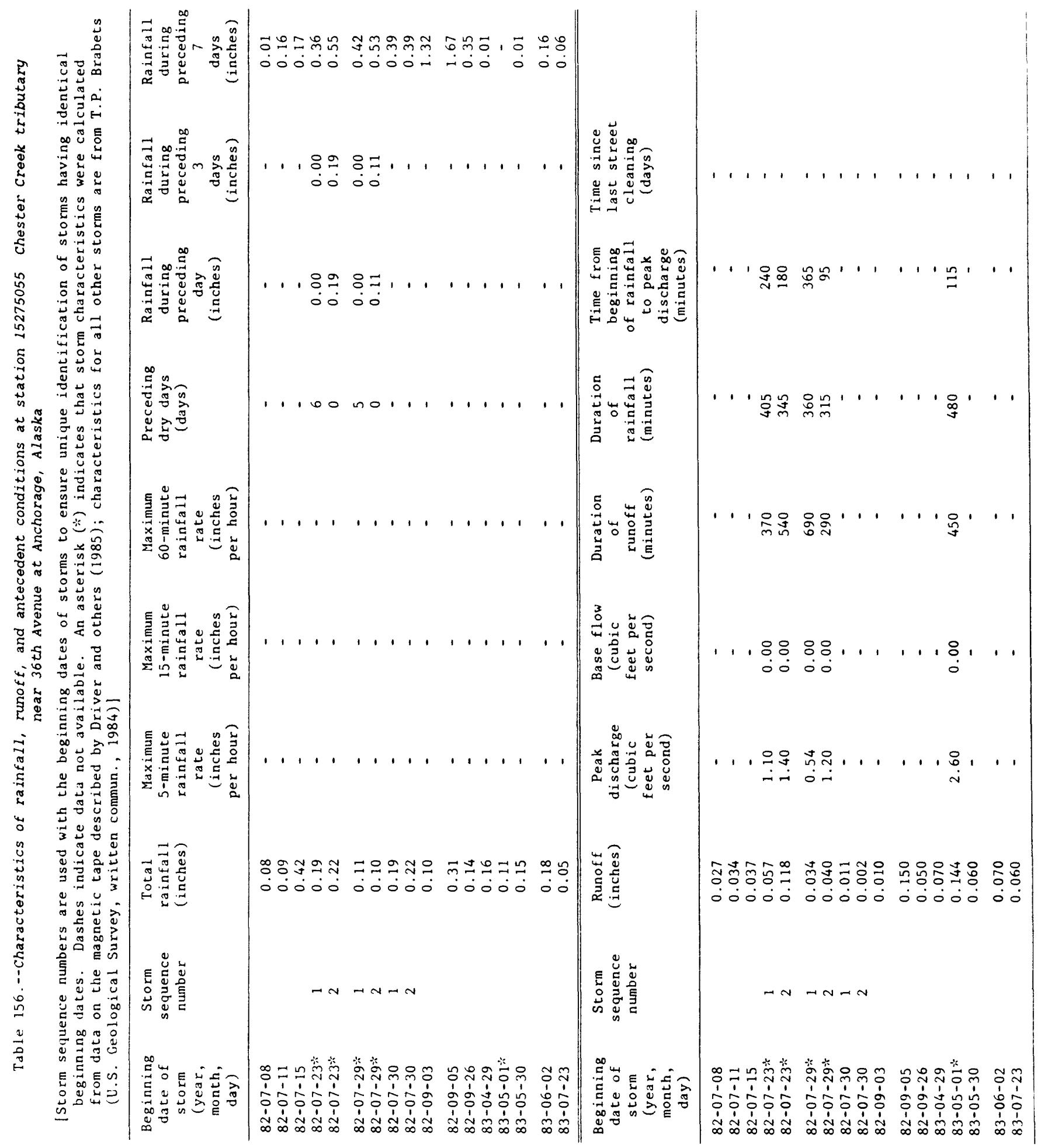




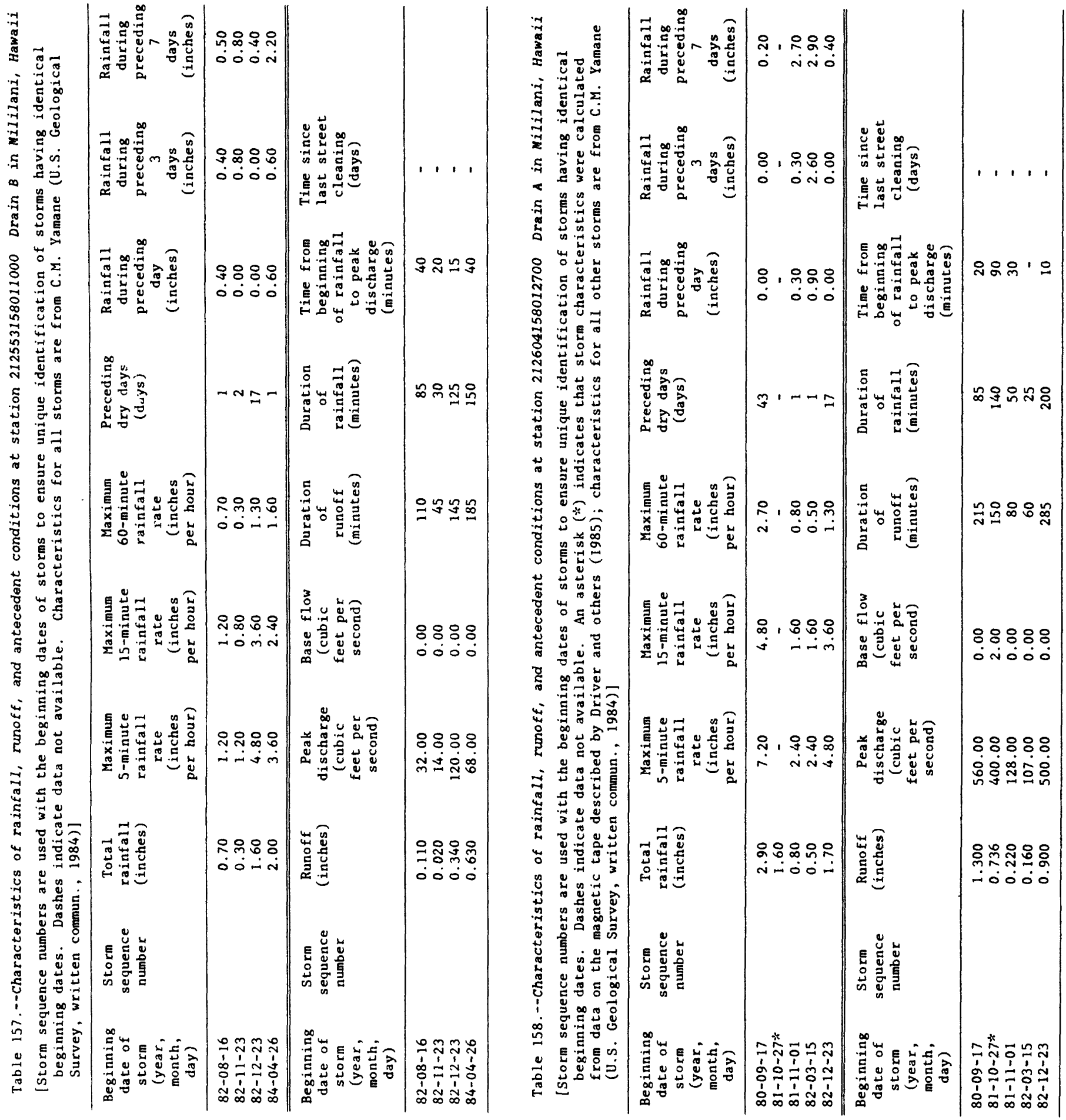




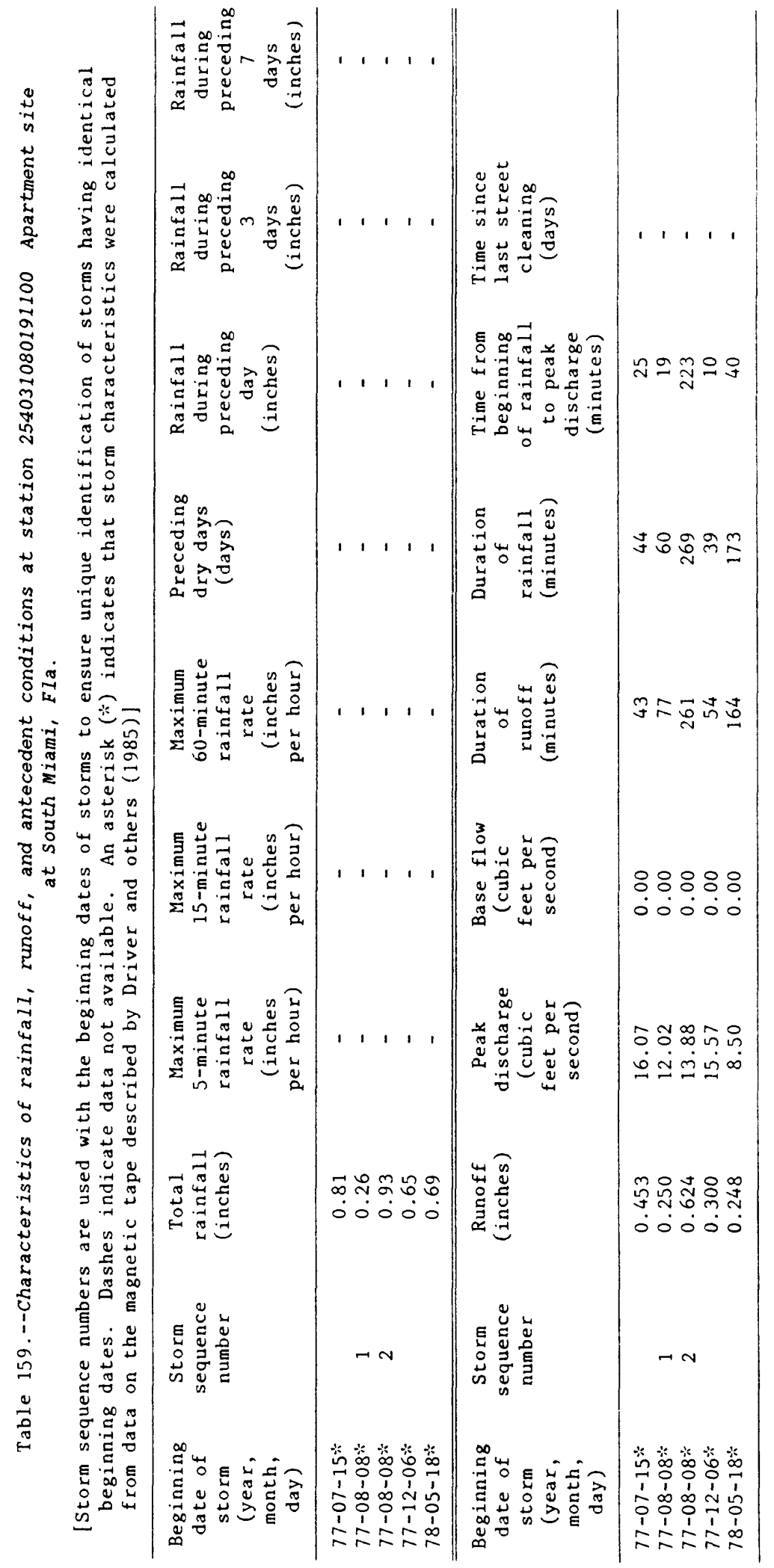




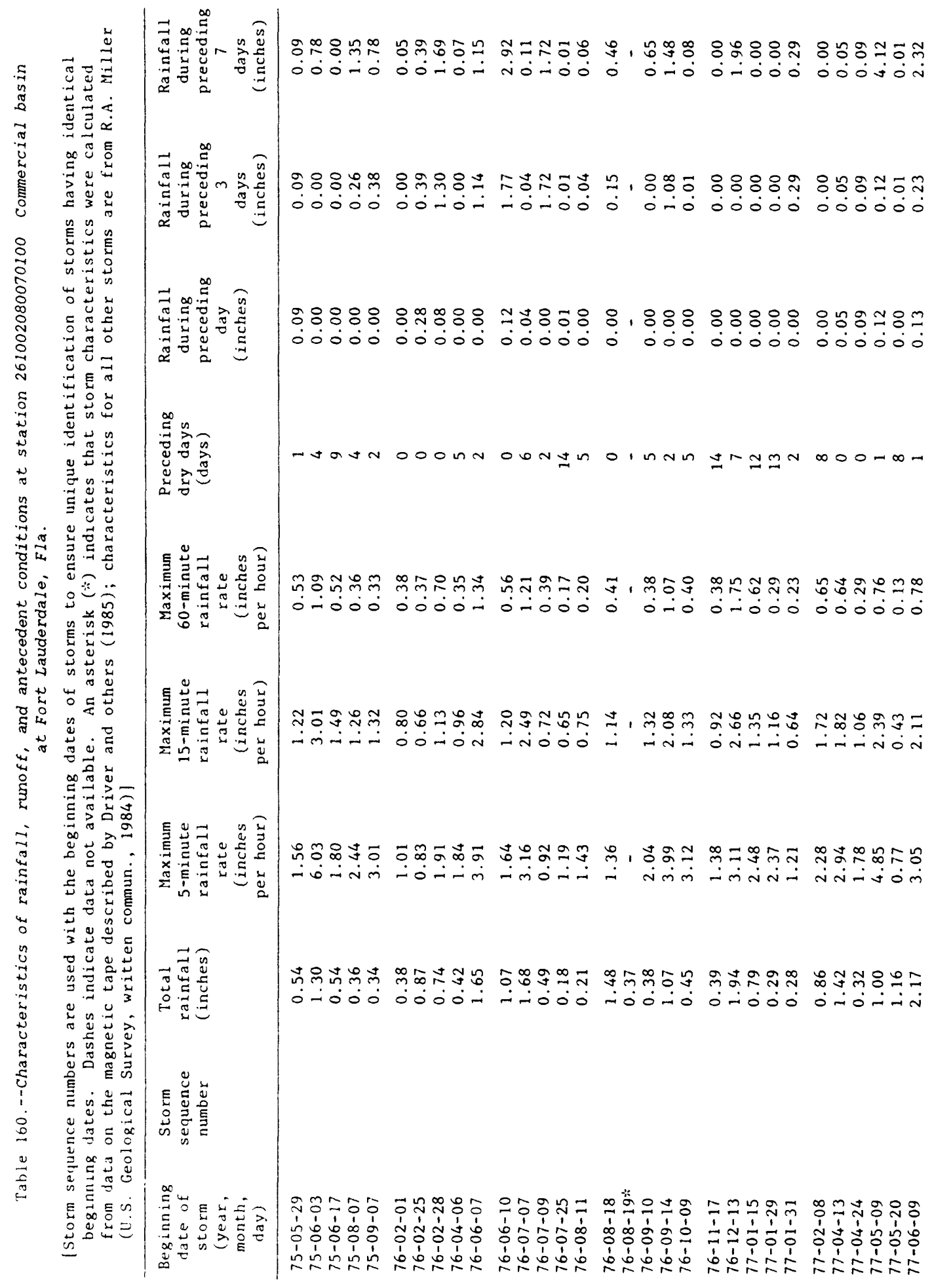




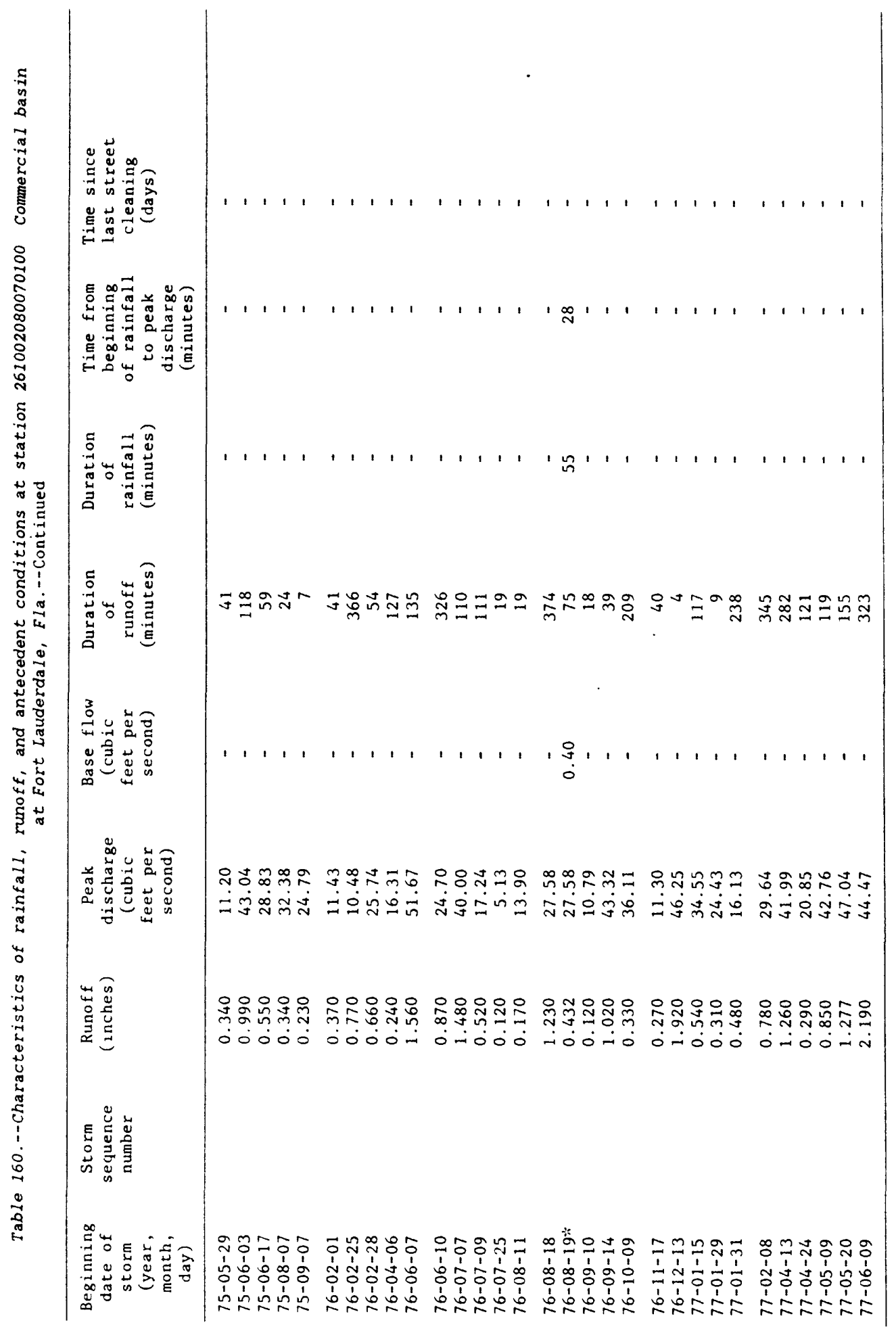




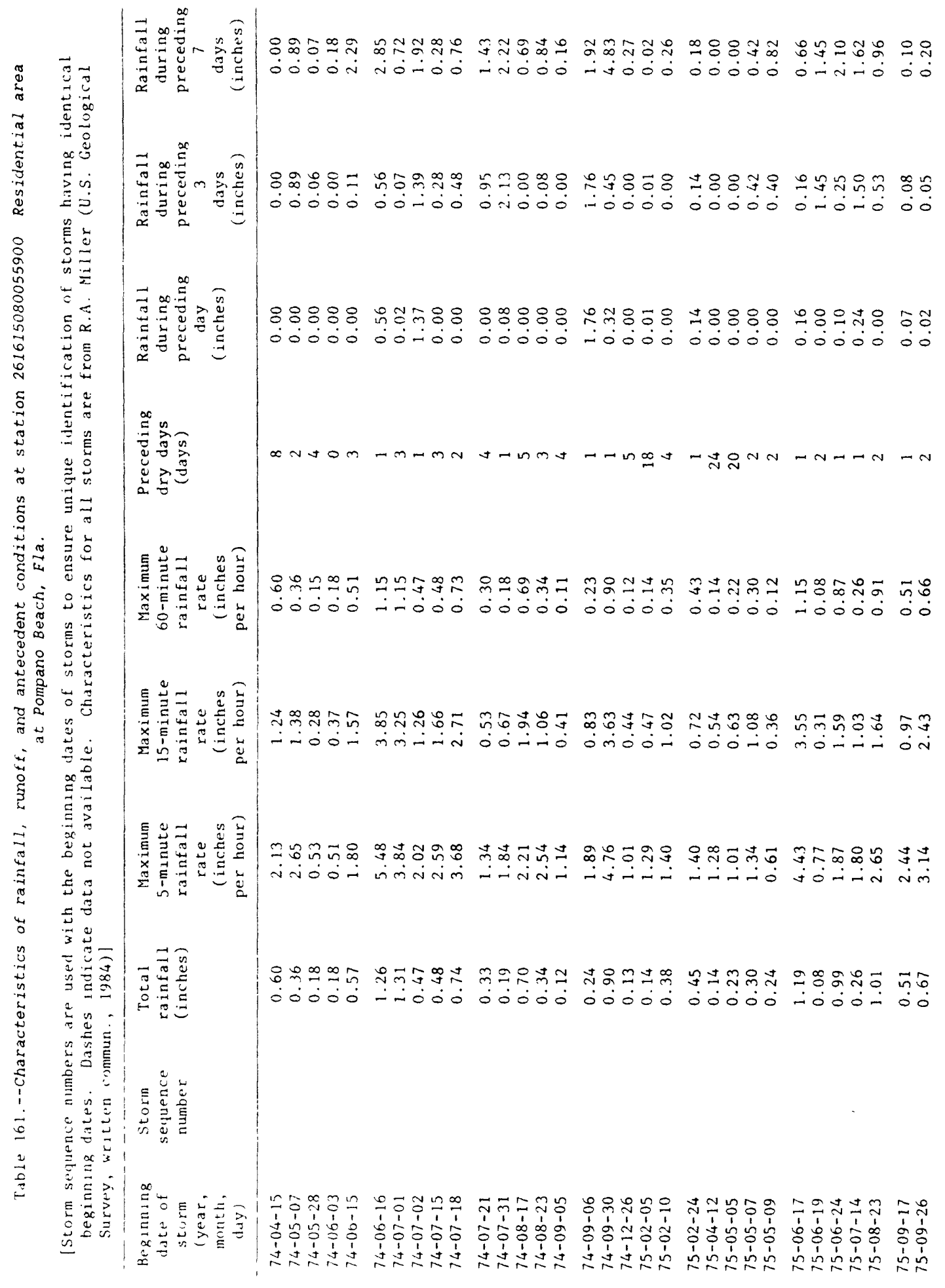




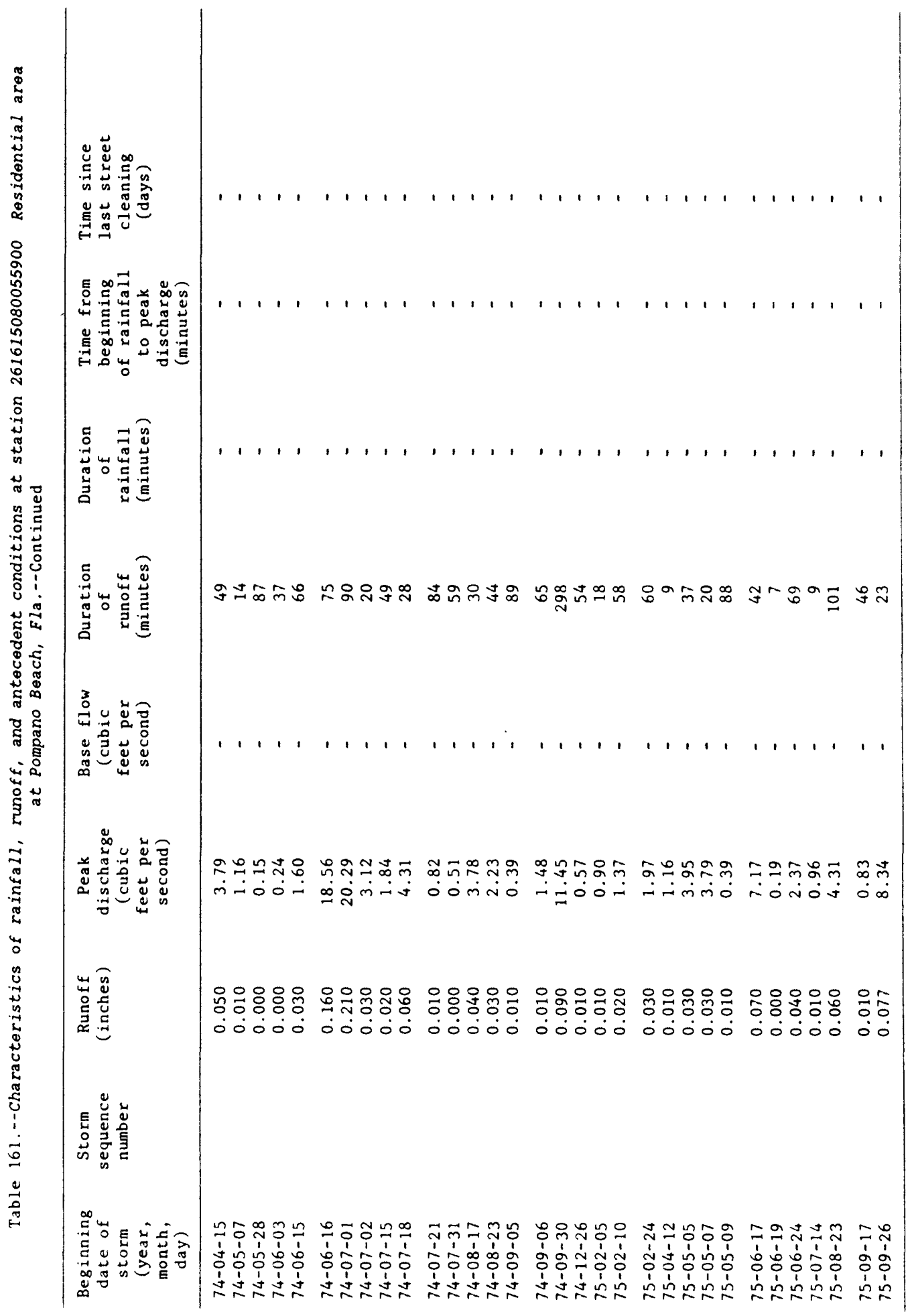




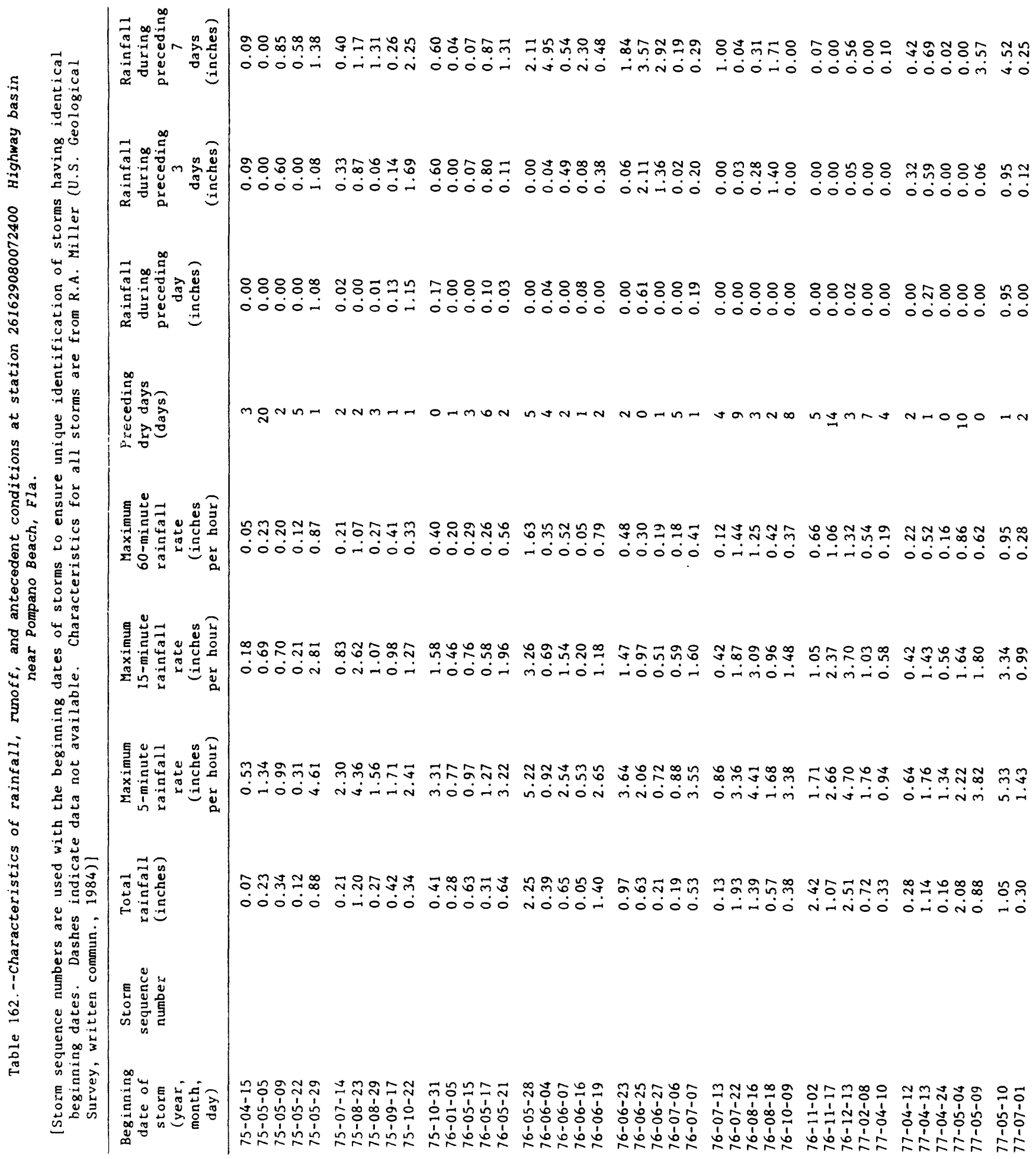




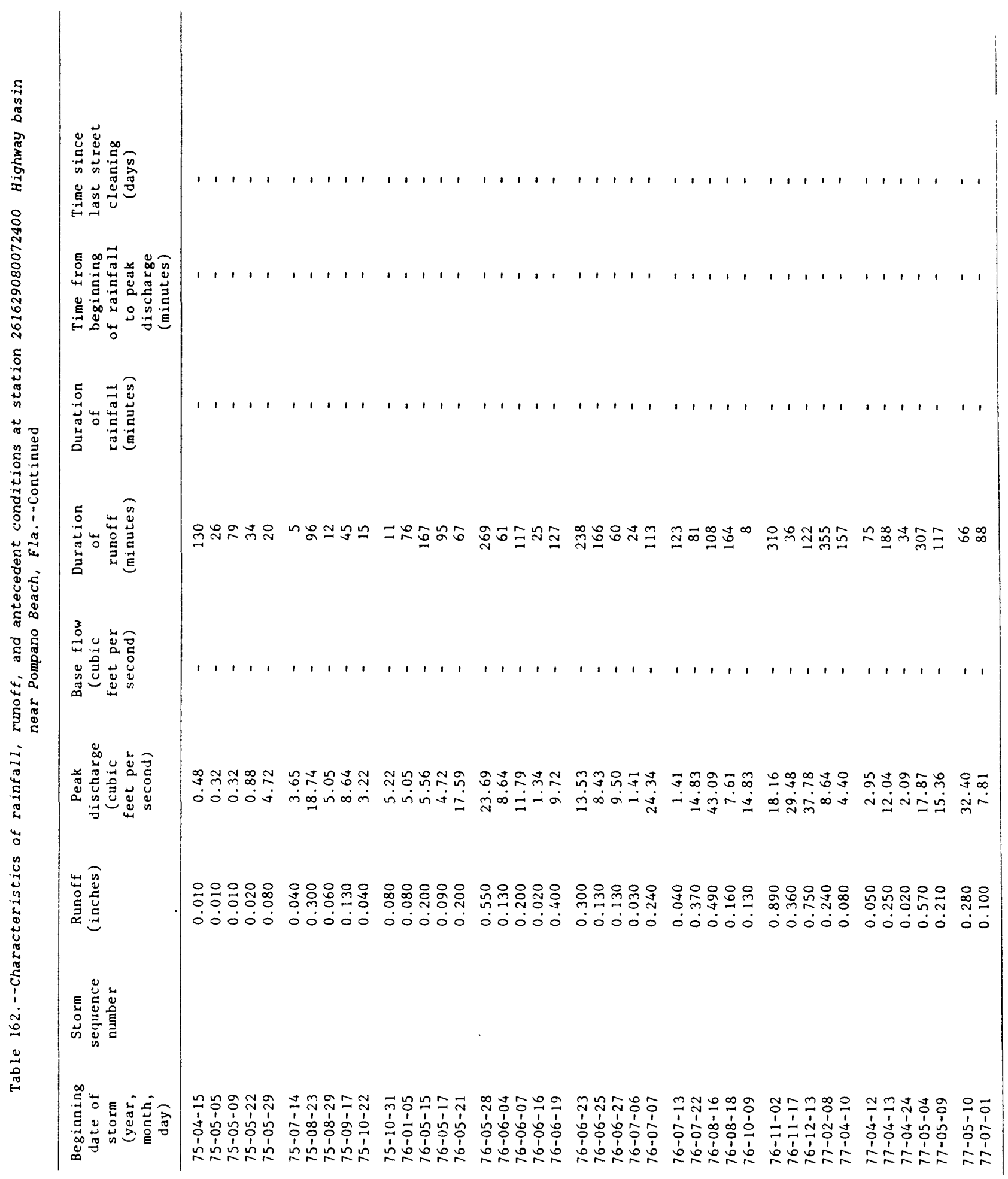




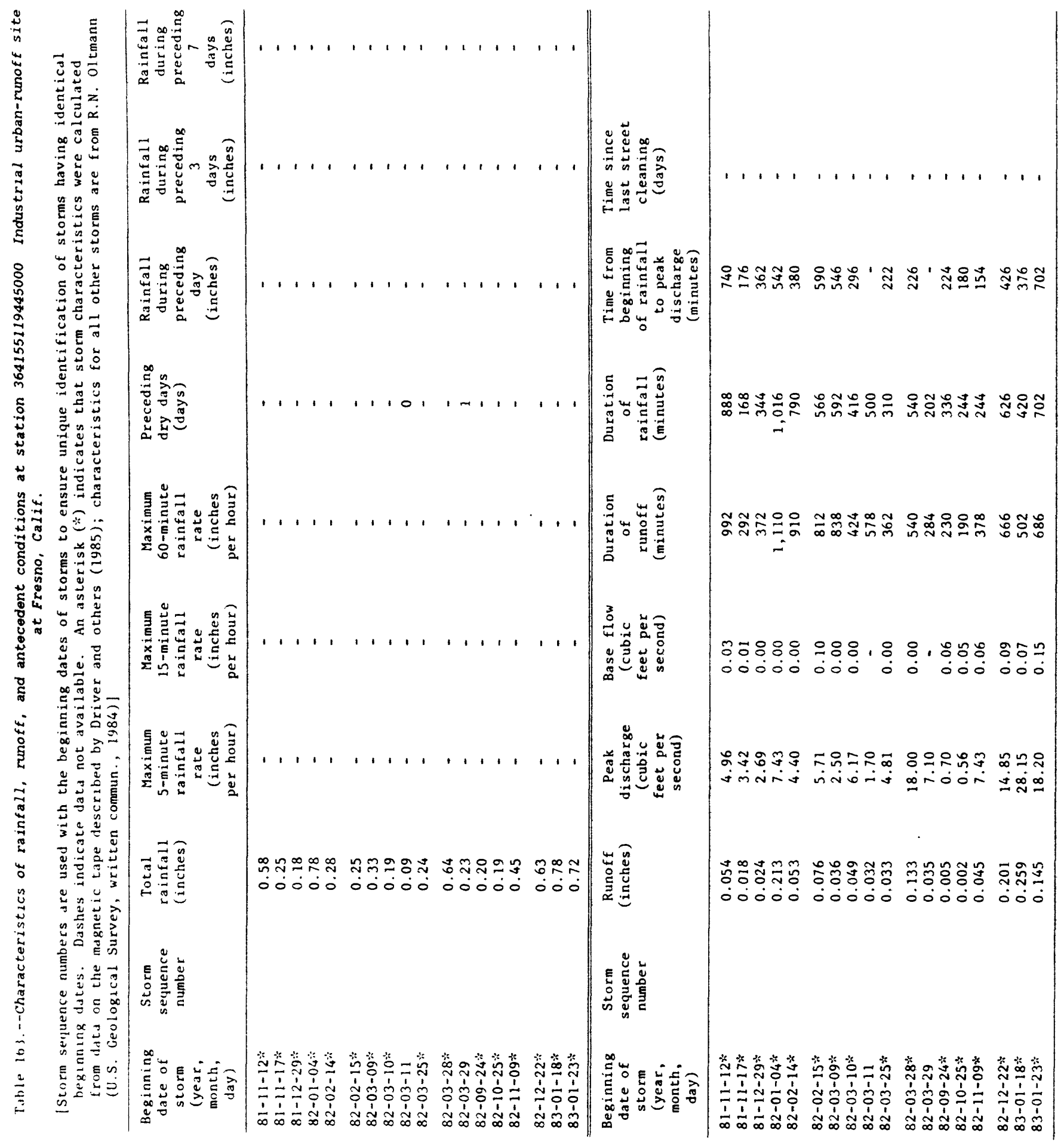




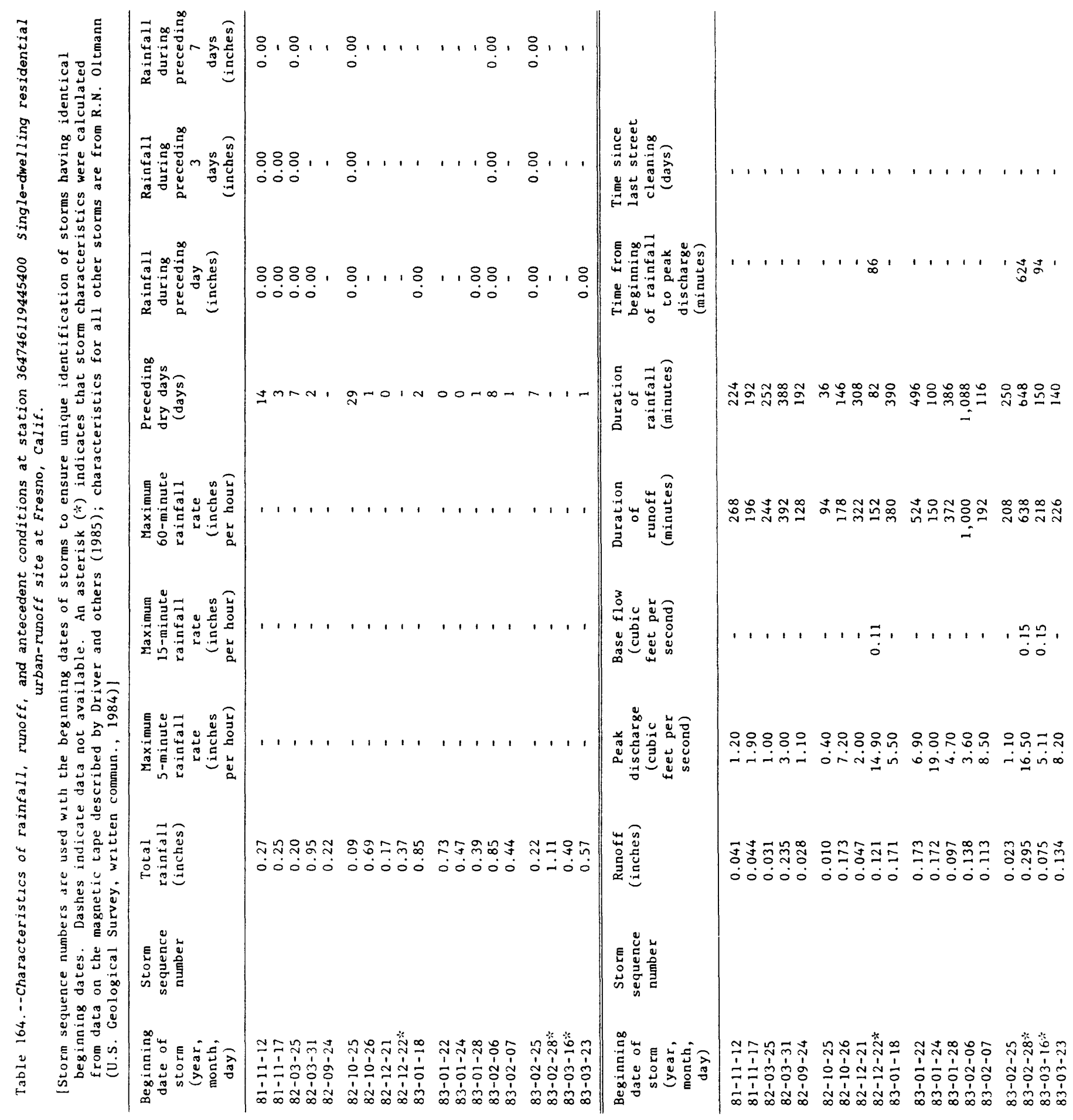




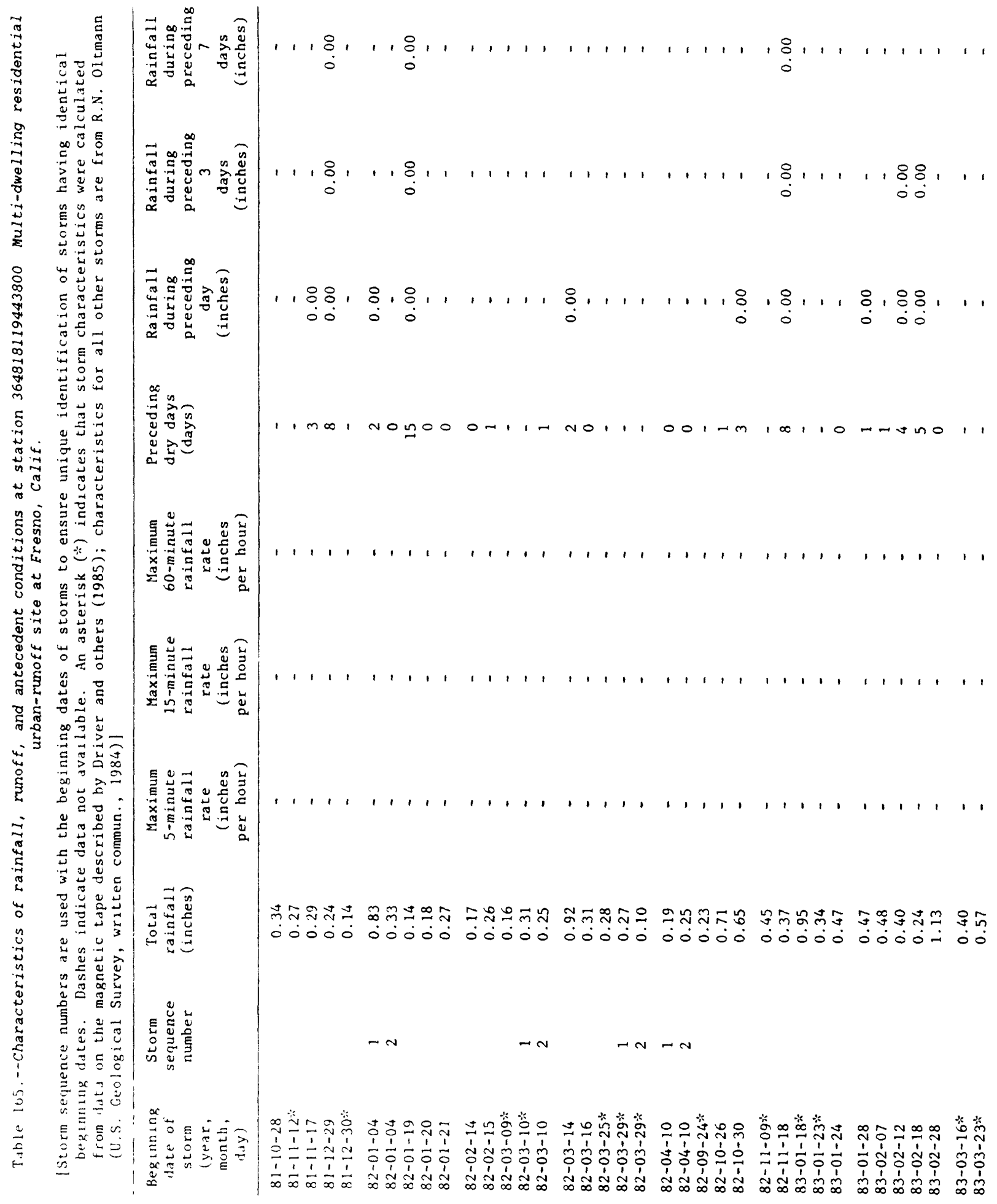




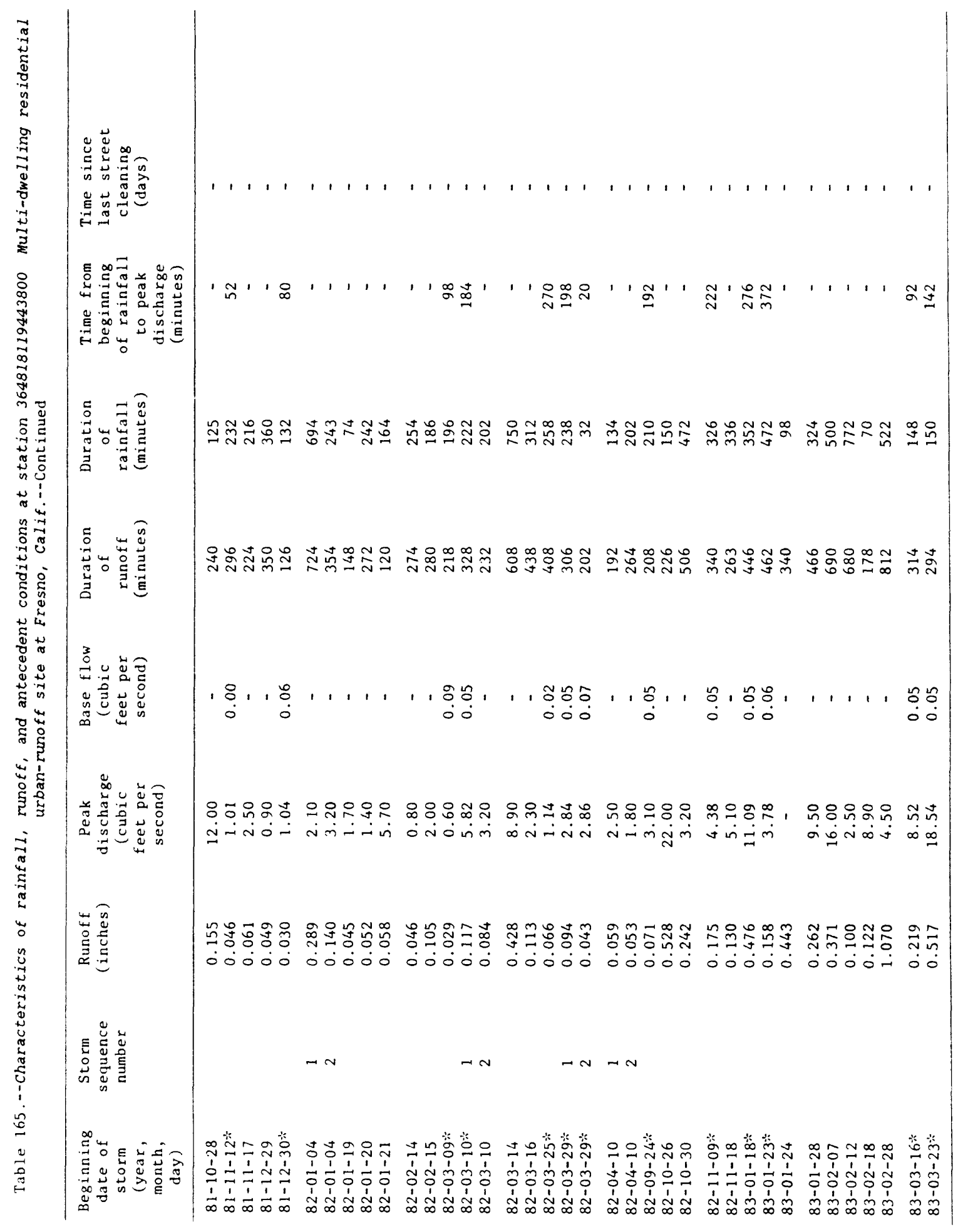




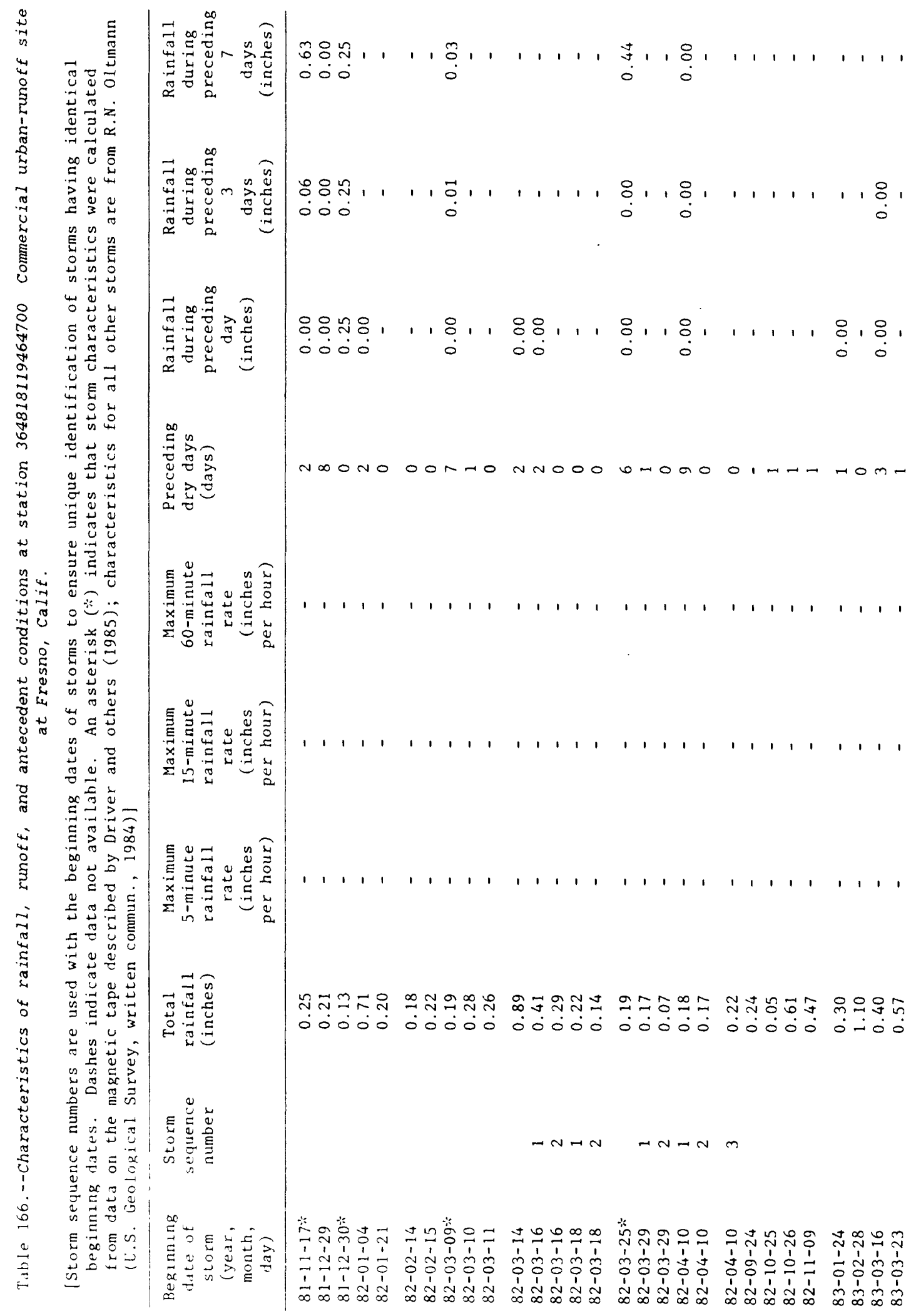




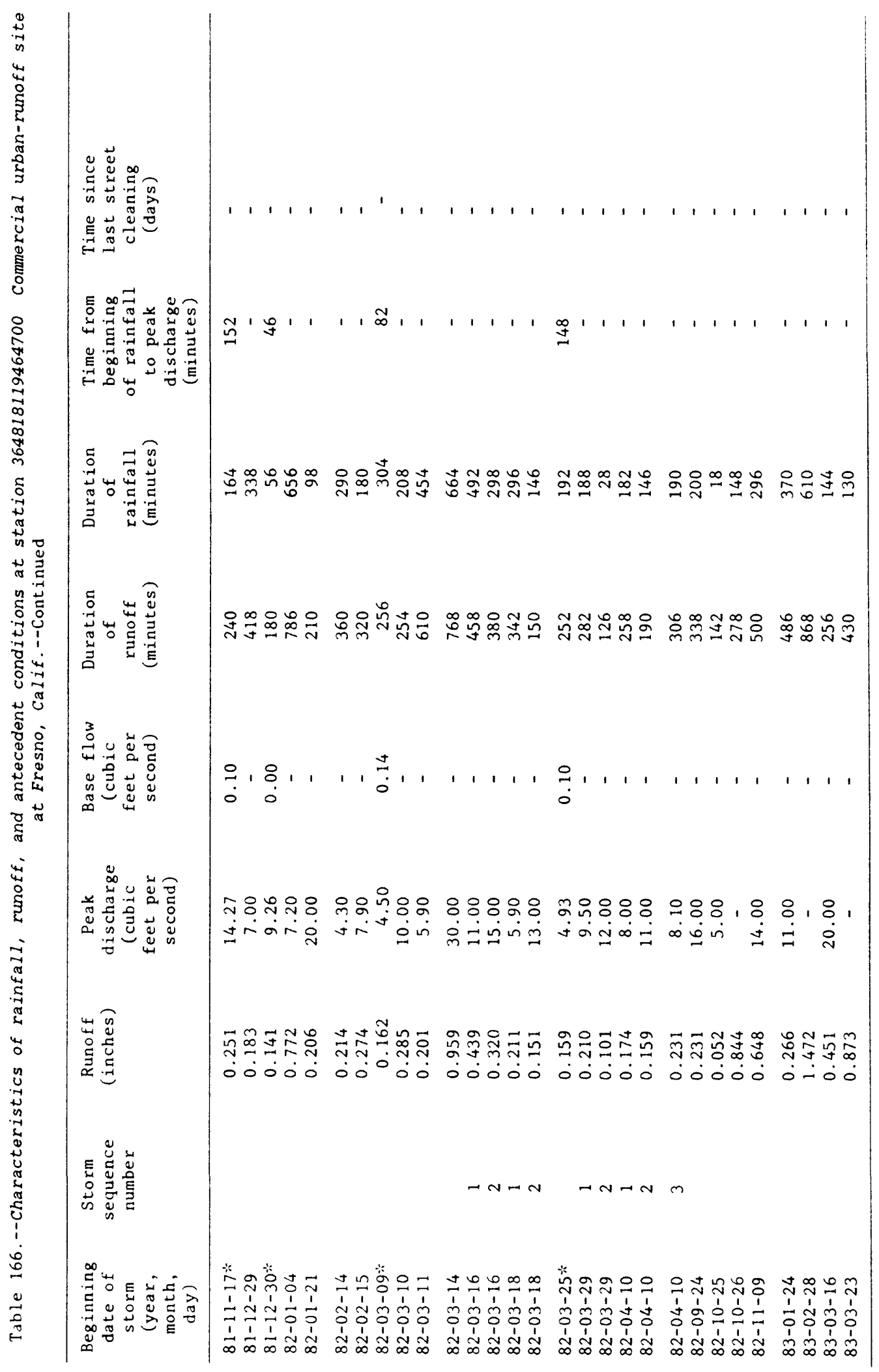




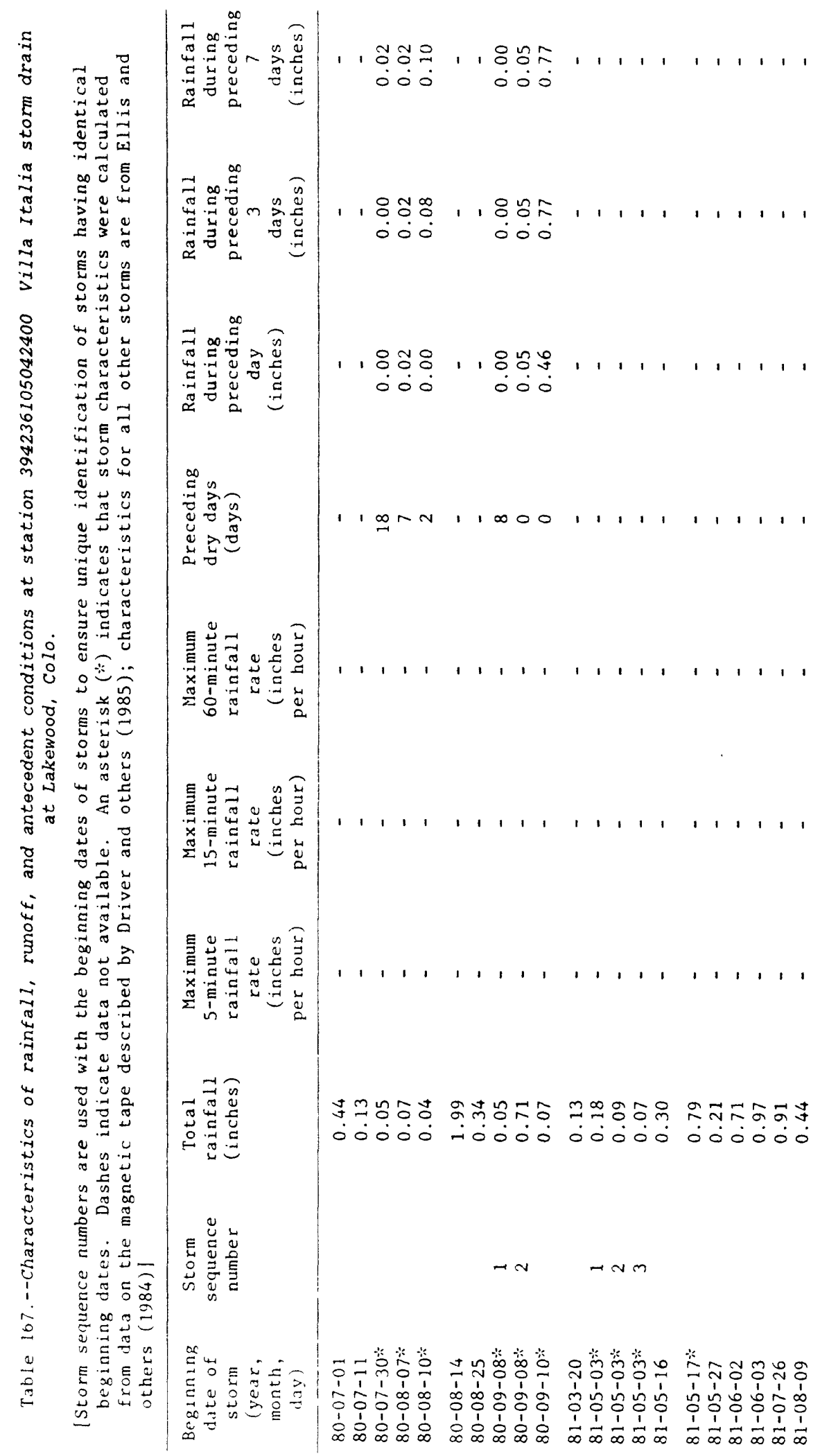




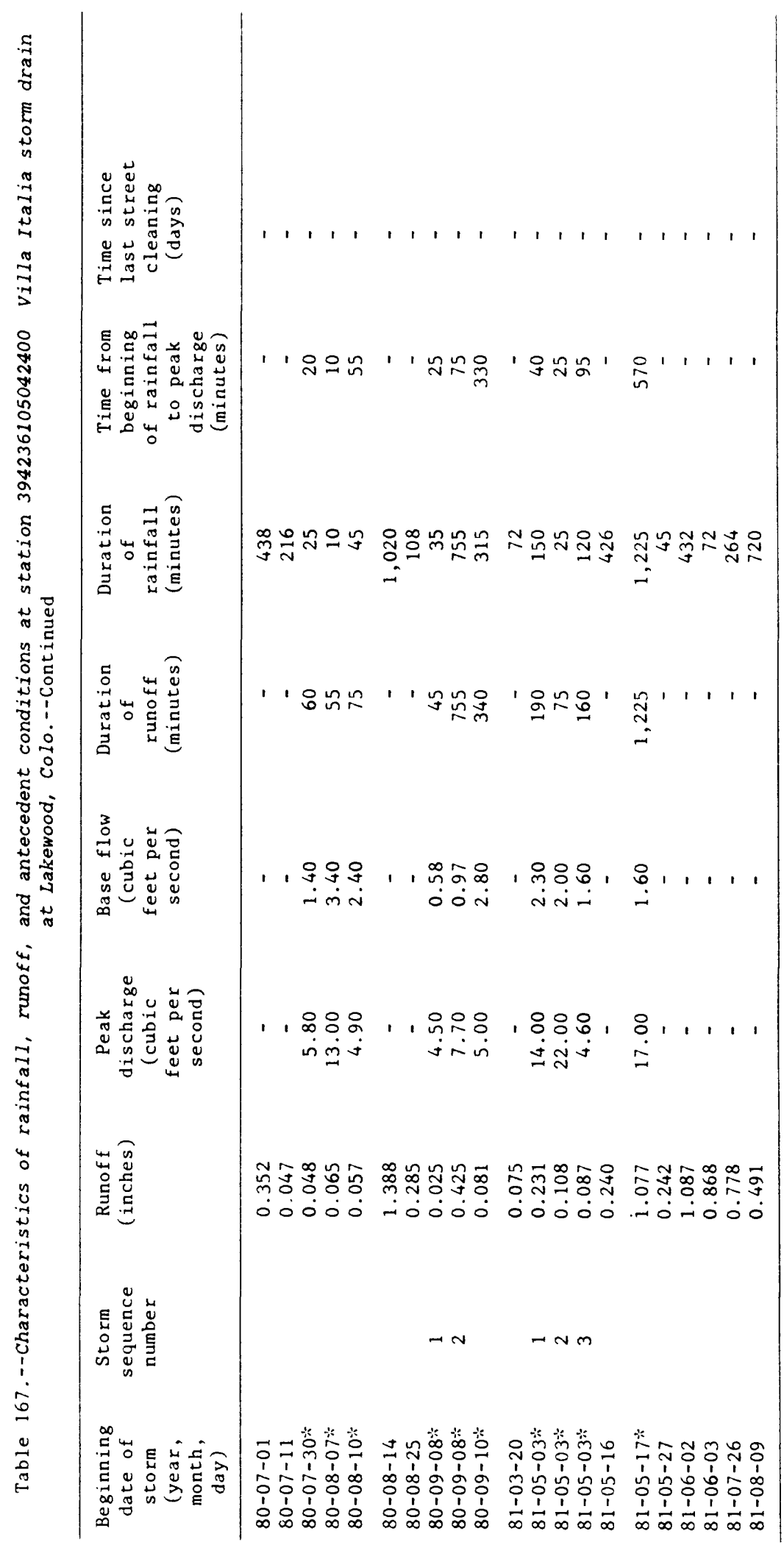



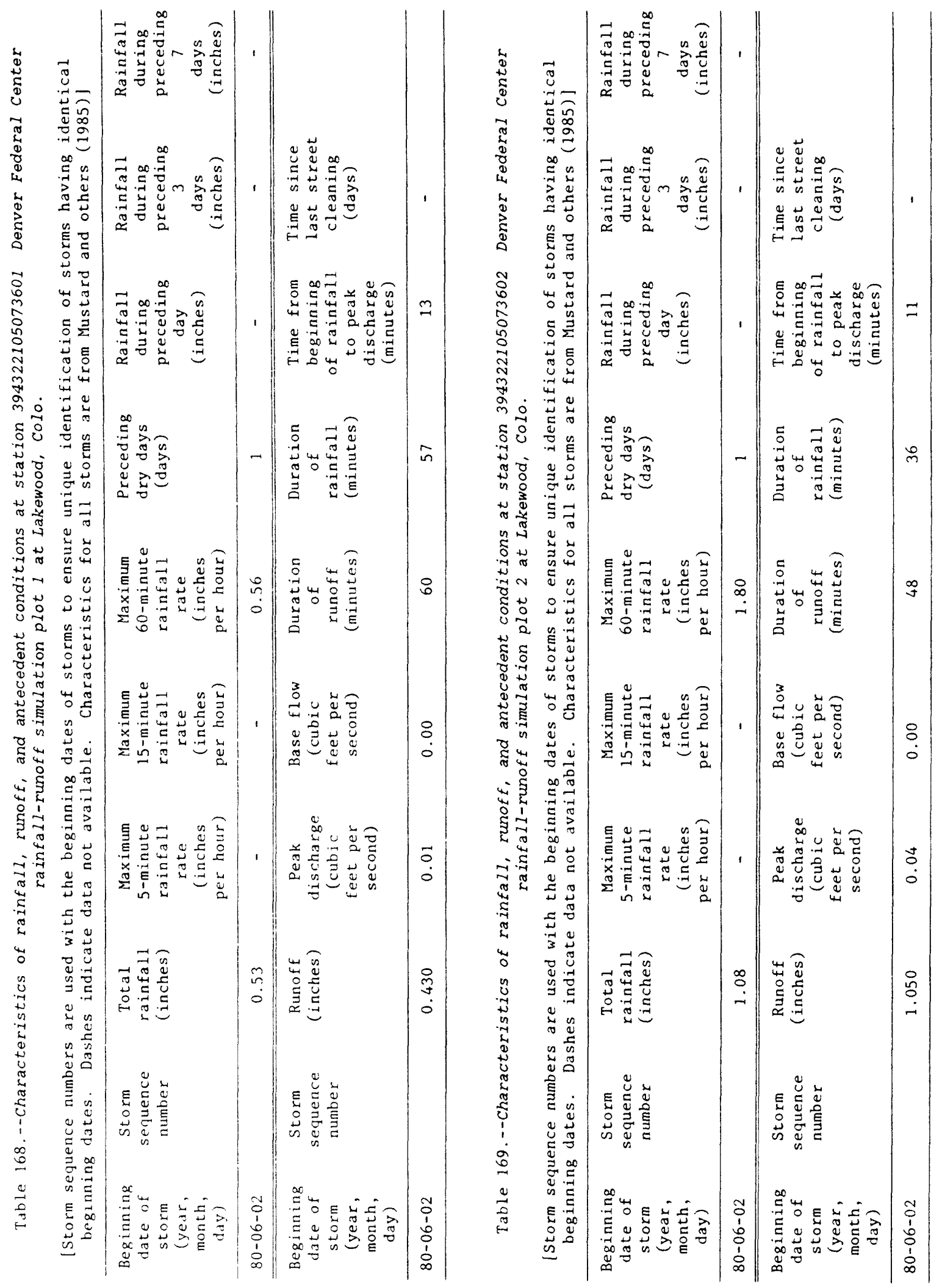


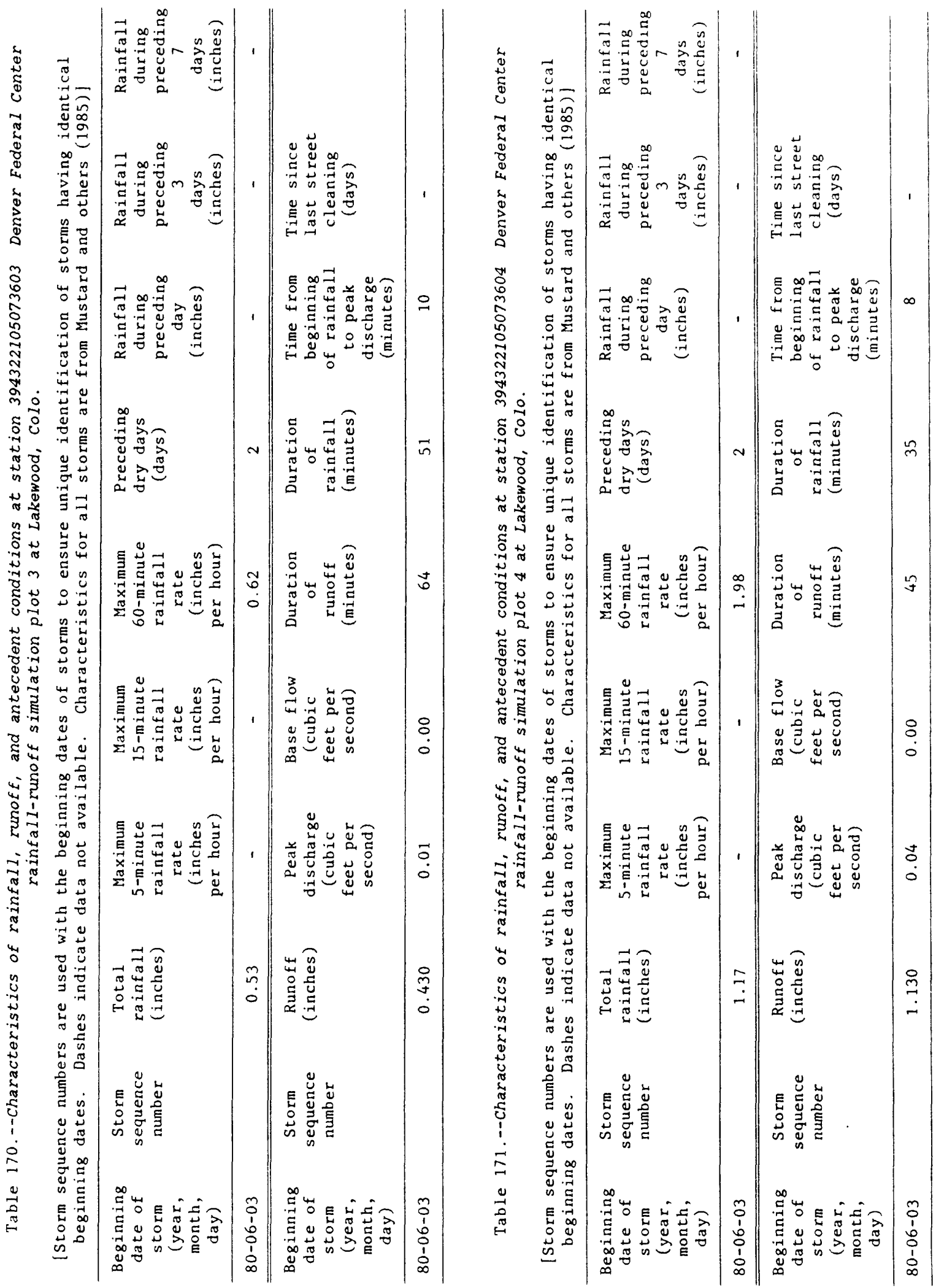



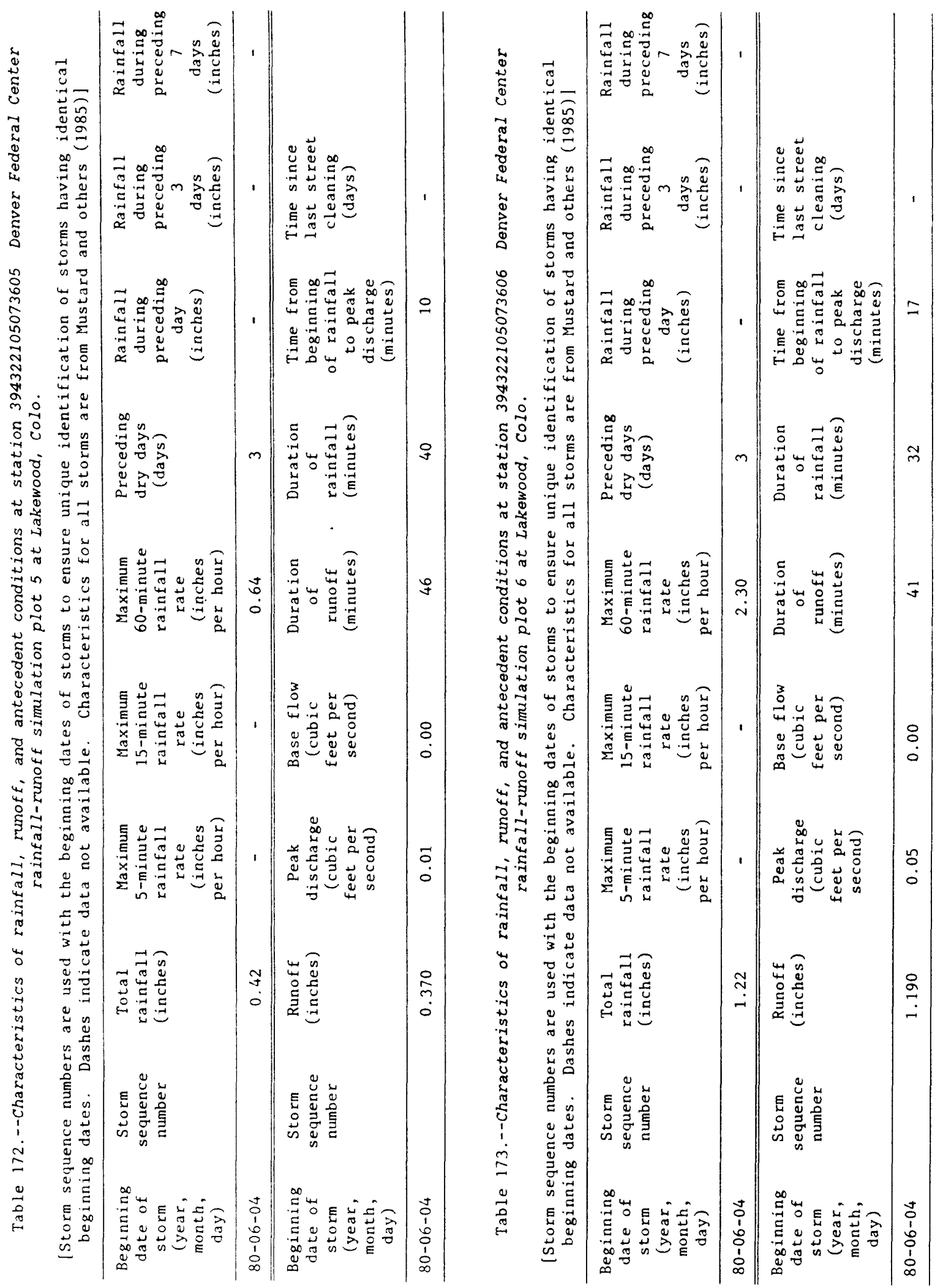


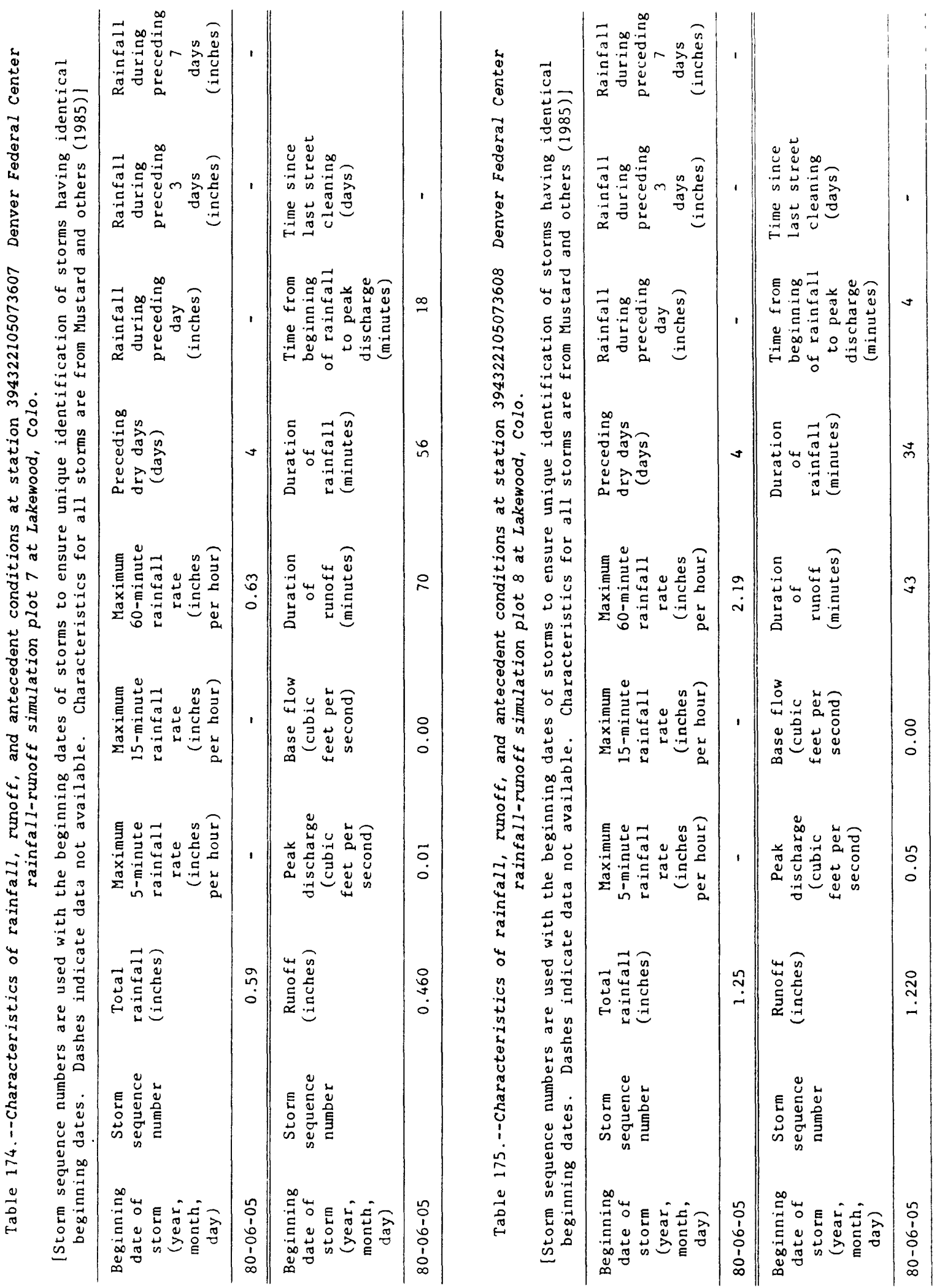




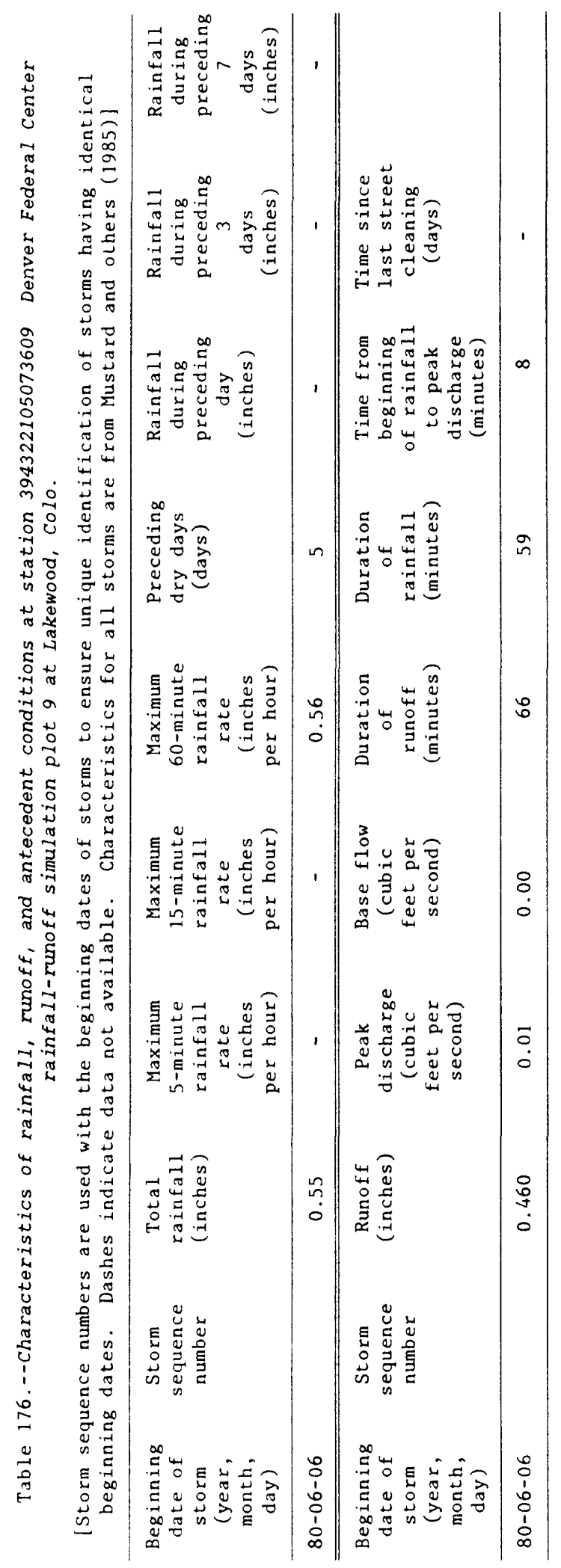




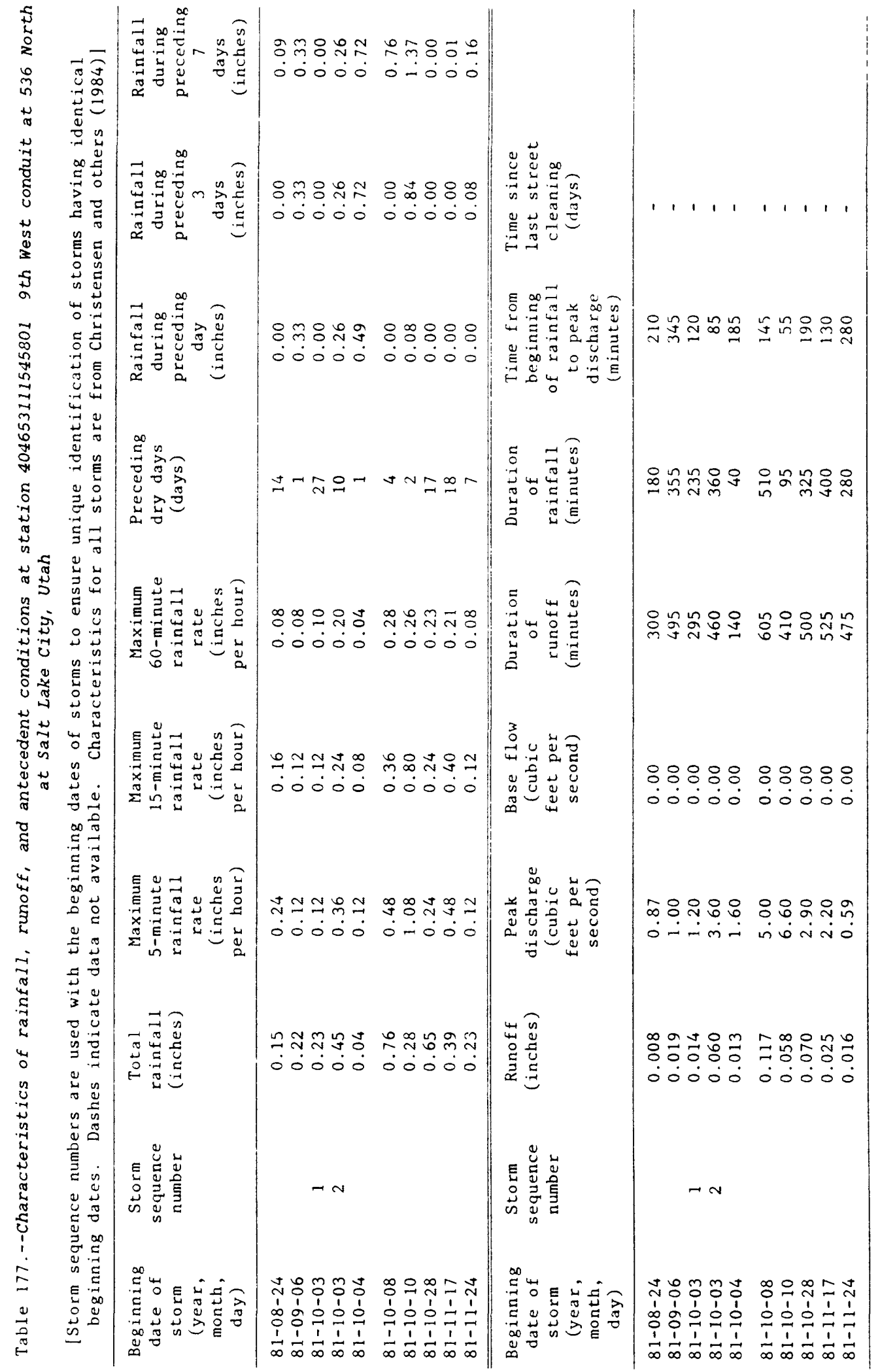




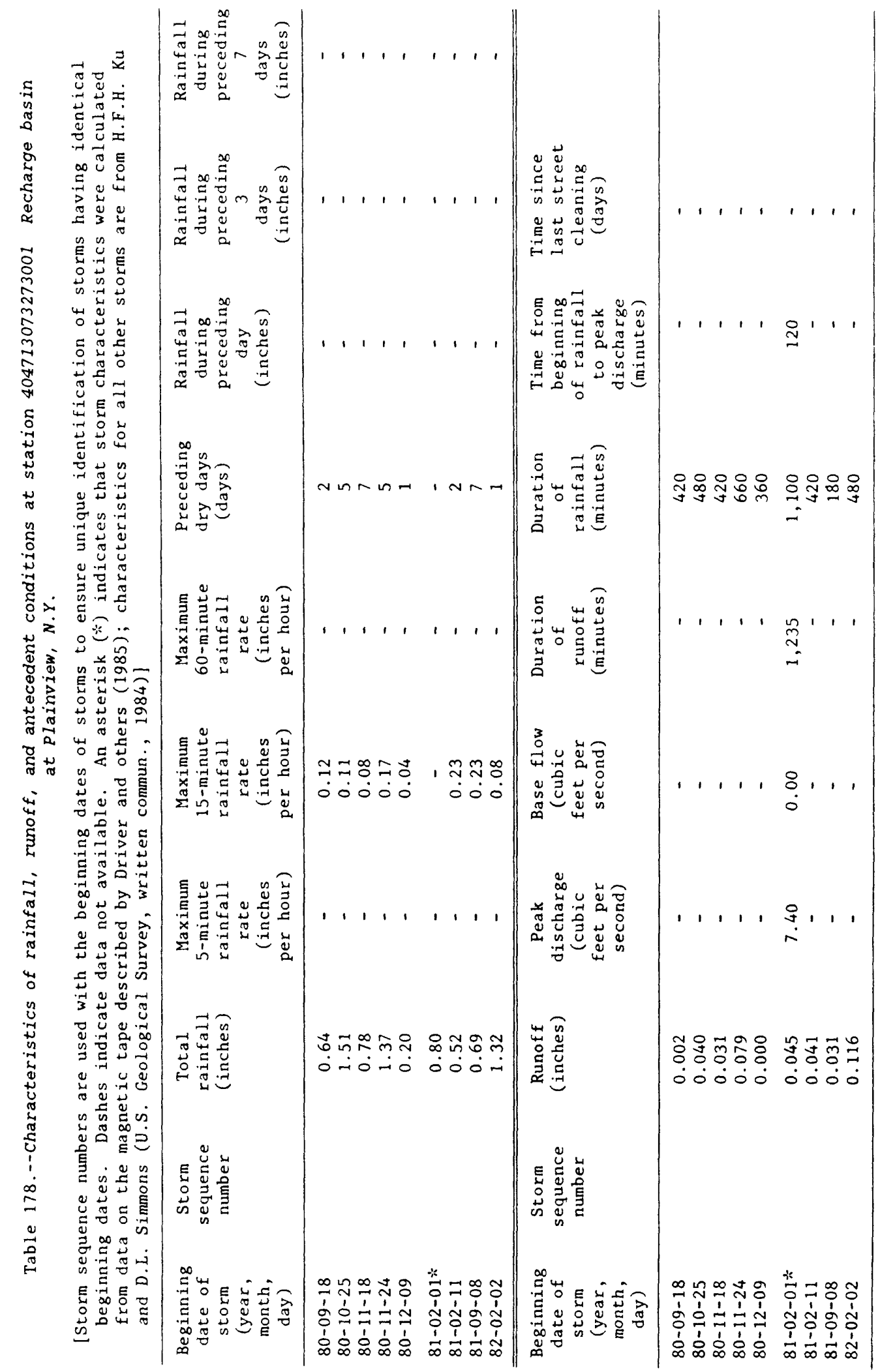




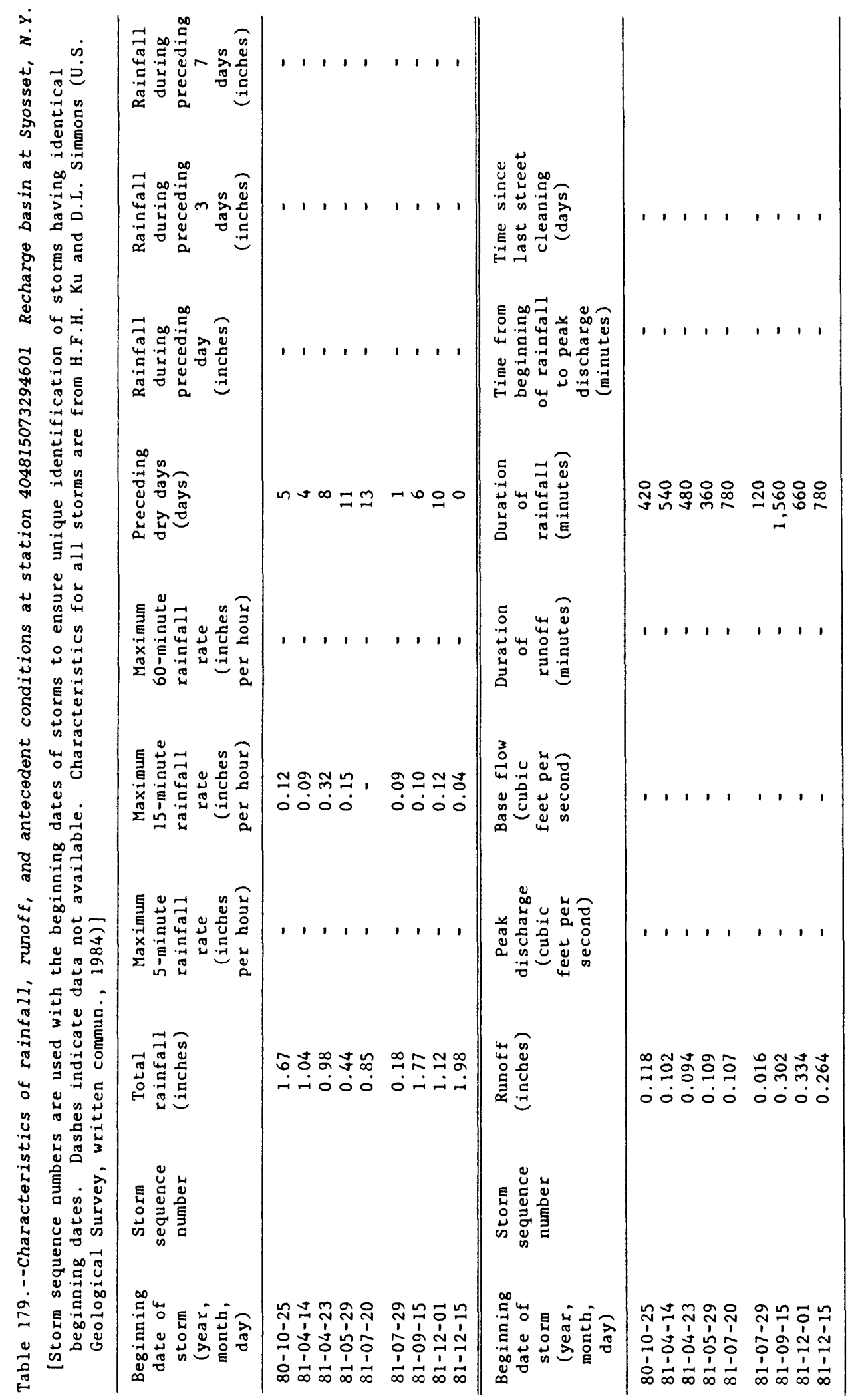




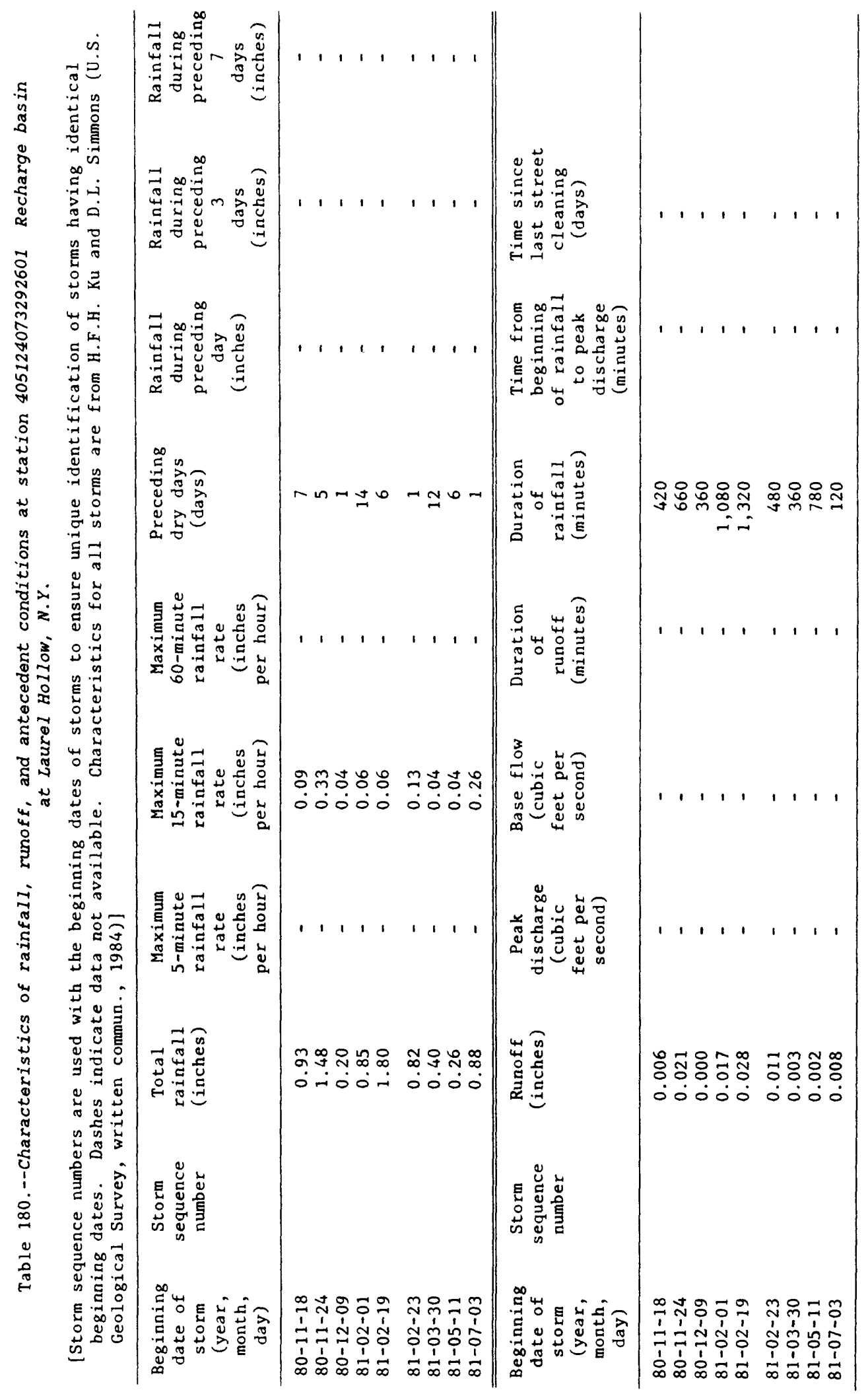




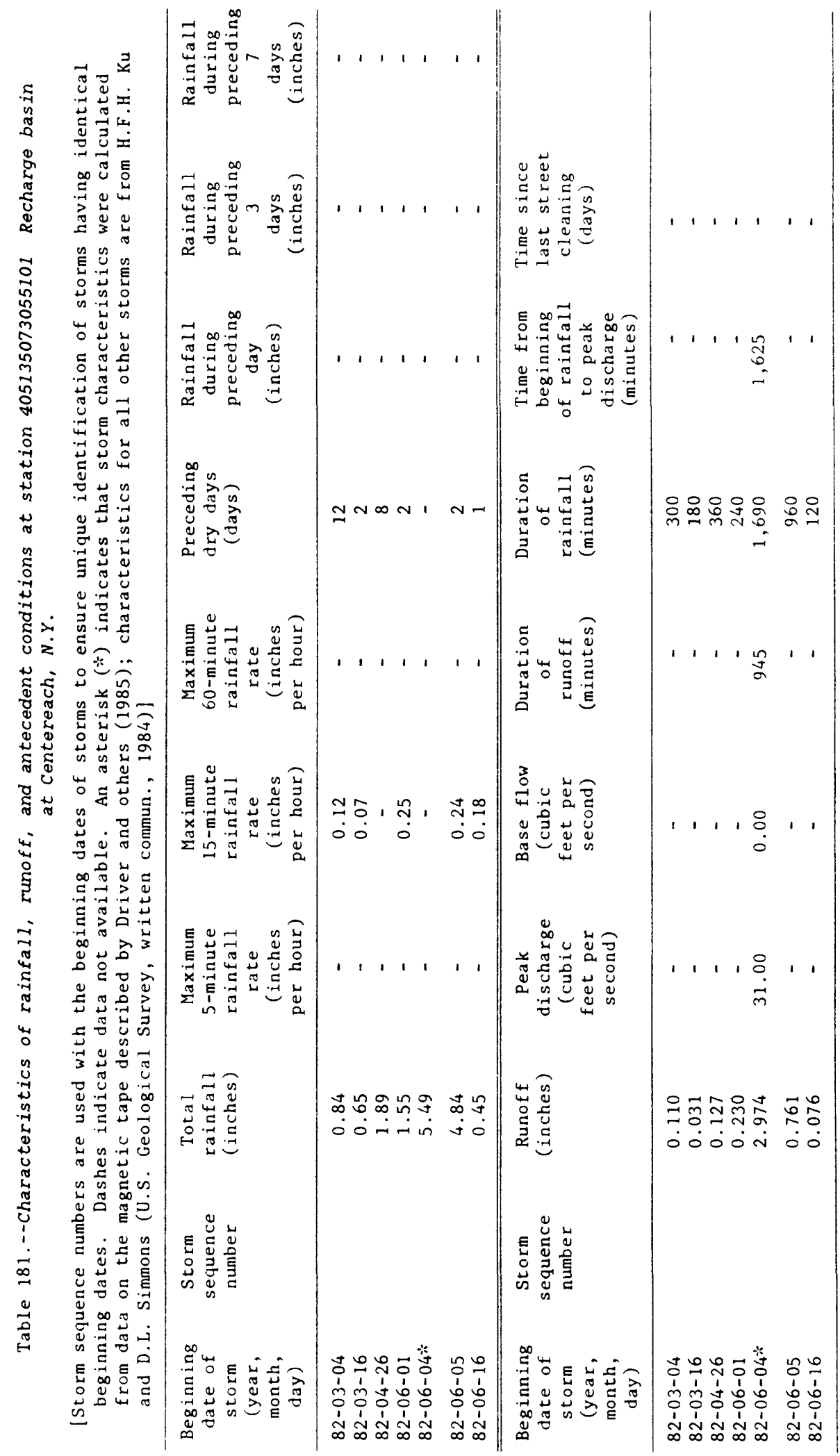




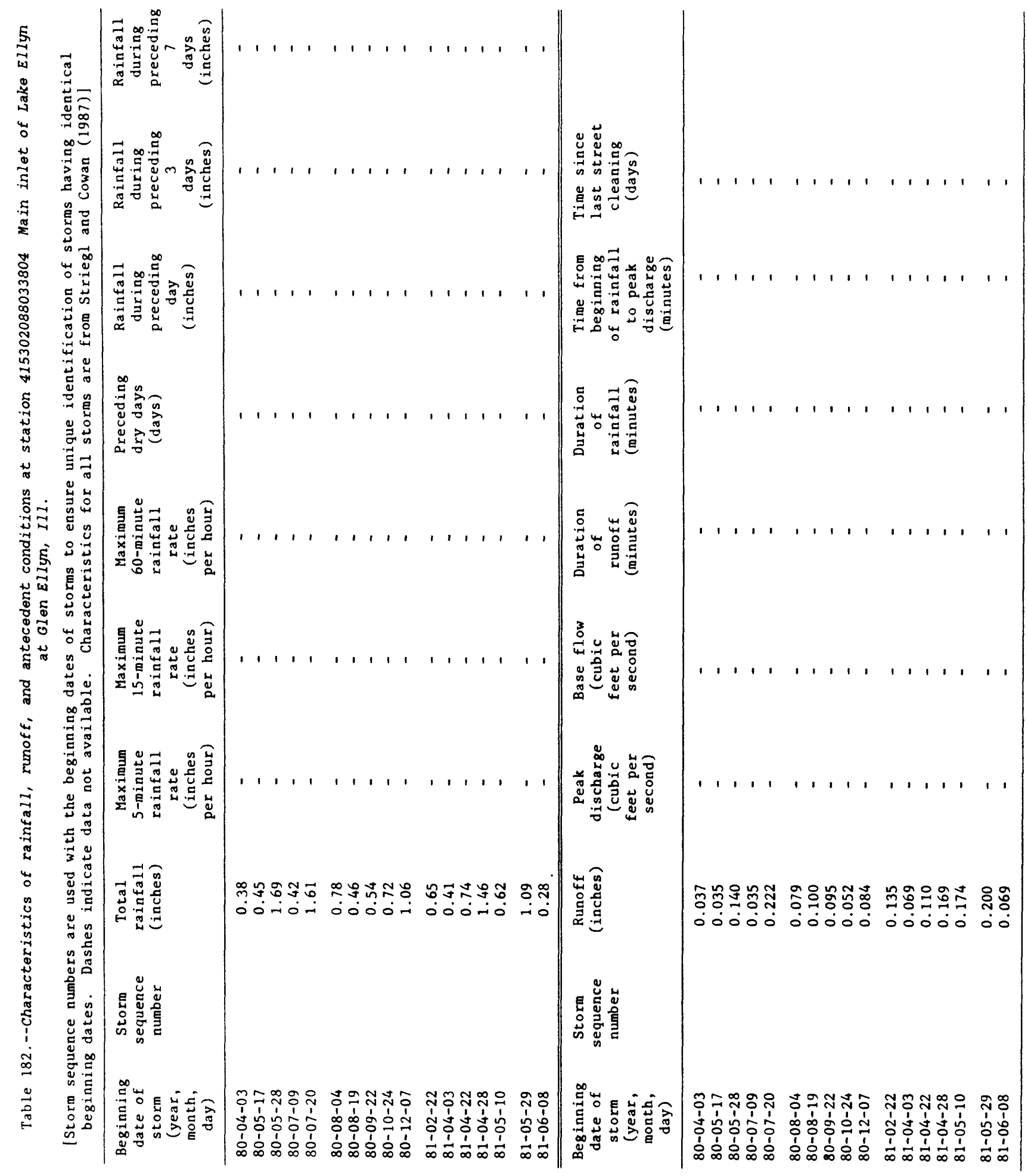




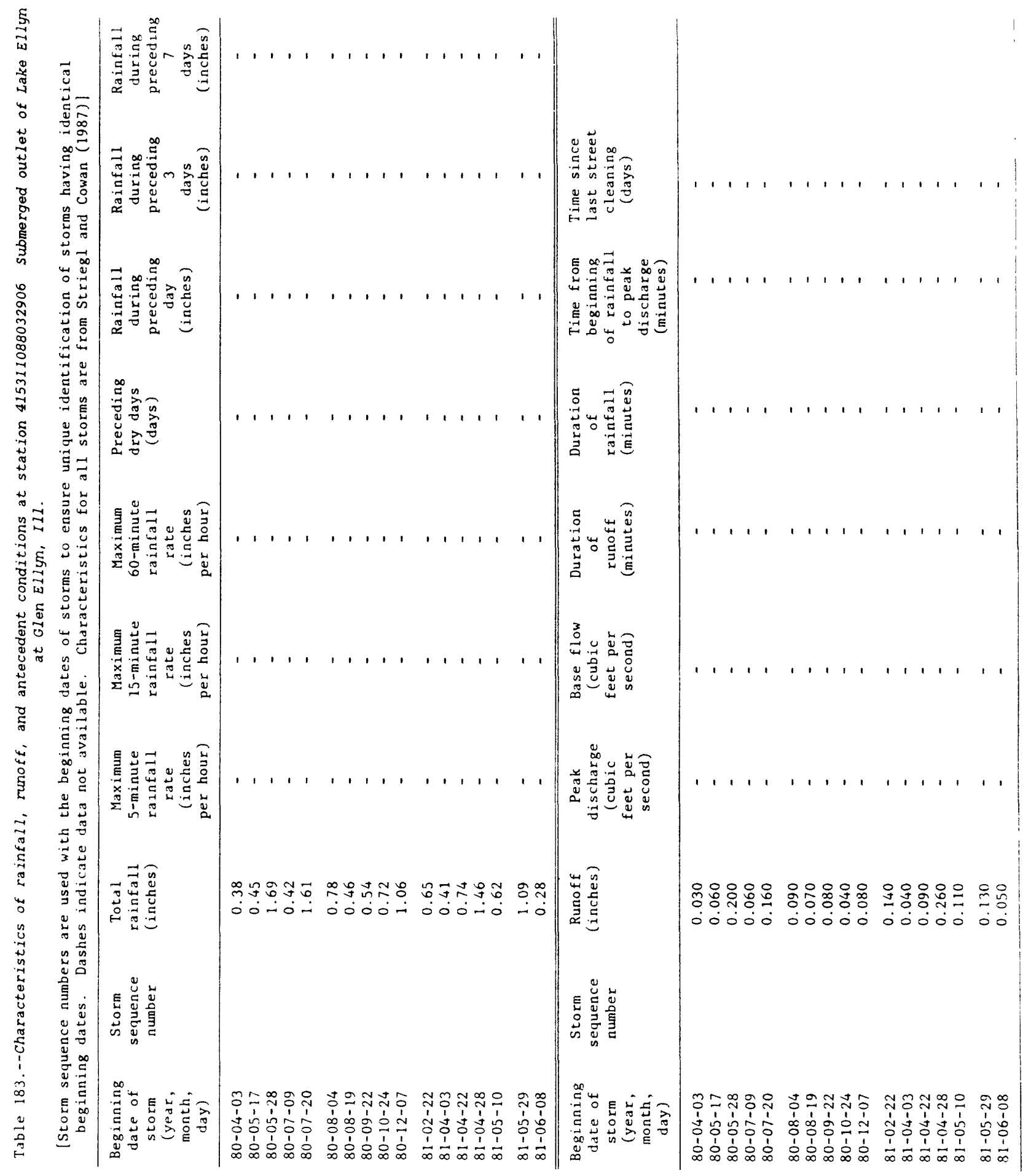




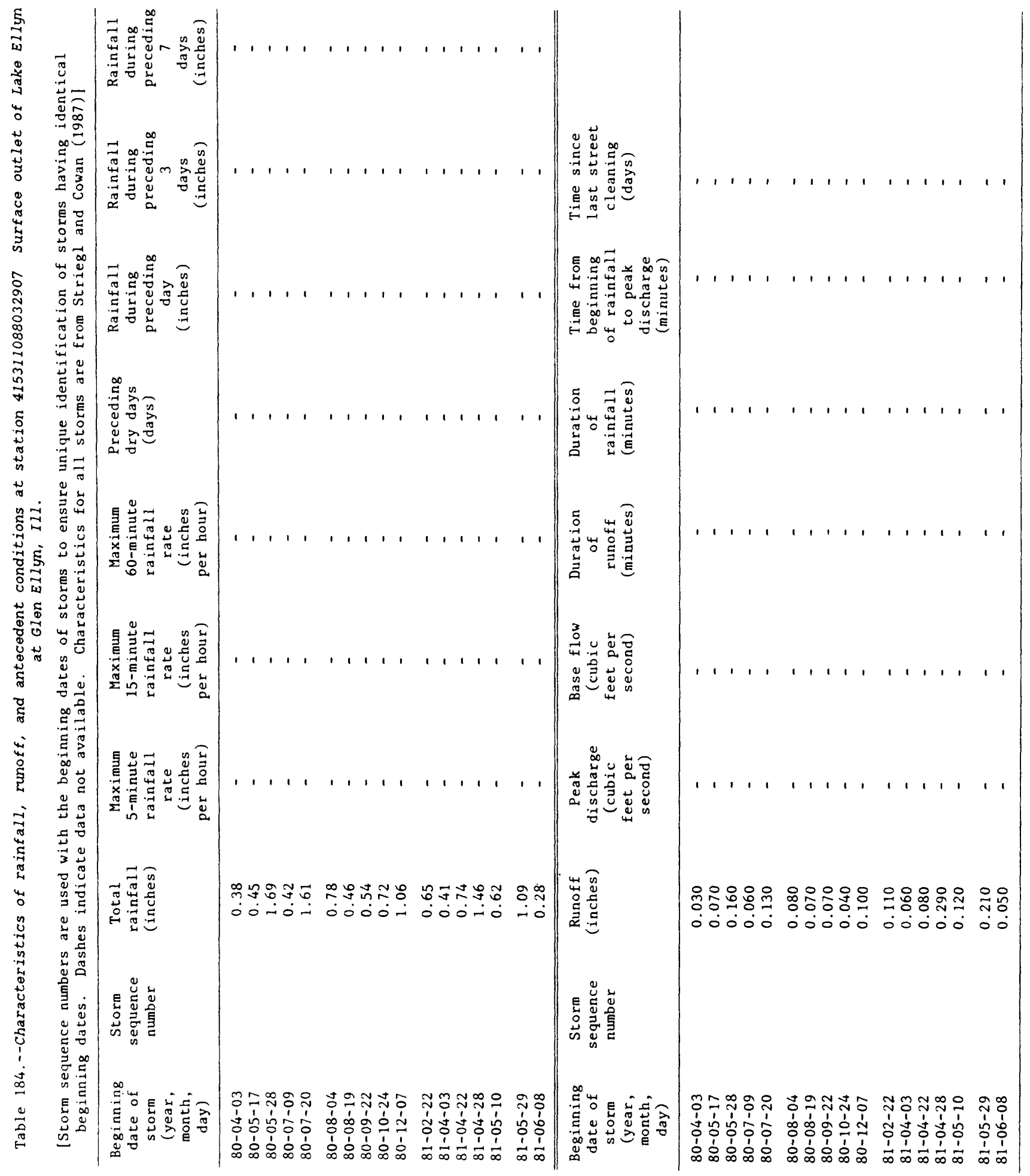




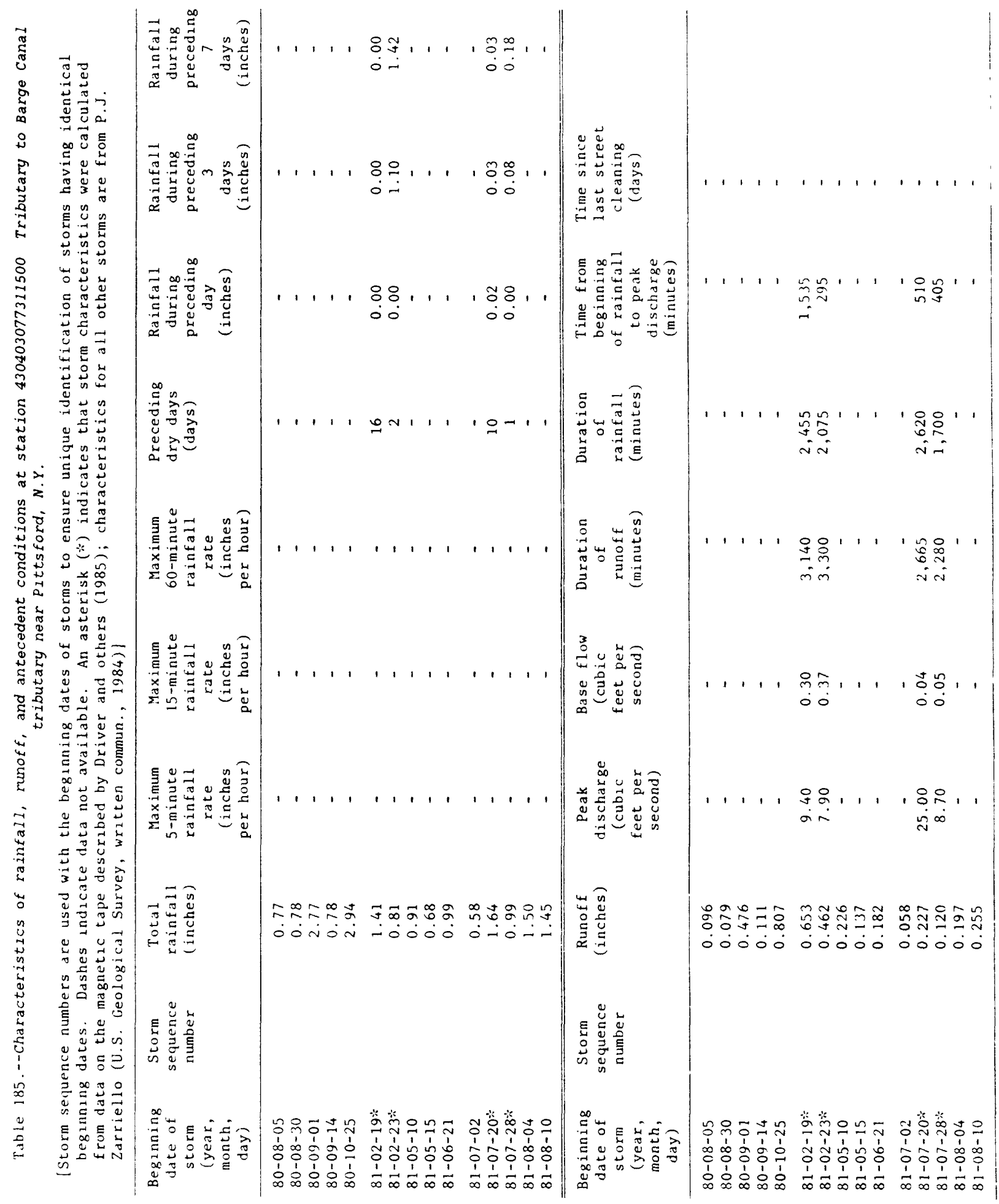




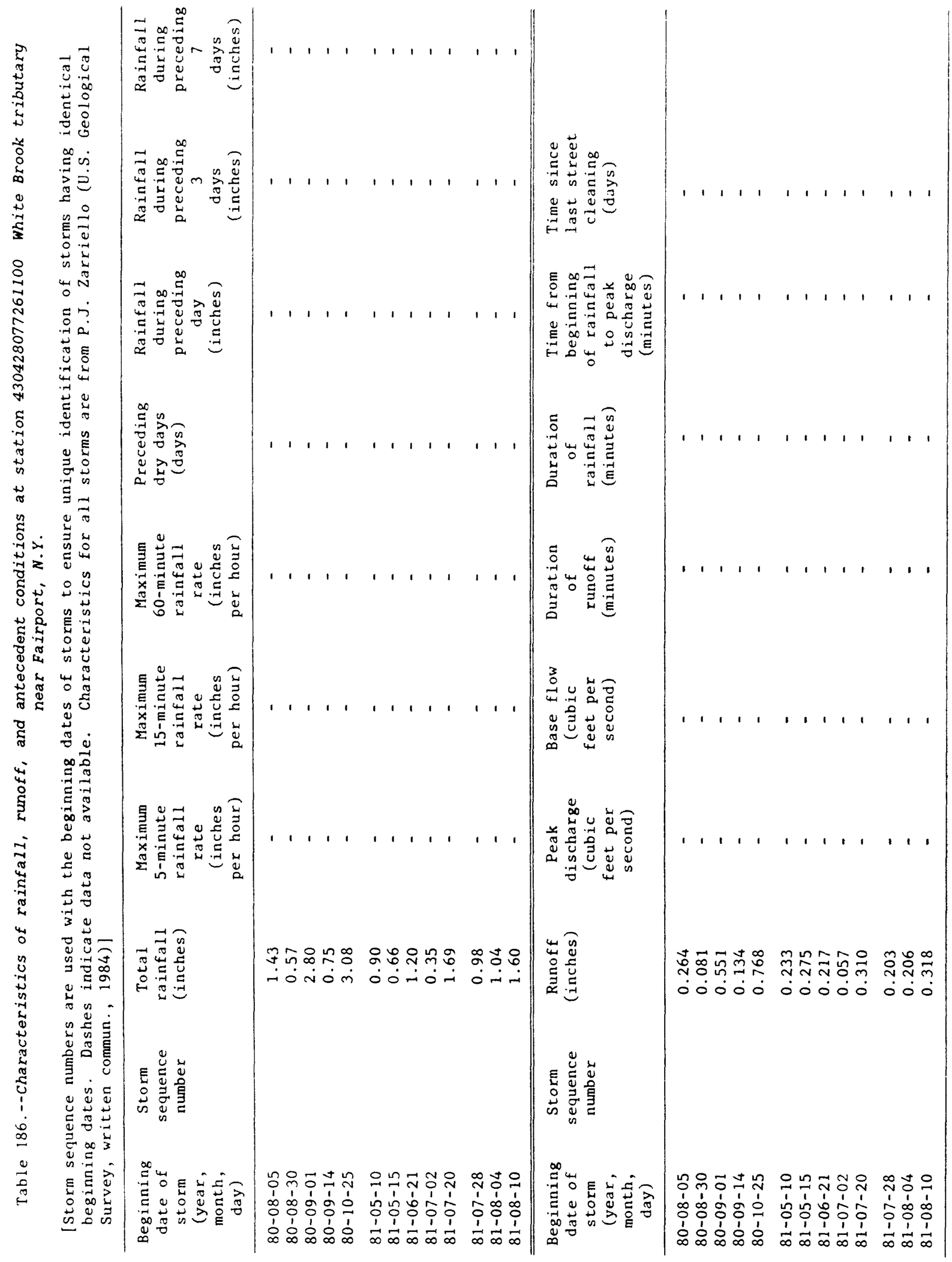




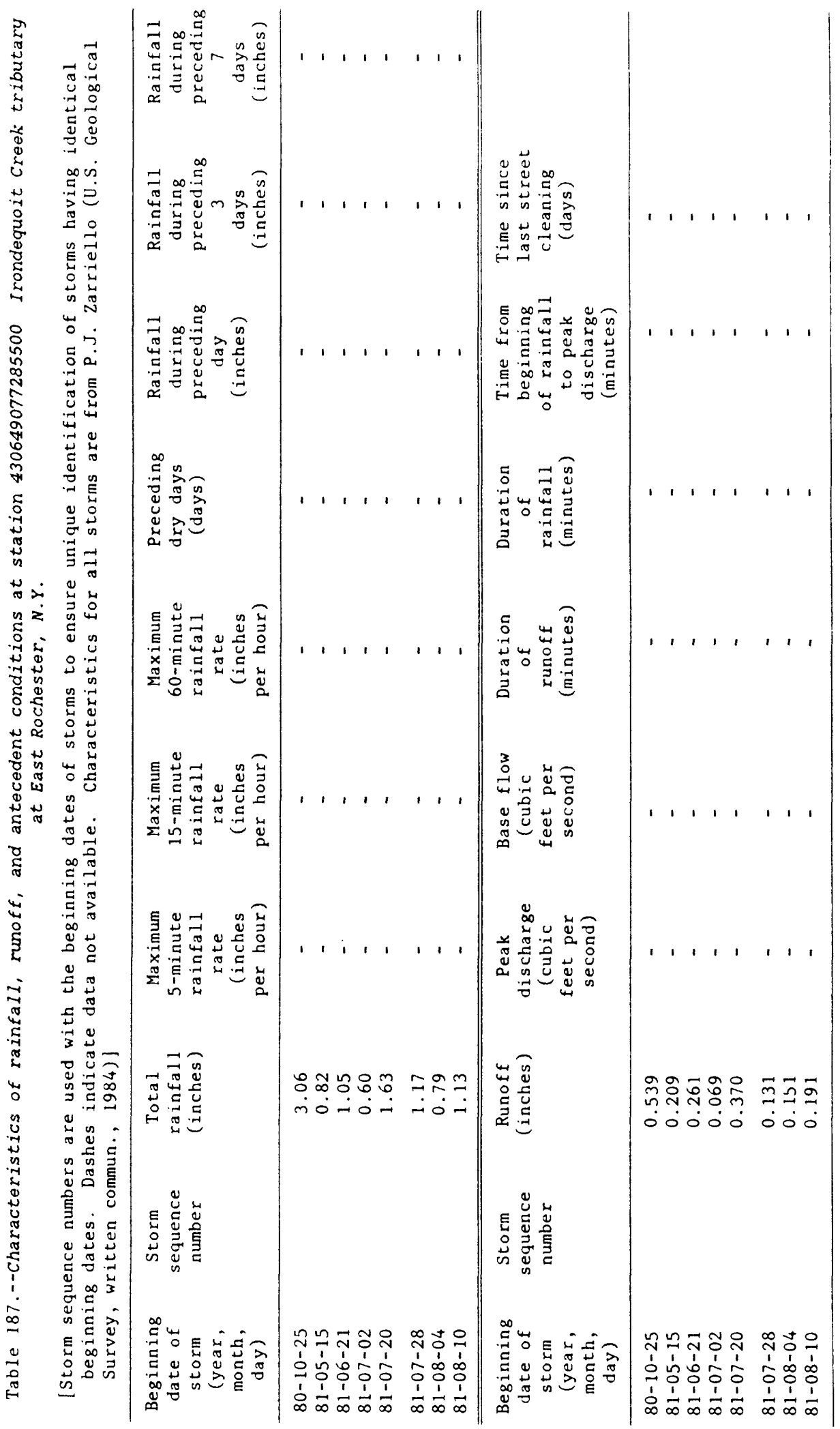




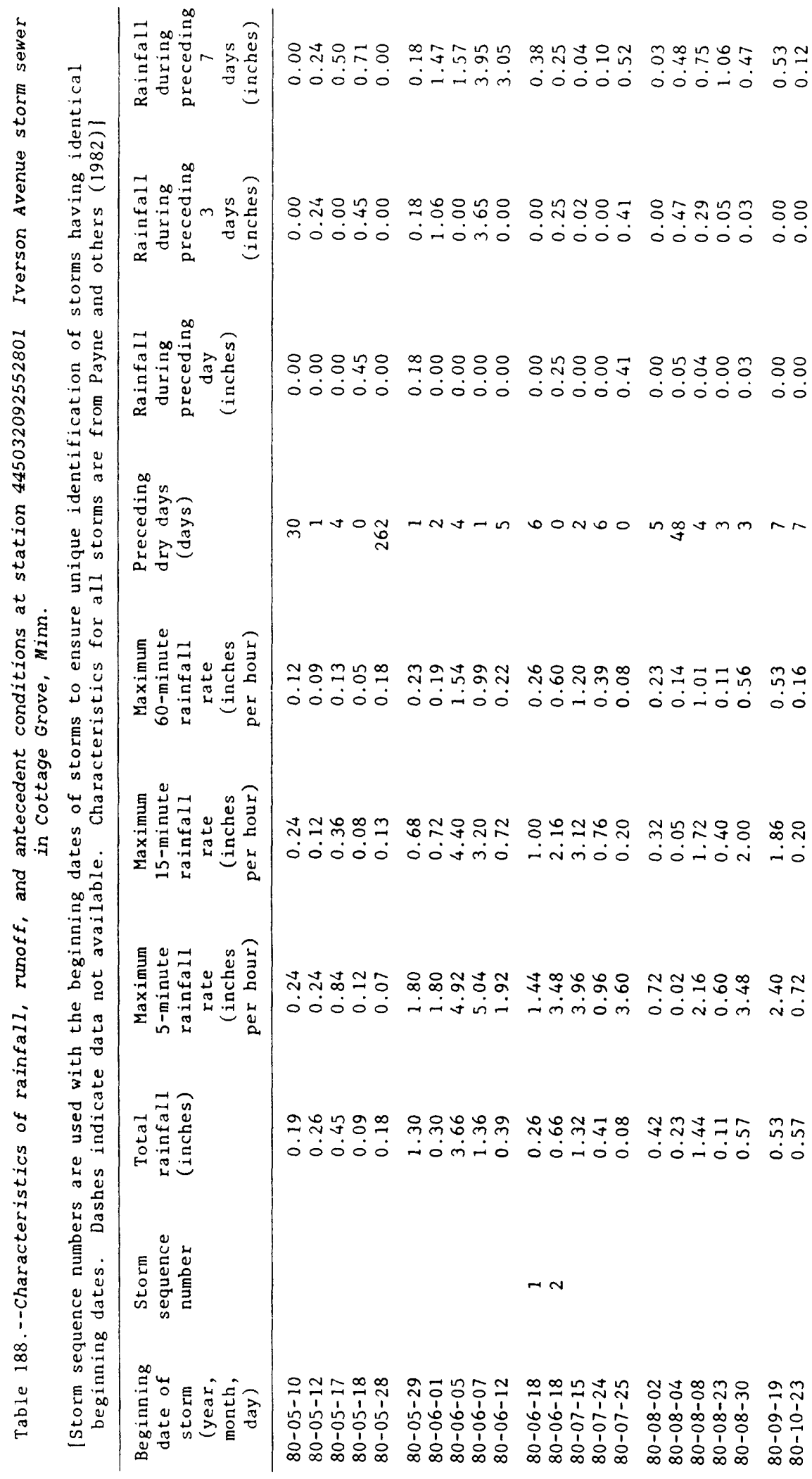




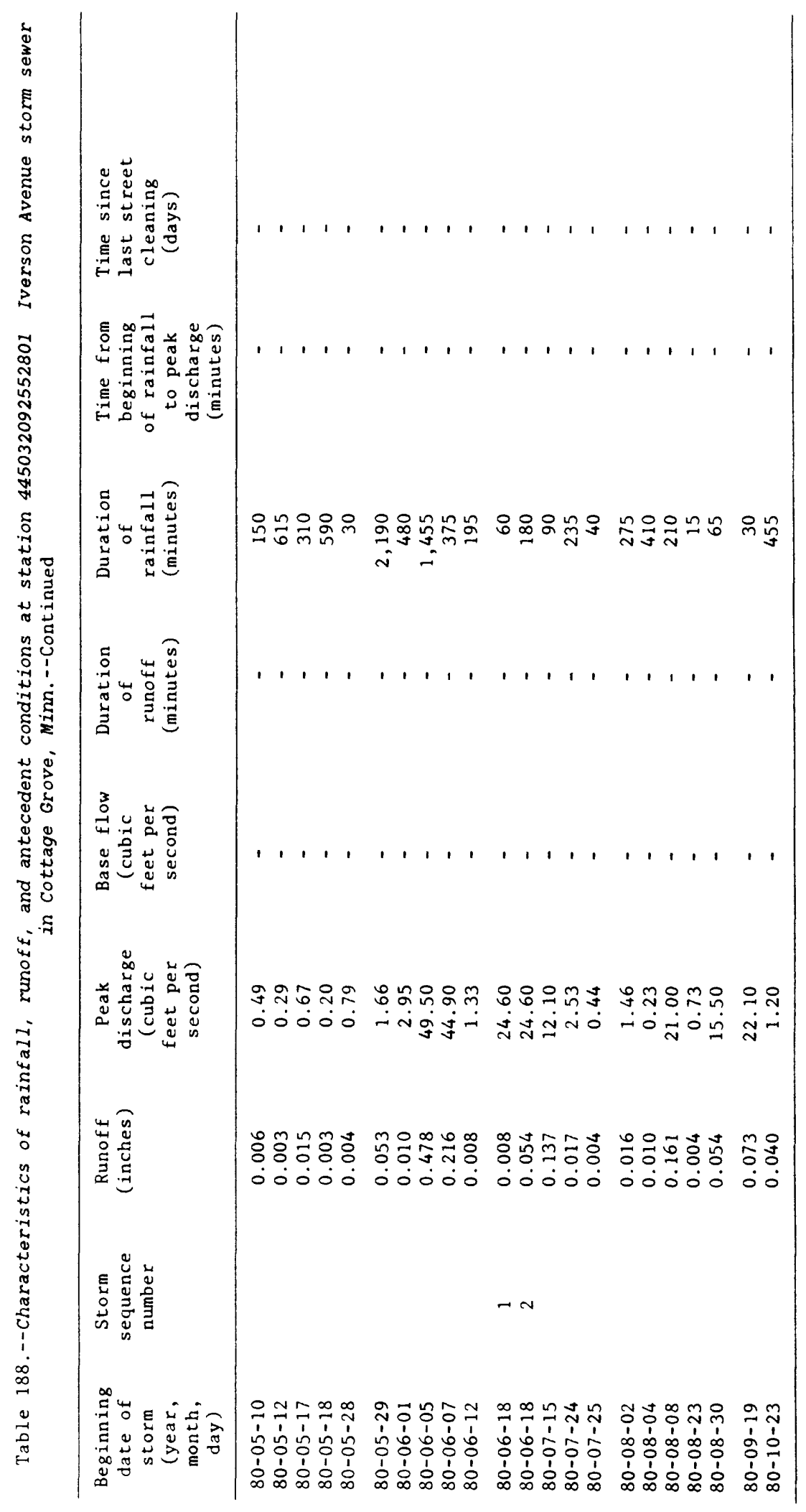




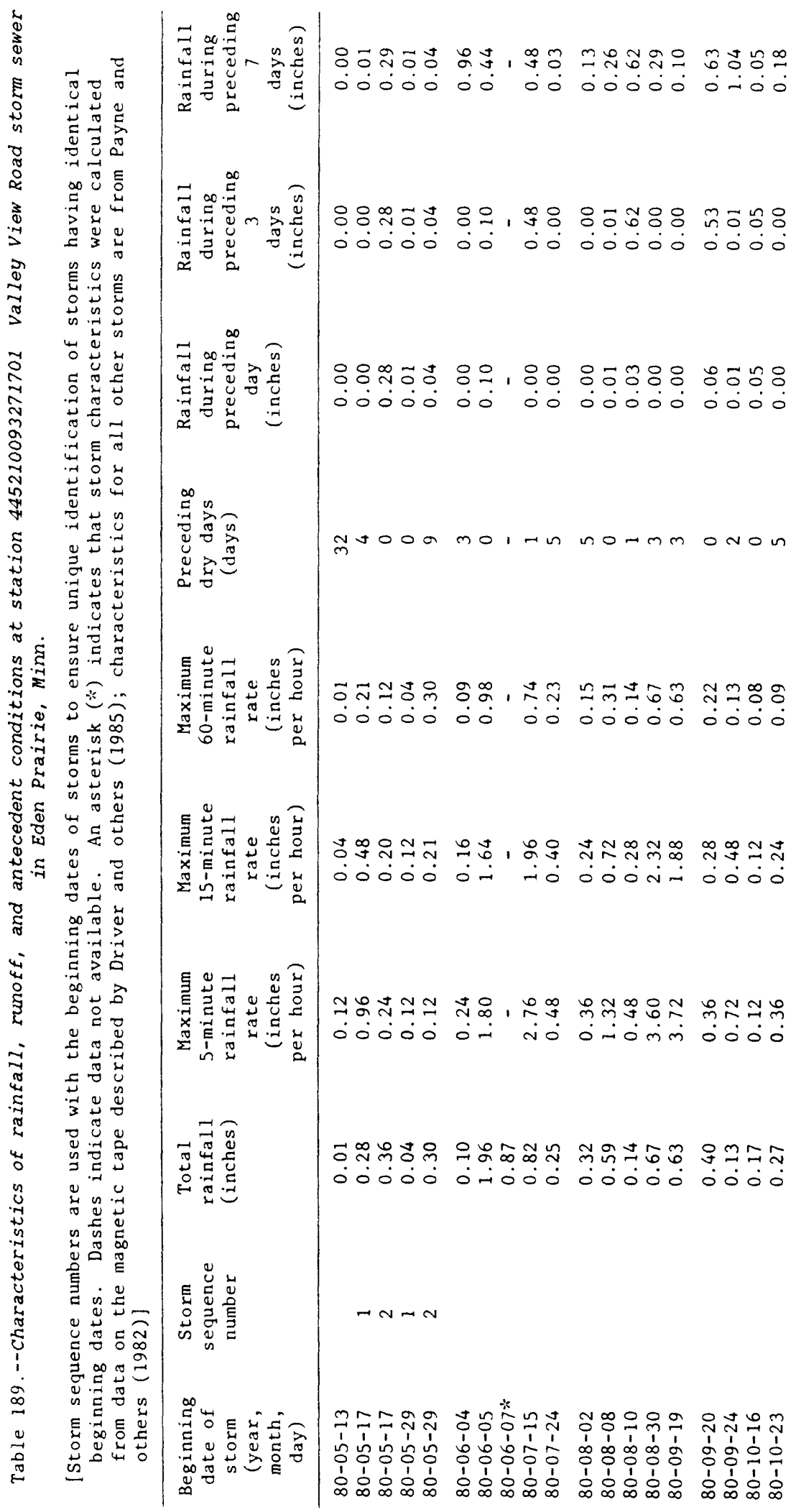




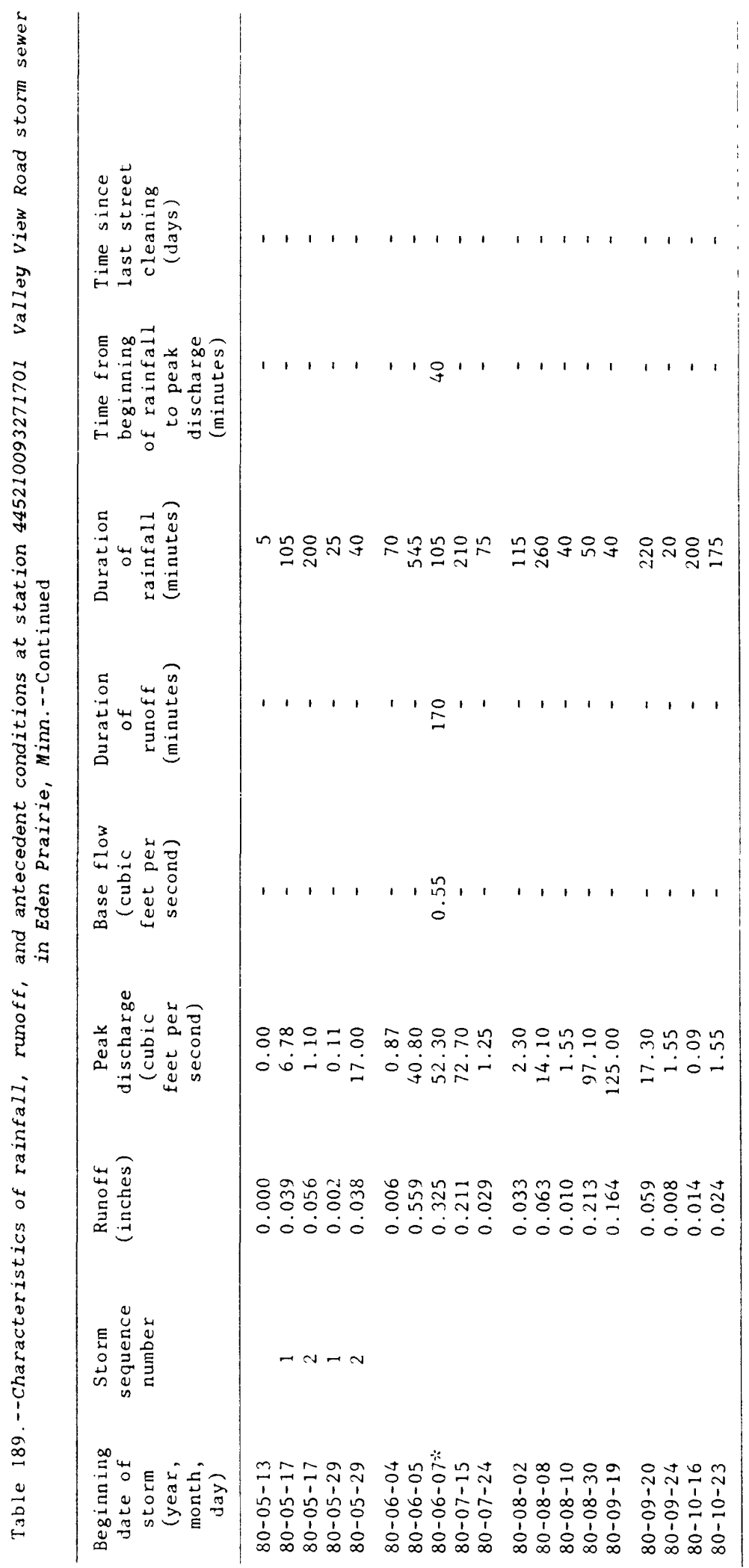




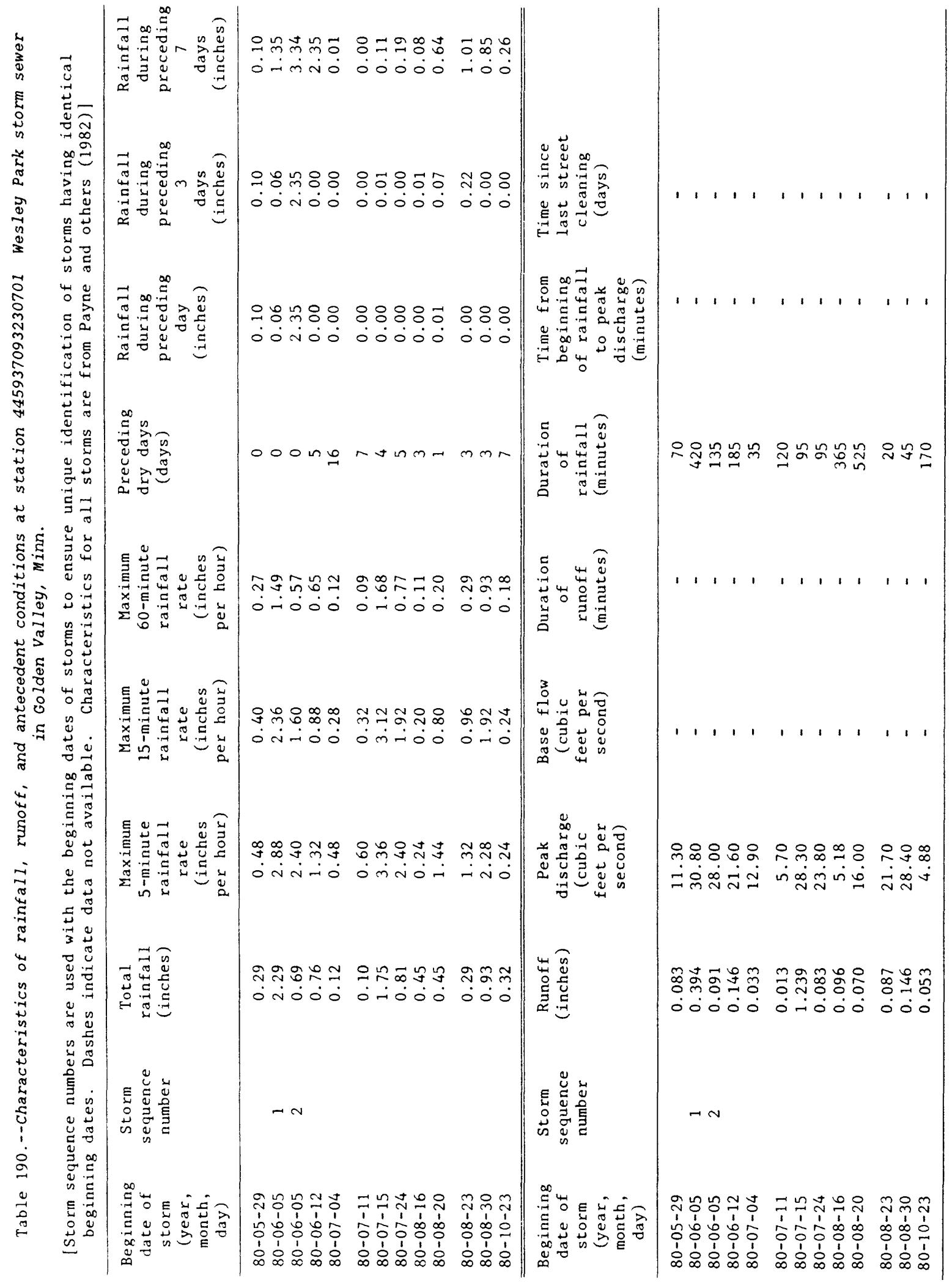




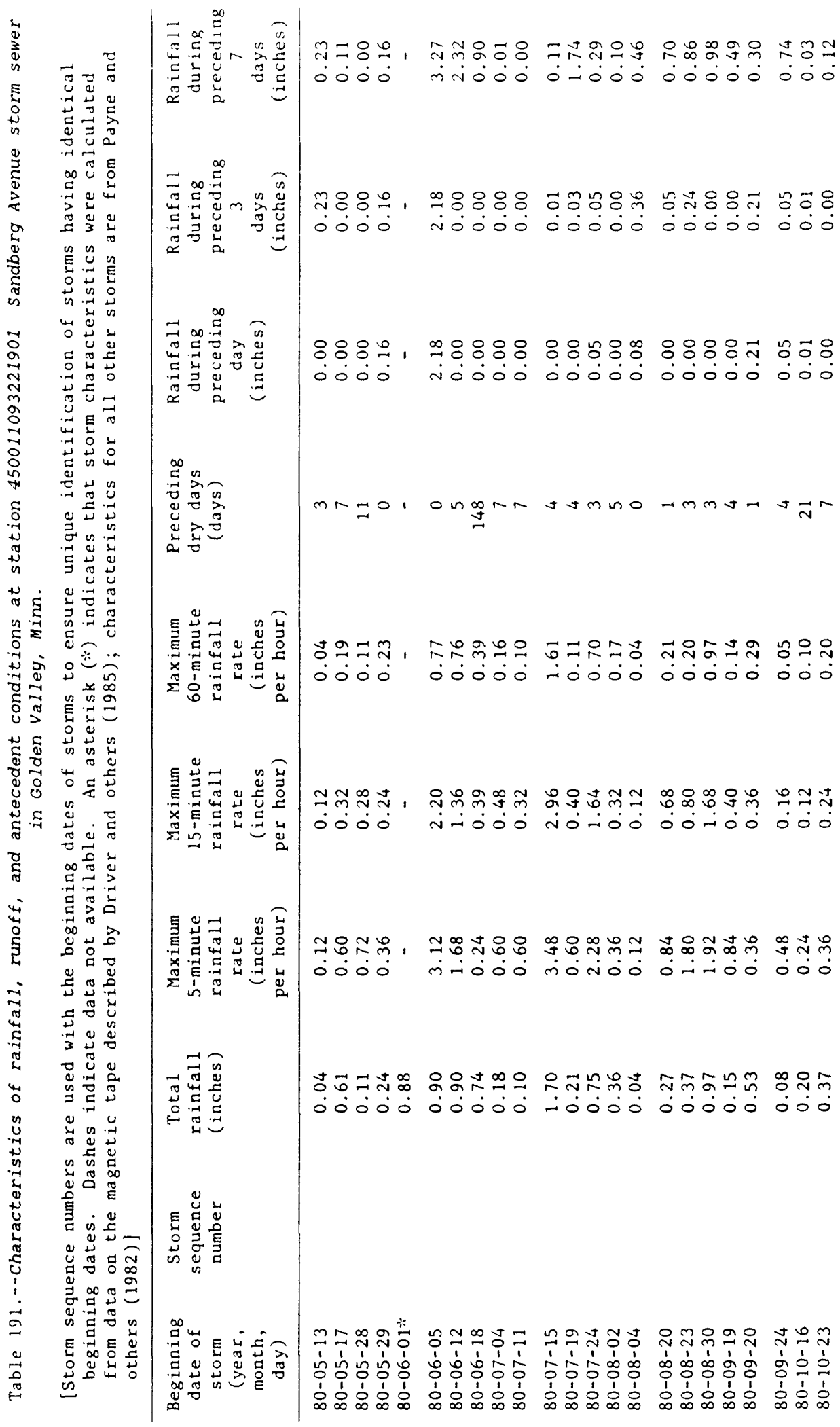




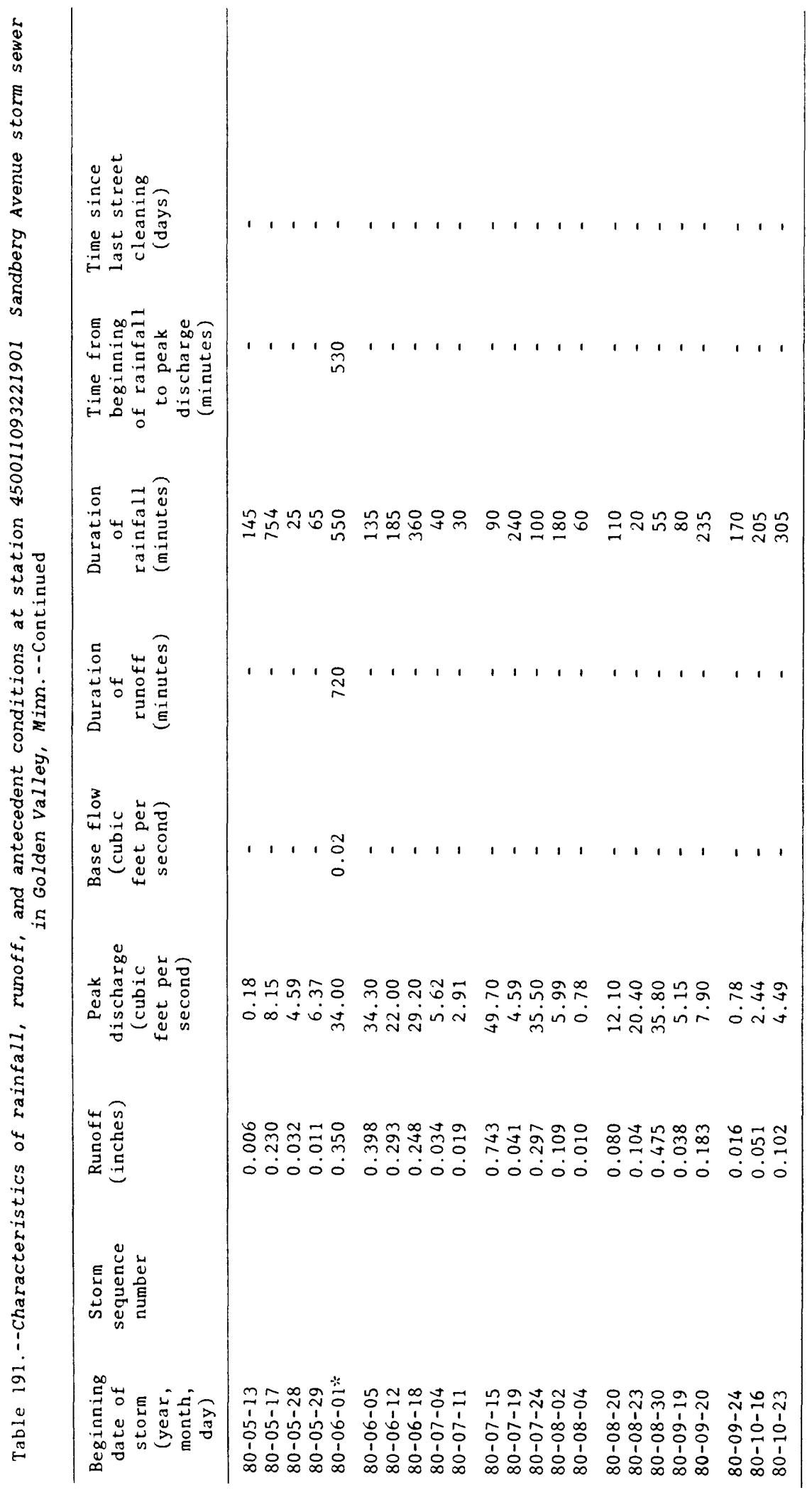




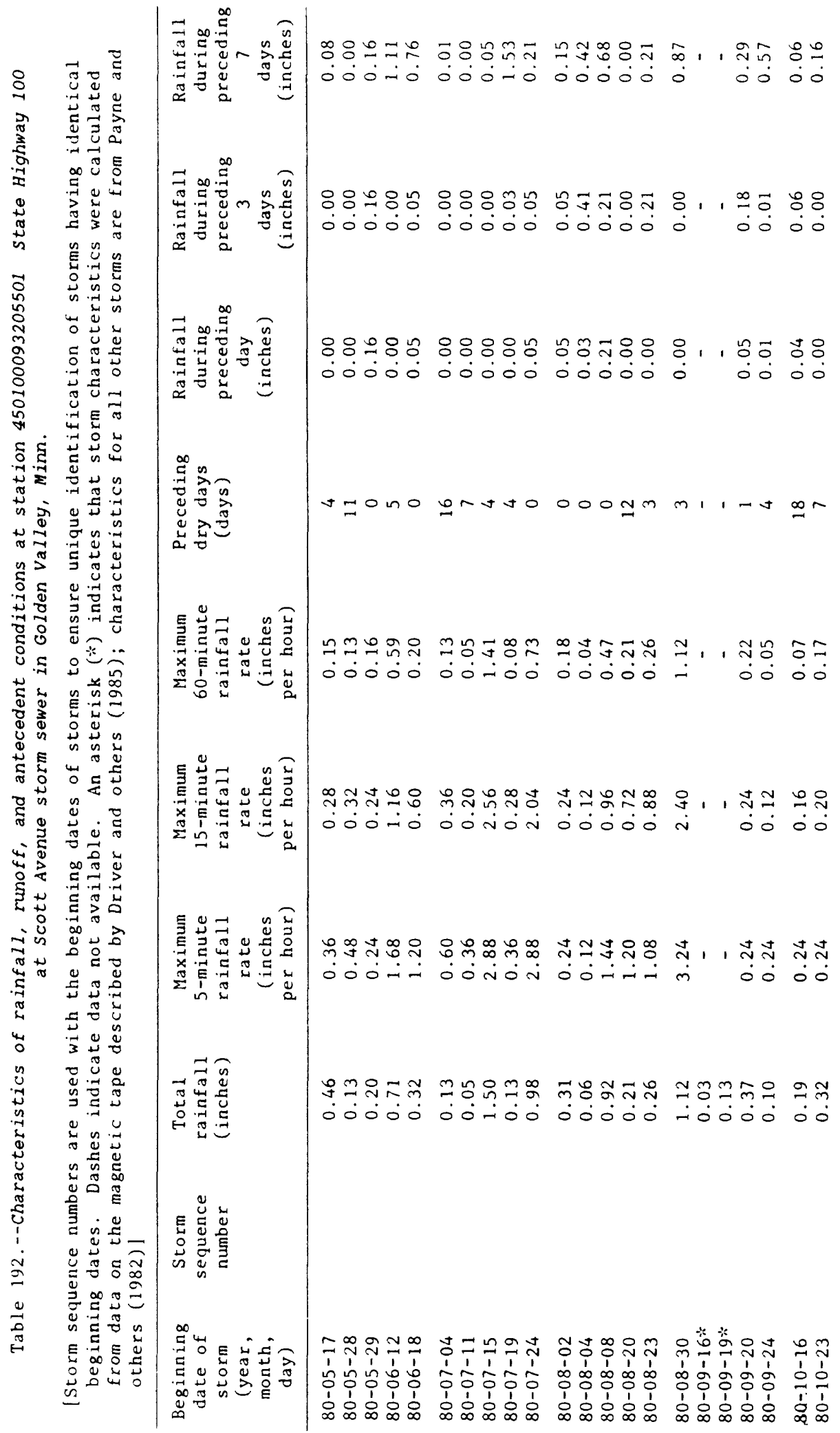




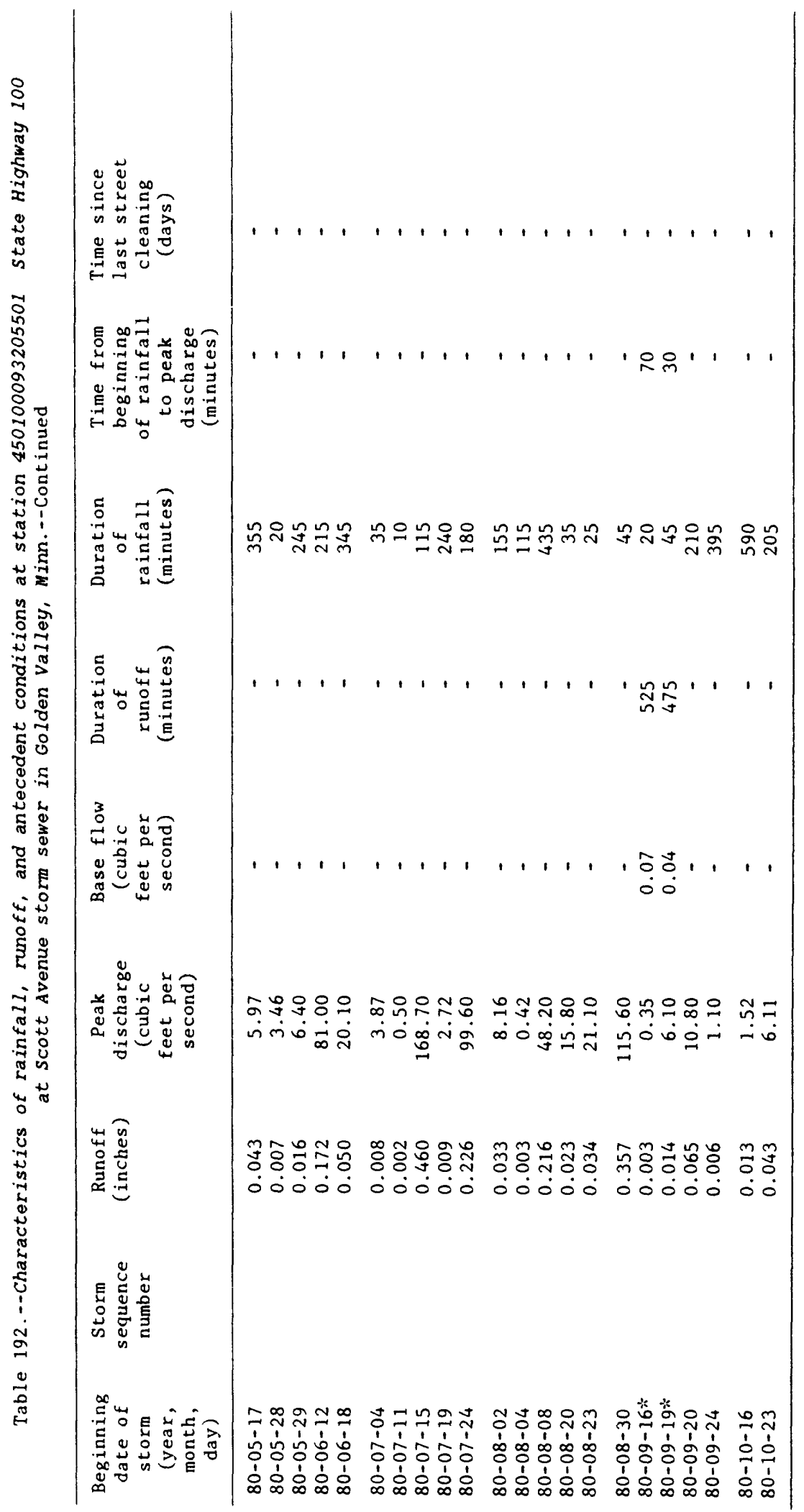




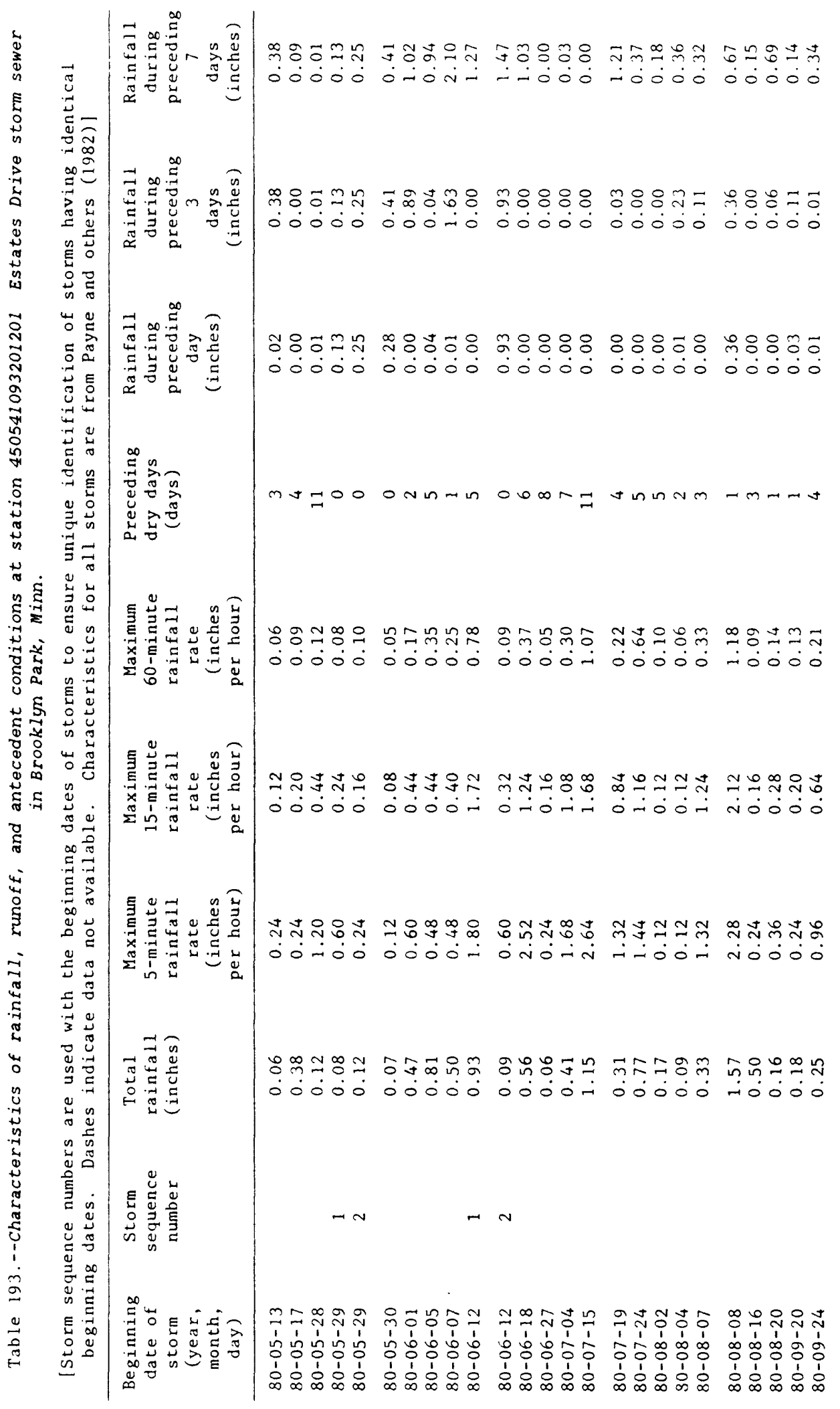




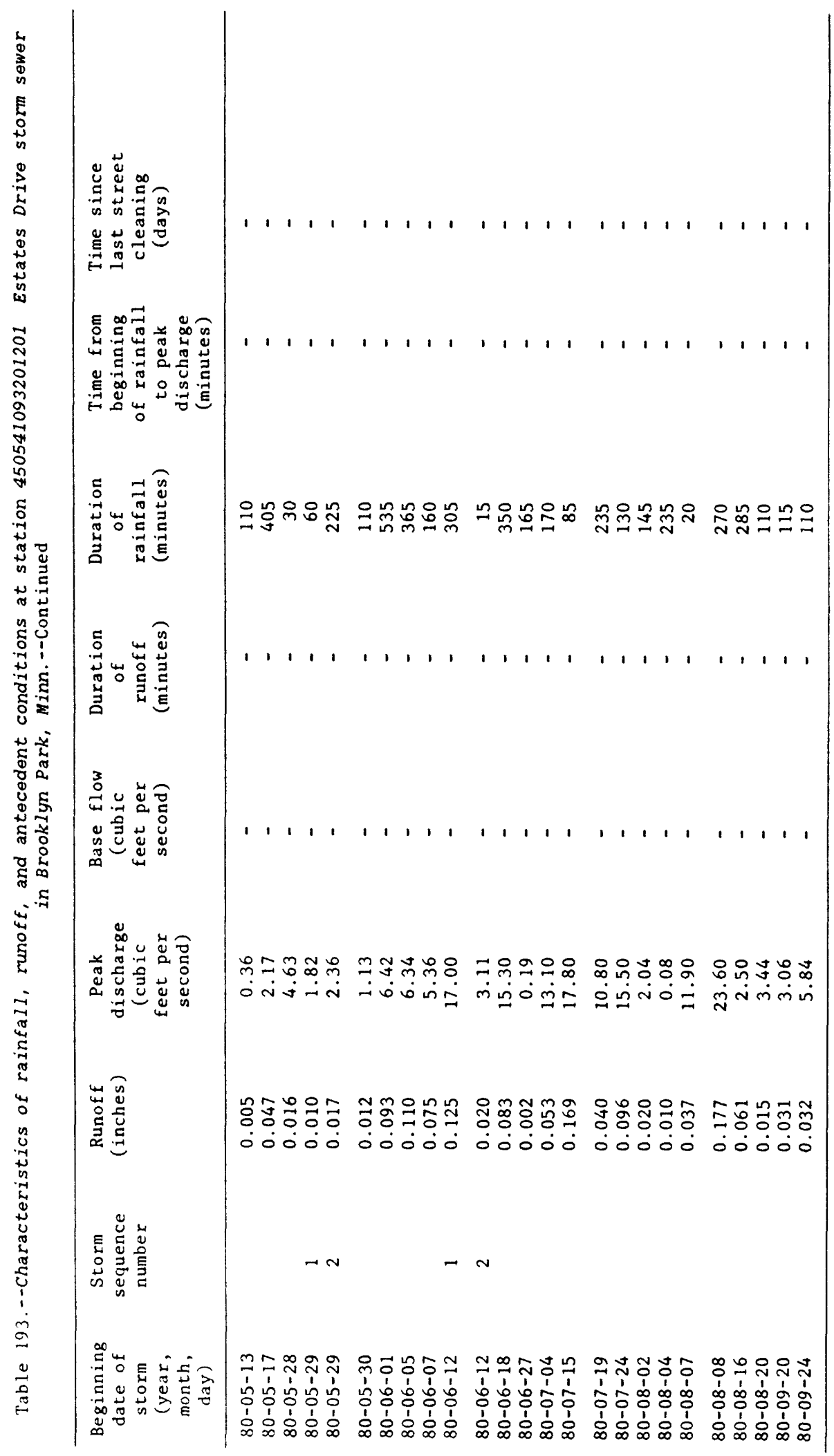




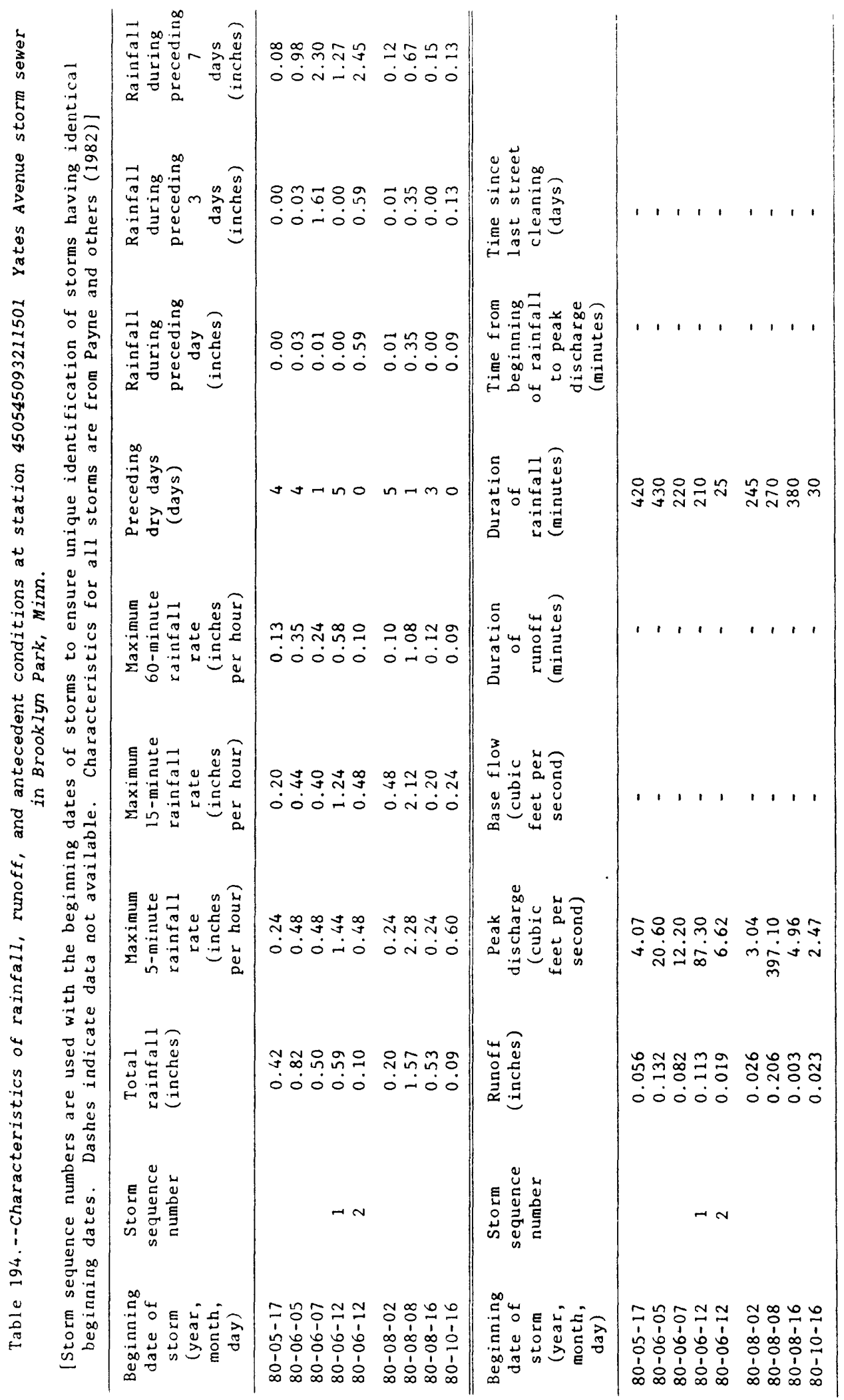




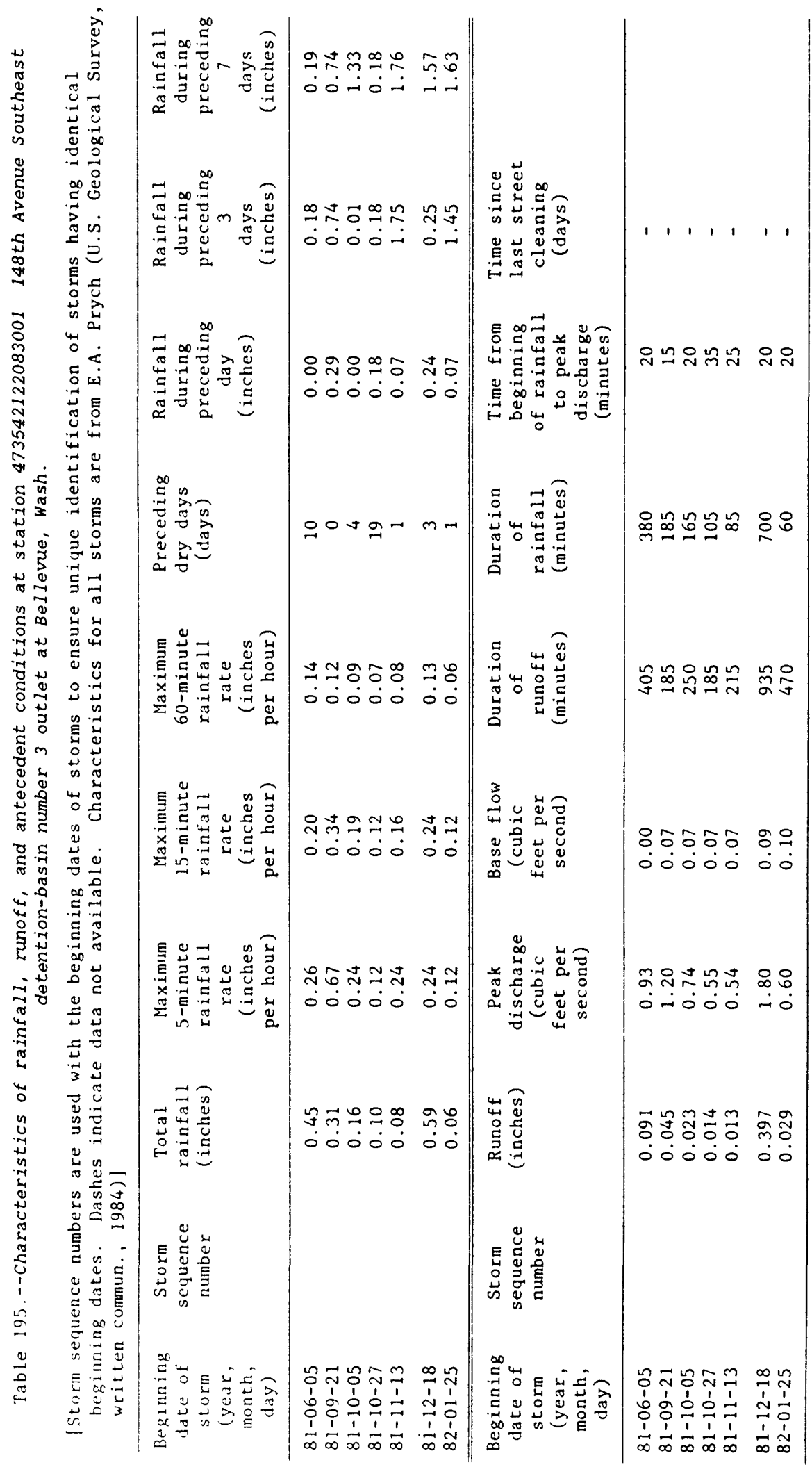




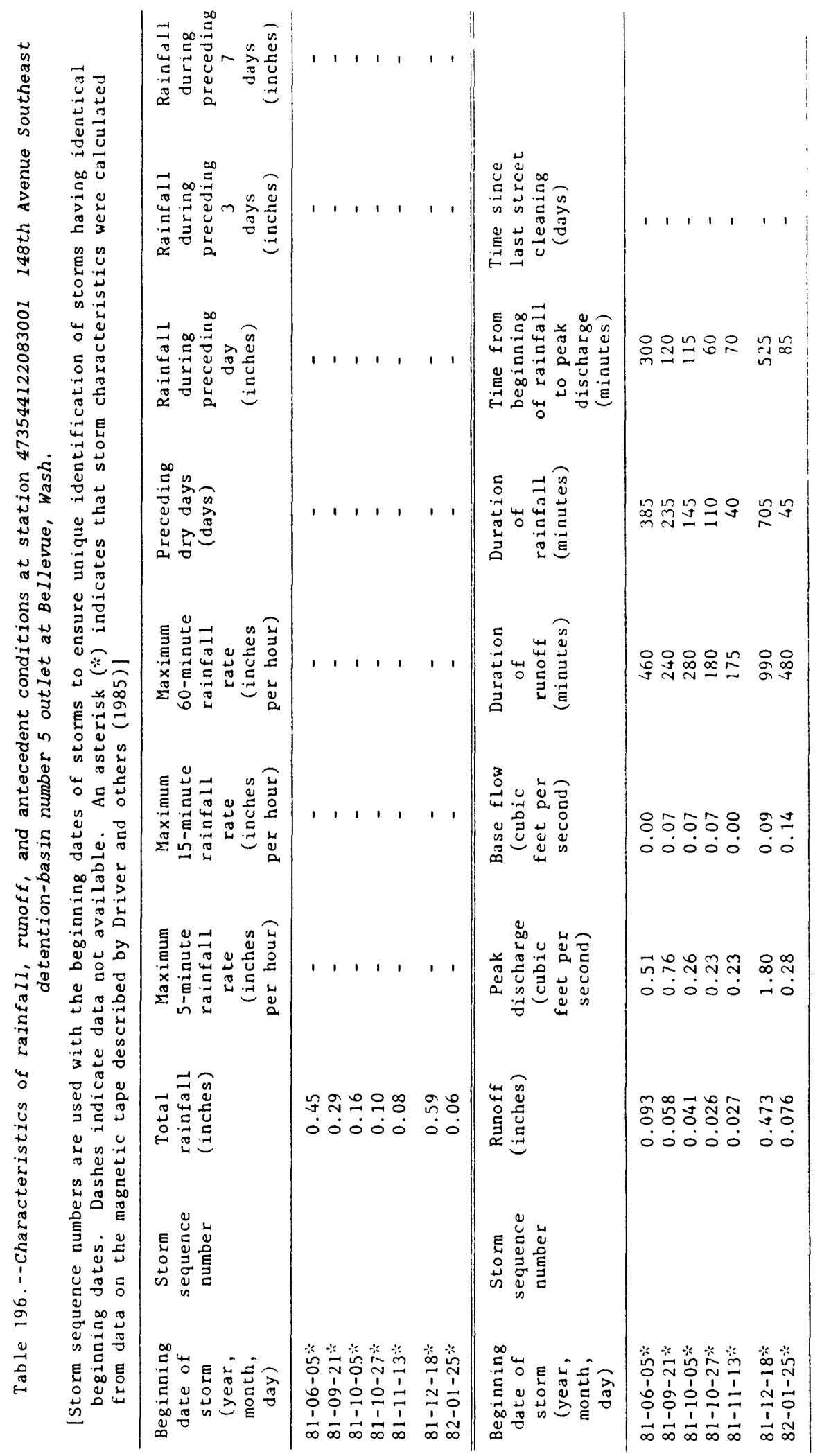


Table 197.--Basin characteristics of station 01589460

Hampden storm sewer at Baltimore, $M d$.

[Dashes indicate data not available. Except where footnoted, data is from magnetic tape described by Driver and others $(1985)$ ]

\begin{tabular}{|c|c|}
\hline Characteristic & Value \\
\hline Contributing drainage area, in acres & 17 \\
\hline $\begin{array}{l}\text { Impervious area, in percentage of contributing } \\
\text { drainage area }\end{array}$ & 72 \\
\hline $\begin{array}{l}\text { Effective impervious area, in percentage of } \\
\text { contributing drainage area }\end{array}$ & 72 \\
\hline Average basin slope, in feet per mile & 206 \\
\hline Main conveyance slope, in feet per mile & 188 \\
\hline $\begin{array}{l}\text { Population density, in number of people per } \\
\text { square mile }\end{array}$ & 35,800 \\
\hline Street density, in miles of lanes per square mile & 112 \\
\hline $\begin{array}{l}\text { Percentage of contributing drainage area with the } \\
\text { following land uses: }\end{array}$ & \\
\hline Rural and pasture & 0 \\
\hline Agricultural & 0 \\
\hline Low-density residential & 0 \\
\hline Medium-density residential & 0 \\
\hline High-density residential & 84 \\
\hline Commercial & 16 \\
\hline Industrial & 0 \\
\hline Construction & 0 \\
\hline Idle or vacant & 0 \\
\hline Wet 1 and & 0 \\
\hline Parkland & 0 \\
\hline Detention storage, in acre-feet & 0 \\
\hline $\begin{array}{l}\text { Percentage of contributing drainage area upstream of } \\
\text { detention storage }\end{array}$ & 0 \\
\hline $\begin{array}{l}\text { Percentage of contributing drainage area drained by a } \\
\text { storm-sewer system }\end{array}$ & 100 \\
\hline Percentage of streets with curb and gutter drainage & 100 \\
\hline Percentage of streets with ditch and swale drainage & 0 \\
\hline Mean annual rainfall, in inches 1 & 40.46 \\
\hline Ten-year, 1-hour rainfall intensity, in inches per hour & 2.5 \\
\hline Mean minimum January temperature, in degrees Fahrenheit ${ }^{1}$ & 24.9 \\
\hline Two-year, 24-hour rainfall, in inches ${ }^{2}$ & 3.3 \\
\hline $\begin{array}{l}\text { Mean annual nitrogen precipitation load, in pounds of } \\
\text { nitrogen per acre }\end{array}$ & 7.00 \\
\hline
\end{tabular}

${ }^{1}$ Data from National Oceanic and Atmospheric Administration, 1980 .

${ }^{2}$ Data from Hershfield, 1961.

${ }^{3}$ Data derived as sum of total deposition of ammonia and of nitrate (National Atmospheric Deposition Program, NADP Subcommittee Number 3 -- Data Management and Analysis, 1985). 
Table 198.--Basin characteristics of station 01589470 Reservoir Hill storm sewer at Baltimore, Md.

[Dashes indicate data not available. Except where footnoted, data is from magnetic tape described by Driver and others (1985)]

\begin{tabular}{|c|c|}
\hline Characteristic & Value \\
\hline Contributing drainage area, in acres & 10 \\
\hline $\begin{array}{l}\text { Impervious area, in percentage of contributing } \\
\text { drainage area }\end{array}$ & 76 \\
\hline $\begin{array}{l}\text { Effective impervious area, in percentage of } \\
\text { contributing drainage area }\end{array}$ & 76 \\
\hline Average basin slope, in feet per mile & 169 \\
\hline Main conveyance slope, in feet per mile & 97 \\
\hline $\begin{array}{l}\text { Population density, in number of people per } \\
\text { square mile }\end{array}$ & 47,300 \\
\hline $\begin{array}{l}\text { Street density, in miles of lanes per square mile } \\
\text { Percentage of contributing drainage area with the } \\
\text { following land uses: }\end{array}$ & 128 \\
\hline Rural and pasture & 0 \\
\hline Agricultural & 0 \\
\hline Low-density residential & 0 \\
\hline Medium-density residential & 0 \\
\hline High-density residential & 100 \\
\hline Commercial & 0 \\
\hline Industrial & 0 \\
\hline Construction & 0 \\
\hline Idle or vacant & 0 \\
\hline Wet 1 and & 0 \\
\hline Parkland & 0 \\
\hline Detention storage, in acre-feet & 0 \\
\hline $\begin{array}{l}\text { Percentage of contributing drainage area upstream of } \\
\text { detention storage }\end{array}$ & 0 \\
\hline $\begin{array}{l}\text { Percentage of contributing drainage area drained by a } \\
\text { storm-sewer system }\end{array}$ & 100 \\
\hline Percentage of streets with curb and gutter drainage & 100 \\
\hline $\begin{array}{l}\text { Percentage of streets with ditch and swale drainage } \\
\text { Mean annual rainfall, in inches } 1\end{array}$ & $\begin{array}{c}0 \\
40.46\end{array}$ \\
\hline Ten-year, 1-hour rainfall intensity, in inches per hour & 2.5 \\
\hline Mean minimum January temperature, in degrees Fahrenheit ${ }^{1}$ & 24.9 \\
\hline Two-year, 24-hour rainfall, in inches ${ }^{2}$ & 3.3 \\
\hline $\begin{array}{l}\text { Mean annual nitrogen precipitation load, in pounds of } \\
\text { nitrogen per acre }\end{array}$ & 7.00 \\
\hline
\end{tabular}

${ }^{1}$ Data from National Oceanic and Atmospheric Administration, 1980 .

${ }^{2}$ Data from Hershfield, 1961.

${ }^{3}$ Data derived as sum of total deposition of ammonia and of nitrate (National Atmospheric Deposition Program, NADP Subcommittee Number 3 -- Data Management and Analysis, 1985). 
Table 199.--Basin characteristics of station 01589475

Bolton Hill storm sewer at Baltimore, Md.

[Dashes indicate data not available. Except where footnoted, data is from magnetic tape described by Driver and others (1985)]

\begin{tabular}{|c|c|}
\hline Characteristic & Value \\
\hline Contributing drainage area, in acres & 14 \\
\hline $\begin{array}{l}\text { Impervious area, in percentage of contributing } \\
\text { drainage area }\end{array}$ & 61 \\
\hline $\begin{array}{l}\text { Effective impervious area, in percentage of } \\
\text { contributing drainage area }\end{array}$ & 61 \\
\hline Average basin slope, in feet per mile & 185 \\
\hline Main conveyance slope, in feet per mile & 86 \\
\hline $\begin{array}{l}\text { Population density, in number of people per } \\
\text { square mile }\end{array}$ & 17,800 \\
\hline $\begin{array}{l}\text { Street density, in miles of lanes per square mile } \\
\text { Percentage of contributing drainage area with the } \\
\quad \text { following land uses: }\end{array}$ & 98 \\
\hline Rural and pasture & 0 \\
\hline Agricultural & 0 \\
\hline Low-density residential & 0 \\
\hline lensity residential & 0 \\
\hline High-density residential & 100 \\
\hline i 1 & 0 \\
\hline Ind & 0 \\
\hline ction & 0 \\
\hline Idle or vacant & 0 \\
\hline Wet 1 and & 0 \\
\hline Parkland & 0 \\
\hline Detention storage, in acre-feet & 0 \\
\hline $\begin{array}{l}\text { Percentage of contributing drainage area upstream of } \\
\text { detention storage }\end{array}$ & 0 \\
\hline $\begin{array}{l}\text { Percentage of contributing drainage area drained by a } \\
\text { storm-sewer system }\end{array}$ & 100 \\
\hline Percentage of streets with curb and gutter drainage & 100 \\
\hline Percentage of streets with ditch and swale drainage & 0 \\
\hline Mean annual rainfall, in inches 1 & 40.46 \\
\hline Ten-year, 1 -hour rainfall intensity, in inches per hour & 2.5 \\
\hline Mean minimum January temperature, in degrees Fahrenheit ${ }^{1}$ & 24.9 \\
\hline Two-year, 24-hour rainfall, in inches ${ }^{2}$ & 3.3 \\
\hline $\begin{array}{l}\text { Mean annual nitrogen precipitation load, in pounds of } \\
\text { nitrogen per acre }\end{array}$ & 7.00 \\
\hline
\end{tabular}
1980.

${ }^{1}$ Datia from National Oceanic and Atmospheric Administration,

2Dala from Hershfield, 1961.

Data derived as sum of total deposition of ammonia and of nitrate (National Atmospheric Deposition Program, NADP Subcommittee Number 3 -- Data Management and Analysis, 1985). 
Table 200.--Basin characteristics of station 02306021

st. Louis street drainage ditch at Tampa, Fla.

[Dashes indicate data not available. Except where footnoted, data is from magnetic tape described by Driver and others (1985)]

\begin{tabular}{|c|c|}
\hline Characteristic & Value \\
\hline Contributing drainage area, in acres & 330 \\
\hline $\begin{array}{l}\text { Impervious area, in percentage of contributing } \\
\text { drainage area }\end{array}$ & 27 \\
\hline $\begin{array}{l}\text { Effective impervious area, in percentage of } \\
\text { contributing drainage area }\end{array}$ & 9 \\
\hline Average basin slope, in feet per mile & - \\
\hline $\begin{array}{l}\text { Main conveyance slope, in feet per mile } \\
\text { Population density, in number of people per }\end{array}$ & 10 \\
\hline $\begin{array}{l}\text { square mile } \\
\text { squer fin number of people per }\end{array}$ & 5,200 \\
\hline $\begin{array}{l}\text { Street density, in miles of lanes per square mile } \\
\text { Percentage of contributing drainage area with the } \\
\text { following land uses: }\end{array}$ & - \\
\hline Rural and pasture & 0 \\
\hline Agricultural & 0 \\
\hline Low-density residential & 0 \\
\hline Medium-density residential & 68 \\
\hline High-density residential & 0 \\
\hline Commercial & 22 \\
\hline Industrial & 0 \\
\hline Construction & 0 \\
\hline Idle or vacant & 8 \\
\hline Wetland & 0 \\
\hline Parkland & 2 \\
\hline Detention storage, in acre-feet & - \\
\hline $\begin{array}{l}\text { Percentage of contributing drainage area upstream of } \\
\text { detention storage }\end{array}$ & - \\
\hline $\begin{array}{l}\text { Percentage of contributing drainage area drained by a } \\
\text { storm-sewer system }\end{array}$ & 100 \\
\hline Percentage of streets with curb and gutter drainage & 100 \\
\hline $\begin{array}{l}\text { Percentage of streets with ditch and swale drainage } \\
\text { Mean annual rainfall, in inches } 1\end{array}$ & $\begin{aligned} 0 \\
10\end{aligned}$ \\
\hline Ten-year, 1-hour rainfall intensity, in inches per hour & $\begin{array}{c}49.30 \\
3.3\end{array}$ \\
\hline Mean minimum January temperature, in degrees Fahrenheit ${ }^{1}$ & 50.1 \\
\hline $\begin{array}{l}\text { Two-year, } 24 \text {-hour rainfall, in inches }{ }^{2} \\
\text { Mean annual nitrogen precipitation load, in pounds of }\end{array}$ & 5.1 \\
\hline
\end{tabular}

${ }^{1}$ Data from National Oceanic and Atmospheric Administration, 1980.

${ }^{2}$ Data from Hershfield, 1961.

${ }^{3}$ Data derived as sum of total deposition of ammonia and of nitrate (National Atmospheric Deposition Program, NADP Subcommittee Number 3 -- Data Management and Analysis, 1985). 
Table 201.--Basin characteristics of station 02307731

Allen Creek near Largo, Fla.

[Dashes indicate data not available. Except where footnoted, data is from magnetic tape described by Driver and others (1985)]

\begin{tabular}{|c|c|}
\hline Characteristic & Value \\
\hline Contributing drainage area, in acres & 1,100 \\
\hline $\begin{array}{l}\text { Impervious area, in percentage of contributing } \\
\text { drainage area }\end{array}$ & 36 \\
\hline $\begin{array}{l}\text { Effective impervious area, in percentage of } \\
\text { contributing drainage area }\end{array}$ & 26 \\
\hline Average basin slope, in feet per mile & - \\
\hline Main conveyance slope, in feet per mile & 23 \\
\hline $\begin{array}{l}\text { Population density, in number of people per } \\
\text { square mile }\end{array}$ & 4,500 \\
\hline $\begin{array}{l}\text { Street density, in miles of lanes per square mile } \\
\text { Percentage of contributing drainage area with the } \\
\text { following land uses: }\end{array}$ & - \\
\hline Rural and pasture & 0 \\
\hline Agricultural & 0 \\
\hline Low-density residential & 0 \\
\hline Medium-density residential & 59 \\
\hline High-density residential & 4 \\
\hline Commercial & 25 \\
\hline Industrial & 0 \\
\hline Construction & 0 \\
\hline Idle or vacant & 11 \\
\hline Wetl and & 0 \\
\hline Parkland & 1 \\
\hline Detention storage, in acre-feet & - \\
\hline $\begin{array}{l}\text { Percentage of contributing drainage area upstream of } \\
\text { detention storage }\end{array}$ & - \\
\hline $\begin{array}{l}\text { Percentage of contributing drainage area drained by a } \\
\text { storm-sewer system }\end{array}$ & - \\
\hline Percentage of streets with curb and gutter drainage & - \\
\hline Percentage of streets with ditch and swale drainage & - \\
\hline Mean annual rainfall, in inches ${ }^{1}$ & 49.38 \\
\hline Ten-year, 1-hour rainfall intensity, in inches per hour & 3.3 \\
\hline Mean minimum January temperature, in degrees Fahrenheit ${ }^{1}$ & 50.1 \\
\hline Two-year, 24-hour rainfall, in inches ${ }^{2}$ & 5.1 \\
\hline $\begin{array}{l}\text { Mean annual nitrogen precipitation load, in pounds of } \\
\text { nitrogen per acre }\end{array}$ & 2.60 \\
\hline
\end{tabular}

${ }^{1}$ Data from National Oceanic and Atmospheric Administration, 1980 .

${ }^{2}$ Data from Hershfield, 1961.

${ }^{3}$ Data derived as sum of total deposition of ammonia and of nitrate (National Atmospheric Deposition Program, NADP Subcommittee Number 3 -- Data Management and Analysis, 1985). 
Table 202.--Basin characteristics of station 03226900

Fishinger Road Creek at Upper Arlington, Ohic

[Dashes indicate data not available. Except where footnoted, data is from magnetic tape described by Driver and others (1985)]

Characteristic

liallue

Contributing drainage area, in acres 290

Impervious area, in percentage of contributing drainage area

60

Effective impervious area, in percentage of contributing drainage area

Average basin slope, in feet per mile

Main conveyance slope, in feet per mile

Population density, in number of people per square mile

Street density, in miles of lanes per square mile

Percentage of contributing drainage area with the following land uses:

Rural and pasture

Agricultural

Low-density residential

Medium-density residential

High-density residential

Commercial

Industrial

Construction

Idle or vacant

Wet land

Parkland

Detention storage, in acre-feet

Percentage of contributing drainage area upstream of detention storage

Percentage of contributing drainage area drained by a storm-sewer system

100

Percentage of streets with curb and gutter drainage

Percentage of streets with ditch and swale drainage

Mean annual rainfall, in inches ${ }^{1}$

Ten-year, 1-hour rainfall intensity, in inches per hour

Mean minimum January temperature, in degrees Fahrenheit ${ }^{1}$

Two-year, 24-hour rainfall, in inches ${ }^{2}$

0

0

80

1

13

0

0

0

0

0

0

Mean annual nitrogen precipitation load, in pounds of nitrogen per acre ${ }^{3}$

${ }^{1}$ Data from National Oceanic and Atmospheric Administration, 1980 .

${ }^{2}$ Data from Hershfield, 1961.

${ }^{3}$ Data derived as sum of total deposition of ammonia and of nitrate (National Atmospheric Deposition Program, NADP Subcommittee Number 3 -- Data Management and Analysis, 1985). 
Table 203.--Basin characteristics of station 03227050

Norman ditch at Chambers Circle at Columbus, Ohio

[Dashes indicate data not available. Except where footnoted, data is from magnetic tape described by Driver and others $(1985)$ ]

\begin{tabular}{|c|c|}
\hline Characteristic & Value \\
\hline Contributing drainage area, in acres & 380 \\
\hline $\begin{array}{l}\text { Impervious area, in percentage of contributing } \\
\text { drainage area }\end{array}$ & 85 \\
\hline $\begin{array}{l}\text { Effective impervious area, in percentage of } \\
\text { contributing drainage area }\end{array}$ & - \\
\hline Average basin slope, in feet per mile & 2 \\
\hline Main conveyance slope, in feet per mile & 67 \\
\hline $\begin{array}{l}\text { Population density, in number of people per } \\
\text { square mile }\end{array}$ & - \\
\hline Street density, in miles of lanes per square mile & - \\
\hline $\begin{array}{l}\text { Percentage of contributing drainage area with the } \\
\text { following land uses: }\end{array}$ & \\
\hline Rural and pasture & 4 \\
\hline Agricultural & 0 \\
\hline Low-density residential & 0 \\
\hline Medium-density residential & 53 \\
\hline High-density residential & 20 \\
\hline Commercial & 23 \\
\hline Industrial & 0 \\
\hline Construction & 0 \\
\hline Idle or vacant & 0 \\
\hline Wet land & 0 \\
\hline Parkland & 0 \\
\hline Detention storage, in acre-feet & 0 \\
\hline $\begin{array}{l}\text { Percentage of contributing drainage area upstream of } \\
\text { detention storage }\end{array}$ & 0 \\
\hline $\begin{array}{l}\text { Percentage of contributing drainage area drained by a } \\
\text { storm-sewer system }\end{array}$ & 100 \\
\hline Percentage of streets with curb and gutter drainage & 100 \\
\hline Percentage of streets with ditch and swale drainage & 0 \\
\hline Mean annual rainfall, in inches 1 & 37.01 \\
\hline Ten-year, 1 -hour rainfall intensity, in inches per hour & 1.9 \\
\hline Mean minimum January temperature, in degrees Fahrenheit ${ }^{1}$ & 20.4 \\
\hline Two-year, 24-hour rainfall, in inches ${ }^{2}$ & 2.5 \\
\hline $\begin{array}{l}\text { Mean annual nitrogen precipitation load, in pounds of } \\
\text { nitrogen per acre }\end{array}$ & 4.70 \\
\hline
\end{tabular}

${ }^{1}$ Data from National Oceanic and Atmospheric Administration, 1980 .

${ }^{2}$ Data from Hershfield, 1961.

${ }^{3}$ Data derived as sum of total deposition of ammonia and of nitrate (National Atmospheric Deposition Program, NADP Subcommittee Number 3 -- Data Management and Analysis, 1985). 
Table 204.--Basin characteristics of station 04086941

Lincoln Creek at 54th Street at Milwaukee, Wis.

[Dashes indicate data not available. Except where footnoted, data is from magnetic tape described by Driver and others (1985)]

Characteristic

Value

Contributing drainage area, in acres

38

Impervious area, in percentage of contributing

drainage area

Effective impervious area, in percentage of contributing drainage area

Average basin slope, in feet per mile

Main conveyance slope, in feet per mile

Population density, in number of people per

square mile

Street density, in miles of lanes per square mile

Percentage of contributing drainage area with the

following land uses:

Rural and pasture

Agricultural

Low-density residential

Medium-density residential

High-density residential

Commercial

Industrial

Construction

Idle or vacant

Wet land

Parkland

Detention storage, in acre-feet

Percentage of contributing drainage area upstream of detention storage

Percentage of contributing drainage area drained by a storm-sewer system

Percentage of streets with curb and gutter drainage Percentage of streets with ditch and swale drainage Mean annual rainfall, in inches 1

Ten-year, 1-hour rainfall intensity, in inches per hour

Mean minimum January temperature, in degrees Fahrenheit ${ }^{1} \quad 11.4$

Two-year, 24-hour rainfal1, in inches ${ }^{2}$

2.6

Mean annual nitrogen precipitation load, in pounds of nitrogen per acre ${ }^{3}$

6.10

${ }^{1}$ Data from National Oceanic and Atmospheric Administration, 1980.

${ }^{2}$ Data from Hershfield, 1961

${ }^{3}$ Data derived as sum of total deposition of ammonia and of nitrate (National Atmospheric Deposition Program, NADP Subcommittee Number 3 -- Data Management and Analysis, 1985). 
Table 205.--Basin characteristics of station 04086943

Lincoln Creek tributary at Milwaukee, Wis.

[Dashes indicate data not available. Except where footnoted, data is from magnetic tape described by Driver and others (1985)]

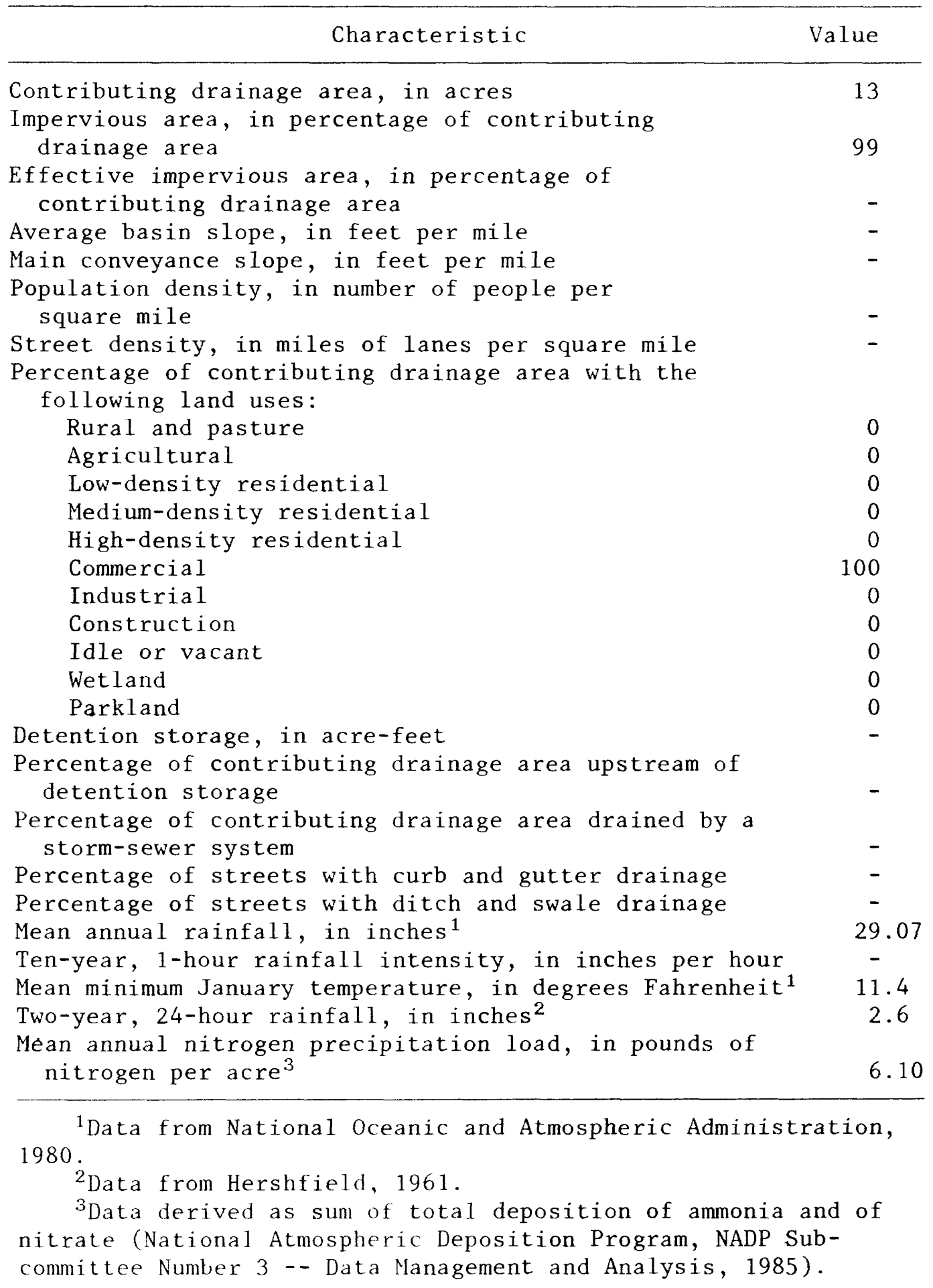


Table 206.--Basin characteristics of station 04086945

Lincoln Creek tributary at Milwaukee, Wis.

[Dashes indicate data not available. Except where footnoted, data is from magnetic tape described by Driver and others $(1985)]$

\section{Characteristic}

Vit lut

Contributing drainage area, in acres

Impervious area, in percentage of contributing

drainage area

Effective impervious area, in percentage of

contributing drainage area

Average basin slope, in feet per mile

Main conveyance slope, in feet per mile

Population density, in number of people per square mile

Street density, in miles of lanes per square mile

Percentage of contributing drainage area with the

following land uses:

Rural and pasture

Agricultural

Low-density residential

Medium-density residential

High-density residential

Commercial

Industrial

Construction

Idle or vacant

Wetland

Parkland

Detention storage, in acre-feet

Percentage of contributing drainage area upstream of detention storage

Percentage of contributing drainage area drained by a storm-sewer system

Percentage of streets with curb and gutter drainage

Percentage of streets with ditch and swale drainage

Mean annual rainfall, in inches ${ }^{1}$

Vit
13
98

Ten-year, 1-hour rainfall intensity, in inches per hour

Mean minimum January temperature, in degrees Fahrenheit ${ }^{1}$

Two-year, 24-hour rainfall, in inches ${ }^{2}$

Mean annual nitrogen precipitation load, in pounds of nitrogen per acre ${ }^{3}$

\footnotetext{
${ }^{1}$ Data from National Oceanic and Atmospheric Administration, 1980.

${ }^{2}$ Data from Hershfield, 1961

${ }^{3}$ Data derived as sum of total deposition of ammonia and of nitrate (National Atmospheric Deposition Program, NADP Subcommittee Number 3 -- Data Management and Analysis, 1985).
} 
Table 207.--Basin characteristics of station 04087056 Tributary to Little Menomonee River tributary at Milwaukee, Wis.

[Dashes indicate data not available. Except where footnoted, data is from magnetic tape described by Driver and others (1985)]

\begin{tabular}{|c|c|}
\hline Characteristic & Value \\
\hline Contributing drainage area, in acres & 64 \\
\hline $\begin{array}{l}\text { Impervious area, in percentage of contributing } \\
\text { drainage area }\end{array}$ & 30 \\
\hline $\begin{array}{l}\text { Effective impervious area, in percentage of } \\
\text { contributing drainage area }\end{array}$ & - \\
\hline Average basin slope, in feet per mile & - \\
\hline Main conveyance slope, in feet per mile & - \\
\hline $\begin{array}{l}\text { Population density, in number of people per } \\
\text { square mile }\end{array}$ & - \\
\hline $\begin{array}{l}\text { Street density, in miles of lanes per square mile } \\
\text { Percentage of contributing drainage area with the } \\
\text { following land uses: }\end{array}$ & - \\
\hline Rural and pasture & 0 \\
\hline Agricultural & 0 \\
\hline Low-density residential & 0 \\
\hline Medium-density residential & 100 \\
\hline High-density residential & 0 \\
\hline Commercial & 0 \\
\hline Industrial & 0 \\
\hline Construction & 0 \\
\hline Idle or vacant & 0 \\
\hline Wetland & 0 \\
\hline Parkland & 0 \\
\hline Detention storage, in acre-feet & - \\
\hline $\begin{array}{l}\text { Percentage of contributing drainage area upstream of } \\
\text { detention storage }\end{array}$ & - \\
\hline $\begin{array}{l}\text { Percentage of contributing drainage area drained by a } \\
\text { storm-sewer system }\end{array}$ & - \\
\hline Percentage of streets with curb and gutter drainage & - \\
\hline Percentage of streets with ditch and swale drainage & - \\
\hline Mean annual rainfall, in inches ${ }^{1}$ & 29.07 \\
\hline Ten-year, 1 -hour rainfall intensity, in inches per hour & - \\
\hline Mean minimum January temperature, in degrees Fahrenheit ${ }^{1}$ & 11.4 \\
\hline Two-year, 24-hour rainfall, in inches ${ }^{2}$ & 2.6 \\
\hline $\begin{array}{l}\text { Mean annual nitrogen precipitation load, in pounds of } \\
\text { nitrogen per acre }\end{array}$ & 6.10 \\
\hline
\end{tabular}

${ }^{1}$ Data from National Oceanic and Atmospheric Administration, 1980 .

${ }^{2}$ Data from Hershfield, 1961.

${ }^{3}$ Data derived as sum of total deposition of ammonia and of nitrate (National Atmospheric Deposition Program, NADP Subcommittee Number 3 -- Data Management and Analysis, 1985). 
Table 208.--Basin characteristics of station 04087057

Little Menomonee River tributary at Milwaukee, Wis.

[Dashes indicate data not available. Except where footnoted, data is from magnetic tape described by Driver and others (1985)]

\begin{tabular}{lc}
\hline \multicolumn{1}{c}{ Characteristic } & lalue \\
\hline Contributing drainage area, in acres & 32 \\
Impervious area, in percentage of contributing & 30 \\
drainage area & - \\
Effective impervious area, in percentage of & - \\
contributing drainage area & - \\
Average basin slope, in feet per mile & - \\
Main conveyance slope, in feet per mile & - \\
Population density, in number of people per & \\
square mile & \\
Street density, in miles of lanes per square mile & 0 \\
Percentage of contributing drainage area with the & 0 \\
following land uses: & 0 \\
Rural and pasture & 100 \\
Agricultural & 0 \\
Low-density residential & 0 \\
Hedium-density residential & 0 \\
Commercial & 0 \\
Industrial & 0 \\
Construction & - \\
Idle or vacant &
\end{tabular}

\footnotetext{
${ }^{1}$ Data from National Oceanic and Atmospheric Administration, 1980 .

${ }^{2}$ Data from Hershfield, 1961.

${ }^{3}$ Data derived as sum of total deposition of ammonia and of nitrate (National Atmospheric Deposition Program, NADP Subcommittee Number 3 -- Data Management and Analysis, 1985).
} 
Table 209.--Basin characteristics of station 04087115

Honey Creek tributary at West Allis, Wis.

[Dashes indicate data not available. Except where footnoted, data is from magnetic tape described by Driver and others (1985)]

\begin{tabular}{|c|c|}
\hline Characteristic & Value \\
\hline Contributing drainage area, in acres & 26 \\
\hline $\begin{array}{l}\text { Impervious area, in percentage of contributing } \\
\text { drainage area }\end{array}$ & 77 \\
\hline $\begin{array}{l}\text { Effective impervious area, in percentage of } \\
\text { contributing drainage area }\end{array}$ & - \\
\hline Average basin slope, in feet per mile & - \\
\hline Main conveyance slope, in feet per mile & - \\
\hline $\begin{array}{l}\text { Population density, in number of people per } \\
\text { square mile }\end{array}$ & - \\
\hline Street density, in miles of lanes per square mile & - \\
\hline $\begin{array}{l}\text { Percentage of contributing drainage area with the } \\
\text { following land uses: }\end{array}$ & \\
\hline Rural and pasture & 0 \\
\hline Agricultural & 0 \\
\hline Low-density residential & 0 \\
\hline Medium-density residential & 0 \\
\hline High-density residential & 20 \\
\hline Commercial & 80 \\
\hline Industrial & 0 \\
\hline Construction & 0 \\
\hline Idle or vacant & 0 \\
\hline Wet 1 and & 0 \\
\hline Parkland & 0 \\
\hline Detention storage, in acre-feet & - \\
\hline $\begin{array}{l}\text { Percentage of contributing drainage area upstream of } \\
\text { detention storage }\end{array}$ & - \\
\hline $\begin{array}{l}\text { Percentage of contributing drainage area drained by a } \\
\text { storm-sewer system }\end{array}$ & - \\
\hline Percentage of streets with curb and gutter drainage & - \\
\hline Percentage of streets with ditch and swale drainage & - \\
\hline Mean annual rainfall, in inches ${ }^{1}$ & 29.07 \\
\hline Ten-year, 1 -hour rainfall intensity, in inches per hour & - \\
\hline Mean minimum January temperature, in degrees Fahrenheit ${ }^{1}$ & 11.4 \\
\hline Two-year, 24-hour rainfall, in inches $^{2}$ & 2.6 \\
\hline $\begin{array}{l}\text { Mean annual nitrogen precipitation load, in pounds of } \\
\text { nitrogen per acre }\end{array}$ & 6.10 \\
\hline
\end{tabular}

${ }^{1}$ Data from National Oceanic and Atmospheric Administration, 1980 .

${ }^{2}$ Data from Hershfield, 1961.

${ }^{3}$ Data derived as sum of total deposition of ammonia and of nitrate (National Atmospheric Deposition Program, NADP Subcommittee Number 3 -- Data Management and Analys is, 1985). 
Table 210.--Basin characteristics of station 04087133

Menomonee River tributary at West Allis, Wis.

[Dashes indicate data not available. Except where footnoted, data is from magnetic tape described by Driver and others (1985)]

\begin{tabular}{|c|c|}
\hline Characteristic & Value \\
\hline Contributing drainage area, in acres & 45 \\
\hline $\begin{array}{l}\text { Impervious area, in percentage of contributing } \\
\text { drainage area }\end{array}$ & 81 \\
\hline $\begin{array}{l}\text { Effective impervious area, in percentage of } \\
\text { contributing drainage area }\end{array}$ & - \\
\hline Average basin slope, in feet per mile & - \\
\hline Main conveyance slope, in feet per mile & - \\
\hline $\begin{array}{l}\text { Population density, in number of people per } \\
\text { square mile }\end{array}$ & - \\
\hline Street density, in miles of lanes per square mile & - \\
\hline $\begin{array}{l}\text { Percentage of contributing drainage area with the } \\
\text { following land uses: }\end{array}$ & \\
\hline Rural and pasture & 0 \\
\hline Agricultural & 0 \\
\hline Low-density residential & 0 \\
\hline Medium-density residential & 0 \\
\hline High-density residential & 30 \\
\hline Commercial & 70 \\
\hline Industrial & 0 \\
\hline Construction & 0 \\
\hline Idle or vacant & 0 \\
\hline Wetland & 0 \\
\hline Parkland & 0 \\
\hline Detention storage, in acre-feet & - \\
\hline $\begin{array}{l}\text { Percentage of contributing drainage area upstream of } \\
\text { detention storage }\end{array}$ & - \\
\hline $\begin{array}{l}\text { Percentage of contributing drainage area drained by a } \\
\text { storm-sewer system }\end{array}$ & - \\
\hline Percentage of streets with curb and gutter drainage & - \\
\hline Percentage of streets with ditch and swale drainage & - \\
\hline Mean annual rainfall, in inches ${ }^{1}$ & 29.07 \\
\hline Ten-year, 1-hour rainfall intensity, in inches per hour & - \\
\hline Mean minimum January temperature, in degrees Fahrenheit ${ }^{1}$ & 11.4 \\
\hline Two-year, 24-hour rainfall, in inches ${ }^{2}$ & 2.6 \\
\hline $\begin{array}{l}\text { Mean annual nitrogen precipitation load, in pounds of } \\
\text { nitrogen per acre }{ }^{3}\end{array}$ & 6.10 \\
\hline
\end{tabular}

${ }^{1}$ Data from National Oceanic and Atmospheric Administration, 1980 .

${ }^{2}$ Data from Hershfield, 1961.

${ }^{3}$ Data derived as sum of total deposition of ammonia and of nitrate (National Atmospheric Deposition Program, NADP Subcommittee Number 3 -- Data Management and Analysis, 1985). 
Table 211.--Basin characteristics of station 04232040

Irondequoit Creek near Pittsford, N.Y.

[Dashes indicate data not available. Except where footnoted, data is from magnetic tape described by Driver and others (1985)]

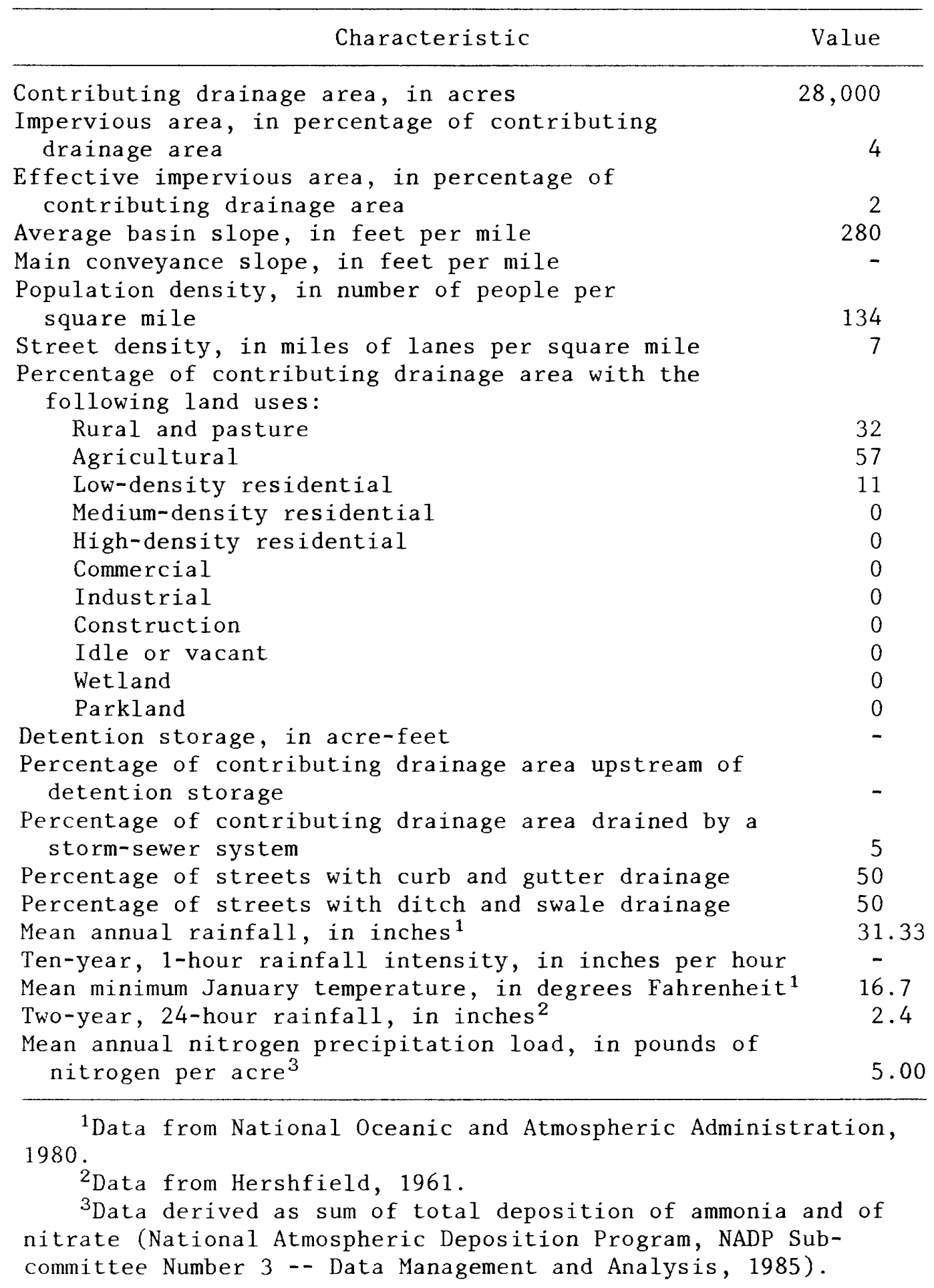


[Dashes indicate data not available. Except where footnoted, data is from magnetic tape described by Driver and others $(1985)$ ]

\begin{tabular}{|c|c|}
\hline Characteristic & Value \\
\hline Contributing drainage area, in acres & 18,000 \\
\hline $\begin{array}{l}\text { Impervious area, in percentage of contributing } \\
\text { drainage area }\end{array}$ & 11 \\
\hline $\begin{array}{l}\text { Effective impervious area, in percentage of } \\
\text { contributing drainage area }\end{array}$ & 2 \\
\hline Average basin slope, in feet per mile & 232 \\
\hline ain conveyance slope, in feet per mile & 16 \\
\hline $\begin{array}{l}\text { Population density, in number of people per } \\
\text { square mile }\end{array}$ & 864 \\
\hline $\begin{array}{l}\text { Street density, in miles of lanes per square mile } \\
\text { Percentage of contributing drainage area with the } \\
\quad \text { following land uses: }\end{array}$ & 7 \\
\hline Rural and pasture & 47 \\
\hline ultural & 26 \\
\hline ensity residential & 23 \\
\hline Medium-density residential & 0 \\
\hline High-density residential & 0 \\
\hline Commercial & 4 \\
\hline Industrial & 0 \\
\hline ruction & 0 \\
\hline Idle or vacant & 0 \\
\hline Wet 1 and & 0 \\
\hline Parkland & 0 \\
\hline Detention storage, in acre-feet & - \\
\hline $\begin{array}{l}\text { Percentage of contributing drainage area upstream of } \\
\text { detention storage }\end{array}$ & - \\
\hline $\begin{array}{l}\text { Percentage of contributing drainage area drained by a } \\
\text { storm-sewer system }\end{array}$ & 10 \\
\hline Percentage of streets with curb and gutter drainage & 50 \\
\hline Percentage of streets with ditch and swale drainage & 50 \\
\hline Mean annual rainfall, in inches ${ }^{1}$ & 31.33 \\
\hline Ten-year, 1-hour rainfall intensity, in inches per hour & - \\
\hline Mean minimum January temperature, in degrees $F$ ahrenheit ${ }^{1}$ & 16.7 \\
\hline Two-year, 24-hour rainfall, in inches ${ }^{2}$ & 2.4 \\
\hline $\begin{array}{l}\text { Mean annual nitrogen } p \\
\text { nitrogen per acre }\end{array}$ & 5.00 \\
\hline
\end{tabular}

${ }^{1}$ Data from National Oceanic and Atmospheric Administration, 1980 .

${ }^{2}$ Data from Hershfield, 1961.

${ }^{3}$ Data derived as sum of total deposition of ammonia and of nitrate (National Atmospheric Deposition Program, NADP Subcommittee Number 3 -- Data Management and Analysis, 1985). 
Table 213.--Basin characteristics of station 06413700 Rapid Creek above water-treatment plant at Rapid City, S. Dak. ${ }^{1}$

[Dashes indicate data not available. Except where footnoted, data is from magnetic tape described by Driver and others (1985)]

\begin{tabular}{|c|c|}
\hline Characteristic & Value \\
\hline Contributing drainage area, in acres & 21,000 \\
\hline $\begin{array}{l}\text { Impervious area, in percentage of contributing } \\
\text { drainage area }\end{array}$ & 9 \\
\hline $\begin{array}{l}\text { Effective impervious area, in percentage of } \\
\text { contributing drainage area }\end{array}$ & - \\
\hline Average basin slope, in feet per mile & - \\
\hline Main conveyance slope, in feet per mile & - \\
\hline $\begin{array}{l}\text { Population density, in number of people per } \\
\text { square mile }\end{array}$ & - \\
\hline Street density, in miles of lanes per square mile & - \\
\hline $\begin{array}{l}\text { Percentage of contributing drainage area with the } \\
\text { following land uses: }\end{array}$ & \\
\hline Rural and pasture & 78 \\
\hline Agricultural & 0 \\
\hline Low-density residential & 9 \\
\hline Medium-density residential & 4 \\
\hline High-density residential & 4 \\
\hline ccial & 0 \\
\hline rial & 0 \\
\hline Construction & 0 \\
\hline Idle or vacant & 1 \\
\hline Wetland & 0 \\
\hline Parkland & 4 \\
\hline Detention storage, in acre-feet & - \\
\hline $\begin{array}{l}\text { Percentage of contributing drainage area upstream of } \\
\text { detention storage }\end{array}$ & - \\
\hline $\begin{array}{l}\text { Percentage of contributing drainage area drained by a } \\
\text { storm-sewer system }\end{array}$ & - \\
\hline Percentage of streets with curb and gutter drainage & - \\
\hline Percentage of streets with ditch and swale drainage & - \\
\hline Mean annual rainfall, in inches ${ }^{2}$ & 17. \\
\hline Ten-year, 1 -hour rainfall intensity, in inches per hour & - \\
\hline Mean minimum January temperature, in degrees Fahrenheit ${ }^{2}$ & 9.6 \\
\hline Two-year, 24-hour rainfall, in inches ${ }^{3}$ & 3.2 \\
\hline Mean annual nitrogen precipitation load, i & \\
\hline
\end{tabular}

${ }^{1}$ Characteristics pertain to the drainage basin below 06412500 Rapid Creek above Canyon Lake near Rapid City.

${ }^{2}$ Data from National Oceanic and Atmospheric Administration, 1980 .

${ }^{3}$ Data from Hershfield, 1961.

${ }^{4}$ Data derived as sum of total deposition of ammonia and of nitrate (National Atmospheric Deposition Program, NADP Subcommittee Number 3 -- Data Management and Analysis, 1985). 
Table 214.--Basin characteristics of station 06414000 Rapid Creek at Rapid City, S. Dak. ${ }^{1}$

[Dashes indicate data not available. Except where footnoted, data is from magnetic tape described by Driver and others $(1.985)$ ]

\begin{tabular}{|c|c|}
\hline Characteristic & Value \\
\hline Contributing drainage area, in acres & 3,900 \\
\hline $\begin{array}{l}\text { Impervious area, in percentage of contributing } \\
\text { drainage area }\end{array}$ & 24 \\
\hline $\begin{array}{l}\text { Effective impervious area, in percentage of } \\
\text { contributing drainage area }\end{array}$ & - \\
\hline Average basin slope, in feet per mile & - \\
\hline Main conveyance slope, in feet per mile & - \\
\hline $\begin{array}{l}\text { Population density, in number of people per } \\
\text { square mile }\end{array}$ & - \\
\hline $\begin{array}{l}\text { Street density, in miles of lanes per square mile } \\
\text { Percentage of contributing drainage area with the } \\
\text { following land uses: }\end{array}$ & - \\
\hline Rura1 and pasture & 50 \\
\hline Agricultural & 10 \\
\hline Low-density residential & 0 \\
\hline Medium-density residential & 1 \\
\hline High-density residential & 1 \\
\hline Commercial & 13 \\
\hline Industrial & 5 \\
\hline Construction & 12 \\
\hline Idle or vacant & 3 \\
\hline Wetland & 0 \\
\hline Parkland & 5 \\
\hline Detention storage, in acre-feet & - \\
\hline $\begin{array}{l}\text { Percentage of contributing drainage area upstream of } \\
\text { detention storage }\end{array}$ & - \\
\hline $\begin{array}{l}\text { Percentage of contributing drainage area drained by a } \\
\text { storm-sewer system }\end{array}$ & - \\
\hline Percentage of streets with curb and gutter drainage & - \\
\hline Percentage of streets with ditch and swale drainage & - \\
\hline Mean annual rainfall, in inches ${ }^{2}$ & 17.12 \\
\hline Ten-year, 1-hour rainfall intensity, in inches per hour & - \\
\hline Mean minimum January temperature, in degrees Fahrenheit ${ }^{2}$ & 9.6 \\
\hline Two-year, 24-hour rainfall, in inches ${ }^{3}$ & 3.2 \\
\hline $\begin{array}{l}\text { Mean annual nitrogen precipitation load, in pounds of } \\
\text { nitrogen per acre } 4\end{array}$ & 1.50 \\
\hline
\end{tabular}

${ }^{1}$ Characteristics pertain to the drainage basin below 06413700 Rapid Creek above water treatment plant at Rapid City.

${ }^{2}$ Data from National Oceanic and Atmospheric Administration, 1980.

${ }^{3}$ Data from Hershfield, 1961.

${ }^{4}$ Data derived as sum of total deposition of ammonia and of nitrate (National Atmospheric Deposition Program, NADP Subcommittee Number 3 -- Data Management and Analysis, 1985). 
Table 215.--Basin characteristics of station 06414700 Rapid Creek at East Main Street at Rapid City, S. Dak. ${ }^{1}$

[Dashes indicate data not available. Except where footnoted, data is from magnetic tape described by Driver and others (1985)]

\begin{tabular}{|c|c|}
\hline Characteristic & Value \\
\hline Contributing drainage area, in acres & 3,600 \\
\hline $\begin{array}{l}\text { Impervious area, in percentage of contributing } \\
\text { drainage area }\end{array}$ & 45 \\
\hline $\begin{array}{l}\text { Effective impervious area, in percentage of } \\
\text { contributing drainage area }\end{array}$ & - \\
\hline Average basin slope, in feet per mile & - \\
\hline Main conveyance slope, in feet per mile & - \\
\hline $\begin{array}{l}\text { Population density, in number of people per } \\
\text { square mile }\end{array}$ & - \\
\hline Street density, in miles of lanes per square mile & - \\
\hline $\begin{array}{l}\text { Percentage of contributing drainage area with the } \\
\text { following land uses: }\end{array}$ & \\
\hline Rural and pasture & 32 \\
\hline Agricultura 1 & 0 \\
\hline Low-density residential & 4 \\
\hline Medium-density residential & 16 \\
\hline High-density residential & 16 \\
\hline Commercial & 13 \\
\hline Indu & 0 \\
\hline ruction & 0 \\
\hline Idle or vacant & 9 \\
\hline Wetland & 0 \\
\hline Parkland & 10 \\
\hline Detention storage, in acre-feet & - \\
\hline $\begin{array}{l}\text { Percentage of contributing drainage area upstream of } \\
\text { detention storage }\end{array}$ & - \\
\hline $\begin{array}{l}\text { Percentage of contributing drainage area drained by a } \\
\text { storm-sewer system }\end{array}$ & - \\
\hline Percentage of streets with curb and gutter drainage & - \\
\hline Percentage of streets with ditch and swale drainage & - \\
\hline Mean annual rainfall, in inches ${ }^{2}$ & 17.1 \\
\hline Ten-year, 1-hour rainfall intensity, in inches per hour & - \\
\hline Mean minimum January temperature, in degrees Fahrenheit ${ }^{2}$ & 9.6 \\
\hline Two-year, 24-hour rainfall, in inches ${ }^{3}$ & 3.2 \\
\hline $\begin{array}{l}\text { Mean annual nitrogen precipitation load, in } \mathrm{p} \\
\text { nitrogen per acre }\end{array}$ & 1.50 \\
\hline
\end{tabular}

${ }^{1}$ Characteristics pertain to the drainage basin below 06414000 Rapid Creek at Rapid City.

${ }^{2}$ Data from National Oceanic and Atmospheric Administration, 1980 .

${ }^{3}$ Data from Hershfield, 1961.

${ }^{4}$ Data derived as sum of total deposition of ammonia and of nitrate (National Atmospheric Deposition Program, NADP Subcommittee Number 3 -- Data Management and Analysis, 1985). 
Table 216.--Basin characteristics of station 06416000 Rapid Creek below Hawthorn ditch at Rapid City, S. Dak. ${ }^{1}$

[Dashes indicate data not available. Except where footnoted, data is from magnetic tape described by Driver and others (1985)]

\begin{tabular}{|c|c|}
\hline Characteristic & Value \\
\hline Contributing drainage area, in acres & 1,600 \\
\hline $\begin{array}{l}\text { Impervious area, in percentage of contributing } \\
\text { drainage area }\end{array}$ & 40 \\
\hline $\begin{array}{l}\text { Effective impervious area, in percentage of } \\
\text { contributing drainage area }\end{array}$ & - \\
\hline Average basin slope, in feet per mile & - \\
\hline Main conveyance slope, in feet per mile & - \\
\hline $\begin{array}{l}\text { Population density, in number of people per } \\
\text { square mile }\end{array}$ & - \\
\hline $\begin{array}{l}\text { Street density, in miles of lanes per square mile } \\
\text { Percentage of contributing drainage area with the } \\
\text { following land uses: }\end{array}$ & - \\
\hline Rural and pasture & 16 \\
\hline Agricultural & 3 \\
\hline Low-density residential & 1 \\
\hline Medium-density residential & 10 \\
\hline High-density residential & 9 \\
\hline Commercial & 26 \\
\hline Industrial & 0 \\
\hline Construction & 0 \\
\hline Idle or vacant & 25 \\
\hline Wetland & 0 \\
\hline Parkland & 10 \\
\hline Detention storage, in acre-feet & - \\
\hline $\begin{array}{l}\text { Percentage of contributing drainage area upstream of } \\
\text { detention storage }\end{array}$ & - \\
\hline $\begin{array}{l}\text { Percentage of contributing drainage area drained by a } \\
\text { storm-sewer system }\end{array}$ & - \\
\hline Percentage of streets with curb and gutter drainage & - \\
\hline Percentage of streets with ditch and swale drainage & - \\
\hline Mean annual rainfall, in inches ${ }^{2}$ & 17.12 \\
\hline Ten-year, 1 -hour rainfall intensity, in inches per hour & - \\
\hline Mean minimum January temperature, in degrees Fahrenheit ${ }^{2}$ & 9.6 \\
\hline Two-year, 24-hour rainfall, in inches ${ }^{3}$ & 3.2 \\
\hline $\begin{array}{l}\text { Mean annual nitrogen precipitation load, in pounds of } \\
\text { nitrogen per acre }\end{array}$ & 1.50 \\
\hline
\end{tabular}

${ }^{1}$ Characteristics pertain to the drainage basin below 06414700 Rapid Creek at East Main Street at Rapid City.

${ }^{2}$ Data from National Oceanic and Atmospheric Administration, 1980 .

${ }^{3}$ Data from Hershfield, 1961.

${ }^{4}$ Data derived as sum of total deposition of ammonia and of nitrate (National Atmospheric Deposition Program, NADP Subcommittee Number 3 -- Data Management and Analysis, 1985). 
Table 217.--Basin characteristics of station 06710200

Big Dry Creek tributary at Littleton, Colo.

[Dashes indicate data not available. Except where footnoted, data is from magnetic tape described by Driver and others (1985)]

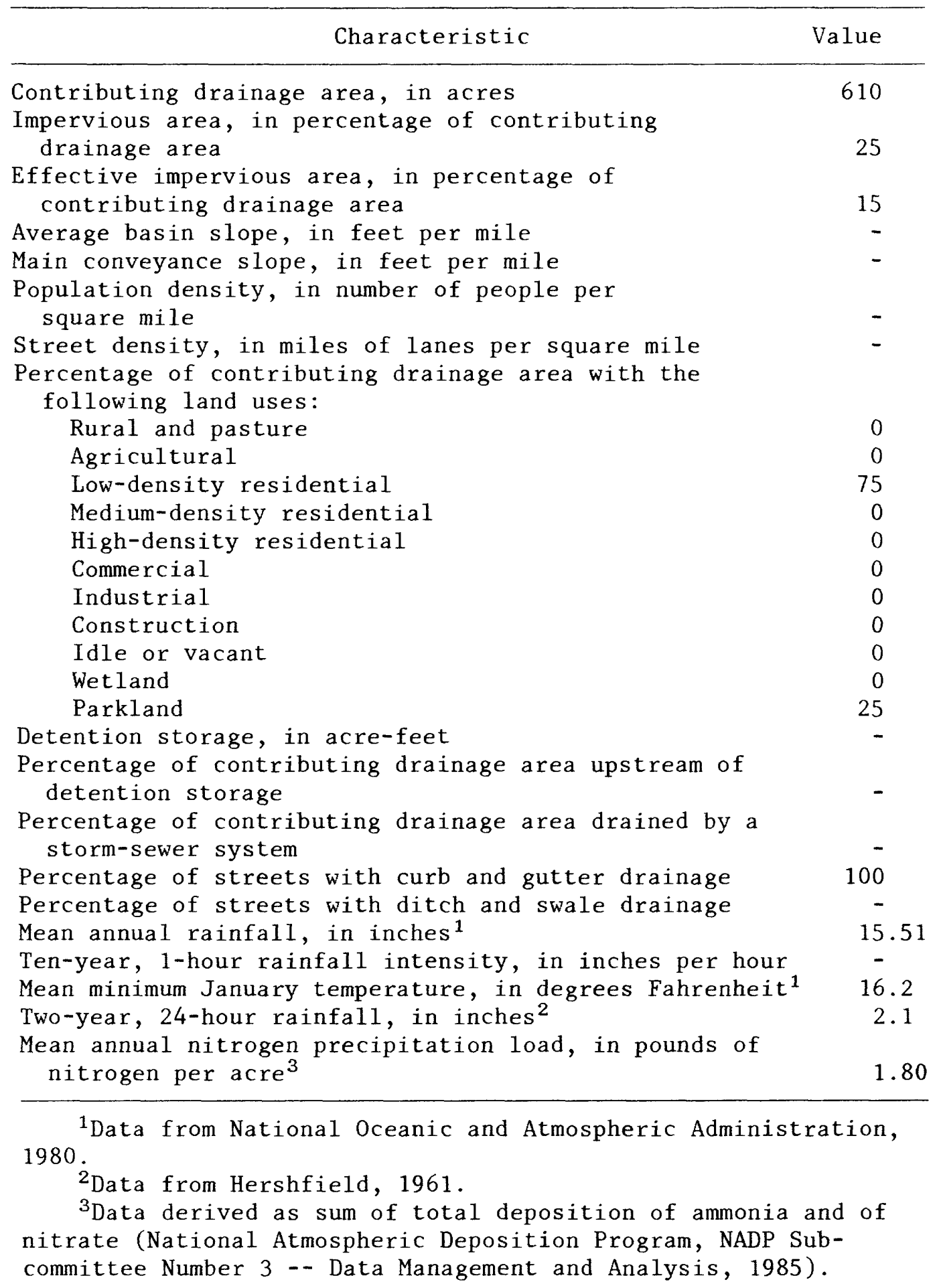


Table 218.--Basin characteristics of station 06710225 Big Dry Creek tributary at Easter street near Littleton, Colo.

[Dashes indicate data not available. Except where footnoted, data is from magnetic tape described by Driver and others (1985)]

\begin{tabular}{|c|c|}
\hline Characteristic & Value \\
\hline Contributing drainage area, in acres & 33 \\
\hline $\begin{array}{l}\text { Impervious area, in percentage of contributing } \\
\text { drainage area }\end{array}$ & 64 \\
\hline $\begin{array}{l}\text { Effective impervious area, in percentage of } \\
\text { contributing drainage area }\end{array}$ & 41 \\
\hline Average basin slope, in feet per mile & - \\
\hline Main conveyance slope, in feet per mile & - \\
\hline $\begin{array}{l}\text { Population density, in number of people per } \\
\text { square mile }\end{array}$ & 12,344 \\
\hline $\begin{array}{l}\text { Street density, in miles of lanes per square mile } \\
\text { Percentage of contributing drainage area with the } \\
\text { following land uses: }\end{array}$ & - \\
\hline Rural and pasture & 0 \\
\hline Agricultural & 0 \\
\hline Low-density residential & 0 \\
\hline Medium-density residential & 0 \\
\hline High-density residential & 100 \\
\hline Commercial & 0 \\
\hline Industrial & 0 \\
\hline Construction & 0 \\
\hline Idle or vacant & 0 \\
\hline Wetland & 0 \\
\hline Parkland & 0 \\
\hline Detention storage, in acre-feet & - \\
\hline $\begin{array}{l}\text { Percentage of contributing drainage area upstream of } \\
\text { detention storage }\end{array}$ & - \\
\hline $\begin{array}{l}\text { Percentage of contributing drainage area drained by a } \\
\text { storm-sewer system }\end{array}$ & - \\
\hline Percentage of streets with curb and gutter drainage & - \\
\hline Percentage of streets with ditch and swale drainage & - \\
\hline Mean annual rainfall, in inches ${ }^{1}$ & 15.51 \\
\hline Ten-year, 1 -hour rainfall intensity, in inches per hour & - \\
\hline Mean minimum January temperature, in degrees Fahrenheit ${ }^{1}$ & 16.2 \\
\hline Two-year, 24-hour rainfall, in inches ${ }^{2}$ & 2.1 \\
\hline $\begin{array}{l}\text { Mean annual nitrogen precipitation load, in pounds of } \\
\text { nitrogen per acre }\end{array}$ & 1.80 \\
\hline
\end{tabular}

${ }^{1}$ Data from National Oceanic and Atmospheric Administration, 1980.

${ }^{2}$ Data from Hershfield, 1961.

${ }^{3}$ Data derived as sum of total deposition of ammonia and of nitrate (National Atmospheric Deposition Program, NADP Subcommittee Number 3 -- Data Management and Analysis, 1985). 
Table 219.--Basin characteristics of station 06710610 Rooney Gulch at Rooney Ranch near Morrison, Colo.

[Dashes indicate data not available. Except where footnoted, data is from magnetic tape described by Driver and others (1985)]

\begin{tabular}{|c|c|}
\hline Characteristic & Value \\
\hline Contributing drainage area, in acres & 400 \\
\hline $\begin{array}{l}\text { Impervious area, in percentage of contributing } \\
\text { drainage area }\end{array}$ & - \\
\hline Effective impervious area, in percentage of & 1 \\
\hline Average basin slope, in feet per mile & - \\
\hline Main conveyance slope, in feet per mile & - \\
\hline $\begin{array}{l}\text { Population density, in number of people per } \\
\text { square mile }\end{array}$ & 0 \\
\hline $\begin{array}{l}\text { Street density, in miles of lanes per square mile } \\
\text { Percentage of contributing drainage area with the } \\
\text { following land uses: }\end{array}$ & - \\
\hline Rural and pasture & 100 \\
\hline Agricultural & 0 \\
\hline Low-density residential & 0 \\
\hline Medium-density residential & 0 \\
\hline High-density residential & 0 \\
\hline Commercial & 0 \\
\hline Industrial. & 0 \\
\hline Construction & 0 \\
\hline Idle or vacant & 0 \\
\hline Wet 1 and & 0 \\
\hline Parkland & 0 \\
\hline Detention storage, in acre-feet & 0 \\
\hline $\begin{array}{l}\text { Percentage of contributing drainage area upstream of } \\
\text { detention storage }\end{array}$ & - \\
\hline $\begin{array}{l}\text { Percentage of contributing drainage area drained by a } \\
\text { storm-sewer system }\end{array}$ & 0 \\
\hline Percentage of streets with curb and gutter drainage & 0 \\
\hline Percentage of streets with ditch and swale drainage & - \\
\hline Mean annual rainfall, in inches 1 & 15.51 \\
\hline Ten-year, 1-hour rainfall intensity, in inches per hour & - \\
\hline Mean minimum January temperature, in degrees Fahrenheit ${ }^{1}$ & 16.2 \\
\hline Two-year, 24-hour rainfall, in inches ${ }^{2}$ & 2.1 \\
\hline $\begin{array}{l}\text { Mean annual nitrogen precipitation load, in pounds of } \\
\text { nitrogen per acre }\end{array}$ & 1.80 \\
\hline
\end{tabular}

${ }^{1}$ Data from National Oceanic and Atmospheric Administration, 1980 .

${ }^{2}$ Data from Hershfield, 1961.

${ }^{3}$ Data derived as sum of total deposition of ammonia and of nitrate (National Atmospheric Deposition Program, NADP Subcommittee Number 3 -- Data Management and Analysis, 1985). 
Table 220.--Basin characteristics of station 06711586 Asbury Park storm drain at Asbury Avenue at Denver, Colo.

[Dashes indicate data not available. Except where footnoted, data is from magnetic tape described by Driver and others (1985)]

\begin{tabular}{|c|c|}
\hline Characteristic & Value \\
\hline Contributing drainage area, in acres & 130 \\
\hline $\begin{array}{l}\text { Impervious area, in percentage of contributing } \\
\text { drainage area }\end{array}$ & 31 \\
\hline $\begin{array}{l}\text { Effective impervious area, in percentage of } \\
\text { contributing drainage area }\end{array}$ & 22 \\
\hline Average basin slope, in feet per mile & - \\
\hline Main conveyance slope, in feet per mile & - \\
\hline $\begin{array}{l}\text { Population density, in number of people per } \\
\text { square mile }\end{array}$ & 5,938 \\
\hline $\begin{array}{l}\text { Street density, in miles of lanes per square mile } \\
\text { Percentage of contributing drainage area with the } \\
\text { following land uses: }\end{array}$ & - \\
\hline Rural and pasture & 0 \\
\hline Agricultural & 0 \\
\hline Low-density residential & 50 \\
\hline Medium-density residential & 32 \\
\hline High-density residential & 0 \\
\hline Commercial & 13 \\
\hline Industrial & 0 \\
\hline Construction & 0 \\
\hline Idle or vacant & 0 \\
\hline Wetland & 0 \\
\hline Parkland & 5 \\
\hline Detention storage, in acre-feet & 2 \\
\hline $\begin{array}{l}\text { Percentage of contributing drainage area upstream of } \\
\text { detention storage }\end{array}$ & 100 \\
\hline $\begin{array}{l}\text { Percentage of contributing drainage area drained by a } \\
\text { storm-sewer system }\end{array}$ & - \\
\hline Percentage of streets with curb and gutter drainage & - \\
\hline Percentage of streets with ditch and swale arainage & - \\
\hline Mean annual rainfall, in inches ${ }^{1}$ & 15.51 \\
\hline $\begin{array}{l}\text { Ten-year, 1-hour rainfall intensity, in inches per hour } \\
\text { Mean minimum January temperature, in degrees Fahrenheit }{ }^{1}\end{array}$ & $\overline{16.2}$ \\
\hline Two-year, 24 -hour rainfall, in inches 2 & 2.1 \\
\hline $\begin{array}{l}\text { Mean annual nitrogen precipitation load, in pounds of } \\
\text { nitrogen per acre }\end{array}$ & \\
\hline
\end{tabular}
1980.

${ }^{1}$ Data from National Oceanic and Atmospheric Administration,

${ }^{2}$ Data from Hershfield, 1961.

${ }^{3}$ Data derived as sum of total deposition of ammonia and of nitrate (National Atmospheric Deposition Program, NADP Subcommittee Number 3 -- Data Management and Analysis, 1985). 
Table 221.--Basin characteristics during 1976-77 of station 06711635 North Avenue storm drain at Denver Federal Center at Lakewood, Colo.

[Dashes indicate data not available. Except where footnoted, data is from magnetic tape described by Driver and others (1985)]

\begin{tabular}{|c|c|}
\hline Characteristic & Value \\
\hline Contributing drainage area, in acres & 77 \\
\hline $\begin{array}{l}\text { Impervious area, in percentage of contributing } \\
\text { drainage area }\end{array}$ & 40 \\
\hline $\begin{array}{l}\text { Effective impervious area, in percentage of } \\
\text { contributing drainage area }\end{array}$ & 30 \\
\hline Average basin slope, in feet per mile & - \\
\hline Main conveyance slope, in feet per mile & - \\
\hline $\begin{array}{l}\text { Population density, in number of people per } \\
\text { square mile }\end{array}$ & - \\
\hline $\begin{array}{l}\text { Street density, in miles of lanes per square mile } \\
\text { Percentage of contributing drainage area with the } \\
\text { following land uses: }\end{array}$ & - \\
\hline Rural and pasture & 0 \\
\hline Agricultural & 0 \\
\hline Low-density residential & 0 \\
\hline Medium-density residential & 0 \\
\hline High-density residential & 30 \\
\hline Commercial & 20 \\
\hline Industrial & 0 \\
\hline Construction & 0 \\
\hline Idle or vacant & 50 \\
\hline Wetland & 0 \\
\hline Parkland & 0 \\
\hline Detention storage, in acre-feet & - \\
\hline $\begin{array}{l}\text { Percentage of contributing drainage area upstream of } \\
\text { detention storage }\end{array}$ & - \\
\hline $\begin{array}{l}\text { Percentage of contributing drainage area drained by a } \\
\text { storm-sewer system }\end{array}$ & - \\
\hline Percentage of streets with curb and gutter drainage & - \\
\hline Percentage of streets with ditch and swale drainage & - \\
\hline Mean annual rainfall, in inches ${ }^{1}$ & 15.51 \\
\hline Ten-year, 1-hour rainfall intensity, in inches per hour & - \\
\hline Mean minimum January temperature, in degrees Fahrenheit ${ }^{1}$ & 16.2 \\
\hline Two-year, 24 -hour rainfall, in inches ${ }^{2}$ & 2.1 \\
\hline $\begin{array}{l}\text { Mean annual nitrogen precipitation load, in pounds of } \\
\text { nitrogen per acre }\end{array}$ & 1.80 \\
\hline
\end{tabular}

${ }^{1}$ Data from National Oceanic and Atmospheric Administration, 1980 .

${ }^{2}$ Data from Hershfield, 1961

${ }^{3}$ Data derived as sum of total deposition of ammonia and of nitrate (National Atmospheric Deposition Program, NADP Subcommittee Number 3 -- Data Management and Analysis, 1985). 
Table 222.--Basin characteristics during 1980-81 of station 06711635 North Avenue storm drain at Denver Federal Center at Lakewood, Colo.

[Dashes indicate data not available. Except where footnoted, data is from magnetic tape described by Driver and others (1985)]

\begin{tabular}{|c|c|}
\hline Characteristic & Value \\
\hline Contributing drainage area, in acres & 69 \\
\hline $\begin{array}{l}\text { Impervious area, in percentage of contributing } \\
\text { drainage area }\end{array}$ & 59 \\
\hline $\begin{array}{l}\text { Effective impervious area, in percentage of } \\
\text { contributing drainage area }\end{array}$ & 50 \\
\hline Average basin slope, in feet per mile & - \\
\hline Main conveyance slope, in feet per mile & - \\
\hline $\begin{array}{l}\text { Population density, in number of people per } \\
\text { square mile }\end{array}$ & 5,938 \\
\hline $\begin{array}{l}\text { Street density, in miles of lanes per square mile } \\
\text { Percentage of contributing drainage area with the } \\
\text { following land uses: }\end{array}$ & - \\
\hline Rural and pasture & 0 \\
\hline Agricultural & 0 \\
\hline Low-density residential & 0 \\
\hline Medium-density residential & 0 \\
\hline High-density residential & 33 \\
\hline Commercial & 30 \\
\hline Industrial & 0 \\
\hline Construction & 0 \\
\hline Idle or vacant & 37 \\
\hline Wetland & 0 \\
\hline Parkland & 0 \\
\hline Detention storage, in acre-feet & 0 \\
\hline $\begin{array}{l}\text { Percentage of contributing drainage area upstream of } \\
\text { detention storage }\end{array}$ & 100 \\
\hline $\begin{array}{l}\text { Percentage of contributing drainage area drained by a } \\
\text { storm-sewer system }\end{array}$ & - \\
\hline Percentage of streets with curb and gutter drainage & - \\
\hline Percentage of streets with ditch and swale drainage & - \\
\hline Mean annual rainfall, in inches 1 & 15.51 \\
\hline $\begin{array}{l}\text { Ten-year, } 1 \text {-hour rainfall intensity, in inches per hour } \\
\text { Mean minimum January temperature, in degrees Fahrenheit }{ }^{1}\end{array}$ & $\overline{16.2}$ \\
\hline Two-year, 24 -hour rainfall, in inches ${ }^{2}$ & 2.1 \\
\hline $\begin{array}{l}\text { Mean annual nitrogen precipitation load, in pounds of } \\
\text { nitrogen per acre }\end{array}$ & \\
\hline
\end{tabular}

${ }^{1}$ Data from National Oceanic and Atmospheric Administration, 1980 .

${ }^{2}$ Data from Hershfield, 1961.

${ }^{3}$ Data derived as sum of total deposition of ammonia and of nitrate (National Atmospheric Deposition Program, NADP Subcommittee Number 3 -- Data Management and Analysis, 1985). 
Table 223.--Basin characteristics of station 06711637

North Avenue storm drain at Denver Federal Center

North Avenue at Lakewood, Colo.

[Dashes indicate data not available. Except where footnoted, data is from magnetic tape described by Driver and others (1985)]

\begin{tabular}{|c|c|}
\hline Characteristic & Value \\
\hline Contributing drainage area, in acres & 80 \\
\hline $\begin{array}{l}\text { Impervious area, in percentage of contributing } \\
\text { drainage area }\end{array}$ & 54 \\
\hline $\begin{array}{l}\text { Effective impervious area, in percentage of } \\
\text { contributing drainage area }\end{array}$ & 46 \\
\hline Average basin slope, in feet per mile & - \\
\hline Main conveyance slope, in feet per mile & - \\
\hline $\begin{array}{l}\text { Population density, in number of people per } \\
\text { square mile }\end{array}$ & 5,000 \\
\hline Street density, in miles of lanes per square mile & - \\
\hline $\begin{array}{l}\text { Percentage of contributing drainage area with the } \\
\text { following land uses: }\end{array}$ & \\
\hline Rural and pasture & 0 \\
\hline Agricultural & 0 \\
\hline Low-density residential & 0 \\
\hline Medium-density residential & 0 \\
\hline High-density residential & 28 \\
\hline Commercial & 26 \\
\hline Industrial & 0 \\
\hline Construction & 7 \\
\hline Idle or vacant & 39 \\
\hline Wetland & 0 \\
\hline Parkland & 0 \\
\hline Detention storage, in acre-feet & 5 \\
\hline $\begin{array}{l}\text { Percentage of contributing drainage area upstream of } \\
\text { detention storage }\end{array}$ & 100 \\
\hline $\begin{array}{l}\text { Percentage of contributing drainage area drained by a } \\
\text { storm-sewer system }\end{array}$ & - \\
\hline Percentage of streets with curb and gutter drainage & - \\
\hline Percentage of streets with ditch and swale drainage & - \\
\hline Mean annual rainfall, in inches ${ }^{1}$ & 15.51 \\
\hline Ten-year, 1 -hour rainfall intensity, in inches per hour & - \\
\hline Mean minimum January temperature, in degrees Fahrenheit ${ }^{1}$ & 16.2 \\
\hline Two-year, 24-hour rainfall, in inches ${ }^{2}$ & 2.1 \\
\hline $\begin{array}{l}\text { Nean anmual nitrogen precipitation load, in pounds of } \\
\text { nitrogen per acre }\end{array}$ & 1.80 \\
\hline
\end{tabular}

${ }^{1}$ Data from National Oceanic and Atmospheric Administration, 1980 .

${ }^{2}$ Data from Hershfield, 1961.

${ }^{3}$ Data derived as sum of total deposition of ammonia and of nitrate (National Atmospheric Deposition Program, NADP Subconmittee Number 3 -- Data Management and Analysis, 1985). 
Table 224.--Basin characteristics of station 06713010

Cherry Knolls storm drain at Denver, Colo.

[Dashes indicate data not available. Except where footnoted, data is from magnetic tape described by Driver and others $(1985)]$

\begin{tabular}{|c|c|}
\hline Characteristic & Value \\
\hline Contributing drainage area, in acres & 57 \\
\hline $\begin{array}{l}\text { Impervious area, in percentage of contributing } \\
\text { drainage area }\end{array}$ & 64 \\
\hline $\begin{array}{l}\text { Effective impervious area, in percentage of } \\
\text { contributing drainage area }\end{array}$ & 38 \\
\hline Average basin slope, in feet per mile & - \\
\hline Main conveyance slope, in feet per mile & - \\
\hline $\begin{array}{l}\text { Population density, in number of people per } \\
\text { square mile }\end{array}$ & 15,625 \\
\hline $\begin{array}{l}\text { Street density, in miles of lanes per square mile } \\
\text { Percentage of contributing drainage area with the } \\
\text { following land uses: }\end{array}$ & - \\
\hline Rural and pasture & 0 \\
\hline Agricultural & 0 \\
\hline Low-density residential & 0 \\
\hline Medium-density residential & 0 \\
\hline High-density residential & 100 \\
\hline Commercial & 0 \\
\hline Industrial & 0 \\
\hline Construction & 0 \\
\hline Idle or vacant & 0 \\
\hline Wetland & 0 \\
\hline Parkland & 0 \\
\hline Detention storage, in acre-feet & - \\
\hline $\begin{array}{l}\text { Percentage of contributing drainage area upstream of } \\
\text { detention storage }\end{array}$ & - \\
\hline $\begin{array}{l}\text { Percentage of contributing drainage area drained by a } \\
\text { storm-sewer system }\end{array}$ & - \\
\hline Percentage of streets with curb and gutter drainage & 100 \\
\hline Percentage of streets with ditch and swale drainage & - \\
\hline Mean annual rainfall, in inches ${ }^{1}$ & 15.51 \\
\hline $\begin{array}{l}\text { Ten-year, 1-hour rainfall intensity, in inches per hour } \\
\text { Mean minimum January temperature, in degrees Fahrenheit }{ }^{1}\end{array}$ & 16.2 \\
\hline Two-year, 24-hour rainfall, in inches ${ }^{2}$ & 2.1 \\
\hline $\begin{array}{l}\text { Mean annual nitrogen precipitation load, in pounds of } \\
\text { nitrogen per acre }\end{array}$ & 1.80 \\
\hline
\end{tabular}

${ }^{1}$ Data from National Oceanic and Atmospheric Administration, 1980 .

${ }^{2}$ Data from Hershfield, 1961.

${ }^{3}$ Data derived as sum of total deposition of ammonia and of nitrate (National Atmospheric Deposition Program, NADP Subcommittee Number 3 -- Data Management and Analysis, 1985). 
Table 225.--Basin characteristics of station 06714100 36 th street storm drain at Denver, Colo.

[Dashes indicate data not available. Except where footnoted, data is from magnetic tape described by Driver and others (1985)]

\begin{tabular}{|c|c|}
\hline Characteristic & Value \\
\hline Contributing drainage area, in acres & 2,200 \\
\hline $\begin{array}{l}\text { Impervious area, in percentage of contributing } \\
\text { drainage area }\end{array}$ & 65 \\
\hline $\begin{array}{l}\text { Effective impervious area, in percentage of } \\
\text { contributing drainage area }\end{array}$ & 40 \\
\hline Average basin slope, in feet per mile & - \\
\hline Main conveyance slope, in feet per mile & - \\
\hline $\begin{array}{l}\text { Population density, in number of people per } \\
\text { square mile }\end{array}$ & - \\
\hline Street density, in miles of lanes per square mile & - \\
\hline $\begin{array}{l}\text { Percentage of contributing drainage area with the } \\
\text { following land uses: }\end{array}$ & \\
\hline Rural and pasture & 0 \\
\hline Agricultural & 0 \\
\hline lensity residential & 0 \\
\hline Medium-density residential & 37 \\
\hline High-density residential & 37 \\
\hline Commercial & 20 \\
\hline Industrial & 0 \\
\hline action & 0 \\
\hline Idle or vacant & 0 \\
\hline Wetland & 0 \\
\hline Parkland & 6 \\
\hline Detention storage, in acre-feet & - \\
\hline $\begin{array}{l}\text { Percentage of contributing drainage area upstream of } \\
\text { detention storage }\end{array}$ & - \\
\hline $\begin{array}{l}\text { Percentage of contributing drainage area drained by a } \\
\text { storm-sewer system }\end{array}$ & - \\
\hline Percentage of streets with curb and gutter drainage & - \\
\hline Percentage of streets with ditch and swale drainage & - \\
\hline Mean annual rainfall, in inches ${ }^{1}$ & 15 . \\
\hline Ten-year, 1-hour rainfall intensity, in inches per hour & - \\
\hline Mean minimum January temperature, in degrees Fahrenheit ${ }^{1}$ & 16.2 \\
\hline Two-year, 24-hour rainfall, in inches ${ }^{2}$ & 2.1 \\
\hline Mean annual nitrogen precipitation load, in pounds of & \\
\hline
\end{tabular}

${ }^{1}$ Data from National Oceanic and Atmospheric Administration, 1980 .

${ }^{2}$ Data from Hershfield, 1961.

${ }^{3}$ Data derived as sum of total deposition of ammonia and of nitrate (National Atmospheric Deposition Program, NADP Subcommittee Number 3 -- Data Management and Analysis, 1985). 
Table 226.--Basin characteristics of station 06720330

Grange Hall Creek at Grant Park at Northglenn, Colo.

[Dashes indicate data not available. Except where footnoted, data is from magnetic tape described by Driver and others (1985)]

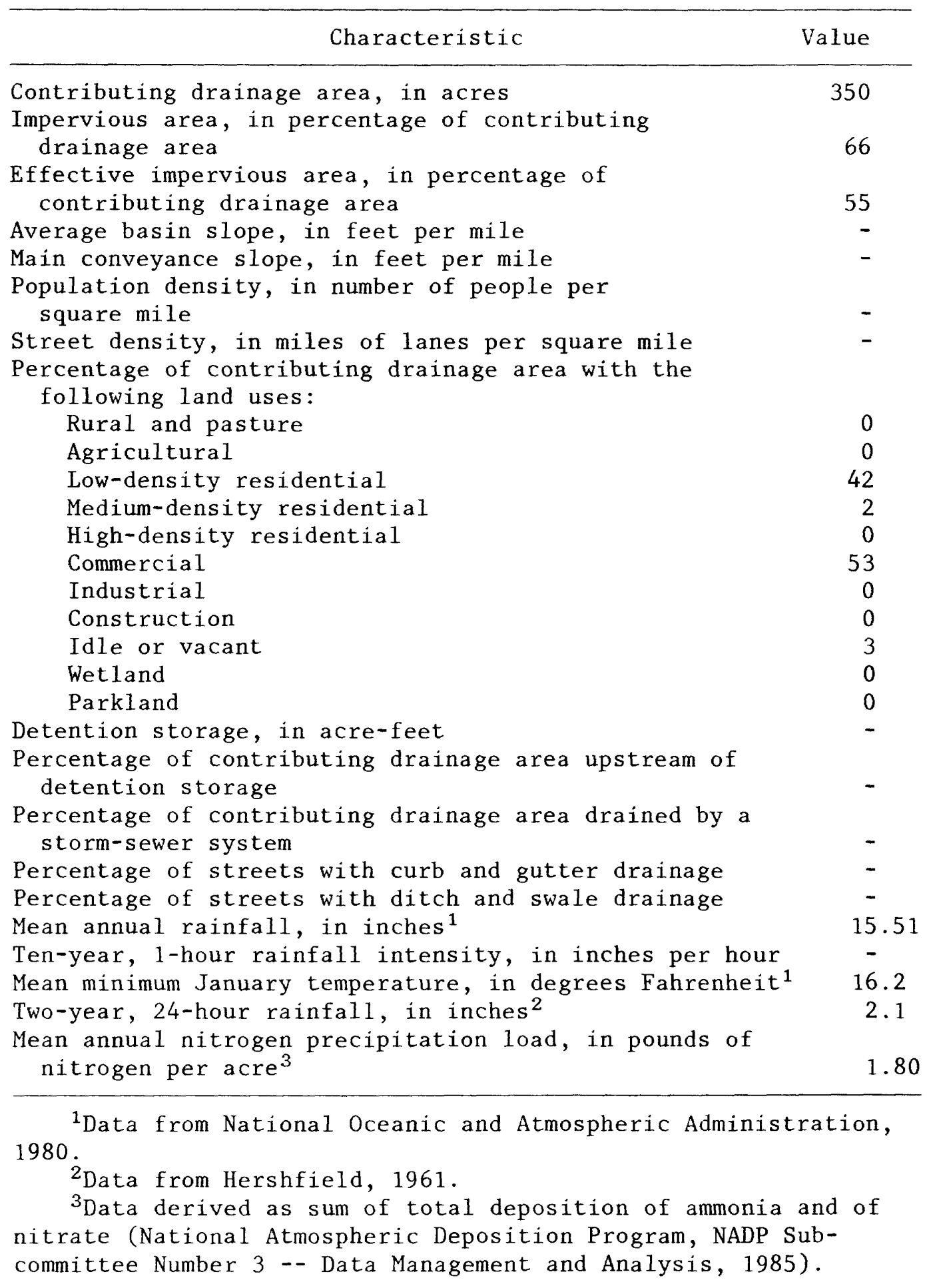


Table 227.--Basin characteristics of station 06720415

Grange Hall Creek at Northglenn, Colo.

[Dashes indicate data not available. Except where footnoted, data is from magnetic tape described by Driver and others (1985)]

\begin{tabular}{|c|c|}
\hline Characteristic & Value \\
\hline Contributing drainage area, in acres & 2,000 \\
\hline $\begin{array}{l}\text { Impervious area, in percentage of contributing } \\
\text { drainage area }\end{array}$ & 34 \\
\hline $\begin{array}{l}\text { Effective impervious area, in percentage of } \\
\text { contributing drainage area }\end{array}$ & 24 \\
\hline Average basin slope, in feet per mile & - \\
\hline Main conveyance slope, in feet per mile & - \\
\hline $\begin{array}{l}\text { Population density, in number of people per } \\
\text { square mile }\end{array}$ & - \\
\hline Street density, in miles of lanes per square mile & - \\
\hline $\begin{array}{l}\text { Percentage of contributing drainage area with the } \\
\text { following land uses: }\end{array}$ & \\
\hline Rural and pasture & 0 \\
\hline Agricultural & 0 \\
\hline Low-density residential & 49 \\
\hline Medium-density residential & 2 \\
\hline High-density residential & 0 \\
\hline Commercial & 14 \\
\hline Industrial & 0 \\
\hline Construction & 0 \\
\hline Idle or vacant & 35 \\
\hline Wetland & 0 \\
\hline Parkland & 0 \\
\hline Detention storage, in acre-feet & - \\
\hline $\begin{array}{l}\text { Percentage of contributing drainage area upstream of } \\
\text { detention storage }\end{array}$ & - \\
\hline $\begin{array}{l}\text { Percentage of contributing drainage area drained by a } \\
\text { storm-sewer system }\end{array}$ & - \\
\hline Percentage of streets with curb and gutter drainage & - \\
\hline Percentage of streets with ditch and swale drainage & - \\
\hline Mean annual rainfall, in inches ${ }^{1}$ & 15.51 \\
\hline Ten-year, 1-hour rainfall intensity, in inches per hour & - \\
\hline Mean minimum January temperature, in degrees Fahrenheit ${ }^{1}$ & 16.2 \\
\hline Two-year, 24 -hour rainfall, in inches ${ }^{2}$ & 2.1 \\
\hline $\begin{array}{l}\text { Mean annual nitrogen precipitation load, in pounds of } \\
\text { nitrogen per acre }\end{array}$ & 1.80 \\
\hline
\end{tabular}

${ }^{1}$ Data from National Oceanic and Atmospheric Administration, 1980 .

${ }^{2}$ Data from Hershfield, 1961.

${ }^{3}$ Data derived as sum of total deposition of ammonia and of nitrate (National Atmospheric Deposition Program, NADP Subcommittee Number 3 -- Data Management and Analysis, 1985). 
Table 228.--Basin characteristics of station 06720420

Storm drain at 116th Avenue and Claude Court

at Northglenn, Colo.

[Dashes indicate data not available. Except where footnoted, data is from magnetic tape described by Driver and others (1985)]

\begin{tabular}{|c|c|}
\hline Characteristic & Value \\
\hline Contributing drainage area, in acres & 170 \\
\hline $\begin{array}{l}\text { Impervious area, in percentage of contributing } \\
\text { drainage area }\end{array}$ & 37 \\
\hline $\begin{array}{l}\text { Effective impervious area, in percentage of } \\
\text { contributing drainage area }\end{array}$ & 24 \\
\hline Average basin slope, in feet per mile & - \\
\hline Main conveyance slope, in feet per mile & - \\
\hline $\begin{array}{l}\text { Population density, in number of people per } \\
\text { square mile }\end{array}$ & 9,219 \\
\hline $\begin{array}{l}\text { Street density, in miles of lanes per square mile } \\
\text { Percentage of contributing drainage area with the } \\
\text { following land uses: }\end{array}$ & - \\
\hline Rural and pasture & 0 \\
\hline Agricultural & 0 \\
\hline Low-density residential & 0 \\
\hline Medium-density residential & 100 \\
\hline High-density residential & 0 \\
\hline Commercial & 0 \\
\hline Industrial & 0 \\
\hline Construction & 0 \\
\hline Idle or vacant & 0 \\
\hline Wetland & 0 \\
\hline Parkland & 0 \\
\hline Detention storage, in acre-feet & - \\
\hline $\begin{array}{l}\text { Percentage of contributing drainage area upstream of } \\
\text { detention storage }\end{array}$ & - \\
\hline $\begin{array}{l}\text { Percentage of contributing drainage area drained by a } \\
\text { storm-sewer system }\end{array}$ & - \\
\hline Percentage of streets with curb and gutter drainage & - \\
\hline Percentage of streets with ditch and swale drainage & - \\
\hline Mean annual rainfall, in inches ${ }^{1}$ & 15.51 \\
\hline $\begin{array}{l}\text { Ten-year, 1-hour rainfall intensity, in inches per hour } \\
\text { Mean minimum January temperature, in degrees Fahrenheit }{ }^{1}\end{array}$ & $\overline{1} \cdot \mathbf{2}$ \\
\hline Two-year, 24-hour rainfall, in inches ${ }^{2}$ & 2.1 \\
\hline $\begin{array}{l}\text { Mean annual nitrogen precipitation load, in pounds of } \\
\text { nitrogen per acre }\end{array}$ & 1.80 \\
\hline
\end{tabular}
1980 .

${ }^{1}$ Data from National Oceanic and Atmospheric Administration,

${ }^{2}$ Data from Hershfield, 1961.

${ }^{3}$ Data derived as sum of total deposition of ammonia and of nitrate (National Atmospheric Deposition Program, NADP Subcommittee Number 3 -- Data Management and Analysis, 1985). 
Table 229.--Basin characteristics of station 06893520

Blue River near Gregory Boulevard at Kansas City, Mo.

[Dashes indicate data not available. Except where footnoted, data is from magnetic tape described by Driver and others (1985)]

\begin{tabular}{lc}
\hline \multicolumn{1}{c}{ Characteristic } & Value \\
\hline Contributing drainage area, in acres & 130,000 \\
Impervious area, in percentage of contributing & \\
drainage area & 70 \\
Effective impervious area, in percentage of & - \\
contributing drainage area & - \\
Average basin slope, in feet per mile & - \\
Main conveyance slope, in feet per mile & - \\
Population density, in number of people per & - \\
square mile & \\
Street density, in miles of lanes per square mile & 0 \\
Percentage of contributing drainage area with the & 7 \\
following land uses: & 8 \\
Rural and pasture & 9 \\
Agricultural & 1 \\
Low-density residential & 2 \\
Medium-density residential & 2 \\
Commercial & 0 \\
Industrial & - \\
Construction & - \\
Idle or vacant &
\end{tabular}
1980 .

${ }^{1}$ Data from National Oceanic and Atmospheric Administration,

${ }^{2}$ Data from Hershfield, 1961.

${ }^{3}$ Data derived as sum of total deposition of ammonia and of nitrate (National Atmospheric Deposition Program, NADP Subcommittee Number 3 -- Data Management and Analysis, 1985). 
Table 230.--Basin characteristics of station 06893566

Blue River at Coal Mine Road at Kansas City, Mo.

[Dashes indicate data not available. Except where footnoted, data is from magnetic tape described by Driver and others (1985)]

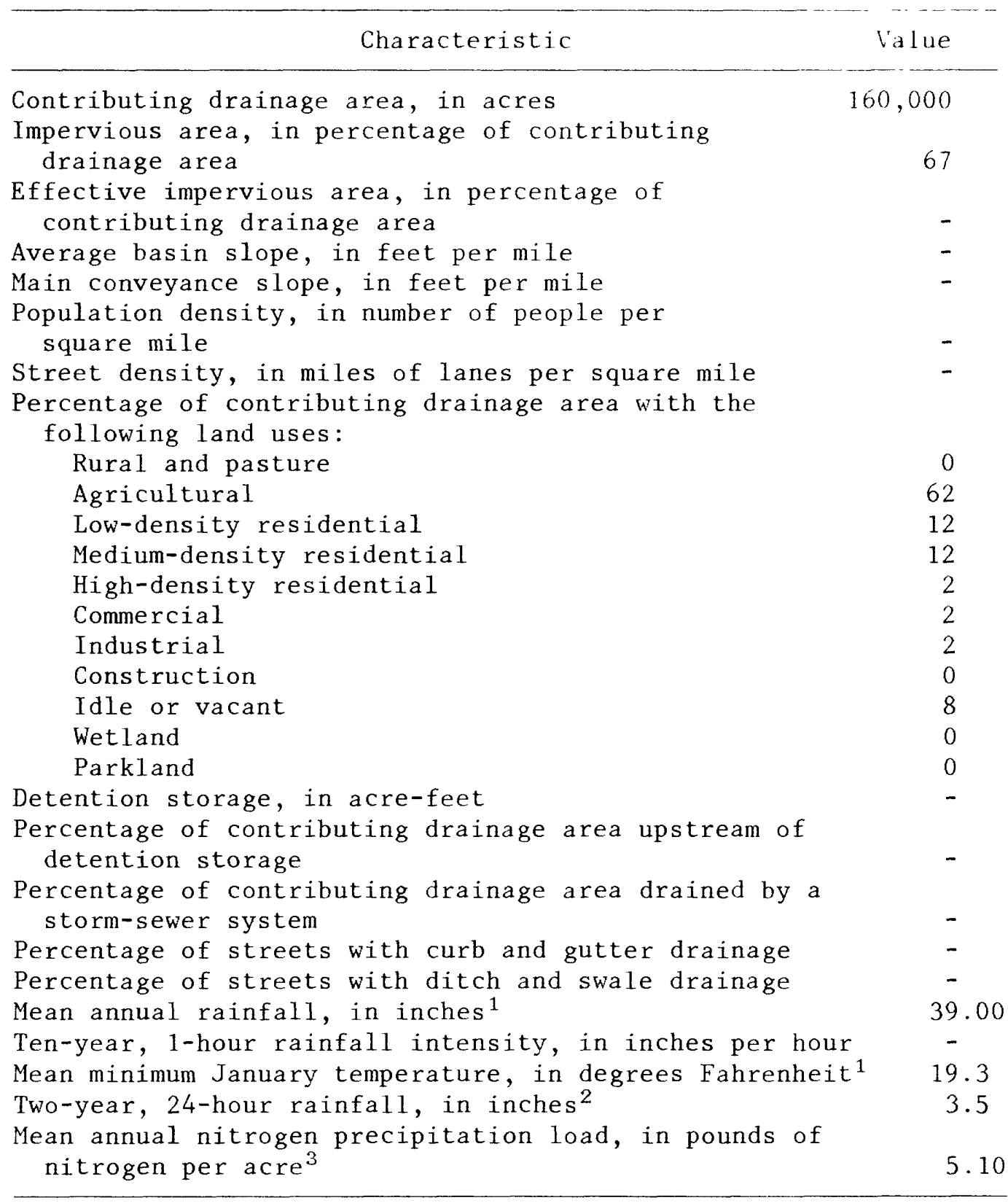
1980

${ }^{1}$ Data from National Oceanic and Atmospheric Administration,

${ }^{2}$ Data from Hershfield, 1961.

${ }^{3}$ Data derived as sum of total deposition of ammonia and of nitrate (National Atmospheric Deposition Program, NADP Subcommittee Number 3 -- Data Management and Analysis, 1985). 
Table 231.--Basin characteristics of station 06893575

Blue Ridge Mall storm sewer at Kansas City, Mo.

[Dashes indicate data not available. Except where footnoted, data is from magnetic tape described by Driver and others (1985)]

\section{Characteristic}

Contributing drainage area, in acres

Impervious area, in percentage of contributing drainage area

Effective impervious area, in percentage of contributing drainage area

Average basin slope, in feet per mile

Main conveyance slope, in feet per mile

Population density, in number of people per square mile

Street density, in miles of lanes per square mile

Percentage of contributing drainage area with the following land uses:

Rural and pasture

Agricultural

Low-density residential

Medium-density residential

High-density residential

Ccmmercial

Industrial

Construction

Idle or vacant

Wetland

Parkland

Detention storage, in acre-feet

Percentage of contributing drainage area upstream of detention storage

Percentage of contributing drainage area drained by a storm-sewer system

Percentage of streets with curb and gutter drainage Percentage of streets with ditch and swale drainage Mean annual rainfall, in inches ${ }^{1}$

Ten-year, 1-hour rainfall intensity, in inches per hour Mean minimum January temperature, in degrees Fahrenheit ${ }^{1}$ Two-year, 24-hour rainfall, in inches?

Mean annual nitrogen precipitation load, in pounds of nitrogen per acre ${ }^{3}$

${ }^{1}$ Data from National Oceanic and Atmospheric Administration, 1980 .

${ }^{2}$ Data from Hershfield, 1961.

${ }^{3}$ Data derived as sum of total deposition of ammonia and of nitrate (National Atmospheric Deposition Program, NADP Subcommittee Number 3 -- Data Management and Analysis, 1985).
80

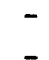

$-$

0

0

0

0

0

100

0

0

0

0

0

0

0

39.00

19.3

3.5

5.10 
Table 232.--Basin characteristics of station 08074400

Lazybrook street storm sewer at Houston, Tex.

[Dashes indicate data not available. Except where footnoted, data is from magnetic tape described by Driver and others (1985)]

\begin{tabular}{|c|c|}
\hline Characteristic & Value \\
\hline Contributing drainage area, in acres & 83 \\
\hline $\begin{array}{l}\text { Impervious area, in percentage of contributing } \\
\text { drainage area }\end{array}$ & 45 \\
\hline $\begin{array}{l}\text { Effective impervious area, in percentage of } \\
\text { contributing drainage area }\end{array}$ & - \\
\hline Average basin slope, in feet per mile & 12 \\
\hline $\begin{array}{l}\text { Main conveyance slope, in feet per mile } \\
\text { Population density, in number of people per }\end{array}$ & 17 \\
\hline square mile & 7,000 \\
\hline $\begin{array}{l}\text { Street density, in miles of lanes per square mile } \\
\text { Percentage of contributing drainage area with the } \\
\text { following land uses: }\end{array}$ & 71 \\
\hline Rural and pasture & 0 \\
\hline Agricultural & 0 \\
\hline Low-density residential & 0 \\
\hline Medium-density residential & 100 \\
\hline High-density residential & 0 \\
\hline Commercial & 0 \\
\hline Industrial & 0 \\
\hline Construction & 0 \\
\hline Idle or vacant & 0 \\
\hline Wet 1 and & 0 \\
\hline Parkland & 0 \\
\hline Detention storage, in acre-feet & - \\
\hline $\begin{array}{l}\text { Percentage of contributing drainage area upstream of } \\
\text { detention storage }\end{array}$ & - \\
\hline $\begin{array}{l}\text { Percentage of contributing drainage area drained by a } \\
\text { storm-sewer system }\end{array}$ & - \\
\hline Percentage of streets with curb and gutter drainage & - \\
\hline Percentage of streets with ditch and swale drainage & - \\
\hline Mean annual rainfall, in inches ${ }^{1}$ & 48.1 \\
\hline Ten-year, 1-hour rainfall intensity, in inches per hour & 3.4 \\
\hline Mean minimum January temperature, in degrees Fahrenheit ${ }^{1}$ & 24.8 \\
\hline Two-year, 24-hour rainfall, in inches ${ }^{2}$ & 5.2 \\
\hline $\begin{array}{l}\text { Mean annual nitrogen precipitation load, in pounds of } \\
\text { nitrogen per acre }\end{array}$ & \\
\hline
\end{tabular}

${ }^{1}$ Data from National Oceanic and Atmospheric Administration, 1980 .

${ }^{2}$ Data from Hershfield, 1961.

${ }^{3}$ Data derived as sum of total deposition of ammonia and of nitrate (National Atmospheric Deposition Program, NADP Subcommittee Number 3 -- Data Management and Analysis, 1985). 
Table 233.--Basin characteristics of station 08155300 Barton Creek at Loop 360 at Austin, Tex.

[Dashes indicate data not available. Except where footnoted, data is from magnetic tape described by Driver and others (1985)]

\begin{tabular}{|c|c|}
\hline Characteristic & Value \\
\hline Contributing drainage area, in acres & 74,000 \\
\hline $\begin{array}{l}\text { Impervious area, in percentage of contributing } \\
\text { drainage area }\end{array}$ & 1 \\
\hline $\begin{array}{l}\text { Effective impervious area, in percentage of } \\
\text { contributing drainage area }\end{array}$ & - \\
\hline Average basin slope, in feet per mile & - \\
\hline Main conveyance slope, in feet per mile & - \\
\hline $\begin{array}{l}\text { Population density, in number of people per } \\
\text { square mile }\end{array}$ & - \\
\hline Street density, in miles of lanes per square mile & - \\
\hline $\begin{array}{l}\text { Percentage of contributing drainage area with the } \\
\text { following land uses: }\end{array}$ & \\
\hline Rural and pasture & 0 \\
\hline Agricultural & 0 \\
\hline Low-density residential & 4 \\
\hline Medium-density residential & 0 \\
\hline High-density residential & 0 \\
\hline Commercial & 1 \\
\hline Industrial & 0 \\
\hline Construction & 0 \\
\hline Idle or vacant & 93 \\
\hline Wetland & 0 \\
\hline Parkland & 2 \\
\hline Detention storage, in acre-feet & - \\
\hline $\begin{array}{l}\text { Percentage of contributing drainage area upstream of } \\
\text { detention storage }\end{array}$ & - \\
\hline $\begin{array}{l}\text { Percentage of contributing drainage area drained by a } \\
\text { storm-sewer system }\end{array}$ & - \\
\hline Percentage of streets with curb and gutter drainage & - \\
\hline Percentage of streets with ditch and swale drainage & - \\
\hline Mean annual rainfall, in inches ${ }^{1}$ & 32.49 \\
\hline Ten-year, 1-hour rainfall intensity, in inches per hour & - \\
\hline Mean minimum January temperature, in degrees Fahrenheit ${ }^{1}$ & 39.3 \\
\hline Two-year, 24 -hour rainfall, in inches $^{2}$ & 4.5 \\
\hline $\begin{array}{l}\text { Mea annual nitrogen precipitation load, in pounds of } \\
\text { nitrogen per acre }\end{array}$ & 2.20 \\
\hline
\end{tabular}

${ }^{1}$ Data from National Oceanic and Atmospheric Administration, 1980 .

${ }^{2}$ Data from Hershfield, 1961.

${ }^{3}$ Data derived as sum of total deposition of ammonia and of nitrate (National Atmospheric Deposition Program, NADP Subcommittee Number 3 -- Data Management and Analysis, 1985). 
Table 234.--Basin characteristics of station 08156800 Shoal Creek at West 12th Street at Austin, Tex.

[Dashes indicate data not available. Except where footnoted, data is from magnetic tape described by Driver and others (1985)]

\begin{tabular}{|c|c|}
\hline Characteristic & Value \\
\hline Contributing drainage area, in acres & 7,900 \\
\hline $\begin{array}{l}\text { Impervious area, in percentage of contributing } \\
\text { drainage area }\end{array}$ & 43 \\
\hline $\begin{array}{l}\text { Effective impervious area, in percentage of } \\
\text { contributing drainage area }\end{array}$ & - \\
\hline Average basin slope, in feet per mile & - \\
\hline Main conveyance slope, in feet per mile & 32 \\
\hline $\begin{array}{l}\text { Population density, in number of people per } \\
\text { square mile }\end{array}$ & - \\
\hline Street density, in miles of lanes per square mile & - \\
\hline $\begin{array}{l}\text { Percentage of contributing drainage area with the } \\
\text { following land uses: }\end{array}$ & \\
\hline Rural and pasture & 0 \\
\hline Agricultural & 0 \\
\hline Low-density residential & 0 \\
\hline Medium-density residential & 42 \\
\hline High-density residential & 0 \\
\hline Commercial & 27 \\
\hline Industrial & 5 \\
\hline Construction & 0 \\
\hline Idle or vacant & 14 \\
\hline Wetland & 0 \\
\hline Parkland & 12 \\
\hline Detention storage, in acre-feet & - \\
\hline $\begin{array}{l}\text { Percentage of contributing drainage area upstream of } \\
\text { detention storage }\end{array}$ & - \\
\hline $\begin{array}{l}\text { Percentage of contributing drainage area drained by a } \\
\text { storm-sewer system }\end{array}$ & - \\
\hline Percentage of streets with curb and gutter drainage & - \\
\hline Percentage of streets with ditch and swale drainage & - \\
\hline Mean annual rainfall, in inches ${ }^{1}$ & 32.49 \\
\hline Ten-year, 1 -hour rainfall intensity, in inches per hour & - \\
\hline Mean minimum January temperature, in degrees Fahrenheit ${ }^{1}$ & 39.3 \\
\hline Two-year, 24-hour rainfall, in inches ${ }^{2}$ & 4.5 \\
\hline $\begin{array}{l}\text { Mean annual nitrogen precipitation load, in pounds of } \\
\text { nitrogen per acre }\end{array}$ & 2.20 \\
\hline
\end{tabular}
1980.

${ }^{1}$ Data from National Oceanic and Atmospheric Administration,

${ }^{2}$ Data from Hershfield, 1961.

${ }^{3}$ Data derived as sum of total deposition of ammonia and of nitrate (National Atmospheric Deposition Program, NADP Subcommittee Number 3 -- Data Management and Analysis, 1985). 
Table 235.--Basin characteristics of station 08329900 North floodway channel near Alameda, N. Mex.

[Dashes indicate data not available. Except where footnoted, data is from magnetic tape described by Driver and others (1985)]

\begin{tabular}{|c|c|}
\hline Characteristic & Value \\
\hline Contributing drainage area, in acres & 52,000 \\
\hline $\begin{array}{l}\text { Impervious area, in percentage of contributing } \\
\text { drainage area }\end{array}$ & 11 \\
\hline $\begin{array}{l}\text { Effective impervious area, in percentage of } \\
\text { contributing drainage area }\end{array}$ & 11 \\
\hline Average basin slope, in feet per mile & 360 \\
\hline Main conveyance slope, in feet per mile & 440 \\
\hline $\begin{array}{l}\text { Population density, in number of people per } \\
\text { square mile }\end{array}$ & 4,880 \\
\hline Street density, in miles of lanes per square mile & 20 \\
\hline $\begin{array}{l}\text { Percentage of contributing drainage area with the } \\
\text { following land uses: }\end{array}$ & \\
\hline Rural and pasture & 0 \\
\hline Agricultural & 0 \\
\hline Low-density residential & 15 \\
\hline Medium-density residential & 48 \\
\hline High-density residential & 0 \\
\hline Commercial & 2 \\
\hline Industrial & 0 \\
\hline Construction & 0 \\
\hline Idle or vacant & 27 \\
\hline Wetland & 0 \\
\hline Parkland & 8 \\
\hline Detention storage, in acre-feet & 2,400 \\
\hline $\begin{array}{l}\text { Percentage of contributing drainage area upstream of } \\
\text { detention storage }\end{array}$ & 50 \\
\hline $\begin{array}{l}\text { Percentage of contributing drainage area drained by a } \\
\text { storm-sewer system }\end{array}$ & - \\
\hline Percentage of streets with curb and gutter drainage & - \\
\hline Percentage of streets with ditch and swale drainage & - \\
\hline Mean annual rainfall, in inches ${ }^{1}$ & 7.77 \\
\hline Ten-year, 1-hour rainfall intensity, in inches per hour & 1.3 \\
\hline Mean minimum January temperature, in degrees Fahrenheit ${ }^{1}$ & 23.5 \\
\hline Two-year, 24 -hour rainfall, in inches ${ }^{2}$ & 1.1 \\
\hline $\begin{array}{l}\text { Mean annual nitrogen precipitation load, in pounds of } \\
\text { nitrogen per acre }{ }^{3}\end{array}$ & 1.80 \\
\hline
\end{tabular}

${ }^{1}$ Data from National Oceanic and Atmospheric Administration, 1980.

${ }^{2}$ Data from Hershfield, 1961.

${ }^{3}$ Data derived as sum of total deposition of ammonia and of nitrate (National Atmospheric Deposition Program, NADP Subcommittee Number 3 -- Data Management and Analysis, 1985). 
Table 236.--Basin characteristics of station 10167220

Bells Canyon conduit at 1000 East and 11000 South at Sandy, Utah

[Dashes indicate data not available. Except where footnoted, data is from magnetic tape described by Driver and others (1985)]

\begin{tabular}{|c|c|}
\hline Characteristic & Value \\
\hline Contributing drainage area, in acres & 64 \\
\hline $\begin{array}{l}\text { Impervious area, in percentage of contributing } \\
\text { drainage area }\end{array}$ & 52 \\
\hline $\begin{array}{l}\text { Effective impervious area, in percentage of } \\
\text { contributing drainage area }\end{array}$ & 23 \\
\hline Average basin slope, in feet per mile & 275 \\
\hline Main conveyance slope, in feet per mile & 312 \\
\hline $\begin{array}{l}\text { Population density, in number of people per } \\
\text { square mile }\end{array}$ & 9,100 \\
\hline $\begin{array}{l}\text { Street density, in miles of lanes per square mile } \\
\text { Percentage of contributing drainage area with the } \\
\text { following land uses: }\end{array}$ & - \\
\hline Rural and pasture & 0 \\
\hline Agricultural & 0 \\
\hline Low-density residential & 0 \\
\hline Medium-density residential & 83 \\
\hline High-density residential & 0 \\
\hline Commercial & 8 \\
\hline Industrial & 0 \\
\hline Construction & 0 \\
\hline Idle or vacant & 0 \\
\hline Wetland & 0 \\
\hline Parkland & 9 \\
\hline Detention storage, in acre-feet & - \\
\hline $\begin{array}{l}\text { Percentage of contributing drainage area upstream of } \\
\text { detention storage }\end{array}$ & - \\
\hline $\begin{array}{l}\text { Percentage of contributing drainage area drained by a } \\
\text { storm-sewer system }\end{array}$ & - \\
\hline Percentage of streets with curb and gutter drainage & - \\
\hline Percentage of streets with ditch and swale drainage & - \\
\hline Mean annual rainfall, in inches 1 & 17.00 \\
\hline Ten-year, 1 -hour rainfall intensity, in inches per hour & 0.86 \\
\hline Mean minimum January temperature, in degrees Fahrenheit ${ }^{1}$ & 18.5 \\
\hline Two-year, 24 -hour rainfall, in inches ${ }^{2}$ & 1.4 \\
\hline $\begin{array}{l}\text { Mean annual nitrogen precipitation load, in pounds of } \\
\text { nitrogen per acre }\end{array}$ & 1.00 \\
\hline
\end{tabular}
1980

${ }^{1}$ Data from National Oceanic and Atmospheric Administration,

${ }^{2}$ Data from Hershfield, 1961.

${ }^{3}$ Data derived as sum of total deposition of ammonia and of nitrate (National Atmospheric Deposition Program, NADP Subcommittee Number 3 -- Data Management and Analysis, 1985). 
Table 237.--Basin characteristics of station 10168000 Little Cottonwood Creek at Jordan River near Salt Lake City, Utah ${ }^{1}$

[Dashes indicate data not available. Except where footnoted, data is from magnetic tape described by Driver and others (1985)]

\begin{tabular}{|c|c|}
\hline Characteristic & Value \\
\hline Contributing drainage area, in acres & 9,000 \\
\hline $\begin{array}{l}\text { Impervious area, in percentage of contributing } \\
\text { drainage area }\end{array}$ & 59 \\
\hline $\begin{array}{l}\text { Effective impervious area, in percentage of } \\
\text { contributing drainage area }\end{array}$ & 16 \\
\hline Average basin slope, in feet per mile & 243 \\
\hline Main conveyance slope, in feet per mile & 60 \\
\hline $\begin{array}{l}\text { Population density, in number of people per } \\
\text { square mile }\end{array}$ & 4,580 \\
\hline Street density, in miles of lanes per square mile & - \\
\hline $\begin{array}{l}\text { Percentage of contributing drainage area with the } \\
\text { following land uses: }\end{array}$ & \\
\hline Rural and pasture & 3 \\
\hline Agricultural & 4 \\
\hline Low-density residential & 5 \\
\hline Medium-density residential & 38 \\
\hline High-density residential & 2 \\
\hline Commercial & 6 \\
\hline Industrial & 3 \\
\hline Construction & 2 \\
\hline Idle or vacant & 20 \\
\hline Wetland & 7 \\
\hline Parkland & 10 \\
\hline Detention storage, in acre-feet & - \\
\hline $\begin{array}{l}\text { Percentage of contributing drainage area upstream of } \\
\text { detention storage }\end{array}$ & - \\
\hline $\begin{array}{l}\text { Percentage of contributing drainage area drained by a } \\
\text { storm-sewer system }\end{array}$ & - \\
\hline Percentage of streets with curb and gutter drainage & - \\
\hline Percentage of streets with ditch and swale drainage & - \\
\hline Mean annual rainfall, in inches $^{2}$ & 18.10 \\
\hline Ten-year, 1-hour rainfall intensity, in inches per hour & 0.83 \\
\hline Mean minimum January temperature, in degrees Fahrenheit ${ }^{2}$ & 18.5 \\
\hline Two-year, 24-hour rainfall, in inches ${ }^{3}$ & 1.4 \\
\hline $\begin{array}{l}\text { Mean annual nitrogen precipitation load, in pounds of } \\
\text { nitrogen per acre } 4\end{array}$ & 1.00 \\
\hline
\end{tabular}

${ }^{1}$ Characteristics pertain only to the urban areas of the drainage basin.

\footnotetext{
${ }^{2}$ Data from National Oceanic and Atmospheric Administration, 1980 .

${ }^{3}$ Data from Hershfield, 1961.

${ }^{4}$ Data derived as sum of total deposition of ammonia and of nitrate (National Atmospheric Deposition Program, NADP Subcommittee Number 3 -- Data Management and Analysis, 1985).
} 
Table 238.--Basin characteristics of station 10168840 Holladay drain at 4800 South at Big Cottonwood Creek near Murray, Utah ${ }^{1}$

[Dashes indicate data not available. Except where footnoted, data is from magnetic tape described by Driver and others (1985)]

\begin{tabular}{|c|c|}
\hline Characteristic & Value \\
\hline Contributing drainage area, in acres & 2,600 \\
\hline $\begin{array}{l}\text { Impervious area, in percentage of contributing } \\
\text { drainage area }\end{array}$ & 54 \\
\hline $\begin{array}{l}\text { Effective impervious area, in percentage of } \\
\text { contributing drainage area }\end{array}$ & 23 \\
\hline Average basin slope, in feet per mile & 474 \\
\hline Main conveyance slope, in feet per mile & 264 \\
\hline $\begin{array}{l}\text { Population density, in number of people per } \\
\text { square mile }\end{array}$ & 8,320 \\
\hline $\begin{array}{l}\text { Street density, in miles of lanes per square mile } \\
\text { Percentage of contributing drainage area with the } \\
\text { following land uses: }\end{array}$ & - \\
\hline Rural and pasture & 1 \\
\hline Agricultural & 1 \\
\hline Low-density residential & 6 \\
\hline Medium-density residential & 69 \\
\hline High-density residential & 2 \\
\hline Commercial & 12 \\
\hline Industrial & 0 \\
\hline Construction & 2 \\
\hline Idle or vacant & 7 \\
\hline Wetland & 0 \\
\hline Parkland & 0 \\
\hline Detention storage, in acre-feet & - \\
\hline $\begin{array}{l}\text { Percentage of contributing drainage area upstream of } \\
\text { detention storage }\end{array}$ & - \\
\hline $\begin{array}{l}\text { Percentage of contributing drainage area drained by a } \\
\text { storm-sewer system }\end{array}$ & - \\
\hline Percentage of streets with curb and gutter drainage & - \\
\hline Percentage of streets with ditch and swale drainage & - \\
\hline Mean annual rainfall, in inches ${ }^{2}$ & 19.00 \\
\hline Ten-year, 1-hour rainfall intensity, in inches per hour & 0.95 \\
\hline Mean minimum January temperature, in degrees Fahrenheit ${ }^{2}$ & 18.5 \\
\hline Two-year, 24-hour rainfall, in inches ${ }^{3}$ & 1.4 \\
\hline $\begin{array}{l}\text { Mean annual nitrogen precipitation load, in pounds of } \\
\text { nitrogen per acre } 4\end{array}$ & 1.00 \\
\hline
\end{tabular}

${ }^{1}$ Characteristics pertain only to the urban areas of the drainage basin.

${ }^{2}$ Data from National Oceanic and Atmospheric Administration, 1980 .

${ }^{3}$ Data from Hershfield, 1961.

${ }^{4}$ Data derived as sum of total deposition of ammonia and of nitrate (National Atmospheric Deposition Program, NADP Subcommittee Number 3 -- Data Management and Analysis, 1985). 
Table 239.--Basin characteristics of station 10169500 Big Cottonwood Creek at Jordan River near Salt Lake City, Utah ${ }^{1}$

[Dashes indicate data not available. Except where footnoted, data is from magnetic tape described by Driver and others (1985)]

\begin{tabular}{|c|c|}
\hline Characteristic & Value \\
\hline Contributing drainage area, in acres & 11,000 \\
\hline $\begin{array}{l}\text { Impervious area, in percentage of contributing } \\
\text { drainage area }\end{array}$ & 54 \\
\hline $\begin{array}{l}\text { Effective impervious area, in percentage of } \\
\text { contributing drainage area }\end{array}$ & 21 \\
\hline Average basin slope, in feet per mile & 274 \\
\hline Main conveyance slope, in feet per mile & 61 \\
\hline $\begin{array}{l}\text { Population density, in number of people per } \\
\text { square mile }\end{array}$ & 8,450 \\
\hline Street density, in miles of lanes per square mile & - \\
\hline $\begin{array}{l}\text { Percentage of contributing drainage area with the } \\
\text { following land uses: }\end{array}$ & \\
\hline Rural and pasture & 2 \\
\hline Agricultural & 2 \\
\hline Low-density residential & 11 \\
\hline Medium-density residential & 57 \\
\hline High-density residential & 3 \\
\hline Commercial & 9 \\
\hline Industrial & 5 \\
\hline Construction & 2 \\
\hline Idle or vacant & 7 \\
\hline Wetland & 1 \\
\hline Parkland & 1 \\
\hline Detention storage, in acre-feet & - \\
\hline $\begin{array}{l}\text { Percentage of contributing drainage area upstream of } \\
\text { detention storage }\end{array}$ & - \\
\hline $\begin{array}{l}\text { Percentage of contributing drainage area drained by a } \\
\text { storm-sewer system }\end{array}$ & - \\
\hline Percentage of streets with curb and gutter drainage & - \\
\hline Percentage of streets with ditch and swale drainage & - \\
\hline Mean annual rainfall, in inches ${ }^{2}$ & 18.50 \\
\hline Ten-year, 1-hour rainfall intensity, in inches per hour & 0.83 \\
\hline Mean minimum January temperature, in degrees Fahrenheit ${ }^{2}$ & 18.5 \\
\hline Two-year, 24-hour rainfall, in inches ${ }^{3}$ & 1.4 \\
\hline $\begin{array}{l}\text { Mean annual nitrogen precipitation load, in pounds of } \\
\text { nitrogen per acre }\end{array}$ & 1.00 \\
\hline
\end{tabular}

${ }^{1}$ Characteristics pertain only to the urban areas of the drainage basin.

${ }^{2}$ Data from National Oceanic and Atmospheric Administration, 1980 .

${ }^{3}$ Data from Hershfield, 1961.

${ }^{4}$ Data derived as sum of total deposition of ammonia and of nitrate (National Atmospheric Deposition Program, NADP Subcommittee Number 3 -- Data Management and Analysis, 1985). 
Table 240.--Basin characteristics of station 10170900 21st South conduit at Jordan River at Salt Lake City, Utah ${ }^{1}$

[Dashes indicate data not available. Except where footnoted, data is from magnetic tape described by Driver and others (1985)]

\begin{tabular}{|c|c|}
\hline Characteristic & Value \\
\hline Contributing drainage area, in acres & 1,200 \\
\hline $\begin{array}{l}\text { Impervious area, in percentage of contributing } \\
\text { drainage area }\end{array}$ & 57 \\
\hline $\begin{array}{l}\text { Effective impervious area, in percentage of } \\
\text { contributing drainage area }\end{array}$ & 47 \\
\hline Average basin slope, in feet per mile & 38 \\
\hline Main conveyance slope, in feet per mile & 35 \\
\hline $\begin{array}{l}\text { Population density, in number of people per } \\
\text { square mile }\end{array}$ & 3,930 \\
\hline Street density, in miles of lanes per square mile & - \\
\hline $\begin{array}{l}\text { Percentage of contributing drainage area with the } \\
\text { following land uses: }\end{array}$ & \\
\hline Rural and pasture & 0 \\
\hline Agricultural & 0 \\
\hline Low-density residential & 0 \\
\hline Medium-density residential & 36 \\
\hline High-density residential & 0 \\
\hline Commercial & 30 \\
\hline Industrial & 32 \\
\hline Construction & 0 \\
\hline Idle or vacant & 0 \\
\hline Wet 1 and & 0 \\
\hline Parkland & 2 \\
\hline Detention storage, in acre-feet & - \\
\hline $\begin{array}{l}\text { Percentage of contributing drainage area upstream of } \\
\text { detention storage }\end{array}$ & - \\
\hline $\begin{array}{l}\text { Percentage of contributing drainage area drained by a } \\
\text { storm-sewer system }\end{array}$ & - \\
\hline Percentage of streets with curb and gutter drainage & - \\
\hline Percentage of streets with ditch and swale drainage & - \\
\hline Mean annual rainfall, in inches ${ }^{2}$ & 15.50 \\
\hline Ten-year, 1-hour rainfall intensity, in inches per hour & 0.88 \\
\hline Mean minimum January temperature, in degrees Fahrenheit ${ }^{2}$ & 18.5 \\
\hline Two-year, 24-hour rainfall, in inches ${ }^{3}$ & 1.4 \\
\hline $\begin{array}{l}\text { Mean annual nitrogen precipitation load, in pounds of } \\
\text { nitrogen per acre } 4\end{array}$ & 1.00 \\
\hline
\end{tabular}

${ }^{1}$ Characteristics pertain only to the urban areas of the drainage basin.

${ }^{2}$ Data from National Oceanic and Atmospheric Administration, 1980 .

${ }^{3}$ Data from Hershfield, 1961.

${ }^{4}$ Data derived as sum of total deposition of ammonia and of nitrate (National Atmospheric Deposition Program, NADP Subcommittee Number 3 -- Data Management and Analysis, 1985). 
Table 241.--Basin characteristics of station 10172372 8th South middle conduit at Jordan River at Salt Lake City, Utah

[Dashes indicate data not available. Except where footnoted, data is from magnetic tape described by Driver and others (1985)]

\begin{tabular}{|c|c|}
\hline Characteristic & Value \\
\hline Contributing drainage area, in acres & 1,500 \\
\hline $\begin{array}{l}\text { Impervious area, in percentage of contributing } \\
\text { drainage area }\end{array}$ & 73 \\
\hline $\begin{array}{l}\text { Effective impervious area, in percentage of } \\
\text { contributing drainage area }\end{array}$ & 44 \\
\hline Average basin slope, in feet per mile & 129 \\
\hline Main conveyance slope, in feet per mile & 72 \\
\hline $\begin{array}{l}\text { Population density, in number of people per } \\
\text { square mile }\end{array}$ & 2,450 \\
\hline $\begin{array}{l}\text { Street density, in miles of lanes per square mile } \\
\text { Percentage of contributing drainage area with the } \\
\text { following land uses: }\end{array}$ & - \\
\hline Rural and pasture & 0 \\
\hline Agricultural & 0 \\
\hline Low-density residential & 0 \\
\hline Medium-density residential & 22 \\
\hline High-density residential & 0 \\
\hline Commercial & 52 \\
\hline Industrial & 0 \\
\hline Construction & 0 \\
\hline Idle or vacant & 20 \\
\hline Wetland & 0 \\
\hline Parkland & 6 \\
\hline Detention storage, in acre-feet & - \\
\hline $\begin{array}{l}\text { Percentage of contributing drainage area upstream of } \\
\text { detention storage }\end{array}$ & - \\
\hline $\begin{array}{l}\text { Percentage of contributing drainage area drained by a } \\
\text { storm-sewer system }\end{array}$ & - \\
\hline Percentage of streets with curb and gutter drainage & - \\
\hline Percentage of streets with ditch and swale drainage & - \\
\hline Mean annual rainfall, in inches ${ }^{1}$ & 18.10 \\
\hline Ten-year, 1 -hour rainfall intensity, in inches per hour & 0.97 \\
\hline Mean minimum January temperature, in degrees Fahrenheit ${ }^{1}$ & 18.5 \\
\hline Two-year, 24-hour rainfall, in inches $^{2}$ & 1.4 \\
\hline $\begin{array}{l}\text { Mean annual nitrogen precipitation load, in pounds of } \\
\text { nitrogen per acre }\end{array}$ & 1.00 \\
\hline
\end{tabular}

${ }^{1}$ Data from National Oceanic and Atmospheric Administration, 1980 .

${ }^{2}$ Data from Hershfield, 1961.

${ }^{3}$ Data derived as sum of total deposition of ammonia and of nitrate (National Atmospheric Deposition Program, NADP Subcommittee Number 3 -- Data Management and Analysis, 1985). 
Table 242.--Basin characteristics of station 10172373 8th South north conduit at Jordan River at Salt Lake City, Utah

[Dashes indicate data not available. Except where footnoted, data is from magnetic tape described by Driver and others (1985)]

\begin{tabular}{|c|c|}
\hline Characteristic & Value \\
\hline Contributing drainage area, in acres & 510 \\
\hline $\begin{array}{l}\text { Impervious area, in percentage of contributing } \\
\text { drainage area }\end{array}$ & 76 \\
\hline $\begin{array}{l}\text { Effective impervious area, in percentage of } \\
\text { contributing drainage area }\end{array}$ & 70 \\
\hline Average basin slope, in feet per mile & 24 \\
\hline Main conveyance slope, in feet per mile & 9 \\
\hline $\begin{array}{l}\text { Population density, in number of people per } \\
\text { square mile }\end{array}$ & 2,010 \\
\hline $\begin{array}{l}\text { Street density, in miles of lanes per square mile } \\
\text { Percentage of contributing drainage area with the } \\
\text { following land uses: }\end{array}$ & - \\
\hline Rural and pasture & 0 \\
\hline Agricultural & 0 \\
\hline Low-density residential & 0 \\
\hline Medium-density residential & 16 \\
\hline High-density residential & 1 \\
\hline Commercial & 60 \\
\hline Industrial & 21 \\
\hline Construction & 0 \\
\hline Idle or vacant & 0 \\
\hline Wetland & 0 \\
\hline Parkland & 2 \\
\hline Detention storage, in acre-feet & - \\
\hline $\begin{array}{l}\text { Percentage of contributing drainage area upstream of } \\
\text { detention storage }\end{array}$ & - \\
\hline $\begin{array}{l}\text { Percentage of contributing drainage area drained by a } \\
\text { storm-sewer system }\end{array}$ & - \\
\hline Percentage of streets with curb and gutter drainage & - \\
\hline Percentage of streets with ditch and swale drainage & - \\
\hline Mean annual rainfall, in inches ${ }^{1}$ & 16.30 \\
\hline Ten-year, 1-hour rainfall intensity, in inches per hour & 0.92 \\
\hline Mean minimum January temperature, in degrees Fahrenheit ${ }^{1}$ & 18.5 \\
\hline Two-year, 24 -hour rainfall, in inches ${ }^{2}$ & 1.4 \\
\hline $\begin{array}{l}\text { Mean annual nitrogen precipitation load, in pounds of } \\
\text { nitrogen per acre }\end{array}$ & 1.00 \\
\hline
\end{tabular}

\footnotetext{
${ }^{1}$ Data from National Oceanic and Atmospheric Administration, 1980 .

${ }^{2}$ Data from Hershfield, 1961

${ }^{3}$ Data derived as sum of total deposition of ammonia and of nitrate (National Atmospheric Deposition Program, NADP Subcommittee Number 3 -- Data Management and Analysis, 1985).
} 
Table 243.--Basin characteristics of station 10172520 North Temple conduit at Jordan River at Salt Lake City, Utah ${ }^{1}$

[Dashes indicate data not available. Except where footnoted, data is from magnetic tape described by Driver and others (1985)]

\begin{tabular}{|c|c|}
\hline Characteristic & Value \\
\hline Contributing drainage area, in acres & 2,000 \\
\hline $\begin{array}{l}\text { Impervious area, in percentage of contributing } \\
\text { drainage area }\end{array}$ & 58 \\
\hline $\begin{array}{l}\text { Effective impervious area, in percentage of } \\
\text { contributing drainage area }\end{array}$ & 17 \\
\hline Average basin slope, in feet per mile & 684 \\
\hline Main conveyance slope, in feet per mile & 46 \\
\hline $\begin{array}{l}\text { Population density, in number of people per } \\
\text { square mile }\end{array}$ & 3,360 \\
\hline Street density, in miles of lanes per square mile & - \\
\hline $\begin{array}{l}\text { Percentage of contributing drainage area with the } \\
\text { following land uses: }\end{array}$ & \\
\hline Rural and pasture & 0 \\
\hline Agricultural & 0 \\
\hline Low-density residential & 16 \\
\hline Medium-density residential & 24 \\
\hline High-density residential & 2 \\
\hline Commercial & 11 \\
\hline Industrial & 6 \\
\hline Construction & 0 \\
\hline Idle or vacant & 30 \\
\hline Wetland & 0 \\
\hline Parkland & 11 \\
\hline Detention storage, in acre-feet & - \\
\hline $\begin{array}{l}\text { Percentage of contributing drainage area upstream of } \\
\text { detention storage }\end{array}$ & - \\
\hline $\begin{array}{l}\text { Percentage of contributing drainage area drained by a } \\
\text { storm-sewer system }\end{array}$ & - \\
\hline Percentage of streets with curb and gutter drainage & - \\
\hline Percentage of streets with ditch and swale drainage & - \\
\hline Mean annual rainfall, in inches ${ }^{2}$ & 19.00 \\
\hline Ten-year, 1-hour rainfall intensity, in inches per hour & 0.99 \\
\hline Mean minimum January temperature, in degrees Fahrenheit ${ }^{2}$ & 18.5 \\
\hline Two-year, 24-hour rainfall, in inches ${ }^{3}$ & 1.4 \\
\hline $\begin{array}{l}\text { Mean annual nitrogen precipitation load, in pounds of } \\
\text { nitrogen per acre } 4\end{array}$ & 1.00 \\
\hline
\end{tabular}

${ }^{1}$ Characteristics pertain only to the urban areas of the drainage basin.

\footnotetext{
${ }^{2}$ Data from National Oceanic and Atmospheric Administration, 1980 .

${ }^{3}$ Data from Hershfield, 1961.

${ }^{4}$ Data derived as sum of total deposition of ammonia and of nitrate (National Atmospheric Deposition Program, NADP Subcommittee Number 3 -- Data Management and Analysis, 1985).
} 
Table 245.--Basin characteristics of station 12119730

148 th Avenue Southeast storm sewer below Lake Hills

Boulevard at Bellevue, Wash.

[Dashes indicate data not available. Except where footnoted, data is from magnetic tape described by Driver and others (1985)]

\begin{tabular}{|c|c|}
\hline Characteristic & Value \\
\hline Contributing drainage area, in acres & 24 \\
\hline $\begin{array}{l}\text { Impervious area, in percentage of contributing } \\
\text { drainage area }\end{array}$ & 57 \\
\hline $\begin{array}{l}\text { Effective impervious area, in percentage of } \\
\text { contributing drainage area }\end{array}$ & 41 \\
\hline Average basin slope, in feet per mile & 92 \\
\hline Main conveyance slope, in feet per mile & 104 \\
\hline $\begin{array}{l}\text { Population density, in number of people per } \\
\text { square mile }\end{array}$ & 4,470 \\
\hline Street density, in miles of lanes per square mile & 81 \\
\hline $\begin{array}{l}\text { Percentage of contributing drainage area with the } \\
\text { following } 1 \text { and uses: }\end{array}$ & \\
\hline Rural and pasture & 0 \\
\hline Agricultural & 0 \\
\hline Low-density residential & 0 \\
\hline Medium-density residential & 2 \\
\hline High-density residential & 15 \\
\hline Commercial & 23 \\
\hline Industrial & 0 \\
\hline Construction & 2 \\
\hline Idle or vacant & 0 \\
\hline Wetland & 0 \\
\hline Parkland & 58 \\
\hline Detention storage, in acre-feet & 0 \\
\hline $\begin{array}{l}\text { Percentage of contributing drainage area upstream of } \\
\text { detention storage }\end{array}$ & 72 \\
\hline $\begin{array}{l}\text { Percentage of contributing drainage area drained by a } \\
\text { storm-sewer system }\end{array}$ & 100 \\
\hline Percentage of streets with curb and gutter drainage & 100 \\
\hline Percentage of streets with ditch and swale drainage & 0 \\
\hline Mean annual rainfall, in inches ${ }^{1}$ & 37.21 \\
\hline Ten-year, 1-hour rainfall intensity, in inches per hour & 0.51 \\
\hline Mean minimum January temperature, in degrees Fahrenheit ${ }^{1}$ & 33.9 \\
\hline Two-year, 24-hour rainfall, in inches ${ }^{2}$ & 2.0 \\
\hline $\begin{array}{l}\text { Mean annual nitrogen precipitation load, in pounds of } \\
\text { nitrogen per acre }\end{array}$ & 0.94 \\
\hline
\end{tabular}

${ }^{1}$ Data from National Oceanic and Atmospheric Administration, 1980.

${ }^{2}$ Data from Hershfield, 1961.

${ }^{3}$ Data derived as sum of total deposition of ammonia and of nitrate (National Atmospheric Deposition Program, NADP Subcommittee Number 3 -- Data Management and Analysis, 1985). 
Table 246.--Basin characteristics of station 12120005

Surrey Downs storm-sewer outfall at Bellevue, Wash.

[Dashes indicate data not available. Except where footnoted, data is from magnetic tape described by Driver and others (1985)]

\begin{tabular}{|c|c|}
\hline Characteristic & Value \\
\hline Contributing drainage area, in acres & 90 \\
\hline $\begin{array}{l}\text { Impervious area, in percentage of contributing } \\
\text { drainage area }\end{array}$ & 31 \\
\hline $\begin{array}{l}\text { Effective impervious area, in percentage of } \\
\text { contributing drainage area }\end{array}$ & 22 \\
\hline Average basin slope, in feet per mile & 570 \\
\hline Main conveyance slope, in feet per mile & 132 \\
\hline $\begin{array}{l}\text { Population density, in number of people per } \\
\text { square mile }\end{array}$ & 5,870 \\
\hline Street density, in miles of lanes per square mile & 45 \\
\hline $\begin{array}{l}\text { Percentage of contributing drainage area with the } \\
\text { following land uses: }\end{array}$ & \\
\hline Rural and pasture & 0 \\
\hline Agricultural & 0 \\
\hline Low-density residential & 2 \\
\hline Medium-density residential & 87 \\
\hline High-density residential & 2 \\
\hline Commercial & 6 \\
\hline Industrial & 0 \\
\hline Construction & 0 \\
\hline Idle or vacant & 1 \\
\hline Wet land & 0 \\
\hline Parkland & 2 \\
\hline Detention storage, in acre-feet & 0 \\
\hline $\begin{array}{l}\text { Percentage of contributing drainage area upstream of } \\
\text { detention storage }\end{array}$ & - \\
\hline $\begin{array}{l}\text { Percentage of contributing drainage area drained by a } \\
\text { storm-sewer system }\end{array}$ & 100 \\
\hline Percentage of streets with curb and gutter drainage & 76 \\
\hline Percentage of streets with ditch and swale drainage & 24 \\
\hline Mean annual rainfall, in inches ${ }^{1}$ & 37.2 \\
\hline Ten-year, 1-hour rainfall intensity, in inches per hour & 0.5 \\
\hline Mean minimum January temperature, in degrees Fahrenheit ${ }^{1}$ & 33.9 \\
\hline Two-year, 24-hour rainfall, in inches $^{2}$ & 2.0 \\
\hline $\begin{array}{l}\text { Mean annual nitrogen precipitation load, in pounds of } \\
\text { nitrogen per acre }\end{array}$ & 0.9 \\
\hline
\end{tabular}

${ }^{1}$ Data from National Oceanic and Atmospheric Administration, 1980 .

${ }^{2}$ Data from Hershfield, 1961.

${ }^{3}$ Data derived as sum of total deposition of ammonia and of nitrate (National Atmospheric Deposition Program, NADP Subcommittee Number 3 -- Data Management and Analysis, 1985). 
Table 247.--Basin characteristics of station 14206330

Beaverton Creek tributary at Southwest Murray

Boulevard in Beaverton, oreg.

[Dashes indicate data not available. Except where footnoted, data is from magnetic tape described by Driver and others (1985)]

\begin{tabular}{|c|c|}
\hline Characteristic & Value \\
\hline Contributing drainage area, in acres & 130 \\
\hline $\begin{array}{l}\text { Impervious area, in percentage of contributing } \\
\text { drainage area }\end{array}$ & 19 \\
\hline $\begin{array}{l}\text { Effective impervious area, in percentage of } \\
\text { contributing drainage area }\end{array}$ & - \\
\hline Average basin slope, in feet per mile & 390 \\
\hline Main conveyance slope, in feet per mile & 180 \\
\hline $\begin{array}{l}\text { Population density, in number of people per } \\
\text { square mile }\end{array}$ & - \\
\hline Street density, in miles of lanes per square mile & 37 \\
\hline $\begin{array}{l}\text { Percentage of contributing drainage area with the } \\
\text { following land uses: }\end{array}$ & \\
\hline Rural and pasture & 21 \\
\hline Agricultural & 0 \\
\hline Low-density residential & 0 \\
\hline Medium-density residential & 53 \\
\hline High-density residential & 5 \\
\hline Commercial & 0 \\
\hline Industrial & 0 \\
\hline Construction & 0 \\
\hline Idle or vacant & 0 \\
\hline Wetland & 0 \\
\hline Parkland & 21 \\
\hline Detention storage, in acre-feet & 0 \\
\hline $\begin{array}{l}\text { Percentage of contributing drainage area upstream of } \\
\text { detention storage }\end{array}$ & 0 \\
\hline $\begin{array}{l}\text { Percentage of contributing drainage area drained by a } \\
\text { storm-sewer system }\end{array}$ & 75 \\
\hline Percentage of streets with curb and gutter drainage & 0 \\
\hline Percentage of streets with ditch and swale drainage & 0 \\
\hline Mean annual rainfall, in inches ${ }^{1}$ & 37.61 \\
\hline Ten-year, 1-hour rainfall intensity, in inches per hour & - \\
\hline Mean minimum January temperature, in degrees Fahrenheit ${ }^{1}$ & 32.5 \\
\hline Two-year, 24-hour rainfall, in inches ${ }^{2}$ & 2.6 \\
\hline $\begin{array}{l}\text { Mean annual nitrogen precipitation load, in pounds of } \\
\text { nitrogen per acre }{ }^{3}\end{array}$ & 0.94 \\
\hline \multicolumn{2}{|c|}{$\begin{array}{l}{ }^{1} \text { Data from National Oceanic and Atmospheric Administration, } \\
1980 . \\
{ }^{2} \text { Data from Hershfield, } 1961 \text {. } \\
{ }^{3} \text { Data derived as sum of total deposition of ammonia and of } \\
\text { nitrate (National Atmospheric Deposition Program, NADP Sub- } \\
\text { committee Number } 3 \text {-- Data Management and Analysis, 1985). }\end{array}$} \\
\hline
\end{tabular}


Table 248.--Basin characteristics of station 14206900

Fanno Creek at Southwest 56th Avenue in Portland, Oreg.

[Dashes indicate data not available. Except where footnoted, data is from magnetic tape described by Driver and others (1985)]

\begin{tabular}{|c|c|}
\hline Characteristic & Value \\
\hline Contributing drainage area, in acres & 1,500 \\
\hline $\begin{array}{l}\text { Impervious area, in percentage of contributing } \\
\text { drainage area }\end{array}$ & 32 \\
\hline $\begin{array}{l}\text { Effective impervious area, in percentage of } \\
\text { contributing drainage area }\end{array}$ & - \\
\hline Average basin slope, in feet per mile & 860 \\
\hline Main conveyance slope, in feet per mile & 230 \\
\hline $\begin{array}{l}\text { Population density, in number of people per } \\
\text { square mile }\end{array}$ & - \\
\hline Street density, in miles of lanes per square mile & 26 \\
\hline $\begin{array}{l}\text { Percentage of contributing drainage area with the } \\
\text { following land uses: }\end{array}$ & \\
\hline Rural and pasture & 6 \\
\hline Agricultural & 0 \\
\hline ensity residential & 0 \\
\hline density residential & 76 \\
\hline High-density residential & 6 \\
\hline Commercial & 6 \\
\hline Industrial & 0 \\
\hline cuction & 0 \\
\hline Idle or vacant & 0 \\
\hline Wetland & 0 \\
\hline Parkland & 6 \\
\hline Detention storage, in acre-feet & 0 \\
\hline $\begin{array}{l}\text { Percentage of contributing drainage area upstream of } \\
\text { detention storage }\end{array}$ & 0 \\
\hline $\begin{array}{l}\text { Percentage of contributing drainage area drained by a } \\
\text { storm-sewer system }\end{array}$ & 57 \\
\hline Percentage of streets with curb and gutter drainage & 0 \\
\hline Percentage of streets with ditch and swale drainage & 0 \\
\hline Mean annual rainfall, in inches ${ }^{1}$ & 37.6 \\
\hline $\begin{array}{l}\text { Ten-year, 1-hour rainfall intensity, in inches per hour } \\
\text { Mean minimum January temperature, in degrees Fahrenheit }{ }^{1}\end{array}$ & 32.5 \\
\hline Two-year, 24 -hour rainfall, in inches ${ }^{2}$ & 2.6 \\
\hline $\begin{array}{l}\text { Mean annual nitrogen precipitation load, in pounds of } \\
\text { nitrogen per acre }\end{array}$ & 0.94 \\
\hline
\end{tabular}

${ }^{1}$ Data from National Oceanic and Atmospheric Administration, 1980

${ }^{2}$ Data from Hershfield, 1961.

${ }^{3}$ Data derived as sum of total deposition of ammonia and of nitrate (National Atmospheric Deposition Program, NADP Subcommittee Number 3 -- Data Management and Analysis, 1985). 
Table 249.--Basin characteristics of station 14211120

willamette River tributary on Southeast

River Road in Oak Grove, Oreg.

[Dashes indicate data not available. Except where footnoted, data is from magnetic tape described by Driver and others (1985)]

\begin{tabular}{|c|c|}
\hline Characteristic & Value \\
\hline Contributing drainage area, in acres & 470 \\
\hline $\begin{array}{l}\text { Impervious area, in percentage of contributing } \\
\text { drainage area }\end{array}$ & 36 \\
\hline $\begin{array}{l}\text { Effective impervious area, in percentage of } \\
\text { contributing drainage area }\end{array}$ & - \\
\hline Average basin slope, in feet per mile & 270 \\
\hline Main conveyance slope, in feet per mile & 130 \\
\hline $\begin{array}{l}\text { Population density, in number of people per } \\
\text { square mile }\end{array}$ & - \\
\hline Street density, in miles of lanes per square mile & 0 \\
\hline $\begin{array}{l}\text { Percentage of contributing drainage area with the } \\
\text { following land uses: }\end{array}$ & \\
\hline Rural and pasture & 7 \\
\hline Agricultura 1 & 0 \\
\hline Low-density residential & 0 \\
\hline Medium-density residential & 73 \\
\hline High-density residential & 4 \\
\hline Commercial & 8 \\
\hline Industrial & 0 \\
\hline Construction & 0 \\
\hline Idle or vacant & 0 \\
\hline Wet land & 0 \\
\hline Parkland & 8 \\
\hline Detention storage, in acre-feet & 0 \\
\hline $\begin{array}{l}\text { Percentage of contributing drainage area upstream of } \\
\text { detention storage }\end{array}$ & 0 \\
\hline $\begin{array}{l}\text { Percentage of contributing drainage area drained by a } \\
\text { storm-sewer system }\end{array}$ & 0 \\
\hline Percentage of streets with curb and gutter drainage & 0 \\
\hline Percentage of streets with ditch and swale drainage & 0 \\
\hline Mean annual rainfall, in inches ${ }^{1}$ & 37.61 \\
\hline Ten-year, 1 -hour rainfall intensity, in inches per hour & - \\
\hline Mean minimum January temperature, in degrees Fahrenheit ${ }^{1}$ & 32.5 \\
\hline Two-year, 24 -hour rainfa11, in inches ${ }^{2}$ & 2.6 \\
\hline $\begin{array}{l}\text { Mean annual nitrogen precipitation load, in pounds of } \\
\text { nitrogen per acre } 3\end{array}$ & 0.94 \\
\hline
\end{tabular}

${ }^{1}$ Data from National Oceanic and Atmospheric Administration, 1980 .

${ }^{2}$ Data from Hershfield, 1961

${ }^{3}$ Data derived as sum of total deposition of ammonia and of nitrate (National Atmospheric Deposition Program, NADP Subcommittee Number 3 -- Data Management and Analysis, 1985). 
Table 250.--Basin characteristics of station 14211301

Tryon Creek tributary at Dolph Court at Portland, Oreg.

[Dashes indicate data not available. Except where footnoted, data is from magnetic tape described by Driver and others (1985)]

\begin{tabular}{|c|c|}
\hline Characteristic & Value \\
\hline Contributing drainage area, in acres & 230 \\
\hline $\begin{array}{l}\text { Impervious area, in percentage of contributing } \\
\text { drainage area }\end{array}$ & 32 \\
\hline $\begin{array}{l}\text { Effective impervious area, in percentage of } \\
\text { contributing drainage area }\end{array}$ & - \\
\hline Average basin slope, in feet per mile & 440 \\
\hline Main conveyance slope, in feet per mile & 180 \\
\hline $\begin{array}{l}\text { Population density, in number of people per } \\
\text { square mile }\end{array}$ & - \\
\hline Street density, in miles of lanes per square mile & 10 \\
\hline $\begin{array}{l}\text { Percentage of contributing drainage area with the } \\
\text { following land uses: }\end{array}$ & \\
\hline Rural and pasture & 6 \\
\hline Agricultural & 0 \\
\hline Low-density residential & 0 \\
\hline Medium-density residential & 72 \\
\hline High-density residential & 10 \\
\hline Commercial & 5 \\
\hline Industrial & 0 \\
\hline Construction & 0 \\
\hline Idle or vacant & 0 \\
\hline Wetland & 0 \\
\hline Parkland & 7 \\
\hline Detention storage, in acre-feet & - \\
\hline $\begin{array}{l}\text { Percentage of contributing drainage area upstream of } \\
\text { detention storage }\end{array}$ & - \\
\hline $\begin{array}{l}\text { Percentage of contributing drainage area drained by a } \\
\text { storm-sewer system }\end{array}$ & 29 \\
\hline Percentage of streets with curb and gutter drainage & - \\
\hline Percentage of streets with ditch and swale drainage & - \\
\hline Mean annual rainfall, in inches ${ }^{1}$ & 37.61 \\
\hline Ten-year, 1-hour rainfall intensity, in inches per hour & - \\
\hline Mean minimum January temperature, in degrees Fahrenheit ${ }^{1}$ & 32.5 \\
\hline Two-year, 24-hour rainfall, in inches ${ }^{2}$ & 2.6 \\
\hline $\begin{array}{l}\text { Mean annual nitrogen precipitation load, in pounds of } \\
\text { nitrogen per acre }\end{array}$ & 0.94 \\
\hline
\end{tabular}

${ }^{1}$ Data from National Oceanic and Atmospheric Administration, 1980 .

${ }^{2}$ Data from Hershfield, 1961.

${ }^{3}$ Data derived as sum of total deposition of ammonia and of nitrate (National Atmospheric Deposition Program, NADP Subcommittee Number 3 -- Data Management and Analysis, 1985). 
Table 251.--Basin characteristics of station 15274820

South Branch South Fork Chester Creek tributary near Baxter Road at Anchorage, Alaska

[Dashes indicate data not available. Except where footnoted, data is from magnetic tape described by Driver and others (1985)]

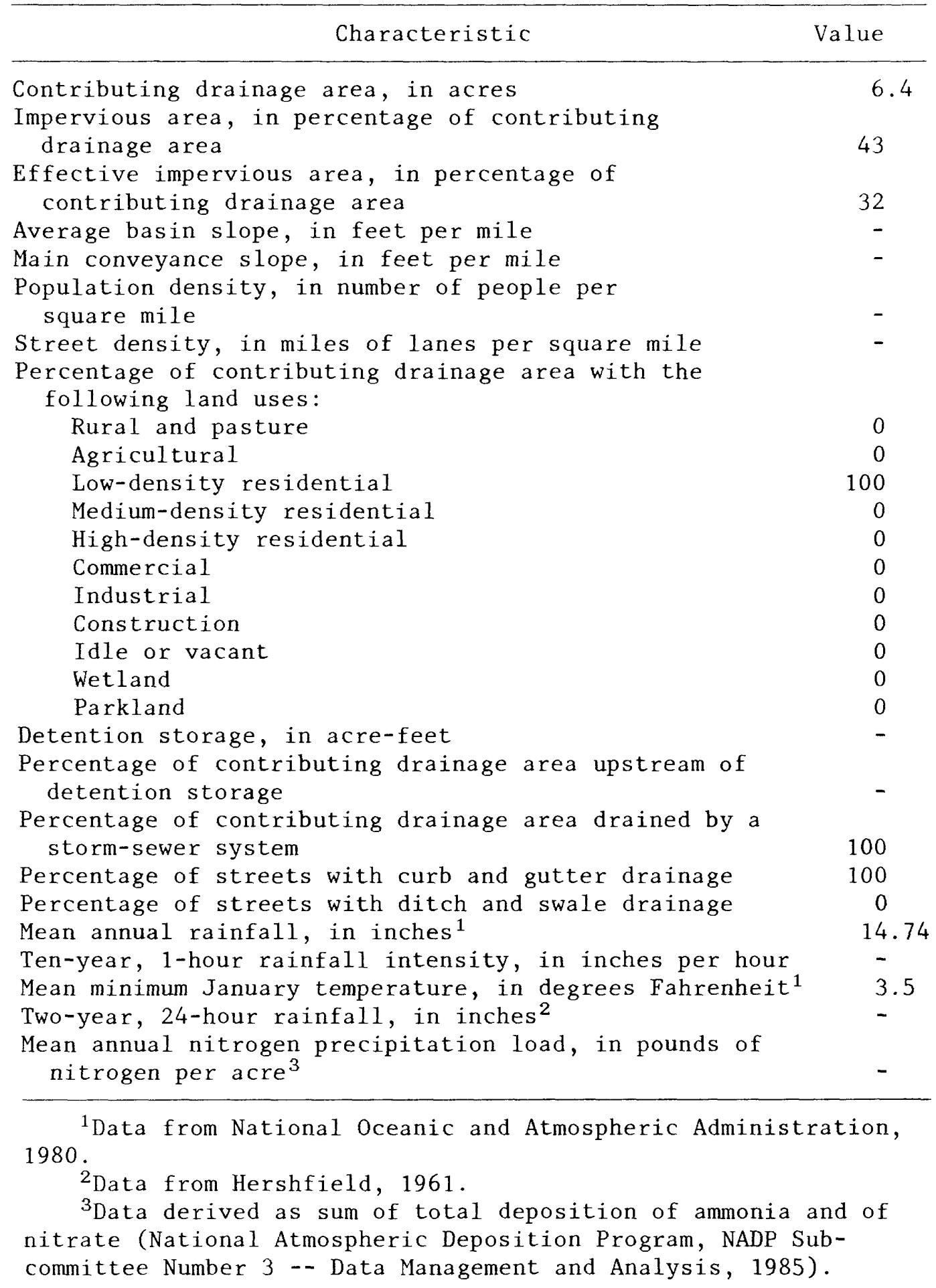


Table 252.--Basin characteristics of station 15275035

North Fork Chester Creek tributary near

20th Avenue at Anchorage, Alaska

[Dashes indicate data not available. Except where footnoted, data is from magnetic tape described by Driver and others (1985)]

\begin{tabular}{|c|c|}
\hline Characteristic & Value \\
\hline Contributing drainage area, in acres & 2.6 \\
\hline $\begin{array}{l}\text { Impervious area, in percentage of contributing } \\
\text { drainage area }\end{array}$ & 66 \\
\hline $\begin{array}{l}\text { Effective impervious area, in percentage of } \\
\text { contributing drainage area }\end{array}$ & 46 \\
\hline Average basin slope, in feet per mile & - \\
\hline Main conveyance slope, in feet per mile & - \\
\hline $\begin{array}{l}\text { Population density, in number of people per } \\
\text { square mile }\end{array}$ & - \\
\hline Street density, in miles of lanes per square mile & - \\
\hline \multicolumn{2}{|l|}{$\begin{array}{l}\text { Percentage of contributing drainage area with the } \\
\text { following land uses: }\end{array}$} \\
\hline Rural and pasture & 0 \\
\hline Agricultural & 0 \\
\hline Low-density residential & 0 \\
\hline Medium-density residential & 100 \\
\hline High-density residential & 0 \\
\hline Commercial & 0 \\
\hline Industrial & 0 \\
\hline Construction & 0 \\
\hline Idle or vacant & 0 \\
\hline Wetland & 0 \\
\hline Parkland & 0 \\
\hline Detention storage, in acre-feet & 0 \\
\hline $\begin{array}{l}\text { Percentage of contributing drainage area upstream of } \\
\text { detention storage }\end{array}$ & 0 \\
\hline $\begin{array}{l}\text { Percentage of contributing drainage area drained by a } \\
\text { storm-sewer system }\end{array}$ & 100 \\
\hline Percentage of streets with curb and gutter drainage & 100 \\
\hline Percentage of streets with ditch and swale drainage & 0 \\
\hline Mean annual rainfall, in inches ${ }^{1}$ & 14.74 \\
\hline Ten-year, 1-hour rainfall intensity, in inches per hour & - \\
\hline Mean minimum January temperature, in degrees Fahrenheit ${ }^{1}$ & 3.5 \\
\hline Two-year, 24-hour rainfall, in inches ${ }^{2}$ & - \\
\hline $\begin{array}{l}\text { Mean annual nitrogen precipitation load, in pounds of } \\
\text { nitrogen per acre }\end{array}$ & - \\
\hline
\end{tabular}
1980 .

${ }^{1}$ Data from National Oceanic and Atmospheric Administration,

${ }^{2}$ Data from Hershfield, 1961.

${ }^{3}$ Data derived as sum of total deposition of ammonia and of nitrate (National Atmospheric Deposition Program, NADP Subcommittee Number 3 -- Data Management and Analysis, 1985). 
Table 253.--Basin characteristics of station 15275055

Chester Creek tributary near 36 th Avenue

at Anchorage, Alaska

[Dashes indicate data not available. Except where footnoted, data is from magnetic tape described by Driver and others (1985)]

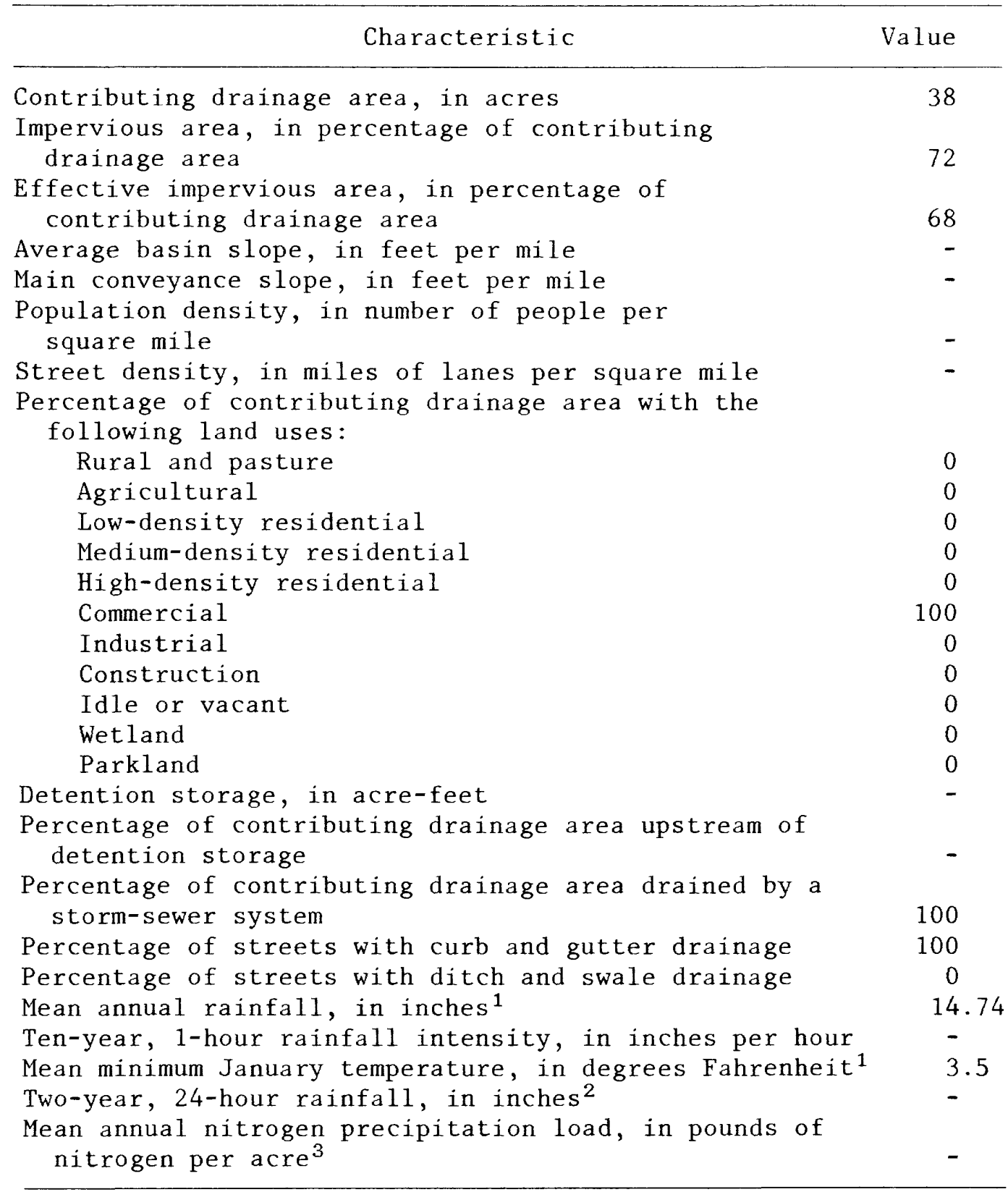

${ }^{1}$ Data from National Oceanic and Atmospheric Administration, 1980 .

${ }^{2}$ Data from Hershfield, 1961.

${ }^{3}$ Data derived as sum of total deposition of ammonia and of nitrate (National Atmospheric Deposition Program, NADP Subcommittee Number 3 -- Data Management and Analysis, 1985). 
Table 254.--Basin characteristics of station 212553158011000 Drain $B$ in Mililani, Hawaii

[Dashes indicate data not available. Except where footnoted, data is from magnetic tape described by Driver and others (1985)]

\begin{tabular}{|c|c|}
\hline Characteristic & Value \\
\hline Contributing drainage area, in acres & 140 \\
\hline $\begin{array}{l}\text { Impervious area, in percentage of contributing } \\
\text { drainage area }\end{array}$ & 49 \\
\hline $\begin{array}{l}\text { Effective impervious area, in percentage of } \\
\text { contributing drainage area }\end{array}$ & 27 \\
\hline Average basin slope, in feet per mile & - \\
\hline Main conveyance slope, in feet per mile & - \\
\hline $\begin{array}{l}\text { Population density, in number of people per } \\
\text { square mile }\end{array}$ & - \\
\hline Street density, in miles of lanes per square mile & - \\
\hline $\begin{array}{l}\text { Percentage of contributing drainage area with the } \\
\text { following land uses: }\end{array}$ & \\
\hline Rural and pasture & - \\
\hline Agricultural & 0 \\
\hline Low-density residential & 0 \\
\hline Medium-density residential & 78 \\
\hline High-density residential & - \\
\hline Commercial & 14 \\
\hline Industrial & - \\
\hline Construction & - \\
\hline Idle or vacant & 1 \\
\hline Wetland & - \\
\hline Parkland & 7 \\
\hline Detention storage, in acre-feet & - \\
\hline $\begin{array}{l}\text { Percentage of contributing drainage area upstream of } \\
\text { detention storage }\end{array}$ & - \\
\hline $\begin{array}{l}\text { Percentage of contributing drainage area drained by a } \\
\text { storm-sewer system }\end{array}$ & - \\
\hline Percentage of streets with curb and gutter drainage & - \\
\hline Percentage of streets with ditch and swale drainage & - \\
\hline Mean annual rainfall, in inches ${ }^{3}$ & 26.69 \\
\hline $\begin{array}{l}\text { Ten-year, } 1 \text {-hour rainfall intensity, in inches per hour } \\
\text { Mean minimum January temperature, in degrees Fahrenheit }{ }^{1}\end{array}$ & $-\overline{6} \cdot 6$ \\
\hline Two-year, 24-hour rainfall, in inches ${ }^{2}$ & - \\
\hline $\begin{array}{l}\text { Mean annual nitrogen precipitation load, in pounds of } \\
\text { nitrogen per acre }\end{array}$ & 0.39 \\
\hline
\end{tabular}

${ }^{1}$ Data from National Oceanic and Atmospheric Administration, 1980 .

${ }^{2}$ Data from Hershfield, 1961.

${ }^{3}$ Data derived as sum of total deposition of ammonia and of nitrate (National Atmospheric Deposition Program, NADP Subcommittee Number 3 -- Data Management and Analysis, 1985). 
Table 255.--Basin characteristics of station 212604158012700

Drain A in Mililani, Hawaii

[Dashes indicate data not available. Except where footnoted, data is from magnetic tape described by Driver and others $(1985)]$

\begin{tabular}{|c|c|}
\hline Characteristic & Value \\
\hline Contributing drainage area, in acres & 290 \\
\hline $\begin{array}{l}\text { Impervious area, in percentage of contributing } \\
\text { drainage area }\end{array}$ & 52 \\
\hline $\begin{array}{l}\text { Effective impervious area, in percentage of } \\
\text { contributing drainage area }\end{array}$ & 26 \\
\hline Average basin slope, in feet per mile & - \\
\hline Main conveyance slope, in feet per mile & - \\
\hline $\begin{array}{l}\text { Population density, in number of people per } \\
\text { square mile }\end{array}$ & - \\
\hline Street density, in miles of lanes per square mile & - \\
\hline $\begin{array}{l}\text { Percentage of contributing drainage area with the } \\
\text { following land uses: }\end{array}$ & \\
\hline Rural and pasture & - \\
\hline Agricultural & 0 \\
\hline Low-density residential & 0 \\
\hline Medium-density residential & 80 \\
\hline High-density residential & 5 \\
\hline Commercial & 4 \\
\hline Industrial & - \\
\hline Construction & - \\
\hline Idle or vacant & 9 \\
\hline Wetland & - \\
\hline Parkland & 2 \\
\hline Detention storage, in acre-feet & - \\
\hline $\begin{array}{l}\text { Percentage of contributing drainage area upstream of } \\
\text { detention storage }\end{array}$ & - \\
\hline $\begin{array}{l}\text { Percentage of contributing drainage area drained by a } \\
\text { storm-sewer system }\end{array}$ & - \\
\hline Percentage of streets with curb and gutter drainage & - \\
\hline Percentage of streets with ditch and swale drainage & - \\
\hline Mean annual rainfall, in inches 1 & 26.69 \\
\hline Ten-year, 1-hour rainfall intensity, in inches per hour & - \\
\hline Mean minimum January temperature, in degrees Fahrenheit ${ }^{1}$ & 67.6 \\
\hline Two-year, 24-hour rainfall, in inches ${ }^{2}$ & - \\
\hline $\begin{array}{l}\text { Mean annual nitrogen precipitation load, in pounds of } \\
\text { nitrogen per acre }\end{array}$ & 0.39 \\
\hline
\end{tabular}

${ }^{1}$ Data from National Oceanic and Atmospheric Administration, 1980 .

${ }^{2}$ Data from Hershfield, 1961.

${ }^{3}$ Data derived as sum of total deposition of ammonia and of nitrate (National Atmospheric Deposition Program, NADP Subcommittee Number 3 -- Data Management and Analysis, 1985). 
Table 256.--Basin characteristics of station 254031080191100 Apartment site at South Miami, Fla.

[Dashes indicate data not available. Except where footnoted, data is from magnetic tape described by Driver and others (1985)]

\begin{tabular}{|c|c|}
\hline Characteristic & Value \\
\hline Contributing drainage area, in acres & 13 \\
\hline $\begin{array}{l}\text { Impervious area, in percentage of contributing } \\
\text { drainage area }\end{array}$ & 71 \\
\hline $\begin{array}{l}\text { Effective impervious area, in percentage of } \\
\text { contributing drainage area }\end{array}$ & 44 \\
\hline Average basin slope, in feet per mile & - \\
\hline Main conveyance slope, in feet per mile & - \\
\hline $\begin{array}{l}\text { Population density, in number of people per } \\
\text { square mile }\end{array}$ & - \\
\hline $\begin{array}{l}\text { Street density, in miles of lanes per square mile } \\
\text { Percentage of contributing drainage area with the } \\
\text { following land uses: }\end{array}$ & - \\
\hline Rural and pasture & 0 \\
\hline Agricultural & 0 \\
\hline Low-density residential & 0 \\
\hline Medium-density residential & 0 \\
\hline High-density residential & 100 \\
\hline Commercial & 0 \\
\hline Industrial & 0 \\
\hline Construction & 0 \\
\hline Idle or vacant & 0 \\
\hline Wetland & 0 \\
\hline Parkland & 0 \\
\hline Detention storage, in acre-feet & - \\
\hline $\begin{array}{l}\text { Percentage of contributing drainage area upstream of } \\
\text { detention storage }\end{array}$ & - \\
\hline $\begin{array}{l}\text { Percentage of contributing drainage area drained by a } \\
\text { storm-sewer system }\end{array}$ & 100 \\
\hline Percentage of streets with curb and gutter drainage & - \\
\hline $\begin{array}{l}\text { Percentage of streets with ditch and swale drainage } \\
\text { Mean annual rainfall, in inches } 1\end{array}$ & $\overline{56.00}$ \\
\hline Ten-year, 1-hour rainfall intensity, in inches per hour & - \\
\hline Mean minimum January temperature, in degrees Fahrenheit ${ }^{1}$ & 58.7 \\
\hline Two-year, 24-hour rainfall, in inches ${ }^{2}$ & 5.8 \\
\hline $\begin{array}{l}\text { Mean annual nitrogen precipitation load, in pounds of } \\
\text { nitrogen per acre }\end{array}$ & 2.60 \\
\hline
\end{tabular}

${ }^{1}$ Data from National Oceanic and Atmospheric Administration, 1980.

${ }^{2}$ Data from Hershfield, 1961.

${ }^{3}$ Data derived as sum of total deposition of ammonia and of nitrate (National Atmospheric Deposition Program, NADP Subcommittee Number 3 -- Data Management and Analysis, 1985). 
Table 257.--Basin characteristics of station 261002080070100 Commercial basin at Fort Lauderdale, Fla.

[Dashes indicate data not available. Except where footnoted, data is from magnetic tape described by Driver and others (1985)]

\begin{tabular}{|c|c|}
\hline Characteristic & Value \\
\hline Contributing drainage area, in acres & 19 \\
\hline $\begin{array}{l}\text { Impervious area, in percentage of contributing } \\
\text { drainage area }\end{array}$ & 98 \\
\hline $\begin{array}{l}\text { Effective impervious area, in percentage of } \\
\text { contributing drainage area }\end{array}$ & 98 \\
\hline Average basin slope, in feet per mile & - \\
\hline Main conveyance slope, in feet per mile & - \\
\hline $\begin{array}{l}\text { Population density, in number of people per } \\
\text { square mile }\end{array}$ & 0 \\
\hline Street density, in miles of lanes per square mile & - \\
\hline \multicolumn{2}{|l|}{$\begin{array}{l}\text { Percentage of contributing drainage area with the } \\
\text { following land uses: }\end{array}$} \\
\hline Rural and pasture & 0 \\
\hline Agricultural & 0 \\
\hline Low-density residential & 0 \\
\hline Medium-density residential & 0 \\
\hline High-density residential & 0 \\
\hline Commercial & 98 \\
\hline Industrial & 0 \\
\hline Construction & 0 \\
\hline Idle or vacant & 0 \\
\hline Wetland & 0 \\
\hline Parkland & 2 \\
\hline Detention storage, in acre-feet & - \\
\hline $\begin{array}{l}\text { Percentage of contributing drainage area upstream of } \\
\text { detention storage }\end{array}$ & - \\
\hline $\begin{array}{l}\text { Percentage of contributing drainage area drained by a } \\
\text { storm-sewer system }\end{array}$ & 100 \\
\hline Percentage of streets with curb and gutter drainage & - \\
\hline Percentage of streets with ditch and swale drainage & - \\
\hline Mean annual rainfall, in inches ${ }^{1}$ & 59.05 \\
\hline Ten-year, 1-hour rainfall intensity, in inches per hour & - \\
\hline Mean minimum January temperature, in degrees Fahrenheit ${ }^{1}$ & 58.7 \\
\hline Two-year, 24-hour rainfall, in inches ${ }^{2}$ & 5.8 \\
\hline $\begin{array}{l}\text { Mean annual nitrogen precipitation load, in pounds of } \\
\text { nitrogen per acre }\end{array}$ & \\
\hline
\end{tabular}
1980

${ }^{1}$ Data from National Oceanic and Atmospheric Administration,

${ }^{2}$ Data from Hershfield, 1961.

${ }^{3}$ Data derived as sum of total deposition of ammonia and of nitrate (National Atmospheric Deposition Program, NADP Subcommittee Number 3 -- Data Management and Analysis, 1985). 
Table 258.--Basin characteristics of station 261615080055900 Residential area at Pompano Beach, Fla.

[Dashes indicate data not available. Except where footnoted, data is from magnetic tape described by Driver and others $(1985)]$

\section{Characteristic}

Contributing drainage area, in acres

Impervious area, in percentage of contributing drainage area

Effective impervious area, in percentage of contributing drainage area

Average basin slope, in feet per mile

Main conveyance slope, in feet per mile

Population density, in number of people per square mile

Street density, in miles of lanes per square mile Percentage of contributing drainage area with the following land uses:

Rural and pasture

Agricultural

Low-density residential

Medium-density residential

High-density residential

Commercial

Industrial

Construction

Idle or vacant

Wetland

Parkland

Detention storage, in acre-feet

Percentage of contributing drainage area upstream of detention storage

Percentage of contributing drainage area drained by a storm-sewer system

Percentage of streets with curb and gutter drainage

Percentage of streets with ditch and swale drainage

Mean annual rainfall, in inches ${ }^{1}$

Ten-year, 1-hour rainfall intensity, in inches per hour

Mean minimum January temperature, in degrees Fahrenheit ${ }^{1}$

Two-year, 24-hour rainfall, in inches ${ }^{2}$

Mean annual nitrogen precipitation load, in pounds of nitrogen per acre ${ }^{3}$
Value

38

44

6

-

-

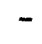

0

0

0

100

0

0

0

0

0

0

0

-

60

0

100

62.00

$-$

58.7

5.8

2.60

${ }^{1}$ Data from National Oceanic and Atmospheric Administration, 1980.

${ }^{2}$ Data from Hershfield, 1961.

${ }^{3}$ Data derived as sum of total deposition of ammonia and of nitrate (National Atmospheric Deposition Program, NADP Subcommittee Number 3 -- Data Management and Analysis, 1985). 
Table 259.--Basin characteristics of station 261629080072400 Highway basin near Pompano Beach, Fla.

[Dashes indicate data not available. Except where footnoted, data is from magnetic tape described by Driver and others (1985)]

\begin{tabular}{|c|c|}
\hline Characteristic & Value \\
\hline Contributing drainage area, in acres & 58 \\
\hline $\begin{array}{l}\text { Impervious area, in percentage of contributing } \\
\text { drainage area }\end{array}$ & 36 \\
\hline $\begin{array}{l}\text { Effective impervious area, in percentage of } \\
\text { contributing drainage area }\end{array}$ & 0 \\
\hline Average basin slope, in feet per mile & - \\
\hline Main conveyance slope, in feet per mile & - \\
\hline $\begin{array}{l}\text { Population density, in number of people per } \\
\text { square mile }\end{array}$ & - \\
\hline Street density, in miles of lanes per square mile & - \\
\hline $\begin{array}{l}\text { Percentage of contributing drainage area with the } \\
\text { following land uses: }\end{array}$ & \\
\hline Rural and pasture & 0 \\
\hline Agricultural & 0 \\
\hline Low-density residential & 0 \\
\hline Medium-density residential & 0 \\
\hline High-density residential & 0 \\
\hline Commercial & 40 \\
\hline Industrial & 0 \\
\hline Construction & 0 \\
\hline Idle or vacant & 60 \\
\hline Wetland & 0 \\
\hline Parkland & 0 \\
\hline Detention storage, in acre-feet & - \\
\hline $\begin{array}{l}\text { Percentage of contributing drainage area upstream of } \\
\text { detention storage }\end{array}$ & - \\
\hline $\begin{array}{l}\text { Percentage of contributing drainage area drained by a } \\
\text { storm-sewer system }\end{array}$ & 60 \\
\hline Percentage of streets with curb and gutter drainage & 100 \\
\hline Percentage of streets with ditch and swale drainage & 0 \\
\hline Mean annual rainfall, in inches ${ }^{1}$ & 62.0 \\
\hline Ten-year, 1-hour rainfall intensity, in inches per hour & - \\
\hline Mean minimum January temperature, in degrees Fahrenheit ${ }^{1}$ & 58.7 \\
\hline Two-year, 24-hour rainfall, in inches ${ }^{2}$ & 5.8 \\
\hline Mean annual nitrogen precipitation load, in pounds of & \\
\hline
\end{tabular}
1980

${ }^{1}$ Data from National Oceanic and Atmospheric Administration,

${ }^{2}$ Data from Hershfield, 1961.

${ }^{3}$ Data derived as sum of total deposition of ammonia and of nitrate (National Atmospheric Deposition Program, NADP Subcommittee Number 3 -- Data Management and Analysis, 1985). 
Table 260.--Basin characteristics of station 364155119445000 Industrial urban-runoff site at Fresno, Calif.

[Dashes indicate data not available. Except where footnoted, data is from magnetic tape described by Driver and others (1985)]

\begin{tabular}{|c|c|}
\hline Characteristic & Value \\
\hline Contributing drainage area, in acres & 280 \\
\hline $\begin{array}{l}\text { Impervious area, in percentage of contributing } \\
\text { drainage area }\end{array}$ & 53 \\
\hline $\begin{array}{l}\text { Effective impervious area, in percentage of } \\
\text { contributing drainage area }\end{array}$ & 0 \\
\hline Average basin slope, in feet per mile & 8 \\
\hline Main conveyance slope, in feet per mile & 8 \\
\hline $\begin{array}{l}\text { Population density, in number of people per } \\
\text { square mile }\end{array}$ & 0 \\
\hline Street density, in miles of lanes per square mile & 16 \\
\hline $\begin{array}{l}\text { Percentage of contributing drainage area with the } \\
\text { following land uses: }\end{array}$ & \\
\hline Rural and pasture & 0 \\
\hline Agricultural & 0 \\
\hline Low-density residential & 0 \\
\hline Medium-density residential & 0 \\
\hline High-density residential & 0 \\
\hline Commercial & 0 \\
\hline Industrial & 66 \\
\hline Construction & 0 \\
\hline Idle or vacant & 34 \\
\hline Wetland & 0 \\
\hline Parkland & 0 \\
\hline Detention storage, in acre-feet & 0 \\
\hline $\begin{array}{l}\text { Percentage of contributing drainage area upstream of } \\
\text { detention storage }\end{array}$ & 0 \\
\hline $\begin{array}{l}\text { Percentage of contributing drainage area drained by a } \\
\text { storm-sewer system }\end{array}$ & 100 \\
\hline Percentage of streets with curb and gutter drainage & 47 \\
\hline Percentage of streets with ditch and swale drainage & 53 \\
\hline Mean annual rainfall, in inches ${ }^{1}$ & 10.2 \\
\hline Ten-year, 1-hour rainfall intensity, in inches per hour & 0.87 \\
\hline Mean minimum January temperature, in degrees Fahrenheit ${ }^{1}$ & 35.8 \\
\hline Two-year, 24-hour rainfall, in inches ${ }^{2}$ & 1.4 \\
\hline $\begin{array}{l}\text { Mean annual nitrogen precipitation load, in pounds of } \\
\text { nitrogen per acre }\end{array}$ & \\
\hline
\end{tabular}
1980.

${ }^{1}$ Data from National Oceanic and Atmospheric Administration,

${ }^{2}$ Data from Hershfield, 1961.

${ }^{3}$ Data derived as sum of total deposition of ammonia and of nitrate (National Atmospheric Deposition Program, NADP Subcommittee Number 3 -- Data Management and Analysis, 1985). 
Table 261.--Basin characteristics of station 364746119445400

Single-dwelling residential urban-runoff site

at Fresno, Calif.

[Dashes indicate data not available. Except where footnoted, data is from magnetic tape described by Driver and others (1985)]

\begin{tabular}{|c|c|}
\hline Characteristic & Value \\
\hline Contributing drainage area, in acres & 96 \\
\hline $\begin{array}{l}\text { Impervious area, in percentage of contributing } \\
\text { drainage area }\end{array}$ & 43 \\
\hline $\begin{array}{l}\text { Effective impervious area, in percentage of } \\
\text { contributing drainage area }\end{array}$ & - \\
\hline Average basin slope, in feet per mile & 8 \\
\hline Main conveyance slope, in feet per mile & 29 \\
\hline $\begin{array}{l}\text { Population density, in number of people per } \\
\text { square mile }\end{array}$ & 7,714 \\
\hline Street density, in miles of lanes per square mile & 47 \\
\hline $\begin{array}{l}\text { Percentage of contributing drainage area with the } \\
\text { following land uses: }\end{array}$ & \\
\hline Rural and pasture & 0 \\
\hline Agricultural & 0 \\
\hline Low-density residential & 9 \\
\hline Medium-density residential & 87 \\
\hline High-density residential & 0 \\
\hline Commercial & 0 \\
\hline Industrial & 0 \\
\hline Construction & 0 \\
\hline Idle or vacant & 4 \\
\hline Wet 1 and & 0 \\
\hline Parkland & 0 \\
\hline Detention storage, in acre-feet & 0 \\
\hline $\begin{array}{l}\text { Percentage of contributing drainage area upstream of } \\
\text { detention storage }\end{array}$ & 0 \\
\hline $\begin{array}{l}\text { Percentage of contributing drainage area drained by a } \\
\text { storm-sewer system }\end{array}$ & 100 \\
\hline Percentage of streets with curb and gutter drainage & 100 \\
\hline Percentage of streets with ditch and swale drainage & 0 \\
\hline Mean annual rainfall, in inches ${ }^{1}$ & 10.24 \\
\hline Ten-year, 1-hour rainfall intensity, in inches per hour & 0.87 \\
\hline Mean minimum January temperature, in degrees Fahrenheit ${ }^{1}$ & 35.8 \\
\hline Two-year, 24-hour rainfall, in inches ${ }^{2}$ & 1.4 \\
\hline $\begin{array}{l}\text { Mean annual nitrogen precipitation load, in pounds of } \\
\text { nitrogen per acre }\end{array}$ & 4.00 \\
\hline
\end{tabular}

${ }^{1}$ Data from National Oceanic and Atmospheric Administration, 1980 .

${ }^{2}$ Data from Hershfield, 1961.

${ }^{3}$ Data derived as sum of total deposition of ammonia and of nitrate (National Atmospheric Deposition Program, NADP Subcommittee Number 3 -- Data Management and Analysis, 1985). 
Table 262.--Basin characteristics of station 364818119443800 Multi-dwelling residential urban-runoff site at Fresno, Calif.

[Dashes indicate data not available. Except where footnoted, data is from magnetic tape described by Driver and others (1985)]

\begin{tabular}{|c|c|}
\hline Characteristic & Value \\
\hline Contributing drainage area, in acres & 45 \\
\hline $\begin{array}{l}\text { Impervious area, in percentage of contributing } \\
\text { drainage area }\end{array}$ & 57 \\
\hline $\begin{array}{l}\text { Effective impervious area, in percentage of } \\
\text { contributing drainage area }\end{array}$ & - \\
\hline Average basin slope, in feet per mile & 7 \\
\hline Main conveyance slope, in feet per mile & 10 \\
\hline $\begin{array}{l}\text { Population density, in number of people per } \\
\text { square mile }\end{array}$ & 16,390 \\
\hline $\begin{array}{l}\text { Street density, in miles of lanes per square mile } \\
\text { Percentage of contributing drainage area with the } \\
\text { following land uses: }\end{array}$ & 39 \\
\hline Rural and pasture & 0 \\
\hline Agricultural & 0 \\
\hline Low-density residential & 0 \\
\hline Medium-density residential & 0 \\
\hline High-density residential & 87 \\
\hline Commercial & 0 \\
\hline Industrial & 0 \\
\hline Construction & 0 \\
\hline Idle or vacant & 13 \\
\hline Wetland & 0 \\
\hline Parkland & 0 \\
\hline Detention storage, in acre-feet & 0 \\
\hline $\begin{array}{l}\text { Percentage of contributing drainage area upstream of } \\
\text { detention storage }\end{array}$ & 0 \\
\hline $\begin{array}{l}\text { Percentage of contributing drainage area drained by a } \\
\text { storm-sewer system }\end{array}$ & 100 \\
\hline Percentage of streets with curb and gutter drainage & 96 \\
\hline Percentage of streets with ditch and swale drainage & 4 \\
\hline Mean annual rainfall, in inches 1 & 10.24 \\
\hline Ten-year, 1-hour rainfall intensity, in inches per hour & 0.87 \\
\hline Mean minimum January temperature, in degrees Fahrenheit ${ }^{1}$ & 35.8 \\
\hline Two-year, 24 -hour rainfall, in inches ${ }^{2}$ & 1.4 \\
\hline $\begin{array}{l}\text { Mean annual nitrogen precipitation load, in pounds of } \\
\text { nitrogen per acre }\end{array}$ & 4.00 \\
\hline
\end{tabular}

${ }^{1}$ Data from National Oceanic and Atmospheric Administration, 1980 .

${ }^{2}$ Data from Hershfield, 1961.

${ }^{3}$ Data derived as sum of total deposition of ammonia and of nitrate (National Atmospheric Deposition Program, NADP Subcommittee Number 3 -- Data Management and Analysis, 1985). 
Table 263.--Basin characteristics of station 364818119464700 Commercial urban-runoff site at Fresno, Calif.

[Dashes indicate data not available. Except where footnoted, data is from magnetic tape described by Driver and others (1985)]

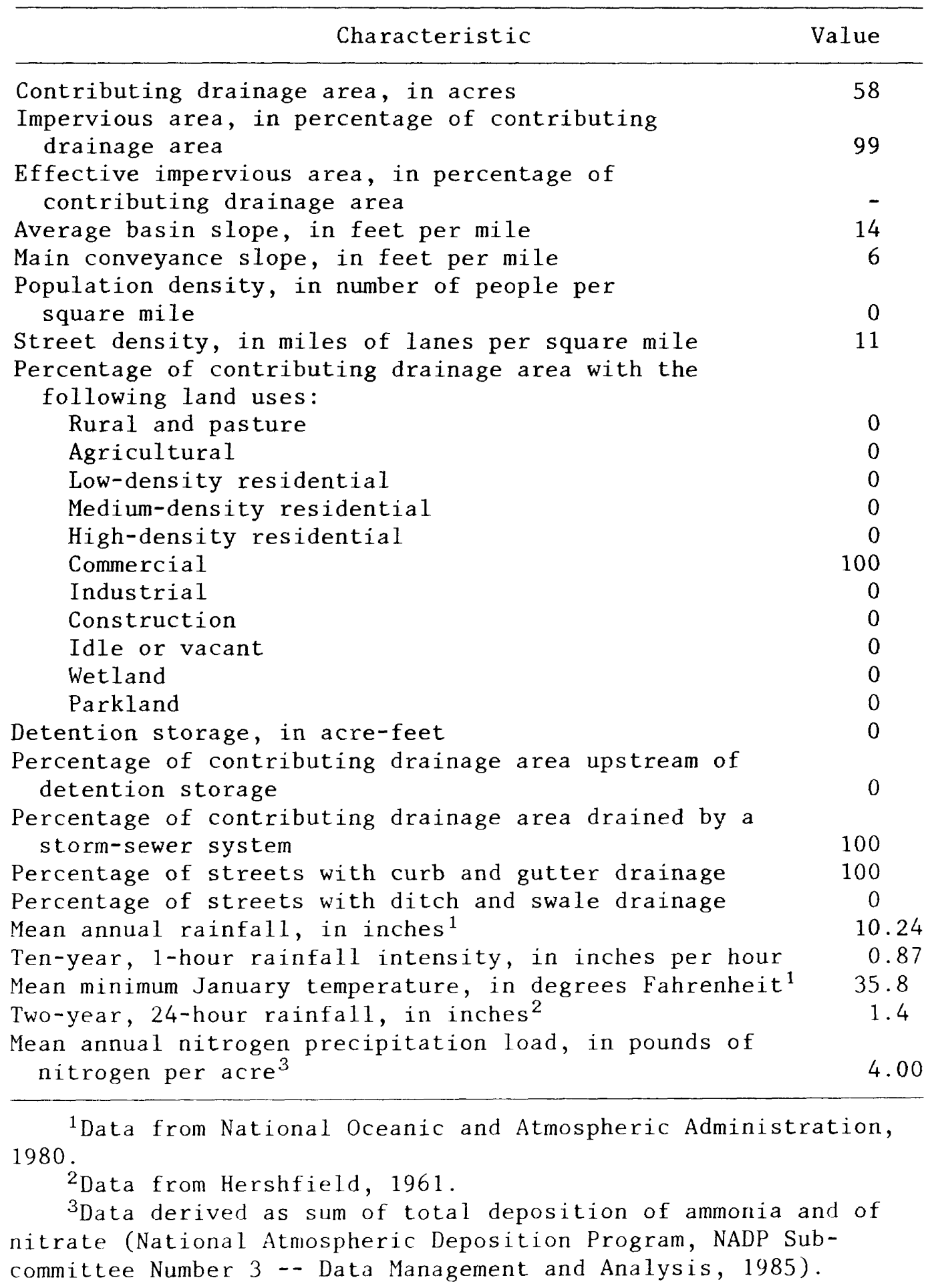


Table 264.--Basin characteristics of station 394236105042400 Villa Italia storm drain at Lakewood, Colo.

[Dashes indicate data not available. Except where footnoted, data is from magnetic tape described by Driver and others (1985)]

\begin{tabular}{|c|c|}
\hline Characteristic & Value \\
\hline Contributing drainage area, in acres & 74 \\
\hline $\begin{array}{l}\text { Impervious area, in percentage of contributing } \\
\text { drainage area }\end{array}$ & 91 \\
\hline $\begin{array}{l}\text { Effective impervious area, in percentage of } \\
\text { contributing drainage area }\end{array}$ & 91 \\
\hline Average basin slope, in feet per mile & - \\
\hline Main conveyance slope, in feet per mile & - \\
\hline $\begin{array}{l}\text { Population density, in number of people per } \\
\text { square mile }\end{array}$ & 0 \\
\hline Street density, in miles of lanes per square mile & - \\
\hline \multicolumn{2}{|l|}{$\begin{array}{l}\text { Percentage of contributing drainage area with the } \\
\text { following land uses: }\end{array}$} \\
\hline Rural and pasture & 0 \\
\hline Agricultural & 0 \\
\hline Low-density residential & 0 \\
\hline Medium-density residential & 0 \\
\hline High-density residential & 0 \\
\hline Commercial & 100 \\
\hline Industrial & 0 \\
\hline Construction & 0 \\
\hline Idle or vacant & 0 \\
\hline Wetland & 0 \\
\hline Parkland & 0 \\
\hline Detention storage, in acre-feet & 0 \\
\hline $\begin{array}{l}\text { Percentage of contributing drainage area upstream of } \\
\text { detention storage }\end{array}$ & - \\
\hline $\begin{array}{l}\text { Percentage of contributing drainage area drained by a } \\
\text { storm-sewer system }\end{array}$ & - \\
\hline Percentage of streets with curb and gutter drainage & - \\
\hline Percentage of streets with ditch and swale drainage & - \\
\hline Mean annual rainfall, in inches ${ }^{1}$ & 15.51 \\
\hline Ten-year, 1-hour rainfall intensity, in inches per hour & - \\
\hline Mean minimum January temperature, in degrees Fahrenheit ${ }^{1}$ & 16.2 \\
\hline Two-year, 24-hour rainfall, in inches ${ }^{2}$ & 2.1 \\
\hline $\begin{array}{l}\text { Mean annual nitrogen precipitation load, in pounds of } \\
\text { nitrogen per acre }\end{array}$ & 1.80 \\
\hline
\end{tabular}
1980.

${ }^{1}$ Data from National Oceanic and Atmospheric Administration,

${ }^{2}$ Data from Hershfield, 1961.

${ }^{3}$ Data derived as sum of total deposition of ammonia and of nitrate (National Atmospheric Deposition Program, NADP Subcommittee Number 3 -- Data Management and Analysis, 1985). 
Table 265.--Basin characteristics of station 394322105073601

Denver Federal Center rainfall-runoff simulation

plot 1 at Lakewood, Colo.

[Dashes indicate data not available. Except where footnoted, data is from magnetic tape described by Driver and others $(1985)$ ]

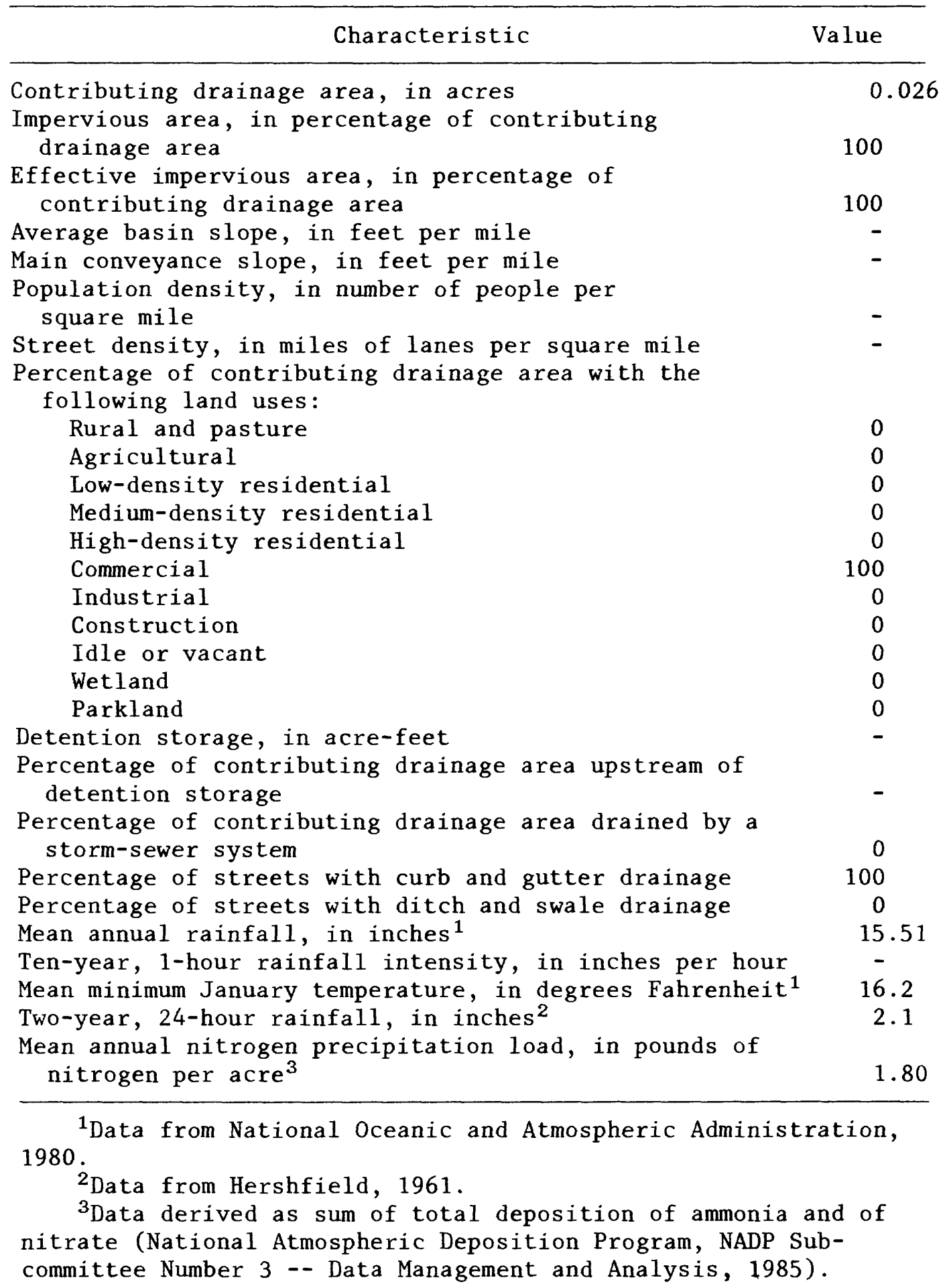


Table 266.--Basin characteristics of station 394322105073602 Denver Federal Center rainfall-runoff simulation plot 2 at Lakewood, Colo.

[Dashes indicate data not available. Except where footnoted, data is from magnetic tape described by Driver and others (1985)]

\begin{tabular}{|c|c|}
\hline Characteristic & Value \\
\hline Contributing drainage area, in acres & 0.026 \\
\hline $\begin{array}{l}\text { Impervious area, in percentage of contributing } \\
\text { drainage area } \\
\text { Effective impervious area, in percentage of }\end{array}$ & 100 \\
\hline contributing drainage area & 100 \\
\hline Average basin slope, in feet per mile & - \\
\hline $\begin{array}{l}\text { Main conveyance slope, in feet per mile } \\
\text { Population density, in number of people per }\end{array}$ & - \\
\hline $\begin{array}{l}\text { Populacion aensity, in number or peopie per } \\
\text { square mile }\end{array}$ & - \\
\hline $\begin{array}{l}\text { Street density, in miles of lanes per square mile } \\
\text { Percentage of contributing drainage area with the } \\
\text { following land uses: }\end{array}$ & - \\
\hline Rural and pasture & 0 \\
\hline Agricultural & 0 \\
\hline Low-density residential & 0 \\
\hline Medium-density residential & 0 \\
\hline High-density residential & 0 \\
\hline Commercial & 100 \\
\hline Industrial & 0 \\
\hline Construction & 0 \\
\hline Idle or vacant & 0 \\
\hline Wetland & 0 \\
\hline Parkland & 0 \\
\hline $\begin{array}{l}\text { Detention storage, in acre-feet } \\
\text { Percentage of contributing drainage area upstream of } \\
\text { detention storage }\end{array}$ & - \\
\hline $\begin{array}{l}\text { Percentage of contributing drainage area drained by a } \\
\text { storm-sewer system }\end{array}$ & 0 \\
\hline Percentage of streets with curb and gutter drainage & 100 \\
\hline Percentage of streets with ditch and swale drainage & 0 \\
\hline Mean annual rainfall, in inches ${ }^{1}$ & 15.51 \\
\hline Ten-year, 1-hour rainfall intensity, in inches per hour & - \\
\hline Mean minimum January temperature, in degrees Fahrenheit ${ }^{1}$ & 16.2 \\
\hline $\begin{array}{l}\text { Two-year, } 24 \text {-hour rainfall, in inches }{ }^{2} \\
\text { Mean annual nitrogen precipitation load, in pounds of }\end{array}$ & 2.1 \\
\hline nitrogen per acre ${ }^{3}$ & 1.80 \\
\hline
\end{tabular}
1980.

${ }^{1}$ Data from National Oceanic and Atmospheric Administration,

${ }^{2}$ Data from Hershfield, 1961.

${ }^{3}$ Data derived as sum of total deposition of ammonia and of nitrate (National Atmospheric Deposition Program, NADP Subcommittee Number 3 -- Data Management and Analysis, 1985). 
Table 267.--Basin characteristics of station 394322105073603

Denver Federal Center rainfall-runoff simulation

plot 3 at Lakewood, Colo.

[Dashes indicate data not available. Except where footnoted, data is from magnetic tape described by Driver and others $(1985)]$

\begin{tabular}{|c|c|}
\hline Characteristic & Value \\
\hline Contributing drainage area, in acres & 0.026 \\
\hline $\begin{array}{l}\text { Imparvious area, in percentage of contributing } \\
\text { drainage area }\end{array}$ & 100 \\
\hline Effective impervious area, in percentage of & \\
\hline contributing drainage area & 100 \\
\hline Average basin slope, in feet per mile & - \\
\hline Main conveyance slope, in feet per mile & - \\
\hline $\begin{array}{l}\text { Population density, in number of people per } \\
\text { square mile }\end{array}$ & - \\
\hline Street density, in miles of lanes per square mile & - \\
\hline $\begin{array}{l}\text { Percentage of contributing drainage area with the } \\
\text { following land uses: }\end{array}$ & \\
\hline Rural and pasture & $\cdots 0$ \\
\hline Agricultural & 0 \\
\hline Low-density residential & 0 \\
\hline Medium-density residential & 0 \\
\hline High-density residential & 0 \\
\hline Commercial & 100 \\
\hline Industrial & 0 \\
\hline Construction & 0 \\
\hline Idle or vacant & 0 \\
\hline Wetland & 0 \\
\hline Parkland & 0 \\
\hline Detention storage, in acre-feet & - \\
\hline $\begin{array}{l}\text { Percentage of contributing drainage area upstream of } \\
\text { detention storage }\end{array}$ & - \\
\hline Percentage of contributing drainage area drained by a & \\
\hline storm-sewer system & 0 \\
\hline Percentage of streets with curb and gutter drainage & 100 \\
\hline Percentage of streets with ditch and swale drainage & 0 \\
\hline Mean annual rainfall, in inches ${ }^{1}$ & 15.51 \\
\hline Ten-year, 1-hour rainfall intensity, in inches per hour & - \\
\hline Mean minimum January temperature, in degrees Fahrenheit ${ }^{1}$ & 16.2 \\
\hline $\begin{array}{l}\text { Two-year, } 24 \text {-hour rainfa11, in inches }{ }^{2} \\
\text { Mean annual nitrogen precipitation load, in pounds of }\end{array}$ & 2.1 \\
\hline nitrogen per acre & 1.80 \\
\hline
\end{tabular}

${ }^{1}$ Data from National Oceanic and Atmospheric Administration, 1980 .

${ }^{2}$ Data from Hershfield, 1961.

${ }^{3}$ Data derived as sum of total deposition of ammonia and of nitrate (National Atmospheric Deposition Program, NADP Subcommittee Number 3 -- Data Management and Analysis, 1985). 
Table 268.--Basin characteristics of station 394322105073604

Denver Federal Center rainfall-runoff simulation

plot 4 at Lakewood, Colo.

[Dashes indicate data not available. Except where footnoted, data is from magnetic tape described by Driver and others (1985)]

Characteristic

Contributing drainage area, in acres

Impervious area, in percentage of contributing drainage area

Effective impervious area, in percentage of contributing drainage area

Average basin slope, in feet per mile

Main conveyance slope, in feet per mile

Population density, in number of people per square mile

Street density, in miles of lanes per square mile

Percentage of contributing drainage area with the

following land uses:

Rural and pasture

Agricultural

Low-density residential

Medium-density residential

High-density residential

Commercial

Industrial

Construction

Idle or vacant

Wetland

Parkland

Detention storage, in acre-feet

Percentage of contributing drainage area upstream of detention storage

Percentage of contributing drainage area drained by a storm-sewer system

Percentage of streets with curb and gutter drainage

Percentage of streets with ditch and swale drainage

Mean annual rainfall, in inches ${ }^{1}$

Ten-year, 1-hour rainfall intensity, in inches per hour

Mean minimum January temperature, in degrees Fahrenheit ${ }^{1}$

Two-year, 24-hour rainfall, in inches ${ }^{2}$

Mean annual nitrogen precipitation load, in pounds of nitrogen per acre ${ }^{3}$
Value

0.026

100

100
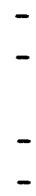

0

0

0

0

0

100

0

0

0

0

0

-

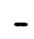

0

100

0

15.51

16.2

2.1

1.80

${ }^{1}$ Data from National Oceanic and Atmospheric Administration, 1980.

${ }^{2}$ Data from Hershfield, 1961.

${ }^{3}$ Data derived as sum of total deposition of ammonia and of nitrate (National Atmospheric Deposition Program, NADP Subcommittee Number 3 -- Data Management and Analysis, 1985). 
Table 269.--Basin characteristics of station 394322105073605

Denver Federal Center rainfall-runoff simulation

plot 5 at Lakewood, Colo.

[Dashes indicate data not available. Except where footnoted, data is from magnetic tape described by Driver and others (1985)]

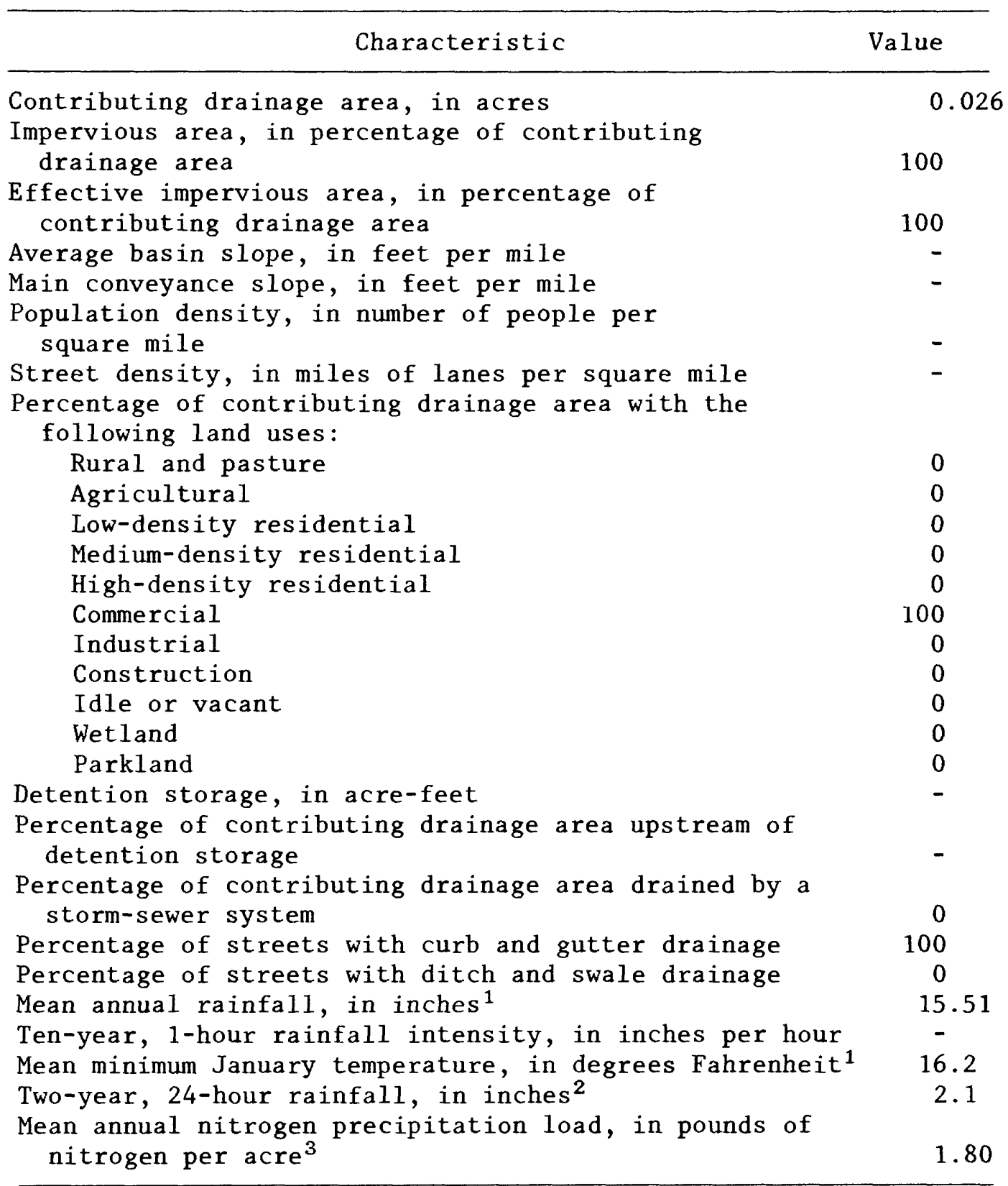
1980 .

${ }^{1}$ Data from National Oceanic and Atmospheric Administration,

${ }^{2}$ Data from Hershfield, 1961.

${ }^{3}$ Data derived as sum of total deposition of ammonia and of nitrate (National Atmospheric Deposition Program, NADP Subcommittee Number 3 -- Data Management and Analysis, 1985). 
Table 270.--Basin characteristics of station 394322105073606

Denver Federal Center rainfall-runoff simulation

plot 6 at Lakewood, Colo.

[Dashes indicate data not available. Except where footnoted, data is from magnetic tape described by Driver and others (1985)]

\begin{tabular}{cc} 
Characteristic Value \\
\hline
\end{tabular}

Contributing drainage area, in acres 0.026

Impervious area, in percentage of contributing

drainage area

Effective impervious area, in percentage of contributing drainage area

Average basin slope, in feet per mile

Main conveyance slope, in feet per mile

Population density, in number of people per square mile

Street density, in miles of lanes per square mile

Percentage of contributing drainage area with the

following land uses:

Rural and pasture

Agricultural

Low-density residential

Medium-density residential

High-density residential

Commercial

Industrial

100

Construction

Idle or vacant

Wetland

Parkland

Detention storage, in acre-feet

Percentage of contributing drainage area upstream of detention storage

Percentage of contributing drainage area drained by a storm-sewer system

Percentage of streets with curb and gutter drainage

Percentage of streets with ditch and swale drainage

Mean annual rainfall, in inches ${ }^{1}$

0

Ten-year, 1-hour rainfall intensity, in inches per hour

Mean minimum January temperature, in degrees Fahrenheit ${ }^{1}$

Two-year, 24-hour rainfall, in inches ${ }^{2}$

16.2

2.1

Mean annual nitrogen precipitation load, in pounds of nitrogen per acre ${ }^{3}$ 1980 .

${ }^{1}$ Data from National Oceanic and Atmospheric Administration,

${ }^{2}$ Data from Hershfield, 1961.

${ }^{3}$ Data derived as sum of total deposition of ammonia and of nitrate (National Atmospheric Deposition Program, NADP Subcommittee Number 3 -- Data Management and Analysis, 1985). 
Table 271.--Basin characteristics of station 394322105073607

Denver Federal Center rainfal1-runoff simulation

plot 7 at Lakewood, Colo.

[Dashes indicate data not available. Except where footnoted, data is from magnetic tape described by Driver and others (1985)]

\begin{tabular}{|c|c|}
\hline Characteristic & Value \\
\hline Contributing drainage area, in acres & 0.026 \\
\hline $\begin{array}{l}\text { Impervious area, in percentage of contributing } \\
\text { drainage area }\end{array}$ & 100 \\
\hline $\begin{array}{l}\text { Effective impervious area, in percentage of } \\
\text { contributing drainage area }\end{array}$ & 100 \\
\hline Average basin slope, in feet per mile & - \\
\hline Main conveyance slope, in feet per mile & - \\
\hline $\begin{array}{l}\text { Population density, in number of people per } \\
\text { square mile }\end{array}$ & - \\
\hline Street density, in miles of lanes per square mile & - \\
\hline \multicolumn{2}{|l|}{$\begin{array}{l}\text { Percentage of contributing drainage area with the } \\
\text { following land uses: }\end{array}$} \\
\hline Rural and pasture & 0 \\
\hline Agricultural & 0 \\
\hline Low-density residential & 0 \\
\hline Medium-density residential & 0 \\
\hline High-density residential & 0 \\
\hline Commercial & 100 \\
\hline Industrial & 0 \\
\hline Construction & 0 \\
\hline Idle or vacant & 0 \\
\hline Wetland & 0 \\
\hline Parkland & 0 \\
\hline Detention storage, in acre-feet & - \\
\hline $\begin{array}{l}\text { Percentage of contributing drainage area upstream of } \\
\text { detention storage }\end{array}$ & - \\
\hline $\begin{array}{l}\text { Percentage of contributing drainage area drained by a } \\
\text { storm-sewer system }\end{array}$ & 0 \\
\hline Percentage of streets with curb and gutter drainage & 100 \\
\hline Percentage of streets with ditch and swale drainage & 0 \\
\hline Mean annual rainfall, in inches ${ }^{1}$ & 15.51 \\
\hline Ten-year, 1-hour rainfal1 intensity, in inches per hour & - \\
\hline Mean minimum January temperature, in degrees Fahrenheit ${ }^{1}$ & 16.2 \\
\hline Two-year, 24-hour rainfall, in inches ${ }^{2}$ & 2.1 \\
\hline $\begin{array}{l}\text { Mean annual nitrogen precipitation load, in pounds of } \\
\text { nitrogen per acre }\end{array}$ & 1.80 \\
\hline
\end{tabular}

${ }^{1}$ Data from National Oceanic and Atmospheric Administration, 1980 .

${ }^{2}$ Data from Hershfield, 1961.

${ }^{3}$ Data derived as sum of total deposition of ammonia and of nitrate (National Atmospheric Deposition Program, NADP Subcommittee Number 3 -- Data Management and Analysis, 1985). 
Table 272.--Basin characteristics of station 394322105073608 Denver Federal Center rainfall-runoff simulation plot 8 at Lakewood, Colo.

[Dashes indicate data not available. Except where footnoted, data is from magnetic tape described by Driver and others (1985)]

\begin{tabular}{lr}
\hline \multicolumn{1}{c}{ Characteristic } & Value \\
\hline Contributing drainage area, in acres & 0.026 \\
Impervious area, in percentage of contributing & \\
drainage area & 100 \\
Effective impervious area, in percentage of & 100 \\
contributing drainage area & - \\
Average basin slope, in feet per mile & - \\
Main conveyance slope, in feet per mile & - \\
Population density, in number of people per & - \\
square mile & \\
Street density, in miles of lanes per square mile & \\
Percentage of contributing drainage area with the & 0 \\
following land uses: & 0 \\
Rural and pasture & 0 \\
Agricultural & 0 \\
Low-density residential & 100 \\
Hedium-density residential & 0 \\
Commercial residential & 0 \\
Industrial & 0 \\
Construction & 0 \\
Idle or vacant &
\end{tabular}
1980

${ }^{1}$ Data from National Oceanic and Atmospheric Administration,

${ }^{2}$ Data from Hershfield, 1961.

${ }^{3}$ Data derived as sum of total deposition of ammonia and of nitrate (National Atmospheric Deposition Program, NADP Subcommittee Number 3 -- Data Management and Analysis, 1985). 
Table 273.--Basin characteristics of station 394322105073609 Denver Federal Center rainfall-runoff simulation plot 9 at Lakewood, Colo.

[Dashes indicate data not available. Except where footnoted, data is from magnetic tape described by Driver and others (1985)]

Characteristic Value

Contributing drainage area, in acres 0.026

Impervious area, in percentage of contributing drainage area

Effective impervious area, in percentage of contributing drainage area

100

Average basin slope, in feet per mile

Main conveyance slope, in feet per mile

Population density, in number of people per square mile

Street density, in miles of lanes per square mile

Percentage of contributing drainage area with the

following land uses:

Rural and pasture

Agricultural

Low-density residential

Medium-density residential

High-density residential

Commercial

Industrial

Construction

Idle or vacant

Wet land

Parkland

Detention storage, in acre-feet

Percentage of contributing drainage area upstream of detention storage

Percentage of contributing drainage area drained by a storm-sewer system

Percentage of streets with curb and gutter drainage

Percentage of streets with ditch and swale drainage

Mean annual rainfall, in inches ${ }^{1}$

Ten-year, 1-hour rainfall intensity, in inches per hour

Mean minimum January temperature, in degrees Fahrenheit ${ }^{1}$

Two-year, 24-hour rainfall, in inches ${ }^{2}$

Mean annual nitrogen precipitation load, in pounds of nitrogen per acre ${ }^{3}$

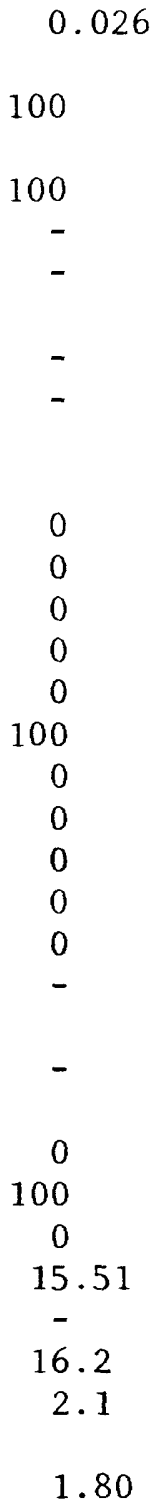

${ }^{1}$ Data from National Oceanic and Atmospheric Administration, 1980 .

${ }^{2}$ Data from Hershfield, 1961.

${ }^{3}$ Data derived as sum of total deposition of ammonia and of nitrate (National Atmospheric Deposition Program, NADP Subcommittee Number 3 -- Data Management and Analysis, 1985). 
Table 274.--Basin characteristics of station 404653111545801

9th West conduit at 536 North at Salt Lake City, Utah

[Dashes indicate data not available. Except where footnoted, data is from magnetic tape described by Driver and others (1985)]

\begin{tabular}{|c|c|}
\hline Characteristic & Value \\
\hline Contributing drainage area, in acres & 150 \\
\hline $\begin{array}{l}\text { Impervious area, in percentage of contributing } \\
\text { drainage area }\end{array}$ & 64 \\
\hline $\begin{array}{l}\text { Effective impervious area, in percentage of } \\
\text { contributing drainage area }\end{array}$ & 39 \\
\hline Average basin slope, in feet per mile & 32 \\
\hline Main conveyance slope, in feet per mile & 11 \\
\hline $\begin{array}{l}\text { Population density, in number of people per } \\
\text { square mile }\end{array}$ & 5,780 \\
\hline $\begin{array}{l}\text { Street density, in miles of lanes per square mile } \\
\text { Percentage of contributing drainage area with the } \\
\text { following land uses: }\end{array}$ & - \\
\hline Rural and pasture & 0 \\
\hline Agricultural & 0 \\
\hline Low-density residential & 0 \\
\hline Medium-density residential & 52 \\
\hline High-density residential & 4 \\
\hline Commercial & 35 \\
\hline Industrial & 0 \\
\hline Construction & 0 \\
\hline Idle or vacant & 5 \\
\hline Wet land & 0 \\
\hline Parkland & 4 \\
\hline Detention storage, in acre-feet & - \\
\hline $\begin{array}{l}\text { Percentage of contributing drainage area upstream of } \\
\text { detention storage }\end{array}$ & - \\
\hline $\begin{array}{l}\text { Percentage of contributing drainage area drained by a } \\
\text { storm-sewer system }\end{array}$ & - \\
\hline Percentage of streets with curb and gutter drainage & - \\
\hline Percentage of streets with ditch and swale drainage & - \\
\hline Mean annual rainfall, in inches ${ }^{1}$ & 15.40 \\
\hline Ten-year, 1-hour rainfall intensity, in inches per hour & 0.94 \\
\hline Mean minimum January temperature, in degrees Fahrenheit ${ }^{1}$ & 18.5 \\
\hline Two-year, 24-hour rainfall, in inches $^{2}$ & 1.4 \\
\hline $\begin{array}{l}\text { Mean annual nitrogen precipitation load, in pounds of } \\
\text { nitrogen per acre }\end{array}$ & 1.00 \\
\hline \multicolumn{2}{|l|}{$\begin{array}{l}{ }^{1} \text { Data from National Oceanic and Atmospheric Administration, } \\
1980 . \\
{ }^{2} \text { Data from Hershfield, } 1961 \text {. } \\
{ }^{3} \text { Data derived as sum of total deposition of ammonia and of } \\
\text { nitrate (National Atmospheric Deposition Program, NADP Sub- } \\
\text { committee Number } 3 \text { - Data Management and Analysis, 1985). }\end{array}$} \\
\hline
\end{tabular}


Table 275.--Basin characteristics of station 404713073273001 Recharge basin at Plainview, N.Y.

[Dashes indicate data not available. Except where footnoted, data is from magnetic tape described by Driver and others (1985)]

\begin{tabular}{|c|c|}
\hline Characteristic & Value \\
\hline Contributing drainage area, in acres & 190 \\
\hline $\begin{array}{l}\text { Impervious area, in percentage of contributing } \\
\text { drainage area }\end{array}$ & 6 \\
\hline $\begin{array}{l}\text { Effective impervious area, in percentage of } \\
\text { contributing drainage area }\end{array}$ & - \\
\hline Average basin slope, in feet per mile & - \\
\hline Main conveyance slope, in feet per mile & - \\
\hline $\begin{array}{l}\text { Population density, in number of people per } \\
\text { square mile }\end{array}$ & - \\
\hline Street density, in miles of lanes per square mile & 27 \\
\hline $\begin{array}{l}\text { ercentage of contributing drainage area with the } \\
\text { following land uses: }\end{array}$ & \\
\hline Rural and pasture & 0 \\
\hline Agricultural & 0 \\
\hline Low-density residential & 0 \\
\hline Medium-density residential & 0 \\
\hline density residential & 0 \\
\hline Com & 100 \\
\hline Industrial & 0 \\
\hline Construction & 0 \\
\hline Idle or vacant & 0 \\
\hline Wetland & 0 \\
\hline Parkland & 0 \\
\hline Detention storage, in acre-feet & - \\
\hline $\begin{array}{l}\text { Percentage of contributing drainage area upstream of } \\
\text { detention storage }\end{array}$ & - \\
\hline $\begin{array}{l}\text { Percentage of contributing drainage area drained by a } \\
\text { storm-sewer system }\end{array}$ & - \\
\hline Percentage of streets with curb and gutter drainage & - \\
\hline Percentage of streets with ditch and swale drainage & - \\
\hline Mean annual rainfall, in inches ${ }^{1}$ & 41.61 \\
\hline $\begin{array}{l}\text { Ten-year, 1-hour rainfall intensity, in inches per hour } \\
\text { Mean minimum January temperature, in degrees Fahrenheit }{ }^{1}\end{array}$ & $-\overline{2} 6.4$ \\
\hline Two-year, 24-hour rainfall, in inches ${ }^{2}$ & 3.4 \\
\hline $\begin{array}{l}\text { lean annual nitrogen precipitation load, in pounds of } \\
\text { nitrogen per acre }\end{array}$ & 4.60 \\
\hline
\end{tabular}

${ }^{1}$ Data from National Oceanic and Atmospheric Administration, 1980 .

${ }^{2}$ Data from Hershfield, 1961.

${ }^{3}$ Data derived as sum of total deposition of ammonia and of nitrate (National Atmospheric Deposition Program, NADP Subcommittee Number 3 -- Data Management and Analysis, 1985). 
Table 276.--Basin characteristics of station 404815073294601 Recharge basin at syosset, N.Y.

[Dashes indicate data not available. Except where footnoted, data is from magnetic tape described by Driver and others $(1985)]$

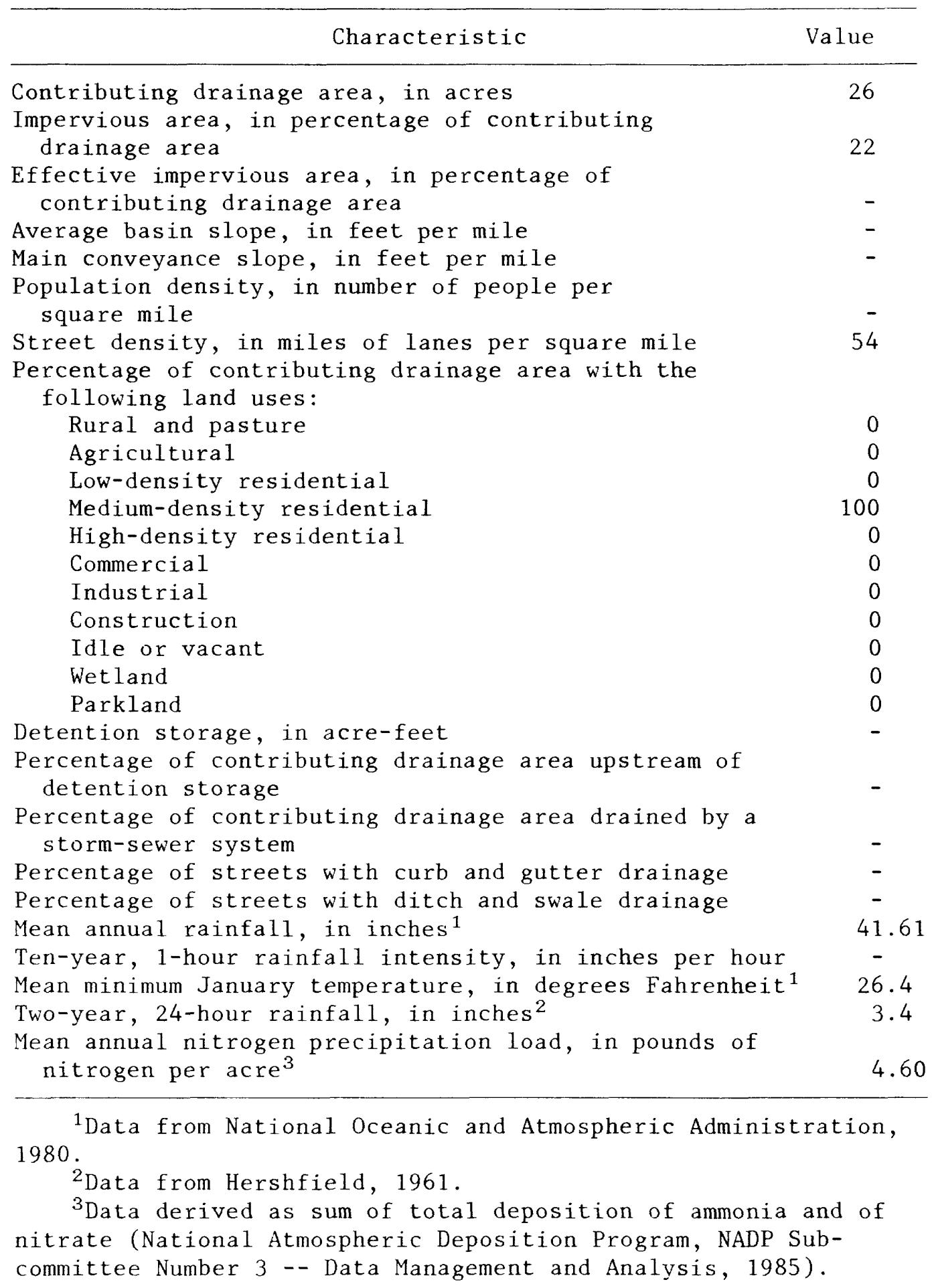


Table 277.--Basin characteristics of station 405124073292601 Recharge basin at Laurel Hollow, N.Y.

[Dashes indicate data not available. Except where footnoted, data is from magnetic tape described by Driver and others $(1985)]$

\begin{tabular}{|c|c|}
\hline Characteristic & Value \\
\hline Contributing drainage area, in acres & 100 \\
\hline $\begin{array}{l}\text { Impervious area, in percentage of contributing } \\
\text { drainage area }\end{array}$ & 5 \\
\hline $\begin{array}{l}\text { Effective impervious area, in percentage of } \\
\text { contributing drainage area }\end{array}$ & - \\
\hline Average basin slope, in feet per mile & - \\
\hline Main conveyance slope, in feet per mile & - \\
\hline $\begin{array}{l}\text { Population density, in number of people per } \\
\text { square mile }\end{array}$ & - \\
\hline Street density, in miles of lanes per square mile & 20 \\
\hline $\begin{array}{l}\text { Percentage of contributing drainage area with the } \\
\text { following land uses: }\end{array}$ & \\
\hline Rural and pasture & 0 \\
\hline Agricultural & 0 \\
\hline Low-density residential & 100 \\
\hline Medium-density residential & 0 \\
\hline High-density residential & 0 \\
\hline Commercial & 0 \\
\hline Industrial & 0 \\
\hline Construction & 0 \\
\hline Idle or vacant & 0 \\
\hline Wetland & 0 \\
\hline Parkland & 0 \\
\hline Detention storage, in acre-feet & - \\
\hline $\begin{array}{l}\text { Percentage of contributing drainage area upstream of } \\
\text { detention storage }\end{array}$ & - \\
\hline $\begin{array}{l}\text { Percentage of contributing drainage area drained by a } \\
\text { storm-sewer system }\end{array}$ & - \\
\hline Percentage of streets with curb and gutter drainage & - \\
\hline Percentage of streets with ditch and swale drainage & - \\
\hline Mean annual rainfall, in inches ${ }^{1}$ & 41.61 \\
\hline Ten-year, 1-hour rainfall intensity, in inches per hour & - \\
\hline Mean minimum January temperature, in degrees Fahrenheit ${ }^{1}$ & 26.4 \\
\hline Two-year, 24-hour rainfall, in inches ${ }^{2}$. & 3.4 \\
\hline $\begin{array}{l}\text { Mean annual nitrogen precipitation load, in pounds of } \\
\text { nitrogen per acre }\end{array}$ & 4.60 \\
\hline
\end{tabular}

${ }^{1}$ Data from National Oceanic and Atmospheric Administration, 1980 .

${ }^{2}$ Data from Hershfield, 1961.

${ }^{3}$ Data derived as sum of total deposition of ammonia and of nitrate (National Atmospheric Deposition Program, NADP Subcommittee Number 3 -- Data Management and Analysis, 1985). 
Table 279.--Basin characteristics of station 415302088033804 Main inlet of Lake Ellyn at Glen Ellyn, III.

[Dashes indicate data not available. Except where footnoted, data is from magnetic tape described by Driver and others (1985)]

\begin{tabular}{|c|c|}
\hline Characteristic & Value \\
\hline Contributing drainage area, in acres & 530 \\
\hline $\begin{array}{l}\text { Impervious area, in percentage of contributing } \\
\text { drainage area }\end{array}$ & 34 \\
\hline $\begin{array}{l}\text { Effective impervious area, in percentage of } \\
\text { contributing drainage area }\end{array}$ & 17 \\
\hline Average basin slope, in feet per mile & 220 \\
\hline Main conveyance slope, in feet per mile & 49 \\
\hline $\begin{array}{l}\text { Population density, in number of people per } \\
\text { square mile }\end{array}$ & 5,000 \\
\hline $\begin{array}{l}\text { Street density, in miles of lanes per square mile } \\
\text { Percentage of contributing drainage area with the } \\
\text { following land uses: }\end{array}$ & 22 \\
\hline Rural and pasture & 0 \\
\hline Agricultural & 0 \\
\hline Low-density residential & 80 \\
\hline Medium-density residential & 0 \\
\hline High-density residential & 3 \\
\hline Commercial & 10 \\
\hline Industrial & 0 \\
\hline Construction & 0 \\
\hline Idle or vacant & 0 \\
\hline Wetland & 2 \\
\hline Parkland & 5 \\
\hline Detention storage, in acre-feet & 0 \\
\hline $\begin{array}{l}\text { Percentage of contributing drainage area upstream of } \\
\text { detention storage }\end{array}$ & 0 \\
\hline Percentage of contributing drainage area drained by a & \\
\hline storm-sewer system & 95 \\
\hline Percentage of streets with curb and gutter drainage & 98 \\
\hline Percentage of streets with ditch and swale drainage & 2 \\
\hline Mean annual rainfall, in inches ${ }^{1}$ & 34.44 \\
\hline Ten-year, 1 -hour rainfall intensity, in inches per hour & 2.0 \\
\hline Mean minimum January temperature, in degrees Fahrenheit ${ }^{1}$ & 17.0 \\
\hline Two-year, 24 -hour rainfall, in inches ${ }^{2}$ & 2.8 \\
\hline $\begin{array}{l}\text { Mean annual nitrogen precipitation load, in pounds of } \\
\text { nitrogen per acre }\end{array}$ & 6.10 \\
\hline
\end{tabular}

${ }^{1}$ Data from National Oceanic and Atmospheric Administration, 1980

2Data from Hershfield, 1961.

${ }^{3}$ Data derived as sum of total deposition of ammonia and of nitrate (National Atmospheric Deposition Program, NADP Subcommittee Number 3 -- Data Management and Analysis, 1985). 
Table 280.--Basin characteristics of station 415311088032906 Submerged outlet of Lake Ellyn at Glen Ellyn, III.

[Dashes indicate data not available. Except where footnoted, data is from magnetic tape described by Driver and others (1985)]

\begin{tabular}{|c|c|}
\hline Characteristic & Value \\
\hline Contributing drainage area, in acres & 530 \\
\hline $\begin{array}{l}\text { Impervious area, in percentage of contributing } \\
\text { drainage area }\end{array}$ & 34 \\
\hline $\begin{array}{l}\text { Effective impervious area, in percentage of } \\
\text { contributing drainage area }\end{array}$ & 17 \\
\hline Average basin slope, in feet per mile & 220 \\
\hline Main conveyance slope, in feet per mile & 49 \\
\hline $\begin{array}{l}\text { Population density, in number of people per } \\
\text { square mile }\end{array}$ & 5,000 \\
\hline $\begin{array}{l}\text { Street density, in miles of lanes per square mile } \\
\text { Percentage of contributing drainage area with the } \\
\text { following land uses: }\end{array}$ & 22 \\
\hline Rural and pasture & 0 \\
\hline Agricultural & 0 \\
\hline Low-density residential & 80 \\
\hline Medium-density residential & 0 \\
\hline High-density residential & 3 \\
\hline Commercial & 10 \\
\hline Industrial & 0 \\
\hline Construction & 0 \\
\hline Idle or vacant & 0 \\
\hline Wetland & 2 \\
\hline Parkland & 5 \\
\hline Detention storage, in acre-feet & 45 \\
\hline $\begin{array}{l}\text { Percentage of contributing drainage area upstream of } \\
\text { detention storage }\end{array}$ & 100 \\
\hline $\begin{array}{l}\text { Percentage of contributing drainage area drained by a } \\
\text { storm-sewer system }\end{array}$ & 95 \\
\hline Percentage of streets with curb and gutter drainage & 98 \\
\hline Percentage of streets with ditch and swale drainage & 2 \\
\hline Mean annual rainfall, in inches ${ }^{1}$ & 34.44 \\
\hline Ten-year, 1-hour rainfall intensity, in inches per hour & 2.0 \\
\hline Mean minimum January temperature, in degrees Fahrenheit ${ }^{1}$ & 17.0 \\
\hline Two-year, 24-hour rainfall, in inches $^{2}$ & 2.8 \\
\hline $\begin{array}{l}\text { Mean annual nitrogen precipitation load, in pounds of } \\
\text { nitrogen per acre }\end{array}$ & 6.10 \\
\hline
\end{tabular}

${ }^{1}$ Data from National Oceanic and Atmospheric Administration, 1980 .

${ }^{2}$ Data from Hershfield, 1961.

${ }^{3}$ Data derived as sum of total deposition of ammonia and of nitrate (National Atmospheric Deposition Program, NADP Subcommittee Number 3 -- Data Management and Analysis, 1985). 
Table 281.--Basin characteristics of station 415311088032907 Surface outlet of Lake Ellyn at Glen Ellyn, III.

[Dashes indicate data not available. Except where footnoted, data is from magnetic tape described by Driver and others (1985)]

\begin{tabular}{|c|c|}
\hline Characteristic & Value \\
\hline Contributing drainage area, in acres & 530 \\
\hline $\begin{array}{l}\text { Impervious area, in percentage of contributing } \\
\text { drainage area }\end{array}$ & 34 \\
\hline $\begin{array}{l}\text { Effective impervious area, in percentage of } \\
\text { contributing drainage area }\end{array}$ & 17 \\
\hline Average basin slope, in feet per mile & 220 \\
\hline Main conveyance slope, in feet per mile & 49 \\
\hline $\begin{array}{l}\text { Population density, in number of people per } \\
\text { square mile }\end{array}$ & 5,000 \\
\hline Street density, in miles of lanes per square mile & 22 \\
\hline $\begin{array}{l}\text { Percentage of contributing drainage area with the } \\
\text { following land uses: }\end{array}$ & \\
\hline and pasture & 0 \\
\hline ultural & 0 \\
\hline Low-density res & 80 \\
\hline Medium-density residential & 0 \\
\hline High-density residential & 3 \\
\hline Comn & 10 \\
\hline Ind & 0 \\
\hline Col & 0 \\
\hline or vacant & 0 \\
\hline Wetland & 2 \\
\hline Parkland & 5 \\
\hline Detention storage, in acre-feet & 45 \\
\hline $\begin{array}{l}\text { Percentage of contributing drainage area upstream of } \\
\text { detention storage }\end{array}$ & 100 \\
\hline $\begin{array}{l}\text { Percentage of contributing drainage area drained by a } \\
\text { storm-sewer system }\end{array}$ & 95 \\
\hline Percentage of streets with curb and gutter drainage & 98 \\
\hline Percentage of streets with ditch and swale drainage & 2 \\
\hline Mean annual rainfall, in inches ${ }^{1}$ & 34.44 \\
\hline Ten-year, 1-hour rainfall intensity, in inches per hour & 2.0 \\
\hline Mean minimum January temperature, in degrees Fahrenheit ${ }^{1}$ & 17.0 \\
\hline Two-year, 24 -hour rainfall, in inches ${ }^{2}$ & 2.8 \\
\hline $\begin{array}{l}\text { Mean annual nitrogen precipitation load, in pounds of } \\
\text { nitrogen per acre }\end{array}$ & 6.10 \\
\hline
\end{tabular}

${ }^{1}$ Data from National Oceanic and Atmospheric Administration, 1980 .

${ }^{2}$ Data from Hershfield, 1961.

${ }^{3}$ Data derived as sum of total deposition of ammonia and of nitrate (National Atmospheric Deposition Program. NADP Subcommittee Number 3 -- Data Management and Analysis, 1985). 
Table 282.--Basin characteristics of station 430403077311500 Tributary to Barge Canal tributary near Pittsford, N.Y.

[Dashes indicate data not available. Except where footnoted, data is from magnetic tape described by Driver and others (1985)]

\begin{tabular}{|c|c|}
\hline Characteristic & Value \\
\hline Contributing drainage area, in acres & 170 \\
\hline $\begin{array}{l}\text { Impervious area, in percentage of contributing } \\
\text { drainage area }\end{array}$ & 22 \\
\hline $\begin{array}{l}\text { Effective impervious area, in percentage of } \\
\text { contributing drainage area }\end{array}$ & 15 \\
\hline Average basin slope, in feet per mile & 174 \\
\hline Main conveyance slope, in feet per mile & 84 \\
\hline $\begin{array}{l}\text { Population density, in number of people per } \\
\text { square mile }\end{array}$ & 3,460 \\
\hline $\begin{array}{l}\text { Street density, in miles of lanes per square mile } \\
\text { Percentage of contributing drainage area with the } \\
\quad \text { following land uses: }\end{array}$ & 33 \\
\hline Rural and pasture & 0 \\
\hline Agricultural & 0 \\
\hline Low-density residential & 100 \\
\hline Medium-density residential & 0 \\
\hline High-density residential & 0 \\
\hline Commercial & 0 \\
\hline Industrial & 0 \\
\hline Construction & 0 \\
\hline Idle or vacant & 0 \\
\hline Wetland & 0 \\
\hline Parkland & 0 \\
\hline Detention storage, in acre-feet & - \\
\hline $\begin{array}{l}\text { Percentage of contributing drainage area upstream of } \\
\text { detention storage }\end{array}$ & - \\
\hline $\begin{array}{l}\text { Percentage of contributing drainage area drained by a } \\
\text { storm-sewer system }\end{array}$ & 90 \\
\hline Percentage of streets with curb and gutter drainage & 75 \\
\hline Percentage of streets with ditch and swale drainage & 25 \\
\hline Mean annual rainfall, in inches ${ }^{1}$ & 31.33 \\
\hline Ten-year, 1 -hour rainfall intensity, in inches per hour & - \\
\hline Mean minimum January temperature, in degrees Fahrenheit ${ }^{1}$ & 16.7 \\
\hline Two-year, 24-hour rainfall, in inches ${ }^{2}$ & 2.4 \\
\hline $\begin{array}{l}\text { Mean annual nitrogen precipitation load, in pounds of } \\
\text { nitrogen per acre }\end{array}$ & 5.00 \\
\hline
\end{tabular}

${ }^{1}$ Data from National Oceanic and Atmospheric Administration, 1980 .

${ }^{2}$ Data from Hershfield, 1961.

${ }^{3}$ Data derived as sum of total deposition of ammonia and of nitrate (National Atmospheric Deposition Program, NADP Subcommittee Number 3 -- Data Management and Analysis, 1985). 
Table 283.--Basin characteristics of station 430428077261100 White Brook tributary near Fairport, N.Y.

[Dashes indicate data not available. Except where footnoted, data is from magnetic tape described by Driver and others (1985)]

\begin{tabular}{|c|c|}
\hline Characteristic & Value \\
\hline Contributing drainage area, in acres & 230 \\
\hline $\begin{array}{l}\text { Impervious area, in percentage of contributing } \\
\text { drainage area }\end{array}$ & 16 \\
\hline $\begin{array}{l}\text { Effective impervious area, in percentage of } \\
\text { contributing drainage area }\end{array}$ & 15 \\
\hline Average basin slope, in feet per mile & 301 \\
\hline Main conveyance slope, in feet per mile & 36 \\
\hline $\begin{array}{l}\text { Population density, in number of people per } \\
\text { square mile }\end{array}$ & 722 \\
\hline $\begin{array}{l}\text { Street density, in miles of lanes per square mile } \\
\text { Percentage of contributing drainage area with the } \\
\text { following land uses: }\end{array}$ & 8 \\
\hline Rural and pasture & 0 \\
\hline Agricultural & 0 \\
\hline Low-density residential & 28 \\
\hline Medium-density residential & 0 \\
\hline High-density residential & 0 \\
\hline Commercial & 37 \\
\hline Industrial & 0 \\
\hline Construction & 0 \\
\hline Idle or vacant & 35 \\
\hline Wetland & 0 \\
\hline Parkland & 0 \\
\hline Detention storage, in acre-feet & - \\
\hline $\begin{array}{l}\text { Percentage of contributing drainage area upstream of } \\
\text { detention storage }\end{array}$ & - \\
\hline $\begin{array}{l}\text { Percentage of contributing drainage area drained by a } \\
\text { storm-sewer system }\end{array}$ & 60 \\
\hline Percentage of streets with curb and gutter drainage & 95 \\
\hline Percentage of streets with ditch and swale drainage & 5 \\
\hline Mean annual rainfall, in inches 1 & 31.33 \\
\hline $\begin{array}{l}\text { Ten-year, 1-hour rainfall intensity, in inches per hour } \\
\text { Mean minimum January temperature, in degrees Fahrenheit }{ }^{1}\end{array}$ & $\overline{16} .7$ \\
\hline Two-year, 24-hour rainfall, in inches ${ }^{2}$ & 2.4 \\
\hline $\begin{array}{l}\text { Mean annual nitrogen precipitation load, in pounds of } \\
\text { nitrogen per acre }\end{array}$ & 5.00 \\
\hline \multicolumn{2}{|c|}{$\begin{array}{l}{ }^{1} \text { Data from National Oceanic and Atmospheric Administration, } \\
1980 . \\
{ }^{2} \text { Data from Hershfield, } 1961 \text {. } \\
{ }^{3} \text { Data derived as sum of total deposition of ammonia and of } \\
\text { nitrate (National Atmospheric Deposition Program, NADP Sub- } \\
\text { committee Number } 3 \text {-- Data Management and Analysis, 1985). }\end{array}$} \\
\hline
\end{tabular}


Table 284.--Basin characteristics of station 430649077285500 Irondequoit Creek tributary at East Rochester, N.Y.

[Dashes indicate data not available. Except where footnoted, data is from magnetic tape described by Driver and others (1985)]

Characteristic

Contributing drainage area, in acres

Impervious area, in percentage of contributing drainage area

Effective impervious area, in percentage of contributing drainage area

Average basin slope, in feet per mile

Main conveyance slope, in feet per mile

Population density, in number of people per square mile

Street density, in miles of lanes per square mile

Percentage of contributing drainage area with the

following land uses:

Rural and pasture

Agricultural

Low-density residential

Medium-density residential

High-density residential

Commercial

Industrial

Construction

Idle or vacant

Wet land

Parkland

Detention storage, in acre-feet

Percentage of contributing drainage area upstream of detention storage

Percentage of contributing drainage area drained by a storm-sewer system

Percentage of streets with curb and gutter drainage

Percentage of streets with ditch and swale drainage Mean annual rainfall, in inches ${ }^{1}$

Ten-year, 1-hour rainfall intensity, in inches per hour

Mean minimum January temperature, in degrees Fahrenheit ${ }^{1}$

Two-year, 24-hour rainfall, in inches ${ }^{2}$

Mean annual nitrogen precipitation load, in pounds of nitrogen per acre ${ }^{3}$
Value

360

38

17

58

15

11,400

42

0

0

0

88

12

0

0

0

0

0

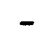

100

90

10

31.33

16.7

2.4

5.00

${ }^{1}$ Data from National Oceanic and Atmospheric Administration, 1980

${ }^{2}$ Data from Hershfield, 1961.

${ }^{3}$ Data derived as sum of total deposition of ammonia and of nitrate (National Atmospheric Deposition Program, NADP Subcommittee Number 3 -- Data Management and Analysis, 1985). 
Table 285.--Basin characteristics of station 445032092552801 Iverson Avenue storm sewer in Cottage Grove, Minn.

[Dashes indicate data not available. Except where footnoted, data is from magnetic tape described by Driver and others (1985)]

Characteristic

Value

Contributing drainage area, in acres 96

Impervious area, in percentage of contributing drainage area

Effective impervious area, in percentage of contributing drainage area

Average basin slope, in feet per mile

Main conveyance slope, in feet per mile

Population density, in number of people per square mile

Street density, in miles of lanes per square mile

Percentage of contributing drainage area with the following land uses:

Rural and pasture

Agricultura 1

Low-density residential

Medium-density residential

High-density residential

Commercial

Industrial

Construction

Idle or vacant

Wetland

Parkland

Detention storage, in acre-feet

Percentage of contributing drainage area upstream of detention storage

Percentage of contributing drainage area drained by a storm-sewer system

Percentage of streets with curb and gutter drainage

Percentage of streets with ditch and swale drainage

Mean annual rainfall, in inches ${ }^{1}$

Ten-year, 1-hour rainfall intensity, in inches per hour

0

0

2

14

Mean minimum January temperature, in degrees Fahrenheit ${ }^{1}$

0

Two-year, 24-hour rainfall, in inches ${ }^{2}$

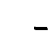

Mean annual nitrogen precipitation load, in pounds of nitrogen per acre ${ }^{3}$

${ }^{1}$ Data from National Oceanic and Atmospheric Administration, 1980.

${ }^{2}$ Data from Hershfield, 1961.

${ }^{3}$ Data derived as sum of total deposition of ammonia and of nitrate (National Atmospheric Deposition Program, NADP Subcommittee Number 3 -- Data Management and Analysis, 1985). 
Table 286.--Basin characteristics of station 445210093271701

Valley View Road storm sewer in Eden Prairie, Minn.

[Dashes indicate data not available. Except where footnoted, data is from magnetic tape described by Driver and others (1985)]

Characteristic

Value

Contributing drainage area, in acres 83

Impervious area, in percentage of contributing drainage area

Effective impervious area, in percentage of contributing drainage area

Average basin slope, in feet per mile

Main conveyance slope, in feet per mile

103

Population density, in number of people per

square mile

Street density, in miles of lanes per square mile

Percentage of contributing drainage area with the following land uses:

Rural and pasture

Agricultural

Low-density residential

Medium-density residential

High-density residential

Commercial

Industrial

Construction

Idle or vacant

Wet 1 and

Parkland

Detention storage, in acre-feet

Percentage of contributing drainage area upstream of detention storage

Percentage of contributing drainage area drained by a storm-sewer system

Percentage of streets with curb and gutter drainage Percentage of streets with ditch and swale drainage Mean annual rainfall, in inches ${ }^{1}$

Ten-year, 1-hour rainfall intensity, in inches per hour

Mean minimum January temperature, in degrees Fahrenheit ${ }^{1}$

Two-year, 24-hour rainfall, in inches ${ }^{2}$

Mean annual nitrogen precipitation load, in pounds of nitrogen per acre ${ }^{3}$ 1980 .

${ }^{1}$ Data from National Oceanic and Atmospheric Administration,

${ }^{2}$ Data from Hershfield, 1961.

${ }^{3}$ Data derived as sum of total deposition of ammonia and of nitrate (National Atmospheric Deposition Program, NADP Subcommittee Number 3 -- Data Management and Analysis, 1985). 
Table 287.--Basin characteristics of station 445937093230701 Wesley Park storm sewer in Golden Valley, Minn.

[Dashes indicate data not available. Except where footnoted, data is from magnetic tape described by Driver and others (1985)]

\begin{tabular}{|c|c|}
\hline Characteristic & Value \\
\hline ontributing drainage area, in acres & 210 \\
\hline $\begin{array}{l}\text { Impervious area, in percentage of contributing } \\
\text { drainage area }\end{array}$ & 22 \\
\hline $\begin{array}{l}\text { Effective impervious area, in percentage of } \\
\text { contributing drainage area }\end{array}$ & 15 \\
\hline Average basin slope, in feet per mile & 60 \\
\hline Main conveyance slope, in feet per mile & 43 \\
\hline $\begin{array}{l}\text { Population density, in number of people per } \\
\text { square mile }\end{array}$ & 6 \\
\hline $\begin{array}{l}\text { Street density, in miles of lanes per square mile } \\
\text { Percentage of contributing drainage area with the } \\
\text { following land uses: }\end{array}$ & - \\
\hline Rural and pasture & 6 \\
\hline Agricultural & 0 \\
\hline Low-density residential & 0 \\
\hline Medium-density residential & 83 \\
\hline High-density residential & 0 \\
\hline Commercial & 0 \\
\hline Industrial & 0 \\
\hline Construction & 0 \\
\hline Idle or vacant & 0 \\
\hline Wetland & 4 \\
\hline Parkland & 7 \\
\hline Detention storage, in acre-feet & - \\
\hline $\begin{array}{l}\text { Percentage of contributing drainage area upstream of } \\
\text { detention storage }\end{array}$ & - \\
\hline $\begin{array}{l}\text { Percentage of contributing drainage area drained by a } \\
\text { storm-sewer system }\end{array}$ & - \\
\hline Percentage of streets with curb and gutter drainage & - \\
\hline Percentage of streets with ditch and swale drainage & - \\
\hline lean annual rainfall, in inches ${ }^{1}$ & 30.00 \\
\hline $\begin{array}{l}\text { Ten-year, 1-hour rainfall intensity, in inches per hour } \\
\text { Mean minimum January temperature, in degrees Fahrenheit }{ }^{1}\end{array}$ & - \\
\hline Two-year, 24-hour rainfall, in inches ${ }^{2}$ & 2.8 \\
\hline $\begin{array}{l}\text { ean annual nitrogen } \\
\text { nitrogen per acre }\end{array}$ & \\
\hline
\end{tabular}

${ }^{1}$ Data from National Oceanic and Atmospheric Administration, 1980.

${ }^{2}$ Data from Hershfield, 1961.

${ }^{3}$ Data derived as sum of total deposition of ammonia and of nitrate (National Atmospheric Deposition Program, NADP Subcommittee Number 3 -- Data Management and Analysis, 1985). 
Table 288.--Basin characteristics of station 450011093221901

Sandberg Avenue storm sewer in Golden Valley, Minn.

[Dashes indicate data not available. Except where footnoted, data is from magnetic tape described by Driver and others (1985)]

\begin{tabular}{|c|c|}
\hline Characteristic & Value \\
\hline Contributing drainage area, in acres & 77 \\
\hline $\begin{array}{l}\text { Impervious area, in percentage of contributing } \\
\text { drainage area }\end{array}$ & 70 \\
\hline $\begin{array}{l}\text { Effective impervious area, in percentage of } \\
\text { contributing drainage area }\end{array}$ & 54 \\
\hline Average basin slope, in feet per mile & 45 \\
\hline Main conveyance slope, in feet per mile & 34 \\
\hline $\begin{array}{l}\text { Population density, in number of people per } \\
\text { square mile }\end{array}$ & 0 \\
\hline $\begin{array}{l}\text { Street density, in miles of lanes per square mile } \\
\text { Percentage of contributing drainage area with the } \\
\text { following land uses: }\end{array}$ & - \\
\hline Rural and pasture & 0 \\
\hline Agricultural & 6 \\
\hline Low-density residential & 10 \\
\hline Medium-density residential & 0 \\
\hline High-density residential & 0 \\
\hline Commercial & 84 \\
\hline Industrial & 0 \\
\hline Construction & 0 \\
\hline Idle or vacant & 0 \\
\hline Wetland & 0 \\
\hline Parkland & 0 \\
\hline Detention storage, in acre-feet & - \\
\hline $\begin{array}{l}\text { Percentage of contributing drainage area upstream of } \\
\text { detention storage }\end{array}$ & - \\
\hline $\begin{array}{l}\text { Percentage of contributing drainage area drained by a } \\
\text { storm-sewer system }\end{array}$ & - \\
\hline Percentage of streets with curb and gutter drainage & - \\
\hline Percentage of streets with ditch and swale drainage & - \\
\hline Mean annual rainfall, in inches 1 & 30.00 \\
\hline Ten-year, 1 -hour rainfall intensity, in inches per hour & - \\
\hline Mean minimum January temperature, in degrees Fahrenheit ${ }^{1}$ & 3.2 \\
\hline Two-year, 24 -hour rainfall, in inches ${ }^{2}$ & 2.8 \\
\hline $\begin{array}{l}\text { Mean annual nitrogen precipitation load, in pounds of } \\
\text { nitrogen per acre }\end{array}$ & 4.50 \\
\hline
\end{tabular}

\footnotetext{
${ }^{1}$ Data from National Oceanic and Atmospheric Administration, 1980.

${ }^{2}$ Data from Hershfield, 1961.

${ }^{3}$ Data derived as sum of total deposition of ammonia and of nitrate (National Atmospheric Deposition Program, NADP Subcommittee Number 3 -- Data Management and Analysis, 1985).
} 
Table 289.--Basin characteristics of station 450100093205501 State Highway 100 at Scott Avenue storm sewer in Golden Valley, Minn.

[Dashes indicate data not available. Except where footnoted, data is from magnetic tape described by Driver and others (1985)]

\begin{tabular}{|c|c|}
\hline Characteristic & Value \\
\hline Contributing drainage area, in acres & 300 \\
\hline $\begin{array}{l}\text { Impervious area, in percentage of contributing } \\
\text { drainage area }\end{array}$ & 35 \\
\hline $\begin{array}{l}\text { Effective impervious area, in percentage of } \\
\text { contributing drainage area }\end{array}$ & 26 \\
\hline Average basin slope, in feet per mile & 45 \\
\hline Main conveyance slope, in feet per mile & 85 \\
\hline $\begin{array}{l}\text { Population density, in number of people per } \\
\text { square mile }\end{array}$ & 6 \\
\hline Street density, in miles of lanes per square mile & - \\
\hline $\begin{array}{l}\text { Percentage of contributing drainage area with the } \\
\text { following land uses: }\end{array}$ & \\
\hline Rural and pasture & 0 \\
\hline Agricultural & 0 \\
\hline Low-density residential & 0 \\
\hline Medium-density residential & 80 \\
\hline High-density residential & 0 \\
\hline Commercial & 19 \\
\hline Industrial & 0 \\
\hline Construction & 1 \\
\hline Idle or vacant & 0 \\
\hline Wet 1 and & 0 \\
\hline Parkland & 0 \\
\hline Detention storage, in acre-feet & - \\
\hline $\begin{array}{l}\text { Percentage of contributing drainage area upstream of } \\
\text { detention storage }\end{array}$ & - \\
\hline $\begin{array}{l}\text { Percentage of contributing drainage area drained by a } \\
\text { storm-sewer system }\end{array}$ & - \\
\hline Percentage of streets with curb and gutter drainage & - \\
\hline Percentage of streets with ditch and swale drainage & - \\
\hline Mean annual rainfall, in inches 1 & 30.00 \\
\hline Ten-year, 1 -hour rainfall intensity, in inches per hour & - \\
\hline Mean minimum January temperature, in degrees Fahrenheit ${ }^{1}$ & 3.2 \\
\hline Two-year, 24-hour rainfall, in inches ${ }^{2}$ & 2.8 \\
\hline $\begin{array}{l}\text { Mean annual nitrogen precipitation load, in pounds of } \\
\text { nitrogen per acre }{ }^{3}\end{array}$ & 4.50 \\
\hline
\end{tabular}
1980.

${ }^{1}$ Data from National Oceanic and Atmospheric Administration,

${ }^{2}$ Data from Hershfield, 1961.

${ }^{3}$ Data derived as sum of total deposition of ammonia and of nitrate (National Atmospheric Deposition Program, NADP Subcommittee Number 3 -- Data Management and Analysis, 1985). 
Table 290.--Basin characteristics of station 450541093201201 Estates Drive storm sewer in Brooklyn Park, Minn.

[Dashes indicate data not available. Except where footnoted, data is from magnetic tape described by Driver and others (1985)]

\begin{tabular}{|c|c|}
\hline Characteristic & Value \\
\hline Contributing drainage area, in acres & 140 \\
\hline $\begin{array}{l}\text { Impervious area, in percentage of contributing } \\
\text { drainage area }\end{array}$ & 29 \\
\hline $\begin{array}{l}\text { Effective impervious area, in percentage of } \\
\text { contributing drainage area }\end{array}$ & 17 \\
\hline Average basin slope, in feet per mile & 16 \\
\hline Main conveyance slope, in feet per mile & 23 \\
\hline $\begin{array}{l}\text { Population density, in number of people per } \\
\text { square mile }\end{array}$ & 7 \\
\hline $\begin{array}{l}\text { Street density, in miles of lanes per square mile } \\
\text { Percentage of contributing drainage area with the } \\
\text { following land uses: }\end{array}$ & - \\
\hline Rural and pasture & 0 \\
\hline Agricultural & 0 \\
\hline Low-density residential & 3 \\
\hline ity residential & 94 \\
\hline High-density residential & 0 \\
\hline Commercial & 3 \\
\hline Industrial & 0 \\
\hline Construction & 0 \\
\hline Idle or vacant & 0 \\
\hline Wet 1 and & 0 \\
\hline Parkland & 0 \\
\hline Detention storage, in acre-feet & - \\
\hline $\begin{array}{l}\text { Percentage of contributing drainage area upstream of } \\
\text { detention storage }\end{array}$ & - \\
\hline $\begin{array}{l}\text { Percentage of contributing drainage area drained by a } \\
\text { storm-sewer system }\end{array}$ & - \\
\hline Percentage of streets with curb and gutter drainage & - \\
\hline Percentage of streets with ditch and swale drainage & - \\
\hline Mean annual rainfall, in inches ${ }^{1}$ & 30 . \\
\hline Ten-year, 1-hour rainfall intensity, in inches per hour & - \\
\hline Mean minimum January temperature, in degrees Fahrenheit ${ }^{1}$ & 3.2 \\
\hline Two-year, 24 -hour rainfall, in inches ${ }^{2}$ & 2.8 \\
\hline $\begin{array}{l}\text { Mean annual nitrogen precipitation load, in pounds of } \\
\text { nitrogen per acre }\end{array}$ & 4.5 \\
\hline
\end{tabular}

${ }^{1}$ Data from National Oceanic and Atmospheric Administration, 1980 .

${ }^{2}$ Data from Hershfield, 1961.

${ }^{3}$ Data derived as sum of total deposition of ammonia and of nitrate (National Atmospheric Deposition Program, NADP Subcommittee Number 3 -- Data Management and Analysis, 1985). 
Table 291.--Basin characteristics of station 450545093211501 Yates Avenue storm sewer in Brooklyn Park, Minn.

[Dashes indicate data not available. Except where footnoted, data is from magnetic tape described by Driver and others (1985)]

\begin{tabular}{|c|c|}
\hline Characteristic & Value \\
\hline Contributing drainage area, in acres & 220 \\
\hline $\begin{array}{l}\text { Impervious area, in percentage of contributing } \\
\text { drainage area }\end{array}$ & 23 \\
\hline $\begin{array}{l}\text { Effective impervious area, in percentage of } \\
\text { contributing drainage area }\end{array}$ & 13 \\
\hline Average basin slope, in feet per mile & 7 \\
\hline Main conveyance slope, in feet per mile & 10 \\
\hline $\begin{array}{l}\text { Population density, in number of people per } \\
\text { square mile }\end{array}$ & 7 \\
\hline Street density, in miles of lanes per square mile & - \\
\hline $\begin{array}{l}\text { Percentage of contributing drainage area with the } \\
\text { following land uses: }\end{array}$ & \\
\hline Rural and pasture & 18 \\
\hline Agricultural & 17 \\
\hline Low-density residential & 2 \\
\hline Medium-density residential & 52 \\
\hline High-density residential & 0 \\
\hline Commercial & 2 \\
\hline Industrial & 0 \\
\hline Construction & 7 \\
\hline Idle or vacant & 2 \\
\hline Wetland & 0 \\
\hline Parkland & 0 \\
\hline Detention storage, in acre-feet & - \\
\hline $\begin{array}{l}\text { Percentage of contributing drainage area upstream of } \\
\text { detention storage }\end{array}$ & - \\
\hline $\begin{array}{l}\text { Percentage of contributing drainage area drained by a } \\
\text { storm-sewer system }\end{array}$ & - \\
\hline Percentage of streets with curb and gutter drainage & - \\
\hline Percentage of streets with ditch and swale drainage & - \\
\hline Mean annual rainfall, in inches ${ }^{1}$ & 29.00 \\
\hline Ten-year, 1-hour rainfall intensity, in inches per hour & - \\
\hline Mean minimum January temperature, in degrees Fahrenheit ${ }^{1}$ & 3.2 \\
\hline Two-year, 24-hour rainfall, in inches ${ }^{2}$ & 2.8 \\
\hline $\begin{array}{l}\text { Mean annual nitrogen precipitation load, in pounds of } \\
\text { nitrogen per acre }\end{array}$ & 4.50 \\
\hline
\end{tabular}
1980

${ }^{1}$ Data from National Oceanic and Atmospheric Administration,

${ }^{2}$ Data from Hershfield, 1961.

${ }^{3}$ Data derived as sum of total deposition of ammonia and of nitrate (National Atmospheric Deposition Program, NADP Subcommittee Number 3 -- Data Management and Analysis, 1985). 
Table 292.--Basin characteristics of station 473542122083001 148th Avenue Southeast detention-basin number 3 outlet at Bellevue, Wash.

[Dashes indicate data not available. Except where footnoted, data is from magnetic tape described by Driver and others (1985)]

\begin{tabular}{|c|c|}
\hline Characteristic & Value \\
\hline Contributing drainage area, in acres & 17 \\
\hline $\begin{array}{l}\text { Impervious area, in percentage of contributing } \\
\text { drainage area }\end{array}$ & 50 \\
\hline $\begin{array}{l}\text { Effective impervious area, in percentage of } \\
\text { contributing drainage area }\end{array}$ & 35 \\
\hline Average basin slope, in feet per mile & 83 \\
\hline Main conveyance slope, in feet per mile & 113 \\
\hline $\begin{array}{l}\text { Population density, in number of people per } \\
\text { square mile }\end{array}$ & 4,200 \\
\hline $\begin{array}{l}\text { Street density, in miles of lanes per square mile } \\
\text { Percentage of contributing drainage area with the } \\
\text { following land uses: }\end{array}$ & 72 \\
\hline Rural and pasture & 0 \\
\hline Agricultural & 0 \\
\hline Low-density residential & 0 \\
\hline Medium-density residential & 2 \\
\hline High-density residential & 15 \\
\hline Commercial & 15 \\
\hline Indust & 0 \\
\hline Construction & 3 \\
\hline Idle or vacant & 0 \\
\hline Wetland & 0 \\
\hline Parkland & 65 \\
\hline Detention storage, in acre-feet & 0 \\
\hline $\begin{array}{l}\text { Percentage of contributing drainage area upstream of } \\
\text { detention storage }\end{array}$ & - \\
\hline $\begin{array}{l}\text { Percentage of contributing drainage area drained by a } \\
\text { storm-sewer system }\end{array}$ & 100 \\
\hline Percentage of streets with curb and gutter drainage & 100 \\
\hline Percentage of streets with ditch and swale drainage & 0 \\
\hline Mean annual rainfall, in inches 1 & 37.21 \\
\hline Ten-year, 1-hour rainfall intensity, in inches per hour & 0.5 \\
\hline Mean minimum January temperature, in degrees Fahrenheit ${ }^{1}$ & 33.9 \\
\hline Two-year, 24-hour rainfall, in inches ${ }^{2}$ & 2.0 \\
\hline $\begin{array}{l}\text { Mean annual nitrogen precipitation load, in pounds of } \\
\text { nitrogen per acre }\end{array}$ & 0.94 \\
\hline
\end{tabular}

${ }^{1}$ Data from National Oceanic and Atmospheric Administration, 1980 .

${ }^{2}$ Data from Hershfield, 1961.

${ }^{3}$ Data derived as sum of total deposition of ammonia and of nitrate (National Atmospheric Deposition Program, NADP Subcommittee Number 3 -- Data Management and Analysis, 1985). 
Table 293.--Basin characteristics of station 473544122083001 148th Avenue Southeast detention-basin number 5 outlet at Bellevue, Wash.

[Dashes indicate data not available. Except where footnoted, data is from magnetic tape described by Driver and others (1985)]

\begin{tabular}{|c|c|}
\hline Characteristic & Value \\
\hline Contributing drainage area, in acres & 17 \\
\hline $\begin{array}{l}\text { Impervious area, in percentage of contributing } \\
\text { drainage area }\end{array}$ & 50 \\
\hline $\begin{array}{l}\text { Effective impervious area, in percentage of } \\
\text { contributing drainage area }\end{array}$ & 35 \\
\hline Average basin slope, in feet per mile & 83 \\
\hline Main conveyance slope, in feet per mile & 113 \\
\hline $\begin{array}{l}\text { Population density, in number of people per } \\
\text { square mile }\end{array}$ & 4,200 \\
\hline Street density, in miles of lanes per square mile & 72 \\
\hline $\begin{array}{l}\text { Percentage of contributing drainage area with the } \\
\text { following land uses: }\end{array}$ & \\
\hline Rural and pasture & 0 \\
\hline Agricultural & 0 \\
\hline Low-density residential & 0 \\
\hline Medium-density residential & 2 \\
\hline High-density residential & 15 \\
\hline Commercial & 15 \\
\hline Industrial & 0 \\
\hline Construction & 3 \\
\hline Idle or vacant & 0 \\
\hline Wetland & 0 \\
\hline Parkland & 65 \\
\hline Detention storage, in acre-feet & 0 \\
\hline $\begin{array}{l}\text { Percentage of contributing drainage area upstream of } \\
\text { detention storage }\end{array}$ & 100 \\
\hline $\begin{array}{l}\text { Percentage of contributing drainage area drained by a } \\
\text { storm-sewer system }\end{array}$ & 100 \\
\hline Percentage of streets with curb and gutter drainage & 100 \\
\hline Percentage of streets with ditch and swale drainage & 0 \\
\hline Mean annual rainfall, in inches 1 & 37.21 \\
\hline Ten-year, 1-hour rainfall intensity, in inches per hour & 0.51 \\
\hline Mean minimum January temperature, in degrees Fahrenheit ${ }^{1}$ & 33.9 \\
\hline Two-year, 24-hour rainfall, in inches ${ }^{2}$ & 2.0 \\
\hline $\begin{array}{l}\text { Mean annual nitrogen precipitation load, in pounds of } \\
\text { nitrogen per acre }\end{array}$ & 0.94 \\
\hline
\end{tabular}
1980 .

${ }^{1}$ Data from National Oceanic and Atmospheric Administration,

${ }^{2}$ Data from Hershfield, 1961.

${ }^{3}$ Data derived as sum of total deposition of ammonia and of nitrate (National Atmospheric Deposition Program, NADP Subcommittee Number 3 -- Data Management and Analysis, 1985). 